\title{
WestVirginiaUniversity
}

THE RESEARCH REPOSITORY @ WVU

Graduate Theses, Dissertations, and Problem Reports

2000

\section{Contrawound toroidal helical antenna modeling using the FDTD method}

\author{
Khaled Mohammad ElSherbini \\ West Virginia University
}

Follow this and additional works at: https://researchrepository.wvu.edu/etd

\section{Recommended Citation}

EISherbini, Khaled Mohammad, "Contrawound toroidal helical antenna modeling using the FDTD method" (2000). Graduate Theses, Dissertations, and Problem Reports. 2616.

https://researchrepository.wvu.edu/etd/2616

This Dissertation is protected by copyright and/or related rights. It has been brought to you by the The Research Repository @ WVU with permission from the rights-holder(s). You are free to use this Dissertation in any way that is permitted by the copyright and related rights legislation that applies to your use. For other uses you must obtain permission from the rights-holder(s) directly, unless additional rights are indicated by a Creative Commons license in the record and/ or on the work itself. This Dissertation has been accepted for inclusion in WVU Graduate Theses, Dissertations, and Problem Reports collection by an authorized administrator of The Research Repository @ WVU.

For more information, please contact researchrepository@mail.wvu.edu. 


\title{
Contrawound Toroidal Helical Antenna Modeling Using the FDTD Method
}

\author{
Khaled Mohammad ElSherbini
}

\author{
Dissertation submitted to the \\ College of Engineering and Mineral Resources \\ At West Virginia University \\ in partial fulfillment of the requirements \\ for the degree of
}

Doctor of Philosophy

in

Engineering

\author{
James E. Smith, Ph.D., Chairman \\ Roy Nutter, Ph.D. \\ Lawrence Hornak, Ph.D. \\ Michael Palmer, Ph.D. \\ Gregory Thompson, Ph.D.
}

Morgantown, WV

2000

Keywords: PCB-CTHA, FDTD, Antenna Modeling, Feed Study 


\title{
Contrawound Toroidal Helical Antenna Modeling Using the FDTD Method
}

\section{By: Khaled Mohammad EISherbini}

\begin{abstract}
The contrawound toroidal helical antenna (CTHA) is a patented antenna developed at the Center for Industrial Research Applications (CIRA) of West Virginia University. In this study, a computer numerical model was built to simulate the behavior of printed circuit board versions of the CTHA. The numerical model was built upon the finite difference time domain method (FDTD). The results were compared to results from physical antennas, obtained from the CIRA anechoic chamber located in the WVU hanger, to verify the validity of the results of the numerical model. The results from both the numerical and experimental models were also used to reach an understanding of the general performance of the PCB-CTHA and its behavior under different feed configurations.

In order to understand the behavior of the antenna under different feed configurations, a feed study was conducted to check for all the possible feeds of a PCBCTHA. These were found to be thirteen different feeds and were named feeds 1 through 13. Thorough numerical and experimental analysis was done on all thirteen feeds for both understanding the behavior of the antenna under these different feeds, and also comparing the predictions of the numerical model to the data obtained from the anechoic chamber.
\end{abstract}

It was found that the reactance curves can be predicted to an accuracy of $3 \%$ or better by the developed numerical model. The far field gains, on the other hand, are far from being reliably predicted by the model. The study also found that significant differences in the behavior of the PCB-CTHA occur when the feed configuration is changed, and three different feed configurations were recommended for future consideration, feeds 7,12 , and 13 . 


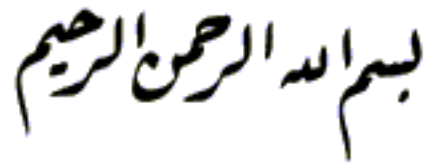

\section{To my brothers in Chechnya}

May Allah give you victory

In this world and in the Hereafter 


\section{Acknowledgments}

I would like to thank the members of the advisory committee, Drs. Roy Nutter, Lawrence Hornak, Michael Palmer, Gregory Thompson, and James Smith for agreeing to be on my committee and for their assistance and critical comments. I express my great appreciation to Dr. James Smith for providing an excellent environment for the research, for his continuous encouragement and support, and for always being available when I needed him. Thanks also to the Center of Industrial Research Applications' members and graduate students, especially Ahmed ElSherbini, my younger brother and dearest friend, and Robert Craven who has been of great help to me with his continuous support, advice, and information.

I would also like to sincerely thank all my brothers at the Islamic Center whom have made these years of my life unforgettable. Without all your love and support life in Morgantown would have never been so pleasant. Special thanks to Drs. Hussein Ghazi, Hany Ammar, and Ahmed Gomaa, my elder brothers whom I highly appreciate and highly respect.

To my wife Magy, without your support and encouragement I would have never made it to the end. You have sacrificed your time and health for me, and I can never reward you enough for all that you have done. All my love and wishes to my three daughters, Mariam, Sarah, and Esraa, and to my brother Ahmed, and my sister Heba.

I owe this work to my father, Mohammad, and my mother, Zeinab. Although I can't thank you enough for all what you did, I would like to express my sincere gratitude and deep love and respect to you both.

All praise is due to Allah, al7amdu lillah, The Creator, The Sustainer, The Compassionate, The Merciful. I witness that there is no God but Allah, and that Mohammad, peace and blessings of Allah be upon him, is Allah's Messenger and Prophet.

\section{Sob7anak Allahoma wa bi7amdika, ashhado alla ilaha illa anta, astaghfiroka wa atoobo ilayk.}




\section{Contents}

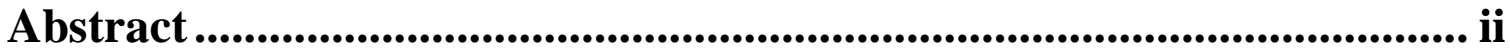

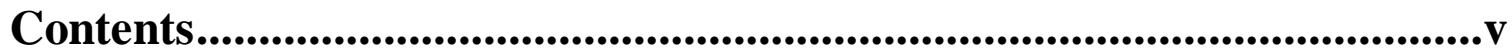

List of Tables.....................................................................................................

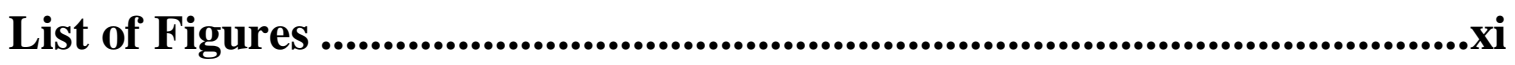

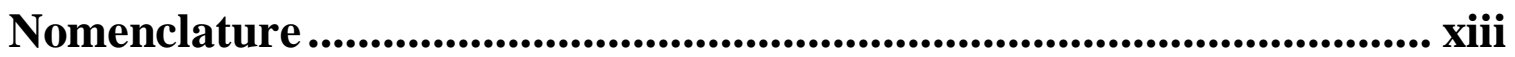

1. Introduction to Antennas..............................................................1

1.1. Fundamentals of Electromagnetics............................................................... 1

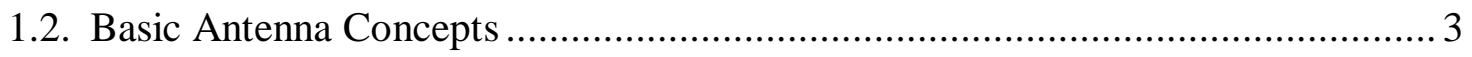

1.2.1. Transmission Lines and Resonators ....................................................... 3

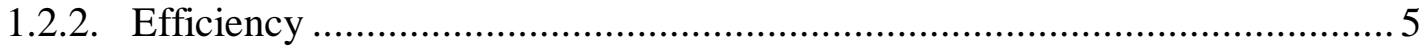

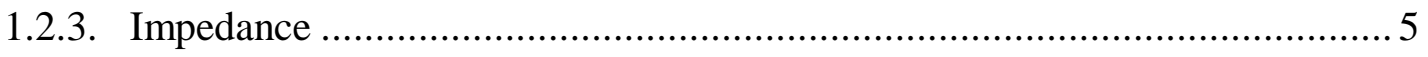

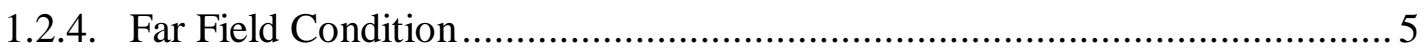

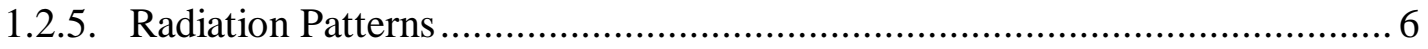

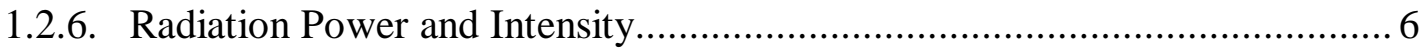

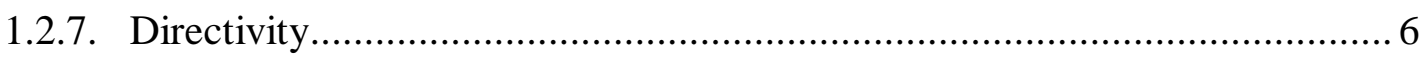

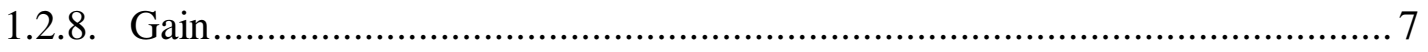

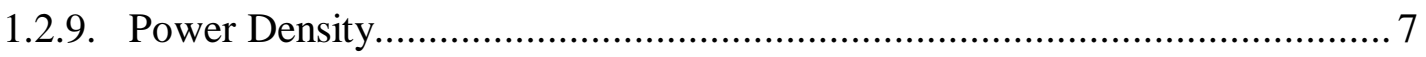

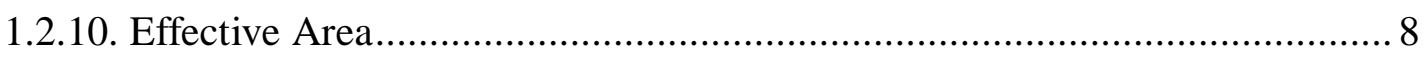

1.2.11. The Aperture Concept ............................................................................ 8

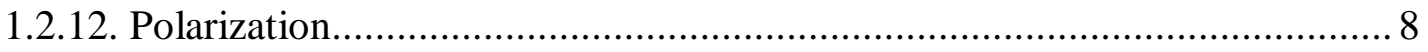

2. The Contrawound Toroidal Helical Antenna ......................................10

2.1. History of the Toroidal Helical Antenna …….................................................... 10

2.2. The Contrawound Toroidal Helical Antenna........................................................ 11

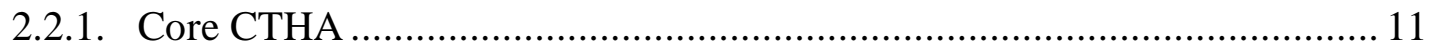

2.2.2. The Printed Circuit Board CTHA............................................................ 12

2.3. Advantages of the CTHA............................................................................ 12 


\section{Research Objective...................................................................................14}

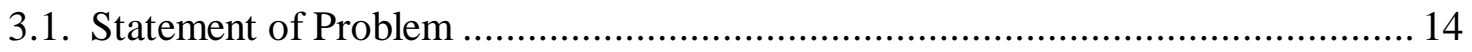

3.2. Create a PCB-CTHA Finite Difference Time Domain Model: ........................... 14

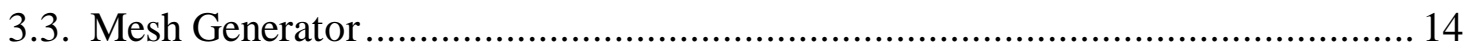

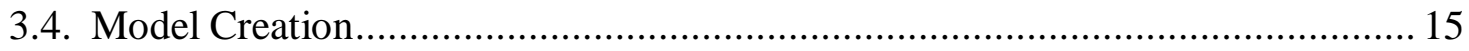

3.5. Practical Application: The Feed Study …………........................................... 15

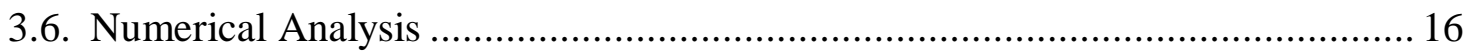

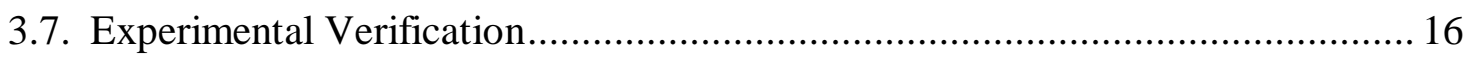

\section{The PCB-CTHA Geometry and Feed Study .....................................17}

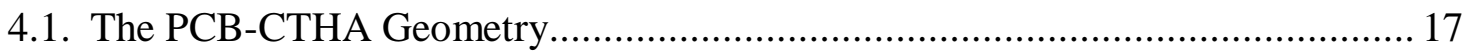

4.2. PCB-CTHA Feed Study................................................................................ 20

4.2.1. CTHA Feed Points Analysis ............................................................... 20

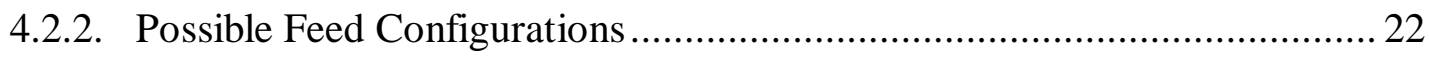

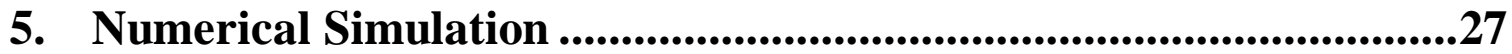

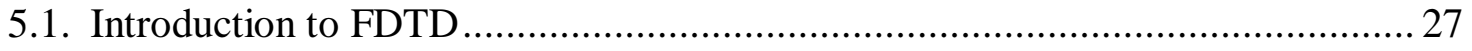

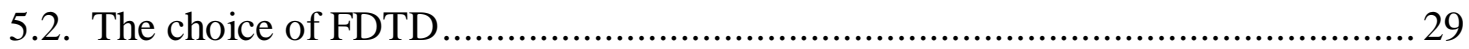

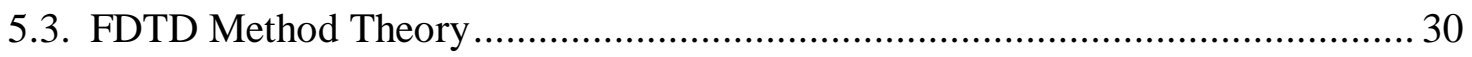

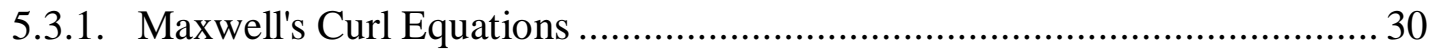

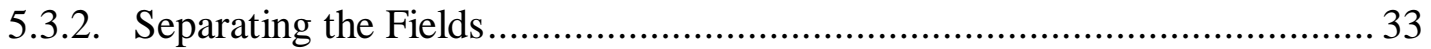

5.3.3. The Finite Difference Method .................................................................... 36

5.3.4. FDTD Formulation of the Scattering Equations for Lossy Materials ........... 37

5.3.5. FDTD Formulation of Scattering Equations for a Perfect Conductor ........... 38

5.4. Related Research Using FDTD ........................................................................ 39

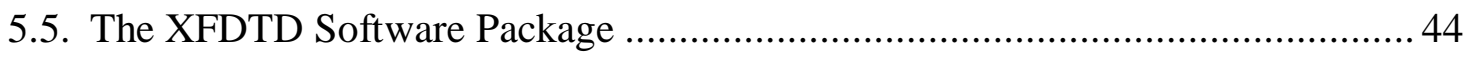

5.5.1. The General Method for Performing FDTD Calculations Using XFDTD ... 45

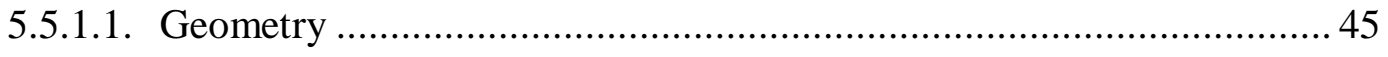

5.5.1.2. Calculation Parameters ........................................................................ 47

5.5.1.3. Post Processing the Results............................................................... 47

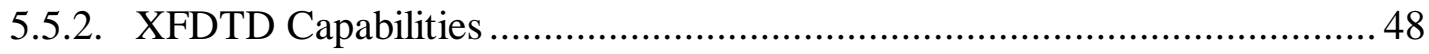

5.5.3. Description of the XFDTD Input Files …………....................................... 49 


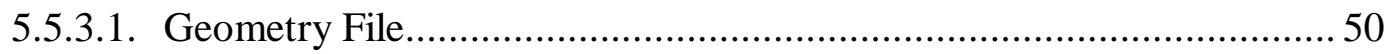

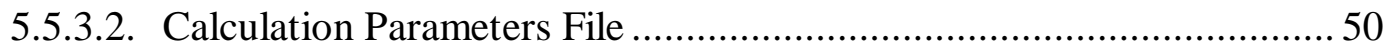

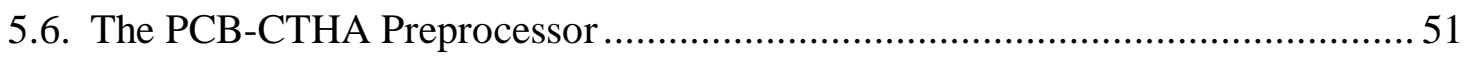

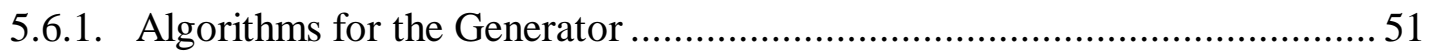

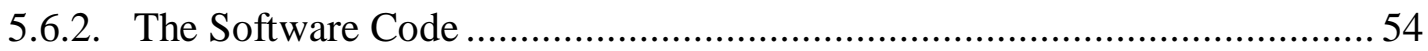

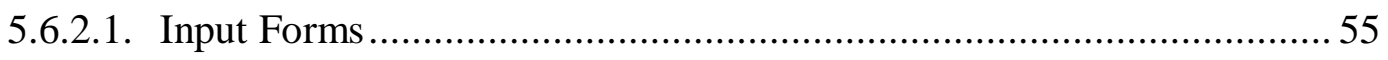

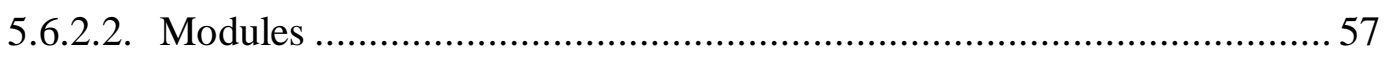

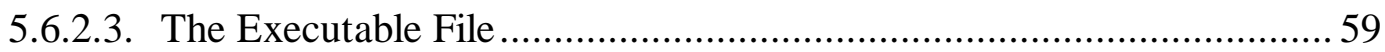

6. Model Creation and Development.........................................................60

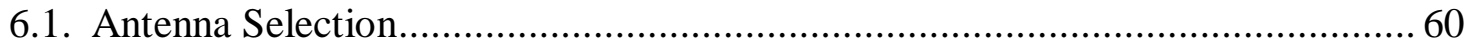

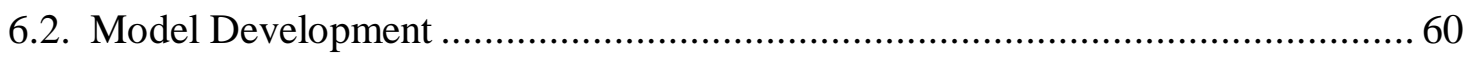

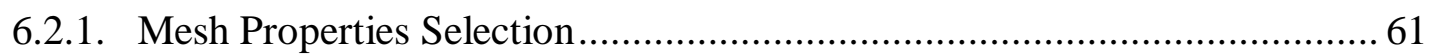

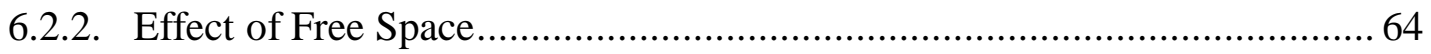

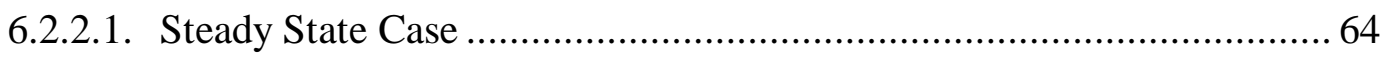

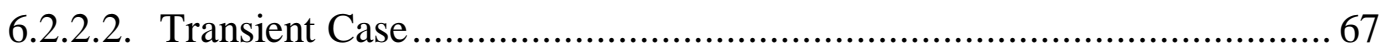

6.2.2.3. Effect of Free Space Conclusions ...................................................... 76

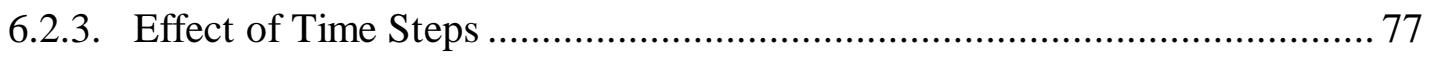

6.2.4. Effect of the Relative Permittivity P....................................................... 79

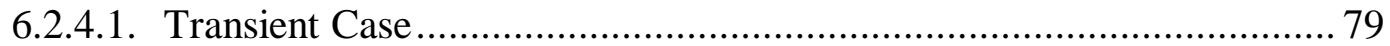

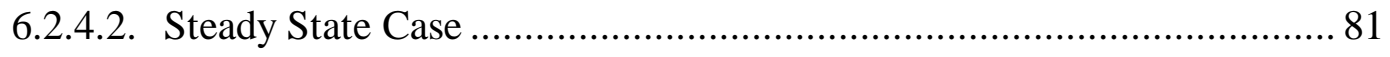

6.2.4.3. Effect of Relative Permittivity Conclusions ......................................... 84

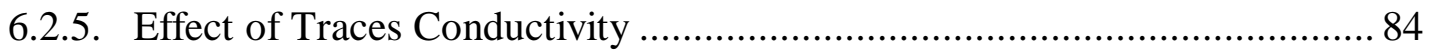

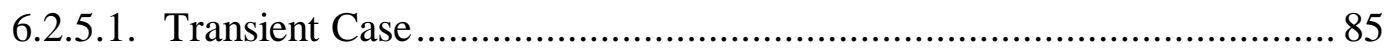

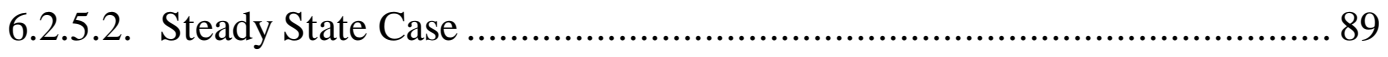

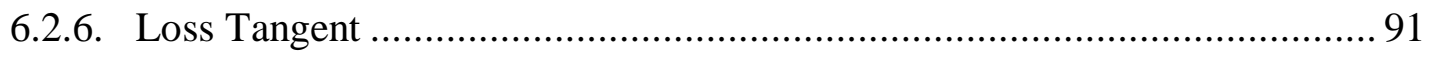

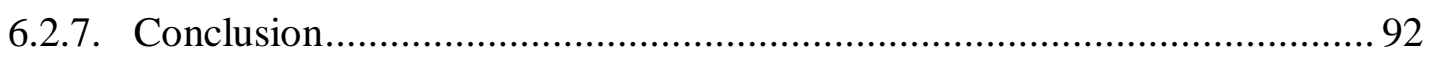

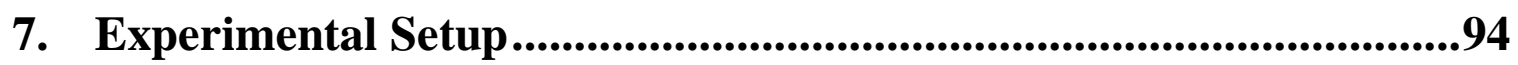

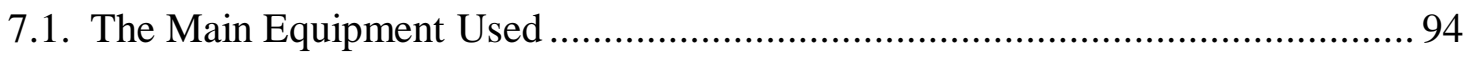

7.1.1. The Anechoic Chamber .......................................................................... 94

7.1.2. The HP8753D Network Analyzer ..........................................................96 


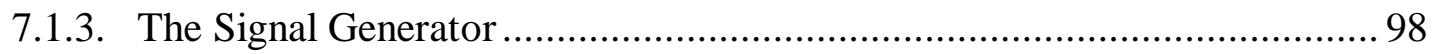

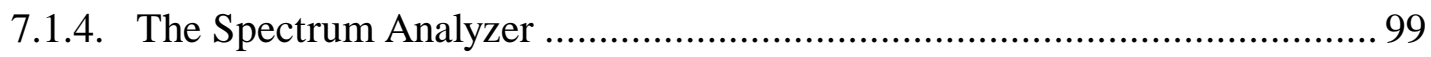

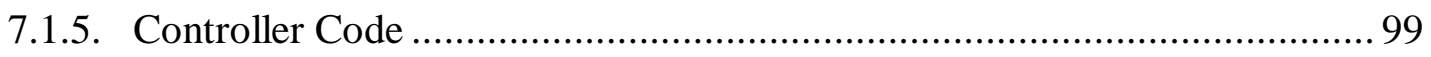

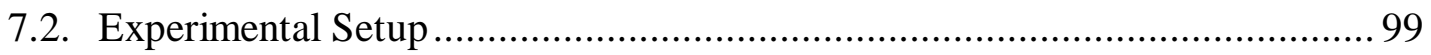

7.2.1. Input Impedance Experiments ................................................................... 99

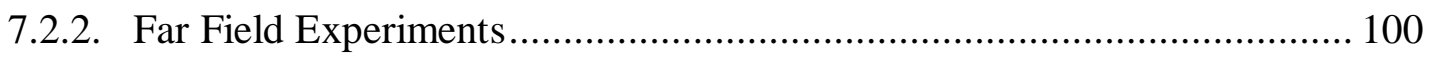

8. Feed Study Results and Validation............................................102

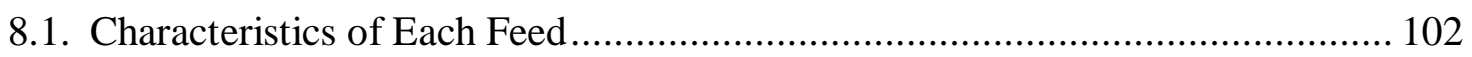

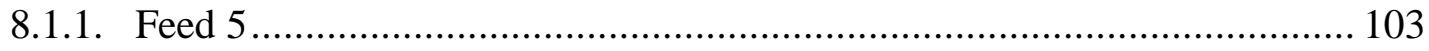

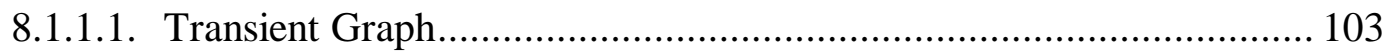

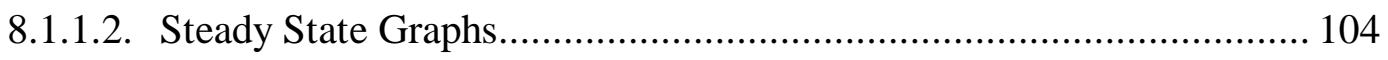

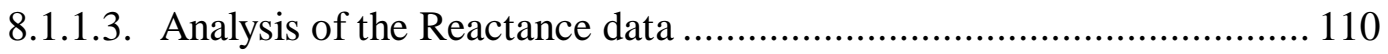

8.1.1.4. Analysis of the Gain Data............................................................... 111

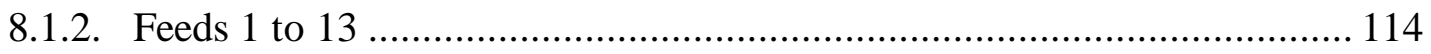

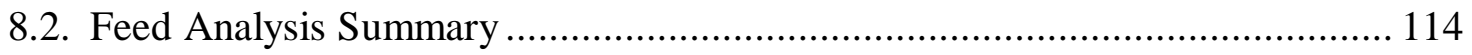

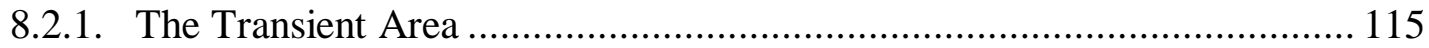

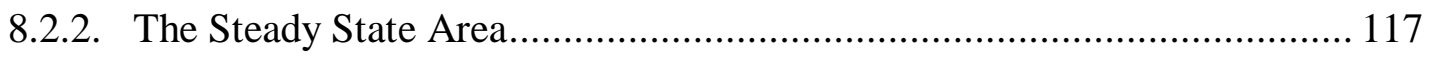

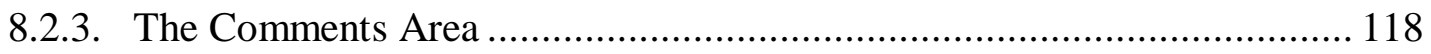

8.3. Feed Study Comments and Conclusions ………............................................ 120

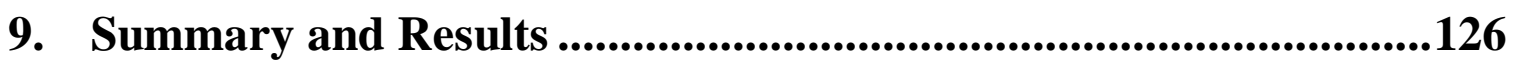

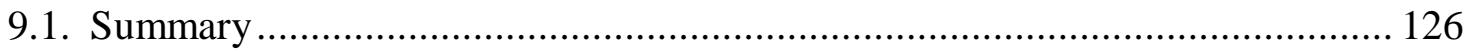

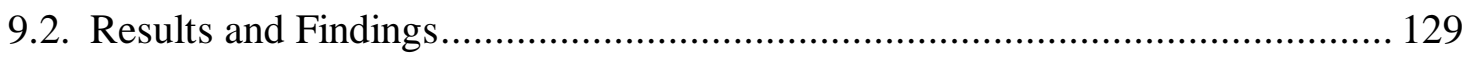

10. Conclusion and Recommendations ...........................................136

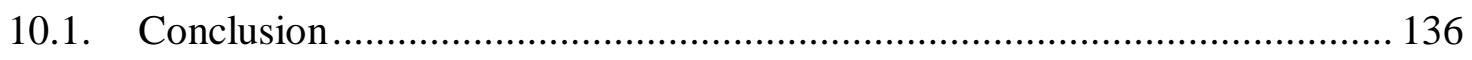

10.2. Recommendations for Future Work...................................................... 136

References

Appendix A: The CTHA Preprocessor Source Code.............................145

Appendix B: The Preprocessor Input Forms .......................................170 
Appendix C: Sample XFDTD Input Files.............................................177

Appendix D: Analyzer.exe Code ......................................................181

Appendix E Photos for the Experimental Analysis Equipment ..........186

Appendix F: Analysis of All 13 Feeds .......................................................194

Appendix G: CIRA Internal Reports .....................................................264 


\section{List of Tables}

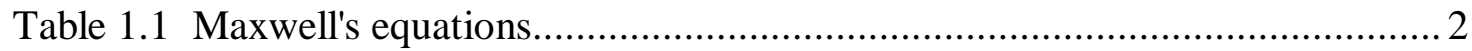

Table 1.2 The significance of Maxwell's equations .............................................. 3

Table 5.1 The user defined material properties in XFDTD ................................ 46

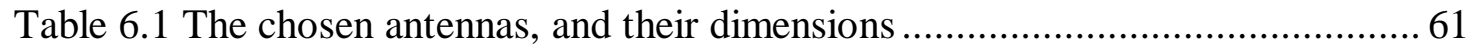

Table 6.2 The computational time required for all 10 transient runs....................... 73

Table 6.3 percent error of resonant frequencies results, compared to 50n20k run...... 73

Table 8.1 Comparing the experimental resonance frequencies for feed 5 with the numerically predicted resonance frequencies............................................ 104

Table 8.2 Analysis of the resonance frequencies obtained from the transient graph. 111

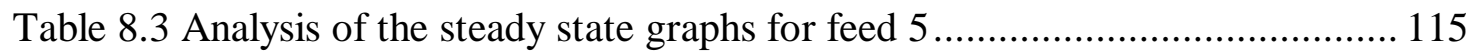

Table 8.4 Summary of the overall performance of all 13 feeds, and how well they were predicted. 


\section{List of Figures}

Figure 2.1 The core CTHA: a) wire only, b) full core, c) hollow core ..................... 12

Figure 2.2 The PCB-CTHA: a) wire only, b) complete CTHA............................... 13

Figure 4.1 Layer 2, showing the traces and the holes .......................................... 18

Figure 4.2 Layer 4, showing one type of feed configuration ............................... 20

Figure 4.3 The standard orientation of the feed points ...................................... 22

Figure 4.4 Visualization of the possible configurations problem ............................ 23

Figure 4.5 Example of one type of feed configuration .................................... 24

Figure 4.6 CTHA feeds with two feed source connections ................................... 25

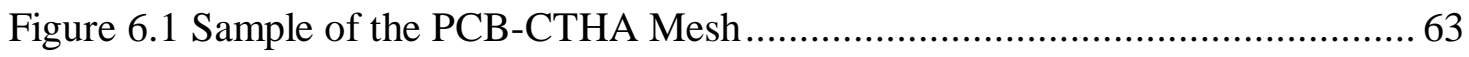

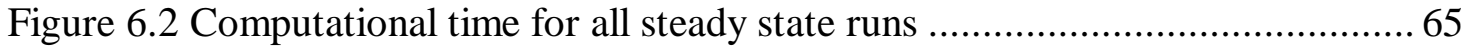

Figure 6.3 Input Impedance for all steady state runs ....................................... 65

Figure 6.4 Effect of free cells on the far field patterns ...................................... 68

Figure 6.5 The input impedance for CB108, 50 free space cells, 20,000 time steps,

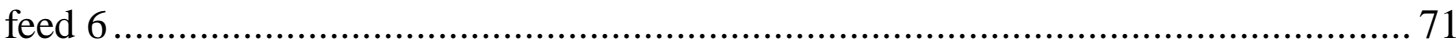

Figure 6.6 Effect of free space and time steps on the reactance of CB0108 feed6 ..... 72

Figure 6.7 The input impedance for CB108, 10 free space cells, 2,000 time steps, and

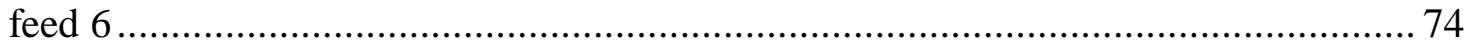

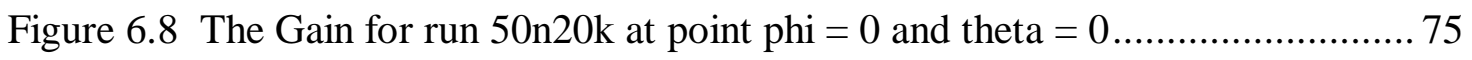

Figure 6.9 Effect of time steps on the far field patterns, Ephi plot, phi $=0 \ldots \ldots \ldots \ldots \ldots . . .78$

Figure 6.10 Effect of time steps on the far field patterns, Etheta plot, phi $=0 \ldots \ldots \ldots . .79$

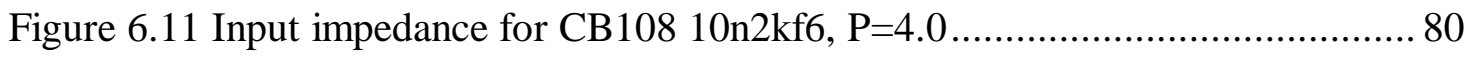

Figure 6.12 Effect of time steps on $\mathrm{CB} 108$ with $\mathrm{P}=4$ and 30 free space .................... 81

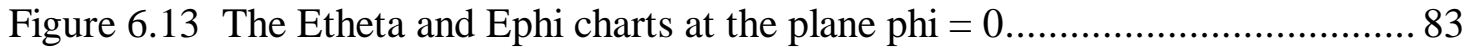

Figure 6.14 Effect of conductivity on all transient results, Input impedance chart,

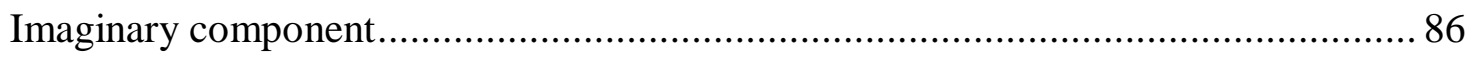

Figure 6.15 Comparison between the resonant frequencies for all five transient runs 87

Figure 6.16 Input Impedance Graph showing the effect of conductivity of the traces. 88

Figure 6.17 Effect of the free space on the conductivity runs .............................. 90

Figure 6.18 The loss tangent for the binding material ................................... 91 


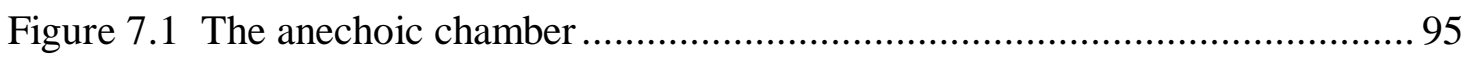

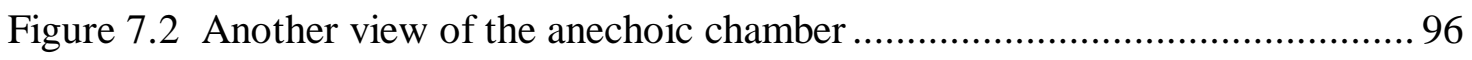

Figure 7.3 The design of the anechoic chamber ............................................... 96

Figure 7.4 The experimental setup for the transient experiments......................... 100

Figure 7.5 The experimental setup for the far field experiments.......................... 100

Figure 8.1 The Reactance for feed 5 - experimental vs. numerical ....................... 104

Figure 8.2 The Phi $=0$ plane for feed 5 - experimental ................................. 108

Figure 8.3 Comparison Between the Reactance Curves for Feed 12 and Feed 13.... 123 


\section{Nomenclature}

$\eta=$ Radiation efficiency, unitless

$\mu=$ Permeability, unitless

$\varepsilon=$ Permittivity, $\mathrm{F} / \mathrm{m}$

$\rho=$ Volume charge density, $\mathrm{C} / \mathrm{m}^{3}$

$\operatorname{Ae}(\theta, \phi)=$ Effective area, $\mathrm{m}^{2}$

$\mathrm{B}=$ Magnetic flux density, $\mathrm{T}$

$\mathrm{D}(\theta, \phi)=$ Directivity, unitless

$\mathrm{D}=$ Electric flux density, $\mathrm{C} / \mathrm{m}^{2}$

$\mathrm{E}=$ Electric field intensity, $\mathrm{V} / \mathrm{m}$

$\mathrm{H}=$ Magnetic field intensity, $\mathrm{A} / \mathrm{m}$

$\mathrm{J}=$ Current density, $\mathrm{A} / \mathrm{m}$

$\mathrm{P}_{0}=$ Power accepted by the antenna, watts

$\mathrm{P}_{\mathrm{r}}=$ Power radiated by the antenna, watts

$\mathrm{Z}=$ Impedance, ohms 


\section{Introduction to Antennas}

\subsection{Fundamentals of Electromagnetics}

A field is a spatial distribution of a quantity, which may or may not be a function of time. A time-varying electric field is accompanied by a magnetic field, and vice versa. So, time-varying electric and magnetic fields are coupled, resulting in electromagnetic fields. The source of an electromagnetic field is invariably electric charges at rest or in motion. However, an electromagnetic field may cause a redistribution of charges, which will in turn change the field [1].

The principle of conservation of electric charge, like the principle of conservation of momentum, is a fundamental law of physics. It states that electric charge is conserved, so it can neither be created nor destroyed. Electric charges can move from one place to another, and can be redistributed under the influence of an electromagnetic field, but the sum of the positive and negative charges in an isolated system remain unchanged. The principle of conservation of electric charge must be satisfied at all times and under all circumstances, and is mathematically represented by the equation of continuity shown later.

There are four fundamental vector field quantities in electromagnetics: electric field intensity $\mathbf{E}$, electric flux density or electric displacement $\mathbf{D}$, magnetic flux density $\mathbf{B}$, and magnetic field intensity $\mathbf{H}$. All four quantities are point functions; they are defined at every point in space, and are functions of space coordinates. Another vector point function known as the volume current density is also defined, or simply the current density $\mathbf{J}$. The current density measures the amount of current flowing through a unit area normal to the direction of the current flow. These five electromagnetic quantities do 
not exist independent of one another, but certain relations among them are imposed by the medium in which they exist. These relations are called the constitutive relations of a medium [2].

Table 1.1 Maxwell's Equations [1].

\begin{tabular}{|l|l|l|}
\hline Differential Form & Integral form & Name \\
\hline$\nabla \times \mathbf{E}=-\frac{\partial \mathbf{B}}{\partial t}$ & $\oint_{C} \mathbf{E} \cdot d l=-\frac{d \Phi}{d t}$ & Faraday's law \\
\hline$\nabla \times \mathbf{H}=\mathbf{J}+\frac{\partial \mathbf{D}}{\partial t}$ & $\oint_{C} \mathbf{H} \cdot d l=I+\int_{s} \frac{d \mathbf{D}}{d t} \cdot d \mathbf{s}$ & Ampere's circuital law \\
\hline$\nabla \cdot \mathbf{D}=\rho$ & $\oint_{S} \mathbf{D} \cdot d \mathbf{s}=Q$ & Gauss's law \\
\hline$\nabla \cdot \mathbf{B}=0$ & $\oint_{S} \mathbf{B} \cdot d \mathbf{s}=0$ & No isolated magnetic charge \\
\hline$\nabla \cdot \mathbf{J}=-\frac{\partial \rho}{\partial t}$ & $\oint_{S} \mathbf{J} \cdot d \mathbf{s}=-\iiint_{V} \frac{\partial \rho}{\partial t} \cdot d v$ & Continuity principle \\
\hline
\end{tabular}

During the 1860s and 1870s James Maxwell developed a complete set of four physical equations that fully describe the time and space relations between the timevarying electric and the magnetic fields $[3,4]$. This set of equations that governs the electromagnetic phenomena is known as the Maxwell's equations. These equations are based on the early work of Coulomb, Gauss, Ampere and Faraday. Maxwell's equations also include in them the continuity equation mentioned above $[5,6,7]$. The four Maxwell's equations and the continuity equation are shown in Table 1.1 in both their differential and integral forms [1], while Table 1.2 states the significance of each of these equations [8]. 
Table 1.2 The significance of Maxwell's equations [8].

\begin{tabular}{|l|l|}
\hline law & Significance \\
\hline Faraday's law & $\begin{array}{l}\text { The electromotive force around a closed path is equal to the } \\
\text { negative time derivative of the magnetic flux emerging through } \\
\text { any surface bounded by this path. }\end{array}$ \\
\hline Ampere's law & $\begin{array}{l}\text { The magnetomotive force around a closed path is equal to the } \\
\text { conduction current plus the time derivative of the electric } \\
\text { displacement emerging through any surface bounded by this path. }\end{array}$ \\
\hline Gauss' electric law & $\begin{array}{l}\text { The net electric displacement emerging through a closed surface is } \\
\text { equal to the net electric charge in the volume enclosed by this } \\
\text { surface. }\end{array}$ \\
\hline Gauss' magnetic law & $\begin{array}{l}\text { The net magnetic flux emerging through any closed surface is } \\
\text { equal to zero. }\end{array}$ \\
\hline Continuity principle & $\begin{array}{l}\text { The net electric current emerging through a closed surface is equal } \\
\text { to the negative of the rate of change of the net electric charge in } \\
\text { the volume enclosed by this surface. }\end{array}$ \\
\hline
\end{tabular}

\subsection{Basic Antenna Concepts}

An antenna, in general, is a special component of an electric circuit, in which the antenna is connected to a source through a transmission line. Under ideal conditions, the energy generated by the source is totally transferred to the antenna through a transmission line, and then radiated to space. But practically there are conduction and dielectric losses due to the transmission line and the antenna, as well as reflection losses at the interface between the transmission line and the antenna due to mismatches [9]. This section will present basic concepts and definitions related to antennas that will be used throughout the text.

\subsubsection{Transmission Lines and Resonators}

A transmission line is a device for transmitting or guiding energy from one point to another. It is usually most desirable to transmit the energy with the minimum attenuation possible, thus heat and radiation losses should be as small as possible. To 
achieve the least attenuation, the wave should not spread in space, but should be confined to the transmission line or be bound closely to it.

An energy generator connected to an infinite, lossless transmission line produces a uniform travelling wave along the line. If the line is short circuited, the outgoing travelling wave is reflected, producing a standing wave on the line due to the interference between the outgoing and reflected waves. A standing wave has associated with it local concentrations of energy. If the reflected wave is equal to the outgoing wave, a pure standing wave is obtained. The energy concentrations in such a wave oscillate from entirely electric to entirely magnetic and back twice per cycle. Such energy behavior is characteristic of a resonant circuit, or a resonator [10]. From these definitions it is understood that:

- Antennas radiate, or receive, energy;

- Transmission lines guide energy; and,

- Resonators store energy.

A simple example is a transmission line connected to a dipole antenna. The dipole acts as an antenna because it launches a free space wave. However, it may also be regarded as a section of an open-ended transmission line. It also exhibits many of the characteristics of a resonator since energy reflected from the ends of the dipole gives rise to a standing wave and energy storage near the antenna. Thus a single dipole exhibits, simultaneously, properties characteristic of an antenna, a transmission line and a resonator [10]. 


\subsubsection{Efficiency}

The radiation efficiency of a transmitting antenna is defined as the ratio of the input power accepted by the antenna to the output power radiated by the antenna. The efficiency is always less than or equal to unity. Let

$\mathrm{P}_{0}=$ power accepted by the antenna, watts

$\mathrm{P}_{\mathrm{r}}=$ power radiated by the antenna, watts

$\eta=$ radiation efficiency, unitless

Thus, the efficiency is given as,

$$
\eta=\frac{P_{r}}{P_{0}} .
$$

\subsubsection{Impedance}

The antenna input impedance is a fundamental parameter when designing efficient coupling networks and matching circuits in order to achieve maximum power transfer. The resistive component $\mathrm{R}_{\mathrm{r}}$ of the impedance $\mathrm{Z}$ is called the radiation resistance. This resistive component corresponds to the radiated power, that is the equivalent resistance that would dissipate an amount of power equal to the total radiated power when the current through the resistance is equal to the current at the antenna input terminals $[8]$.

\subsubsection{Far Field Condition}

At distances that are large compared to the size of the antenna and large compared to the operating wavelength, the shape of the field pattern is independent of the distance. This is called the far field condition [10,12]. It can be expressed as;

$$
r>\lambda,
$$


where $r$ is the observation point, or the distance radially from the antenna, and $\lambda$ is the operating wavelength.

\subsubsection{Radiation Patterns}

An antenna radiation pattern is defined as "a mathematical function or graphical representation of the radiation properties of the antennas as a function of space coordinates. In most cases, the radiation pattern is determined in the far field region and is represented as a function of the directional coordinates" [13]. Radiation properties include radiation intensity, power density, directivity, gain and polarization. These properties are described later. Most patterns have a single main lobe and several minor lobes.

\subsubsection{Radiation Power and Intensity}

Let $\Phi(\theta, \phi)$ be the radiation intensity (watts/steradian), and assuming far field conditions so that $\Phi$ is independent from $r$, the total power radiated from the antenna can be given as,

$$
P_{r}=\int_{0}^{2 \pi} \int_{0}^{\pi} \Phi(\theta, \phi) \sin \theta d \theta d \phi .
$$

and the average radiation intensity is given as,

$$
\Phi_{\text {avg }}=\frac{P_{r}}{4 \pi}
$$

\subsubsection{Directivity}

The directivity $\mathrm{D}(\theta, \phi)$ is a unitless measure of the ability of an antenna to concentrate radiated power in a particular direction [13]. It can be understood as the ratio of the power radiated by a given antenna in a certain direction to the power radiated by an 
imaginary isotropic antenna having the same total radiated power [8]. The directivity can thus be expressed as,

$$
D(\theta, \phi)=\frac{\Phi(\theta, \phi)}{\Phi_{\text {avg }}}=\frac{\Phi(\theta, \phi)}{P_{r} / 4 \pi} .
$$

When an antenna is said to have a certain directivity value, this is understood to be the maximum directivity of the antenna $\mathrm{D}(\theta, \phi)_{\max }[10]$.

\subsubsection{Gain}

The gain of an antenna $\mathrm{G}(\theta, \phi)$ is defined as the directivity of the antenna multiplied by its efficiency as follows,

$$
G(\theta, \phi)=\eta D(\theta, \phi)=\eta \frac{\Phi(\theta, \phi)}{P_{r} / 4 \pi}
$$

Substituting the value of $\eta$ from equation 1.1,

$$
G(\theta, \phi)=\frac{\Phi(\theta, \phi)}{P_{0} / 4 \pi}
$$

Thus the gain is a measure of the ability to concentrate in a particular direction the power accepted by the antenna [13].

\subsubsection{Power Density}

The power density $\mathrm{P}(\theta, \phi)$ (watts $\left./ \mathrm{m}^{2}\right)$ is the ratio of the radiation intensity to the square of the radial distance from the antenna. This can be expressed as,

$$
P(\theta, \phi)=\frac{\Phi(\theta, \phi)}{r^{2}}=G(\theta, \phi) \frac{P_{0}}{4 \pi r^{2}}
$$

where the factor $P_{0} / 4 \pi r^{2}$ represents the power density that would result if the power accepted by the antenna were radiated by a lossless isotropic antenna. 


\subsubsection{Effective Area}

The effective area $\mathrm{A}_{\mathrm{e}}(\theta, \phi)$ (meters square) is best understood when considering a receiving antenna. In this case, the effective area is a measure of the effective absorption area presented by an antenna to an incident plane wave. The effective area is related to the gain and wavelength by the following relationship,

$$
A_{e}(\theta, \phi)=\frac{\lambda^{2}}{4 \pi} G(\theta, \phi) .
$$

\subsubsection{The Aperture Concept}

The aperture is defined as that portion of a plane surface near the antenna, perpendicular to the direction of maximum radiation, through which most of the radiation flows. Many high-gain antennas such as horns, reflectors, and lenses are called aperturetype antennas. The antenna efficiency of an aperture-type antenna $\eta_{a}$ (unitless), also called the aperture efficiency, is defined as the ratio of the effective area of the antenna $A_{e}$ to the physical area of the antenna aperture A (meters square) as follows:

$$
\eta_{a}=\frac{A_{e}}{A},
$$

substituting from equation 1.9, the gain of an aperture antenna can be given as,

$$
G=\frac{4 \pi}{\lambda^{2}} A \eta_{a} .
$$

\subsubsection{Polarization}

The polarization of an electromagnetic wave is a property of a single frequency electromagnetic wave that describes the shape and orientation of the electric field vectors $\mathrm{E}_{\theta}$ and $\mathrm{E}_{\phi}$ as a function of time. If the phase angle between the two vectors $\mathrm{E}_{\theta}$ and $\mathrm{E}_{\phi}$ is constant in time then a linearly polarized wave is obtained, otherwise an elliptically 
polarized wave is obtained. The plane containing the electric and magnetic fields is called the plane of polarization, and is orthogonal to the direction of propagation. The tip of the electric field vector moves, in general, along an elliptical path in the plane of polarization. The shape of the ellipse is specified by its axial ratio, the ratio of its major to minor axis, while the orientation is specified by the tilt angle, an angle between the major axis and a reference direction when viewed looking in the direction of propagation.

The polarization of an antenna in a specific direction is defined to be the polarization of the far field wave radiated in that direction from the antenna. An antenna that radiates a linearly polarized wave, like a dipole, is called a linearly polarized antenna, while an antenna that radiates a circularly polarized wave, where the ratio of the major to minor axis is 1 , is called a circularly polarized antenna. For more details on polarization see IEEE and Hollis $[14,15]$. 


\section{The Contrawound Toroidal Helical Antenna}

In this chapter, the history of the invention and development of the Toroidal Helical Antenna (THA), and its main class the Contrawound Toroidal Helical Antenna (CTHA), is discussed. The two main types of CTHAs, the core CTHA and the Printed Circuit Board CTHA (PCB-CTHA) are also introduced, and a general description of the geometry of the CTHA is provided.

\subsection{History of the Toroidal Helical Antenna}

The Toroidal Helical Antenna (THA) was invented by Corum in the early 1980s

$[8,16,18]$ during his investigations on the principle of global wireless power transfer which was investigated by Nikola Tesla and others around the turn of the $20^{\text {th }}$ century. Corum introduced the THA as an excitation element for the earth cavity resonance which is in the very low frequency VLF band. He based his work on the previous research done by others on the linear helical antenna $[10,19,20]$.

The THA is a wire antenna which consists of one or more conductor helices wrapped about a toroidal core usually with different pitch senses that are fed from one or more ports. The cross section of the torrid is an ellipse or a rectangle, but circles and squares can also be used. The core material is usually made of a dielectric material [21]. The most important class of the THA is the Contrawound Toroidal Helical Antenna (CTHA) which is the main focus of this investigation.

In 1991, Smith proposed the usage of the THA as an application to the Ground Wave Emergency Network (GWEN) [22]. This lead to a detailed investigation of the applicability of Corum's THA to GWEN at the Center for Industrial Research Applications (CIRA) at West Virginia University. But the THA described by Corum, 
which was a monofilar toroidal element, or a single winding of wire around a toroidal core, showed difficulties in achieving the desired results. Thus, the bifilar contrawound toroidal helix, with two monofilar structures of opposite pitch senses and multifeed ports, was suggested and patented by Smith and his then doctoral student Kurt Van Voorhies $[23,24,25]$. The resulting antenna was named the contrawound toroidal helical antenna (CTHA).

\subsection{The Contrawound Toroidal Helical Antenna}

The CTHA consists essentially of two helical toroidal windings of wire, with opposite pitch, supported by a binding material. The binding material, in addition to holding the antenna together, also plays a significant role in the characteristics of the antenna, namely affecting its input impedance and gain [26]. CIRA has been carrying out an extensive research program aiming to characterize and improve the performance of the CTHA. During this extensive research several new shapes of the CTHA were designed and constructed. The two most significant of these types, the core CTHA and the printed circuit board CTHA, are briefly described below.

\subsubsection{Core CTHA}

The core CTHA, shown in Figure 2.1, consists of two helical windings of wire supported upon a central core. The binding material of the core CTHA is a full block of material. Figure 2.1 shows three instances of the core CTHA. The first, for clarity, is just the wire windings with no support material. The second shows the CTHA with full core material, and the third is for a core CTHA with a hollow center. For more references on the core CTHA and insights on the effect of its different parameters on its characteristics and properties see CIRA internal reports [32, 29, 30, 27]. 
a)

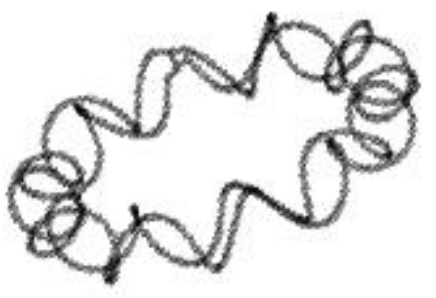

b)

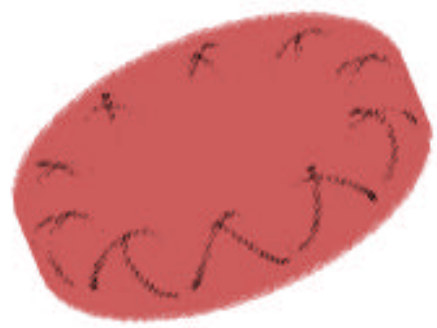

c)

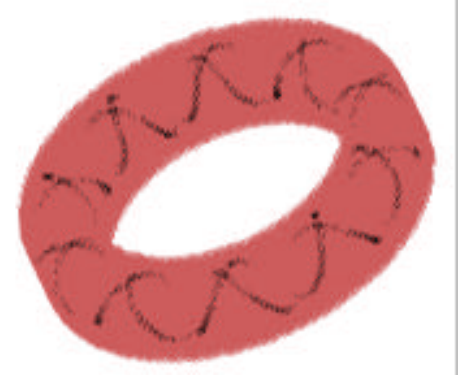

Figure 2.1 The core CTHA: a) wire only, b) full core, c) hollow core

\subsubsection{The Printed Circuit Board CTHA}

The PCB-CTHA, on the other hand, as is clear from its name, has the two wire windings in the shape of traces upon a PCB and holes drilled through the board. The holes can either be filled with conducting material, or hollow but with traces of the conducting material painted within to provide contact between the layers of traces. Figure. 2.2 shows two instances of the PCB-CTHA; the first shows the traces alone with no binding material, while the second shows the complete PCB-CTHA. A thorough investigation of the effect of the different PCB-CTHA parameters on its performance can be found in references $[32,28]$.

\subsection{Advantages of the CTHA}

The main advantage of the CTHA, and the THA family in general, is the small physical profile compared to a linear dipole, or monopole, having the same operating frequency and operating conditions. Ellithy [8] gives a detailed analysis, and comparison, of the length of wire and dimensions of a CTHA compared to a regular dipole. This small physical profile makes it attractive in many applications over the larger antennas in situations like when the operating environment does not permit large 
structures, when the aerodynamic drag on the antenna is a major factor, or for stationary structures where it is more economical to manufacture smaller structures.

In comparison tests conducted by the Navy in 1994 [21], the CTHA outperformed several other antennas, including a standard military antenna and other antennas of larger physical dimensions, in long range tests. In addition to the small physical size, and the long range performance, the results of the tests showed the CTHA to be favorable in several features. These features included its omnidirectionality in multiple axis making it a good isotropic radiator, and its elliptical polarization.

a)

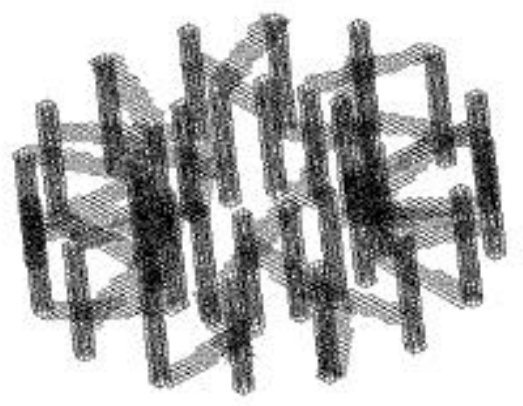

b)

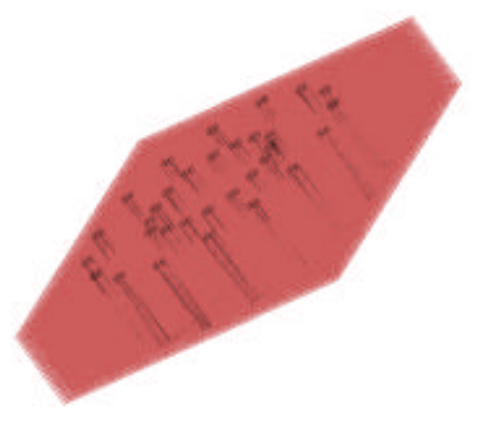

Figure.2.2 The PCB-CTHA: a) wire only, b) complete CTHA 


\section{Research Objective}

\subsection{Statement of Problem}

The objective of this research is to build a numerical model that is capable of predicting the electromagnetic characteristics of PCB-CTHAs. The numerical model will use the finite difference time domain method for all calculations. The numerical-derived results will be compared to experimental-derived results for PCB-CTHA antennas to reach an understanding of the general performance of the PCB-CTHA and its behavior under different feed configurations. The resulting general model of a PCB-CTHA will assist future PCB-CTHA designers in choosing geometry and appropriate feed types for their own application.

In order to achieve this objective, the following process is proposed:

\subsection{Create a PCB-CTHA Finite Difference Time Domain Model:}

In order to create a PCB-CTHA numerical model, it is required to create a finite difference mesh. Numerical computations are then processed upon this preliminary mesh, and corrections and refinements are performed until the computations converge within an acceptable accuracy and processing time. The specifications of this final outcome are used as the model for performing all later analysis.

\subsection{Mesh Generator}

Due to the complexity of the geometry of the PCB-CTHA, the manual mesh generation process for each antenna would require a significant amount of time and effort. Thus, an automatic mesh generator for the XFDTD input files would reduce the time and effort for a new PCB-CTHA design. This mesh generator must have a flexible user friendly interface that would accept different geometric parameters of the PCB- 
CTHA and generate output files ready for numerical analysis. The sole aim of this code is to provide a user friendly, efficient, and flexible package that would increase the accuracy of the PCB-CTHA models while saving modeling time.

\subsection{Model Creation}

Analysis will be performed using XFDTD, an X-Windows finite difference time domain software package sold by REMCOM [44] that uses the finite difference time domain numerical method (FDTD) to simulate the antenna characteristics. The generated mesh files will be read by XFDTD and calculations performed. Although the FDTD method is very flexible and powerful, it can be numerically unstable. Results can be highly affected by minor changes in the mesh characteristics such as the free space around the antenna, the cell size, and the number of time steps. The FDTD method may also require a significant amount of computational time. The initial mesh needs to be modified through an analysis of the results until a PCB-CTHA FDTD model can be designed that will converge and give acceptable results using a minimal amount of processing time.

\subsection{Practical Application: The Feed Study}

The above FDTD model will then be used for studying the characteristics of the PCBCTHA antenna with different feed configurations. In order to achieve this objective a feed analysis is performed, modeled, and experimentally verified.

The complex geometry of the PCB-CTHA allows for numerous methods of connecting the feed points together. Until recently, only one feed method was typically used. A thorough feed study is to be performed in order to define all possible feed configurations. A group of acceptable feeds will then be selected from among them. 


\subsection{Numerical Analysis}

The selected feeds will be modeled using the numerical XFDTD model developed

earlier in the research process. A detailed numerical investigation will be performed on the computational results to find and identify the characteristics of each feed method.

\subsection{Experimental Verification}

Field experiments will be performed upon a selected group of antennas and feed methods to verify the results obtained from the numerical model. The experimental investigation will help ensure that the numerical results obtained correctly predict the behavior of the PCB-CTHA. 


\section{The PCB-CTHA Geometry and Feed Study}

\subsection{The PCB-CTHA Geometry}

PCB-CTHAs are composed of multi layers of printed circuit boards that are combined together to form the shape of the PCB-CTHA. The wires of the core CTHA are found as conducting material traces on the boards of the PCB-CTHA. The conducting material on each of the layers is printed on the layer prior to applying the adhesive to combine the layers together. These traces on all the layers are connected together by introducing holes of conducting material into the board shape, which form the final shape of the PCB-CTHA.

The PCB-CTHA is actually produced out of three segments of printed boards. The upper and lower segments are both printed on both sides with the conducting traces, while the middle segment has no traces printed on it. Thus, the traces of the CTHA are printed on four layers: the lower, the higher, and two intermediate layers. These layers are called layers 1 to 4 from bottom to top.

The dielectric constant (Permittivity) of the board material plays a major role in defining the characteristics of the PCB-CTHA [26], while other parameters, like the spacer segment thickness, the diameter of the holes, and the spacing between the traces also play significant roles in defining these characteristics. Thorough investigation into the effects of these parameters is being conducted at CIRA-WVU [28].

The traces are actually connected in two toroids that go in opposite directions (thus the name contrawound). The first wound goes between layers 1 and 3, while the second goes between layers 2 and 4 . Also, on each layer, each of the two wounds connects between two sets of holes, where each set is located on a perimeter of a circle. 
For each wound the cycle goes as follows: the traces start at one point of the highest layer of this wound, say on a hole in the inner circle. They are then connected to a hole on the outer circle of the same layer. The trace then drops down to the lower layer of this wound, and then it is connected to a hole on the inner circle. This same procedure is repeated until the whole wound is finished and the trace returns to the starting point. The same procedure will be applied to the other wound thus generating two separate wounds that are totally independent. Figure 4.1 shows layer 2 where traces are connected between the inner and outer circle forming the lower layer of one of the wounds. Of course the cycle is connected by the upper layer of the same wound which will be the top layer of the CTHA in this case since layers 2 and 4 together contribute to the same wound.

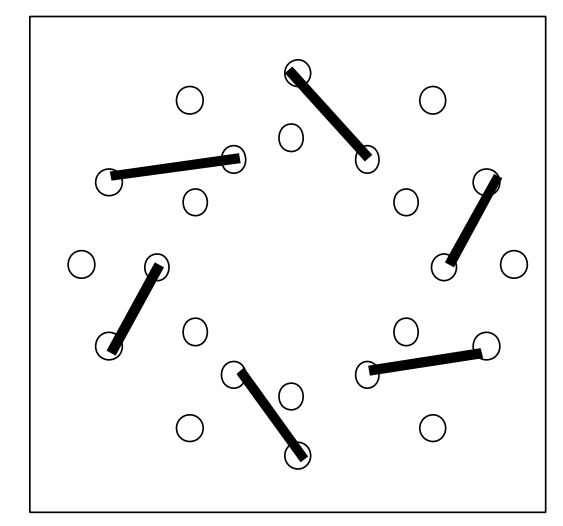

Figure 4.1 Layer 2, showing the traces and the holes 
The two contrawounds have to be connected together to introduce the feed point. In order to do that, a slight modification has to be made to the last cycle in the wound that runs between layers 1 and 3. One of the traces that is supposed to fit on layer 3 is selected, and this trace is eliminated. The two holes that are supposed to connect this trace are marked and identified on the top layer. If these two identified holes were to be connected with each other on layer 4 they would cross over another trace from the second wound. This other trace from the second wound is also eliminated and the two holes connecting it are also marked on layer 4 . So now 4 points have been marked on layer 4 , these will be called feed points A, B, C, and D, as shown in Figure 4.2, where feed points A and $\mathrm{C}$ represent one wound, and feed points B and D represent the other.

Depending upon how these feed points are connected, different shapes of feeds can be obtained. Figure 4.2 shows one way of connecting these feed points, where points $\mathrm{A}$ and $\mathrm{B}$ are connected together by a trace line, and points $\mathrm{C}$ and $\mathrm{D}$ are also connected together by another trace line. Then a third trace line is drawn between these two traces, thus finalizing the shape of the feed. This is just an example of one type of possible feed configuration. A detailed analysis of all possible types of feed configurations is provided next. 


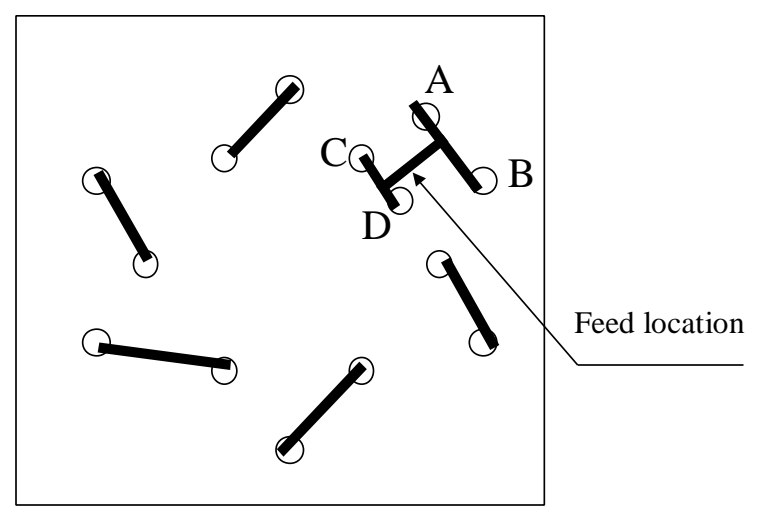

Figure 4.2 Layer 4, showing one type of feed configuration

\subsection{PCB-CTHA Feed Study}

In his report on CTHA feeds, Craven [56] showed how different modes of operation of the CTHA can be induced by different feed configurations. Craven states that "a great deal of variety can be introduced both to the input impedance characteristics and to the polarization and radiation pattern of an antenna via the feed selection." In this section, a detailed study of the CTHA feeds is developed. The study focuses on the different configurations that can be achieved by the four feed points defined in the previous chapter, and the differences between these configurations. The configurations are then grouped into classes that have similar physical properties.

\subsubsection{CTHA Feed Points Analysis}

This analysis focuses on all the possible physical connections of the four feed points. To demonstrate these possible connections all the different points of the geometry 
have to be defined, and what each point can be connected to. The method developed by Craven [56] will be considered as the standard for the feed points orientation. Craven examined the antenna with the four feed points at the lowest edge of the antenna, as shown in Figure 4.3. The difference is that Craven did his study on the core CTHA, thus he had several ways of locating the windings' crossings, including the "polar crossings" and the "equatorial crossings." But in this study, focused on PCB-CTHAs, the shape of the windings is different, and the location of the feeds is restricted to the upper layer of conductor traces. The two CTHA wire windings end at the feed points, where each two points actually represent the two ends of a single wire. So points A and D represent the ends of one wire, and points B and C represent the ends of the other wire. Since the feed trace needs to be connected to both sides of the voltage source that feeds the antenna, then it can also be considered that two points, resembling the two ends of the feed source, also exist. From this it is understood that the four wire ends can be:

$>$ Connected to another feed point

$>$ Connected to one of the two feed sources

> Or, left unconnected or open.

On the other hand, the two ends of the feed source can be:

$>$ Connected to a feed point

> Connected to a group of feed points

$>$ Left unconnected or open. 


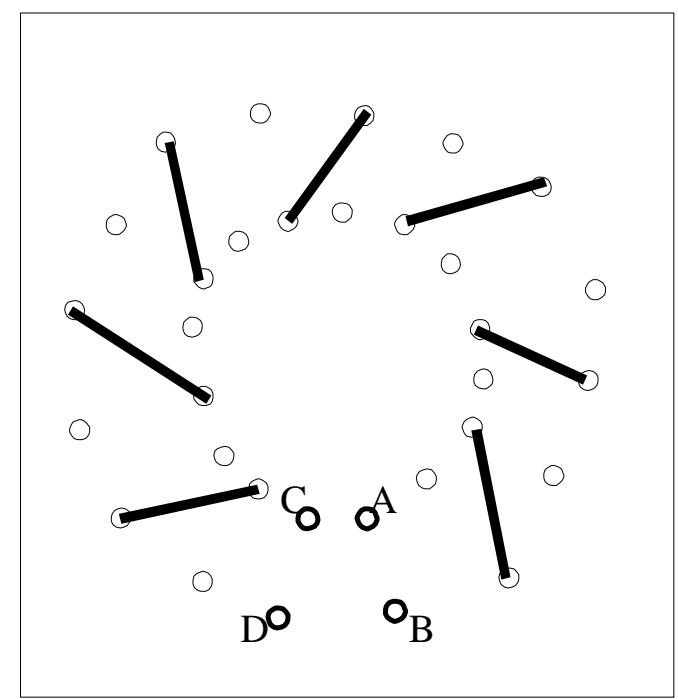

Figure 4.3 The standard orientation of the feed points

\subsubsection{Possible Feed Configurations}

From the previous section, the problem can be imagined as demonstrated in Figure 4.4, where five imaginary terminals were identified. The first terminal, terminal 0 , is a "no connection terminal" where a wire between a feed point and this terminal is just as if the feed point is not connected to anything. Terminals 1 and 2 are the two sides of the feed source, so if one feed point is connected to one of these terminals and the other feed point is connected to the second, then the feed source exists between these two feed points. Terminals 3 and 4 are two connection terminals, so if any two feed points or more are connected to any one of these terminals, then it is as if these feed points are directly connected to each other, while if only one feed point alone is connected to any of these terminals then it is as if it is left unconnected or open, or to point 0. 


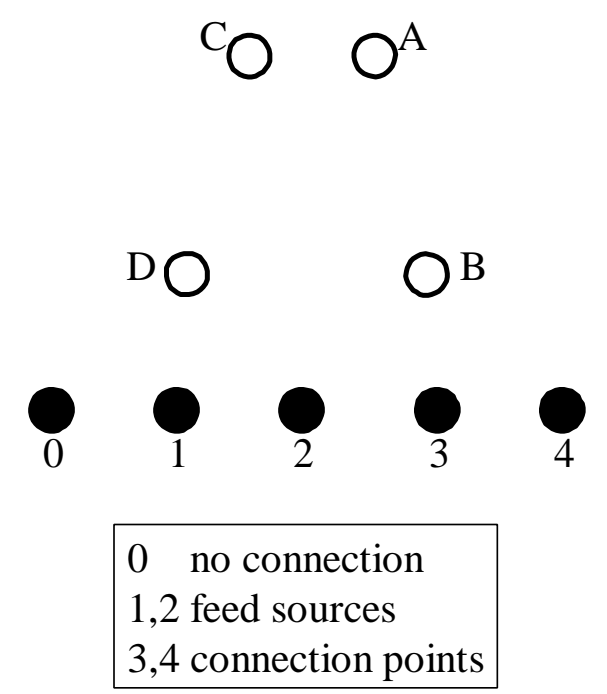

Figure 4.4 Visualization of the possible configurations problem

As an example, if it is desired to describe the feed shown in Figure 4.5, then it can be said that feed points $\mathrm{A}$ and $\mathrm{B}$ are both connected to terminal 3 , feed point $\mathrm{C}$ is connected to terminal 1 , and feed point $\mathrm{D}$ is connected to terminal 2, and so on. From this it is found that there are four feed points, each point can be connected to any of 5 terminals, and the number of possible combinations is $5^{4}=625$ possible combinations. 


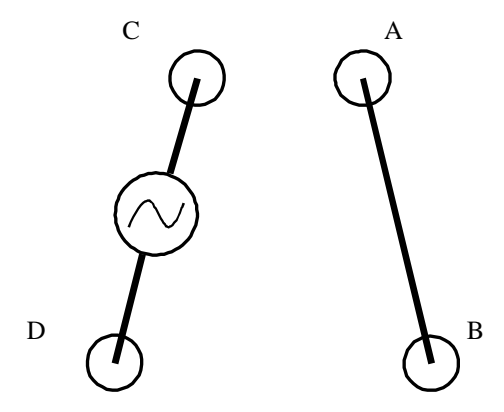

Figure 4.5 Example of one type of feed configuration

But some conditions exist to ensure that no redundancy occurs, these conditions were noted in Craven's report, and are repeated for clarity:

$>$ No one wire alone can be connected to terminals 3 or 4 . If this occurs then it is just as if this wire was connected to terminal 0.

$>$ terminals 3 and 4 are interchangeable

$>$ terminals 1 and 2 are interchangeable

> Symmetry 1: feed points A \& B can be swapped for feed points C \& D.

$>$ Symmetry 2: feed points A \& D can be swapped for feed points B \& C.

> Symmetry 3: feed points A \& B can be swapped for feed points D \& C.

By applying all these rules to the core CTHA, Craven found the unique number of feed configurations to be only 35 out of the 625 possible configurations. This would be the same case for the PCB-CTHA. Craven then classifies the 35 different configurations into 3 categories depending upon the number of connections to the feed sources terminals. The three categories were

$>$ Two feed source connections 
$>$ One feed source connection

$>$ No feed source connections

Both the second and the third categories rely upon alternative feed techniques, which will not be discussed in this study. Since 13 of the 35 feeds are in category 2, and 9 in category 3, this leaves 13 unique feeds. These feeds, from Cravens report, are shown in Figures 4.7.

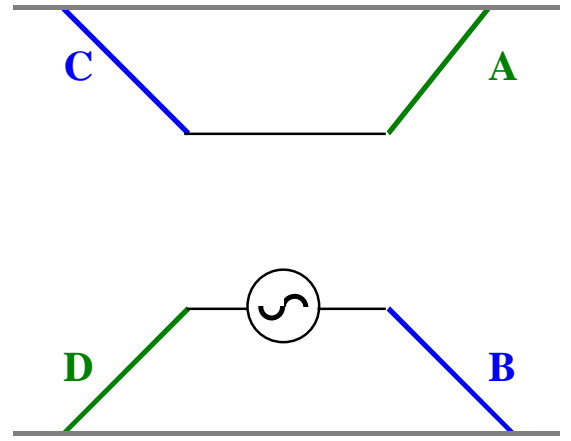

Feed 1

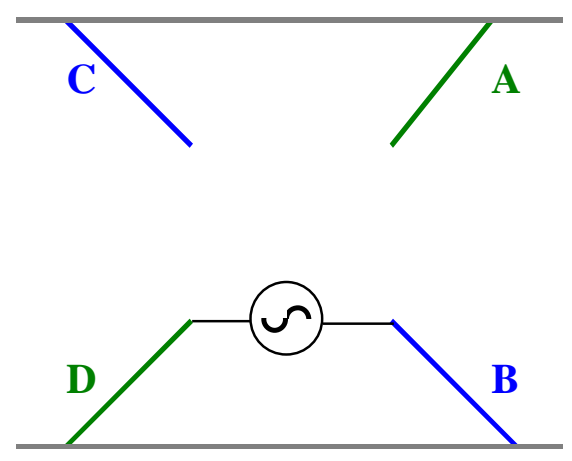

Feed 3

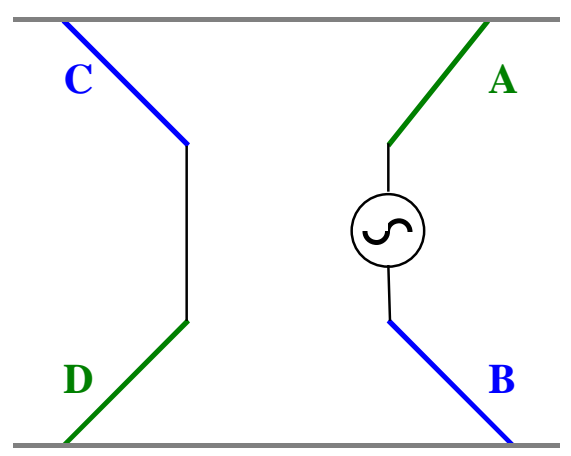

Feed 5

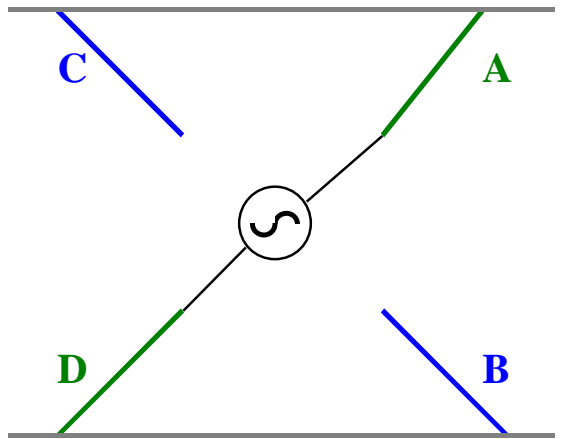

Feed 2

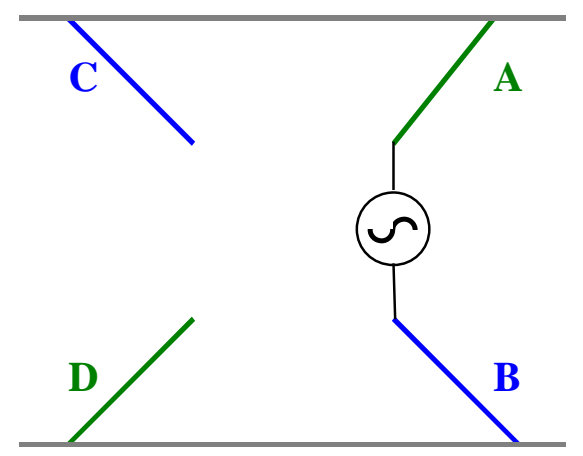

Feed 4

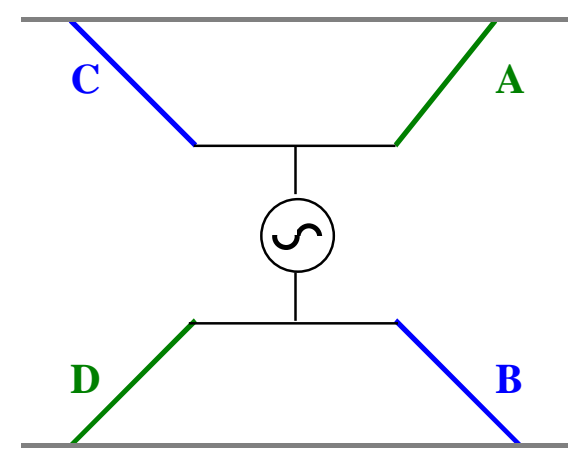

Feed 6

Figure 4.6 CTHA feeds with two feed source connections [56] 


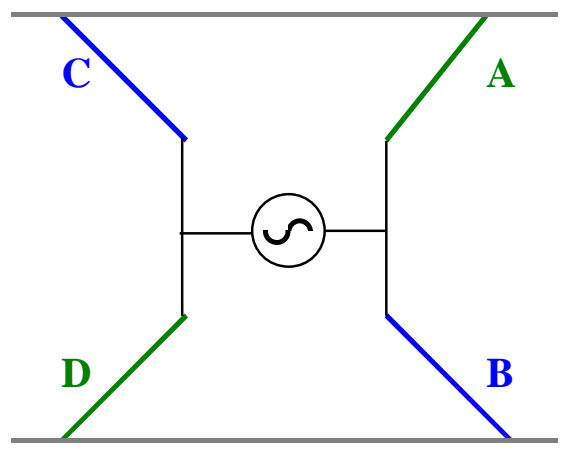

Feed 7

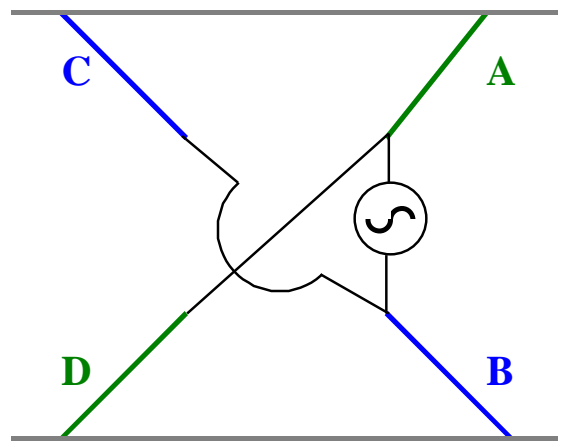

Feed 9

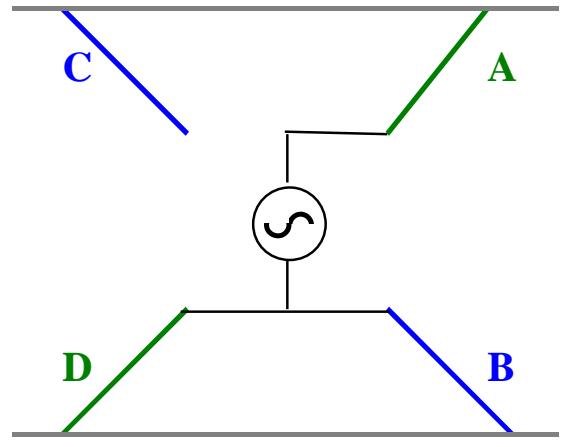

Feed 11

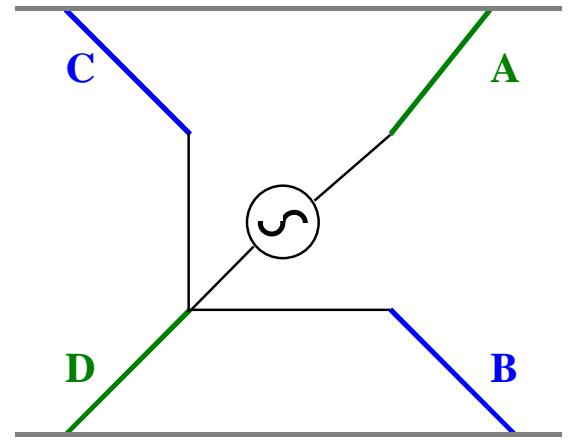

Feed 13

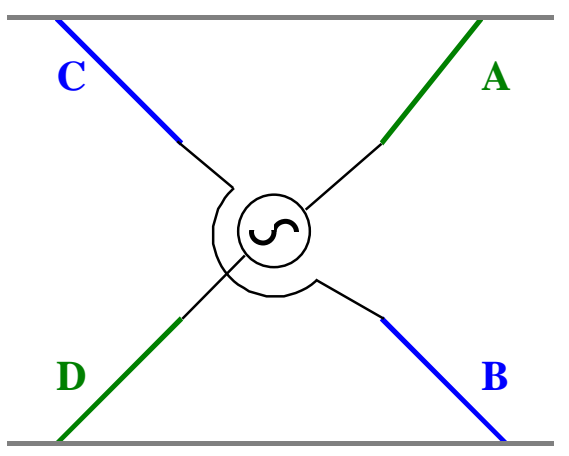

Feed 8

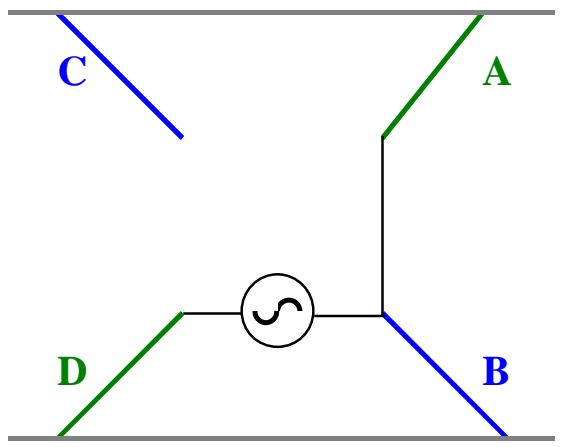

Feed 10

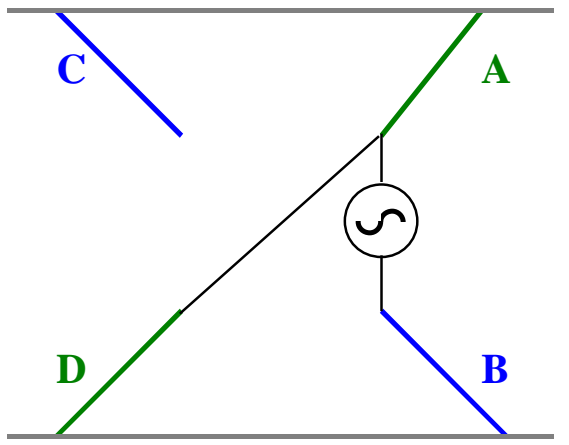

Feed 12

Figure 4.6 CTHA feeds with two feed source connections [56] (cont.) 


\section{Numerical Simulation}

In this chapter, a theoretical overview of the finite difference time domain method (FDTD) is provided. This will be followed by an introduction to the numerical code XFDTD (X-Windows Finite Difference Time Domain) which will be used throughout this study for the numerical calculations. The introduction to XFDTD will also include a brief look at the input files and formats accepted by the package, since this will help make the CTHA geometry modeler code described in the last section of this chapter better understood. The chapter will also include a literature review of some of the work done using FDTD. And finally, the Geometry modeler developed to provide a user friendly method for creating XFDTD input files will be described.

\subsection{Introduction to FDTD}

Among the several techniques and approaches that exist for electromagnetic computations, including the method of moment, finite element, geometric theory of diffraction, physical optics and finite difference time domain, the latter is the technique applicable to the widest range of electromagnetic problems [43]. The FDTD method was first formulated for electromagnetics by Yee in 1966 as a numerical solution of Maxwell's equations in isotropic media [33]. The method was then refined and used by many other authors to solve different electromagnetic problems $[34,35]$. One of the primary contributions to the technique was the introduction of the integral equation approach by Taflove et al. [36] in 1988.

The FDTD method is a transient, marching-in-time approach in which time is divided into small discrete steps, and the electric and magnetic fields on a grid are calculated at each time step [44]. Early applications of this approach were mainly in the 
areas of electromagnetic pulse response and penetration of electromagnetic fields into various materials including human tissues [37] where the near zone electromagnetic fields were of primary interest. But later, however, FDTD was applied to antenna and radar problems, where the far zone electromagnetic fields are of prime interest. Currently, the technique is being widely applied to antennas and microstrip calculations [38], including microstrip patch antennas [39], radiating waveguides, horn antennas [40], monopole antennas, and conical antennas [41].

Electromagnetic computation includes the processes of electromagnetic modeling, simulation, and analysis of the electromagnetic responses of complex systems to various electromagnetic stimuli. It also provides an understanding of the system response that allows for optimizing designs. The FDTD method is a powerful tool for all of the above characteristics of an electromagnetic computational package, and offers many advantages in the areas of electromagnetic modeling, simulation, and analysis. Some of the capabilities of FDTD include [41]:

- FDTD allows for the modeling of any arbitrary 3D objects;

- Frequency dependent parameters allow for the modeling of a wide variety of materials, including magnetic materials, lossy dielectrics, anisotropic plasmas, and magnetized ferrites;

- Predict broadband responses centered about the system resonances;

- Many types of responses can be computed, including far fields derived from near fields, scattered fields, antenna patterns, radar cross section, surface response, currents, and power density; 
- Availability of a wide variety of stimuli covering a broad range of frequencies, including electromagnetic pulses, high power microwave, lightning, radar, and lasers;

- The systems that respond to the stimuli are also of a wide range. Examples include aerosols, shelters, aircraft, humans, satellites, and buried antennas; and

- FDTD can treat several kinds of electromagnetic computational problems, including problems of antennas (reception, detection, and radiation), transmission (transmission lines, and waveguides), coupling, shielding, penetration, scattering, switching and nonlinearities.

Although, as shown above, almost all kinds of antennas, and all types of electromagnetic computational problems can be solved using FDTD, the method may become costly in computation time and memory storage when it comes to complex structures.

\subsection{The choice of FDTD}

The choice of the FDTD method as the numerical method to be used for this study came from several points, the most important of these points are:

- The capability of FDTD to model dielectric material: all the previous work done on the CTHA was done using the method of moments. The method of moments, though, is not capable of modeling dielectric materials. Thus all previous numerical work was done with an assumption of a hollow core.

- The power and flexibility of the FDTD method: as mentioned later in detail, the FDTD method is currently the most powerful and most flexible numerical method for modeling electromagnetic problems. Since the PCB-CTHA is a 
complex geometry, it was suggested that this method might be the most appropriate modeling method for this antenna.

- The availability of the modeling program: CIRA already owns a powerful FDTD software called XFDTD to be discussed later in this chapter. The ownership of this software package was from the factors that encouraged the use of the FDTD method for modeling the PCB-CTHA.

\subsection{FDTD Method Theory}

In this section a detailed description of the FDTD method theory is provided. Most of the work in this section was based on references [43, 42, 39].

\subsubsection{Maxwell's Curl Equations}

The FDTD method consists of a discretization and solution of the Maxwell's curl equation in derivative form in the time domain. These equations are expressed in a linearized form by means of central finite differencing. The fields are advanced temporally in discrete time steps over spatial cells of rectangular shape, thus only nearest neighboring interactions need to be considered.

The curl of any vector field $\mathbf{A}$, denoted by curl $\boldsymbol{A}$ or $\nabla \times \mathbf{A}$, is a vector whose magnitude is the maximum net circulation of $\mathbf{A}$ per unit area as the area tends to zero, and whose direction is the normal direction of the area when the area is oriented to make the net circulation maximum [1]. For Cartesian coordinates $\mathrm{x}, \mathrm{y}$, and $\mathrm{z}$ the curl is given as:

$$
\nabla \times \mathbf{A}=\left|\begin{array}{ccc}
\mathbf{a}_{\mathbf{x}} & \mathbf{a}_{\mathbf{y}} & \mathbf{a}_{\mathbf{z}} \\
\frac{\partial}{\partial x} & \frac{\partial}{\partial y} & \frac{\partial}{\partial z} \\
A_{x} & A_{y} & A_{z}
\end{array}\right|
$$


From Table 2.2 it is known that Maxwell's equations in differential form in a linear medium are given as:

$$
\begin{gathered}
\nabla \times \mathbf{E}=-\frac{\partial \mathbf{B}}{\partial t}, \\
\nabla \times \mathbf{H}=\mathbf{J}+\frac{\partial \mathbf{D}}{\partial t}, \\
\nabla \cdot \mathbf{D}=\rho, \text { and } \\
\nabla \cdot \mathbf{B}=0 .
\end{gathered}
$$

It is also known that:

$$
\begin{gathered}
\nabla \cdot \mathbf{J}=-\frac{\partial \rho}{\partial t}, \\
\mathbf{D}=\varepsilon \mathbf{E}, \text { and } \\
\mathbf{B}=\mu \mathbf{H} .
\end{gathered}
$$

If the initial field distribution is known and satisfies Maxwell's equations, then the above equations are sufficient enough, for linear isotropic materials, to completely specify the field behavior over time. Usually, the field and sources are set to zero at initial time, time equal zero. Thus, equations 5.4 and 5.5 become redundant in this case since they are contained within the curl equations and the initial boundary conditions. For proof of their redundancy see [43]. Thus two curl equations remain. Let the following parameters be defined:

$\sigma:$ the conductivity,

$$
\sigma_{\mathrm{m}} \text { : the magnetic conductivity, }
$$

and

$$
\mathbf{J}=\sigma \mathbf{E} .
$$


Then the two curl equations can be rewritten as follows:

$$
\begin{gathered}
\nabla \times \mathbf{E}=-\frac{\partial \mathrm{B}}{\partial t}=-\frac{\partial}{\partial t}(\mu \mathrm{H})=-\mu \frac{\partial \mathrm{H}}{\partial t}-\mathrm{H} \frac{\partial \mu}{\partial t}, \\
-\mu \frac{\partial \mathrm{H}}{\partial t}=\nabla \times \mathbf{E}+\mathrm{H} \frac{\partial \mu}{\partial t}, \\
\frac{\partial \mathrm{H}}{\partial t}=\frac{-1}{\mu}(\nabla \times \mathbf{E})-\frac{1}{\mu} \frac{\partial \mu}{\partial t} \mathrm{H} \\
\frac{\partial \mathbf{H}}{\partial t}=\frac{-1}{\mu}(\nabla \times \mathbf{E})-\frac{\sigma_{m}}{\mu} \mathbf{H}
\end{gathered}
$$

Similarly for the second equation:

$$
\begin{gathered}
\nabla \times \mathbf{H}=\mathbf{J}+\frac{\partial \mathbf{D}}{\partial t}=\sigma \mathbf{E}+\frac{\partial}{\partial t}(\varepsilon \mathbf{E}), \\
\nabla \times \mathbf{H}=\sigma \mathrm{E}+\mathrm{E} \frac{\partial \varepsilon}{\partial t}+\varepsilon \frac{\partial \mathrm{E}}{\partial t}, \\
\varepsilon \frac{\partial \mathrm{E}}{\partial t}=\nabla \times \mathbf{H}-\sigma \mathrm{E}, \\
\frac{\partial \mathbf{E}}{\partial t}=\frac{1}{\varepsilon}(\nabla \times \mathbf{H})-\frac{\sigma}{\varepsilon} \mathbf{E}
\end{gathered}
$$

Equations 5.13 and 5.17 are the starting point for the FDTD formulations. The formulations only treat the electric and magnetic fields $\mathrm{E}$ and $\mathrm{H}$, while they do not treat the fluxes D and B. Any linear isotropic material property can be specified using these formulations since all four constitutive parameters $\varepsilon, \mu, \sigma$, and $\sigma_{\mathrm{m}}$ are available in the equations. 


\subsubsection{Separating the Fields}

Instead of discretizing the two curl equations to obtain a total field FDTD formulation, the fields will be separated as follows:

$$
\begin{gathered}
\mathbf{E}=\mathbf{E}^{\text {total }} \equiv \mathbf{E}^{\text {incident }}+\mathbf{E}^{\text {scattered }}, \text { and } \\
\mathbf{H}=\mathbf{H}^{\text {total }} \equiv \mathbf{H}^{\text {incident }}+\mathbf{H}^{\text {scattered }} .
\end{gathered}
$$

The separate field approach is followed since this formulation allows the incident field components to be specified analytically throughout the problem space while the scattered fields are found computationally and only the scattered fields need to be absorbed at the problem space outer boundaries.

The scattered field arises in response to the incident field so as to satisfy the boundary conditions, which in this case are Maxwell's equations. For a perfect conductor media, Maxwell's equations require that,

$$
\mathbf{E}^{\text {scattered }}=-\mathbf{E}^{\text {incident }}
$$

For any other case, the scattered fields depend upon the constitutive parameters of the material. At any time the scattered and incident fields can be added to obtain the total field.

When in the media, the scattered fields follow the Maxwell's equations of the media, while outside the media they follow the Maxwell's equations for free space. The incident field, on the other hand, is defined as the field that would be present in the absence of the scatterer, so it always propagates in free space, and it always follows the Maxwell equations for free space.

For linear materials, the incident and scattered fields must satisfy Maxwell's equations independently. In the media of the scatterer, the total field satisfies equations 5.13 and 5.17 as follows 


$$
\begin{gathered}
\nabla \times \mathbf{E}^{\text {total }}=-\mu \frac{\partial \mathbf{H}^{\text {total }}}{\partial t}-\mathbf{H}^{\text {total }} \sigma_{m}, \text { and } \\
\nabla \times \mathbf{H}^{\text {total }}=\varepsilon \frac{\partial \mathbf{E}^{\text {total }}}{\partial t}+\sigma \mathbf{E}^{\text {total }} .
\end{gathered}
$$

By substituting from Equations 5.18 and 5.19,

$$
\begin{gathered}
\nabla \times\left(\mathbf{E}^{\text {incident }}+\mathbf{E}^{\text {scattered }}\right)=-\mu \frac{\partial\left(\mathbf{H}^{\text {incident }}+\mathbf{H}^{\text {scattered }}\right)}{\partial t}-\left(\mathbf{H}^{\text {incident }}+\mathbf{H}^{\text {scattered }}\right) \sigma_{m}, \text { and } \\
\nabla \times\left(\mathbf{H}^{\text {incident }}+\mathbf{H}^{\text {scattered }}\right)=\varepsilon \frac{\partial\left(\mathbf{E}^{\text {incident }}+\mathbf{E}^{\text {scattered }}\right)}{\partial t}+\sigma\left(\mathbf{E}^{\text {incident }}+\mathbf{E}^{\text {scattered }}\right)
\end{gathered}
$$

Equations 5.23 and 5.24 are for the total field behavior in the media. The equations for the incident fields in free space, which are the same as those of the incident waves in the media, are:

$$
\begin{gathered}
\nabla \times \mathbf{E}^{\text {incident }}=-\mu_{0} \frac{\partial \mathbf{H}^{\text {incident }}}{\partial t}, \text { and } \\
\nabla \times \mathbf{H}^{\text {incident }}=\varepsilon_{0} \frac{\partial \mathbf{E}^{\text {incident }}}{\partial t} .
\end{gathered}
$$

where $\varepsilon_{0}$ and $\mu_{0}$ are the permittivity and permeability of the free space, respectively. The conduction terms have been taken out of these free space equations. Now that the total field behavior in the media is given, and the incident field behavior in the media has also been derived, the scattered field behavior in the media can be found by subtracting the latter from the former, as follows:

$$
\begin{gathered}
\nabla \times\left(\mathbf{E}^{\text {incident }}+\mathbf{E}^{\text {scattered }}\right)-\nabla \times \mathbf{E}^{\text {inc }}=-\mu \frac{\partial\left(\mathbf{H}^{\text {incident }}+\mathbf{H}^{\text {scattered }}\right)}{\partial t}-\left(\mathbf{H}^{\text {incident }}+\mathbf{H}^{\text {scattered }}\right) \sigma_{m}+\mu_{0} \frac{\partial \mathrm{H}^{\text {incident }}}{\partial t} \\
\nabla \times \mathbf{E}^{\text {scattered }}=-\mu \frac{\partial \mathbf{H}^{\text {scattered }}}{\partial t}-\mu \frac{\partial \mathbf{H}^{\text {incident }}}{\partial t}+\mu_{0} \frac{\partial \mathbf{H}^{\text {incident }}}{\partial t}-\mathbf{H}^{\text {incident }} \sigma_{m}-\mathbf{H}^{\text {scattered }} \sigma_{m}
\end{gathered}
$$

Regrouping these terms, 


$$
\nabla \times \mathbf{E}^{\text {scattered }}=-\mu \frac{\partial \mathbf{H}^{\text {scattered }}}{\partial t}-\sigma_{m} \mathbf{H}^{\text {scattered }}-\left[\left(\mu-\mu_{0)} \frac{\partial \mathbf{H}^{\text {incident }}}{\partial t}-\sigma_{m} \mathbf{H}^{\text {incident }}\right]\right.
$$

using the same previous analysis,

$$
\nabla \times \mathbf{H}^{\text {scattered }}=\varepsilon \frac{\partial \mathbf{E}^{\text {scattered }}}{\partial t}+\sigma \mathbf{E}^{\text {scattered }}+\left[\left(\varepsilon-\varepsilon_{0)} \frac{\partial \mathbf{E}^{\text {incident }}}{\partial t}-\sigma \mathbf{E}^{\text {incident }}\right]\right.
$$

Now the equations for the behavior of the total fields, 5.21 and 5.22, the incident fields, 5.25 and 5.26, and the scattered fields, 5.28 and 5.29, inside the media have been obtained. The same analysis can be repeated for the free space, but it is easier just to substitute into the previous equations found for the media case with the values of the parameters in the free space:

$$
\begin{gathered}
\mu=\mu_{0}, \\
\varepsilon=\varepsilon_{0,} \text { and } \\
\sigma=\sigma_{\mathrm{m}}=0 .
\end{gathered}
$$

Substituting these values into equations 5.21 and 5.22 the total field behavior in free space are:

$$
\begin{gathered}
\nabla \times \mathbf{E}^{\text {total }}=-\mu_{0} \frac{\partial \mathbf{H}^{\text {total }}}{\partial t}, \text { and } \\
\nabla \times \mathbf{H}^{\text {total }}=\varepsilon_{0} \frac{\partial \mathbf{E}^{\text {total }}}{\partial t} .
\end{gathered}
$$

The incident field behavior is the same as that shown in equations 5.25, and 5.26. As for the scattered field behavior, this becomes

$$
\begin{gathered}
\nabla \times \mathbf{E}^{\text {scattered }}=-\mu_{0} \frac{\partial \mathbf{H}^{\text {scattered }}}{\partial t}, \text { and } \\
\nabla \times \mathbf{H}^{\text {scattered }}=\varepsilon_{0} \frac{\partial \mathbf{E}^{\text {scattered }}}{\partial t} .
\end{gathered}
$$


By examining all the above pairs of equations, it can be seen that

> The equations in free space are special cases of the equations in the scattering media, with $\mu=\mu_{0,} \varepsilon=\varepsilon_{0}$, and $\sigma=\sigma_{\mathrm{m}}=0$.

$>$ The equations for the total field are a summation of those in the scattering field and those in the incident field.

> And the equations for the incident field are only a reminder that the incident field must satisfy Maxwell's equations.

This leaves the equations in the media, equations 5.28 and 5.29. Only these equations need to be determined with the scattered field computationally. And by doing so all the fields would thus be determined.

The next step is to generate the difference equations from equations 5.28 and 5.29. But to make the differencing process easier, the equations should be rearranged such that the time derivative of the field is expressed as a function of the remaining terms. After rearranging the equations the final shape is:

$$
\frac{\partial \mathbf{H}^{\text {scattered }}}{\partial t}=-\frac{\sigma_{m}}{\mu} \mathbf{H}^{\text {scattered }}-\frac{\sigma_{m}}{\mu} \mathbf{H}^{\text {incident }}-\frac{\left(\mu-\mu_{0}\right)}{\mu} \frac{\partial \mathbf{H}^{\text {incident }}}{\partial t}-\frac{1}{\mu}\left(\nabla \times \mathbf{E}^{\text {scattered }}\right)
$$

$$
\frac{\partial \mathbf{E}^{\text {scattered }}}{\partial t}=-\frac{\sigma}{\varepsilon} \mathbf{E}^{\text {scattered }}-\frac{\sigma}{\varepsilon} \mathbf{E}^{\text {incident }}-\frac{\left(\varepsilon-\varepsilon_{0}\right)}{\varepsilon} \frac{\partial \mathbf{E}^{\text {incident }}}{\partial t}+\frac{1}{\varepsilon}\left(\nabla \times \mathbf{H}^{\text {scattered }}\right)
$$

The next step is to difference this set of scattering field equations.

\subsubsection{The Finite Difference Method}

The main concept behind finite difference is the approximation of derivatives in terms of differences [45], so that 


$$
\begin{aligned}
& \frac{\partial f}{\partial t} \equiv \lim _{\Delta t \rightarrow 0} \frac{f\left(x, t_{2}\right)-f\left(x, t_{1}\right)}{\Delta t} \approx \frac{f\left(x, t_{2}\right)-f\left(x, t_{1}\right)}{\Delta t}, \text { and } \\
& \frac{\partial f}{\partial x} \equiv \lim _{\Delta x \rightarrow 0} \frac{f\left(x_{2}, t\right)-f\left(x_{1}, t\right)}{\Delta x} \approx \frac{f\left(x_{2}, t\right)-f\left(x_{1}, t\right)}{\Delta x} .
\end{aligned}
$$

By doing this calculus is converted to algebra since $\Delta \mathrm{t}$ and $\Delta \mathrm{x}$ are finite rather than infinitesimal. This method of differencing is called the explicit central difference scheme.

\subsubsection{FDTD Formulation of the Scattering Equations for Lossy Materials}

By applying the explicit central difference scheme to the scattering Equations 5.34 and 5.35, the required FDTD formulation is achieved. This formulation is derived for any lossy dielectric or lossy magnetic material since it is in its most general form. In the next section the equations derived in this section are used to derive the FDTD formulation for a perfect conductor material.

In the following equations " $\mathrm{i}$ " will be used for incident and "s" will be used for scattering. The analysis will only be given for Equation 5.34, while the final answer for Equation 5.28 will be given directly since it follows the same method of deduction from Equation 5.35:

$$
\begin{gathered}
\frac{\partial \mathbf{H}^{\mathrm{s}}}{\partial t}=-\frac{\sigma_{m}}{\mu} \mathbf{H}^{\mathrm{s}}-\frac{\sigma_{m}}{\mu} \mathbf{H}^{\mathrm{i}}-\frac{\left(\mu-\mu_{0}\right)}{\mu} \frac{\partial \mathbf{H}^{\mathrm{i}}}{\partial t}-\frac{1}{\mu}\left(\nabla \times \mathbf{E}^{\mathrm{s}}\right), \\
\mu \frac{\partial \mathbf{H}^{\mathrm{s}}}{\partial t}=-\sigma_{m} \mathbf{H}^{\mathrm{s}}-\sigma_{m} \mathbf{H}^{\mathrm{i}}-\left(\mu-\mu_{0}\right) \frac{\partial \mathbf{H}^{\mathbf{i}}}{\partial t}-\left(\nabla \times \mathbf{E}^{\mathrm{s}}\right), \\
\mu \frac{\partial \mathbf{H}^{\mathrm{s}}}{\partial t}+\sigma_{m} \mathbf{H}^{\mathrm{s}}=-\sigma_{m} \mathbf{H}^{\mathrm{i}}-\left(\mu-\mu_{0}\right) \frac{\partial \mathbf{H}^{\mathrm{i}}}{\partial t}-\left(\nabla \times \mathbf{E}^{\mathrm{s}}\right),
\end{gathered}
$$

approximating using finite differences leads to 


$$
\begin{gathered}
\mu \frac{\left(\mathbf{H}^{s, n}-\mathbf{H}^{s, n-1}\right)}{\Delta t}+\sigma_{m} \mathbf{H}^{\mathrm{s}, \mathbf{n}}=-\sigma_{m} \mathbf{H}^{\mathrm{i}, \mathbf{n}}-\left(\mu-\mu_{0}\right) \dot{\mathbf{H}}^{\mathrm{i}, \mathbf{n}}-\left(\nabla \times \mathbf{E}^{\mathrm{s}, \mathbf{n}-\frac{1}{2}}\right), \\
\mu\left(\mathbf{H}^{s, n}-\mathbf{H}^{s, n-1}\right)+\Delta t \sigma_{m} \mathbf{H}^{\mathrm{s}, \mathbf{n}}=-\Delta t \sigma_{m} \mathbf{H}^{\mathrm{i}, \mathbf{n}}-\Delta t\left(\mu-\mu_{0}\right) \dot{\mathbf{H}}^{\mathrm{i}, \mathbf{n}}-\Delta t\left(\nabla \times \mathbf{E}^{\mathbf{s}, \mathbf{n}-\frac{1}{2}}\right), \\
\left(\mu+\Delta t \sigma_{m}\right) \mathbf{H}^{s, n}=\mu \mathbf{H}^{s, n-1}-\Delta t \sigma_{m} \mathbf{H}^{\mathrm{i}, \mathbf{n}}-\Delta t\left(\mu-\mu_{0}\right) \dot{\mathbf{H}}^{\mathrm{i}, \mathbf{n}}-\Delta t\left(\nabla \times \mathbf{E}^{\mathbf{s}, \mathbf{n}-\frac{1}{2}}\right),
\end{gathered}
$$

and rearranging this final equation gives:

$$
\mathbf{H}^{s, n}=\left(\frac{\mu}{\left(\mu+\Delta t \sigma_{m}\right)}\right) \mathbf{H}^{s, n-1}-\left(\frac{\Delta t \sigma_{m}}{\left(\mu+\Delta t \sigma_{m}\right)}\right) \mathbf{H}^{\mathbf{i , n}}-\left(\frac{\Delta t\left(\mu-\mu_{0}\right)}{\left(\mu+\Delta t \sigma_{m}\right)}\right) \dot{\mathbf{H}}^{\mathbf{i}, \mathbf{n}}-\frac{\Delta t}{\left(\mu+\Delta t \sigma_{m}\right)}\left(\nabla \times \mathbf{E}^{\mathbf{s}, \mathbf{n}-\frac{1}{2}}\right)
$$

using the same analysis on equation 5.35 gives:

$$
\mathbf{E}^{s, n}=\left(\frac{\varepsilon}{(\varepsilon+\Delta t \sigma)}\right) \mathbf{E}^{s, n-1}-\left(\frac{\Delta t \sigma}{(\varepsilon+\Delta t \sigma)}\right) \mathbf{E}^{\mathbf{i}, \mathbf{n}}-\left(\frac{\Delta t\left(\varepsilon-\varepsilon_{0}\right)}{(\varepsilon+\Delta t \sigma)}\right) \dot{\mathbf{E}}^{\mathbf{i}, \mathbf{n}}-\frac{\Delta t}{(\varepsilon+\Delta t \sigma)}\left(\nabla \times \mathbf{H}, \mathbf{n}-\frac{\mathbf{1}}{\mathbf{2}}\right)
$$

Equations 5.43 and 5.44 are the FDTD formulation for a lossy material. By defining the parameters $\mu, \mu_{0}, \varepsilon, \varepsilon_{0}, \sigma$, and $\sigma_{\mathrm{m}}$ the computations can proceed on these equations.

\subsubsection{FDTD Formulation of Scattering Equations for a Perfect Conductor}

For a perfect conductor $\sigma=\infty$, by substituting this in the equation 5.35 above it is found that,

$$
\mathbf{E}^{\text {scattered }}=-\mathbf{E}^{\text {incident }}
$$

Also, by substituting $\sigma=\infty$ in equation 5.44 the same result is achieved which confirms the analysis. Using this result, and decomposing the vector Maxwell curl equations governing the scattered fields into their component scalar parts, then differencing as in the previous section the final results are achieved, for all the components, as 


\section{$\underline{\text { Electrical }}$}

$$
\frac{\mathbf{E}_{x}^{s, n}-\mathbf{E}_{x}^{s, n-1}}{\Delta t}=\frac{1}{\varepsilon_{0}}\left[\frac{\Delta \mathbf{H}_{z}^{s, n-\frac{1}{2}}}{\Delta y}-\frac{\Delta \mathbf{H}_{y}^{s, n-\frac{1}{2}}}{\Delta z}\right]
$$$$
\frac{\mathbf{E}_{y}^{s, n}-\mathbf{E}_{y}^{s, n-1}}{\Delta t}=\frac{1}{\varepsilon_{0}}\left[\frac{\Delta \mathbf{H}_{x}^{s, n-\frac{1}{2}}}{\Delta z}-\frac{\Delta \mathbf{H}_{z}^{s, n-\frac{1}{2}}}{\Delta x}\right]
$$$$
\frac{\mathbf{E}_{z}^{s, n}-\mathbf{E}_{z}^{s, n-1}}{\Delta t}=\frac{1}{\varepsilon_{0}}\left[\frac{\Delta \mathbf{H}_{y}^{s, n-\frac{1}{2}}}{\Delta x}-\frac{\Delta \mathbf{H}_{x}^{s, n-\frac{1}{2}}}{\Delta y}\right]
$$

\section{$\underline{\text { Magnetic }}$}

$$
\frac{\mathbf{H}_{x}^{s, n+\frac{1}{2}}-\mathbf{H}_{x}^{s, n-\frac{1}{2}}}{\Delta t}=\frac{1}{\mu_{0}}\left[\frac{\Delta \mathbf{E}_{y}^{s, n}}{\Delta z}-\frac{\Delta \mathbf{E}_{z}^{s, n}}{\Delta y}\right]
$$

These are the perfect conductor field formulations, a special case of the field formulations of the scattering equations for the lossy materials.

\subsection{Related Research Using FDTD}

Numerous research has been done using the FDTD method. In this section a brief view on some of this research in different fields is provided, and most specifically in the field of antennas.

In his research on feed models using FDTD, Luebbers [38] suggests several relatively straight forward approaches to accurately and efficiently model antenna and microstrip feeds within the constraints of FDTD approximations. The methods suggested include simple approaches, as gap and frill feeds, in addition to some complicated approaches that involve including the coaxial cable in the FDTD calculation space. The reduction of the required time steps to reach convergence of the calculations was one of the important points of interest in this study. But Luebbers teaches that, for most of the antenna geometries, a simple gap model with an internal source resistance provides 
accurate results while greatly reducing the number of time steps required for convergence.

Luebbers also uses the FDTD method to report calculations of absolute gain and radiation efficiency for a variety of antenna geometries [50]. A pulsed excitation is used in order to report the gain and efficiency within a wide band from a single FDTD computation. Luebbers then confirms his findings by comparing the results from his method with those obtained using the method of moments. In another study [37], he provides a detailed description of the FDTD transformation from near zone to far zone in the time domain. The results are validated by comparing the results of far zone scattering of a flat plate transformed to the frequency domain with the same case using the method of moments.

Reineix and Jecko [39] performed an analysis of the characteristics of microstrip patch antennas using the FDTD method. They modified the method in order to apply it to the microstrip patch antennas, and with appropriate choices of excitation methods the frequency dependence of several parameters was found using Fourier transformation of the transient current. Different types of excitations were used, including coaxial excitation, and microstrip lines. They plotted the spatial distribution of the current density, which gave information on the resonance modes. And finally, several frequency dependent parameters were provided, including the input impedance and the radiation pattern.

When time domain electromagnetic field equations are solved using finite difference techniques in unbounded space, a method for limiting the domain in which the field is computed has to exist. This is achieved by using absorbing boundary conditions 
as described in previous sections of this chapter. In his study, Mur [42] presents highly absorbing boundary conditions for electromagnetic field equations that can be used for both $2 \mathrm{D}$ and $3 \mathrm{D}$ configurations. He also provides numerical results that clearly exhibit the accuracy and limits of applicability of highly absorbing boundary conditions.

Chen et al. used the FDTD method to analyze a dielectric leaky-wave antenna comprising metal strips etched on a rectangular dielectric rod [49]. They determined the radiation patterns of the leaky-wave antenna, and discussed the effects of the launching discontinuity on the performance of the antenna. They also described the method of applying the perfect matched layer (PML) technique to the 3D dielectric waveguide and its performance. The effects caused by the perturbation on the wave propagation characteristics of dielectric waveguides were also discussed. They verified there results using experimental results, and found them to be in good agreement.

Other research includes the research done by Kashiwa et al. on the analysis of microstrip antennas on a curved surface [51]. They developed a curvilinear FDTD method, and showed how it provided excellent versatility in modeling and analyzing microstrip antennas mounted on curved surfaces. They further confirmed their method by comparing the numerical results to experimental results and showed how they agree within acceptable limits.

Taflove et al. provided a detailed analysis of electromagnetic fields penetrating narrow slots and lapped joints in thick conducting screens using the FDTD method [36]. They used a Faraday's law counter-integral approach to develop new and simple modifications of the basic FDTD algorithm to properly model the slot physics. They then validated their results using samples of method-of-moment models. 
The ability of the FDTD method to calculate radiation patterns, input impedance, and gain for a monopole antenna on a conducting box is demonstrated in another research project by Luebbers [52]. Results of this study are provided for both a bare box, and a box coated with a dielectric layer. Radiation patterns are compared with experimental measurements, and with the results using the method of moments for the bare box. Radiation patterns for the dielectric covered box, and all impedance and gain results are compared with experimental results. The study shows good agreement in all of the above mentioned cases. The study also shows how it is much easier to include the dielectric on the covered box in the FDTD approach than it would be for the method of moments approach.

A theory for a complete far field transmit-receive system characterization of short pulse antennas is derived in the time domain by Shlivinski [48]. The transmit-receive antenna system is characterized by a set of cascaded operators, which transform the source waveform and power into similar quantities at the receiving antenna terminals. Two sets are defined, the first is in terms of the wave-type time dependent effective height operator, while the second is defined in terms of the energy type gain operator. These operators are derived entirely in the context of the time domain field equation. Shlivinski demonstrates the concepts developed in his research on a simple short dipole antenna.

In another study on applying the FDTD method for antenna radiation, Tirkas and Balanis [53] use the FDTD method to model and predict the radiation patterns of wire and aperture antennas of three basic configurations. They also provide a critical approach to modeling the feed. The results they obtain are compared to those obtained using the 
geometrical theory of diffraction (GTD). They showed that the computed radiation patterns compared well with measurements.

The stability condition and the accuracy of the exponential and centered timedifferencing schemes for FDTD in an isotropic homogeneous lossy dielectric where the electric and magnetic conductivities are determined and analyzed by Petropoulos [54]. He shows that these schemes are equivalent, and shows that both schemes must be used with a time step that resolves the electric and magnetic conduction current relaxation time scales. He also discusses the implications of these results for PML type absorbing boundary conditions.

In another study on curved surfaces, a hybrid FDTD method is proposed by $\mathrm{Wu}$ [55] for solving transient electromagnetic problems associated with structures of curved surfaces. The method employs the conventional FDTD method for most of the regular region but introduces the tetrahedral edge-based finite-element scheme to model the region near the curved surfaces. Wu finds that, without any interpolation of the fields on the curved surface or any additional stability constraint due to the finer division near the curved surface, the finite element scheme has a high accuracy and unconditional stability. The scheme is also shown to have high computational efficiency and programming ease. He applies the hybrid method to find the electromagnetic scattering of 3D arbitrarily shaped dielectric objects to demonstrate its superior performance.

This was just a brief sample of some of the extensive research that is being applied on antennas using the finite difference time domain method. It is out of the scope of this study to go into more details in any of these studies, since the aim behind providing this section was just to show how the applications in this field are oriented in 
general. Numerous other studies can be found in the IEEE transactions on antennas and propagation, the IEEE transactions on microwave theory and techniques, and in the IEEE transactions on electromagnetic compatibility.

\subsection{The XFDTD Software Package}

XFDTD is an X-Windows finite difference time domain graphical user interface for electromagnetic calculations. The version described in this section is Remcom's version 5.0. The code is very general in the materials and geometries that it can handle, due to the power and simplicity of the FDTD method as shown in the previous section. But the code requires a very powerful computer to be able to solve for even the simplest of problems due to the high memory and processor usage. A server, workstation, or at least an extremely powerful up-to-date PC is required, or else the solvable problems will be very limited and would require extremely long hours of computation. At CIRA-WVU a powerful Digital Alpha workstation is used, with a 64 bit architecture Alpha processor of $500 \mathrm{MHz}$ speed and a random access memory of 1GB. But even with this extremely powerful machine complicated problems can sometimes take hundreds of $\mathrm{cpu}$ hours to solve. An average PCB-CTHA transient problem, with a 30 cell empty space from each direction and 20,000 time steps, usually takes about 12 hours of run time on this workstation. In the following section the general method for performing FDTD calculations using XFDTD is discussed, the XFDTD capabilities are listed, and the input files required to perform a calculation are described. 


\subsubsection{The General Method for Performing FDTD Calculations Using XFDTD}

\subsubsection{Geometry}

In XFDTD the entire working space is approximated in the shape of nodes in Cartesian coordinates. Each node holds an address depending on its location in these coordinates. All geometries are then defined as discrete cell edges, where a cell edge is defined as the edge of a cell initiating from a certain node in the positive coordinate direction from that node. So every node in the working space needs six parameters to define it; three to define the $\mathrm{x}, \mathrm{y}$, and $\mathrm{z}$ location of the node, and three to define the material of the cell edge initiating from that node in the positive $\mathrm{x}, \mathrm{y}$, and $\mathrm{z}$ directions. Thus, all the geometry has to be approximated into a number of nodes and edges, surfaces, or volumes of rectangular cells.

Each cell edge is defined by a certain material on that edge, as stated above. These materials are identified by different properties that can be defined by the user. XFDTD provides two predefined materials, these are materials for empty space and for a perfect conductor. The user, on the other hand, is provided with fourteen other materials that can be user defined, in addition to four wire thickness materials. The empty space takes a material number 0 , the perfect conductor takes a material number 1 , the 14 user defined materials take the numbers 2 to 15 , and the thick wire materials take numbers 16 to 19 . Table 5.1 shows a list of all sixteen material properties that can be defined by the user. In the current problems, the only material property that required to be defined is the relative permittivity, or the dielectric constant of the material.

For accurate results, the cell edges must be smaller than approximately one tenth the operating wavelength [44]. They should also be small enough to approximate the 
geometrical features of the desired model. The graphical user interface provides several alternatives for meshing the desired models, including manual modeling using the mouse, using predefined shapes in XFDTD to build up the mesh, like plates, cylinders, and spheres, or importing predefined mesh geometry files created by another application. In these cases, due to the complexity of the geometry of the PCB-CTHA, the latter approach was chosen where a flexible mesh generator was created. This mesh generator is described in the next section.

Table 5.1 The user defined material properties in XFDTD [44]

\begin{tabular}{|l|l|}
\hline Relative Permittivity & Damping Coefficient \\
\hline Conductivity S/m & Type (Normal, Debye, or Lorentz) \\
\hline Relative Permeability & Larmor Precision Frequency \\
\hline Magnetic Conductivity & Saturation Magnetization rad/sec \\
\hline Material Density kg/m ${ }^{3}$ & Magnetic Damping Coefficient \\
\hline Static Permittivity & Magnetic Field Theta \\
\hline Relaxation Time & Magnetic Field Phi \\
\hline Resonant Frequency & $\begin{array}{l}\text { Magnetic Ferrite Type } \\
\text { (normal or anisotropic ferrite) }\end{array}$ \\
\hline
\end{tabular}

The outer boundary of the XFDTD mesh is, by default, defined as an unbounded space, so it is set to absorb all the radiated and scattered fields that reach it. This absorption mechanism requires a free space of cells around the modeled geometry and the outer boundary limits. Although the recommended space by Remcom [44] is 6 to 15 cells, from previous experience of the researchers in CIRA it was found that at least 20 cells are required for the PCB-CTHA calculations in order to get accurate results. This 
free space boundary is extremely important in the case of far zone fields and radiation patterns.

\subsubsection{Calculation Parameters}

After defining the geometry, the next step is to define the FDTD calculation parameters upon which the desired run will be performed. The first parameter to be defined is the excitation method. XFDTD allows for several excitation methods, including excitation by an incident plane wave, for scattering or penetration problems, or excitation by a voltage source connected to the geometry, for microstrip or antenna radiation parameters. In the problems of this report the voltage source excitation method is always used.

Next, the form of the wave has to be defined. The wave can either be a transient pulse or a sine wave. The output quantities required also have to be defined by the user. XFDTD provides several output quantities, but increasing the output quantities requested increases the calculations to be performed by XFDTD and thus increases the run time of the problem. Output quantities include far zone fields in particular directions, near zone fields at particular locations or in specific planes, input impedance, and many more. The quantity of greatest interest in studying CTHAs is the far zone fields.

\subsubsection{Post Processing the Results}

After defining the geometry and the calculation parameters, the FDTD calculations take place. Once the calculations are complete, the graphical user interface is once again used to post process the results of the calculations, and to display the required graphs and quantities. The output files can also be custom processed by the user if desired, since the files are provided in text format. Due to the repetitive nature of the post processing 
required in this research, custom built modules were developed to post-process, analyze, and rearrange the output files. These custom built modules included modules for finding the resonant frequencies, plotting the far field information, calculating the input impedance charts, and interpolating between the results to find the desired result at any specific point.

\subsubsection{XFDTD Capabilities}

Following is a list of some of the most important capabilities of the XFDTD graphical user interface and calculation engine as described in the XFDTD user manual [44]. Many other features exist, but these are the capabilities most important for the specific calculations required in this text. Using XFDTD the user can:

> Model lossy dielectric, lossy magnetic, and perfect conductors,

> Include dielectric materials with frequency dependent constitutive parameters,

> Display, generate, and modify meshed geometries graphically,

> Merge several meshes together to combine objects,

$>$ Display geometries in 3D, or in 2D slices,

> Use already built libraries of basic geometry shapes,

> Define the outer boundary of the working space as either an absorbing boundary or a perfect conductor boundary,

> Choose an incident plane wave, or near zone voltage source excitation,

> Choose the excitation method for the voltage source from several methods, including a Gaussian pulse, a Gaussian derivative pulse, a modulated Gaussian pulse, or a sinusoidal excitation, 
> Transient near zone to far zone transformation for transient scattering and radiation calculations,

$>$ Steady state near zone to far zone transformation for efficient calculation of single frequency radiation patterns, including circular polarization, with user adjusted angle increments,

> Calculation of wide bandwidth impedance from Fast Fourier Transformations of transient voltage and current,

> Calculation of single frequency, steady state, antenna impedance, input power, and efficiency,

> Near zone sampling of electric and magnetic fields and currents,

> Color intensity graphical display of near zone field parameters,

$>$ Line plots of near zone and far zone transient fields vs. time, impedance vs. frequency, radiation patterns, and other results,

Display and operate on user generated meshed geometries, and

> Output files available for processing with user-generated post-processing software.

\subsubsection{Description of the XFDTD Input Files}

Only two types of input files are needed for this research, the geometry file and the calculation parameters file. Following is a brief description of each of these files, while Appendix $\mathrm{C}$ shows a sample of these input files. Although the input files described in Appendix $\mathrm{C}$ were generated using the flexible mesh generator discussed next, they are exactly identical to the files created by the XFDTD graphical user interface. 


\subsubsection{Geometry File}

The geometry file starts with defining the grid size which is the number of cells in the $\mathrm{x}, \mathrm{y}$, and $\mathrm{z}$ directions. The cell sizes in the $\mathrm{x}, \mathrm{y}$ and $\mathrm{z}$ directions are then defined, followed by the location of this geometry if it is to be used as a sub grid. Then the material properties are specified for all the 14 user defined materials, and for the four thick wire materials if needed. These are followed by the number of nodes that have other than empty space material on the edges coming out of these nodes in any direction. And finally there is a listing of all the nodes and the material types on the edges generating from them.

\subsubsection{Calculation Parameters File}

For flexibility, XFDTD uses flags in its calculation parameters files to indicate if a feature is to be used or not. A "0" flag indicates that a feature is not to be used, while a "1" flag indicates that it is to be used. This helps in making one type of file for any kind of input source.

It is to be noted that XFDTD makes its calculations based on time steps of size $\Delta \mathrm{t}$ that can either be user defined, or left to the processing module to determine. The number of time steps is also user defined, but the code provides a recommended number of time steps according to the required frequency. This recommendation is to ensure that steady state would occur. Also, although a 3D view of the geometry can be shown for the geometry, all radiation patterns and graphs and plots are only shown in 2D sections. The main method of viewing any object is in $2 \mathrm{D}$ slices of that object. The slices can only be perpendicular to the three main axis $\mathrm{x}, \mathrm{y}$, and $\mathrm{z}$.

The following parameters are a list of some of the main parameters that are user defined in the specific cases found in this report: 
> The name and path of the file(s),

> The pulse width of the Gaussian pulse,

$>$ The total number of time steps,

$>$ The wavelength,

$>$ The input form of the source,

The planes upon which the output is desired to be saved,

> The far zone locations required, and

> The FFT size for input impedance calculations.

\subsection{The PCB-CTHA Preprocessor}

Due to the complexity of the geometry of the PCB-CTHA, the modeling process for each antenna would require a significant amount of time and effort. Thus, a computer code was developed to function as a mesh generator for XFDTD input files. The sole aim of this code is to provide a user friendly, efficient, and flexible package that would increase the accuracy of the PCB-CTHA models while saving modeling time.

In this section a detailed look at the geometry of the PCB-CTHA will be provided. This will be followed by a thorough description of the developed code. A complete listing of the code can be found in Appendix A. Appendix B shows a capture of the forms of the executable package, while Appendix $\mathrm{C}$ provides sample output files from an actual execution process of the package.

\subsubsection{Algorithms for the Generator}

In this section, the basic algorithms upon which the flexible mesh generator code was developed will be discussed. The software developed is able to create both the geometry file and calculation parameter file for any PCB-CTHA. It is to be made clear 
however, that although the geometry is very complicated as shown earlier, the software has been made extremely general to accommodate any shape, size, or specification of a PCB-CTHA required, while at the same time providing ease of use, and reliable output files.

After the user provides all the required data, the program calculates the optimum cell size and optimum number of cells in each direction for the given data. This choice is based on three criterions that are each evaluated separately, and then the safest of these three criterions is selected. The three criterions are that the wavelength should be at least made of ten cells, as advised by XFDTD [44] for accurate calculations, that the width of the wire should be made of at least the number of cells specified by the user, and that the hole diameter should have no less than three nodes.

The program then creates a matrix that resembles the whole space. This matrix is initialized to all zeros. The size of the matrix is equal to the number of nodes in the $\mathrm{x}$ direction times the number of nodes in the $\mathrm{y}$ direction times the number of nodes in the $\mathrm{z}$ direction. Each memory location in this three dimensional matrix includes three values, which represent the information of the material of the edge going out in each of the three directions $\mathrm{x}, \mathrm{y}$, and $\mathrm{z}$ from this specific node.

The next step for the program is to define all the points from which all the traces and holes will start. Each one of these traces or holes is defined by the $\mathrm{x}, \mathrm{y}$, and $\mathrm{z}$ location of its center. These defined center points are then saved in another matrix. The program then starts by defining the entire geometry of all the holes required for the mesh. To do this, the code has to continuously check for the center locations of the starting and 
ending point of each hole from the locations matrix, and draw the hole between these two points based on the diameter provided by the user.

The process of "drawing the holes" is actually a process of defining all the nodes in the mesh matrix that are to be located on the surface of a cylinder. Then for each node, the code has to determine the type of material to assign to each direction, should it be a conductor material, a board material, or empty space material, since all edges in XFDTD are defined by material coming out of defined nodes. But due to the complexity of the previous process, it will sometimes be found that some extra edges will exist coming out of the holes and standing in the air. This is due to the extra cautiousness put in the code to ensure that no edges are left out, causing gaps in the model that could affect the results. So a process of "cleaning" is defined where the program has to go around all holes and smooth their surfaces to ensure no extra residual edges still exist.

The next step after all the holes are defined and all the nodes information are saved in the geometry matrix is to define the traces of conducting material on all four layers of the board. First, the traces for the first wound are defined by a special algorithm that, based on the current location of the trace, calculates the end point of the trace and defines all the nodes of a polygon inside which the trace should be located. The same process is then repeated for the second wound by using the same algorithm. In both cases, the wound ends at the trace that is to be eliminated, and the four feed point locations are identified and labeled A through D. Finally, depending upon the type of feed chosen by the user, the code generates the traces for the feed location. The code also calculates the exact location of the center of the feed. This location will later be used while generating the calculation parameters output ".fdtd" file. 
As with the holes, the traces also need to be cleaned up from all the extra extensions coming out of them. In doing this, all the nodes defining the traces and holes are rechecked by a special algorithm that "cleans" them up. By doing this then all the nodes, edges, and materials would be defined, and the whole model is now fully defined in one large matrix.

The final step is to print out all the output files. All the data provided by the user, in addition to all the calculated data are modified to the form that is accepted by XFDTD. The space matrix is read node by node and checked for any nodes that have no material defined on their edges. These nodes are skipped, and only the nodes with at least one defined edge are regarded in the output.

The two output files, the ".id" file and the ".fdtd" file, are generated and saved in the desired location specified by the user. These files are now ready to be processed by XFDTD. If any minor changes need to be made, especially in the calculation parameters, then these can be done directly from the XFDTD user interface.

\subsubsection{The Software Code}

The developed PCB-CTHA preprocessor and mesh generator was created using Visual Basic 5.0 (VB). The software developed in this research is made up of 12 modules; 7 forms and 5 program codes. Following is a brief description of each of these

modules, while Appendix A shows a complete listing of all the code for each of these 12 modules. All of the following input forms and modules were developed by the author for the sake of creating a user friendly interface that can more easily generate a PCB-CTHA mesh. 


\subsubsection{Input Forms}

Forms in VB are the means by which the program communicates with the user. They are just like the windows that are used in all Windows Operating System programs. Forms are also code of programming language, but the difference between them and the code modules is that they provide an output interface for accepting and providing data, while code modules mainly utilize this data to perform calculations upon them and provide results $[46,47]$. Seven forms were created by the author for this software, all for the sake of input, since the output, as previously mentioned, is in the shape of two files. Following is a description of these seven forms, while the shapes of these forms are provided in Appendix B,

1. Filename: This form allows the user to identify the folder in which he wants the output files to be saved, and a generic name for the output files. The program takes this name and adds to it the extensions ".id" for the geometry file and ".fdtd" for the calculations parameters file.

2. Mainsub: This is the main form from which all the other forms are called. It provides access to the geometry, material properties, and calculation parameters forms. In addition it gives the order for the program modules to start doing the calculations. This form remains on the screen until all the calculations are finished to notify the user that the calculations are still in progress. Quitting either the filename form or the mainsub form ends the program.

3. CTHA: This form allows the user to input and modify all the geometry parameters of the PCB-CTHA. These parameters include: The diameter of the holes, the width of the traces and the minimum allowed number of cells per trace width, the length, the width, and the height of the CTHA, the four planes upon 
which the traces will be identified, the diameter of the inner and outer circles upon which the holes will be identified, the hole type (filled or hollow), the number of holes per revolution, the highest working frequency under which this CTHA will be evaluated, the desired number of free cells between the geometry borders and the mesh outer border, and the required feed type. Some predefined antennas are already available in a drop box for repeated usage, and default data already exists for rapid usage, so that at any time the user can go back to the default values and use them without modification.

4. Specs1: On this form all the material properties of both the traces and the binder material are specified. The default for the traces is a perfect conductor, and the default for the binder is empty space. At any time the user can return to these defaults, or he can specify properties of his own.

5. Gaussfdtd: This form is used to specify the calculation parameters of the run. This information will be used essentially to generate the ".fdtd" file. Information includes: The pulse width, the time steps, the FFT size for impedance calculations, and the information for the number of postprocessor files to be generated after the calculations are made. Again, default values are given for the user to use at any time.

6. Testprint: This form is used by the user to check all the input parameters that have been provided to the package just before starting the calculations. This is a listing of all the main properties required, and their values upon which the calculations will proceed. It allows the user to check for any errors and return to correct them before program execution. 
7. Suggfreq: After the code has done all of its calculations, and just before quitting, this form is displayed. It provides four pieces of information that describe the antenna. These are the total length of all the conductor material, how much of this total length is in the shape of holes, and how much is in the shape of traces, in addition to the "suggested" resonance frequency based on half the wavelength. Of course the real frequency will only be found after the actual execution of XFDTD, but this is a rough figure for the user to start his trial and error procedure if he wishes to do so.

It is important to note that the forms have been designed to check for any errors done by the user and correct them automatically. The forms also provide suggestions for some values when changes are made in other values. Thus automatically convert the type of the data, in most cases, from those given by the user to those required by the program if this needs to be done.

\subsubsection{Modules}

As previously stated, the program calculations are really made in the code modules. These are the modules that take the data provided by the user to the input forms described above and perform the required calculations using this data to reach the desired output. Intense calculations are made with the data since the geometry is very complicated as described previously. Following is a brief description of the five modules developed by the author, while a complete commented listing of the code of these modules is provided in Appendix A: 
1. Mainmod: This is a very short module that acts as the focus for the whole program. It is this module that controls the display of the forms and the execution of the functions at the desired times.

2. Properties: In this module all the parameters that were inputed by the user are modified to take the shapes that can later be of use to the other modules. The parameters are also assigned there respective variables, and formatted in their required data types. This module also controls the initialization of all the property variables, and holds the default values that would be called if required by the user.

3. Holes: This module does all the calculations from the identification of the centers of the holes, to drawing the holes, to calling the cleaning function for the holes. In this module, the initial empty space of the geometry is declared and initialized. The program leaves this module after all the holes have been identified and cleaned up and ready for the traces to be added.

4. Wires: As with the holes module, this module identifies all the traces, draws them in the space matrix, and calls the function that cleans them up. At the end of this module all the geometry would be defined and the software would be ready to print out the results.

5. Printout: Finally, this is the module that controls the output shape of the files. In this module the ".id" file is first printed out, followed by the ".fdtd" file. Both files are then located in the desired place initially specified by the user.

From within XFDTD, the two files are automatically called just by opening the ".fdtd" file. By opening this file it first loads the geometry file, followed by the calculation parameters. Even though the calculations can be held out without even 
opening XFDTD by calling the ".fdtd" file from the prompt, but it is usually better to check in XFDTD if any modifications are required, then perform the computations.

\subsubsection{The Executable File}

The user does not have to go through all the modules listed above to be able to activate the program. All the modules are compiled and linked together to make one executable file that executes the program just by a click. The executable file, which has already been created by the author, also prevents the user from performing any modifications to the original files. The executable file will show on the windows desktop with an icon just like any other program, and will have the extension ".exe". This file was given the name "PCB-CTHA" 


\section{Model Creation and Development}

In this chapter the FDTD model of the PCB-CTHA will go under extensive numerical investigation seeking to find the best model parameters for future PCB-CTHA evaluations. First, several PCB-CTHA antennas will be selected and their dimensions and characteristics will be defined. Then one of these chosen antennas will be modeled using the preprocessor designed in the previous chapter. The selected antenna design will also be examined experimentally in an anechoic chamber. These parameters include the effect of the free space, the time steps, and the binding material. From the results of this investigation an optimum design will be defined, and this optimum model design will be later used in all the numerical calculations.

\subsection{Antenna Selection}

The CIRA inventory contains numerous PCB-CTHA antennas that have been manufactured, but none of which has been numerically evaluated before. One antenna from the CIRA inventory was selected, this antenna had the code number CB108. Table 6.1 shows the geometrical parameters of this antenna.

\subsection{Model Development}

It is desired in this section to design an optimum PCB-CTHA FDTD model that would yield accurate results while maintaining the lowest possible computational time. CB108, defined above, will be used for all the runs made in this section. All the results obtained on CB108 in this section are assumed to apply to the other PCB-CTHA's, too.

The first steps in developing and understanding the characteristics of an FDTD model of a PCB-CTHA will be to define the relative permittivity of the material to be equal to that of empty space $(\mathrm{P}=1)$, and the traces to be perfect conductors. These 
parameters are the basic configuration for the simplest model. In this case the focus will be on the effect of the time steps and the free space on both the transient and the steady state cases.

Table 6.1 The chosen antenna, and its dimensions

\begin{tabular}{|l|c|}
\hline Dimension & CB108 \\
\hline Length/Width (mm) & 45.72 \\
\hline Height (mm) & 2.54 \\
\hline $\begin{array}{l}\text { Diameter of Holes } \\
\text { (mm) }\end{array}$ & 1.676 \\
\hline $\begin{array}{l}\text { Thickness of traces } \\
\text { (mm) }\end{array}$ & 0.847 \\
\hline $\begin{array}{l}\text { Height of layer 2 of } \\
\text { traces (mm) }\end{array}$ & 1.693 \\
\hline $\begin{array}{l}\text { height of Layer 3 of } \\
\text { traces (mm) }\end{array}$ & 33 \\
\hline $\begin{array}{l}\text { Diameter of Inner } \\
\text { circle of holes (mm) }\end{array}$ & 16.5 \\
\hline $\begin{array}{l}\text { Diameter of Outer } \\
\text { circle of holes (mm) }\end{array}$ & Hollow \\
\hline $\begin{array}{l}\text { Number of holes per } \\
\text { circle (mm) }\end{array}$ & \begin{tabular}{l} 
Type of holes (mm) \\
\hline
\end{tabular} \\
\hline
\end{tabular}

After reaching an optimum configuration for this ideal case, a further investigation will then be done upon the effect of the relative permittivity, and how this will reflect upon the previous findings for the time steps and free space. Finally the same will be done with the conductivity of the traces.

\subsubsection{Mesh Properties Selection}

The fundamental constraint in XFDTD for the cell size is that it must be less than the smallest wavelength for which accurate results are desired, with 10 cells per wavelength recommended [44]. Since the frequency range of concern is from $900 \mathrm{MHz}$ 
to $2 \mathrm{GHz}$, then the smallest wavelength would be correspondent to the highest frequency, which is $2 \mathrm{GHz}$. The wavelength at $2 \mathrm{Ghz}$ is 0.15 meters, thus one tenth of the highest frequency is 0.015 meters, or $15 \mathrm{~mm}$.

The other important constraint when using XFDTD for predicting far field patterns is that an outer boundary of empty cells, which is required around the antenna model in all directions. According to the XFDTD manual "a minimum spacing is 10 cells, with 15 or even more sometimes required for accurate results ... separating the geometry from the outer boundary by approximately $1 / 3$ wavelength at the highest frequency of interest is a good rule to follow." [44]

Since the wavelength is $150 \mathrm{~mm}$, then one third of a wavelength is $50 \mathrm{~mm}$. Dividing that by 10 cells, as a minimum number of spacing cells, would provide a maximum cell size of $5 \mathrm{~mm}$. Since the first constraint yielded a maximum cell size of 15 $\mathrm{mm}$, which is more than the $5 \mathrm{~mm}$ restraint in the second point, then the $5 \mathrm{~mm}$ maximum cell size is used.

When modeling the antenna though, a third geometry constraint appeared. It was found that in order to model the traces on the printed circuit board accurately the traces need a width of at least 2 cells. The width of the traces, from Table 6.1, is $1.676 \mathrm{~mm}$, thus a maximum cell size of $0.838 \mathrm{~mm}$ needs to be used. Since $0.838 \mathrm{~mm}$ satisfies the first two conditions mentioned earlier, then it is found that satisfying this condition alone is enough to satisfy all the cell size requirements.

In the PCB-CTHA preprocessor developed, the user is asked to define the traces width, the required number of cells per trace width, the desired frequency range, and the number of spacing cells around the model. Based on these inputs the three mentioned 
conditions are checked and the optimum cell size is found. The cell sizes used were within the range of 0.4 to $0.8 \mathrm{~mm}$, since 2 to 4 cells per trace width were used in all the models.

Figure 6.1 shows an $\mathrm{X}-\mathrm{Y}$ plane view of an actual model of the PCB-CTHA CB108. The model includes an outer spacing of 30 free cells, and the edge size for each of these cells is $0.4 \mathrm{~mm}$.

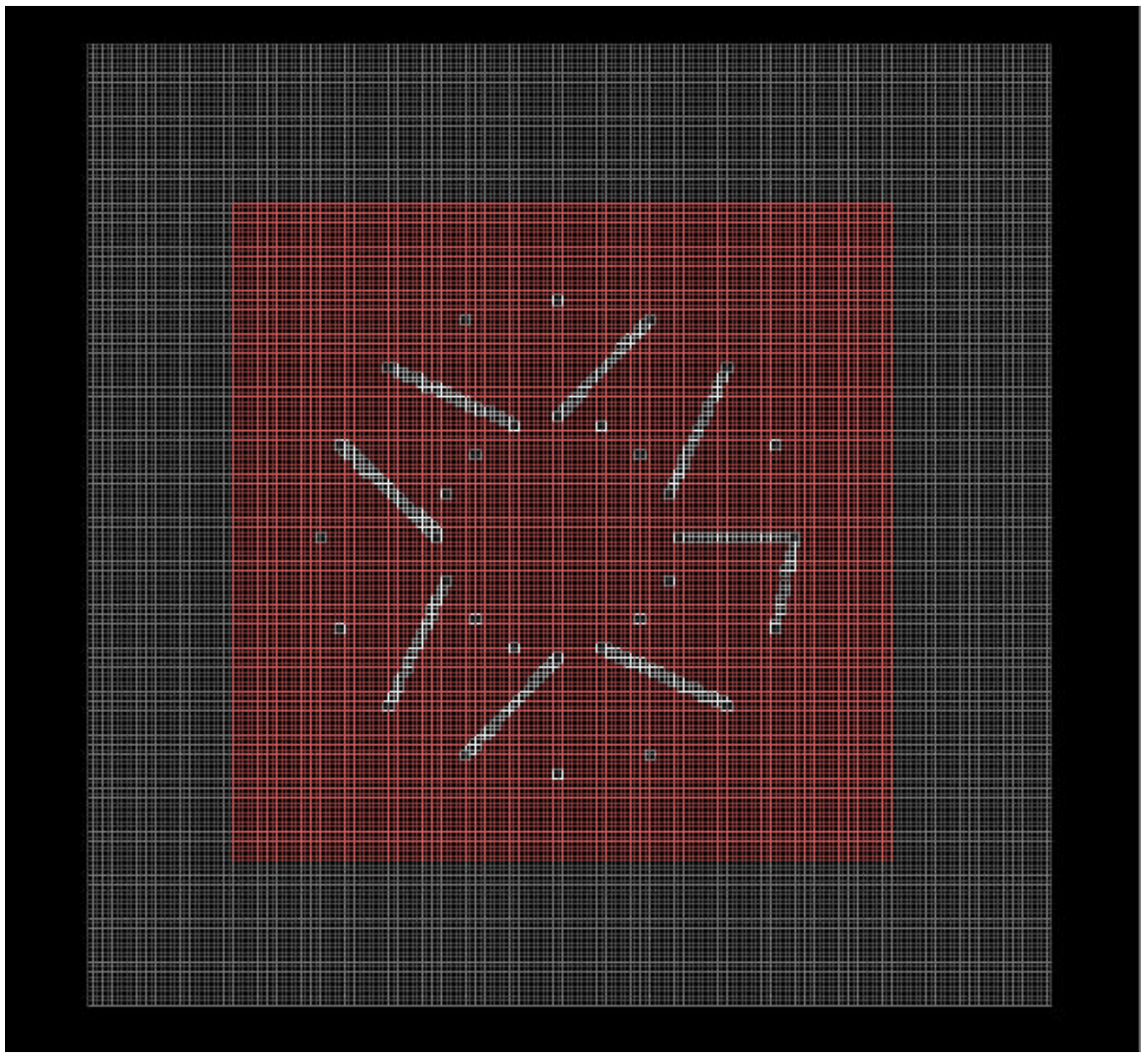

Figure 6.1 Sample of the PCB-CTHA Mesh 


\subsubsection{Effect of Free Space}

The effect of free space on a PCB-CTHA with perfectly conducting traces and $\mathrm{P}=1$ was studied on both steady state and transient cases.

\subsubsection{Steady State Case}

For the steady state case, a random frequency of $1 \mathrm{GHz}$ was chosen. The only basis of choosing this frequency was because it is a desirable test frequency within the CIRA anechoic chamber. The PCB-CTHA was modeled five times using the preprocessor, where for each time an equal empty space of 10 cells to 50 cells, in increments of 10 cells, was defined in each direction.

The following parameters were monitored for comparison in each of these five models:

1. Computational time,

2. Input impedance, real and imaginary components, and

3. Far Field Patterns, both Ephi and Etheta plots in three planes: Phi $=0$ plane, $\mathrm{Phi}=90$ plane, and Theta $=90$ plane.

Figure 6.2 shows a comparison of the computational time required for each of the five runs. It is clear that there is an exponential increase in the time required as the grid size becomes larger, which was expected. Thus the grid size has to be optimized to save processor time. This optimization will depend on the other parameters monitored. 


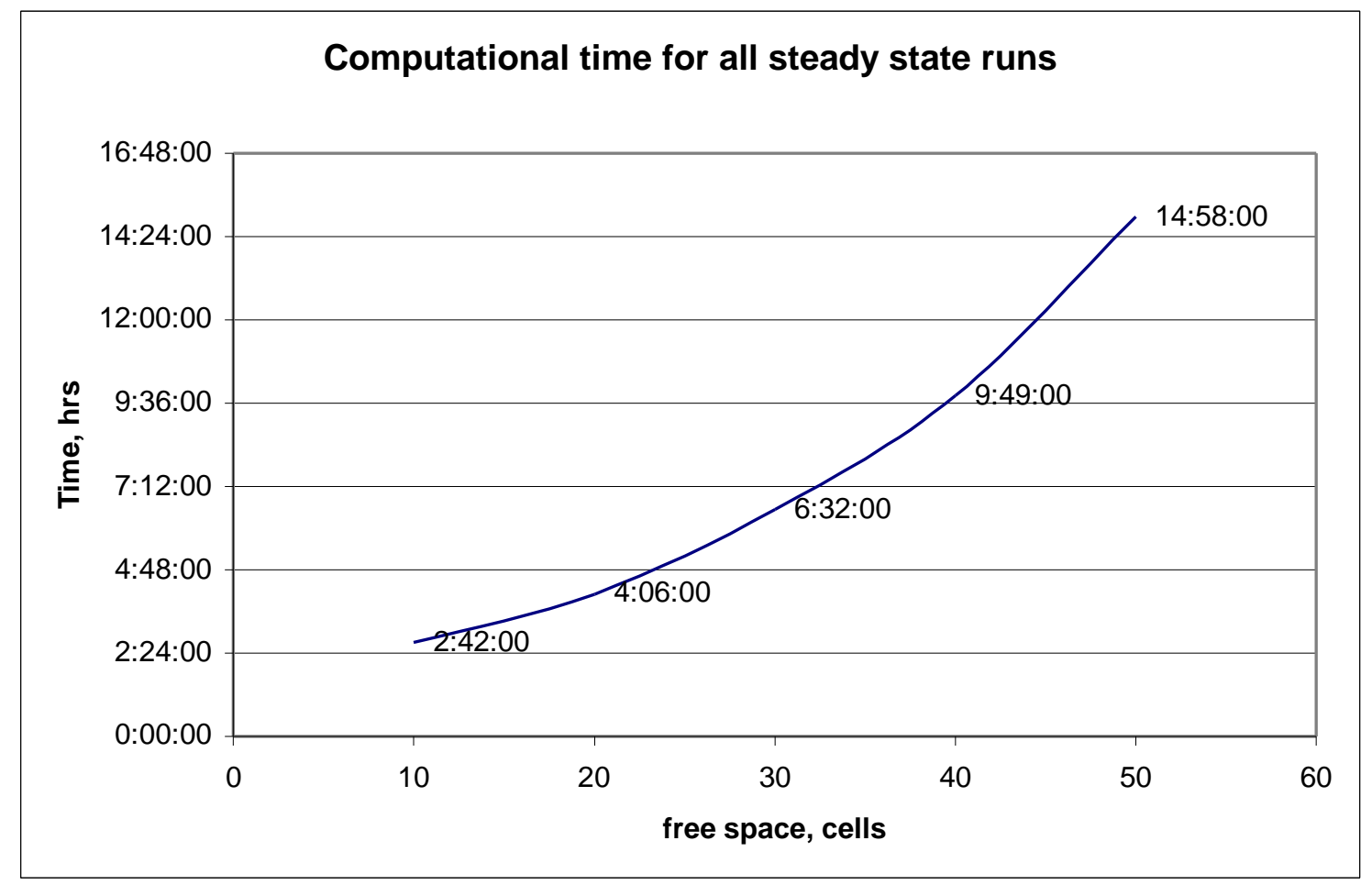

Figure 6.2 Computational time for all steady state runs

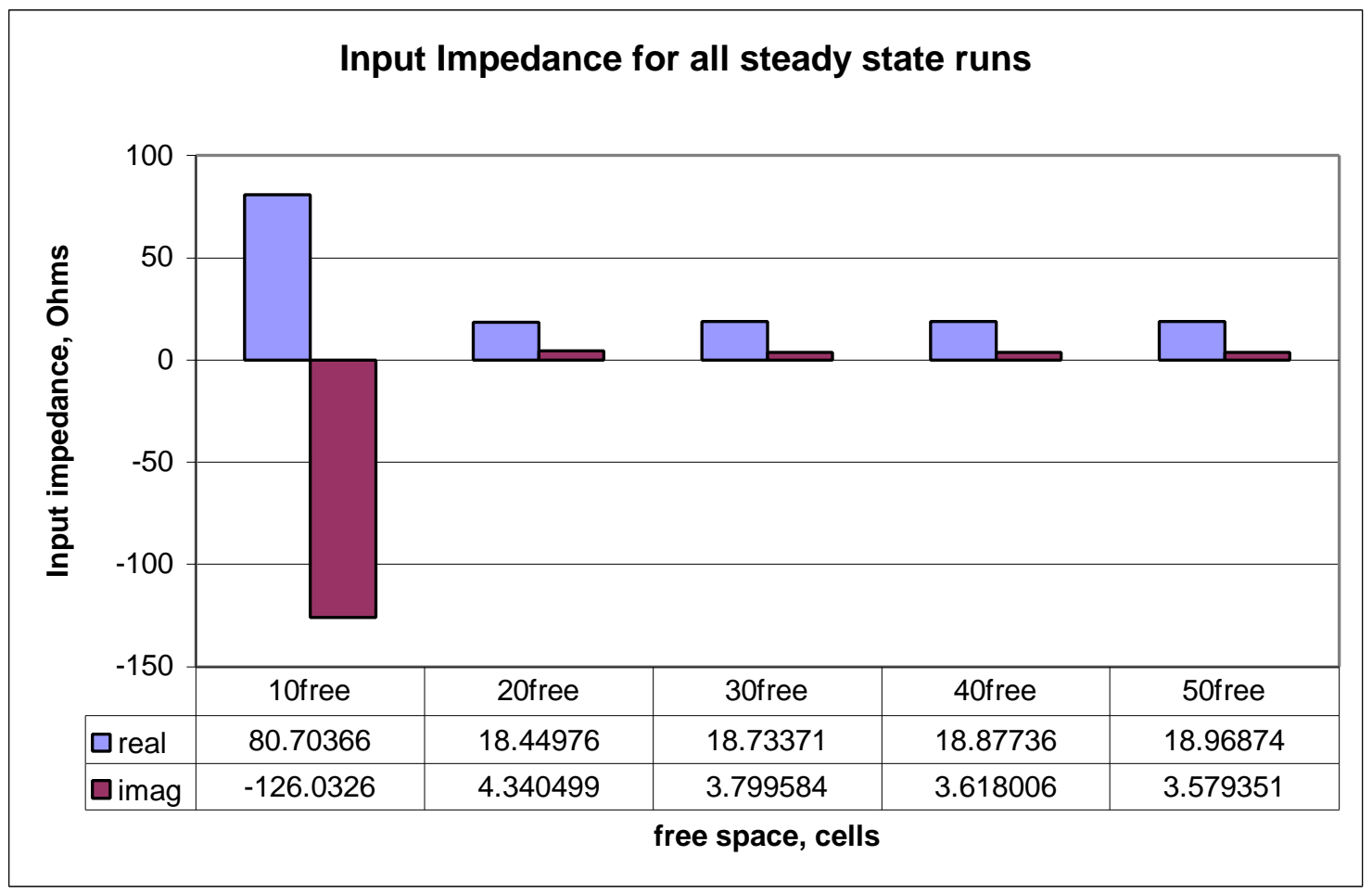

Figure 6.3 Input Impedance for all steady state runs 
Figure 6.3 shows the input impedance for all five cases. It is clear that the input impedance is very different in the case of the 10 free cells than the other cases. While the input impedance is clearly converging with the increase of the number of cells, but the accuracy gain after the 30 free cells case does not justify the high computational extra time required. The difference between the 20 cell case and the 30 cell case, on the other hand, seems slightly significant, but it cost an extra two and a half hours, or almost $60 \%$ increase in required processor time. Thus the choice of 20 or 30 cells free space depends on a balance between the degree of accuracy required by the designer versus the time available. Currently, the 20-cell case will be considered an adequate starting point, but later in the study it will be shown that this configuration does not hold for different parameter definitions of the PCB-CTHA.

The six far field patterns obtained from these runs at $1 \mathrm{GHz}$ are shown in Figure 6.4. It is worth noting that in this figure, and in all the figures to come, the location of the feed point is at angle zero. These are the Ephi and Etheta far field patterns at phi $=0^{\circ}$, phi $=90^{\circ}$, and theta $=90^{\circ}$. Once again, as with the input impedance results shown in Figure 6.3, the far field patterns for the 10 free cell case showed a big discrepancy from the rest of the runs, while the other four cases are very close to each other. It is also clear that with the increase in free cells the result tends to converge to a certain answer, but the amount of change is insignificant above 20 free cells, and once again it is confirmed that 20 free cells provide acceptable results for the least required run time.

All the results provided here in the steady state case give a conclusion that the optimum time/results combination for the $\mathrm{CB} 108$ model with $\mathrm{P}=1$ and perfect conductor traces is a model with 20 free cells in all directions of the antenna. Further investigations performed 
in the following sections by changing the model parameters will show that this configuration does not always hold, and that it will be affected by the change of the parameters. The amount of change of these parameters will be determined depending upon the parameter changes.

\subsubsection{Transient Case}

The transient runs are used to identify the resonant frequencies of a certain antenna so that the steady state runs can later be made at these resonant frequencies or at desired percentages of shift from them. From these runs the input impedance of the antenna at all frequencies were identified, and thus the design points and other points of interest were defined.

The transient runs can also be used to find the far field patterns at all frequencies, which is an extremely powerful tool that would eliminate the need for the steady state runs if the results are acceptable. The problem however with using transient runs for achieving far fields is that the run time becomes prohibitly long, in addition to the fact that the runs become unstable and usually cause the results to fail. Extensive work has been done to try to achieve acceptable and repeatable far field results from the transient runs, and the scientists at REMCOM, the designers of the XFDTD package, were contacted for assistance. Finally it was accepted by both sides, CIRA and REMCOM, that the PCB-CTHA design is too complex that it is "exceeding the limits of the power of FDTD transient analysis" as one scientist at REMCOM put it. Thus it was agreed to abandon any more trials to try to achieve far field results from the transient runs, and they would only be used to identify the input impedance of the antenna at all frequencies. A sample of the output of the far fields from a transient run are shown later. 
Effect of free space on the far field patterns, Ephi plot, phi $=0,1 \mathrm{GHz}$

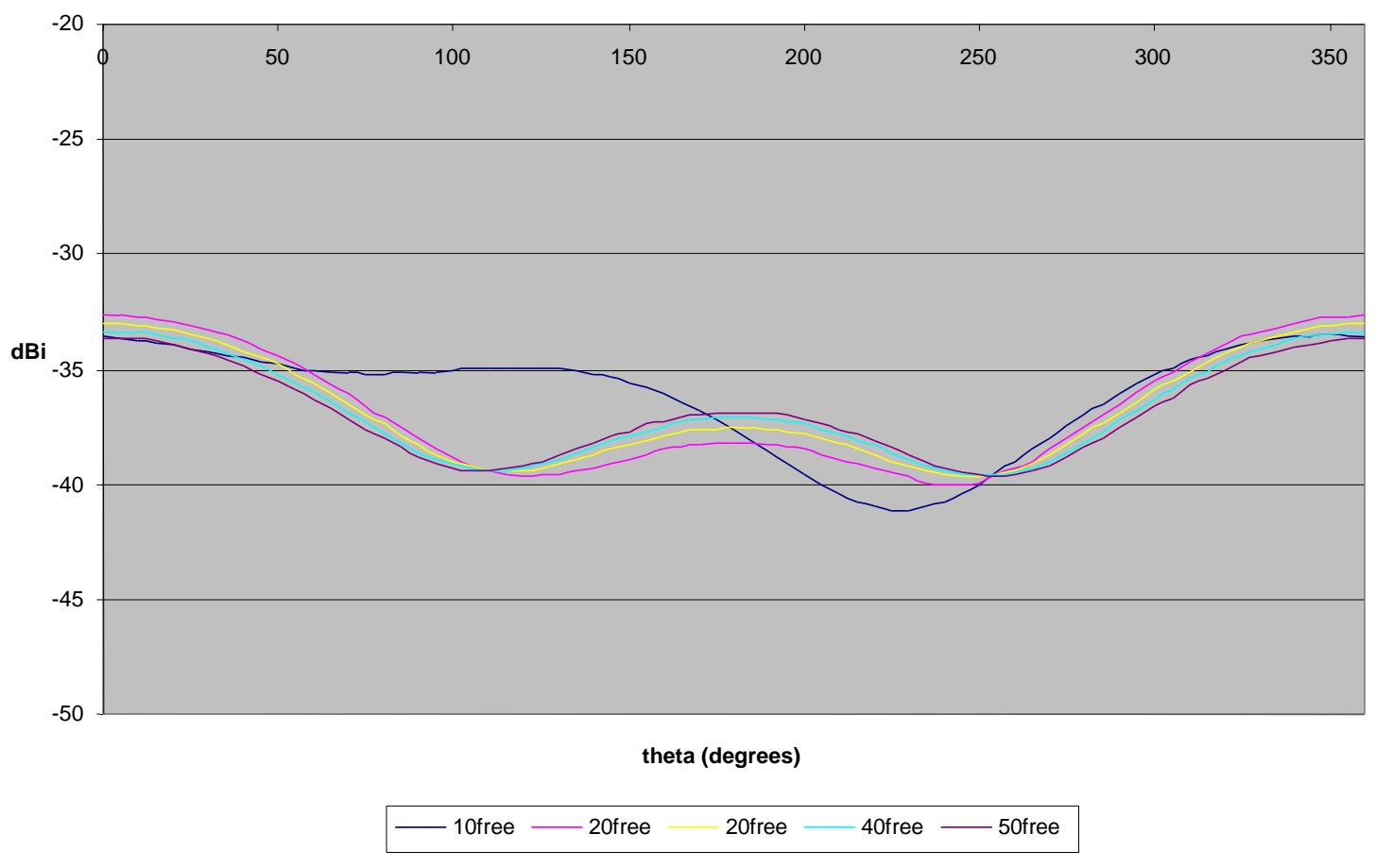

Effect of free space on the far field patterns, Etheta plot, phi=0, $1 \mathrm{GHz}$

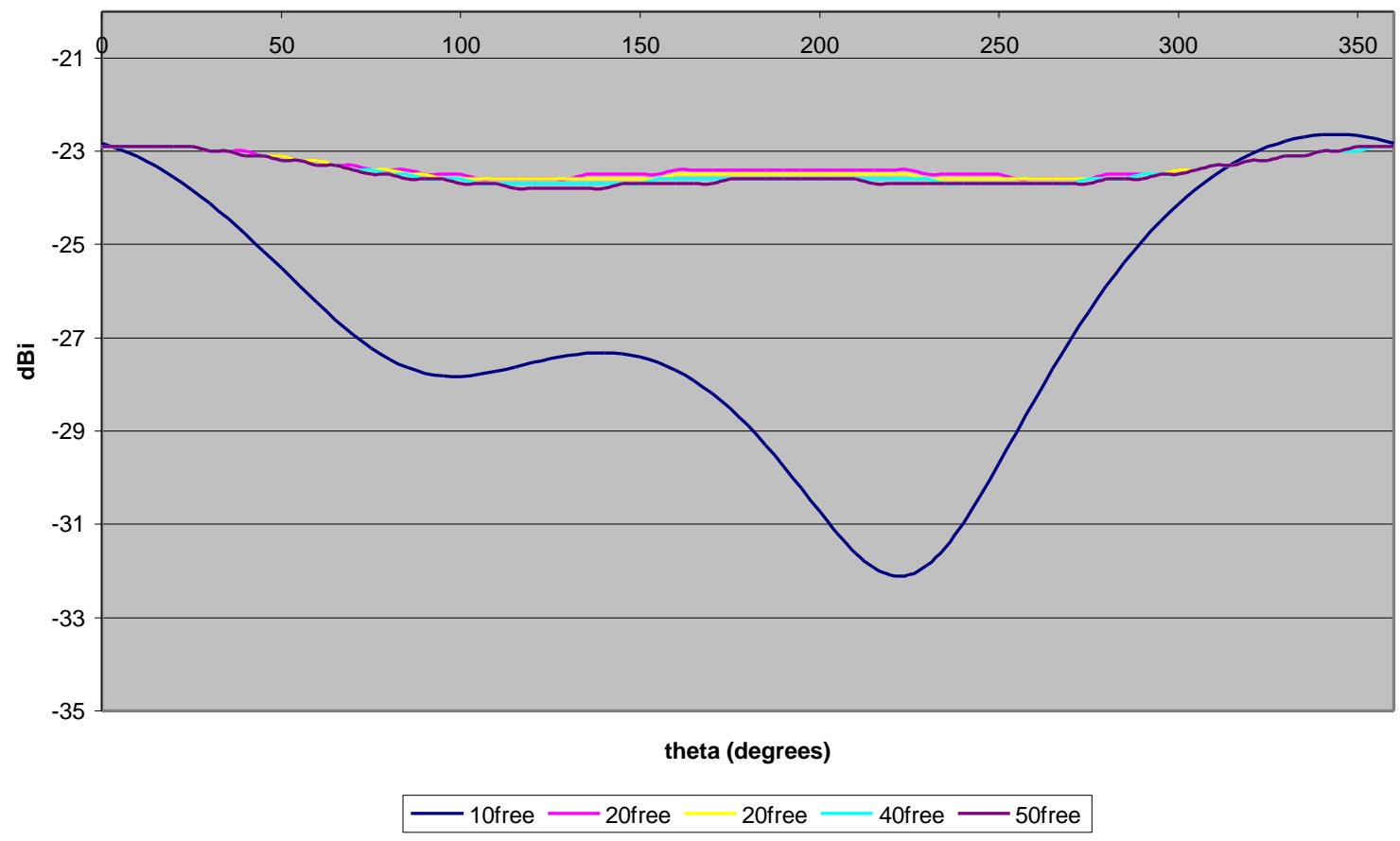

Figure 6.4 Effect of free cells on the far field patterns at $1 \mathrm{GHz}$ 
Effect of free space on the far field patterns, Ephi plot, phi=90, $1 \mathrm{GHz}$

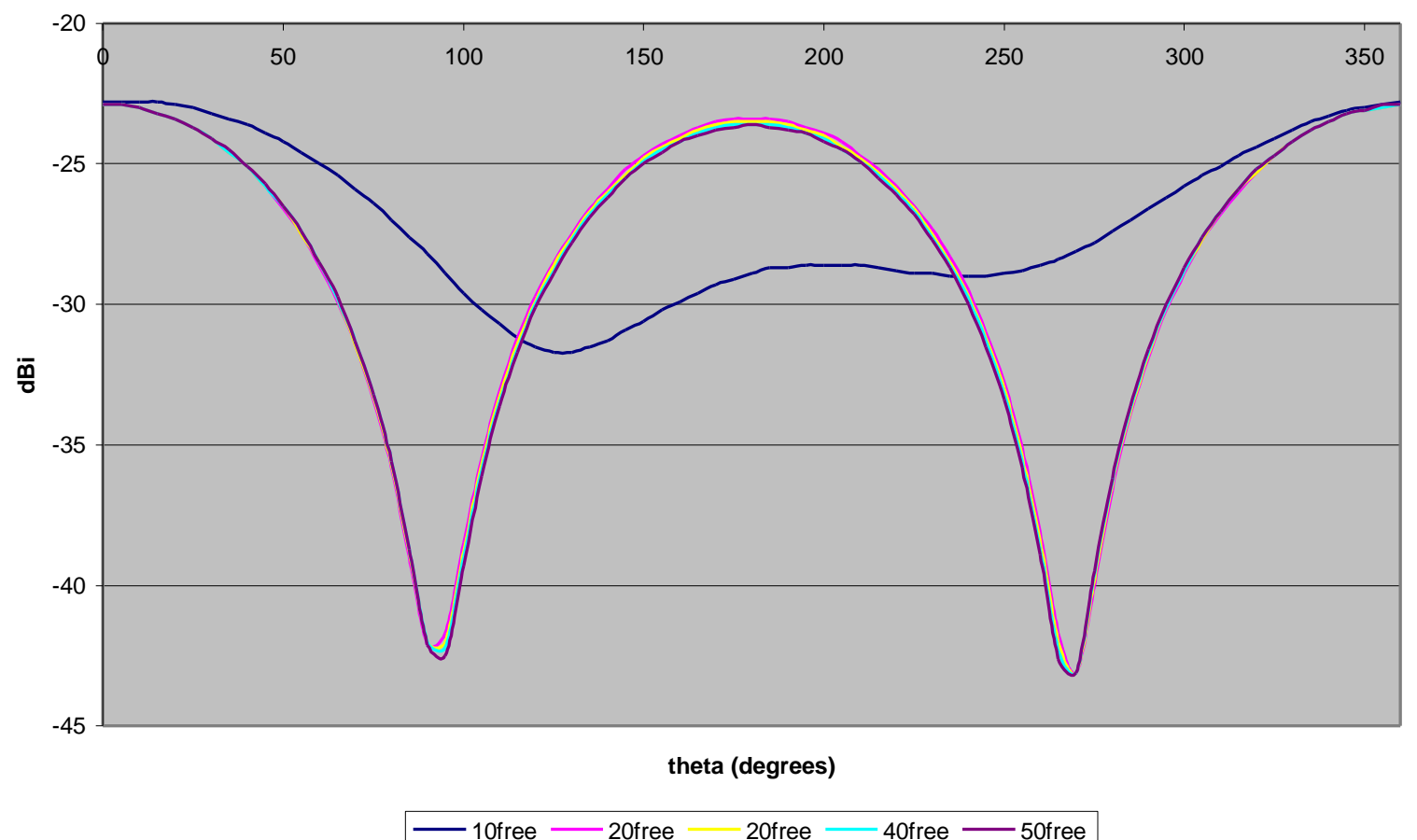

Effect of free space on the far field patterns, Etheta plot, phi=90, $1 \mathrm{GHz}$

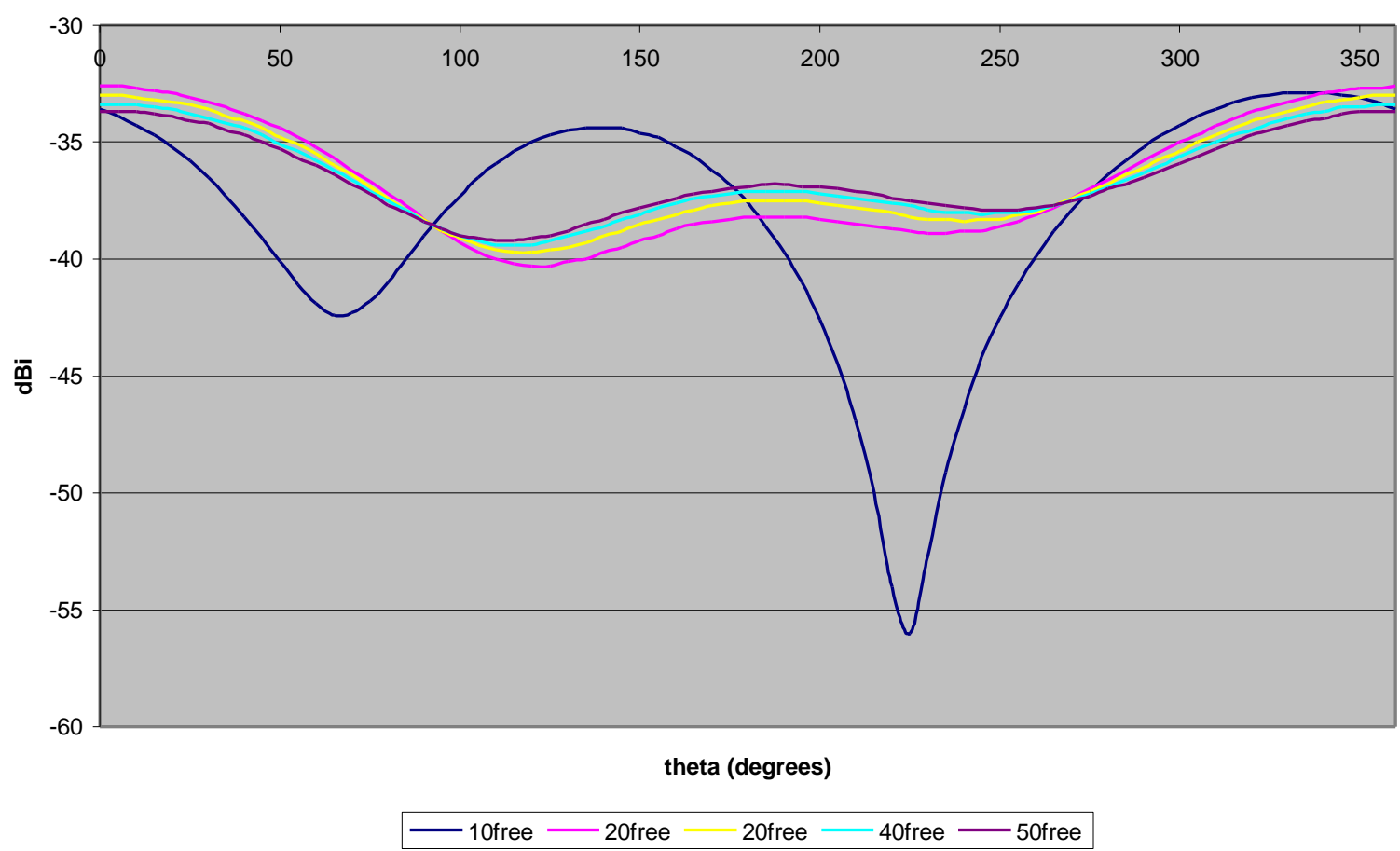

Figure 6.4 Effect of free cells on the far field patterns at $1 \mathrm{GHz}$ (cont.) 
Effect of free space on the far field patterns, Ephi plot, theta=90, $1 \mathrm{GHz}$

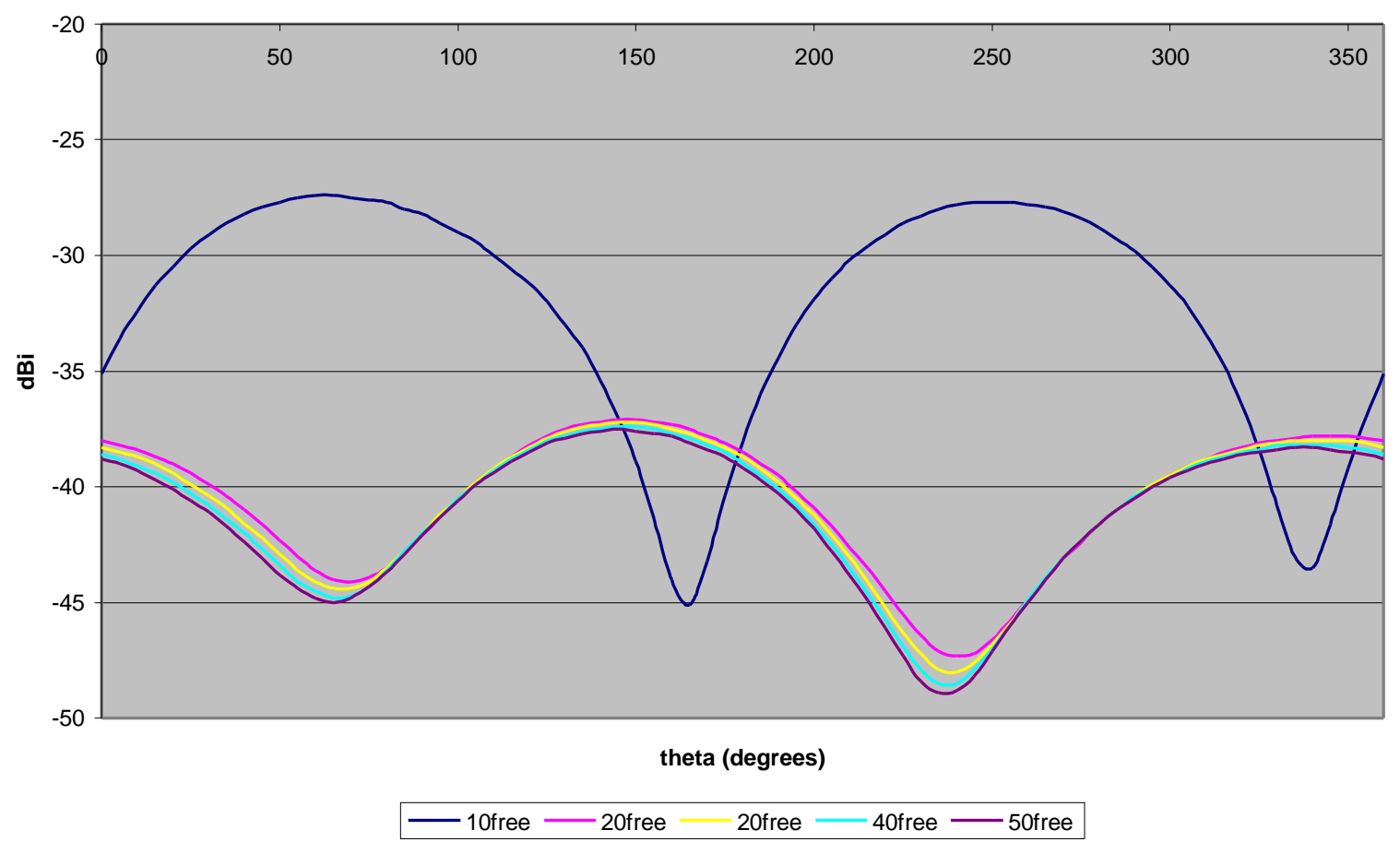

Effect of free space on the far field patterns, Etheta plot, theta=90, $1 \mathrm{GHz}$

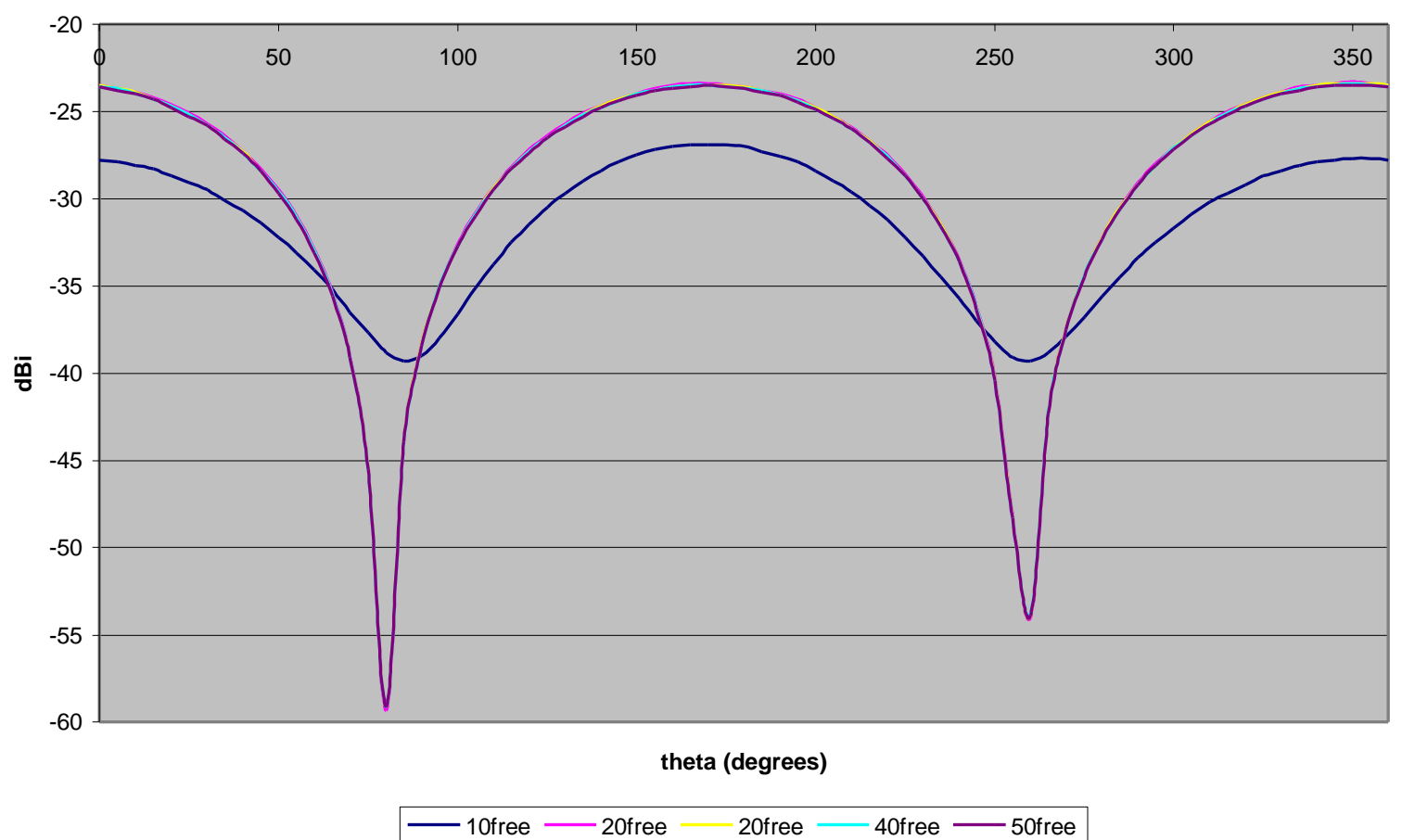

Figure 6.4 Effect of free cells on the far field patterns at $1 \mathrm{GHz}$ (cont.) 
Once again, the five different models of CB108 with different free cell sizes were used, and transient runs were performed upon them. Each model was tested under two time step lengths, 2,000 time steps and 20,000 time steps, since the effect of time steps is still unknown, and the optimum time step length is still to be found.

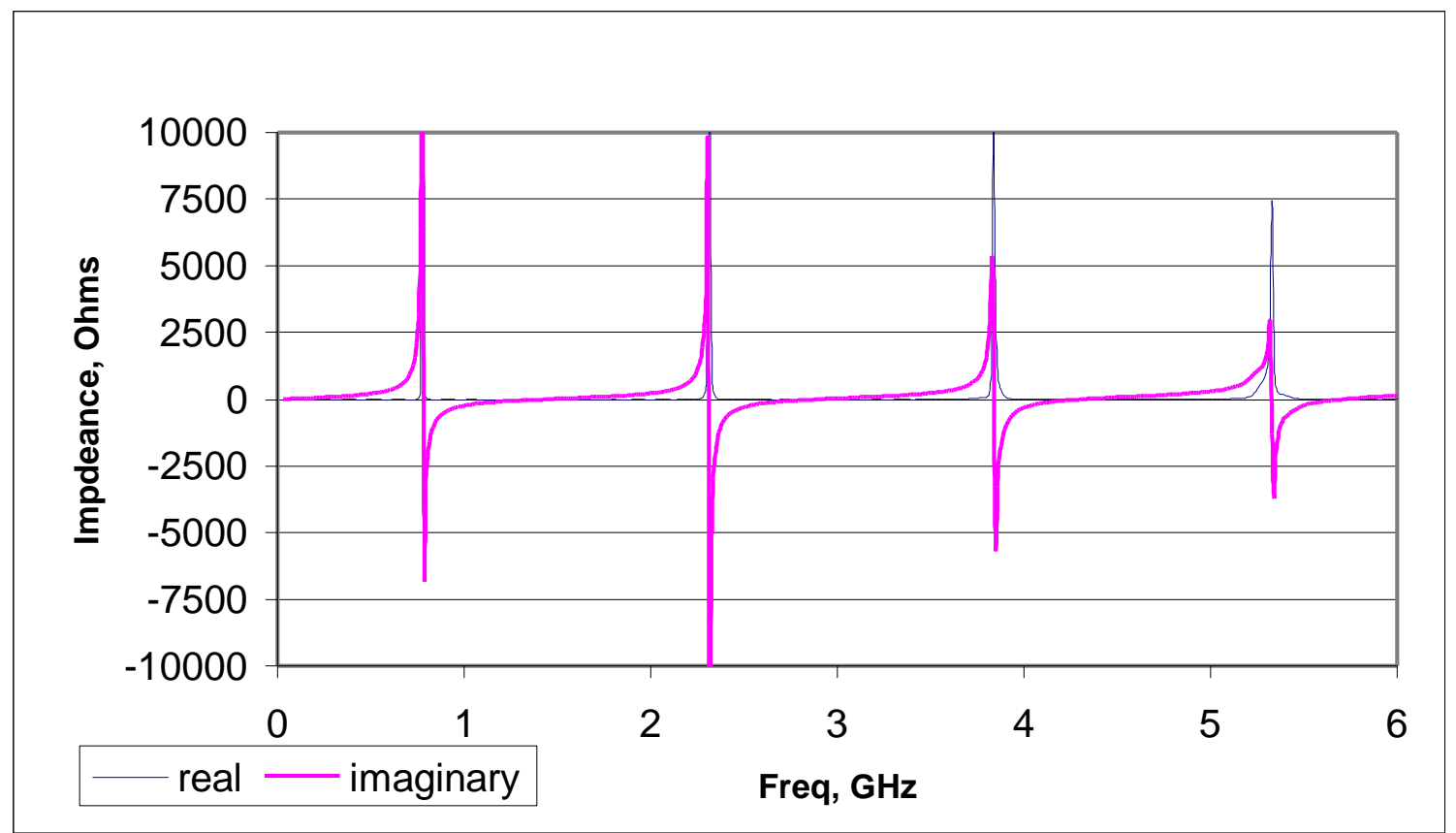

Figure 6.5 The input impedance for CB108, 50 free space cells, 20,000 time steps, feed 6

Figure 6.5 shows the input impedance graph achieved from the transient run on the model with 50 free cells around CB108 using 20,000 time steps. The data for this graph is provided by XFDTD in a text file, with input impedance results at approximately $10 \mathrm{MHz}$ intervals. Only the first $6 \mathrm{GHz}$ of the results are shown here.

A custom built program was developed to analyze the data in the XFDTD impedance file and extract from it the resonant frequencies. This program outputs two files, one containing the complete set of data from which the above graph was generated, and the other contains only the resonant frequencies up to $4 \mathrm{GHz}$. A complete listing of the code of this program is shown in Appendix D. As Figure 6.5 shows, the PCB-CTHA 
has two types of resonant frequencies. The first type has the imaginary component of the input impedance graph pass smoothly across the $\mathrm{x}$-axis from the negative side to the positive side, while the second type makes an instantaneous shift from $+\infty$ to $-\infty$. It has

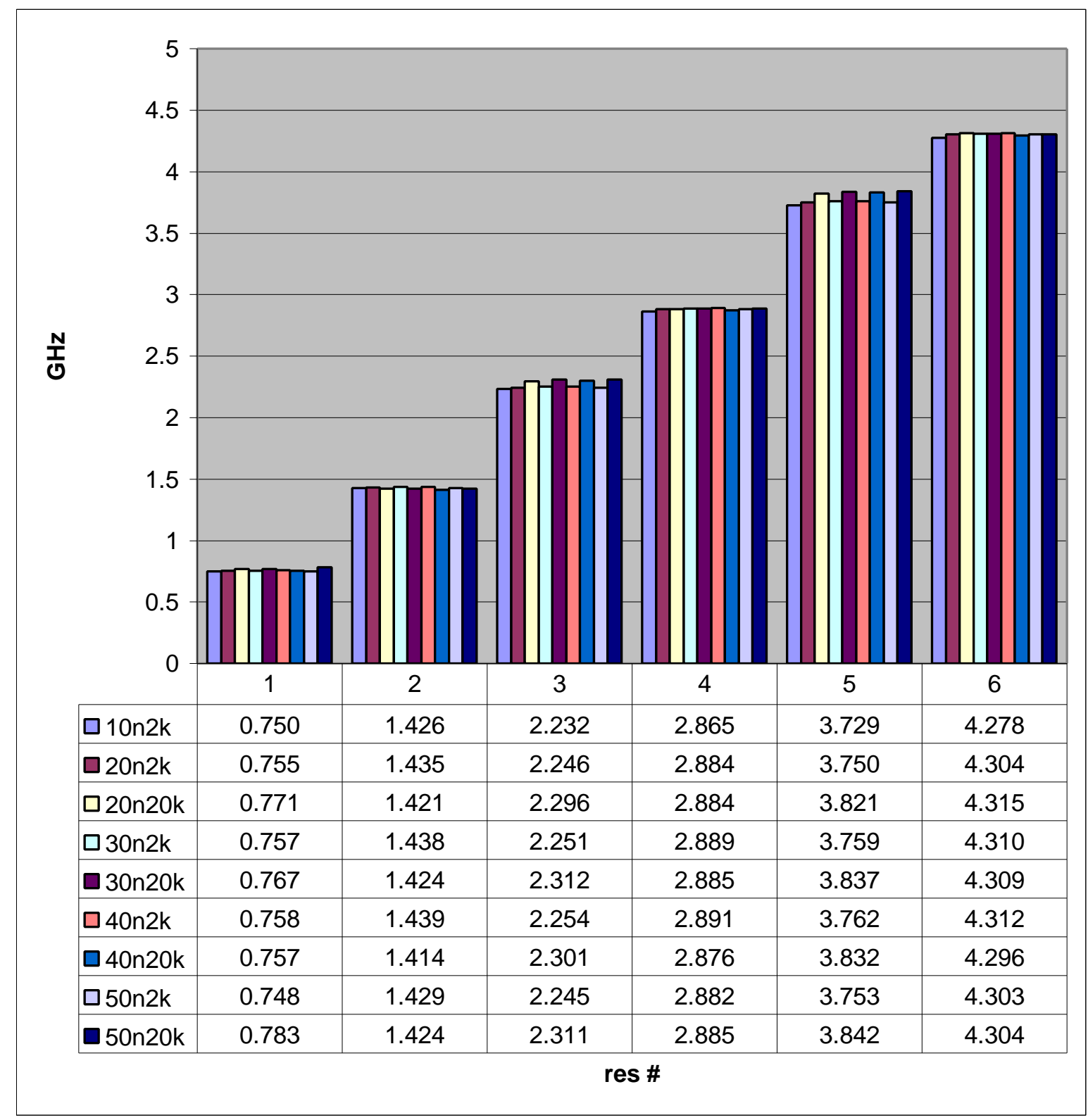

Figure 6.6 Effect of free space and time steps on the resonances of CB0108 feed6 been agreed upon within the CIRA group to call the first type as real resonances and the second type as anti-resonances. Thus, to make things clear, in Figure 6.5 it is found that 
there exists an anti resonance at slightly below $1 \mathrm{GHz}$, a real resonance at about $1.5 \mathrm{GHz}$, and another anti resonance at approximately $2.2 \mathrm{GHz}$.

The first six resonant frequencies for all 10 runs can be found in Figure 6.6. The run with only 10 free cells, and for 20,000 time steps diverged and gave no results. The run with 20 free cells and 20,000 time steps also diverged, but provided a few results at the lower frequencies, then collapsed after that. In the legend, the first two digits refer to the number of free cells, while the digits after the $\mathrm{n}$ refer to the number of time steps, so for example 40n20k would refer to the run with 40 free cells and 20,000 time steps. Table 6.2 shows the computational time required for all 10 transient runs.

Table 6.2 The computational time required for all 10 transient runs.

\begin{tabular}{|c|c|c|c|c|}
\hline run \# & time hr:min:sec & run \# & time hr:min:sec \\
\hline 10n2k & $0: 45: 01$ & & 40n2k & $2: 44: 13$ \\
\hline 10n20k & $6: 44: 30$ & & 40n20k & $24: 37: 57$ \\
\hline 20n2k & $1: 12: 41$ & $50 \mathrm{n} 2 \mathrm{k}$ & $4: 09: 37$ \\
\hline 20n20k & $10: 55: 05$ & $50 \mathrm{n} 20 \mathrm{k}$ & $37: 26: 33$ \\
\hline 30n2k & $1: 54: 07$ & & \\
\hline 30n20k & $17: 06: 04$ & & \\
\hline
\end{tabular}

Table 6.3 percent difference of resonant frequencies results, compared to $50 \mathrm{n} 20 \mathrm{k}$ run

\begin{tabular}{|c|c|c|c|c|c|c|c|c|}
\hline res \# & 10n2k & 20n2k & 20n20k & 30n2k & 30n20k & 40n2k & 40n20k & 50n2k \\
\hline 1 & 4.22 & 3.56 & 1.55 & 3.27 & 2.00 & 3.16 & 3.24 & 4.36 \\
\hline 2 & 0.10 & 0.74 & 0.20 & 0.94 & 0.05 & 1.01 & 0.70 & 0.34 \\
\hline 3 & 3.41 & 2.82 & 0.65 & 2.57 & 0.03 & 2.48 & 0.42 & 2.87 \\
\hline 4 & 0.70 & 0.05 & 0.06 & 0.14 & 0.01 & 0.20 & 0.34 & 0.12 \\
\hline 5 & 2.94 & 2.39 & 0.55 & 2.15 & 0.12 & 2.08 & 0.27 & 2.31 \\
\hline 6 & 0.62 & 0.01 & 0.25 & 0.13 & 0.10 & 0.18 & 0.20 & 0.03 \\
\hline
\end{tabular}

In order to understand the data in Figure 6.6, the results in Table 6.3 were calculated. Here the percent difference in reference to run $50 \mathrm{n} 20 \mathrm{k}$, which is considered be the most stable and most accurate, was calculated. These data show that the input 
impedance is slightly affected by the number of time steps and the free space area. The percent difference for the real resonances was less then $1 \%$, while that for the anti resonances was $3 \%$ to $4 \%$, which is logical due to the imaginative nature of the point of change of sign from an extremely high value to an extremely low value. This means that a huge saving on the run time can be gained by minimizing the time steps and the free space for the transient run, and save the time for the steady state runs. So the conclusion is that if the only thing required from a transient run is the resonant frequencies, then a $10 \mathrm{n} 2 \mathrm{k}$ run is sufficient.

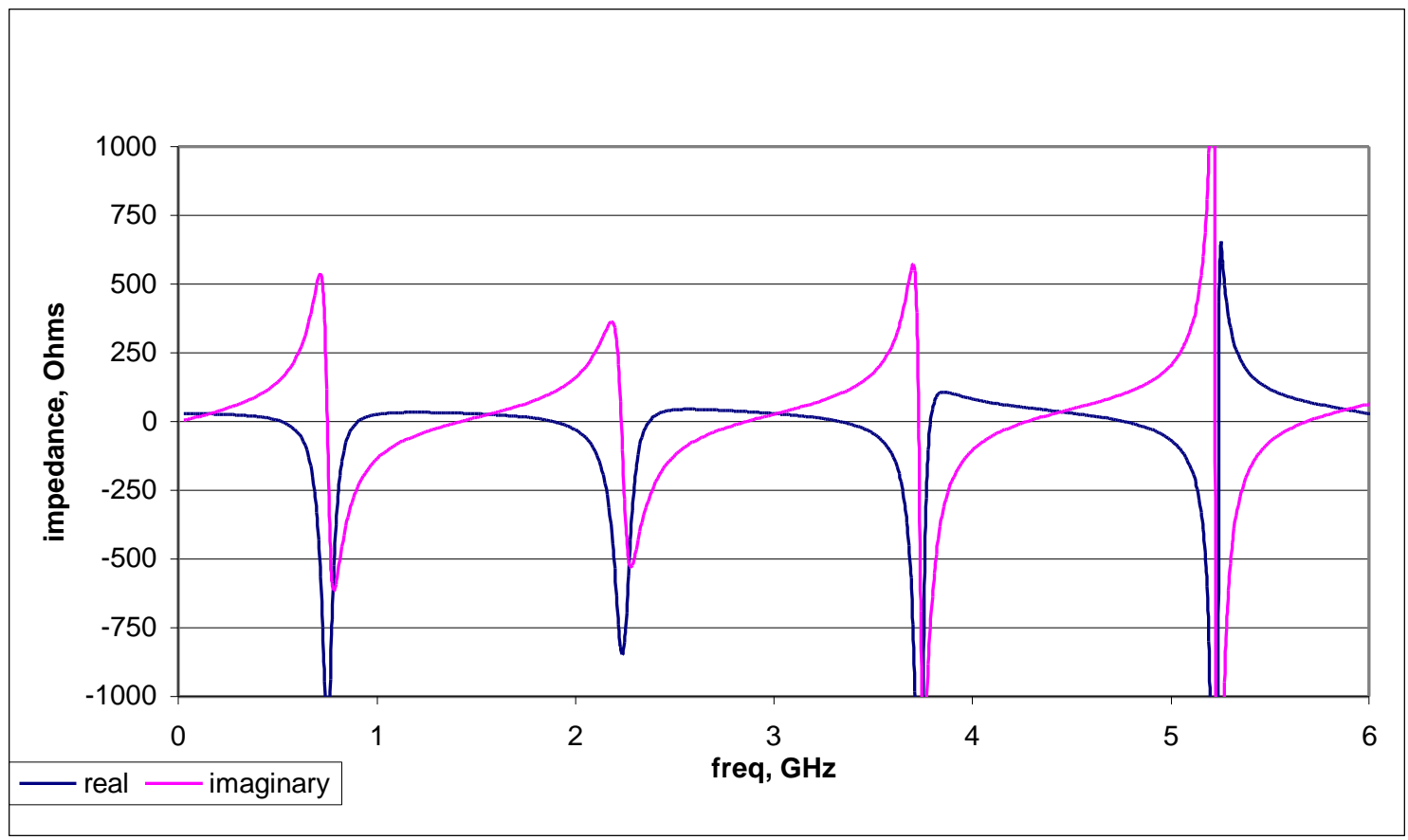

Figure 6.7 The input impedance for CB108, 10 free space cells, 2,000 time steps, f6

It must be noted however that although the $10 \mathrm{n} 2 \mathrm{k}$ run with only 10 free space cells and as few as 2000 time steps yielded resonant frequencies almost identical to those for the 50 free cell space and 20,000 time steps, the same is not the case if the entire input impedance chart is desired not just the resonance frequency points. Figure 6.7 shows the input impedance graph for the run $10 \mathrm{n} 2 \mathrm{k}$ up to $6 \mathrm{GHz}$. This can be directly compared to 
Figure 6.5. It can be seen that the real component in Figure 6.7 is affected by the anti resonance and drops way within the negative, which is an unrealistic case. This same phenomena appears in all the runs, but keeps decreasing with the increase in free space and time steps, until the shape shown in Figure 6.5 is obtained where this case is totally eliminated. The imaginary component on the other hand seems to be unaffected by the different runs, which is why the resonant frequencies, which depend only on the imaginary component, showed no change between both runs. From this it is concluded that the $10 \mathrm{n} 2 \mathrm{k}$, and above, can be used only to determine the resonant frequencies, while if the designer desires the real component for the input impedance then larger runs as the 50n20k should be used.

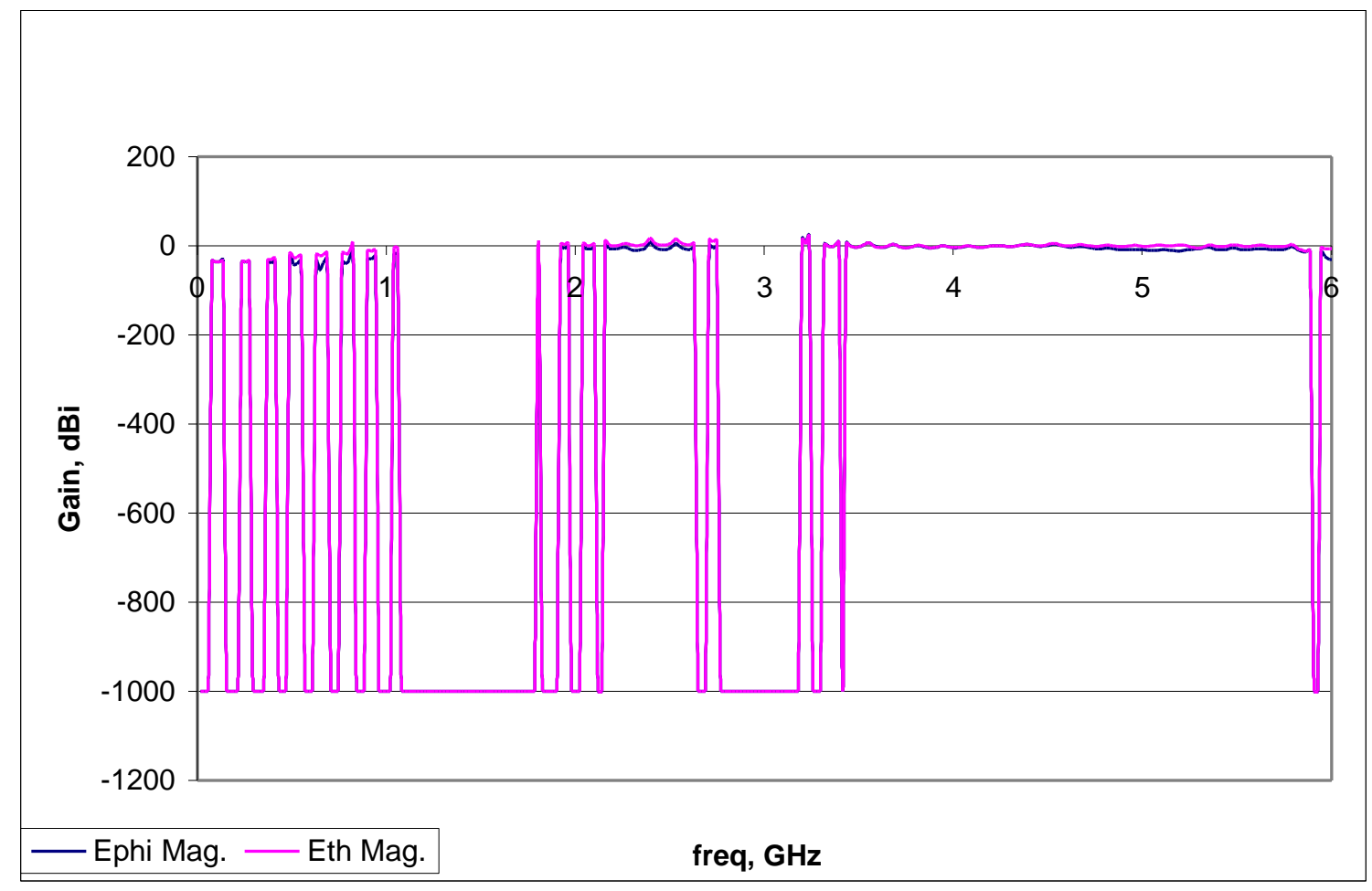

Figure 6.8 The Gain for run 50n20k at point phi $=0$ and theta $=0$

Also to demonstrate the problem of obtaining the far fields from the transient runs Figures 6.8 is shown. In this Figure the far field result for one point, $\mathrm{phi}=0$ and theta $=$ 
0 , is shown for run $50 \mathrm{n} 20 \mathrm{k}$. At several points along the chart the gain suddenly drops to an error value of -999 , this, according to the scientists at REMCOM, means an error value, and that XFDTD could not get the results at these values. At other ranges, on the other hand, the results were fine, and the gain at these frequencies could be obtained, as is the case for the range between 3.43 to $5.88 \mathrm{GHz}$. These same patterns would appear in all runs, with the error values both changing location and increasing or decreasing in range, thus rendering the output of these runs totally unpredictable. As it was earlier mentioned, these types of runs were totally abandoned, since that they required a large amount of run time, and the steady state runs were used to obtain the far field patterns at the required frequencies.

\subsubsection{Effect of Free Space Conclusions}

Several conclusions can be reached from the above steady state and transient studies. All these runs were done on CB108 with a relative permittivity for the binder of unity. In the next section the relative permittivity will be changed to see if the same conclusions hold, or will differ with the change of the relative permittivity. The following is a list of the conclusions obtained in this section:

1. The input impedance and far field patterns in the steady state runs can not be predicted by only a 10 free cell space in all directions. At least 20 free cells are required, with results improving if the free space is increased.

2. The run time required increases exponentially with the increase in the free space around the antenna model. Thus the slight increase obtained in the steady state runs accuracy above 20 free cell space is not justified, and 20 free cells are considered appropriate. 
3. The resonant frequencies obtained from the transient runs are almost unaffected by the free space and the number of time steps, thus the minimum tested model can be used to save on run time. A free space of 10 cells with 2000 time steps is considered enough.

4. To unify the model, and avoid any problems that could occur from using two different models, only one model with 20 free cell space is enough. This model can be used for both the steady state and the transient runs. For the transient runs it should be used at 2000 time steps, while for the steady state the number suggested by XFDTD has always been used up to this point. The effect of different time steps on steady state runs will be studied next.

\subsubsection{Effect of Time Steps}

The effect of the time steps on transient runs has already been covered in the previous section where it was shown that this effect is negligible. But in all the steady state runs only the recommended time steps by XFDTD were used, and the effect of increasing these time steps still needs to be checked. Again CB108 will be used with a relative permittivity of unity and a free cell space of 20 cells as was defined in the previous section.

For the above mentioned model XFDTD suggested 9021 time steps for the runs. Four runs were conducted, the first using the suggested number of 9021, and then this number was multiplied by $1.25,1.5$, and 2.0 and advanced to the closest odd number, yielding 11277,13533 , and 18043 respectively. The model can not be run with time steps less than those suggested by XFDTD since in this case the run does not reach steady state and no output results are provided by XFDTD. The run at 18043 diverged, and 
yielded a continuous error value of -999 . Figures 6.9 and 6.10 show the far field Ephi and Etheta patterns at phi $=0$ for the other three runs. As shown, with the increase in time steps the pattern retains its shape but provides larger gain values. This same behavior was experienced time and time again when time steps were increased on steady state runs, the gain for the far field pattern would increase continuously until it crashes at about twice the recommended value by XFDTD.

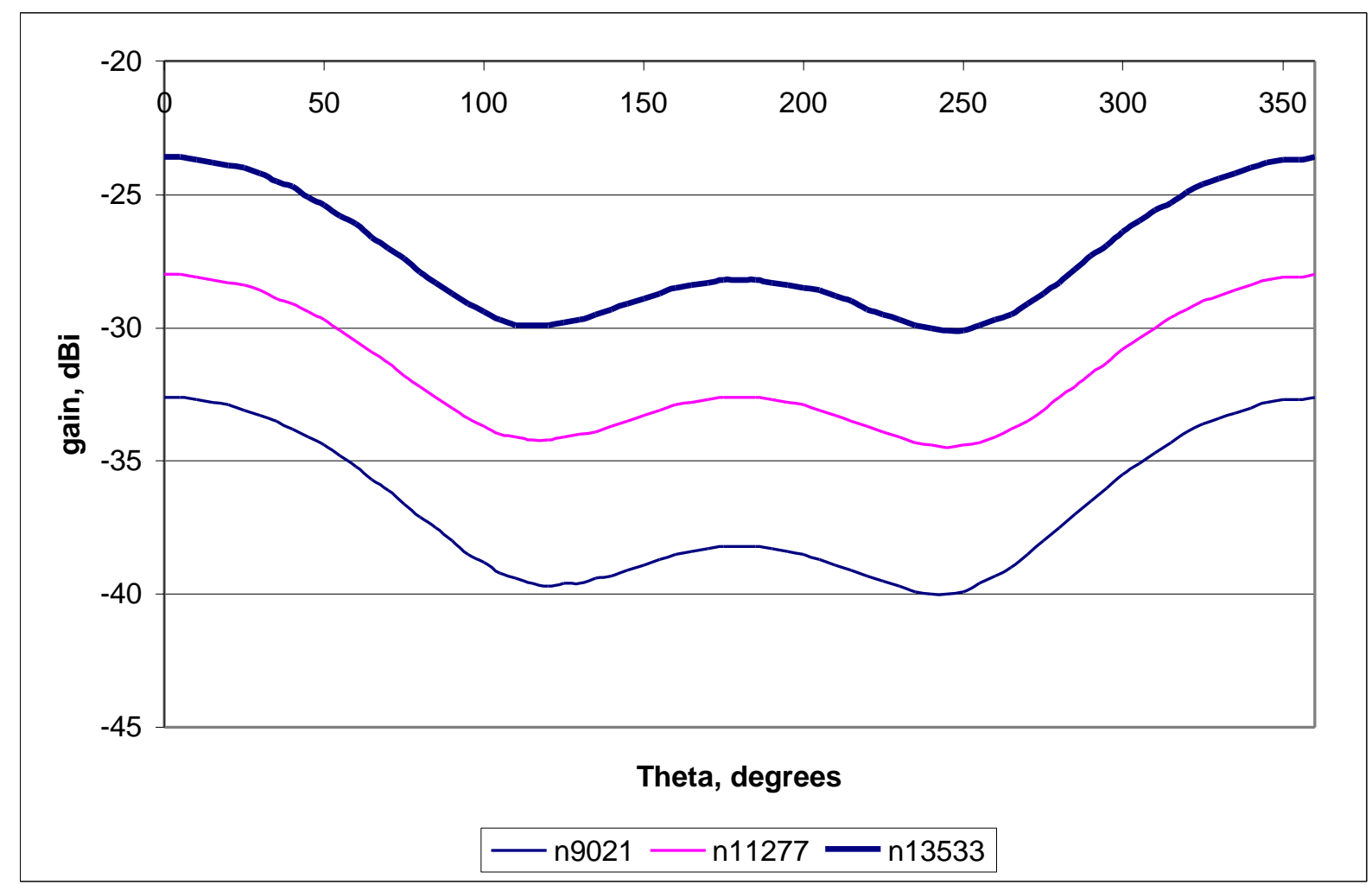

Figure 6.9 Effect of time steps on the far field patterns, Ephi plot, phi $=0$

Since the results yield continuous difference when the number of time steps are increased, it will be assumed that increasing the time steps yields errors in the results, and that the best results are for the least number, which is the number suggested by XFDTD. This conclusion can only be verified by experimental data, which will come later in the text. If the experimental data shows the values to be higher or lower then a multiplication 
by, or addition of, a constant factor might be able to solve the problem, since as shown in Figures 6.9 and 6.10 the pattern keeps its shape and is only shifted by a constant amount.

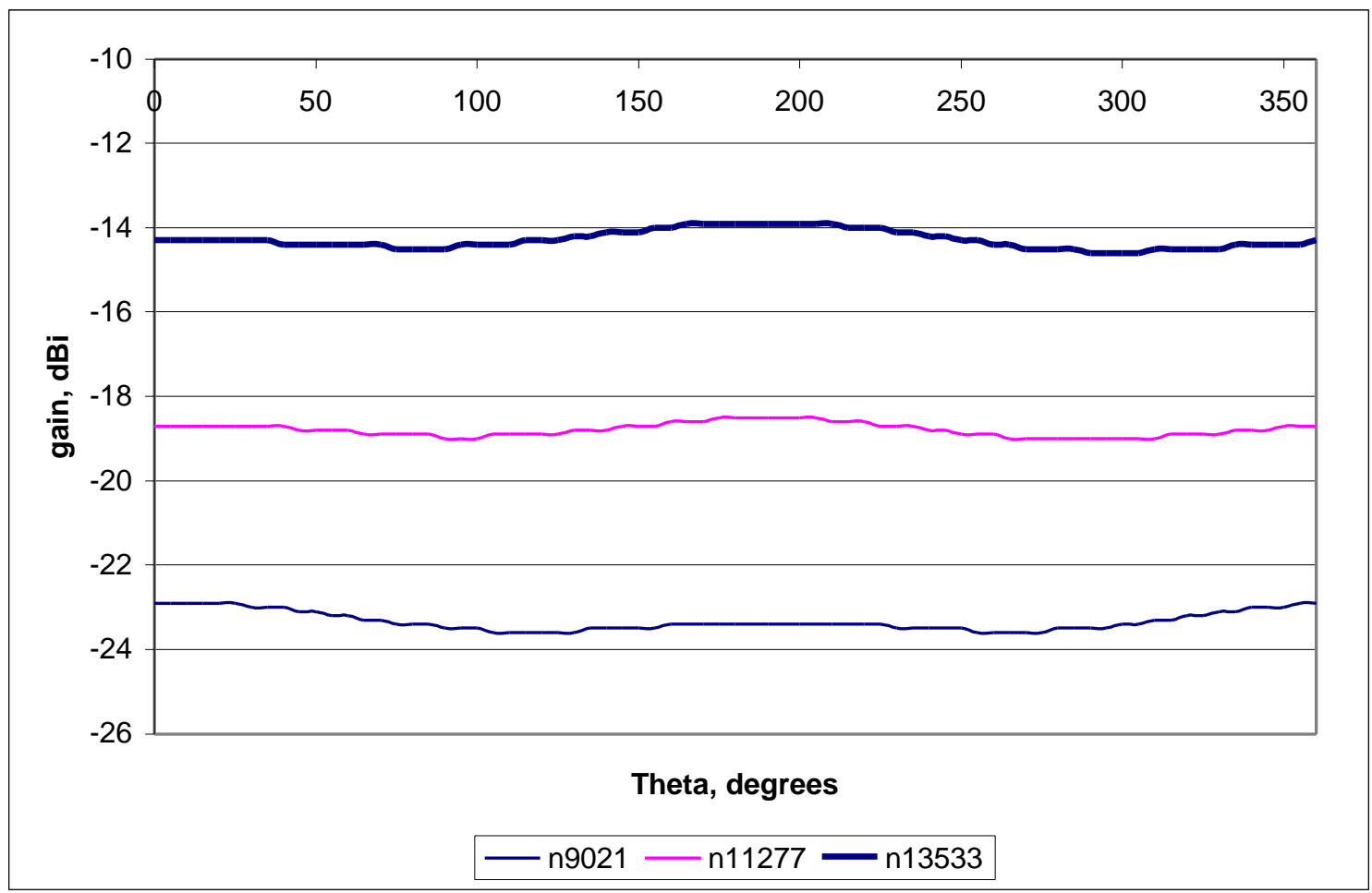

Figure 6.10 Effect of time steps on the far field patterns, Etheta plot, phi $=0$

\subsubsection{Effect of the Relative Permittivity $P$}

\subsubsection{Transient Case}

All the previous results were for an antenna with a binding material relative permittivity $(\mathrm{P})$ of unity. But the actual relative permittivity of the antennas available at CIRA is approximately 4, so the effect of the change in P, if any, must be identified. CB108 was once again used in a transient run, with feed 6, 10 cells free space, and 2000 time steps, but this time with a value of $\mathrm{P}=4.0$. The run is identical to the run that yielded the chart in Figure 6.7, but with the only difference of the value of the relative permittivity. Figure 6.11 shows that the run totally failed to calculate the input 
impedance for this model, suggesting that the time steps and free space need adjustment. The same was the case for all the other feed types tested.

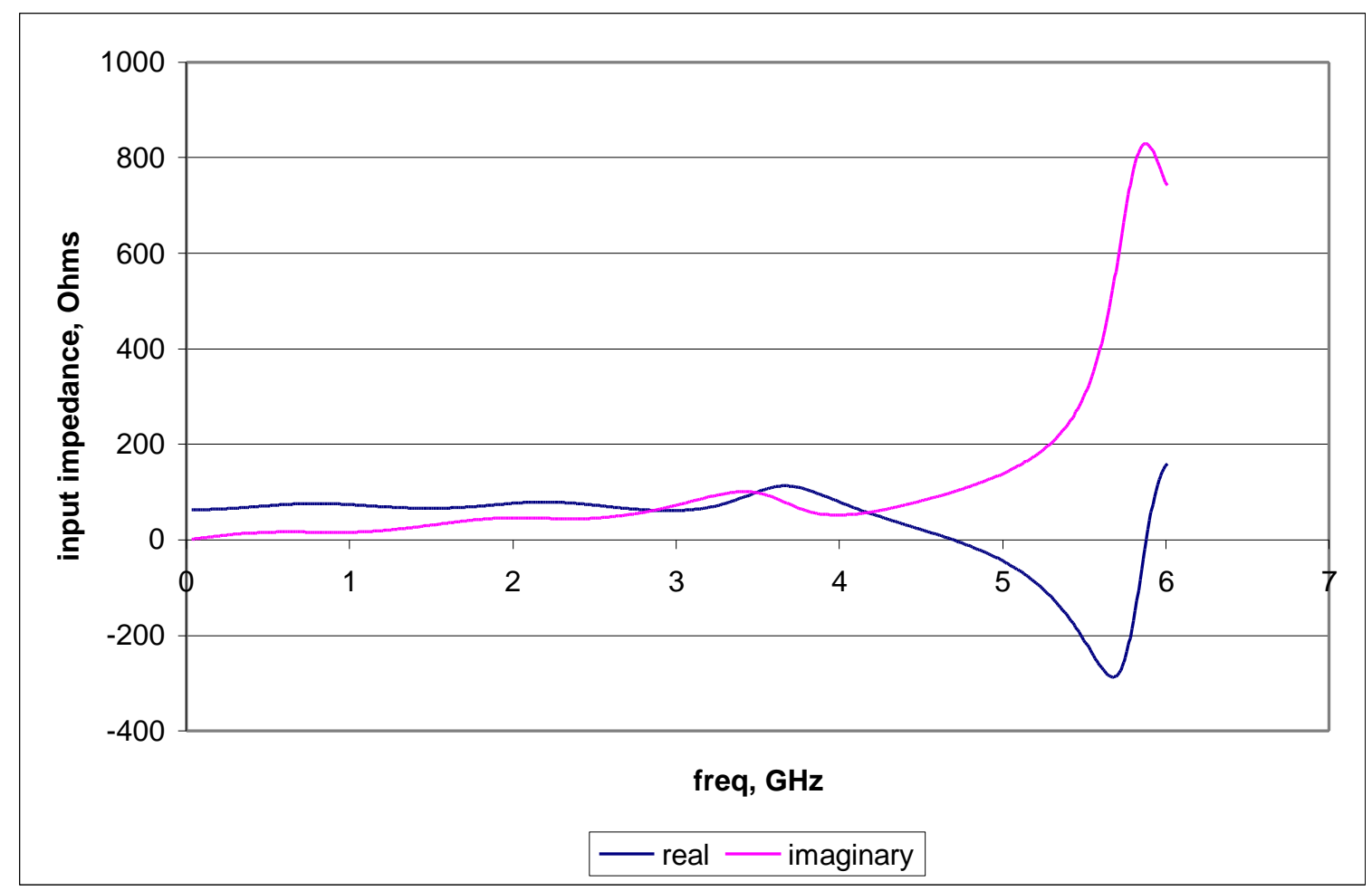

Figure 6.11 Input impedance for CB108 10n2kf6, $P=4.0$

The number of free space cells was increased to 30 cells, and a full study of the effect of the time steps at 30 free cells was conducted. Six runs were performed with time steps of 2000, 3000, 4000, 5000, 10,000, and 20,000. The first two, the 2000 and 3000 time step cases, yielded no results, as with the case shown in Figure 6.12, while the other four cases all yielded results that are almost identical as shown in Figure 6.12. This implies that the runs for the cases where no input impedance results were obtained had not yet reached a required convergence limit. But once this limit is reached, as in the other cases that did yield results, then any further increase in time steps or in free space is only a waste of processor run time. 
This analysis proves that the increase in $\mathrm{P}$ required an increase in both the free space area and the number of time steps in order to achieve convergence in the results. The previous recommendation of a free space of 10 cells and 2000 time steps, obtained for a relative permittivity of unity, thus no longer holds, and the numbers have to be increased to a free space of 30 cells and 4000 time steps for a relative permittivity of 4 . Since all the antennas that will be tested have a relative permittivity less than 4 , then it can be safely said that the setting of 30 cells and 4000 time steps should hold for all PCBCTHAs in this study.

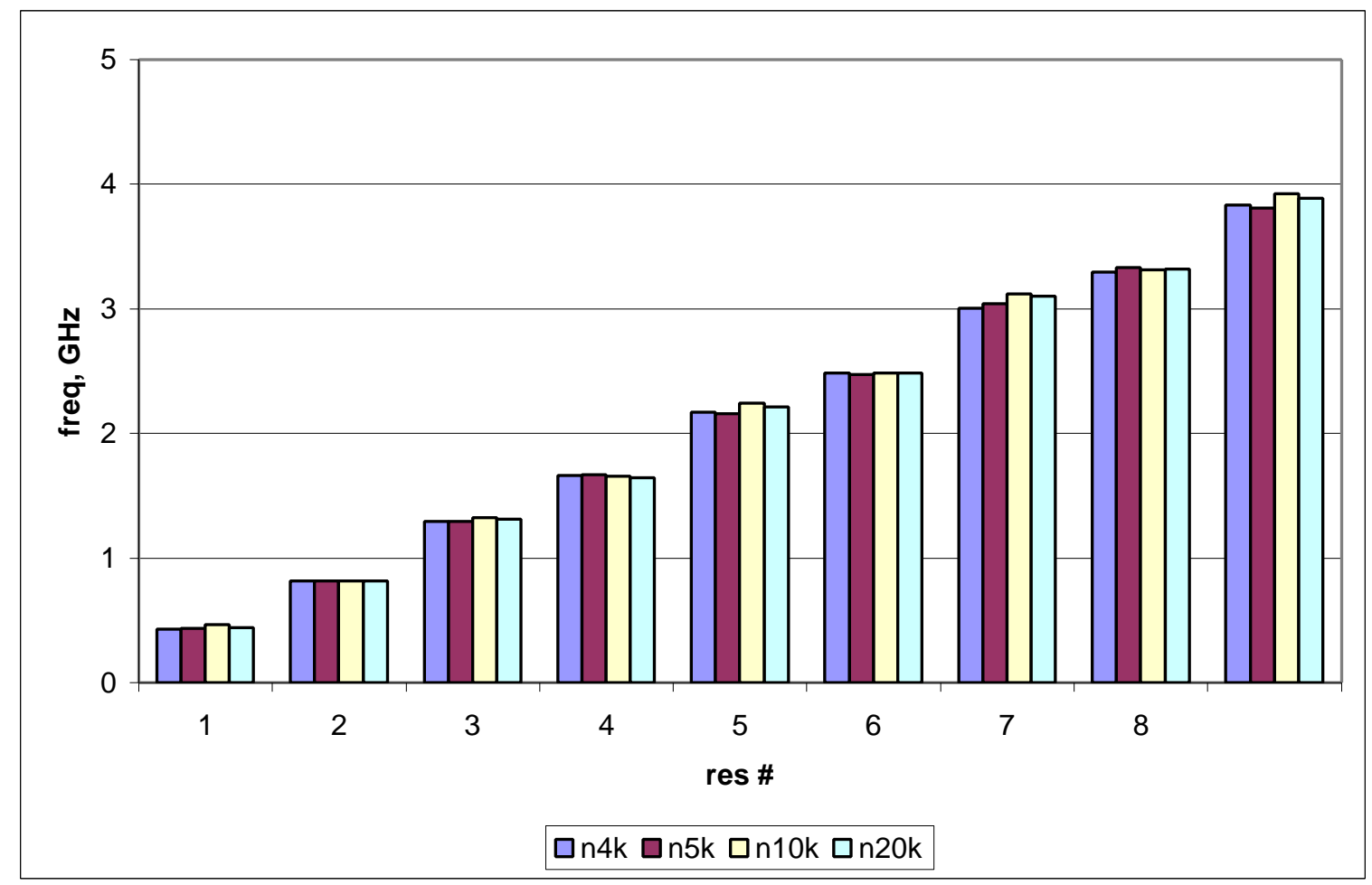

Figure 6.12 Effect of time steps on CB108 with $P=4$ and 30free space

\subsubsection{Steady State Case}

Using the same previous model with a relative permittivity of 4.0 , the steady state case was studied with 20, 30, 40, 50, and 60 free space cells. The 10 free space cell case was not tested since it already failed in the $\mathrm{P}=1$ case, and is expected to fail here too as 
shown in the transient case before. All four runs were applied with the number of time steps recommended by XFDTD, which has already been proven to be the best number of time steps to use.

Figure 6.13 shows the Ephi and Etheta patterns for all 5 cases at the plane phi=0. For the sake of comparison, the case with 50 free space cells and a relative permittivity of unity was plotted on the same chart. This Figure clearly demonstrates the large effect of the change of the relative permittivity on the far fields of the PCB-CTHA, with the gain significantly dropping and the pattern shape changing too.

But when it comes to the effect of the free space cells, the differences are not so clear. While the 20 cell case followed almost the same pattern as the other 4 cases, it could be easily distinguished as being separate from the other charts. The other 4 cases, on the other hand, were very close to the extent that makes the extra time required for the larger grids unnecessary since the same results can be achieved with the smaller grids. From this it can be concluded that the 30 free space cells case is sufficient enough to yield acceptable results for the steady state case with a binder relative permittivity of 4.0. 

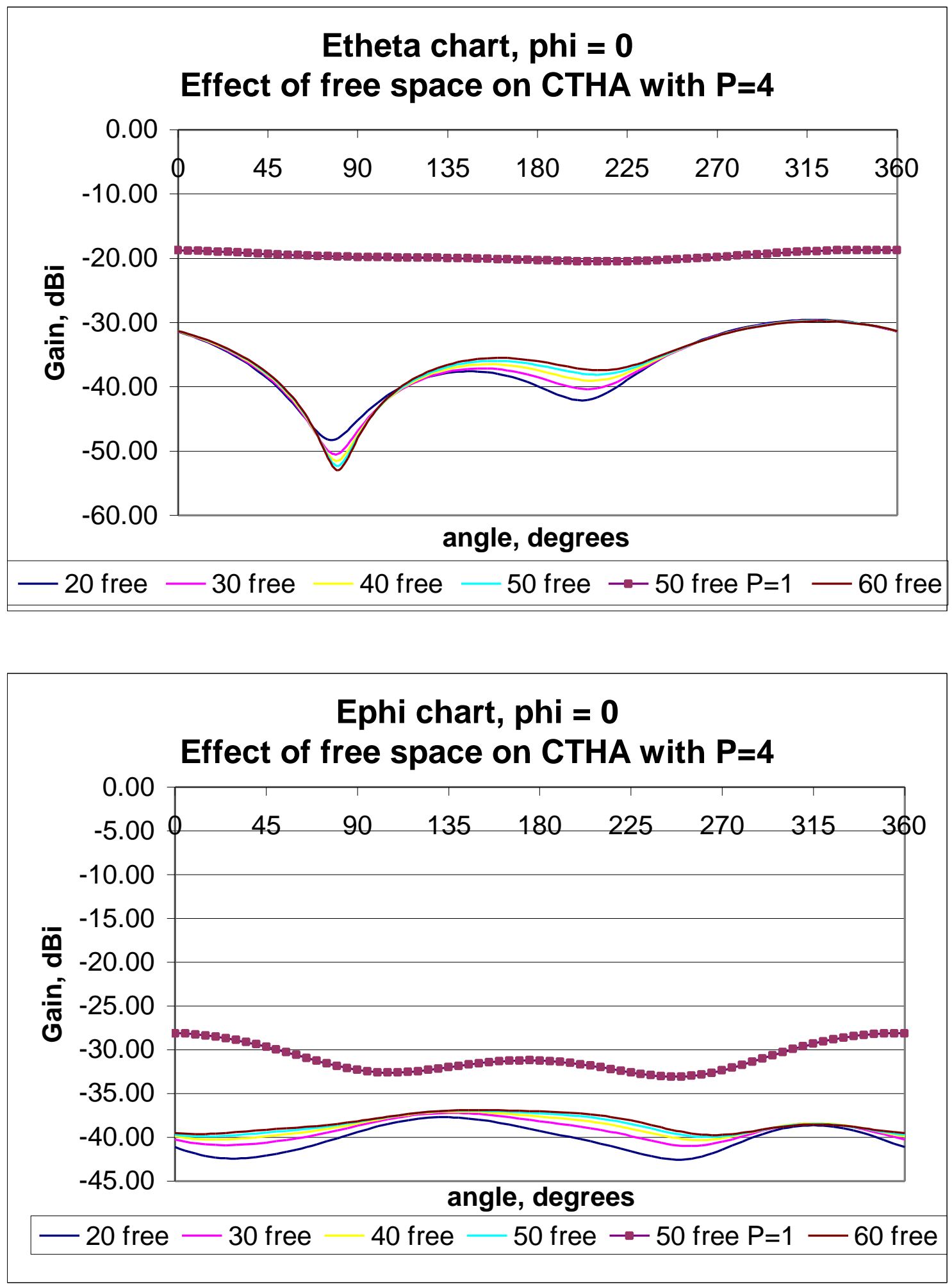

Figure 6.13 The Etheta and Ephi charts at the plane phi $=0$ 


\subsubsection{Effect of Relative Permittivity Conclusions}

From this it is clear that the area of free space cells around the antenna is extremely critical, and that increasing the area helps in stabilizing the results. On the other hand, the time steps have been proven to be only important up to the case where results reach convergence, after which increasing the time steps has no effect, or might even lead to errors as was shown in the steady state run.

For both the steady state and the transient cases of a PCB-CTHA model with binder permittivity of 4.0 a free space area of 30 cells was found to be the optimum case. Since all the antennas that will be tested have a binder permittivity that is less than 4.0 , then it can be safely concluded that this configuration should hold for all the current PCB-CTHA antennas.

As for the number of time steps, once again it was found that the optimum number for the steady state runs is that suggested by XFDTD. For the transient runs, on the other hand, 4000 time steps was found to be an acceptable number, after which increasing the number of time steps has no significant effect.

\subsubsection{Effect of Traces Conductivity}

The final parameter that the model needs to be optimized for is the conductivity of the traces. Although the PCB-CTHA traces are copper traces, the value used in all the previous models was that of a perfect conductor. In this section the latest optimum model found in the previous section is used, but the traces will be given a conductivity as that of copper, and according to this change in conductivity the model will be optimized. As with all the previous sections, this will be done for both the transient and steady state cases. 
It is worth noting, however, that Kunz states in [43] that the conductivity feature provided by XFDTD is for dielectric material, and is not designed for conducting material. In order to use it for conducting material an extremely dense grid needs to be used and a dielectric value for the conducting material needs to be specified. For the PCB-CTHA case, using an extremely dense grid would exceed the capabilities of both the workstation and XFDTD, thus a regular grid has to be used. Thus the results that will be obtained from this section need to be then verified to find if they are valid or not, and to also decide upon which more accurately models a PCB-CTHA: the model using a perfect conductor or the model using the conductive material.

\subsubsection{Transient Case}

A model with 30 free space cells was generated as in the previous section, but this time the copper traces were given a conductivity of $5.8 * 10^{7}(\mathrm{~S} / \mathrm{m})$ which is the value of copper conductivity as given in [1]. Models were also generated with 40, 50, and 60 free space cells with the same conductivity value. In order to create these models the original perfect conducting material used in all previous XFDTD geometry files had to be changed. The perfect conducting material uses material number 1 in XFDTD, but for the conductive material model material number 3 was used. Material number 2 is already being used for the binding material, and is given a relative permittivity of 4.0 as mentioned earlier.

All four runs were made with 10,000 time steps, and the default relative permittivity value of 1.0 was used for the conductive material. Another set of four runs was also made but with the relative permittivity value changed from default to a number of 2000 to check the effect of changing the relative permittivity value in these runs. Both 
sets of runs were then compared to the perfect conductivity runs made in the previous section.

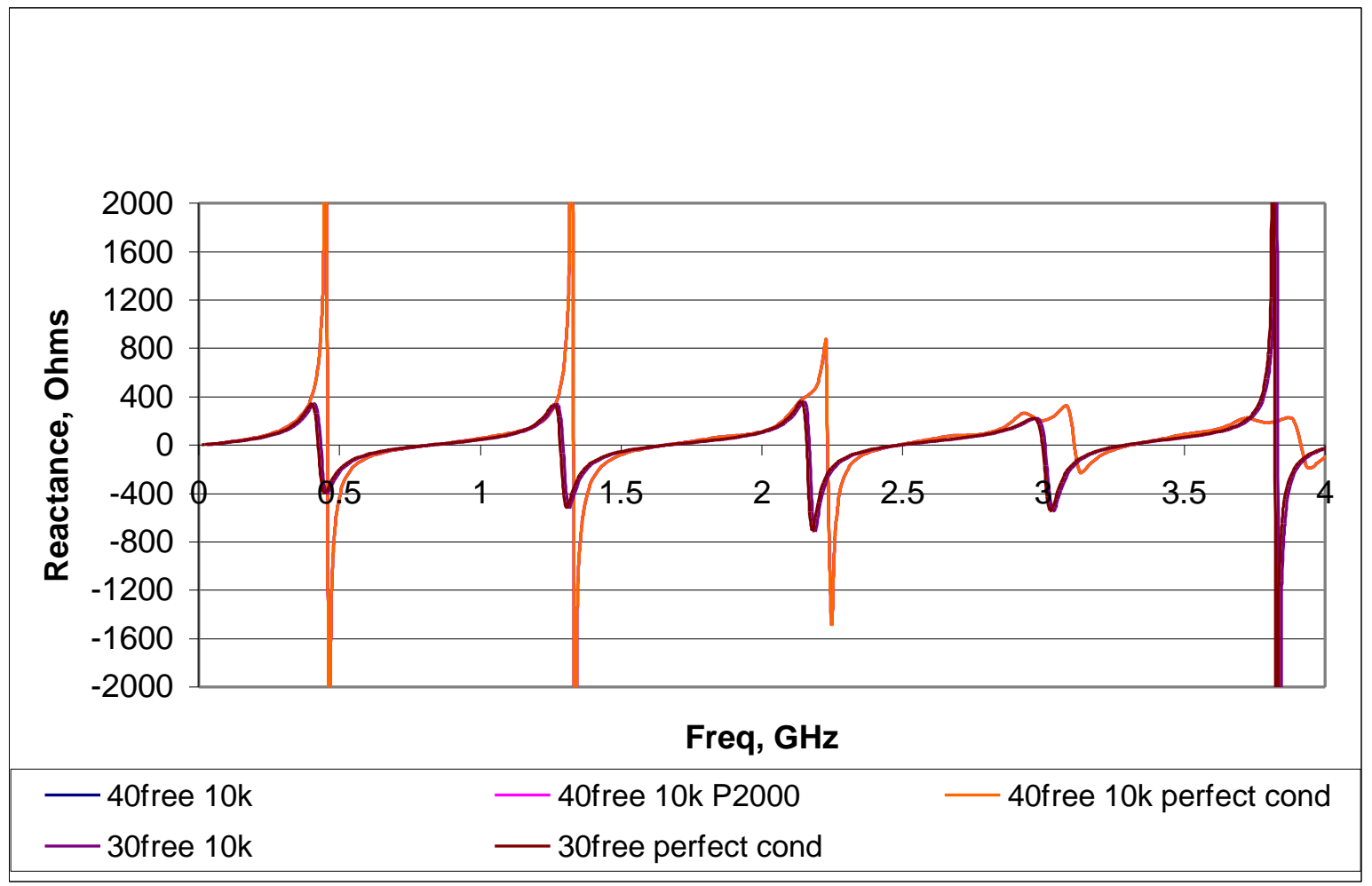

Figure 6.14 Effect of conductivity on the Reactance

Figure 6.14 shows the imaginary component of the input impedance chart for five of the runs, the 40 free space cells model with default relative permittivity, $\mathrm{P}=2000$, and with a perfect conductor, and the 30 free space cells model with default relative permittivity and with a perfect conductor. The other runs gave identical results to these runs, thus were excluded from the chart for clarity.

All three runs done with 40 free space cells followed exactly the same path and gave very close results. While the case of a perfect conductor and the case of a run with P $=2000$ were identical, the case with a default permittivity showed resonance frequencies of about $0.6 \%$ less on the average. The runs with 30 free space cells also showed the same results. Both runs were almost identical, and followed closely the results of the 40 
free space cells runs, with an average difference of $1 \%$ for the perfect conductor run, and $2 \%$ for the default relative permittivity run. Figure 6.15 shows a comparison between the resonance frequencies of all five runs.

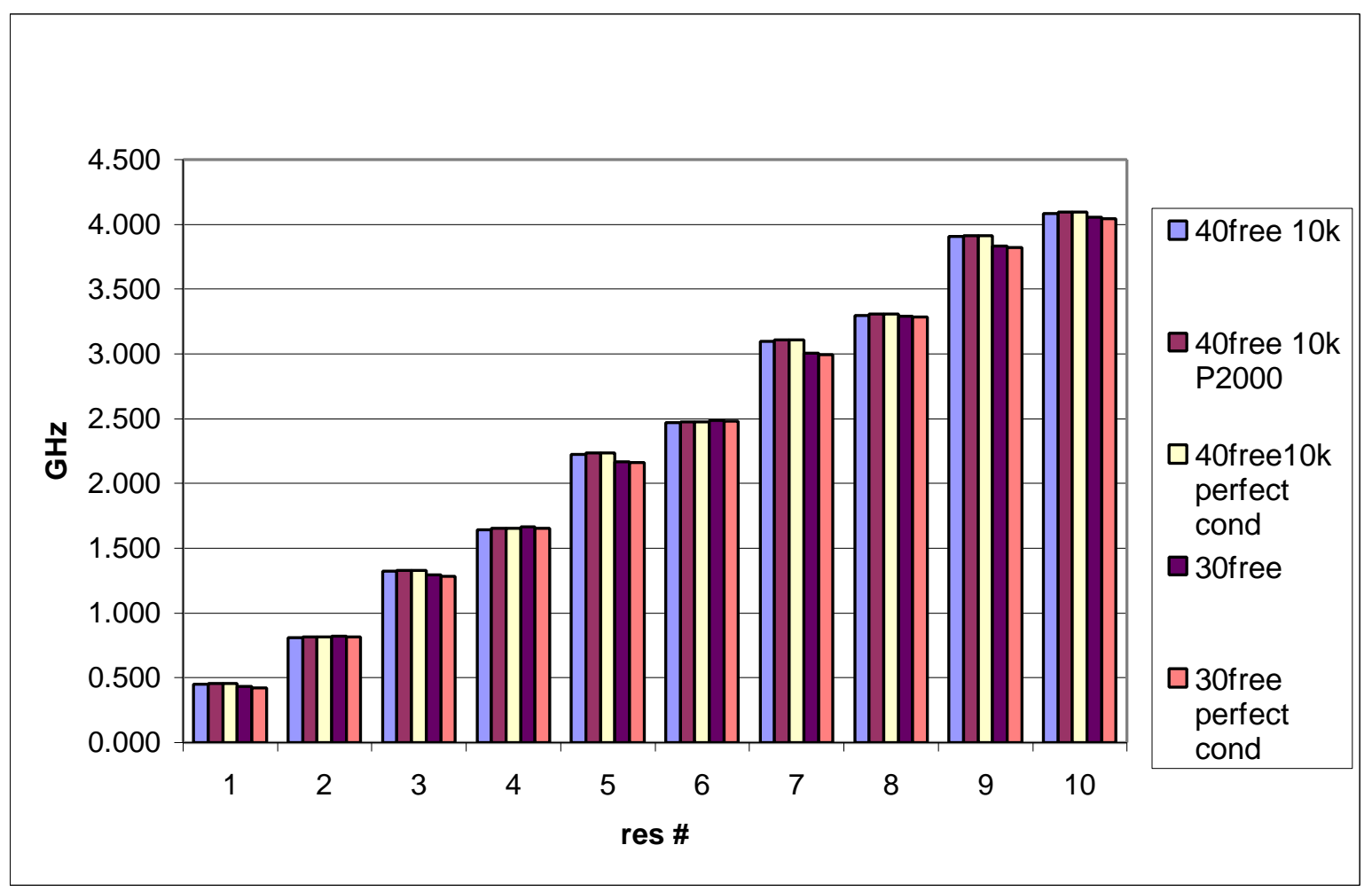

Figure 6.15 Comparison between the resonant frequencies for all five transient runs

In Figure 6.15 it can be seen that the resonant frequencies at the real resonances are almost identical for all runs. As with the anti-resonances, the values differed up to $2 \%$ as mentioned above. This is due to the approximations made by the software in order to model and draw a sudden change from a large positive number to a large negative number.

The above results were also found for both the 50 free space cells and the 60 free space cells. This indicates that XFDTD failed to model the conductivity effect of the traces of the PCB-CTHA in the transient runs. Since the results do not differ from those obtained through perfect conductor models, and the perfect conductor models were 
thoroughly checked for stability and convergence, it is recommended all transient runs on PCB-CTHA be performed using the model developed in the previous section.

To further prove on the above mentioned findings, Figure 6.16 shows both the real and the imaginary components of the input impedance for two cases. The first is for CTHA 108 with 30 free cells, feed 6, and perfect conductor traces. The second is the same antenna but with traces defined for copper. It is clear from the graph that, as shown above, the conductivity of the traces has minimal effect on the charts, since neither the real nor the imaginary components are affected.

Input impedance of model with 30 free space cells and defined conductivity, $\mathbf{6} 6 \mathbf{4 k}$

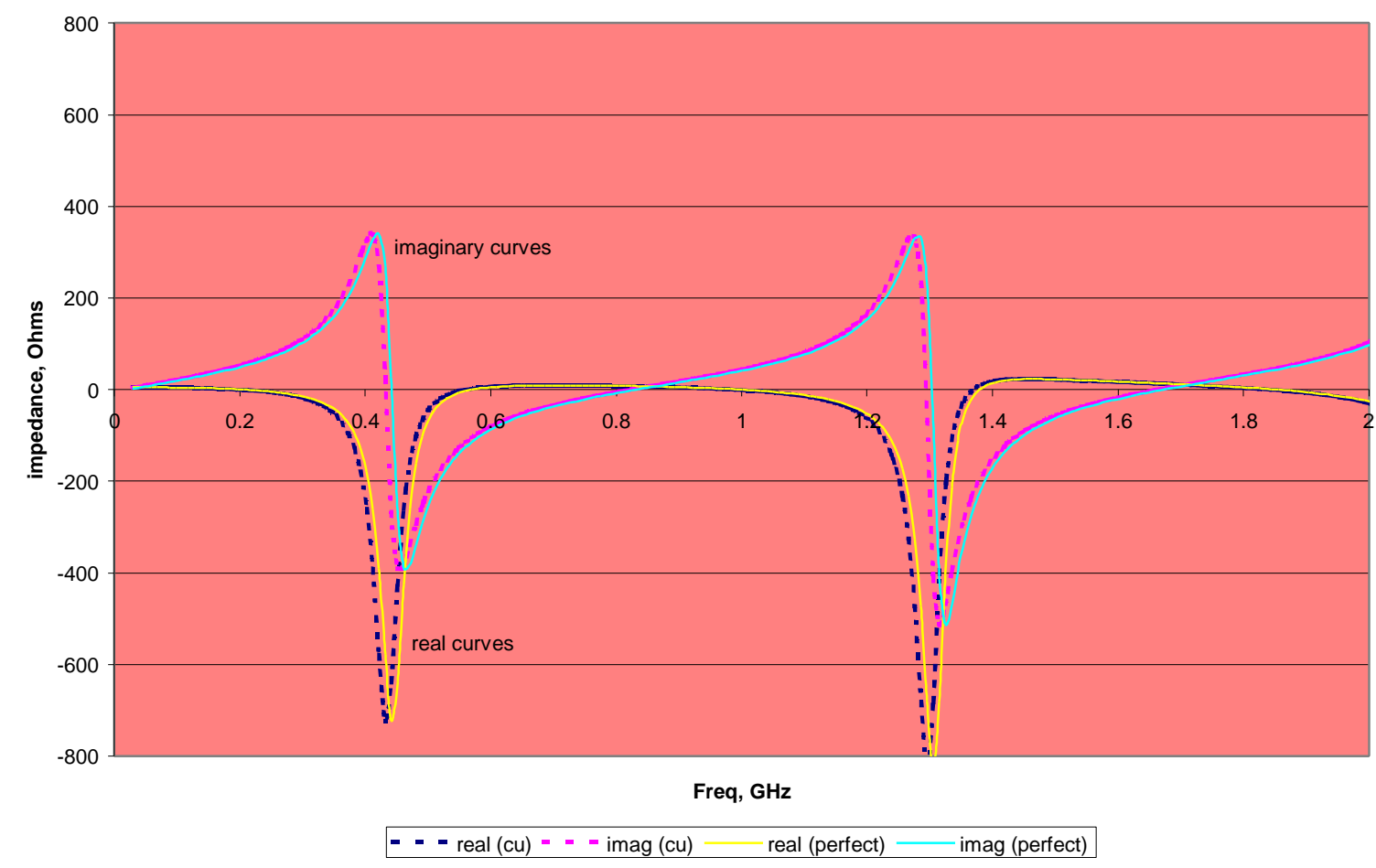

Figure 6.16 Input Impedance Graph showing the effect of conductivity of the traces 


\subsubsection{Steady State Case}

As with the transient runs, the steady state runs were also modeled using the same geometries. The numerical package XFDTD suggested 7741 time steps for all models, which is the same number of time steps suggested earlier for the runs without conductivity, and that number was used for all runs. Figure 6.17 shows the Ephi and Etheta graphs for the 30,40 , and 50 free space cells cases at the plane phi $=0$. On the same Figure the values for the 30 free space cells without conductivity were graphed for sake of comparison.

It is clear from the Figure that the introduction of conductivity had no effect whatsoever on the steady state results. Neither the Ephi nor the Etheta values were affected by the conductivity, nor did the modification of the number of free space cells introduce any significant change in the results.

The above result confirms the conclusion that was reached through the transient runs, and the studies of Kunz [43] on the FDTD method. XFDTD fails to show the effect of the conductivity of the traces of the PCB-CTHA. Since the runs with conductivity consumed much more time than the perfect conductor runs, the optimum model for a PCB-CTHA would be that found for the perfect conductor model. 

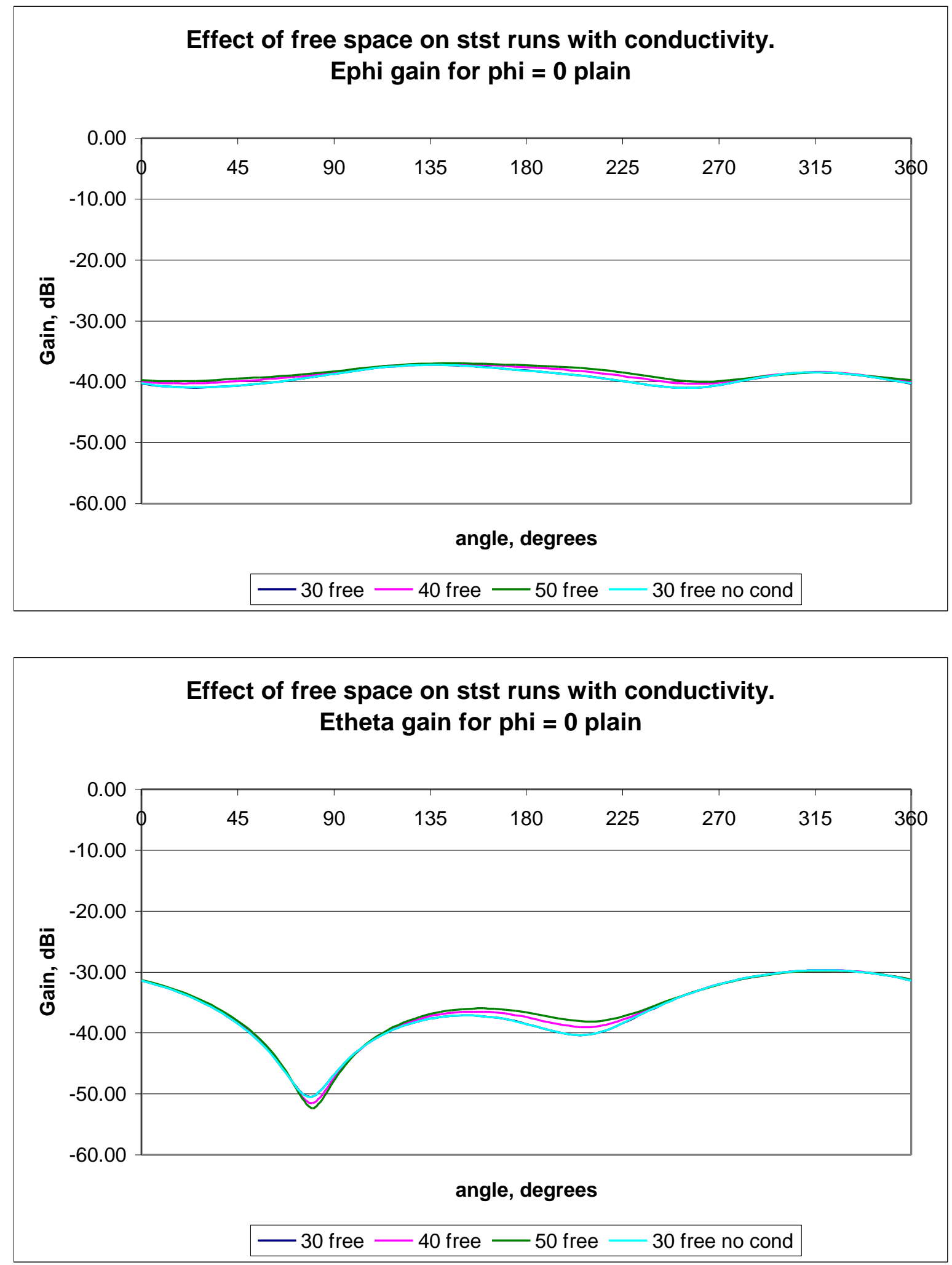

Figure 6.17 Effect of the free space on the conductivity runs. 


\subsubsection{Loss Tangent}

The loss tangent measures the power loss in the medium. It is defined as:

$$
\tan \delta_{c}=\frac{\sigma}{\omega \varepsilon_{0} \varepsilon_{r}}
$$

where:

$\tan \delta_{c}:$ The loss tangent

$\sigma:$ The effective conductivity, $\mathrm{S} / \mathrm{m}$

$\omega$ : The angular frequency $=2 \pi \mathrm{f}$, where $\mathrm{f}$ is the frequency in $\mathrm{Hz}$

$\varepsilon_{0}$ : Permittivity of free space, $=\frac{10^{-9}}{36 \pi} \mathrm{F} / \mathrm{m}$

$\varepsilon_{r}$ : Relative Permittivity, also called the dielectric constant.

The quantity $\delta_{c}$ is called the loss angle. A medium is said to be a "good conductor" if $\sigma>>\varepsilon[1]$, and is said to be a "good insulator" if $\sigma<<\omega \varepsilon$. Figure 6.18 below shows the loss tangent for the binding material within the range from 0.9 to $2 \mathrm{GHz}$, which is the working range of the anechoic chamber.

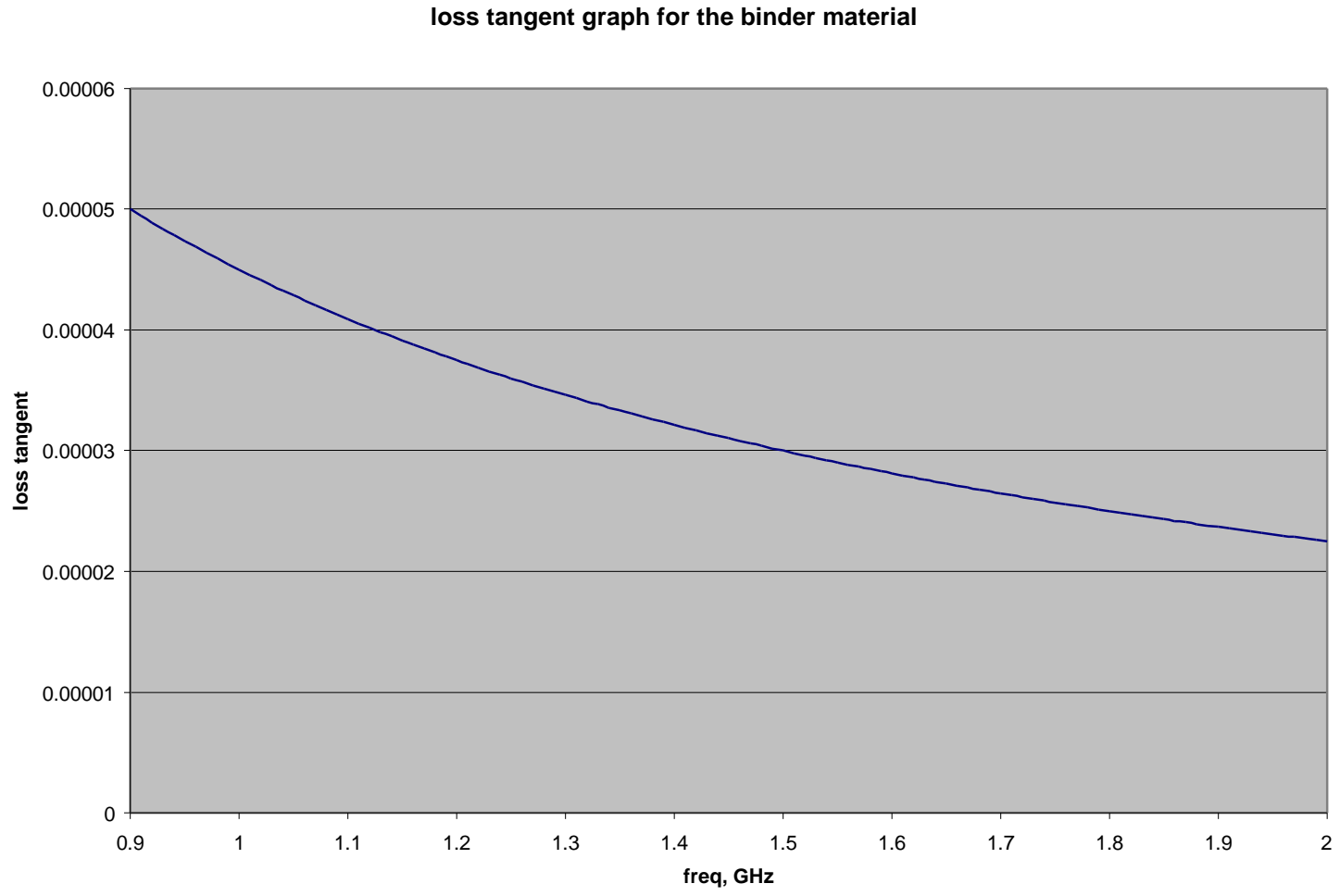

Figure 6.18 The loss tangent for the binding material. 


\subsubsection{Conclusion}

In this chapter, the effect of the different parameters of the model of a PCBCTHA were investigated, and an analysis of these effects was performed in order to reach an optimum model of the PCB-CTHA that would provide the best results with the least required processor time. The following conclusions were reached:

1. In all transient runs it is enough to use the minimum number of time steps and free space cells that would produce steady results. Any increase after that will have no effect on the results as they will stay the same.

2. Contrary to the above, increasing the time steps of a steady state run will cause the far field patterns to increase in value, while keeping their patterns the same. This will continue until approximately twice the number of time steps suggested by XFDTD are used, after which the run will crash. Thus it is advised to always use the number of time steps suggested by XFDTD for all steady state runs.

3. For a PCB-CTHA model where no relative permittivity is defined for the binding material, and the traces are modeled as perfect conductors, only 20 free space cells are required for the geometry file. Two thousand time steps are enough for the transient runs to yield results, while the number of time steps suggested by XFDTD should be used for the steady state runs.

4. If the relative permittivity of the binder material is introduced in the model, then the requirements for both the free space and the transient time steps increase. In this case 30 free space cells are needed in order to reach stable results, and 4000 time steps are required for the transient runs. These 
numbers apply up to a relative permittivity value of 4.0 , after which the requirements for the free space and time steps might differ.

5. XFDTD has failed to predict the effect of introducing a conductivity value for the traces of the PCB-CTHA. All runs made with conductivity gave the exact same results as if a perfect conductor material was used. Thus this feature should not be used.

6. The configuration described in item 4 above will be used as the default model format in all PCB-CTHA models for the rest of this study. And this same configuration is recommended for all future PCB-CTHA models. So unless otherwise mentioned, all runs will be performed using a 30 free space cells model, with perfect conducting traces, and a binding material with a relative permittivity of 4.0. The steady state runs will use the number of time steps suggested by XFDTD, while the transient runs will use 4000 time steps. 


\section{Experimental Setup}

For validating the numerical results obtained by XFDTD an experimental analysis on the PCB-CTHA needs to be performed. In this chapter the equipment used for the experiments will be described. The required setup for each type of experiment will also be shown along with the test procedures used.

\subsection{The Main Equipment Used}

In this section a brief introduction to all the equipment used in the experimental set up are introduced. These include the anechoic chamber, the network analyzer, the spectrum analyzer, and the frequency generator. In addition to this equipment, the software used for the far field experiments, which was developed within the CIRA group by Gururajan [63] will also be described.

\subsubsection{The Anechoic Chamber}

All the experimental work was done at the CIRA anechoic chamber located at the WVU hanger. The anechoic chamber is a closed room that has all its walls covered with an absorbing, non-reflecting, material that provides shielding for the room from all external electromagnetic interferences. These interferences exist in regular environments and could be either natural, as extraterrestrial radio waves or from thunderstorms, or they could be man-made like radio or communication waves, or they could even be resulting from the surrounding environment by reflecting the test waves generated thus causing interference. By conducting the experiments in an anechoic chamber, the effects of these external interferences are screened out, and the results can be considered to accurately represent the tested antennas. 


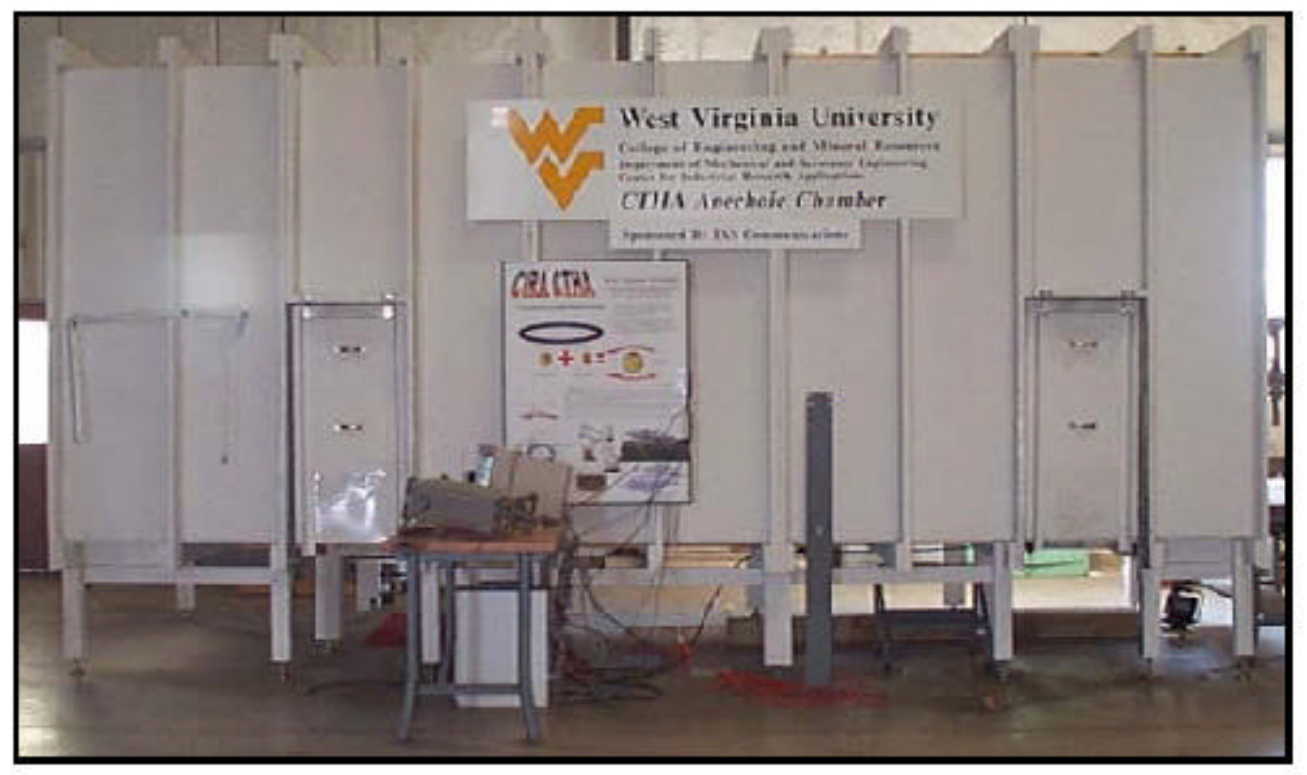

Figure 7.1 The anechoic chamber

The CIRA anechoic chamber was constructed by Dr. Gregory Thompson in the spring of 1998. The chamber's internal wall-to-wall dimensions are $18 \mathrm{ft}$ long $* 8 \mathrm{ft}$ deep * $8 \mathrm{ft}$ high. EHP-12 wedge absorber material was used as the internal absorbing material. This material was used to cover all the internal walls of the chamber, including the ceiling and the ground. The material was provided by RANTEC, and is marketed under the commercial name of RANTEC FerroSorb. Since the length of the cone of the absorber material is one foot, the resulting cone tip-to-cone tip length is $16 \mathrm{ft}$ long $* 6 \mathrm{ft}$ deep $* 6 \mathrm{ft}$ high.

As discussed by Pertl [58], The chamber is not recommended for use at frequencies below $900 \mathrm{MHz}$ since the reflections of the chamber walls will become significant and will no longer simulate a free space environment. Figure 7.1 and Figure 7.2 show photos of the anechoic chamber, while Figure 7.3, taken from [59], shows the dimensions and design of the chamber. 


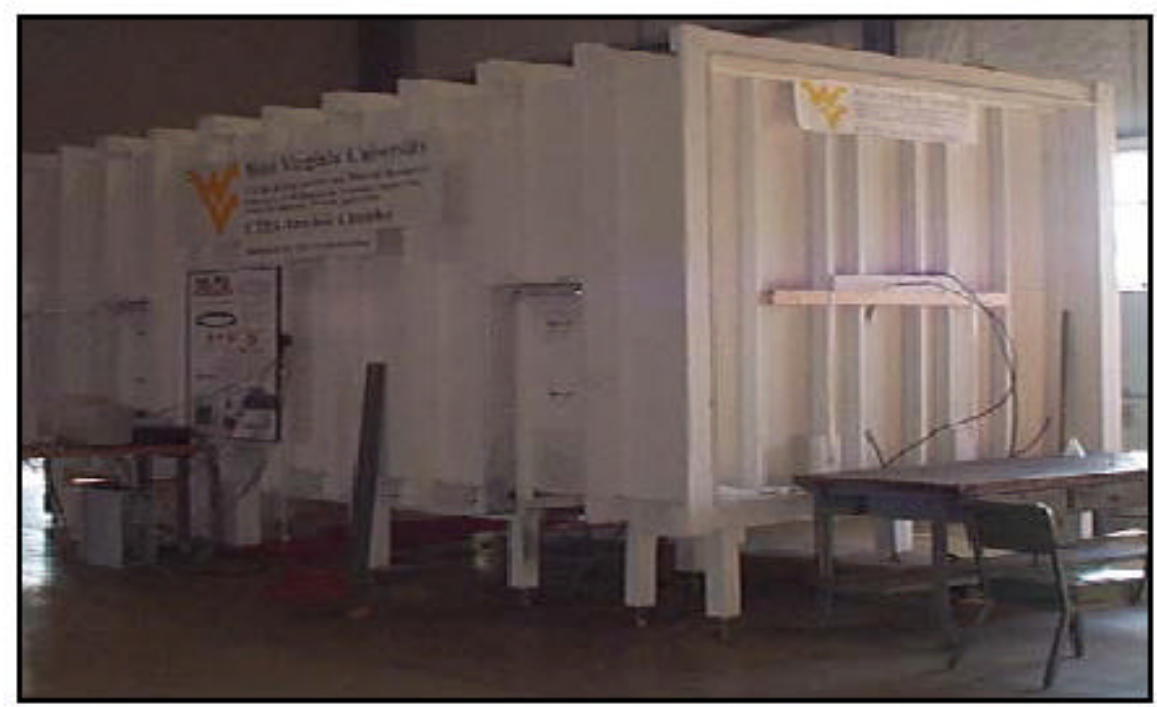

Figure 7.2 Another view of the anechoic chamber
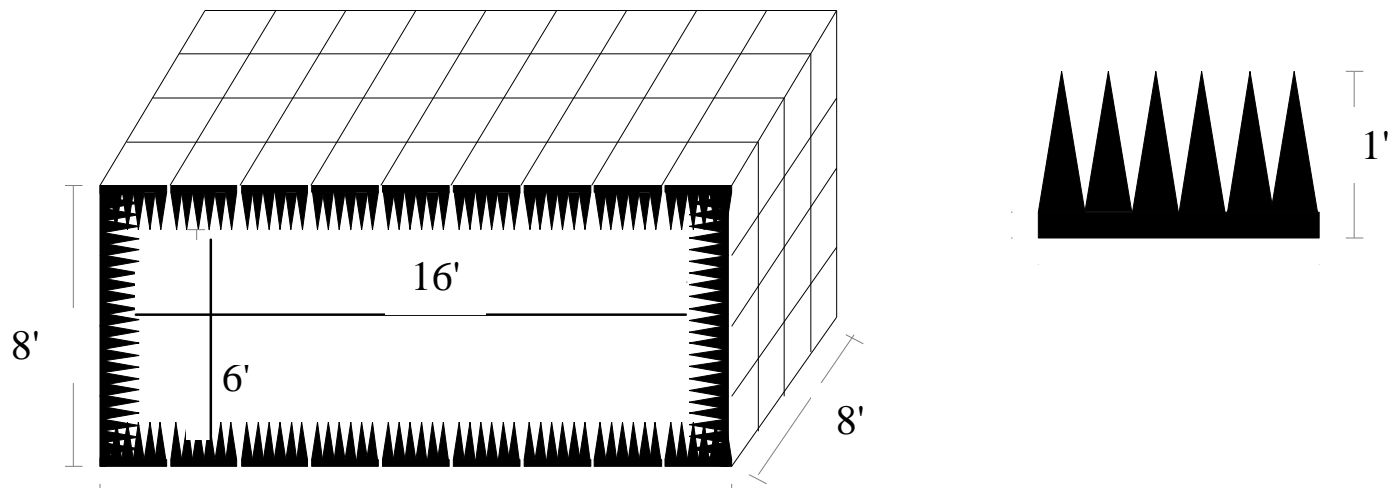

$18^{\prime}$

Figure 7.3 The design of the anechoic chamber

\subsubsection{The HP8753D Network Analyzer}

The Network analyzer available at the WVU-CIRA labs is the HP 8753D option

011. This is a high performance network analyzer that provides combined digital signal processing and microprocessor controls for easy operation and measurement improvement [60]. It integrates a high resolution synthesized RF source and a dual 
channel three-input receiver to measure and display magnitude, phase, and group delay responses of active and passive RF networks. The network analyzer was used for measuring the input impedance for the PCB-CTHA.

Measurement calibration is an accuracy enhancement procedure that effectively removes the system errors that cause uncertainty in measuring a test device. It measures known standard devices and uses the results of these measurements to characterize the system [60]. For calibrating the network analyzer in all the input impedance experiments, the standard HP 85033D 3.5mm female calibration kit was used. The frequency range of the calibration kit is $30 \mathrm{KHz}$ to $6 \mathrm{GHz}$, which covers the desired range of $900 \mathrm{MHz}$ to $2 \mathrm{GHz}$.

The results of the experiments are provided by the network analyzer in a formatted data array that adjusts for corrections, electrical delays, and other processing requirements. The formatted data array file has an extension of .f1 if channel 1 on the network analyzer is used, and .f2 if channel 2 is used. Within the formatted data file, the input impedance values are recorded in real and imaginary data pairs Re and Im. To calculate the resistance and reactance from this data pair equations 7.1 and 7.2 shown below are used:

$$
\begin{gathered}
\text { Resistance }=\left(1-\operatorname{Re}^{2}-\operatorname{Im}^{2}\right) /\left((1-R e)^{2}+\operatorname{Im}^{2}\right) * \mathrm{Z}_{0} \\
\text { Reactance }=(2 * \mathrm{Im}) /\left((1-\mathrm{Re})^{2}+\mathrm{Im}^{2}\right) * \mathrm{Z}_{0}
\end{gathered}
$$

Where $\mathrm{Z}_{0}$, the characterized impedance of the system, is $50 \mathrm{Ohms}$. A Visual Basic code was written that searches for .f1 and .f2 files in a certain directory, reads the pairs of real and imaginary data within the file, converts them to resistance and reactance, and creates an Excel file of the data that is ready to be graphed in Excel by the user. 
The maximum number of points per range on the network analyzer is 1601 points. Usually the desired test range is large, and testing it all at once would yield low accuracy since the distance between each point and the following point would be large. If the desired resolution is higher than that which can be attained by doing the test on the full range, then the range is cut into several sub-ranges, and the experiment is conducted on each sub-range independently, and each sub-range is saved in its own .f1 or .f2 file. For example if the test is desired to be between $800 \mathrm{MHz}$ and $4 \mathrm{GHz}$, as is the case in the impedance tests shown later, then testing all the range once would yield a resolution of about $2 \mathrm{MHz}$. But if the range is cut into two sub-ranges, from $800 \mathrm{MHz}$ to $2.4 \mathrm{GHz}$, and from $2.4 \mathrm{GHz}$ to $4 \mathrm{GHz}$, then the resolution would drop to $1 \mathrm{MHz}$. The Visual Basic program described above also takes the sub-ranging concept into consideration and checks for the different .f1 or .f2 files in each folder, then it assumes that all the .f1 and .12 files in each folder are all sub-ranges for one run or for one antenna. So the program processes all the data and merges all the results together and sorts them to produce one file. But the user has to be careful to include in each folder only the related .fx files for each run, since if runs for different antennas are located together in the same folder, the program will not understand this and will group them thus giving incorrect results. Appendix D provides the Visual Basic code for this program.

\subsubsection{The Signal Generator}

For generation of the signals used in the far fields tests in the anechoic chamber, the HP 8648C signal generator was used. The HP 8648C has a frequency range of 100 $\mathrm{kHz}$ to $3.2 \mathrm{GHz}$, a resolution of $1 \mathrm{~Hz}$, and a display resolution of $10 \mathrm{~Hz}$. If the signal 
generator is given adequate warm up time, usually one hour, its typical accuracy of frequency generation is $\pm 3 \mathrm{E}-06$ times the carrier frequency in $\mathrm{Hz}$ [61].

\subsubsection{The Spectrum Analyzer}

The Spectrum analyzer available at the WVU-CIRA labs is the HP 8590E. This spectrum analyzer covers the RF and microwave frequency ranges from $9 \mathrm{kHz}$ to 6.5 $\mathrm{GHz}$ [62]. It was used to graph and save the far field data for the antennas.

\subsubsection{Controller Code}

For controlling the far field experiments, the code written by Gururajan [63] was used. This code controls both the signal generator and the spectrum analyzer described above. It also signals to a motor that provides the rotation movement of the antenna, so that the far field pattern can be studied in any desired plane. The code was written in Visual Basic, and provides complete control to all the components from the available computer. For more details on the program and its code refer to [63].

\subsection{Experimental Setup}

Two types of experiments were conducted to be able to find the input impedance of an antenna within a certain frequency range, and to plot its far field graph at a certain frequency in a defined plane. Following is a description of the experimental setup for both types of experiments, showing the equipment used, and the basic connection diagram.

\subsubsection{Input Impedance Experiments}

For conducting the transient experiments and finding the input impedance within a certain range only the network analyzer is needed. Figure 7.5 below shows the setup, 
where the PCB-CTHA is located inside the anechoic chamber, and the network analyzer is located outside. The antenna is connected to the network analyzer via a coax cable.

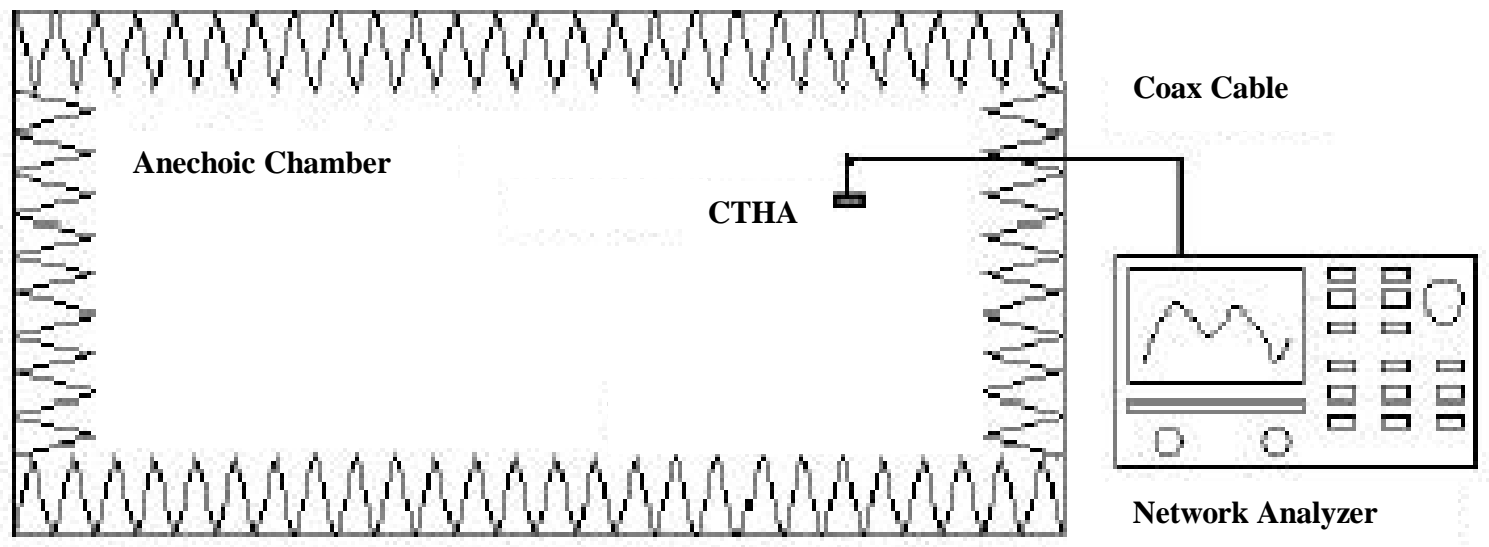

Figure 7.4 The experimental setup for the transient experiments

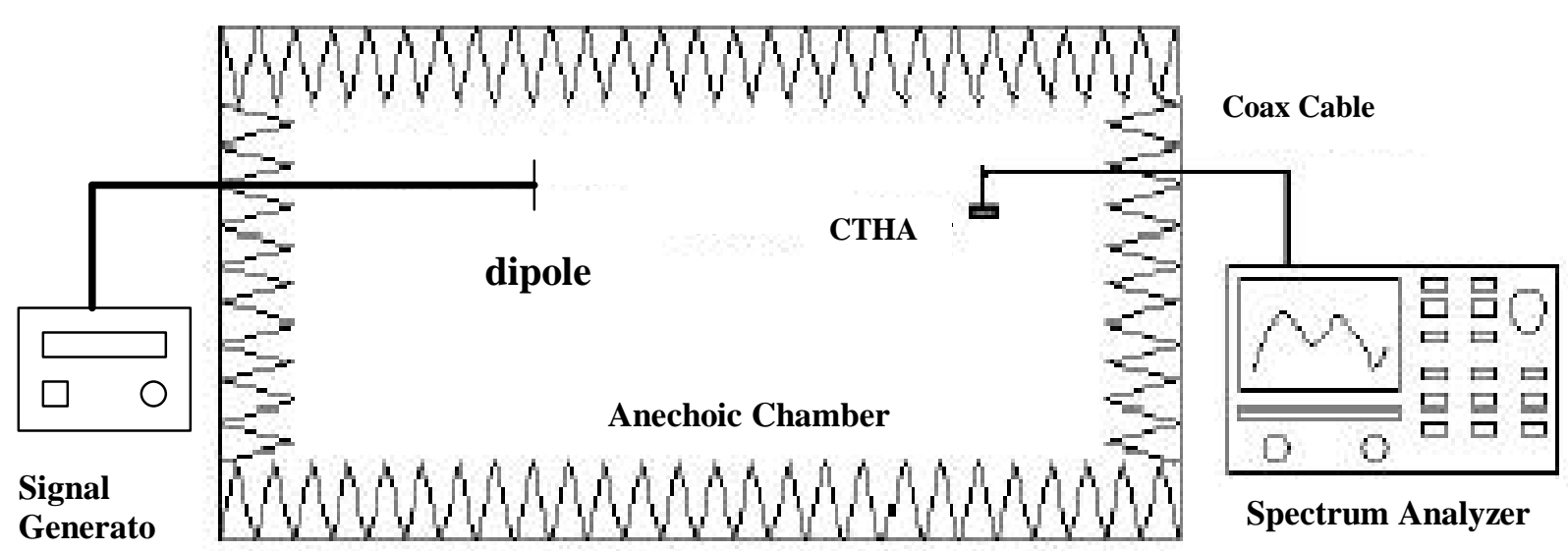

Figure 7.5 The experimental setup for the far field experiments

\subsubsection{Far Field Experiments}

For the far field experiments, both the spectrum analyzer and the signal generator are used. The signal generator is placed outside the chamber at one end, and the spectrum analyzer is located at the other end. The signal generator is connected to a dipole antenna that is used for sending the wave, while the PCB-CTHA is connected to 
the spectrum analyzer and is used for receiving the wave. Figure 7.5 shows this configuration.

Appendix E contains a collection of photos taken at the anechoic chamber site. These photos show the anechoic chamber and the equipment used for the experimental analysis that have been described in this chapter. 


\section{Feed Study Results and Validation}

In this chapter the results of both the numerical and experimental feed investigation will be shown. From these results the validity of the numerical model will be determined. In addition, the results will also provide a full analysis of the feed shape effect on the input impedance and the far field patterns of the PCB-CTHAs.

First, the chapter will start with a detailed analysis of one of the feeds to demonstrate all charts and methods of comparison and validation. The remaining 12 feeds will be provided in Appendix F, but a summarized analysis in this chapter will show the main conclusions obtained from these feeds. The result of the analysis of all 13 feeds will be used to reach conclusions on grouping the feeds. General comments will also be provided for these feeds.

\subsection{Characteristics of Each Feed}

In this section each of the 13 feeds will be studied individually. The general method followed for all feeds was identical. First a transient run is conducted on the antenna. The results of this run are then compared to the experimental results to validate the numerical process and determine how close it compares to experimental data. The resonance frequencies are identified for both the numerical and experimental results. Two resonant frequencies, one real and one anti, that fall between 0.9 and $2 \mathrm{GHz}$ are chosen to compare the numerical and the experimental results. Steady state runs are conducted at the two resonance frequencies calculated by the numerical analysis. Far field experiments were conducted in the anechoic chamber at the experimental resonance frequencies. The two sets of results are then compared to validate the accuracy of the numerical predictions of the model. Finally, conclusions are drawn on how well the 
numerical model simulates both the steady state and the transient runs, and general conclusions and comments on the characteristics of the feed are then deduced.

\subsubsection{Feed 5}

\subsubsection{Transient Graph}

As stated above, the first step in the analysis of each feed is to make a transient run and to compare it to the transient results obtained from the network analyzer with the antenna in the anechoic chamber. Since the least acceptable frequency for the anechoic chamber is $0.9 \mathrm{GHz}$, the results obtained from both sets, the numerical and the experimental, started from $0.9 \mathrm{GHz}$ and were limited to a maximum of $2 \mathrm{GHz}$. Figure 8.1 shows the imaginary component of the input impedance for feed 5 for both the experimental and the numerical cases.

Figure 8.1 shows that there is an excellent match between the numerical and the experimental results for the imaginary component of the input impedance graph for this feed. The numerical graph intersected the $\mathrm{x}$-axis at almost the exact places the experimental graph did, thus predicting the input impedances with high accuracy. Table 8.1 shows the input impedance frequencies found from both these graphs and the calculated deviations.

It is also clear from the graph that the impedance maximum values at the anti resonances did not always match, which is especially clear in Figure 8.1 at the first and third anti resonances. This is the same finding that was earlier noted in Chapter 6. 


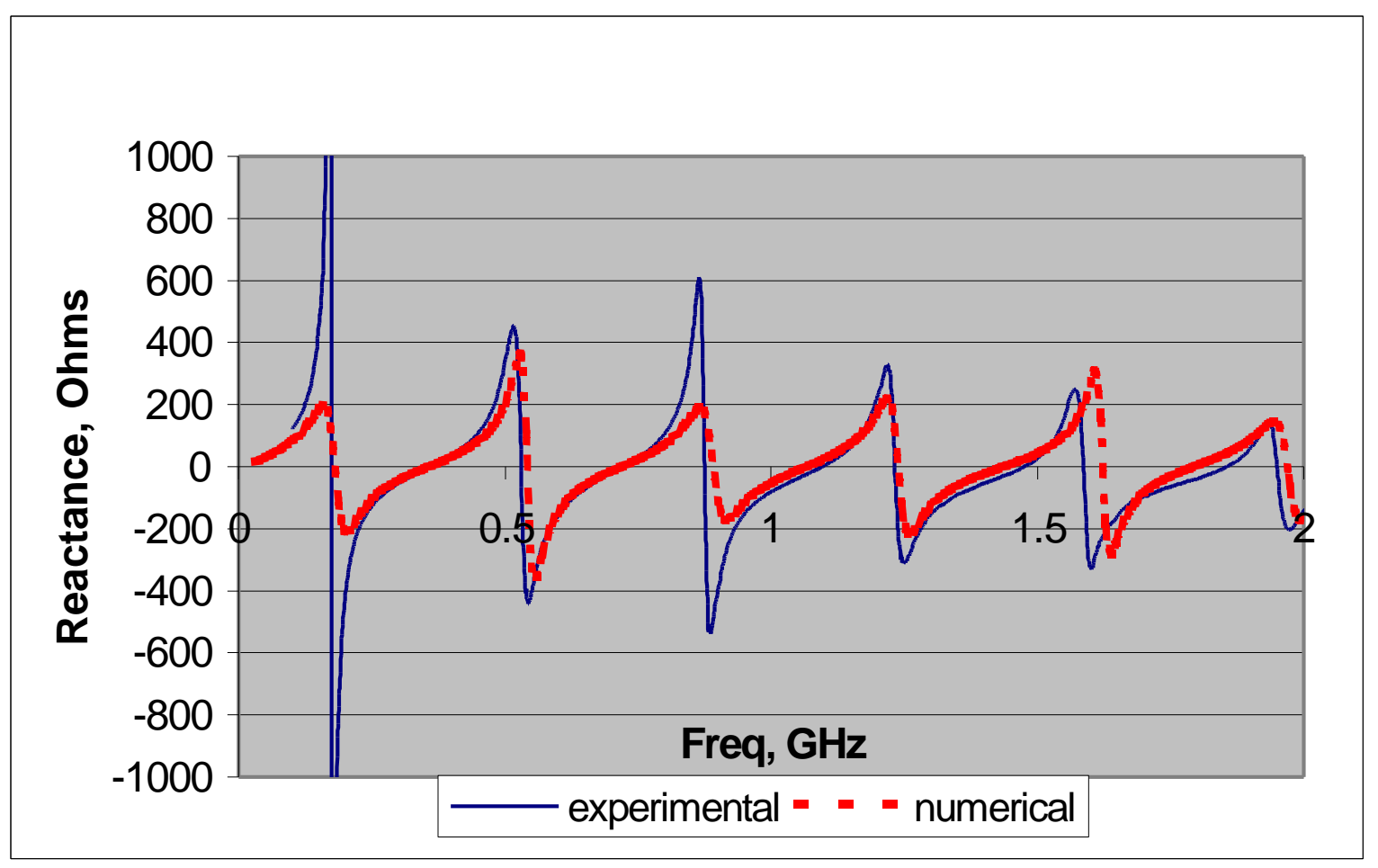

Figure 8.1 The Reactance for feed 5 - experimental vs. numerical

Table 8.1 Comparing the experimental resonance frequencies for feed 5 with the numerically predicted resonance frequencies.

\begin{tabular}{|c|c|c|}
\hline$f 5$ exp. $(\mathrm{GHz})$ & $f 5$ num. $(\mathrm{GHz})$ & \% difference \\
\hline 0.174 & 0.186 & 6.90 \\
\hline 0.358 & 0.366 & 2.23 \\
\hline 0.53 & 0.549 & 3.58 \\
\hline 0.719 & 0.728 & 1.25 \\
\hline 0.875 & 0.904 & 3.31 \\
\hline 1.096 & 1.086 & 0.91 \\
\hline 1.233 & 1.263 & 2.43 \\
\hline 1.476 & 1.46 & 1.08 \\
\hline 1.587 & 1.646 & 3.72 \\
\hline 1.856 & 1.822 & 1.83 \\
\hline
\end{tabular}

\subsubsection{Steady State Graphs}

The next step was to perform the far field experiments for this same feed. Two resonant frequencies were chosen for these experiments, these are the frequencies shown in bold in Table 8.1. The experimental runs were performed at the experimental resonant 
frequencies, which are 1.096 and $1.233 \mathrm{GHz}$, while the numerical runs were performed at the numerically predicted resonant frequencies of 1.086 and 1.263 . Although any other resonant frequencies could have been chosen, these two sets were specifically chosen for the combination of the following reasons:

1. They fall well within the anechoic chamber preferred range,

2. They represent two consecutive real and imaginary resonances, so that both cases can be studied, and

3. The good match between the numerical and the experimental resonant frequencies at these points, since the difference is $0.9 \%$ for the real resonances, and $2.4 \%$ for the anti resonances.

The experimental setup in the anechoic chamber was prepared to test the antenna in two convenient orientations. The first has the antenna mounted horizontally, with the axis of the holes in the antenna parallel to the axis of the mounting pole, refer to Figure E.6 in Appendix E. The second has the antenna mounted vertically, as in Figures E.4 and E.5. The horizontal orientation provides the far field patterns at the theta $=90$ plane, while the vertical orientation provides the patterns at the phi $=0$ plane.

It has to be also noted from the orientation of the antennas, and for sake of comparison with the numerical results, that the starting point of the horizontal experimental results, where the angle $=0$, is equal to an angle of $180^{\circ}$ in the numerical results. Similarly, the starting point of the vertical experimental results, where the angle $=0$, is also equal to an angle of $180^{\circ}$ in the numerical results. This is due to the physical orientation of the antenna fixture in the anechoic chamber, in contrast to the axis definitions in XFDTD. This difference can be clearly visualized by comparing the 
antenna orientation in Figures E.4 through E.6 with the standard definition of the phi and theta axis, which is what is used in the numerical analysis.

The orientation of the transmitting dipole is also of great importance. For a horizontally mounted CTHA, and when the dipole is also horizontally mounted then the far field pattern obtained is the Ephi pattern. On the other hand, when the dipole is vertically mounted, also with a horizontally mounted CTHA, then the resulting pattern is the Etheta patterns for the same plane. Figure E.7 shows the dipole mounted horizontally, while Figure E. 8 shows it mounted vertically. When the CTHA is mounted vertically the effect of the orientation of the dipole will be the opposite. So when the CTHA is mounted vertically and the dipole is horizontal then the resulting pattern is the Etheta pattern. When the CTHA is vertical and the dipole is also vertical then the pattern is the Ephi pattern. Any other orientation of the dipole will result in a pattern that is a mix between the Ephi and the Etheta patterns and will be of no use when compared to the numerical results.

Thus, from the above analysis, it is found that for each antenna four different experimental sets of runs need to be performed at each frequency. Two with the CTHA mounted horizontally, the first with a horizontal dipole and the second with a vertical dipole. And the other two with the CTHA mounted vertically, also the first with a horizontal dipole and the second with a vertical dipole. Then all of these results need to be adjusted so that the starting point in the experimental results is made equivalent to angle $180^{\circ}$, and the rest of the graphs adjusted thereupon. Since the experiments will be done at two different frequencies, one at the real resonance and one at the anti resonance, there will be eight experiments for each of the thirteen feeds. 
The controller code described in Chapter 7 allows for three different settings for the angular separation at $0.9,1.8$ and 4.5 degrees. The 1.8 separation is recommended by Gururajan [67] and is thus used in all the analysis. Similarly, all the numerical graphs were adjusted to provide results at $1.8^{\circ}$ angular increments to be consistent with their experimental equivalents.

An important point also worth mentioning is that the experimental results are provided in $\mathrm{dBm}$, while the numerical results are provided in $\mathrm{dBi}$. Figure 8.2 below shows the steady state results for feed 5. As shown in the graphs, the experimental results are plotted in $\mathrm{dBm}$, while the numerical results are plotted in $\mathrm{dBi}$. As stated earlier, two resonant frequencies were chosen from the transient results, one real and one anti. For each of these two frequencies two graphs are plotted, one for the phi $=0$ plane and one for the theta $=90$ plane. Each of these two planes is graphed separately; thus there are four graphs, with the Ephi and Etheta gains plotted on each graph per frequency per feed.

To be able to easily differentiate between the Ephi and the Etheta curves, the Ephi curves will always be plotted with thick lines, while the Etheta curves will always be plotted with thin lines. On the other hand, the difference between the numerical and the experimental curves is that the numerical curves will always be higher in value, i.e. closer to the $\mathrm{x}$-axis, than the experimental curves. 


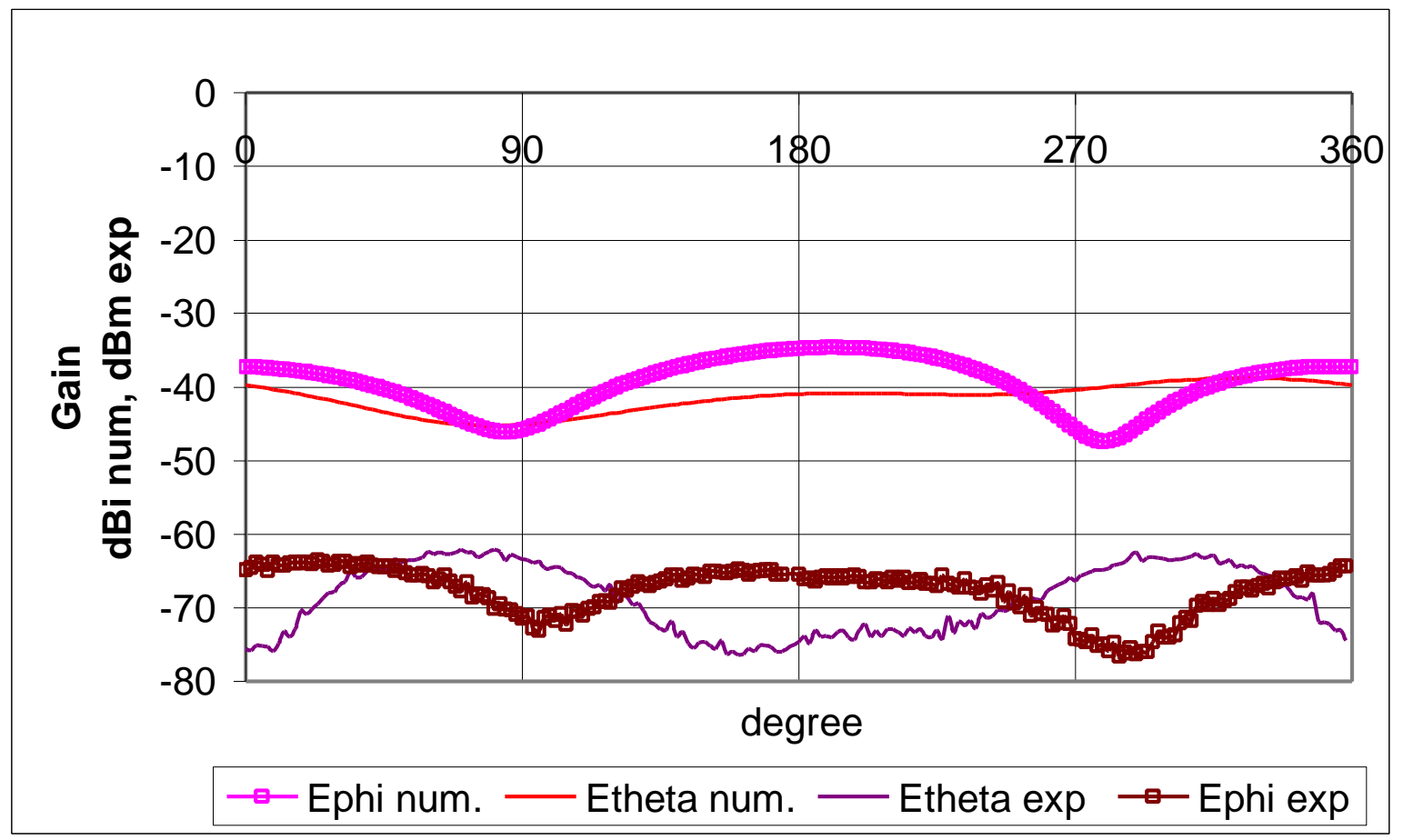

Figure 8.2a The Phi = O plane for feed 5 at $1086 \mathrm{MHz}$ num and $1096 \mathrm{MHz}$ exp

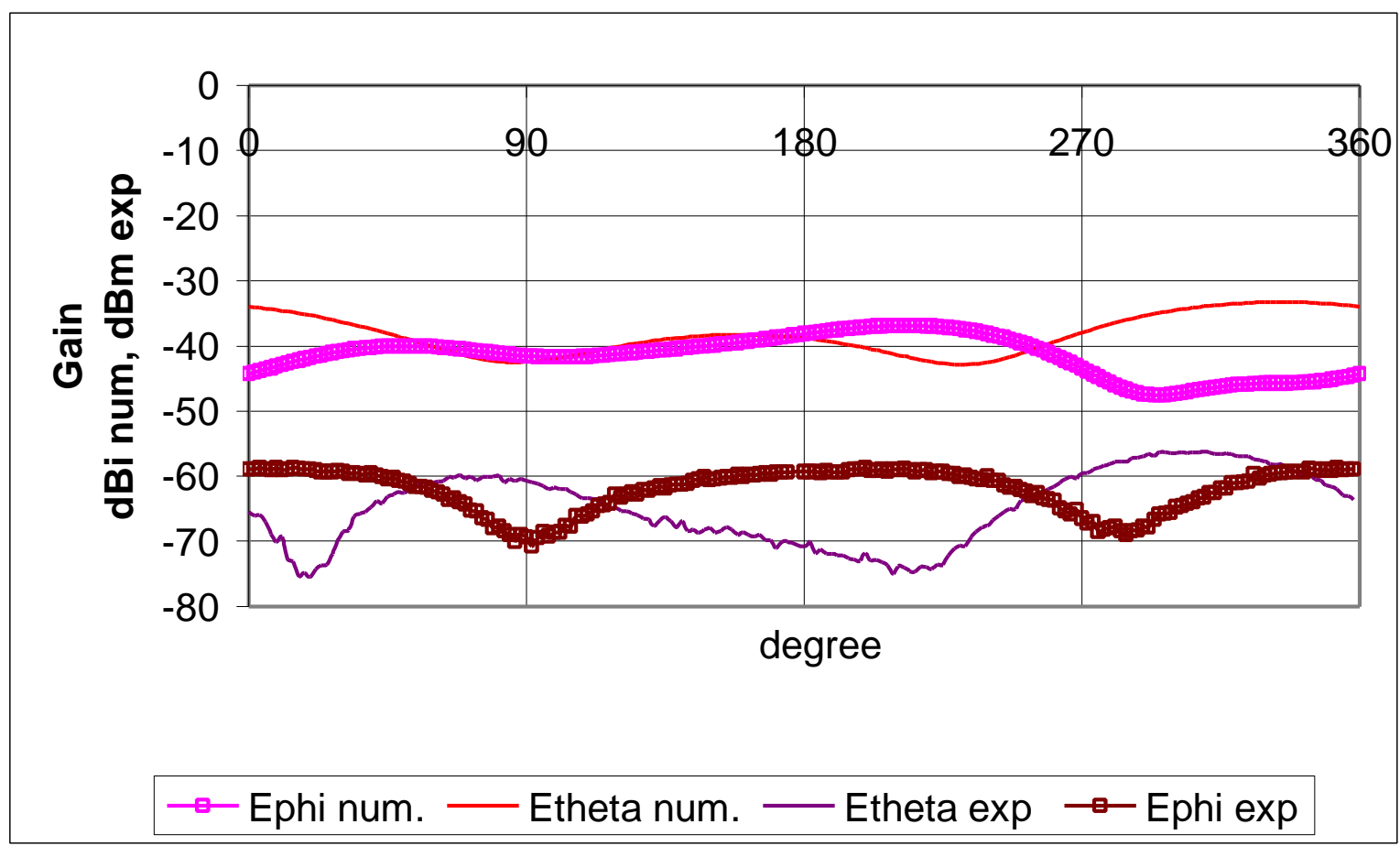

Figure $8.2 \mathrm{~b}$ The Phi $=0$ plane for feed 5 at $1263 \mathrm{MHz}$ num and $1233 \mathrm{MHz}$ exp 


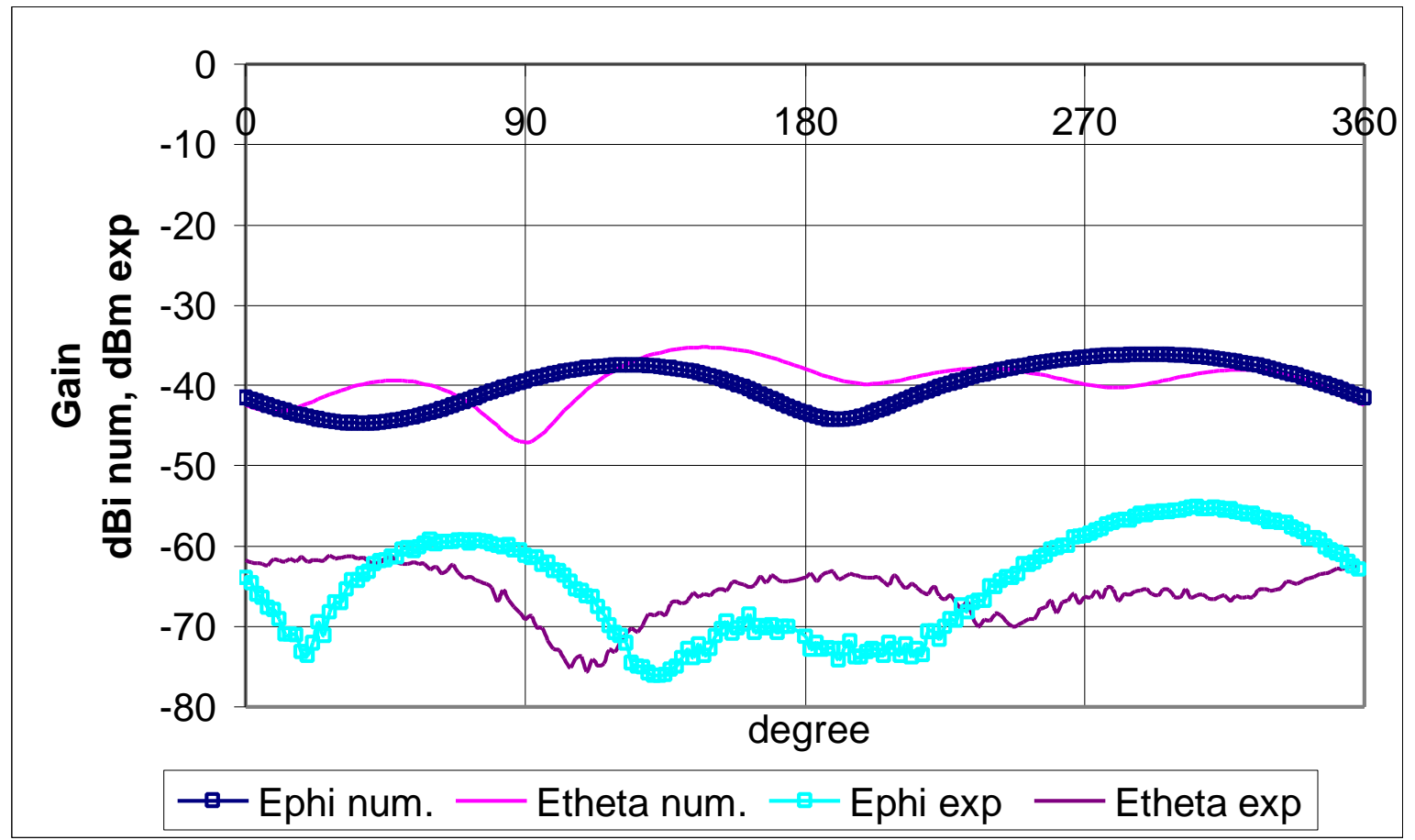

Figure 8.2c The Theta $=90$ plane for feed 5 at $1263 \mathrm{MHz}$ num and $1233 \mathrm{MHz}$ exp

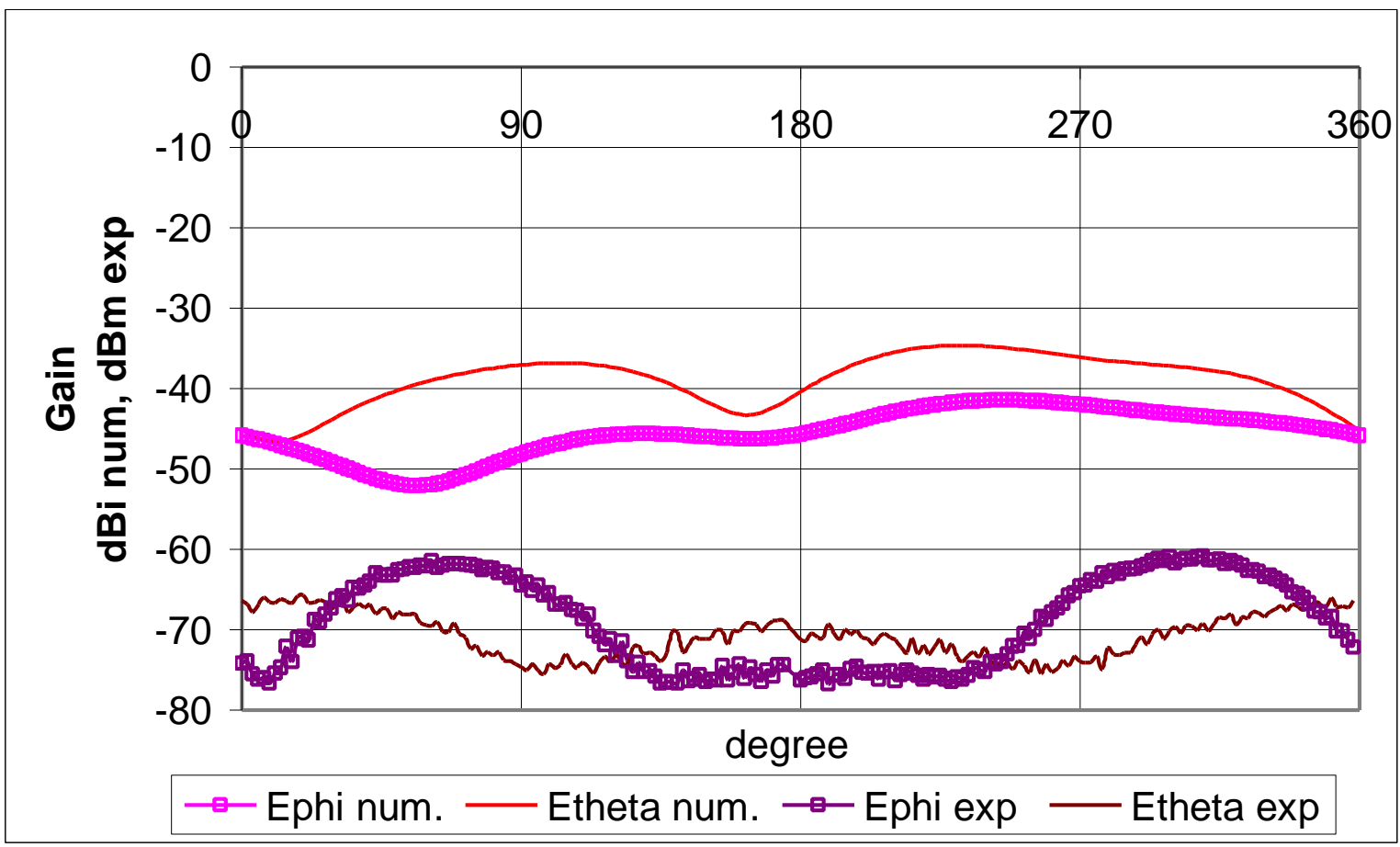

Figure 8.2d The Theta $=90$ plane for feed 5 at $1086 \mathrm{MHz}$ num and $1096 \mathrm{MHz}$ exp 


\subsubsection{Analysis of the Reactance data}

The above three sections showed the data that was collected from the different tests, how it was collected, and how it was presented. In this and the following sections the data will be analyzed so that the behavior of the PCB-CTHA with this specific feed can be understood.

Again, as stated earlier, the same method of analysis that will be performed here on feed 5 will be performed on the rest of the feeds that are presented in Appendix F. The detailed method of analysis will only be mentioned in this location, while Appendix F will only list the results.

Referring to Figure 8.1 it is clear that the match between the experimental and the numerical results is excellent. Table 8.1 shows that the resonant frequencies predicted by the numerical analysis, except for the first which is at a frequency of less than $200 \mathrm{MHz}$ and is thus out of the reliable range for the anechoic chamber, are all within $4 \%$ of the actual experiments.

The real resonances were found to be predicted better than the anti resonances. The real resonances difference was always less than $2 \%$, while the anti resonances difference was between $2 \%$ and $4 \%$.

From the above it can be stated that for this specific feed the transient behavior of the PCB-CTHA is well predicted. As for the behavior of the antenna, the graph shows that there is a uniform pattern for the graph as a whole. The resonant frequencies tend to repeat themselves at equally spaced intervals. Table 8.2, which was calculated from the results in Table 8.1 above, shows the intervals between the frequencies for both the experimental and the numerical results. 
Table 8.2 Analysis of the resonance frequencies obtained from the transient graph

Experimental

\begin{tabular}{|c|c|c|}
\hline $\begin{array}{c}\text { f5 exp. } \\
(\mathrm{GHz})\end{array}$ & $\begin{array}{c}\text { diff. between } \\
\text { res freq's }\end{array}$ & $\begin{array}{c}\text { diff. between } \\
\text { same type freq's }\end{array}$ \\
\hline 0.174 & & \\
\hline 0.358 & 0.184 & \\
\hline 0.53 & 0.172 & 0.356 \\
\hline 0.719 & 0.189 & 0.361 \\
\hline 0.875 & 0.156 & 0.345 \\
\hline 1.096 & 0.221 & 0.377 \\
\hline 1.233 & 0.137 & 0.358 \\
\hline 1.476 & 0.243 & 0.38 \\
\hline 1.587 & 0.111 & 0.354 \\
\hline 1.856 & 0.269 & 0.38 \\
\hline & & \\
\hline mean & 0.187 & 0.364 \\
\hline
\end{tabular}

Numerical

\begin{tabular}{|c|c|c|}
\hline $\begin{array}{c}\text { f5 num. } \\
(\mathrm{GHz})\end{array}$ & $\begin{array}{c}\text { diff between } \\
\text { res freq's }\end{array}$ & $\begin{array}{c}\text { diff between same } \\
\text { type freq's }\end{array}$ \\
\hline 0.186 & & \\
\hline 0.366 & 0.180 & \\
\hline 0.549 & 0.183 & 0.363 \\
\hline 0.728 & 0.179 & 0.362 \\
\hline 0.904 & 0.176 & 0.355 \\
\hline 1.086 & 0.182 & 0.358 \\
\hline 1.263 & 0.177 & 0.359 \\
\hline 1.46 & 0.197 & 0.374 \\
\hline 1.646 & 0.186 & 0.383 \\
\hline 1.822 & 0.176 & 0.362 \\
\hline & & \\
\hline mean & 0.182 & 0.365 \\
\hline
\end{tabular}

The first column in Table 8.2, labeled "diff between res freq's" was calculated by subtracting every resonant frequency from the frequency that followed it. The other column, labeled "diff. between same type freq's" was calculated by subtracting every resonant frequency from the frequency of its same type that follows it, so in this case one frequency is skipped and then the following is used.

Table 8.2 shows that the resonant frequencies do have a uniform pattern that is regularly followed. The table also shows that this pattern is also well predicted by the numerical analysis. The numerical analysis tends to predict a perfect cycle, where the spacing between every two resonant frequencies is constantly $182 \mathrm{MHz}$. The experimental analysis, on the other hand, has much more deviations from the mean value, but still tends to always be around the same previous Figure.

\subsubsection{Analysis of the Gain Data}

Figures $8.2 \mathrm{a}$ through $8.2 \mathrm{~d}$ show the comparison between the numerical and experimental far field patterns for the PCB-CTHA at two different resonant frequencies, 
one at a real resonance and the other at an anti resonance. Each of the four graphs shows the experimental and numerical pattern per plane per frequency. As stated earlier, the Ephi patterns are graphed in thick lines, while the Etheta patterns are in thin lines. Also the experimental patterns are in $\mathrm{dBm}$ and are always lower than the numerical patterns which are in $\mathrm{dBi}$.

Figure 8.2a shows a good agreement between the numerical and the experimental Ephi data, with both having similar patterns, and the difference in $\mathrm{dB}(\mathrm{dBd})$ almost constant between 27 and 30. The Etheta pattern though did not show the same similarity. The peaks on both graphs did not match, and the patterns were very different. The difference between both graphs ranged from 17 to $35 \mathrm{dBd}$. So for the Phi $=0$ plane at the real resonance the numerical tests were able to predict the Ephi patterns but failed to predict the Etheta patterns.

Figure $8.2 \mathrm{~b}$ shows the $\mathrm{Phi}=0$ plane also but at the anti resonance. Here again the numerical simulation was also able to predict the Ephi pattern well. Except for the low peak at 90 degrees which was not predicted deep enough, but the rest of the graph followed closely with a constant difference of 15 to $20 \mathrm{dBd}$. The Etheta pattern prediction on the other hand was not a total failure as in the last graph. Here the Etheta numerical pattern well predicted the experimental pattern between 180 and 360 degrees, with a constant difference of approximately $30 \mathrm{dBd}$. The first 180 degrees though were falsely predicted, with a low peak at 23 degrees that was not predicted at all, and a peak at 90 degrees that was a high peak on the experimental graph but was predicted a low peak on the numerical graph. 
Figures 8.2c and 8.2d showed the Theta $=90$ plane for the anti and real resonances, respectively. This time Figure $8.2 \mathrm{c}$ predicted the Etheta pattern well. Both the numerical and the experimental graphs matched, with a difference of 20 to $25 \mathrm{dBd}$. The Ephi pattern was also predicted well, with the patterns from 180 to 360 degrees well matching and having a mean difference of $25 \mathrm{dBd}$. The pattern from 0 to 180 degrees did not match so well though. The experimental graph showed a steep low peak at about 20 degrees, while the numerical graph had a shallow broad low peak at 41 degrees. The experimental graph also showed a broad high peak at 80 degrees, while the numerical had that broad high peak at 115 degrees. The mean difference between the numerical and experimental Ephi graphs ranged between 20 and $30 \mathrm{dBd}$.

The Ephi pattern on Figure 8.2d showed the same characteristics as those found for Figure 8.2c, where the patterns matched, but poorly, between 180 and 360 degrees, but did not match for the first 180 degrees. On the other hand, the Etheta patterns did not match at all between both graphs. Actually the experimental Etheta pattern looked as if it were a mirror image of the numerical Etheta pattern, with the highs on one graph coinciding with the lows on the other. The mean difference between both Ephi graphs was between 15 and $25 \mathrm{dBd}$, while that for the Etheta graphs was between 20 and 30 $\mathrm{dBd}$.

The above results are all summarized in Table 8.3. In this table the quality of the prediction, called the score, was given either poor, fair, good, or very good, depending on how well the numerical graph qualitatively predicted the experimental graph. The $\mathrm{dBd}$ column showed the difference between the experimental graph, in $\mathrm{dBm}$, and the numerical graph, in $\mathrm{dBi}$, along the curve. The smaller the range between the higher and 
lower values in the $\mathrm{dBd}$ column the better the match between the numerical and experimental curves.

In general it can be said that the numerical graphs provided a fair prediction of the experimental graphs. Although nowhere comparable to the results obtained from the transient runs, but the predictions showed a reasonable qualitative insight to what the experimental graphs looked like, with a general difference of 20 to $30 \mathrm{dBd}$.

\subsubsection{Feeds 1 to 13}

The same method of analysis that was performed on feed 5 in the previous pages was performed on all 13 feeds. This detailed analysis of all feeds can be found in Appendix F. The detailed description will not be provided since it has already been shown here, but all the results and graphs and tables will be shown. For the transient results a comparative graph will be shown similar to Figure 8.1 for each feed, in addition to a table similar to Table 8.2 but with the addition of the error column as in Table 8.1.

As for the steady state cases, four Figures similar to Figures 8.2a through 8.2d will also be shown for each feed. And their summary will also be provided in a table similar to Table 8.3. Finally all results from Appendix $F$ will be summarized and provided in a simplified form.

\subsection{Feed Analysis Summary}

The following Table 8.4, attempts to summarize all the above information in one easy to read and analyze sheet. The table is divided into three main areas, the transient analysis, the steady state analysis, and the comments. It is very important to have full understanding of the following comments before attempting to read the table, since these 
comments shed light on several points that may be confusing or misunderstood if taken directly from the table.

Table 8.3 Analysis of the steady state graphs for feed 5

\begin{tabular}{|c|c|c|c|c|c|c|}
\hline & & & & & \multicolumn{2}{|c|}{$\mathrm{dBd}$} \\
\hline Plane & res. type & Graph & angles & score & $\min$ & $\max$ \\
\hline \multirow{8}{*}{ Phi $=0$} & \multirow{4}{*}{ real } & \multirow{2}{*}{ Ephi } & $0-180$ & v. good & 21 & 32 \\
\hline & & & $180-360$ & v. good & 27 & 37 \\
\hline & & \multirow{2}{*}{ Etheta } & $0-180$ & poor & 17 & 35 \\
\hline & & & $180-360$ & fair & 23 & 40 \\
\hline & \multirow{4}{*}{ anti } & \multirow{2}{*}{ Ephi } & $0-180$ & good & 15 & 29 \\
\hline & & & $180-360$ & v. good & 13 & 45 \\
\hline & & \multirow{2}{*}{ Etheta } & $0-180$ & poor & 18 & 40 \\
\hline & & & $180-360$ & v. good & 21 & 34 \\
\hline \multirow{8}{*}{ Theta $=90$} & \multirow{4}{*}{ real } & \multirow{2}{*}{ Ephi } & $0-180$ & poor & 10 & 32 \\
\hline & & & $180-360$ & fair & 17 & 46 \\
\hline & & \multirow{2}{*}{ Etheta } & $0-180$ & poor & 19 & 39 \\
\hline & & & $180-360$ & poor & 22 & 45 \\
\hline & \multirow{4}{*}{ anti } & \multirow{2}{*}{ Ephi } & $0-180$ & fair & 16 & 38 \\
\hline & & & $180-360$ & v. good & 19 & 42 \\
\hline & & \multirow{2}{*}{ Etheta } & $0-180$ & v. good & 18 & 36 \\
\hline & & & $180-360$ & v. good & 20 & 42 \\
\hline
\end{tabular}

\subsubsection{The Transient Area}

The columns of this area are divided into two groups. The first group focuses on the comparison between the numerical and the experimental curve, while the second group focuses on the behavior of the transient curve. The following is a definition of each column in this area:

1. Overall matching: gives a rating of how well the numerical curve predicts the experimental curve. The ratings, as with all the other columns that will provide ratings, are "poor", "fair", "good", "v. good", and "excellent". 
2. Acceptable range: this is the range of frequencies that are included in the study. The minimum acceptable range is $900 \mathrm{MHz}$ to $2 \mathrm{GHz}$ as stated earlier, which is the acceptable range for the anechoic chamber. But sometimes the results were acceptable even outside this range, thus the limits were expanded to include all this into the acceptable range.

3. Prediction error: is the range of errors, from lowest error to highest error, between the numerically and experimentally predicted resonant frequencies within the acceptable range defined above.

4. Repetitive: provides a "yes" or "no" answer to whether the resonant frequencies of this feed have a repetitive nature or not.

5. Diff between res freq's: provides the average difference between each two resonant frequencies regardless of their type. The following conditions apply:

- If the "repetitive" column has a "no" in it for this feed, then this column is left blank.

- If the feed is repetitive, but the frequency of repetitiveness is inconsistent, then no value can be plugged into this cell, and the value "NC", for "Non Consistent" is used.

- If the spacing between the real and the following anti resonance is not equal to the spacing between the anti and the following real resonance, but this behavior is consistent, then two values are entered with a slash "/" in between in the following format "real to anti / anti to real"

- If the spacing between all resonances is consistent and approximately equal then only one value is shown in this column. 
6. Diff between same type freq's: provides the average difference between each two resonant frequencies of the same type. The value in this column should be approximately twice the value in the previous column if only one value exists in it, or the summation of the values in the previous column if two values exist in it. The same conditions mentioned in the previous column apply for this column too.

\subsubsection{The Steady State Area}

The columns of this group also concentrate on both the performance of the feed and how well the experimental curve is predicted by the numerical curve. The following is a definition of each column in this area:

1. Overall prediction: provides a general rating to how well the numerical curves, as a whole, were able to predict the experimental curves. The rating in this column is taken as an average between all the steady state ratings from the tables in Appendix F.

2. Highest gain value: gives a value in $\mathrm{dBm}$ for the highest gain achieved from among all the experimental curves.

3. High gain range: is the mean range of values for the best performing curve(s) for this feed, excluding all sudden narrow high or low peaks. The purpose of this column is to show the overall performance of the high gain curves, in contrast to the previous column that might show a high value which came from a sudden high peak, while the rest of the curve provided very low gain.

4. Best curves: lists the curves that performed the best for this feed, if any, even if their prediction by the numerical curve was poor. The curves listed in this 
column are matched with the ranges from the previous column. If all the curves for this feed provided the regular gains of -60 to $-75 \mathrm{dBm}$, which was seen frequently in the analysis, then no curves are listed in this area. The following abbreviations were used in this and the following columns:

- $\quad$ P for phi $=0$ plane, and $\mathrm{T}$ for theta $=90$ plane

- Eph for the Ephi curve, and Eth for the Etheta curve

- Real for the real resoance, and anti for the anti resonance

5. Well predicted: lists the curves that were predicted best, even if their performance was poor.

\subsubsection{The Comments Area}

This area provides general comments on the performance of the feed, and how well the numerical curves predicted the experimental results for each feed. 
Table 8.4 Summary of the overall performance of all 13 feeds, and how well they were predicted.

\begin{tabular}{|c|c|c|c|c|c|c|c|c|c|c|c|c|}
\hline & \multicolumn{6}{|c|}{ Transient analysis } & \multicolumn{5}{|c|}{ steady state } & \multirow[t]{2}{*}{ comments } \\
\hline Feed & \begin{tabular}{|c|} 
overall \\
matching
\end{tabular} & $\begin{array}{c}\text { acceptable } \\
\text { range } \\
\mathrm{GHz}\end{array}$ & \begin{tabular}{|c} 
prediction \\
difference \\
$\%$
\end{tabular} & repetitive & \begin{tabular}{|c|} 
diff betweer \\
res freq's \\
$\mathrm{MHz}$
\end{tabular} & \begin{tabular}{|c|} 
diff between \\
same type freq's \\
$\mathrm{MHz}$
\end{tabular} & \begin{tabular}{|c|} 
overall \\
prediction
\end{tabular} & \begin{tabular}{|c|} 
highest \\
gain value \\
$\mathrm{dBm}$ \\
\end{tabular} & \begin{tabular}{|c|} 
high gain \\
range \\
$\mathrm{dBm}$
\end{tabular} & $\begin{array}{c}\text { best } \\
\text { curves }\end{array}$ & $\begin{array}{c}\text { well } \\
\text { predicted }\end{array}$ & \\
\hline 1 & fair & $0.8-1.8$ & $2.2-8.5$ & no & - & - & poor & -55 & -60 to -70 & $\mathrm{~T}$ anti Eph & - & poor overal \\
\hline \multirow[t]{2}{*}{2} & poor & $0.5-2$ & $0.7-6.7$ & no & - & - & good & -55 & -60 to -70 & P real Eth & $\mathrm{P}$ real Eth & poor overal \\
\hline & & & & & & & & & & & T anti Eph & \\
\hline \multirow[t]{2}{*}{3} & good & $0.6-2.4$ & $1.6-4.2$ & no & - & - & fair & -53 & -53 to -62 & T real Eph & $\mathrm{P}$ real Eth & less than a \\
\hline & & & & & & & & & & & & no speacial \\
\hline \multirow[t]{3}{*}{4} & excellent & $0-2$ & $0.7-3.3$ & yes & 179 & 366 & v. good & -50 & -50 to -60 & P real Eph & $\mathrm{P}$ anti Eph & one of the \\
\hline & & & & & & & & & & Treal Eph & T real Eth & one of the \\
\hline & & & & & & & & & & & T anti Eph & very consis \\
\hline \multirow[t]{2}{*}{5} & excellent & $0.2-2$ & $0.9-3.7$ & yes & 182 & 365 & good & -55 & -60 to -70 & P anti Eph & P real Eph & consistent : \\
\hline & & & & & & & & & & T anti Eph & T anti Eth & average st \\
\hline \multirow[t]{2}{*}{6} & good & $0-2$ & $1-9$ & yes & $\mathrm{NC}$ & $\mathrm{NC}$ & poor & -54 & -55 to -65 & P anti Eph & - & less than a' \\
\hline & & & & & & & & & & $\mathrm{P}$ anti Eth & & poor steads \\
\hline \multirow[t]{4}{*}{7} & v. good & $0-2$ & * & no & - & - & v. good & -55 & -55 to -60 & Treal Eph & P real Eth & very unique \\
\hline & & & & & & & & & & P real Eth & $\mathrm{P}$ anti Eth & above aver \\
\hline & & & & & & & & & & & T real Eph & \\
\hline & & & & & & & & & & & T anti Eph & \\
\hline \multirow[t]{2}{*}{8} & poor & $0.6-2$ & $0.1-4.4$ & yes & $180 / \mathrm{NC}$ & $\mathrm{NC}$ & fair & -60 & -60 to -70 & P real Eth & $\mathrm{P}$ real Eth & extremely \\
\hline & & & & & & & & & & T real Eph & & poor overal \\
\hline \multirow[t]{2}{*}{9} & poor & $0.9-2$ & $0.7-5.3$ & yes & $\mathrm{NC}$ & $\mathrm{NC}$ & fair & -58 & -58 to -65 & $\mathrm{P}$ anti Eph & $\mathrm{P}$ anti Eph & poor overal \\
\hline & & & & & & & & & & $\mathrm{P}$ anti Eth & $\mathrm{T}$ anti Eph & \\
\hline \multirow[t]{2}{*}{10} & v. good & $0.3-2$ & $1.2-2.7$ & no & - & - & fair & -52 & -55 to -65 & $\mathrm{P}$ anti Eph & - & unique tran \\
\hline & & & & & & & & & & $\mathrm{T}$ anti Eph & & average ste \\
\hline \multirow[t]{3}{*}{11} & v. good & $0.5-1.7$ & $0-2.8$ & yes & 172 & 342 & fair & -50 & -50 to -60 & Treal Eph & T anti Eth & consistent $t$ \\
\hline & & & & & & & & & & P real Eth & & very good s \\
\hline & & & & & & & & & & P real Eph & & \\
\hline \multirow[t]{3}{*}{12} & excellent & $0-2$ & $0.2-3.1$ & yes & 172 & 355 & good & -50 & -50 to -58 & P real Eph & P real Eph & consistent : \\
\hline & & & & & & & & & & $\mathrm{P}$ real Eth & T anti Eth & one of the \\
\hline & & & & & & & & & & Treal Eph & & best real re \\
\hline \multirow[t]{2}{*}{13} & excellent & $0-2$ & $0-2.6$ & yes & 173 & 345 & good & -50 & -52 to -60 & P anti Eph & P real Eph & consistent \\
\hline & & & & & & & & & & & T anti Eth & above aver \\
\hline
\end{tabular}

* Due to the unique behavior of feed 7 , only four resonances were available within the range. The numerical curve was able to predict only two of them with high : resonances, the numerical curve did not cut the x-axis, thus the two resonances were not predicted, and the error can be considered infinite. 


\subsection{Feed Study Comments and Conclusions}

From the analysis that has been conducted through this chapter, the following comments and conclusions can be made:

1. The overall numerical prediction of the transient behavior of the PCB-CTHA is highly accurate for most of the feeds up to $2 \mathrm{GHz}$. It can be relied upon to predict the resonant frequencies for most of the feeds, especially feeds 4,5 , and 10 to 13 . The lower limit for acceptable numerical prediction differs from feed to feed, but the range from $0.5 \mathrm{GHz}$ to 2 $\mathrm{GHz}$ was found to be acceptable for all the feeds.

2. The six above mentioned feeds, 4,5 , and 10 to 13 , all showed prediction errors less than $4 \%$ along the whole acceptable range. Even for the other feeds the error was always less than $8 \%$ except for two frequencies in feeds 1 and 6 that showed an 8.5 and 9.1 error consecutively.

3. The steady state behavior prediction is far from being reliable. Most of the predictions were far off from even representing the correct pattern of behavior. The best numerical predictions found were able to show a very similar pattern to the experimental pattern, and correctly define where the high and low peak gains would exist. But even these best predicted curves usually failed to show exactly how high or low these peaks would be relative to the rest of the curve, and all predictions remained qualitative.

4. Feeds 4 and 12 showed identical transient behavior and resonant points, but feed 12 showed a slightly better steady state performance. Comparing the physical shape of these two feeds it can be seen that the only difference is that points A and D are shorted in feed 12 while they are left open in feed 4. Both feeds' transient behavior were excellently predicted by the numerical model. The steady state behavior prediction for these two feeds was also much higher than average, with feed 4 having one of the best steady state predictions. 
5. It is also noticed that for these two feeds the real resonance performance curves were significantly higher than the anti resonance. While the anti resonance curves all showed a low -60 to $-75 \mathrm{dBm}$ range, the real resonances reached as high as $-50 \mathrm{dBm}$ and ranged in general between -50 and $-60 \mathrm{dBm}$

6. As with the previous feeds, feeds 5 and 13 also showed identical behavior. Comparing the physical shapes of the two feeds, and reducing feed 13 to an equivalent connection of having points $\mathrm{A}$ and $\mathrm{B}$ connected to the feed points, and points $\mathrm{B}$ and $\mathrm{D}$ shorted, it is then seen that the only difference between feeds 5 and 13 is that feed 13 has points B and D shorted, while they are open in feed 5. This is a very similar differentiation to that found between feeds 4 and 12. And also the effect is very similar. Both feeds showed identical transient behavior and resonant points. The steady state prediction and behavior of both feeds was above average, with the performance of feed 13 clearly better than feed 5 .

7. Contrary to feeds 4 and 12 , these two feeds, feeds 5 and 13 , showed a significantly better performance for the anti resonance over the real resonance. The real resonance gains were all between -60 and $-75 \mathrm{dBm}$, while the anti resonance gains reached as high as $-50 \mathrm{dBm}$, and a few of the curves ranged between -50 and $-60 \mathrm{dBm}$.

8. Things get even more interesting when both of the previous sets are compared to each other, i.e. feeds 4 and 12 versus feeds 5 and 13. Both sets yielded identical resonant frequencies, but of opposite type. So when feeds 4 and 12 showed a real resonance, feeds 5 and 13 showed an anti resonance, and vice versa. This can be seen clearly in Figure 8.6 below, which the transient behavior for feeds 12 and 13. It is clear from this Figure that the resonances are almost identical along the whole curve, but of different types. Event the high and low peaks of each two anti resonances seem to provide very close values. To see the 
physical aspect behind this, the best thing is to compare feeds 5 and 12 . In both feeds, the feed points are located between points A and B, but for feed 5 the only other connection is points $\mathrm{C}$ and $\mathrm{D}$, while for feed 12 it is points $\mathrm{A}$ and $\mathrm{D}$. This physically means that for feed 5 both loops are shorted at the start and the end of the loop, thus creating one long loop with the feed point within that loop. While for feed 12 only one loop is complete, and that is the loop containing points $\mathrm{A}$ and $\mathrm{D}$, while the other loop is open and is only connected to the closed loop at one point, points B. This slight change between both feed shapes had the effect of reversing the type of resonances while keeping the frequencies still, and also switched the better performing gain patterns between the different types of resonances.

9. These four feeds, feeds $4,5,12$, and 13 , are also seen to be the best performing and best predicted feeds among all the feeds that had a repetitive resonant frequency nature. Thus all the other feeds with repetitive nature can be disregarded and all studies can be focused on these four feeds.

10. From the previous points it can also be deducted that when an extra connection was added to feed 4 to yield feed 12, and when an extra connection was added to feed 5 to yield feed 13 , in both cases the extra connection increased the gain output of the antenna while not affecting its transient behavior. So definitely feeds 12 and 13 are preferred over feeds 4 and 5 due to this increased gain and identical resonances. 


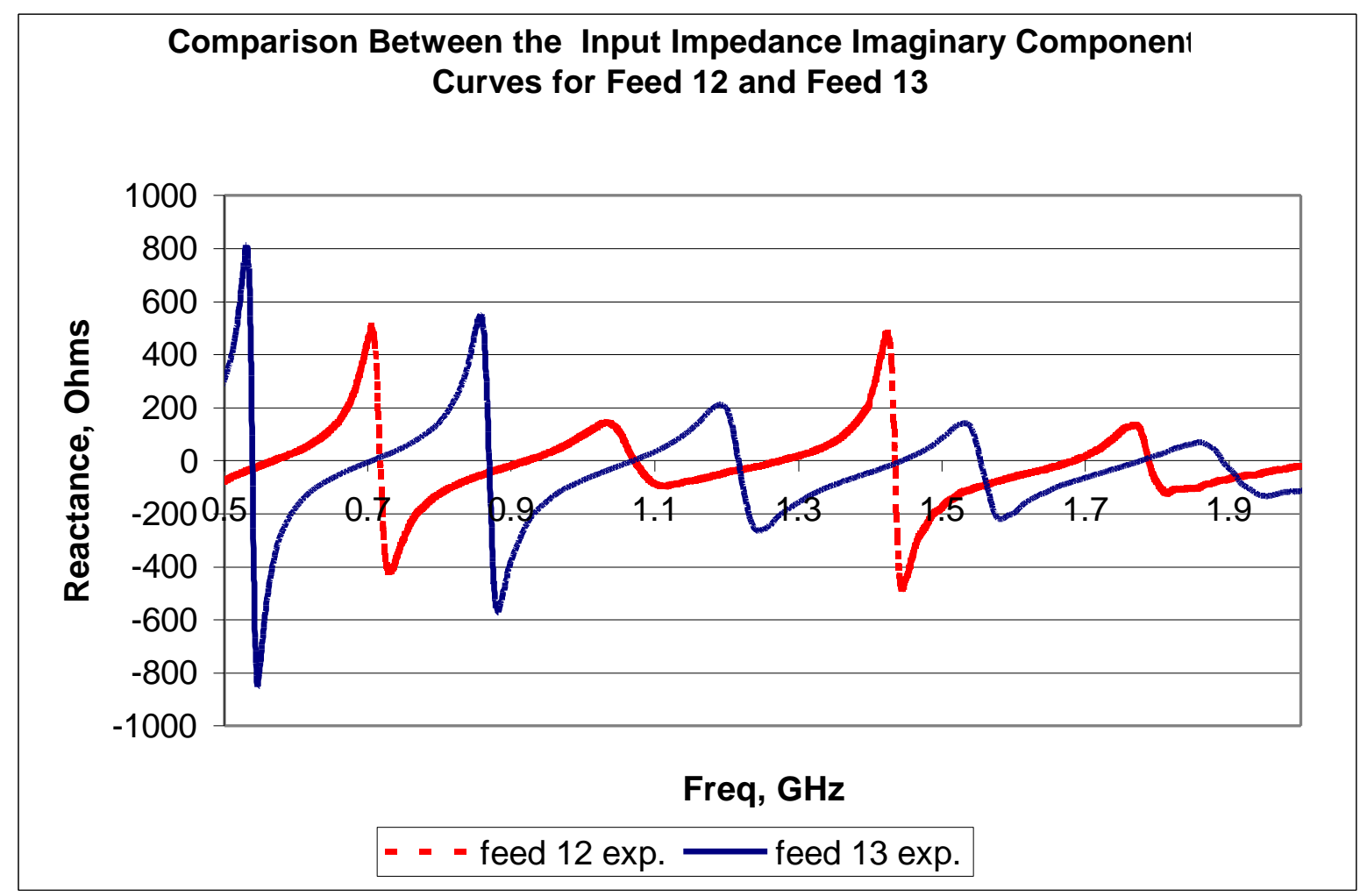

Figure 8.3 Comparison Between the Reactance Curves for Feed 12 and Feed 13

11. Feeds 1 to 3 showed a semi repetitive nature, where several resonant frequencies existed but at non consistent intervals. The spacing between the resonances had no clear relation to govern it. The overall performance and numerical predictions for the three feeds was relatively poor, where the predictions for feeds 1 and 2 were from among the worst found, both transient and steady state. No special features or behaviors can be identified in these feeds, and they can all be safely disregarded.

12. The two remaining feeds, feeds 7 and 10, showed a unique transient behavior not seen in any of the other feeds. One clear distinct anti resonant frequency was seen at about $500 \mathrm{MHz}$, then no other resonances are found until close to the end of the range of $2 \mathrm{GHz}$ where the transient curves starts crawling around and crossing over the $\mathrm{x}$-axis and forming some other resonances. This behavior can be found useful if an antenna with only one resonant frequency over a whole $1 \mathrm{GHz}$ range is required, and the location of this resonance can be 
controlled by the methods described in [35]. The behavior could also be useful if an area where the imaginary component of the input impedance is always close to zero is required, like what is seen for these two feeds from 1 to $2 \mathrm{GHz}$, also approximately a $1 \mathrm{GHz}$ range.

13. Once again it can be seen that the only difference between these two feeds, feeds 7 and 10 , is an extra short connection. Reducing feed 7 to a connection between A and B, another between $\mathrm{C}$ and $\mathrm{D}$, and having points $\mathrm{D}$ and $\mathrm{B}$ connected to the feed point, it then becomes clear that the difference between feed 7 and feed 10 is an extra short connection between points $\mathrm{C}$ and $\mathrm{D}$ in feed 7 that is not found in feed 10 .

14. And surprisingly enough, the extra short connection provides the same behavior change that was seen before between feeds 4 and 12 and between feeds 5 and 13. The steady state gain from the feed with the extra connection, feed 7 , showed best results at the real resonance frequency, while the other feed showed the best results at the anti resonance frequency. The steady state curve prediction for feed 7 was among the best, if not the best, steady state prediction between all the feeds, while the prediction for feed 10 was below average. The numerical model however predicted the transient behavior of both feeds very well. The steady state performance of both feeds, on the other hand, was very similar, with feed 7 being slightly better overall than feed 10.

15. No clear choice can be made as to which of these two feeds is better, especially that one performs best at the real resonances, while the other performs best at the anti resonances, so each may have its different usage. But as a choice for further investigation it would be feed 7 due to its slightly better steady state performance, and its clearly better steady state prediction. The results of the extra research on feed 7 can then be reapplied on feed 10 and should expect to yield the same results due to the similar nature of these two feeds. 
16. Ironically, feed 6, which was the only feed that was being used previously within CIRA, showed less than average predictions and performance, and a poor steady state behavior. At least 7 other feeds had a better transient predicted curve. Feed 6 also had a relatively nonconsistent repetitive shape. The steady state behavior was also poorly predicted, and the steady state performance was clearly less than average. It is highly recommended to substitute feed 6 in further studies and usage with either feed 12 or feed 13.

17. From all of the above points it can finally be concluded that in the future only feeds 12,13 , and 7 should be concentrated upon. All research and experimentation should be directed towards these three feeds, and the further improvements in the numerical model should be directed towards better simulating their behavior. Concentrating on these three feeds alone will save valuable time and money and will accelerate the further development and understanding of the PCB-CTHA. The numerical simulation of the transient behavior of these three feeds is already almost perfect, but the steady state simulation still has a long way to go. 


\section{Summary and Results}

The goal of this study was to validate the accuracy of a numerical model for the PCBCTHA using the FDTD method. This model predicts the input impedance and far field gains of a PCB-CTHA. The predictions from the FDTD model were then compared to experimental measurements for several feed configurations. The following sections provide a summary of this study, and all the steps it passed through during its development. This is followed by a list of results and contributions.

\subsection{Summary}

1. The study starts by an introduction to antennas in general, in Chapter 1 , and to the CTHA specifically in Chapter 2. The introduction to antennas includes a look at the fundamentals of electromagnetics, and definitions of the basic antenna concepts. Next, an introduction to the CTHA is provided, showing the history of development of the CTHA, the different types of CTHAs, including the PCB-CTHA which is the focus of this study, and finally outlining some of the advantages of the CTHA.

2. After introducing the CTHA, and understanding its geometry, a feed study was conducted on the PCB-CTHA to determine all the possible feed configurations. From these possible feed configurations, only the practical configurations were selected. These turned out to be 13 different feeds. The selected feeds were numbered 1 to 13 , and their geometry was defined and sketched in Figure 4.6.

3. The following section of the study concentrated on the numerical model and the method used to create the model. This method is the finite difference time domain method, FDTD. An introduction to the FDTD method is provided, followed by a detailed analysis of the method theory in which the FDTD equations are formulated from scratch. This is followed 
by a literature survey that was performed to find and understand some of the related research using FDTD that has been performed.

4. The same previous section is then followed by a description of the software package that uses the FDTD equations to solve electromagnetic problems; this package is XFDTD version 5.0 developed by Remcom. The section described the general method used by XFDTD to perform FDTD calculations, the XFDTD capabilities, and the inputs required by XFDTD and the outputs achieved from its calculations. Although XFDTD includes both an input preprocessor and an output postprocessor, but these are very general and do not achieve the requirements of this research. Thus both the preprocessor and the post processor were ignored, and custom preprocessors and post processors were developed especially for the PCB-CTHA. These developed modules are also described in detail in this section. Thus only the core calculation engine within XFDTD is used in this study, but all input and output interfaces and modules are custom built.

5. The creation and the development of the PCB-CTHA numerical model built on the previously described method, and utilizing the XFDTD calculation engine and the custom built preprocessor and post processors, is described in chapter 6. The development of the model passes through several stages, checking the effect of the different parameters of the PCB-CTHA model on the output results of the calculation, and defining the best method for selecting these parameters, and their optimum values. The first of these parameters was the free space that is left around the PCB-CTHA geometry in order to be able to calculate the far field gains and patterns accurately. The number of calculation time steps that are required until a converged output result is achieved follows this. Also studied was the relative permittivity of the binding material of the PCB-CTHA and how it affects the findings of the 
two previous parameters, the free space and the time steps. And finally checked is the effect of altering the conductivity of the traces of the model, and checking if this alteration has any effect on the results.

6. After the creation and development of the numerical model, the experimental setup was designed and described in chapter 7. In this chapter the main equipment used for the experiments was described, including the anechoic chamber, the network analyzer, the spectrum analyzer, and the signal generator. This was followed by a description of the experimental setup for both the input impedance and the far field experiments.

7. And finally, the results of the feed study, and the validation of the numerical code, were established in chapter 8 . In this chapter the numerical and experimental results for both the input impedance and the far field gains for all 13 feeds were graphed and compared together. Due to the large amount of graphs and tables, most of the Figures were listed in Appendix F to increase the readability of this chapter. Based on the analysis done in this chapter, both the validity of the numerical code, and the performance of the different feeds was understood.

8. Five other appendices, other than Appendix F stated above, were included in the text of this study. The first three of these five were related to the code developed in this study. In Appendix A, the preprocessor source code is listed. As stated earlier, this code was written in visual basic. The following Appendix showed actual grabbed images of the input forms of the preprocessor. Appendix $\mathrm{C}$ then showed sample XFDTD input files. These input files are in the format required by the FDTD engine to perform the calculation. These sample files can be identically generated by both the XFDTD preprocessor, and the specially designed PCB-CTHA preprocessor. But what can be done in the latter preprocessor in less than two 
minutes requires at least several hours to be done by the former. The fourth Appendix, Appendix D then provides a code listing of the program developed to analyze the output files from the network analyzer. And finally Appendix E shows some photos for the anechoic chamber, and the experimental analysis equipment and setup described in chapter 7.

\subsection{Results and Findings}

Following is a list of the main results and findings achieved from this study. They are basically listed in the same order they were provided in the text. These results are considered some of the contributions of this research, since they are all unique results that are found for the first time:

- From the analysis of all the possible physical connections of the four feed points of the PCBCTHA it was found that, theoretically speaking, 625 possible feed configurations exist. But by ruling out all the existing redundancies and similarities, only 35 feeds remain. These feed configurations are of three types; feeds that have connections to both feed source points, feeds that have connection to one feed source point, and feeds that have no connections to the feed source points. But since the second and the third types listed above both rely on alternative feed techniques and excitation methods, then only the feeds of the first type were considered. These were found to be 13 different feeds, and their physical shapes are shown in Figure 4.6. These 13 feeds were given numbers from 1 to 13 to identify them uniquely.

- The following contribution was the PCB-CTHA FDTD preprocessor. The PCB-CTHA's geometry was found to be extremely complicated for the available general purpose preprocessors, thus a custom built preprocessor was developed. This preprocessor facilitated the development of the numerical grid for different PCB-CTHAs in a few minutes, a task that would required hours on general purpose preprocessors. All 13 feed configurations were 
then provided as built in feed options in the preprocessor, in addition to some built in default antenna configurations that represent some of the main antennas in the CIRA labs.

- A numerical model based on the FDTD method is created for the PCB-CTHA. This model attempts to predict both the input impedance behavior and the far field patterns for the PCBCTHA. By studying the effect of altering the different model and antenna parameters on the model output, an optimum parameter combination for the available antennas was produced. In addition to this optimum combination, guidelines were provided in the text to the advised changes to be made to the model parameters if other special antennas are to be modeled. These other special antennas could have different characteristics, like a relative permittivity far outside the range considered in this study, etc. The optimum combination found included a 30 free space cells model, with perfect conducting traces, and a binding material with a relative permittivity of 4.0. The steady state runs are to be conducted using the number of time steps suggested by XFDTD, while the transient runs will use 4000 time steps.

- Other results and advises obtained from the numerical model development process included:

1. In all transient runs it is enough to use the minimum number of time steps and free space cells that would produce steady results. Any increase after that will have no effect on the results as they will stay the same.

2. Contrary to the above, increasing the time steps of a steady state run will cause the far field patterns to increase in value, while keeping their patterns. This will continue until approximately twice the number of time steps suggested by XFDTD are used, after which the run will crash. Thus it is advised to always use the number of time steps suggested by XFDTD for all steady state runs. 
3. For a PCB-CTHA model where no relative permittivity is defined for the binding material, and the traces are modeled as perfect conductors, only 20 free space cells are required for the geometry file. 2000 time steps are enough for the transient runs to yield results, while the number of time steps suggested by XFDTD should be used for the steady state runs.

4. If the relative permittivity of the binder material is introduced in the model, then the requirements for both the free space and the transient time steps increase. In this case 30 free space cells are needed in order to reach stable results, and 4000 time steps are required for the transient runs. These numbers apply up to a relative permittivity value of 4.0, after which the requirements for the free space and time steps might differ.

5. XFDTD has failed to predict the effect of introducing a conductivity value for the traces of the PCB-CTHA. All runs made with conductivity gave the exact same results as if a perfect conductor material was used. Thus this feature should not be used.

- Several custom built programs were developed for the sake of post processing the data of both the numerical and the experimental runs. These programs are available with the model, and highly facilitate the task of future research in this field. These programs include:

1. a program that analyses the input impedance files from XFDTD and convert the text files to formatted Microsoft excel files with the data ready to be graphed.

2. a program that analyses the input impedance data, both numerical and experimental, and provides a list of all the resonant frequencies available within a specified range 
3. A program that converts the input impedance data from the network analyzer into Ohms, and also reformats the data and provides it in an excel worksheet ready to be graphed.

4. A program that reformats the numerical far field pattern data into an excel sheet, where several columns are disregarded and others are reorganized.

5. Another similar program was developed for the experimental results, but since the format of the experimentally obtained files is totally different from the numerically obtained file, thus a separate program was required.

- From the feed study conducted in chapter 8, the following results and contributions are obtained:

- The most important contribution of this work is that it has provided an accurate tool for predicting the resonant frequencies for any PCB-CTHA. The overall numerical prediction of the input impedance behavior of the PCB-CTHA is highly accurate for most of the feeds up to $2 \mathrm{GHz}$. It can be relied upon to predict the resonant frequencies for most of the feeds, especially feeds 4,5 , and 10 to 13 . The lower limit for acceptable numerical prediction differs from feed to feed, but it is safe to say that a range from $0.5 \mathrm{GHz}$ to $2 \mathrm{GHz}$ is acceptable for all the feeds.

- The six feeds mentioned above, 4, 5, and 10 to 13, all showed prediction errors less than $4 \%$ along the whole acceptable range. Even for the other feeds the error was always less than $8 \%$ except for two frequencies in feeds 1 and 6 that showed an 8.5 and 9.1 error consecutively.

- The steady state behavior numerical prediction is far from being reliable, and further improvements can still be made regarding this point. Most of the predictions were far off from even representing the correct pattern of behavior. The best numerical predictions found 
were able to show a very similar pattern to the experimental pattern, and correctly define where the high and low peak gains would exist. But even these best predicted curves usually failed to show exactly how high or low these peaks would be relative to the rest of the curve, and all predictions remained qualitative.

- The four feeds, $4,5,12$, and 13 , were found to be the best performing and best predicted feeds among all the feeds that had a repetitive resonant frequency nature. Thus all the other feeds with repetitive nature can be disregarded and all studies can be focused on these four feeds.

- Feeds 1 to 3 showed a semi repetitive nature, where several resonant frequencies existed but at non consistent intervals. The spacing between the resonances had no clear relation to govern it. The overall performance and numerical predictions for the three feeds was relatively poor, where the predictions for feeds 1 and 2 were from among the worst found, both transient and steady state. No special features or behaviors can be identified in these feeds, and they can all be safely disregarded.

- The two remaining feeds, feeds 7 and 10 , showed a unique transient behavior not seen in any of the other feeds. One clear distinct anti resonant frequency was seen at about 500 $\mathrm{MHz}$, then no other resonances are found until close to the end of the range of $2 \mathrm{GHz}$ where the transient curves starts crawling around and crossing over the $\mathrm{x}$-axis and forming some other resonances. This behavior can be found useful if an antenna with only one resonant frequency over a whole $1 \mathrm{GHz}$ range is required, and the location of this resonance can be controlled by the methods described in [35]. The behavior could also be useful if an area where the imaginary component of the input impedance is always close to zero is required, like what is seen for these two feeds from 1 to $2 \mathrm{GHz}$, also approximately a $1 \mathrm{GHz}$ range. 
- The steady state gain from feed 7 showed best results at the real resonance frequency, while the other feed, feed 10, showed the best results at the anti resonant frequency. The steady state curve prediction for feed 7 was among the best, if not the best, steady state prediction between all the feeds, while the prediction for feed 10 was below average. The numerical model however very well predicted the transient behavior of both feeds. The steady state performance of both feeds, on the other hand, was very similar, with feed 7 being slightly better overall than feed 10 . No clear choice can be made as to which of these two feeds is better, thus disregard the other, since one performs best at the real resonances, while the other performs best at the anti resonances, so each may have its different usage. But as a choice for further investigation it would be feed 7 due to its better steady state performance and prediction. The results of the extra research on feed 7 can then be reapplied on feed 10 and should expect to yield the same results due to the similar nature of these two feeds.

- Feed 6, which was the only feed that was being used previously within CIRA, showed less than average predictions and performance, and a poor steady state behavior. At least 7 other feeds had a better transient predicted curve. Feed 6 also had a relatively non-consistent repetitive shape. The steady state behavior was also poorly predicted, and the steady state performance was clearly less than average. It is highly recommended to substitute feed 6 in further studies and usage with either feed 12 or feed 13.

- It can finally be concluded that in the future only feeds 12,13 , and 7 should be concentrated upon. All research and experimentation should be directed towards these three feeds, and the further improvements in the numerical model should be directed towards better simulating their behavior. Concentrating on these three feeds alone will save lots of valuable time and money and will accelerate the further development and understanding of the PCB-CTHA. 
The numerical simulation of the transient behavior of these three feeds is already almost perfect, but the steady state simulation still has a long way to go. 


\section{Conclusion and Recommendations}

\subsection{Conclusion}

The goal of this study was to validate the accuracy of a numerical model for the printed circuit board contrawound toroidal helical antenna (PCB-CTHA) using the finite difference time domain (FDTD) method. This model predicts the input impedance and far field gains of a PCBCTHA. The predictions from the FDTD model were then compared to experimental measurements for several feed configurations. It was found that although the input impedance curves can be very accurately predicted by the developed numerical model, the steady state far field gains are far from being reliable. The study also found that significant differences in the behavior of the PCB-CTHA occur when the feed configuration is changed, and three feed configurations were recommended for future consideration, feeds 7, 12, and 13.

\subsection{Recommendations for Future Work}

Following is a list of some recommended points of research that require future investigation:

1. Further investigation is required to determine why the far field numerical curves do not predict the actual far field. Modifying and advancing the techniques used in this study should be able to achieve this.

2. Now that it has been found that feeds 7,12 , and 13 , provide the highest gains and numerical predictability, further investigation and thorough research is required to better understand the performance and behavior of these three feeds. It would be advisable to manufacture several antennas of different geometrical and material properties and perform a parametric study upon them to understand the effect of the change of these parameters on the behavior of the antenna. 
3. Research also needs to be done on the behavior of the above three mentioned curves at different frequencies. This is similar to what has been done in this study, but instead of studying the antenna only at two frequencies the study needs to be done at several real and anti resonances and numerous points between them. From this it can be understood how the antenna performs at different frequencies, so that the most appropriate frequency location can be chosen according to the required function.

4. After all the previously mentioned points have been achieved, then an investigation needs to take place to compare the performance of the PCB-CTHA with that of other available antennas. This study would highlight the powers and the advantages of the PCB-CTHA, and also make its potential market more aware of its strengths and abilities. 


\section{References}

1. Cheng, D.K., Field and Wave Electromagnetics, Addison Wesley Publishing Company, 1992.

2. Harrington, R.F., Time Harmonic Electromagnetic Fields, McGraw-Hill Book Company, Inc., New York, 1961.

3. Stratton, J.A., Electromagnetic Theory, McGraw-Hill Book Company, Inc., New York, 1941.

4. Collin, R.E. and Plonsey, R., Principles and Applications of Electromagnetic Fields, McGraw-Hill Book Company, Inc., 1961.

5. Jordan, E.C. and Balmain, K.G., Electromagnetic Waves and Radiating Systems, PrenticeHall, Inc., Englewood Cliffs, N. J., Second Edition, 1968.

6. Hayt, W.H., Engineering Electromagnetics, McGraw-Hill Company, Inc., 1967.

7. Maxwell J.C., A Treatise on Electricity and Magnetism, Oxford University Press, New York, 1904.

8. Ellithy, W.S., "The Toroidal Helical Antenna Analysis and Performance Evaluation," Ph.D. Dissertation, West Virginia University, 1996.

9. Guan, T., "Impedance Effects of Metallic Ground Plane on the CTHA Antenna," MSME Thesis, West Virginia University, 1998.

10. Kraus, J.D., Antennas, McGraw-Hill Book Company, Inc., New York, 1988.

11. Jackson, J.D., Classical Electromagnetics, John Wiley and Sons, New York, 1975.

12. Elliot, R.S., Antenna Theory and Design, Prentice-Hall, Inc., Englewood Cliffs, N.J., 1981.

13. Johnson, R.C., Antenna Engineering Handbook, McGraw-Hill Book Company, Inc., New York, 1993. 
14. "IEEE standard test Procedures for Antennas," IEEE Std. 149-1979, Institute of Electrical and Electronics Engineers, New York, 1979.

15. Hollis, J. S., Techniques of Microwave Antenna Measurements, John Wiley and Sons, Inc., New York, 1984.

16. Balanis, C.A., Antenna Theory: Analysis and Design, John Wiley and Sons, Inc., New York, 1996.

17. Corum, J.F., "Toroidal Antenna," U. S. Patent 4751515, June 1986.

18. Corum, J.F. and Corum K.L., "Toroidal Helix Antenna," Proceedings of the 1987 IEEE Antennas and Propagation Society International Symposium, pp. 832-835, 1987.

19. Fowler, V.J., Analysis of Helical Transmission Lines by Means of the Complete Circuit Equations, IRE Transactions on Antennas and Propagation, pp. 132-143, October, 1954.

20. Kandoian, A.G. and Sichak W., Wide Frequency Range Tuned Helical Antennas and Circuits. Convention Record of the IRE, National Convention, Part 2- Antennas and Communications, pp. 42-47, 1953.

21. Parsons J., Toroidal Helical Antenna (THA), Development Project. Technical Report, Wintec, Inc., 12805 Old Fort Road, Fort Washington, MD, June 1994.

22. GWEN Research Project Phase I Final Status Report, West Virginia University, College of Engineering, September 1991.

23. Van Voorhis, K.L. and Smith J.E., The Promises and Prospects of Wireless Power Transmission, Proceedings of the 26th IECEC, August 1991.

24. Van Voorhis, K.L., The Segmented Bifilar Contrawound Toroidal Helical Antenna, Ph.D. Thesis, West Virginia University, College of Engineering, 1993.

25. Van Voorhis, K.L. and Smith J.E., Toroidal Antenna, U. S. Patent 5442369, August 1995. 
26. ElSherbini, K.M., Pertl, F.A., Craven, R. and Smith, J.E., "The Effect of the Binding Material Permittivity on the Input Impedance of CTHAs, " Proceedings of ACES '99, 1999.

27. ElSherbini, K.M., "Design of a $433 \mathrm{MHz}$ PCB-CTHA," CTHA Internal Report Series 980806, Center for Industrial Research Applications, West Virginia University, 1998.

28. ElSherbini, K.M., "Effect of the Material Properties and the Holes Geometry on the Input Impedance of a PCB-CTHA," CTHA Internal Report Series 980729, Center for Industrial Research Applications, West Virginia University, 1998.

29. ElSherbini, K.M., "Experiments Conducted on Larry Hawks Antennas," CTHA Internal Report Series 980715, Center for Industrial Research Applications, West Virginia University, 1998.

30. ElSherbini, K.M., "More Experiments on Larry Hawks Antennas," CTHA Internal Report Series 980722, Center for Industrial Research Applications, West Virginia University, 1998.

31. Zheng, L., "Flexible Mesh Generator for CTHA," CTHA Internal Report Series 980805, Center for Industrial Research Applications, West Virginia University, 1998.

32. Zheng, L., "Parametric Study on Resonance Frequency of CTHA, " CTHA Internal Report Series 980812, Center for Industrial Research Applications, West Virginia University, 1998.

33. Yee, K.S., "Numerical solution of initial boundary value problems involving Maxwell's equations in isotropic media," IEEE Transactions on Antennas and Propagation, vol. AP-14, pp. 302-307, May 1966.

34. Hollan, R., Simpson, L. and Kunz, K.S., "Finite Difference Analysis of EMP Coupling to Lossy Dielectric Structures," IEEE transactions on Electromagnetic Compatibility, vol. EMC-22, pp. 203-209, Aug. 1980. 
35. Taflove, A. and Umashankar, K., "A hybrid moment method/finite difference time domain approach to electromagnetic coupling and aperture penetration into complex geometries," IEEE Transactions on Antennas and Propagation, vol. AP-30, pp. 617-627, July 1982.

36. Taflove, A., Umashankar, K., Beker, B., Harfoush, F. and Yee, K., "Detailed FD-TD analysis of electromagnetic fields penetrating narrow slots and lapped joints in thick conducting screens," IEEE Transactions on Antennas and Propagation, vol. 36, pp. 247-257, Feb 1988.

37. Luebbers, R.J., Kunz, K.S., Schneider, M. and Hunsberger, F., "A finite difference timedomain near zone to far zone transformation," IEEE Transactions on Antennas and Propagation, vol. 39, No. 4, pp. 429-433, April 1991.

38. Luebbers, R.J. and Langdon, H.S., "A simple feed model that reduces time steps needed for FDTD antenna and microstrip calculations," IEEE Transactions on Antennas and Propagation, vol. 44, No. 7, pp. 1000-1005, April 1996.

39. Reineix, A. and Jecko, B., "Analysis of microstrip patch antennas using finite difference time domain method," IEEE Transactions on Antennas and Propagation, vol. 37, pp. 1361-1369, Nov. 1989.

40. Katz, D., Piker-May, M., Taflove, A. and Umashankar, K., "FDTD analysis of electromagnetic wave radiation from systems containing horn antennas, " IEEE Transactions on Antennas and Propagation, vol. 39, pp. 1203-1212, Aug 1991.

41. Maloney, J.G., Smith, G.S. and Scott, W.R., "Accurate computation of the radiation from simple antennas using the finite-difference time domain method," IEEE Transactions on Antennas and Propagation, vol. 38, No. 7, pp. 1059-1068, July 1990. 
42. Mur, G., "Absorbing boundary conditions for the finite -difference approximation of the timedomain electromagnetic-field equations," IEEE transactions on Electromagnetic Compatibility, vol. EMC-23, No. 4, pp. 377-382, August, 1981.

43. Kunz, K.S., Luebbers, R.J. The Finite Difference Time Domain Method for Electromagnetics, CRC Press, 1993.

44. "Users Manual for XFDTD, the X-Window Finite Difference Time Domain Graphical User Interface for Electromagnetic Calculations, " Version 4.0, Remcom, Inc., March 1997

45. Myers, G.E., Analytical methods in conduction heat transfer, Genium Publishing Corporation, New York, 1987.

46. Cornell Gray, Visual Basic 5 from the ground up, McGraw-Hill Book company, Inc., New York, 1997.

47. Mckinney, B., Hardcore Visual Basic Version 5.0, Microsoft Press, 1997.

48. Shlivinski, A., Heyman, E. and Kastner, R., "Antenna Characterization in the Time Domain," IEEE Transactions on Antennas and Propagation, vol. 45, No. 7, July, 1997.

49. Chen, M., Houshmand, B. and Itoh, T., "FDTD Analysis of a Metal-Strip-Loaded Dielectric Leaky-Wave Antenna," IEEE Transactions on Antennas and Propagation, vol. 45, No. 8, August, 1997.

50. Luebbers, R.J. and Beggs, J., "FDTD Calculation of Wide-Band Antenna Gain and Efficiency," IEEE Transactions on Antennas and Propagation, vol. 40, No. 11, November, 1992.

51. Kashiwa, T., Onishi, T. and Fukai, I., "Analysis of Microstrip Antennas on a Curved Surface Using the Conformal Grids FD-TD Method," IEEE Transactions on Antennas and Propagation, vol. 42, No. 3, March, 1994. 
52. Luebbers, R., Chen, L., Uno, T. and Saburo, A., "FDTD Calculation of Radiation Patterns, Impedance, and Gain for a monopole Antenna on a Conducting Box, " IEEE Transactions on Antennas and Propagation, vol. 40, No. 12, December, 1992.

53. Tirkas, P.A. and Balanis, C.A., "Finite-Difference Time-Domain Method for Antenna Radiation," IEEE Transactions on Antennas and Propagation, vol. 40, No. 3, March, 1992.

54. Petropoulos, P.G., "Analysis of Exponential Time-Differencing for FDTD in Lossy Dielectrics, " IEEE Transactions on Antennas and Propagation, vol. 45, No. 6, June, 1997.

55. Wu, R. and Itoh, T., "Hybrid Finite-Difference Time-Domain Modeling of Curved Surfaces Using Tetrahedral Edge Elements," IEEE Transactions on Antennas and Propagation, vol. 45, No. 8, August, 1997.

56. Craven, R., "CTHA feed study 1," CTHA Internal Report Series 980325, Center for Industrial Research Applications, West Virginia University, 1998.

57. Jenkins-Jones, S., Random House Webster's Dictionary of Scientists, Random House, New York, 1997.

58. Pertl, F., "Expected Anechoic Chamber Properties," CIRA internal report series 980622, Center for Industrial Research Applications, West Virginia University, 1999.

59. Pertl, F., "Anechoic Chamber Recommendations," CIRA internal report series 980203, Center for Industrial Research Applications, West Virginia University, 1999.

60. "Users Guide - HP 8753D Option 011 Network Analyzer," Hewlett-Packard Company, 1994.

61. "Signal Generator operation and service guide - HP 8648A/B/," Hewlett-Packard Company, 1995.

62. "Users Guide - HP 8590 E-series and L-series spectrum analyzers," Hewlett-Packard Company, 1995. 
63. Gururajan, S., "Design of Anechoic Chamber Instrumentation and Data Acquisition Module for Testing and Analysis of the CTHA," MSME Thesis, West Virginia University, 1999. 


\section{Appendix A: The CTHA Preprocessor Source Code}

Following is a listing of all the code of the CTHA flexible mesh generator described in

chapter 5 .

\section{Form: filename}

File: RunNec.frm

Option Explicit

Private Sub Command1_Click()

End

End Sub

Private Sub Dir1_Change()

UpdateFile

End Sub

Private Sub Dir1_Click()

UpdateFile

End Sub

Private Sub file_txt_Click()

UpdateFile

End Sub

Private Sub File1_Click()

UpdateFile

End Sub

Private Sub UpdateFile()

File1.Path = Dir1.Path

If Dir1.Path = "C:I" Or Dir1.Path = "A:I" Then

filename_txt.Text $=$ Dir1.Path

Else

filename_txt.Text $=$ Dir1.Path + "।"

End If

End Sub

Private Sub Form_Load()

File1.Path $=$ Dir1.Path

filename_txt.Text $=$ Dir1.Path + "।"

UpdateFile

End Sub 


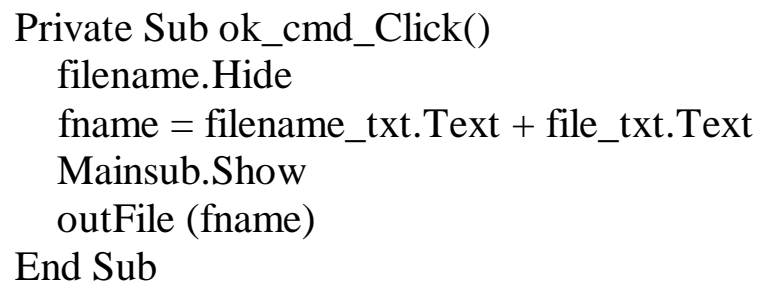

\section{Form: Mainsub}

\section{Filename: Main.frm}

Sub adhesivemat_com_Click() specs1.meshtitle_lab.Caption = " Adhesive" specs1.mat_com.Caption = " Properties for Air" specs1.Caption = "Adhesive Material" specs1. Show CTHA.Hide

End Sub

Sub cancel_com_Click()

End

End Sub

Private Sub cmdfdtd_Click() Gaussfdtd.Show

End Sub

Sub geo_com_Click() CTHA.Show specs1.Hide

End Sub

Sub OK_com_Click() calcs (1)

End Sub

Private Sub testprint_cmd_Click() testprint_update testprint. Show testprint_cmd. Visible $=$ False testprinthide_cmd. Visible $=$ True

End Sub

Private Sub testprinthide_cmd_Click() testprint_cmd. Visible $=$ True 
testprinthide_cmd.Visible $=$ False

testprint.Hide

\section{End Sub}

Sub wiremat_com_Click()

specs1.meshtitle_lab.Caption = " Wire"

specs1.mat_com.Caption = " Perfect Conductor"

specs1.Caption = "Wire Material"

specs1. Show

CTHA.Hide

End Sub

\section{Form: CTHA}

\section{File: CTHA.frm}

Public Feed As Integer

Sub cancel_com_Click()

CTHA.Hide

correctdisplay

End Sub

Private Sub height_dat_Change()

Hplanew1_dat.Text = height_dat.Text

End Sub

Private Sub Form_Load()

lstpresets.AddItem "CB-21"

lstpresets.AddItem "small height"

lstpresets.AddItem "medium height"

lstpresets.AddItem "large height"

lstpresets.AddItem "Default"

1stpresets.Text = "preset CB's"

1stfeeds.AddItem "1"

lstfeeds.AddItem "2"

1stfeeds.AddItem "3"

lstfeeds.AddItem "4"

lstfeeds.AddItem "5"

lstfeeds.AddItem "6"

lstfeeds.AddItem "7"

1stfeeds.AddItem "8"

lstfeeds.Text = "Choose a Feed"

End Sub

Private Sub lstfeeds_Click() 
If lstfeeds. Text $=$ "Choose a Feed" Then 1stfeeds. Text = "7"

Feed $=$ CInt(lstfeeds.Text)

\section{End Sub}

Private Sub lstpresets_Click()

If lstpresets. Text $=$ lstpresets.List $(0)$ Then updateCB21

If lstpresets. Text $=$ lstpresets.List $(1)$ Then updateCBsmall

If lstpresets.Text $=$ lstpresets.List $(2)$ Then updateCBmed

If lstpresets.Text $=$ lstpresets.List(3) Then updateCBlarge

If lstpresets. Text $=$ lstpresets.List(4) Then

restorecthadefs

CTHA.Refresh

End If

End Sub

Sub OK_com_Click()

If lstfeeds. Text $=$ "Choose a Feed" Then lstfeeds.Text = "7"

Feed $=$ CInt(lstfeeds.Text)

propsupdate

CTHA.Hide

End Sub

'Private Sub restoredefs()

' restorecthadefs

' CTHA.Refresh

'End Sub

Sub updateCB21()

CTHA.length_dat.Text $=30.3022$

CTHA. width_dat.Text $=30.3022$

CTHA.height_dat.Text $=3.2258$

CTHA.holedia_dat.Text $=1.1176$

CTHA.Frequency_dat.Text $=4$

CTHA.angle_dat.Text $=16$

CTHA. wirethick_dat.Text $=1.6764$

CTHA.indiameter_dat.Text $=12.3698$

CTHA.outdiameter_dat.Text $=24.892$

CTHA.Lplanew1_dat. Text $=0.8382$

CTHA.Hplanew1_dat.Text $=$ CTHA.height_dat.Text

CTHA.Lplanew2_dat.Text $=0$

CTHA.Hplanew2_dat. Text $=2.3876$

CTHA.holetype_dat.Text $=0$

CTHA.cellperwidth_dat.Text $=1$

CTHA.txtxoffset. Text $=20$

CTHA.txtyoffset. Text $=20$

CTHA.txtzoffset.Text $=20$ 
End Sub

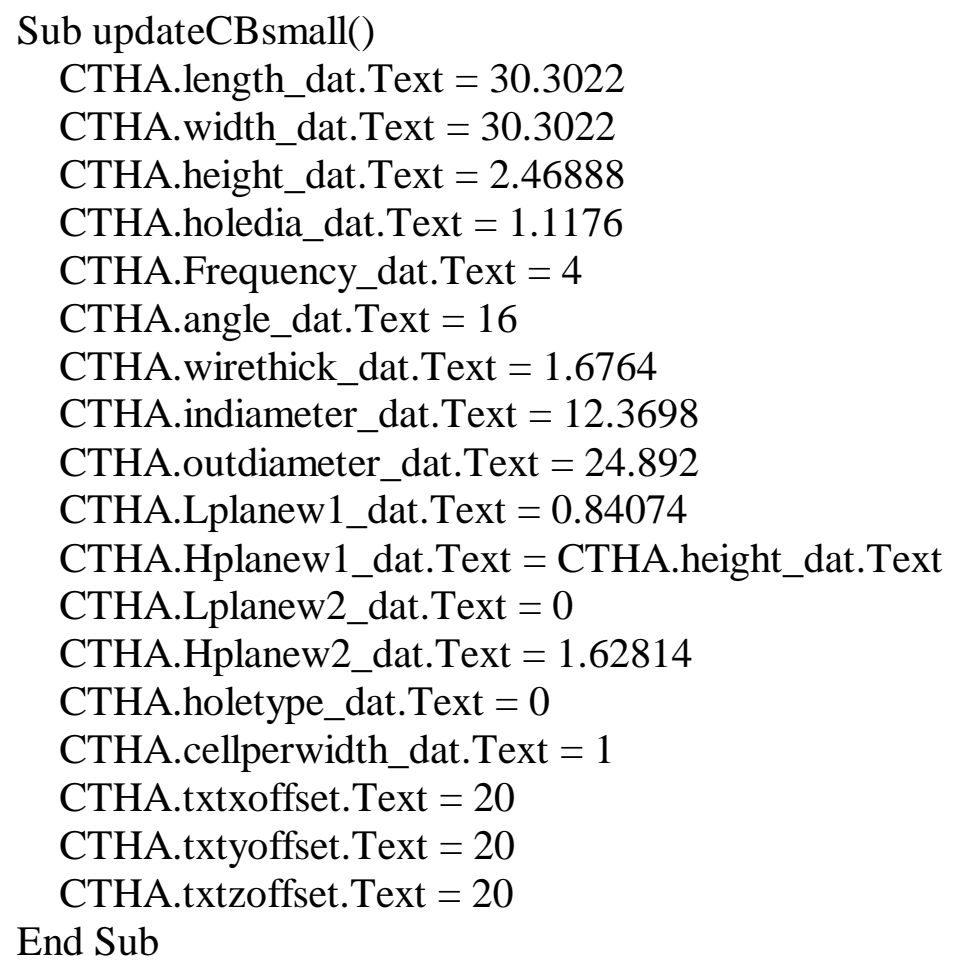

Sub updateCBmed()

CTHA.length_dat.Text $=30.3022$

CTHA.width_dat.Text $=30.3022$

CTHA.height_dat.Text $=3.25628$

CTHA.holedia_dat. Text $=1.1176$

CTHA.Frequency_dat.Text $=4$

CTHA.angle_dat.Text $=16$

CTHA.wirethick_dat.Text $=1.6764$

CTHA.indiameter_dat. Text $=12.3698$

CTHA.outdiameter_dat. Text $=24.892$

CTHA.Lplanew1_dat. Text $=0.84074$

CTHA.Hplanew1_dat.Text $=$ CTHA.height_dat.Text

CTHA.Lplanew2_dat. Text $=0$

CTHA.Hplanew2_dat. Text $=2.41554$

CTHA.holetype_dat.Text $=0$

CTHA.cellperwidth_dat.Text $=1$

CTHA.txtxoffset. Text $=20$

CTHA.txtyoffset. Text $=20$

CTHA.txtzoffset. Text $=20$

End Sub

Sub updateCBlarge()

CTHA.length_dat.Text $=30.3022$ 
CTHA.width_dat. Text $=30.3022$

CTHA.height_dat.Text $=4.85648$

CTHA.holedia_dat.Text $=1.1176$

CTHA.Frequency_dat.Text $=4$

CTHA.angle_dat.Text $=16$

CTHA.wirethick_dat.Text $=1.6764$

CTHA.indiameter_dat.Text $=12.3698$

CTHA.outdiameter_dat. Text $=24.892$

CTHA.Lplanew1_dat. Text $=0.84074$

CTHA.Hplanew1_dat.Text $=$ CTHA.height_dat.Text

CTHA.Lplanew2_dat.Text $=0$

\section{CTHA.Hplanew2_dat.Text $=\mathbf{4 . 0 1 5 7 4}$}

CTHA.holetype_dat.Text $=0$

CTHA.cellperwidth_dat.Text $=1$

CTHA.txtxoffset. Text $=20$

CTHA.txtyoffset. Text $=20$

CTHA.txtzoffset. Text $=20$

End Sub

\section{Form: specs1}

File: specs1.frm

Private Sub cancel_com_Click()

specs1.Hide

End Sub

Private Sub mat_com_Click()

If meshtitle_lab.Caption $=$ " Adhesive" Then matnum $=0$ Else matnum $=1$

selectstandard (matnum)

specs1.Hide

End Sub

Private Sub OK_com_Click()

If meshtitle_lab.Caption $=$ " Adhesive" Then matnum $=2$ Else matnum $=3$

matpropsupdate (matnum)

specs1.Hide

End Sub

\section{Form: Gaussfdtd}

\section{File: Gauss.frm}

Dim A(6) As Long

Private Sub cmdcancel_Click() 


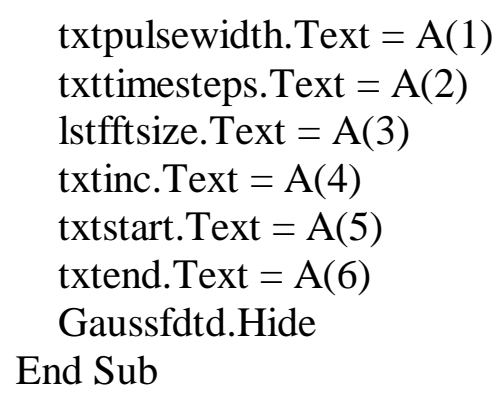

\section{Form: testprint}

\section{File: testprint.frm}

No code was required for this form, since it is just used for display without any interaction from the user. 
Form: Suggfreq

File: Suggfreq.frm

Private Sub cmdok_Click()

Suggfreq.Hide

End Sub

Module: mainmod

File: mainmod.bas

Sub main()

initcthaprops

initmatprops

filename. Show

End Sub

\section{Module: properties}

File: props.bas

Option Base 1

Public Type CTHAprop

lngth As Single 'length of the CTHA

wdth As Single 'width of the CTHA

hight As Single 'height of the CTHA

dia As Single 'Diameter of the holes

freq As Single 'highest working frequency

Angle As Single 'number of holes per revolution

thick As Single 'thickness of the wire

india As Single 'the distance between the center of the ctha

'and the center of the inner holes

outdia As Single '....and the center of the outer holes

elev(4) As Single 'defines four planes for hole starts and ends

holetype As Integer 'type of hole (empty or filled)

cellsperwidth As Single 'number of cells per width of the wire

End Type

Public Type materials

matprop(2 To 15, 1 To 16) As Single

adhmat As Integer

wiremat As Integer

End Type 
Public mat As materials

Public filenam As String

Public props As CTHAprop

Dim defprops As CTHAprop

Public matnum As Integer

Public fname As String

Sub initcthaprops()

defprops.lngth $=32 / 1000$

defprops.wdth $=32 / 1000$

defprops.hight $=2 / 1000$

defprops.dia $=1 / 1000$

defprops.freq $=2$

defprops. Angle $=16$

defprops.thick $=1 / 1000$

defprops.india $=12 / 1000$

defprops.outdia $=25 / 1000$

defprops.elev $(2)=0.5 / 1000$

defprops.elev $(4)=2 / 1000$

defprops.elev $(1)=0 / 1000$

defprops.elev $(3)=1.5 / 1000$

defprops.holetype $=0$

defprops.cellsperwidth $=4$

props $=$ defprops

testprint_update

End Sub

Sub initmatprops()

For $\mathrm{i}=2$ To 15

For $\mathrm{j}=1$ To 16

mat.matprop $(i, j)=0$

Next $\mathrm{j}$

Next i

mat.adhmat $=0$

mat. wiremat $=1$

testprint_update

End Sub

Sub propsupdate()

props.lngth $=$ CSng(CTHA.length_dat.Text $) / 1000$

props.wdth $=$ CSng(CTHA.width_dat.Text $) / 1000$

props.hight $=$ CSng $($ CTHA.height_dat.Text $) / 1000$

props.dia $=$ CSng(CTHA.holedia_dat.Text $) / 1000$

props.freq $=$ CSng $($ CTHA.Frequency_dat.Text $)$

props.Angle $=$ CInt $($ CTHA.angle_dat.Text $)$

If (props.Angle Mod 2) Then 


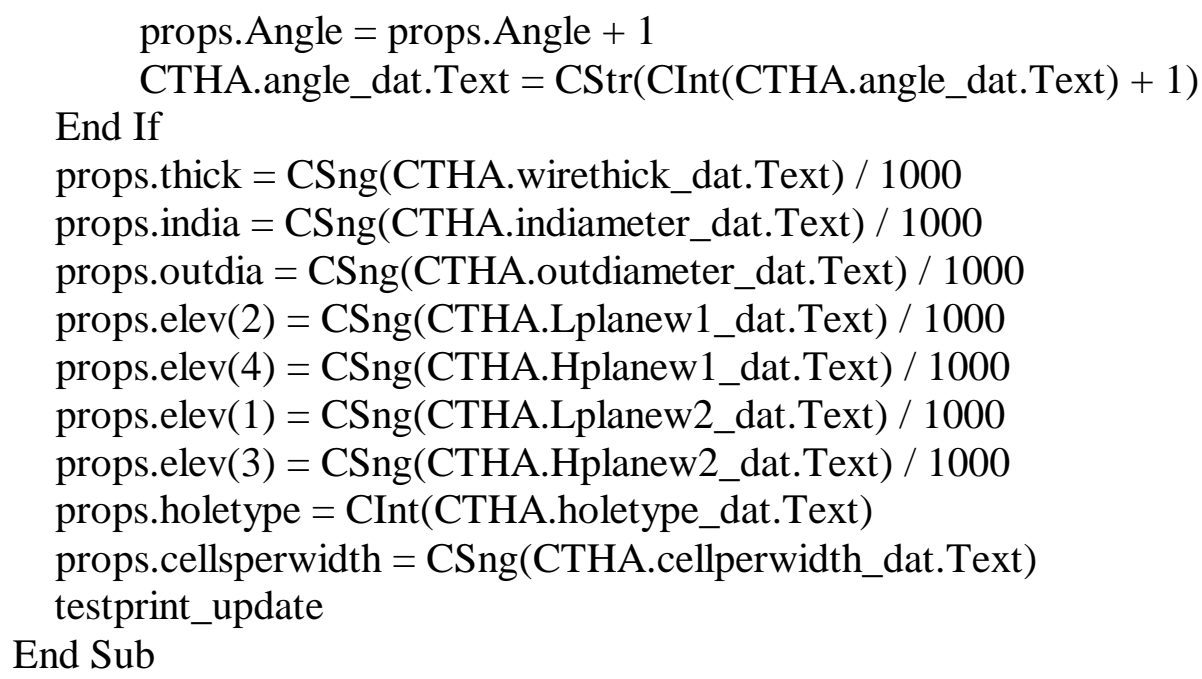



CTHA.Hplanew2_dat.Text $=\operatorname{props.elev}(3) * 1000$
CTHA.holetype_dat.Text = props.holetype
CTHA.cellperwidth_dat.Text $=$ props.cellsperwidth

End Sub

Sub matpropsupdate(matnum As Integer)

mat.matprop $($ matnum, 1$)=$ specs 1 .permittivity_dat.Text

mat.matprop $($ matnum, 2$)=$ specs 1 .conductivity_dat.Text

mat.matprop(matnum, 3) = specs1.permiability_dat.Text

mat.matprop $($ matnum, 4$)=$ specs 1 .magconductivity_dat.Text

mat.matprop $($ matnum, 5) $=$ specs1.density_dat.Text

mat.matprop $($ matnum, 6$)=$ specs 1. statperm_dat.Text

mat.matprop $($ matnum, 7$)=$ specs1.relaxtime_dat.Text

mat.matprop $($ matnum, 8$)=$ specs1.resfreq_dat.Text

mat.matprop $($ matnum, 9$)=$ specs 1 .dampcoeff_dat.Text

mat.matprop $($ matnum, 10) $=$ specs1.mattype_dat.Text

mat.matprop $($ matnum, 11$)=$ specs1.larmor_dat.Text

mat.matprop $($ matnum, 12) = specs1.satmag_dat.Text

mat.matprop $\left(\right.$ matnum, 13) $=$ specs 1. dampcoeff $2 \_$dat.Text

mat.matprop $($ matnum, 14$)=$ specs1.thetastatic_dat.Text

mat.matprop $($ matnum, 15) $=$ specs1.phistatic_dat.Text

mat.matprop (matnum, 16) $=$ specs1.ferrite_dat.Text

If matnum $=2$ Then mat adhmat $=2$ Else mat wiremat $=3$

testprint_update

End Sub

Sub selectstandard(matnum As Integer)

If matnum $=0$ Then mat adhmat $=0$ Else mat.wiremat $=1$

testprint_update

End Sub

Sub testprint_update()

testprint.Text $1(0)$. Text $=$ props. Ingth

testprint. Text1(1).Text $=$ props. wdth

testprint.Text1(2). Text $=$ props.hight

testprint.Text1(3). Text $=$ props.dia

testprint.Text1(4).Text $=$ props. . freq

testprint. Text 1(5). Text $=$ props. Angle

testprint.Text1(6).Text $=$ props.thick

testprint.Text1(7).Text $=$ props.india

testprint. Text $1(8)$. Text $=$ props. outdia

testprint.Text 1(9).Text $=$ props.elev $(2)$

testprint.Text 1(10). Text $=$ props.elev $(4)$

testprint.Text1(11).Text $=$ props.elev $(1)$

testprint.Text1(12).Text $=$ props.elev(3)

testprint. Text1(13). Text $=$ mat. wiremat 


$$
\begin{aligned}
& \text { testprint.Text1(14). Text }=\text { mat.adhmat } \\
& \text { testprint.Text 1(15). Text }=\text { mat } \cdot \operatorname{matprop}(2,1) \\
& \text { testprint.Text 1(16). Text }=\text { mat. } \operatorname{matprop}(2,2) \\
& \text { testprint. Text 1(17). Text }=\text { mat. } \operatorname{matprop}(2,3) \\
& \text { testprint.Text1(18). Text }=\text { mat.matprop }(2,4) \\
& \text { testprint.Text 1(19). Text }=\text { mat. } \operatorname{matprop}(2,5) \\
& \text { testprint.Text 1(26). Text }=\text { mat } \cdot \operatorname{matprop}(2,6) \\
& \text { testprint. Text 1(20). Text }=\text { mat } \cdot \text { matprop }(3,1) \\
& \text { testprint. Text } 1(21) \text {. Text }=\text { mat. } \operatorname{matprop}(3,2) \\
& \text { testprint. Text 1(22). Text }=\text { mat } \cdot \text { matprop }(3,3) \\
& \text { testprint.Text 1(23). Text }=\text { mat. } \operatorname{matprop}(3,4) \\
& \text { testprint.Text 1(24). Text }=\text { mat.matprop }(3,5) \\
& \text { testprint.Text1(27). Text }=\text { mat.matprop }(3,6) \\
& \text { testprint. Text } 1(25)=\text { filenam } \\
& \text { testprint.Text1 }(28)=\text { props.holetype } \\
& \text { ' testprint.Text1(29) = props.cellsperwidth }
\end{aligned}
$$

Sub outFile(outfname As String)

filenam = outfname + ".id"

testprint_update

End Sub

Sub calcs $(\mathrm{x})$

If $x=1$ Then $A=\operatorname{startcalcs}($ props, mat)

\section{End Sub}

\section{Module: holes}

\section{File: Calculations.bas}

Option Base 1

Public Type meshpoints

xmat As Integer

ymat As Integer

zmat As Integer

\section{End Type}

Public mesharea() As meshpoints

Public wavelength, cellsize As Single

Public cell(3) As Single '3 different cell sizes depending on 3 criterias

Public sizes(3) As Single ' size of the cells in the 3 directions

Public cellnos(3) As Integer ' no of cells in each direction

Public theta As Single

Public Type location

xloc As Integer

yloc As Integer 


\section{End Type}

Public holecenters() As location

Public centers() As location

Dim radius, $r$ As Single

Dim holesperrev As Integer

Dim innerdiam, outerdiam As Single

Dim noofcells() As Integer

Dim centerofholes() As location

Public Const PI $=3.141592654$

Public elevation(4) As Integer

Dim z As Integer

Dim plane As Integer

Dim intradius As Integer

Dim zmaterial As Integer

Dim holeradius As Integer

Function startcalcs(props As CTHAprop, mat As materials)

$\mathrm{A}=$ Findcellsizes(props) $\quad$ 'defining the cell sizes in sizes(), and the cell numbers in cellnos()

$\mathrm{b}=$ findholecenters(props) 'defining the centers of all the holes in holecenters()

If CTHA.lstfeeds. Text $=$ "8" Then extra $=1$ Else extra $=0$

ReDim mesharea(cellnos(1) +1 , cellnos $(2)+1$, cellnos(3) $+1+$ extra) 'defining the space array

Initialization

defineholes

End Function

Function Findcellsizes(property As CTHAprop)

wavelength $=0.3 /$ property.freq

cell $(1)=$ wavelength $/ 11$

cell $(2)=$ property.thick $/$ props.cellsperwidth

$\operatorname{cell}(3)=$ property.dia $/ 3$

cellsize $=\operatorname{smallest}(\operatorname{cell}(), 3)$

cellnos $(1)=\operatorname{CInt}($ property.lngth $/$ cellsize $)-1$

cellnos $(2)=\operatorname{CInt}($ property $\cdot \mathrm{wdth} /$ cellsize $)-1$

cellnos $(3)=$ CInt $($ property.hight $/$ cellsize $)-1$

For $\mathrm{i}=1$ To 3

cellnos(i) $=\operatorname{checknos}(\operatorname{cellnos}(i))$

Next i

sizes $(1)=$ property.lngth $/$ cellnos $(1)$

$\operatorname{sizes}(2)=$ property. $w d t h /$ cellnos $(2)$

sizes $(3)=$ property.hight $/$ cellnos $(3)$

End Function

Function findholecenters(property As CTHAprop)

theta $=(2 * \mathrm{PI}) /$ property. Angle 


\section{ReDim holecenters(2, property.Angle)}

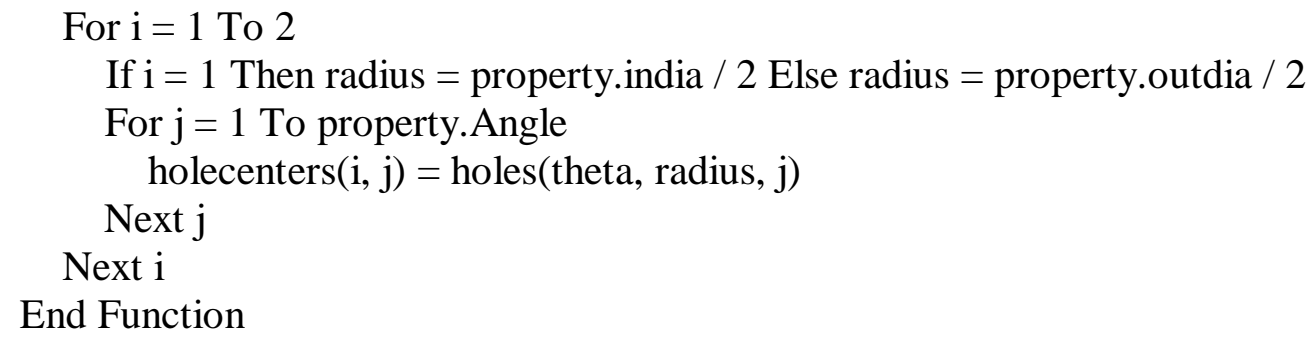

End Function

Function smallest(cell() As Single, j As Integer) As Single

smallest $=\operatorname{cell}(1)$

For $\mathrm{i}=2$ To $\mathrm{j}$

If cell(i) $<$ smallest Then smallest $=$ cell $(\mathrm{i})$

\section{Next i}

End Function

Function checknos(cells As Integer) As Integer

If cells $<20$ Then checknos $=19$ Else checknos $=$ cells

End Function

Function holes(theta As Single, r, j) As location

Dim currenttheta As Single

currentheta $=$ theta $* \mathrm{j}$

holes. $x$ loc $=(\mathrm{r} * \operatorname{Cos}($ currenttheta $)) / \operatorname{sizes}(1)+(\operatorname{cellnos}(1) / 2)$

holes.yloc $=(r * \operatorname{Sin}($ currenttheta $)) / \operatorname{sizes}(2)+(\operatorname{cellnos}(2) / 2)$

End Function

Sub defineholes ()

For $\mathrm{i}=1$ To 4 'convert elevations from meters to cell numbers elevation $(\mathrm{i})=\operatorname{CInt}($ props.elev $(\mathrm{i}) / \operatorname{sizes}(3))+1$

Next i

holeradius $=$ props.dia $/(\operatorname{sizes}(1) * 2)$ 'find hole radius in \# of cells

For $\mathrm{x}=1$ To 2

For $\mathrm{y}=1$ To props.Angle

zmaterial $=$ mat. wiremat

For plane $=$ elevation $(1)$ To elevation $(4)$

If plane $=$ elevation $(4)$ Then zmaterial $=0$

For intradius $=0$ To holeradius mat.adhmat)

$\mathrm{A}=$ drawcircle(plane, holecenters $(\mathrm{x}, \mathrm{y})$, intradius, zmaterial, mat.wiremat, Next

Next

Next

Next 
cleanupholes

connectwires

If props.holetype $=0$ Then drillair

printtofile

End

End Sub

Function drawcircle(z, hole As location, intradius, zmaterial, matofwire As Integer, matofadh As Integer)

Dim x As Integer, y As Integer

Dim stepangle As Single, incangle As Single, endangle As Single

If intradius Then stepangle $=2 *$ PI / ( $8 *$ intradius) Else stepangle $=2 *$ PI

endangle $=(2 * \mathrm{PI})$

For incangle $=0$ To endangle Step stepangle

$\mathrm{x}=\mathrm{CInt}($ intradius $*$ Cos $($ incangle $)+$ hole.xloc $)$

$\mathrm{y}=$ CInt (intradius $*$ Sin(incangle $)+$ hole.yloc)

mesharea $(x, y, z) \cdot x$ mat $=$ matofwire: $\operatorname{mesharea}(x, y, z) . y m a t=$ matofwire: $\operatorname{mesharea}(x, y$,

$\mathrm{z})$.zmat $=$ zmaterial

Next

End Function

Sub Initialization()

For $\mathrm{x}=1$ To cellnos $(1)+1$

For $\mathrm{y}=1$ To cellnos $(2)+1$

For $\mathrm{z}=1$ To cellnos $(3)+1$

mesharea $(x, y, z) \cdot x m a t=$ mat.adhmat

mesharea $(x, y, z) \cdot y m a t=$ mat.adhmat

mesharea $(x, y, z) \cdot z m a t=$ mat.adhmat

Next

Next

Next

End Sub

Sub drillair()

For $\mathrm{x}=1$ To 2

For $\mathrm{y}=1$ To props.Angle

For $\mathrm{z}=$ elevation(1) To elevation(4)

$\mathrm{i}=\operatorname{holecenters}(\mathrm{x}, \mathrm{y})$.xloc

$\mathrm{j}=$ holecenters $(\mathrm{x}, \mathrm{y})$.yloc

mesharea $(i, j, z) \cdot x m a t=0:$ mesharea $(i, j, z)$.ymat $=0$ : mesharea $(i, j, z) \cdot z m a t=0$

mesharea $(\mathrm{i}-1, \mathrm{j}, \mathrm{z})$.xmat $=0$

Next

mesharea $(\mathrm{i}, \mathrm{j}-1, \mathrm{z})$.ymat $=0$

Next

Next

End Sub 
'use the following function to create empty holes for 12 cell thick CTHA models 'but make hole diameter equal to wire width 'remember to only use one of the two functions (above and below)

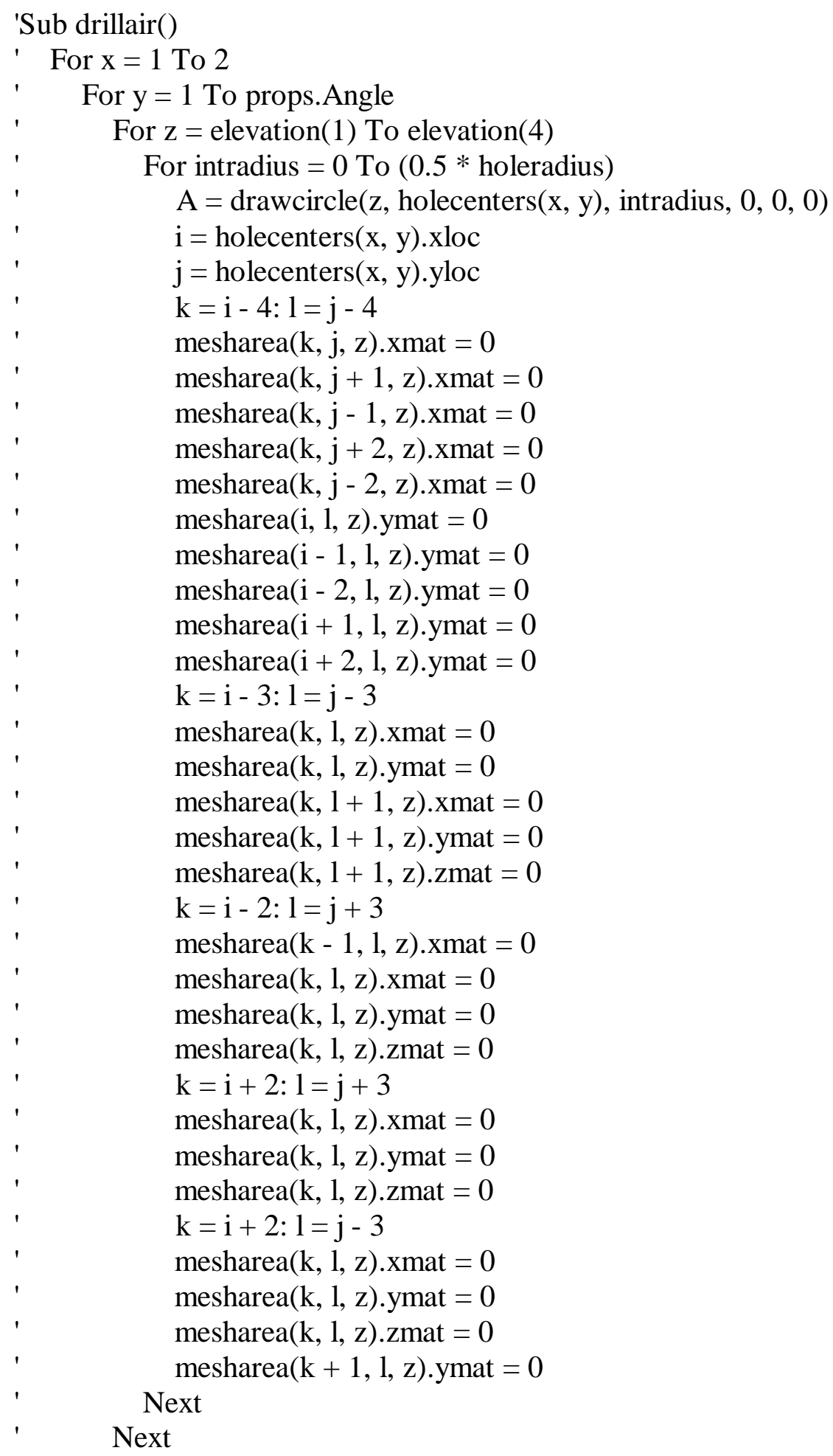


1) Next

Next

'End Sub

\section{Module: wires}

\section{File: wires.bas}

Dim m As Integer, $\mathrm{n}$ As Integer ' m,n to save position of old point locations

Dim i As Integer, $\mathrm{k}$ As Integer ' i,k counters for new point locations

Dim r As Byte, o As Byte ' o for each wind, $r$ for elevations

Dim elev As Integer 'for the elevation of the wire

Dim xstart As Integer, ystart As Integer

Dim xend As Integer, yend As Integer

Dim j As Integer ' j just to help $\mathrm{k}$

Dim q As Integer ' $\mathrm{q}$ to check if this is the first entrance to the big loop

Public Totallength As Single

Sub connectwires( $)$

$\mathrm{r}=0: \mathrm{m}=1: \mathrm{n}=2$

For $\mathrm{o}=1$ To $2: \mathrm{i}=\mathrm{o}: \mathrm{q}=1$

For $\mathrm{j}=2$ To props. Angle +2

If $\mathrm{j}>$ props. Angle Then $\mathrm{k}=\mathrm{j}$ - props. Angle Else $\mathrm{k}=\mathrm{j}$

elev $=\operatorname{checkforelev}(\mathrm{m}, \mathrm{n})$

elev $=$ elevation $($ elev $+r)$

If $\mathrm{q}\langle>1$ Then

$$
r=r+2: \text { If } r=4 \text { Then } r=0
$$

If $m<>i$ Then

$A=$ wire2D(holecenters $(m, n) \cdot x l o c$, holecenters $(m, n) . y l o c$,

holecenters(i, k).xloc, holecenters(i, k).yloc, elev, props.thick / $(2 * \operatorname{sizes}(1)), 0)$ End If

End If

$\mathrm{m}=\mathrm{i}: \mathrm{n}=\mathrm{k}: \mathrm{q}=\mathrm{q}+1$

$\mathrm{i}=\mathrm{i}+1:$ If $\mathrm{i}=3$ Then $\mathrm{i}=1$

If $\mathrm{k}=1$ Then $\mathrm{i}=\mathrm{i}-1$ : If $\mathrm{i}=0$ Then $\mathrm{i}=2$

\section{Next}

Next

drawfeed

cleanupwires

End Sub

Function checkforelev(m As Integer, $\mathrm{n}$ As Integer) As Integer

checkforelev $=2$

If $m=1$ And $(n \operatorname{Mod} 2)=1$ And $n<>1$ Then checkforelev $=1$

If $m=2$ And $(n \operatorname{Mod} 2)=0$ Then checkforelev $=1$

End Function 
Function wire2D(xstart, ystart, xend, yend, z As Integer, thick As Single, flag As Byte)

Dim $r$ As Single, deltax As Integer, deltay As Integer

Dim theta As Single

Dim sox As Integer, soy As Integer

Dim dist As Single, side As Single

Dim xctr As Single, yctr As Single

Dim xa As Single, ya As Single

Dim x As Integer, y As Integer

Dim zmaterial As Byte

deltax $=$ xstart - xend

deltay $=$ ystart - yend

$\mathrm{r}=\operatorname{Sqr}\left((\operatorname{deltax})^{\wedge} 2+(\text { deltay })^{\wedge} 2\right)$

Totallength $=$ Totallength $+\mathrm{r}$

If deltax $=0$ Then theta $=($ PI $/ 2)$ Else theta $=\operatorname{Atn}($ deltay $/$ deltax $)$

If (xstart < cellnos(1) / 2) And (ystart > cellnos(2) / 2) Then theta $=$ theta +3.141592654

If $\operatorname{Sgn}(\operatorname{Cos}($ theta $))=\operatorname{Sgn}($ deltax $)$ Then $\operatorname{sox}=-1 * \operatorname{Sgn}($ deltax $)$ Else sox $=\operatorname{Sgn}($ deltax $)$

If $\operatorname{Sgn}(\operatorname{Sin}($ theta $))=\operatorname{Sgn}($ deltay $)$ Then soy $=-1 * \operatorname{Sgn}($ deltay) Else soy = Sgn(deltay)

For dist $=0$ To $r$ Step 0.5

If dist $>=\mathrm{r} / 2$ And dist $<(\mathrm{r} / 2+0.5)$ And flag $=1$ Then dist $=\operatorname{dist}+1$

$\mathrm{xctr}=\mathrm{xstart}+\operatorname{sox} *$ dist $* \operatorname{Cos}($ theta $) * \operatorname{Sgn}($ deltax $)$

yctr $=$ ystart + soy $*$ dist $* \operatorname{Sin}($ theta $) * \operatorname{Sgn}($ deltay $)$

For side $=0$ To thick Step 0.5

xa $=-1 *$ side $* \operatorname{Sin}($ theta $)$

ya $=$ side $* \operatorname{Cos}($ theta $)$

$\mathrm{x}=\operatorname{CInt}(\mathrm{xctr}+\mathrm{xa})$

$\mathrm{y}=\operatorname{CInt}(\mathrm{yctr}+\mathrm{ya})$

If mesharea $(\mathrm{x}, \mathrm{y}, \mathrm{z}) \cdot \mathrm{zmat}=$ mat. wiremat Then zmaterial $=$ mat. wiremat Else zmaterial $=$ mat.adhmat

mesharea $(\mathrm{x}, \mathrm{y}, \mathrm{z}) \cdot \mathrm{xmat}=$ mat.wiremat: $\operatorname{mesharea}(\mathrm{x}, \mathrm{y}, \mathrm{z}) \cdot \mathrm{ymat}=$ mat.wiremat: mesharea $(x, y, z) \cdot z$ mat $=$ zmaterial

$\mathrm{x}=\operatorname{CInt}(\mathrm{xctr}-\mathrm{xa})$

$\mathrm{y}=\operatorname{CInt}(\mathrm{yctr}-\mathrm{ya})$

If mesharea $(x, y, z) \cdot z$ mat $=$ mat. wiremat Then zmaterial $=$ mat. wiremat Else zmaterial $=$ mat.adhmat

mesharea $(\mathrm{x}, \mathrm{y}, \mathrm{z}) \cdot \mathrm{xmat}=$ mat.wiremat: $\operatorname{mesharea}(\mathrm{x}, \mathrm{y}, \mathrm{z}) \cdot \mathrm{ymat}=$ mat.wiremat: mesharea $(x, y, z) \cdot z m a t=$ zmaterial

Next

Next

End Function

Function findcenter(xstart, ystart, xend, yend) As location

deltax $=$ xstart - xend

deltay $=$ ystart - yend

$\mathrm{r}=\operatorname{Sqr}\left((\text { deltax })^{\wedge} 2+(\text { deltay })^{\wedge} 2\right)$ 
If deltax $=0$ Then theta $=1.5708$ Else theta $=\operatorname{Atn}($ deltay $/$ deltax $)$

If (xstart < cellnos(1) / 2) And (ystart > cellnos(2) / 2) Then theta $=$ theta +3.141592654

dist $=r / 2$

If $\operatorname{Sgn}(\operatorname{Cos}($ theta $))=\operatorname{Sgn}($ deltax $)$ Then $\operatorname{sox}=-1 * \operatorname{Sgn}($ deltax $)$ Else sox $=\operatorname{Sgn}($ deltax $)$

If $\operatorname{Sgn}(\operatorname{Sin}($ theta $))=\operatorname{Sgn}($ deltay $)$ Then soy $=-1 * \operatorname{Sgn}($ deltay $)$ Else soy $=\operatorname{Sgn}($ deltay $)$

findcenter.xloc $=$ xstart $+\operatorname{sox} *$ dist $* \operatorname{Cos}($ theta $) * \operatorname{Sgn}($ deltax $)$

findcenter.yloc $=$ ystart + soy $*$ dist $* \operatorname{Sin}($ theta $) * \operatorname{Sgn}($ deltay $)$

End Function

Sub drawfeed()

If CTHA.lstfeeds.Text = "Choose a Feed" Then CTHA.lstfeeds.Text = "7"

Feed $=$ CInt $($ CTHA.lstfeeds.Text $)$

If Feed $=1$ Then Call feed 1

If Feed $=2$ Then Call feed 2

If Feed $=3$ Then Call feed 3

If Feed $=4$ Then Call feed 4

If Feed $=5$ Then Call feed 5

If Feed $=6$ Then Call feed 6

If Feed $=7$ Then Call feed 7

If Feed $=8$ Then Call feed 8

End Sub

Sub feed1()

$\mathrm{m}=1: \mathrm{n}=1: \mathrm{i}=1: \mathrm{k}=2$

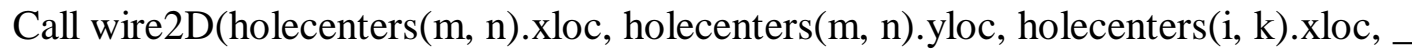
holecenters(i, k).yloc, elevation(4), props.thick / (2* sizes(1)), 0)

$\mathrm{m}=2: \mathrm{i}=2: \mathrm{k}=2: \mathrm{n}=1$

Call wire2D(holecenters (m, n).xloc, holecenters(m, n).yloc, holecenters(i, k).xloc, holecenters(i, k).yloc, elevation(4), props.thick / $(2 * \operatorname{sizes}(1)), 1)$

End Sub

Sub feed2()

$\mathrm{m}=1: \mathrm{n}=1: \mathrm{i}=2: \mathrm{k}=2$

Call wire2D(holecenters (m, n).xloc, holecenters(m, n).yloc, holecenters(i, k).xloc, holecenters(i, k).yloc, elevation(4), props.thick / (2* sizes $(1)), 1)$

End Sub

Sub feed3()

$\mathrm{m}=2: \mathrm{i}=2: \mathrm{k}=2: \mathrm{n}=1$

Call wire2D(holecenters $(m, n) . x l o c$, holecenters $(m, n) . y l o c$, holecenters(i, k).xloc, holecenters(i, k).yloc, elevation(4), props.thick / $(2 * \operatorname{sizes}(1)), 1)$

End Sub

Sub feed4()

$\mathrm{n}=2: \mathrm{i}=2: \mathrm{k}=2: \mathrm{m}=1$

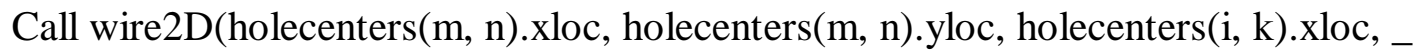


holecenters(i, k).yloc, elevation(4), props.thick / (2* sizes(1)), 1)

End Sub

Sub feed5()

$\mathrm{m}=1: \mathrm{n}=1: \mathrm{k}=1: \mathrm{i}=2$

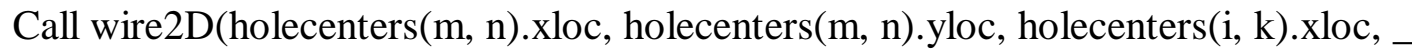
holecenters(i, k).yloc, elevation(4), props.thick / (2* sizes $(1)), 0)$

$\mathrm{n}=2: \mathrm{i}=2: \mathrm{k}=2: \mathrm{m}=1$

Call wire2D(holecenters (m, n).xloc, holecenters(m, n).yloc, holecenters(i, k).xloc, holecenters(i, k).yloc, elevation(4), props.thick / $(2 * \operatorname{sizes}(1)), 1)$

End Sub

Sub feed6()

$\mathrm{m}=1: \mathrm{n}=1: \mathrm{i}=1: \mathrm{k}=2$

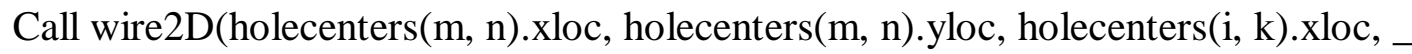
holecenters(i, k).yloc, elevation(4), props.thick / (2* sizes(1)), 0)

$\mathrm{m}=2: \mathrm{i}=2: \mathrm{k}=2: \mathrm{n}=1$

Call wire2D(holecenters $(m, n) . x l o c$, holecenters(m, n).yloc, holecenters(i, k).xloc, _ holecenters(i, k).yloc, elevation(4), props.thick / (2* sizes(1)), 0)

$\operatorname{Dim} \mathrm{A}(2)$ As location

For $\mathrm{i}=1$ To 2

$\mathrm{A}(\mathrm{i})=$ findcenter(holecenters $(\mathrm{i}, 1)$.xloc, holecenters(i, 1).yloc,

Next i holecenters(i, 2).xloc, holecenters(i, 2).yloc)

Call wire2D(A(1).xloc, $\mathrm{A}(1) . y l o c, A(2) . x l o c, A(2) . y l o c$, elevation(4), props.thick / (2* sizes(1)), 1)

End Sub

Sub feed7()

$\mathrm{m}=1: \mathrm{n}=1: \mathrm{k}=1: \mathrm{i}=2$

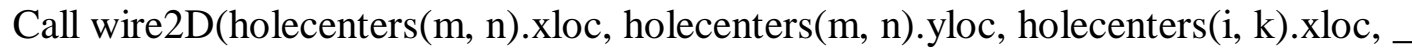
holecenters(i, k).yloc, elevation(4), props.thick / $(2 * \operatorname{sizes}(1)), 0)$

$\mathrm{n}=2: \mathrm{i}=2: \mathrm{k}=2: \mathrm{m}=1$

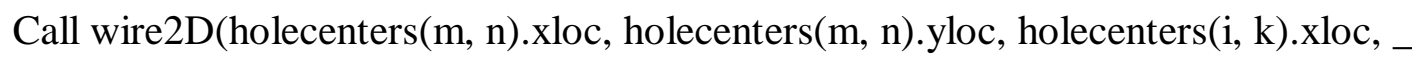
holecenters(i, k).yloc, elevation(4), props.thick / (2* sizes(1)), 0)

$\operatorname{Dim} \mathrm{A}(2)$ As location

For $\mathrm{i}=1$ To 2

$\mathrm{A}(\mathrm{i})=$ findcenter(holecenters $(1, \mathrm{i}) . \mathrm{xloc}$, holecenters $(1, \mathrm{i}) . \mathrm{yloc}$,

Next i holecenters $(2, \mathrm{i}) \cdot x$ loc, holecenters $(2, \mathrm{i}) . \mathrm{yloc})$

Call wire2D(A(1).xloc, A(1).yloc, A(2).xloc, $\mathrm{A}(2)$.yloc, elevation(4), props.thick / (2 * sizes(1)), 1)

End Sub

Sub feed 8()

$\mathrm{m}=1: \mathrm{n}=1: \mathrm{i}=2: \mathrm{k}=2$ 


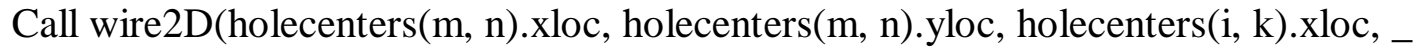
holecenters(i, k).yloc, elevation(4), props.thick / $(2 * \operatorname{sizes}(1)), 1)$

$\mathrm{i}=2: \mathrm{n}=2: \mathrm{m}=1: \mathrm{k}=1$

Call wire2D(holecenters $(m, n) . x l o c$, holecenters(m, n).yloc, holecenters(i, k).xloc, holecenters $(\mathrm{i}, \mathrm{k})$.yloc, elevation $(4)+1$, props.thick / $(2 * \operatorname{sizes}(1)), 0)$

End Sub

\section{Module: printout}

\section{File: printout.bas}

Dim mainfilenam As String

Dim fdtdfilenam As String

Dim noofcell(3) As Integer

Dim holeslength As Single

Dim xoffset As Integer

Dim yoffset As Integer

Dim zoffset As Integer

Sub printtofile()

If CTHA.lstfeeds. Text $=" 8 "$ Then extra $=1$ Else extra $=0$

xoffset $=$ CTHA.txtxoffset. Text $* 2$

yoffset $=$ CTHA.txtyoffset.Text $* 2$

zoffset $=$ CTHA.txtzoffset. Text $* 2$

If zoffset $=0$ And extra $=1$ Then zoffset $=2$

Dim nodes As Long

nodes $=0$

For $\mathrm{x}=1$ To cellnos $(1)+1$

For $\mathrm{y}=1$ To cellnos $(2)+1$

For $\mathrm{z}=1$ To cellnos(3) $+1+$ extra

If mesharea(x, y, z).xmat Or mesharea(x,y, z).ymat Or mesharea(x, y, z).zmat Then nodes $=$ nodes +1

Next

Next

Next

For $\mathrm{i}=1$ To 3

noofcell $(\mathrm{i})=\operatorname{cellnos}(\mathrm{i})$

If $(\operatorname{cellnos}(\mathrm{i}) \operatorname{Mod} 5)<>0$ Then noofcell(i) $=\operatorname{cellnos}(\mathrm{i})+(5-(\operatorname{cellnos}(\mathrm{i}) \operatorname{Mod} 5))$

Next i

Open filenam For Output As \#1

Print \#1, "Geometry file version 3.6"

Print \#1, noofcell(1) + xoffset; noofcell(2) + yoffset; noofcell(3) + zoffset

Print \#1, sizes(1); sizes(2); sizes(3)

Print \#1, CInt $((\operatorname{cellnos}(1)+1) * 2 / 5) ; \operatorname{CInt}((\operatorname{cellnos}(2)+1) * 2 / 5) ; \operatorname{CInt}((\operatorname{cellnos}(3)+1) * 2$ /

5)

Print \#1, 0 
For $\mathrm{i}=2$ To 3

For $\mathrm{j}=1$ To 16

If $\mathrm{j}=10$ Or $\mathrm{j}=16$ Then

Print \#1, CInt(mat.matprop $(i, j))$,

Else

If $(j=1$ Or $j=3$ Or $j=5$ Or $j=6)$ And mat.matprop $(i, j)=0$ Then mat.matprop $(i, j)=1$

Print \#1, Format(mat.matprop(i, j), "\#.000000e+00 ");

End If

Next

If $\mathrm{j}=5$ Or $\mathrm{j}=10$ Then Print \#1,

Print \#1,

Next

For $\mathrm{q}=1$ To 12

Print \#1, "1.000000e+00 0.000000e+00 1.000000e+00 0.000000e+00 1.000000e+00"

Print \#1, "1.000000e+00 0.000000e+00 0.000000e+00 0.000000e+00 0"

Print \#1, "0.000000e+00 0.000000e+00 0.000000e+00 0.000000e+00 0.000000e+00 0"

Next q

Print \#1, 0

Print \#1, "1.000000e+00 0.000000e+00 0.000000e+00"

Print \#1, "0.000000e+00 1.000000e+00 0.000000e+00"

Print \#1, "0.000000e+00 0.000000e+00 1.000000e+00"

Print \#1, "Array format: material"

Print \#1, 1;0

Print \#1, nodes

For $\mathrm{x}=1$ To cellnos $(1)+1$

For $\mathrm{y}=1$ To cellnos $(2)+1$

For $\mathrm{z}=1$ To cellnos $(3)+1+$ extra

If $x=$ cellnos $(1)+1$ Then mesharea $(x, y, z) \cdot x$ mat $=0$

If $y=$ cellnos $(2)+1$ Then mesharea $(x, y, z) \cdot y$ mat $=0$

If $\mathrm{z}=\operatorname{cellnos}(3)+1$ Then mesharea $(\mathrm{x}, \mathrm{y}, \mathrm{z}) \cdot \mathrm{zmat}=0$

If mesharea(x, y, z).xmat Or mesharea(x, y, z).ymat Or mesharea(x, y, z).zmat Then

Print \#1, x + CInt(xoffset / 2); y + CInt(yoffset / 2); z + CInt(zoffset / 2); mesharea(x,

$y, z) . x m a t ;$ mesharea(x, y, z).ymat; mesharea(x, y, z).zmat

End If

Next

Next

Next

Close \#1

'printmainfile

printfdtdfile

Mainsub.Hide

linelength $=$ Totallength $* \operatorname{sizes}(1)$

holeslength $=(($ elevation $(3)-$ elevation $(1)) *$ props.Angle $)+(($ elevation $(4)-$ elevation $(2)) *$ props.Angle $)+(($ elevation $(4)-$ elevation $(3)) * 2)$

holeslength $=$ holeslength $* \operatorname{sizes}(3)$

Totallength $=$ linelength + holeslength 


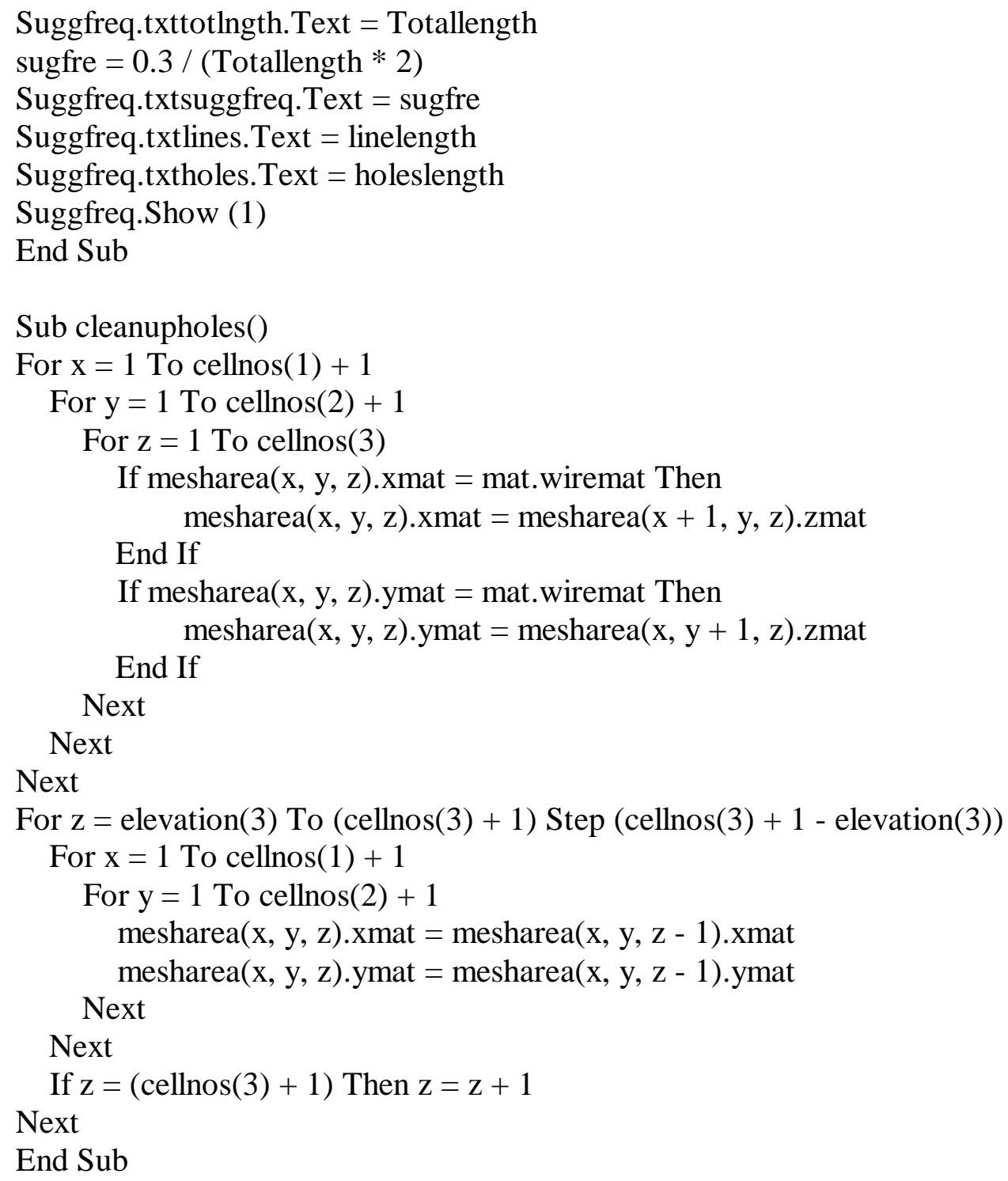


Next

End Sub

Sub printmainfile()

mainfilenam $=$ filename.filename_txt.Text + filename.file_txt.Text + "main.id"

Open mainfilenam For Output As \#1

Print \#1, "Geometry file version 3.6"

Print \#1, cellnos $(1)+1 ; \operatorname{cellnos}(2)+1 ; \operatorname{cellnos}(3)+1$

Print \#1, sizes $(1) * 5 ; \operatorname{sizes}(2) * 5 ; \operatorname{sizes}(3) * 5$

Print \#1, 0;0;0

Print \#1, 0

For $\mathrm{q}=1$ To 14

Print \#1, "1.000000e+00 0.000000e+00 1.000000e+00 $0.000000 \mathrm{e}+001.000000 \mathrm{e}+03 "$

Print \#1, "1.000000e+00 0.000000e+00 0.000000e+00 0.000000e+00 0"

Print \#1, "0.000000e+00 0.000000e+00 0.000000e+00 0.000000e+00 0.000000e+00 0"

Next q

Print \#1, 0

Print \#1, "1.000000e+00 0.000000e+00 0.000000e+00"

Print \#1, "0.000000e+00 1.000000e+00 0.000000e+00"

Print \#1, "0.000000e+00 0.000000e+00 1.000000e+00"

Print \#1, "Array format: material"

Print \#1, 1; 0

Print \#1, 0

Close \#1

End Sub

Sub printfdtdfile()

fdtdfilenam = filename.filename_txt.Text + filename.file_txt.Text + ".fdtd"

Open fdtdfilenam For Output As \#1

Print \#1, "FDTD file version 3.11"

Print \#1, "0 00000008 "

Print \#1, 0 ' number of existing sub files

mainpath $=$ "/usr/users/sherbo/" + filename.file_txt.Text + "main.id"

mainpath $=$ filename.file_txt.Text + ".id"

fdtdfile $=$ filename.file_txt.Text

Print \#1, mainpath

Print \#1, fdtdfile

Print \#1, filename.file_txt.Text + ".id"

' Print \#1, localpath

' Print \#1, fdtdfile

' Print \#1, filename.file_txt.Text + ".id" 
' $\quad$ Print \#1, CInt $((\operatorname{cellnos}(1)+1) * 2 / 5) ; \operatorname{CInt}((\operatorname{cellnos}(2)+1) * 2 / 5) ; \operatorname{CInt}((\operatorname{cellnos}(3)+1) * 2$ / 5) 'location of sub relative to main

' Print \#1, $5 ; 2 \quad$ ' $5=1 / 5$ or $3=1 / 3$, followed by which file $(2=$ second file $)$

Print \#1,0 ' $0=$ gaussian upto $3=$ sinusoid

Print \#1, Format(Gaussfdtd.txtpulsewidth.Text, "\#.000000e+00") ' pulsewidth if previous $=0$, frequensy if previous $=3$

Print \#1, "1.000000e+03"

Print \#1, Gaussfdtd.txttimesteps.Text ' number of time steps

Print \#1, "1.000000e+00" ' wavelength, previously = 7.142857e-01 when sub grid was used

Print \#1, 2 ' 2 indicates voltage source calculations (1=planewave, 3=TEM)

Print \#1, 1 ' number of feeds (if previous is 2 )

'feed data: location $\mathrm{x}, \mathrm{y}, \mathrm{z}$ - direction $(0=\mathrm{x}, 1=\mathrm{y}, 2=\mathrm{z})$ - amplitude, phase, resistance :

Print \#1, $60+C \operatorname{Int}(x o f f s e t ~ / ~ 2) ; 54+C I n t(y o f f s e t ~ / ~ 2) ; 20+C I n t(z o f f s e t ~ / ~ 2) ; 0$; "1.000000e+00 0.000000e+00 5.000000e+01"; 0

Print \#1,-1 ' flag (1=save, $-1=$ dont save)

Print \#1, $-1 \quad$ ' flag for saving fields in slices, -1 or $1=$ no or yes

Print \#1, 2 ' \# of slice planes

'plane info: plane $(1=x y, 2=y z, 3=x z)$, slice, time step increment, starting timestep, ending timestep, grid number

' Print \#1, 1; (cellnos(3) + 1) / 2; Gaussfdtd.txtinc, Gaussfdtd.txtstart, Gaussfdtd.txtend; 0

' Print \#1, 2; (cellnos(1) + 1) / 2; Gaussfdtd.txtinc, Gaussfdtd.txtstart, Gaussfdtd.txtend; 0

For $\mathrm{i}=1$ To $4 \quad$ ' four flags (SAR, Nearzone, ??)

Print \#1, -1

Next i

Print \#1, -1 ' flag for far zone $-1=$ no, 1=yes

Print \#1, $285 \quad$ ' number of far zone directions

For $\mathrm{j}=0$ To 90 Step $45 \quad$ ' far zone phi and theta

For $\mathrm{i}=0$ To 355 Step 5

Print \#1, Format(j, "\#.000000e+00 "); Format(i, "\#.000000e+00 ") constant phi Next $\mathrm{i}$

Next j

For $i=0$ To 355 Step $5 \quad$ 'constant theta

If $\mathrm{i}=0$ Or $\mathrm{i}=45$ Or $\mathrm{i}=90$ Then $\mathrm{i}=\mathrm{i}+5$

Print \#1, Format(i, "\#.000000e+00 "); "9.000000e+01"

Next i

Print \#1, 1

' flag for input impedence

Print \#1, Gaussfdtd.lstfftsize.Text ' size of FFT for impedence calculations

Print \#1, -1

For $\mathrm{i}=1$ To 42

' flag

Print \#1, $1 ; 1$

Next i

Print \#1,

Close \#1

End Sub 


\section{Appendix B: The Preprocessor Input Forms}

In this section a sample printout of all the forms used in the CTHA flexible mesh generator is shown,

\section{Form: filename}

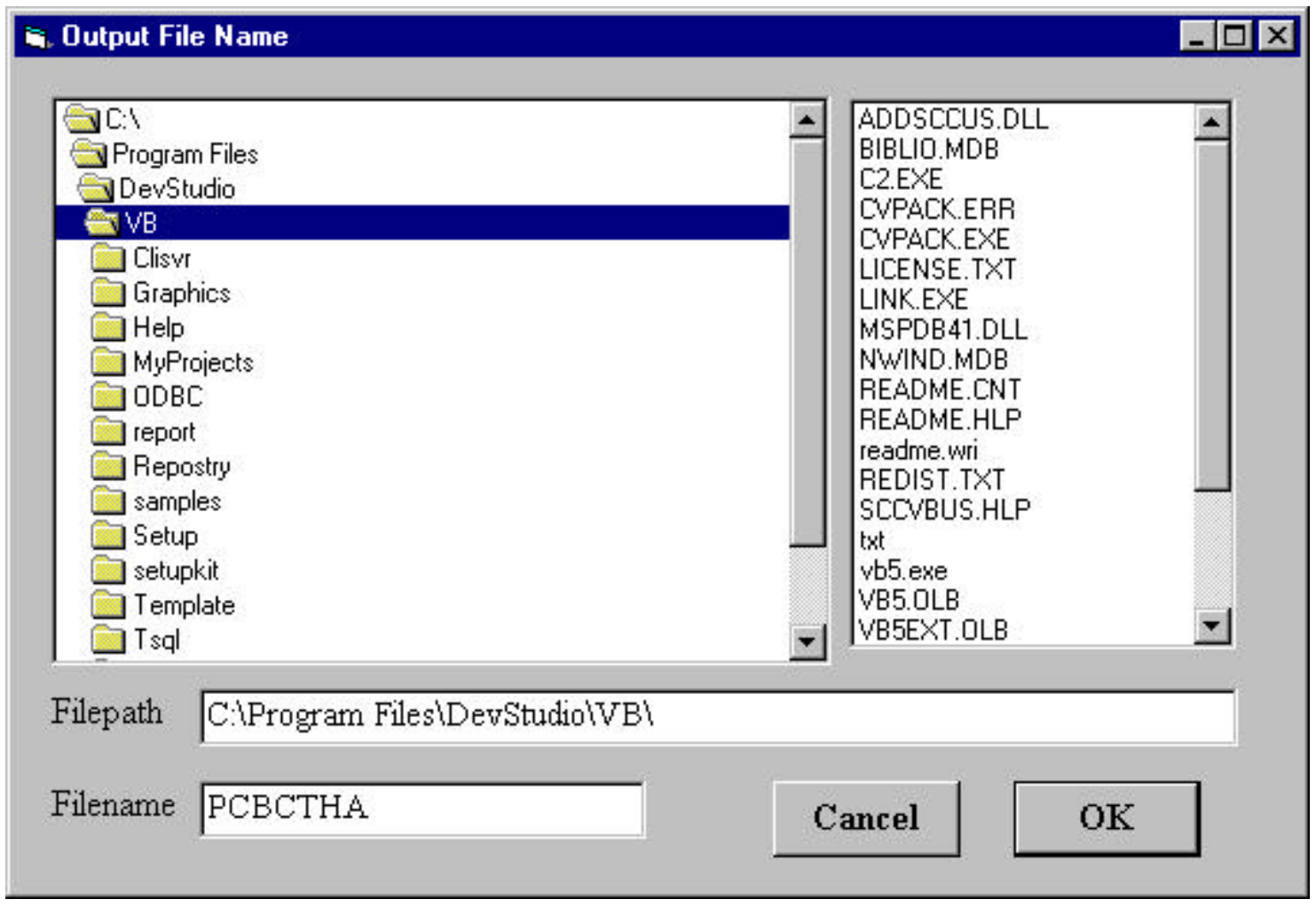

Figure B.1 Form filename 
Form: Mainsub

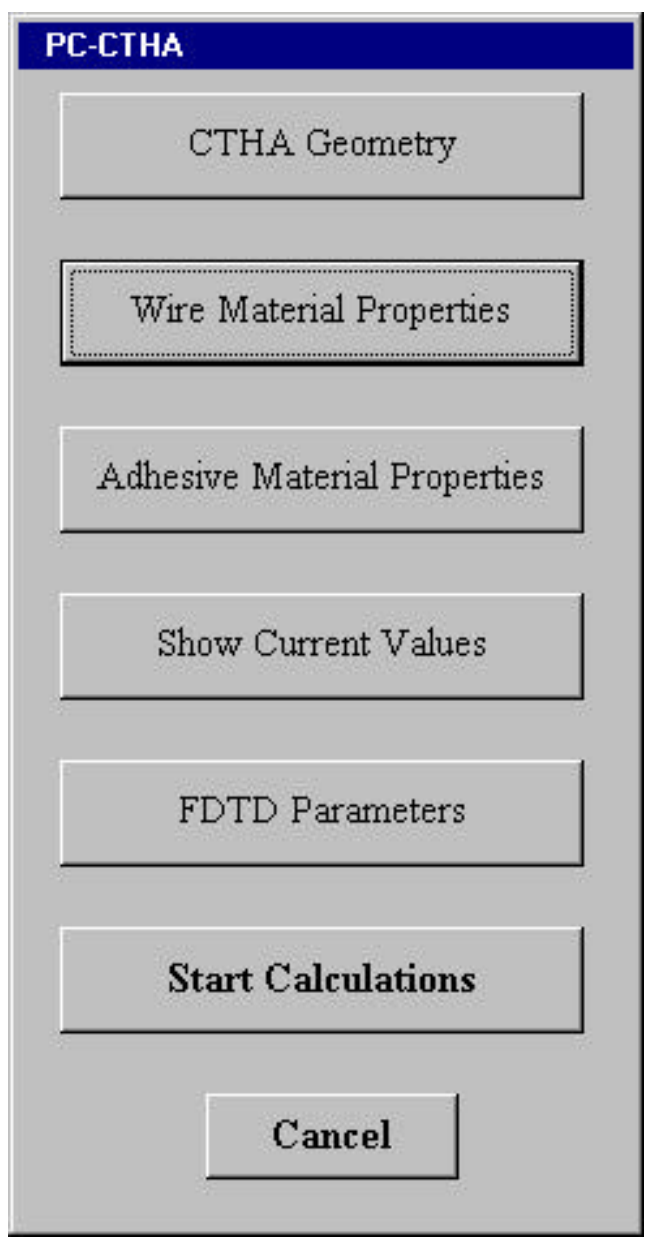

Figure B.2 Form Mainsub 


\section{Form: CTHA}

\begin{tabular}{|c|c|c|c|}
\hline \multicolumn{2}{|c|}{ a. Printed Circuit CTHA Input Parameters } & & \\
\hline \multicolumn{4}{|c|}{ CTHA Parameters: } \\
\hline Diameter of the holes, $\mathrm{mm}$ & 1.1176 & \multirow{3}{*}{$\begin{array}{l}\text { Length, } m \\
\text { Width, mr } \\
\text { Height, } m\end{array}$} & 30.3022 \\
\hline Width of the wire, $\mathrm{mm}$ & 1.6764 & & 30.3022 \\
\hline $\begin{array}{l}\text { approx no. of cells per } \\
\text { wire width }\end{array}$ & 1 & & 3.2258 \\
\hline Lowest plane of wires, $\mathrm{mm}$ & 0 & \multirow{3}{*}{$\begin{array}{l}\text { empty air in } x \\
\text { empty air in } y \\
\text { empty air in } z\end{array}$} & 20 \\
\hline Second plane of wires, $\mathrm{mm}$ & 0.8382 & & 20 \\
\hline Third plane of wires, $\mathrm{mm}$ & 2.3876 & & 20 \\
\hline Highest plane of wires, mm & 3.2258 & \multirow[b]{2}{*}{$\mathrm{CB}-21$} & \\
\hline Inner Holes Diameter, $\mathrm{mm}$ & 12.3698 & & . \\
\hline Outer Holes Diameter, mm & 24.892 & 6 & 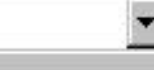 \\
\hline \multicolumn{2}{|c|}{ Type of holes: 0 hollow / 1 filled } & 0 & Cancel \\
\hline \multicolumn{2}{|c|}{ Number of holes per revolution } & 16 & \multirow[b]{2}{*}{ OK } \\
\hline Highest Working Frequency & $\mathrm{GHz}$ & 4 & \\
\hline
\end{tabular}

Figure B.3 Form CTHA 


\section{Form: specs1}

\begin{tabular}{l}
$\begin{array}{l}\text { G. Wire Material } \\
\text { Material Properties for the CTHA Wire }\end{array}$ \\
$\begin{array}{l}\text { Electric Mesh Properties } \\
\text { Relitive Permittivity } \\
\text { Conductivity }\end{array}$ \\
$\begin{array}{l}\text { Magnetic Mesh Properties } \\
\text { Relitive Permeability }\end{array}$ \\
\begin{tabular}{l|c|}
\hline \\
Magnetic Conductivity
\end{tabular} \\
\hline
\end{tabular}

\section{Advanced Material Properties}

Frequency Dependent Material

Static Permittivity

Relaxation Time

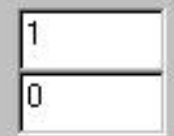

Resonant Frequency

Damping Coefficient

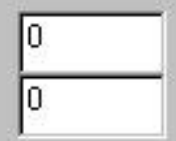

Material Type: (0 normal, 1 Debye, 2 Lorentz) 0

Magnetized Ferrite Material Properties

Damping Coeff.

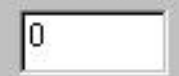

Larmor Precision Freq. 0

Theta Static magnetic

Field Dir.

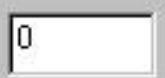

Sat. Mag, radisec

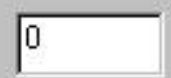

Phi Static magnetic

Field Dir.

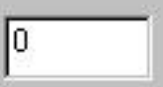

0 normal magnetic mat
1 anisotropic ferrites

Figure B.4 Form specsl 
Form: Gaussian

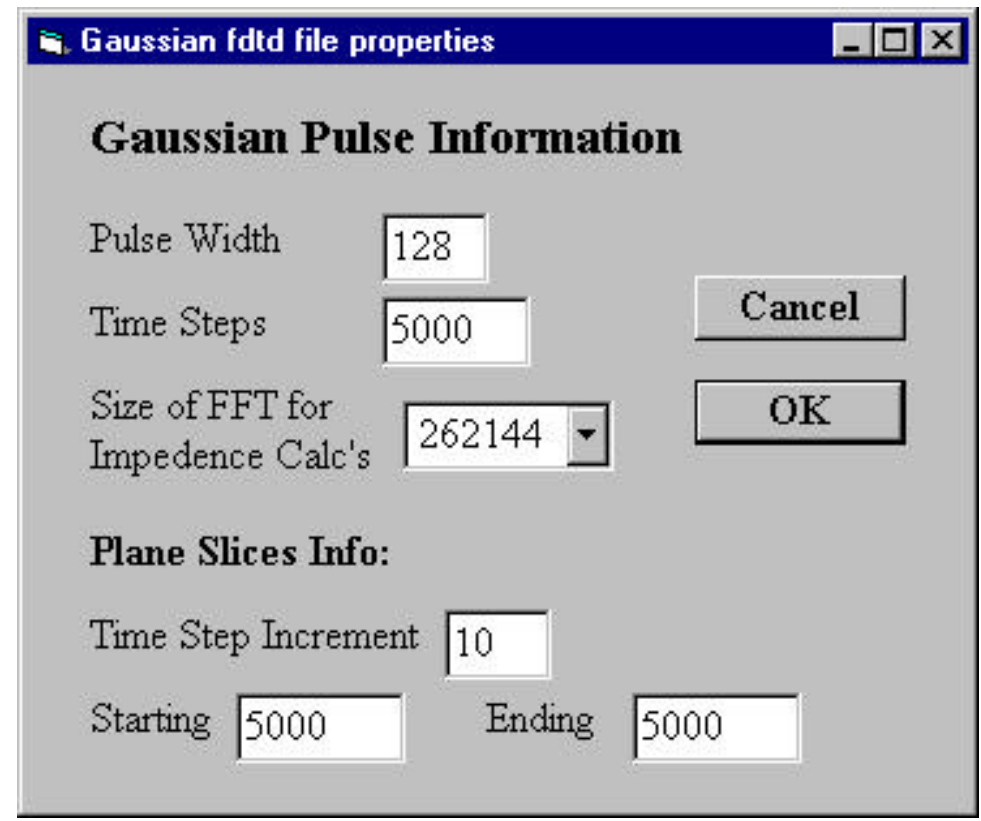

Figure B.5 Form Gaussian 


\section{Form: testprint}

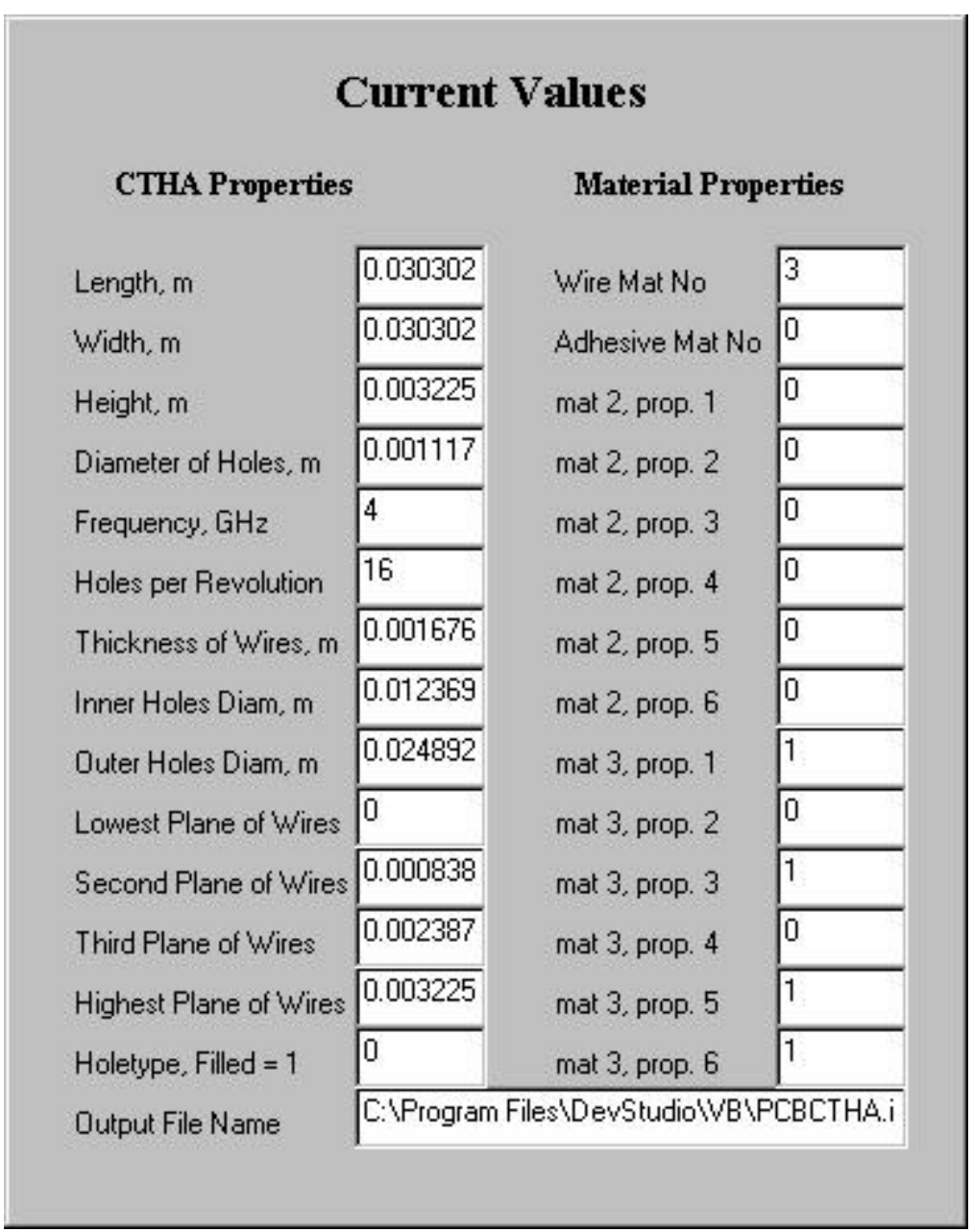

Figure B.6 Form testprint 


\section{Form: Suggfreq}

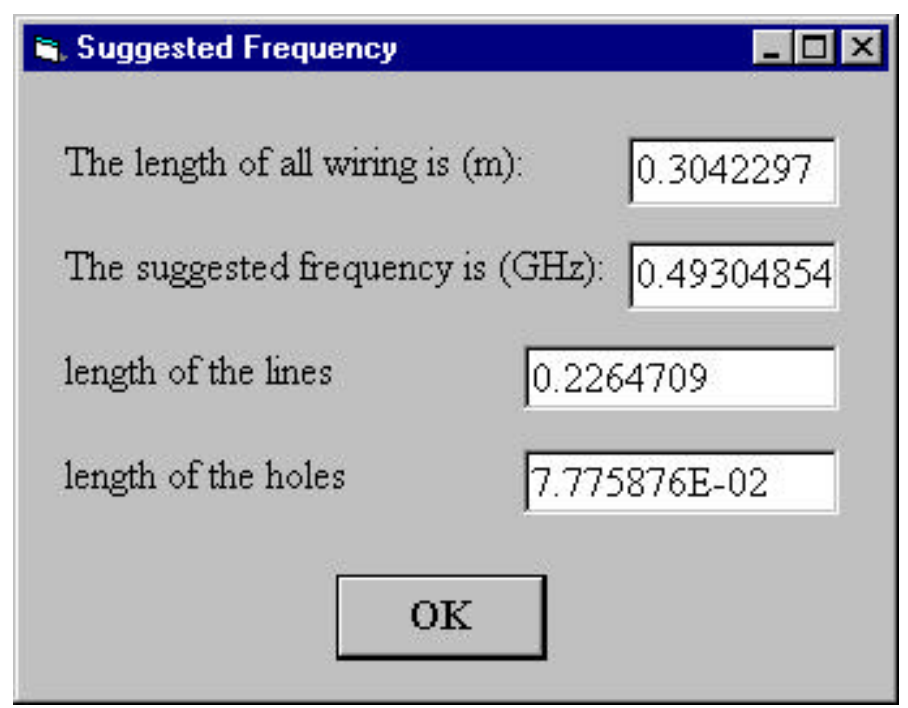

Figure B.7 Form Suggfreq 


\section{Appendix C: Sample XFDTD Input Files}

Following is a sample of the typical input files to XFDTD generated from the PCB-

CTHA Flexible Mesh Generator described in chapter 4. The design parameters for these files are those shown in the figures of the forms in the previous Appendix. Since the file name provided to the code was PCBCTHA, the developed files have the names PCBCTHA.id and PCBCTHA.fdtd.

\section{PCBCTHA.id}

This is the geometry file for the PCBCTHA model. Since this file includes all the geometry characteristics of the CTHA, it is usually a huge file. In this case it turned out to be $3 \mathrm{MB}$ in size, with over 2.4 million characters in about 2300 pages. In this section only the first few pages of this file are shown. The rest of the file is the same as the last page of the provided output, where the coordinates and the material types of the edges are given for all the model.

Geometry file version 3.6

12012060

3.787775E-04 3.787775E-04 1.69779E-04

32328

0

$1.000000 \mathrm{e}+00 \quad 0.000000 \mathrm{e}+00 \quad 1.000000 \mathrm{e}+00 \quad 0.000000 \mathrm{e}+00 \quad 1.000000 \mathrm{e}+00$

$1.000000 \mathrm{e}+00 \quad 0.000000 \mathrm{e}+00 \quad 0.000000 \mathrm{e}+00 \quad 0.000000 \mathrm{e}+00 \quad 0$

$0.000000 \mathrm{e}+00 \quad 0.000000 \mathrm{e}+00 \quad 0.000000 \mathrm{e}+00 \quad 0.000000 \mathrm{e}+00 \quad 0.000000 \mathrm{e}+00 \quad 0$

$1.000000 \mathrm{e}+00 \quad 0.000000 \mathrm{e}+00 \quad 1.000000 \mathrm{e}+00 \quad 0.000000 \mathrm{e}+00 \quad 1.000000 \mathrm{e}+00$

$1.000000 \mathrm{e}+00 \quad 0.000000 \mathrm{e}+00 \quad 0.000000 \mathrm{e}+00 \quad 0.000000 \mathrm{e}+00 \quad 0$

$\begin{array}{llllll}0.000000 e+00 & 0.000000 e+00 & 0.000000 e+00 & 0.000000 e+00 & 0.000000 e+00 & 0\end{array}$

$1.000000 \mathrm{e}+00 \quad 0.000000 \mathrm{e}+001.000000 \mathrm{e}+00 \quad 0.000000 \mathrm{e}+001.000000 \mathrm{e}+00$

$1.000000 \mathrm{e}+000.000000 \mathrm{e}+000.000000 \mathrm{e}+000.000000 \mathrm{e}+000$

$0.000000 \mathrm{e}+00 \quad 0.000000 \mathrm{e}+00 \quad 0.000000 \mathrm{e}+00 \quad 0.000000 \mathrm{e}+00 \quad 0.000000 \mathrm{e}+000$

$1.000000 \mathrm{e}+00 \quad 0.000000 \mathrm{e}+001.000000 \mathrm{e}+00 \quad 0.000000 \mathrm{e}+001.000000 \mathrm{e}+00$

$1.000000 \mathrm{e}+00 \quad 0.000000 \mathrm{e}+00 \quad 0.000000 \mathrm{e}+000.000000 \mathrm{e}+000$

$0.000000 \mathrm{e}+00 \quad 0.000000 \mathrm{e}+00 \quad 0.000000 \mathrm{e}+00 \quad 0.000000 \mathrm{e}+000.000000 \mathrm{e}+000$

$1.000000 \mathrm{e}+00 \quad 0.000000 \mathrm{e}+001.000000 \mathrm{e}+00 \quad 0.000000 \mathrm{e}+001.000000 \mathrm{e}+00$

$1.000000 \mathrm{e}+00 \quad 0.000000 \mathrm{e}+00 \quad 0.000000 \mathrm{e}+00 \quad 0.000000 \mathrm{e}+000$

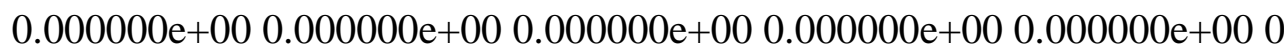

$1.000000 \mathrm{e}+00 \quad 0.000000 \mathrm{e}+00 \quad 1.000000 \mathrm{e}+00 \quad 0.000000 \mathrm{e}+001.000000 \mathrm{e}+00$

$1.000000 \mathrm{e}+000.000000 \mathrm{e}+000.000000 \mathrm{e}+000.000000 \mathrm{e}+000$ 
$0.000000 \mathrm{e}+00 \quad 0.000000 \mathrm{e}+00 \quad 0.000000 \mathrm{e}+00 \quad 0.000000 \mathrm{e}+00 \quad 0.000000 \mathrm{e}+000$ $1.000000 \mathrm{e}+00 \quad 0.000000 \mathrm{e}+001.000000 \mathrm{e}+00 \quad 0.000000 \mathrm{e}+001.000000 \mathrm{e}+00$ $1.000000 \mathrm{e}+000.000000 \mathrm{e}+00 \quad 0.000000 \mathrm{e}+000.000000 \mathrm{e}+000$ $0.000000 \mathrm{e}+00 \quad 0.000000 \mathrm{e}+00 \quad 0.000000 \mathrm{e}+00 \quad 0.000000 \mathrm{e}+00 \quad 0.000000 \mathrm{e}+000$ $1.000000 \mathrm{e}+00 \quad 0.000000 \mathrm{e}+00 \quad 1.000000 \mathrm{e}+00 \quad 0.000000 \mathrm{e}+00 \quad 1.000000 \mathrm{e}+00$ $1.000000 \mathrm{e}+00 \quad 0.000000 \mathrm{e}+00 \quad 0.000000 \mathrm{e}+00 \quad 0.000000 \mathrm{e}+000$ $0.000000 \mathrm{e}+00 \quad 0.000000 \mathrm{e}+00 \quad 0.000000 \mathrm{e}+00 \quad 0.000000 \mathrm{e}+00 \quad 0.000000 \mathrm{e}+000$ $1.000000 \mathrm{e}+00 \quad 0.000000 \mathrm{e}+00 \quad 1.000000 \mathrm{e}+00 \quad 0.000000 \mathrm{e}+00 \quad 1.000000 \mathrm{e}+00$ $1.000000 \mathrm{e}+00 \quad 0.000000 \mathrm{e}+00 \quad 0.000000 \mathrm{e}+00 \quad 0.000000 \mathrm{e}+000$ $\begin{array}{llllll}0.000000 \mathrm{e}+00 & 0.000000 \mathrm{e}+00 & 0.000000 \mathrm{e}+00 & 0.000000 \mathrm{e}+00 & 0.000000 \mathrm{e}+000\end{array}$ $1.000000 \mathrm{e}+00 \quad 0.000000 \mathrm{e}+001.000000 \mathrm{e}+00 \quad 0.000000 \mathrm{e}+001.000000 \mathrm{e}+00$ $1.000000 \mathrm{e}+000.000000 \mathrm{e}+000.000000 \mathrm{e}+000.000000 \mathrm{e}+000$ $0.000000 \mathrm{e}+00 \quad 0.000000 \mathrm{e}+00 \quad 0.000000 \mathrm{e}+00 \quad 0.000000 \mathrm{e}+00 \quad 0.000000 \mathrm{e}+000$ $1.000000 \mathrm{e}+00 \quad 0.000000 \mathrm{e}+00 \quad 1.000000 \mathrm{e}+00 \quad 0.000000 \mathrm{e}+00 \quad 1.000000 \mathrm{e}+00$ $1.000000 \mathrm{e}+000.000000 \mathrm{e}+000.000000 \mathrm{e}+000.000000 \mathrm{e}+000$ $0.000000 \mathrm{e}+00 \quad 0.000000 \mathrm{e}+000.000000 \mathrm{e}+00 \quad 0.000000 \mathrm{e}+00 \quad 0.000000 \mathrm{e}+000$ $1.000000 \mathrm{e}+00 \quad 0.000000 \mathrm{e}+001.000000 \mathrm{e}+00 \quad 0.000000 \mathrm{e}+001.000000 \mathrm{e}+00$ $1.000000 \mathrm{e}+00 \quad 0.000000 \mathrm{e}+00 \quad 0.000000 \mathrm{e}+00 \quad 0.000000 \mathrm{e}+000$ $0.000000 \mathrm{e}+00 \quad 0.000000 \mathrm{e}+00 \quad 0.000000 \mathrm{e}+00 \quad 0.000000 \mathrm{e}+00 \quad 0.000000 \mathrm{e}+000$ $1.000000 \mathrm{e}+00 \quad 0.000000 \mathrm{e}+001.000000 \mathrm{e}+000.000000 \mathrm{e}+001.000000 \mathrm{e}+00$ $1.000000 \mathrm{e}+000.000000 \mathrm{e}+000.000000 \mathrm{e}+000.000000 \mathrm{e}+000$ $0.000000 \mathrm{e}+00 \quad 0.000000 \mathrm{e}+00 \quad 0.000000 \mathrm{e}+00 \quad 0.000000 \mathrm{e}+00 \quad 0.000000 \mathrm{e}+000$ $1.000000 \mathrm{e}+00 \quad 0.000000 \mathrm{e}+00 \quad 1.000000 \mathrm{e}+00 \quad 0.000000 \mathrm{e}+001.000000 \mathrm{e}+00$ $1.000000 \mathrm{e}+00 \quad 0.000000 \mathrm{e}+00 \quad 0.000000 \mathrm{e}+00 \quad 0.000000 \mathrm{e}+000$ $0.000000 \mathrm{e}+00 \quad 0.000000 \mathrm{e}+000.000000 \mathrm{e}+00 \quad 0.000000 \mathrm{e}+00 \quad 0.000000 \mathrm{e}+000$ 0

$1.000000 \mathrm{e}+000.000000 \mathrm{e}+000.000000 \mathrm{e}+00$

$0.000000 \mathrm{e}+001.000000 \mathrm{e}+000.000000 \mathrm{e}+00$

$0.000000 \mathrm{e}+000.000000 \mathrm{e}+001.000000 \mathrm{e}+00$

Array format: material

10

130580

$\begin{array}{llllll}21 & 21 & 21 & 2 & 2 & 2\end{array}$

$\begin{array}{llllll}21 & 21 & 22 & 2 & 2 & 2\end{array}$

$\begin{array}{llllll}21 & 21 & 23 & 2 & 2 & 2\end{array}$

$\begin{array}{llllll}21 & 21 & 24 & 2 & 2 & 2\end{array}$

$\begin{array}{llllll}21 & 21 & 25 & 2 & 2 & 2\end{array}$

$\begin{array}{llllll}21 & 21 & 26 & 2 & 2 & 2\end{array}$

$\begin{array}{llllll}21 & 21 & 27 & 2 & 2 & 2\end{array}$

$\begin{array}{llllll}21 & 21 & 28 & 2 & 2 & 2\end{array}$

$\begin{array}{llllll}21 & 21 & 29 & 2 & 2 & 2\end{array}$

$\begin{array}{llllll}21 & 21 & 30 & 2 & 2 & 2\end{array}$

$\begin{array}{llllll}21 & 21 & 31 & 2 & 2 & 2\end{array}$

$\begin{array}{llllll}21 & 21 & 32 & 2 & 2 & 2\end{array}$

$\begin{array}{llllll}21 & 21 & 33 & 2 & 2 & 2\end{array}$

$\begin{array}{llllll}21 & 21 & 34 & 2 & 2\end{array}$ 
$\begin{array}{lllll}21 & 21 & 35 & 2 & 2\end{array}$

$\begin{array}{lllll}21 & 21 & 36 & 2 & 2\end{array}$

$\begin{array}{lllll}21 & 21 & 37 & 2 & 2\end{array}$

$\begin{array}{lllll}21 & 21 & 38 & 2 & 2\end{array}$

$\begin{array}{lllll}21 & 21 & 39 & 2 & 2\end{array}$

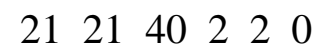

$\begin{array}{llllll}21 & 22 & 21 & 2 & 2 & 2\end{array}$

$\begin{array}{llllll}21 & 22 & 22 & 2 & 2 & 2\end{array}$

$\begin{array}{llllll}21 & 22 & 23 & 2 & 2 & 2\end{array}$

$\begin{array}{llllll}21 & 22 & 24 & 2 & 2 & 2\end{array}$

$\begin{array}{llllll}21 & 22 & 25 & 2 & 2 & 2\end{array}$

$\begin{array}{llllll}21 & 22 & 26 & 2 & 2 & 2\end{array}$

$\begin{array}{llllll}21 & 22 & 27 & 2 & 2 & 2\end{array}$

$\begin{array}{llllll}21 & 22 & 28 & 2 & 2 & 2\end{array}$

$\begin{array}{llllll}21 & 22 & 29 & 2 & 2 & 2\end{array}$

$\begin{array}{llllll}21 & 22 & 30 & 2 & 2 & 2\end{array}$

$\begin{array}{llllll}21 & 22 & 31 & 2 & 2 & 2\end{array}$

$\begin{array}{llllll}21 & 22 & 32 & 2 & 2 & 2\end{array}$

$\begin{array}{llllll}21 & 22 & 33 & 2 & 2 & 2\end{array}$

$\begin{array}{lllll}21 & 22 & 34 & 2 & 2\end{array}$

$\begin{array}{llllll}21 & 22 & 35 & 2 & 2 & 2\end{array}$

$\begin{array}{llllll}21 & 22 & 36 & 2 & 2\end{array}$

$\begin{array}{llllll}21 & 22 & 37 & 2 & 2 & 2\end{array}$

$\begin{array}{lllll}21 & 22 & 38 & 2 & 2\end{array}$

$\begin{array}{llllll}21 & 22 & 39 & 2 & 2 & 2\end{array}$

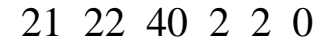

$\begin{array}{llllll}21 & 23 & 21 & 2 & 2 & 2\end{array}$

$\begin{array}{llllll}21 & 23 & 22 & 2 & 2 & 2\end{array}$

$\begin{array}{llllll}21 & 23 & 23 & 2 & 2 & 2\end{array}$

$\begin{array}{llllll}21 & 23 & 24 & 2 & 2 & 2\end{array}$

$\begin{array}{llllll}21 & 23 & 25 & 2 & 2 & 2\end{array}$

$\begin{array}{llllll}21 & 23 & 26 & 2 & 2 & 2\end{array}$

$\begin{array}{llllll}21 & 23 & 27 & 2 & 2 & 2\end{array}$

$\begin{array}{llllll}21 & 23 & 28 & 2 & 2 & 2\end{array}$

$\begin{array}{llllll}21 & 23 & 29 & 2 & 2 & 2\end{array}$

$\begin{array}{llllll}21 & 23 & 30 & 2 & 2 & 2\end{array}$

$\begin{array}{llllll}21 & 23 & 31 & 2 & 2 & 2\end{array}$

$\begin{array}{lllll}21 & 23 & 32 & 2 & 2\end{array}$

$\begin{array}{llllll}21 & 23 & 33 & 2 & 2 & 2\end{array}$

$\begin{array}{llllll}21 & 23 & 34 & 2 & 2 & 2\end{array}$

$\begin{array}{llll}21 & 2335222\end{array}$

\section{PCBCTHA.fdtd}

This file includes all the calculation parameters for the desired run. This file is a much shorter file since it deals with certain flags and options that are almost independent of the 
antenna model. In the example, the ".fdtd" file turned out to be two pages of length. Only the first of these two pages is provided, since the second page is identical to the last group of lines shown below.

FDTD file version 3.11

000000008

0

PCBCTHA.id

PCBCTHA

PCBCTHA.id

0

$1.280000 \mathrm{e}+02$

$1.000000 \mathrm{e}+03$

5000

$1.000000 \mathrm{e}+00$

2

1

$8074 \quad 40 \quad 01.000000 \mathrm{e}+00 \quad 0.000000 \mathrm{e}+005.000000 \mathrm{e}+010$

$-1$

$-1$

$-1$

$-1$

$-1$

$-1$

$-1$

1

262144

$-1$

11

11

11

11

11

11

11

11

11 


\section{Appendix D: Analyzer.exe Code}

Following is a listing of the computer code for the analyzer.exe program. The analyzer.exe program is used to read the pairs of real and imaginary data within the .f1 and .f2 files provided by the network analyzer and convert them to resistance and reactance. The program creates a .xls file of the data that is ready to be graphed in MS Excel by the user.

The program also handles several files that are sub-ranges for one run, as described in chapter 7. It checks for the different .f1 or .f2 files in each folder, then it assumes that all the f1 and .f2 files in each folder are all sub-ranges for one run or for one antenna. So the program processes all the data and merges all the results together and sorts them to produce one file and one smooth chart. But the user has to be careful to include in each folder only the related ff1 or .$f 2$ files for each run, since if runs for different antennas are located together in the same folder, the program will not understand this and will group them thus giving messed up results.

\section{Module mainmod}

Public freq() As Double

Public reals11() As Double

Public imags11() As Double

Public num() As Double

Public denum() As Double

Public real() As Double

Public imag() As Double

Public F() As Double

Public resonance() As Double

Public realres() As Double

Public imagres() As Double

Public SWRres() As Double

Sub main()

filename.File1.ListIndex $=0 \quad$ 'use first file name in the list to generate the output file name Name $=$ filename.File 1

$\mathrm{i}=\mathrm{Len}(\mathrm{Name})$

pos $=\operatorname{InStr}($ Name,,.$")$

Name $=$ Left $($ Name, pos - 1) + "-Z."

'name of file until before the ext plus $-\mathrm{Z}$ 
outname $=$ filename.filename_txt.Text + Name + "xls" $\quad$ 'name of Z file with all the path and extension

Open outname For Output As \#2

Print \#2, "Freq(GHz)", Tab, "Real", Tab, "Imag", Tab, "SWR"

For $\mathrm{i}=0$ To (filename.File1.ListCount - 1) 'will open all the files sequentially

filename.File1.ListIndex $=\mathrm{i}$

fileturn $=$ filename.filename_txt.Text + filename.File 1

Open fileturn For Input As \#1

For $\mathrm{j}=1$ To 7

Line Input \#1, A 'skip the first seven lines

Next

$\begin{array}{ll}\text { pos = InStr(A, " ") } & \text { 'find first space } \\ \text { length = Len(A) } & \text { 'find length of the string } \\ \text { A = Right(A, length - pos) } & \text { 'remove first part of the string } \\ \text { pos = InStr(A, " ") } & \text { 'find second space } \\ \text { length = Len(A) } & \text { 'find length of the string } \\ \text { freqmin = Left(A, pos) } & \text { 'define the min freq } \\ \text { A = Right(A, length - pos) } & \text { 'take the reamaining of the string } \\ \text { pos = InStr(A, " ") } & \text { 'find third space } \\ \text { length = Len(A) } & \text { 'find length of the string } \\ \text { freqmax = Left(A, pos) } & \text { 'define the max freq } \\ \text { points = Right(A, length - pos) } & \text { 'define the number of points }\end{array}$

freqmin $=\mathrm{CDec}($ freqmin $) \quad$ 'convert to decimal

freqmax $=\mathrm{CDec}($ freqmax $)$

points $=\mathrm{CDec}$ (points)

ReDim freq(points)

ReDim reals11(points)

ReDim imags11(points)

ReDim num(points)

ReDim denum(points)

ReDim real(points)

ReDim imag(points)

freq $(1)=$ freqmin $/ 1000000000 \# \quad$ 'convert $\mathrm{Hz}$ to $\mathrm{GHz}$

$\operatorname{div}=($ freqmax - freqmin $) /($ points -1$) \quad$ 'find the division between each two freq's

For $\mathrm{j}=2$ To (points)

freqmin $=$ freqmin + div

freq $(j)=$ freqmin $/ 1000000000 \# \quad$ 'define frequencies in freq ()

Next

Line Input \#1, A

'skip 2 lines

Line Input \#1, A 
For $\mathrm{j}=1$ To points 'read each line, which includes a real and imag number corresponding to the freq at that point

Line Input \#1, A

$\operatorname{pos}=\operatorname{InStr}(\mathrm{A}$, ",")

length $=\operatorname{Len}(\mathrm{A})$

reals $11(\mathrm{j})=\mathrm{CDec}(\operatorname{Left}(\mathrm{A}$, pos -1$)) \quad$ 'real and imag for $\mathrm{s} 11$

$\operatorname{imags} 11(\mathrm{j})=\operatorname{CDec}(\operatorname{Right}(\mathrm{A}$, length $-\operatorname{pos}))$

$\operatorname{num}(\mathrm{j})=1+\operatorname{reals} 11(\mathrm{j}) \quad$ 'find $1+\mathrm{s} 11$ where $1+\mathrm{s} 11=(1+$ real $)+$ imag

$\operatorname{denum}(\mathrm{j})=1-\operatorname{reals} 11(\mathrm{j}) \quad$ 'find $1-\mathrm{s} 11 \quad$ where $1-\mathrm{s} 11=(1-$ real $)+(-1 *$ imag $)$

$\begin{array}{ll}A=\operatorname{num}(j) & \text { 'real for } 1+\mathrm{s} 11 \\ B=\operatorname{imags} 11(j) & \text { 'imag for } 1+\mathrm{s} 11 \\ \mathrm{C}=\operatorname{denum}(\mathrm{j}) & \text { 'real for } 1-\mathrm{s} 11 \\ \mathrm{D}=-1 * \text { imags11(j) } & \text { 'imag for } 1-\mathrm{s} 11\end{array}$

$\operatorname{real}(\mathrm{j})=50 *(\mathrm{~A} * \mathrm{C}+\mathrm{B} * \mathrm{D}) /\left(\mathrm{C}^{\wedge} 2+\mathrm{D}^{\wedge} 2\right) \quad 50 * \mathrm{Z}=50 *$ real $+50 *$ imag where

$50+0 \mathrm{i}$ is the line feed

$\operatorname{imag}(\mathrm{j})=50 *(\mathrm{~B} * \mathrm{C}-\mathrm{A} * \mathrm{D}) /\left(\mathrm{C}^{\wedge} 2+\mathrm{D}^{\wedge} 2\right)$

$\operatorname{refcoeff}=\operatorname{Sqr}\left(\left((\operatorname{real}(\mathrm{j})-50)^{\wedge} 2+\operatorname{imag}(\mathrm{j}) \wedge 2\right) /\left((\operatorname{real}(\mathrm{j})+50)^{\wedge} 2+\operatorname{imag}(\mathrm{j}) \wedge 2\right)\right)$

$\mathrm{SWR}=\operatorname{Abs}((1+$ refcoeff $) /(1-$ refcoeff $))$

Print \#2, freq(j), Tab, real(j), Tab, imag(j), Tab, SWR ' print to file Next

Close \#1

Next

Close \#2

Open outname For Input As \#1

Line Input \#1, xx

newfile = filename.filename_txt.Text + "resonance." + "xls"

Open newfile For Output As \#2

Print \#2, Tab, "res\#", Tab, "freq(GHz)", Tab, "real", Tab, "imag", Tab, "SWR"

lines $=(\mathrm{i}+1) *$ points

ReDim F(lines, 4)

ReDim resonance(points)

ReDim realres(points)

ReDim imagres(points)

ReDim SWRres(points)

$\mathrm{i}=1$

For $\mathrm{j}=1$ To $4 \quad$ 'read first line of data

Input \#1, F( $\mathrm{i}, \mathrm{j}) \quad$ ' $\mathrm{F}$ is an array of 3 columns: freq, real, imag

Next 


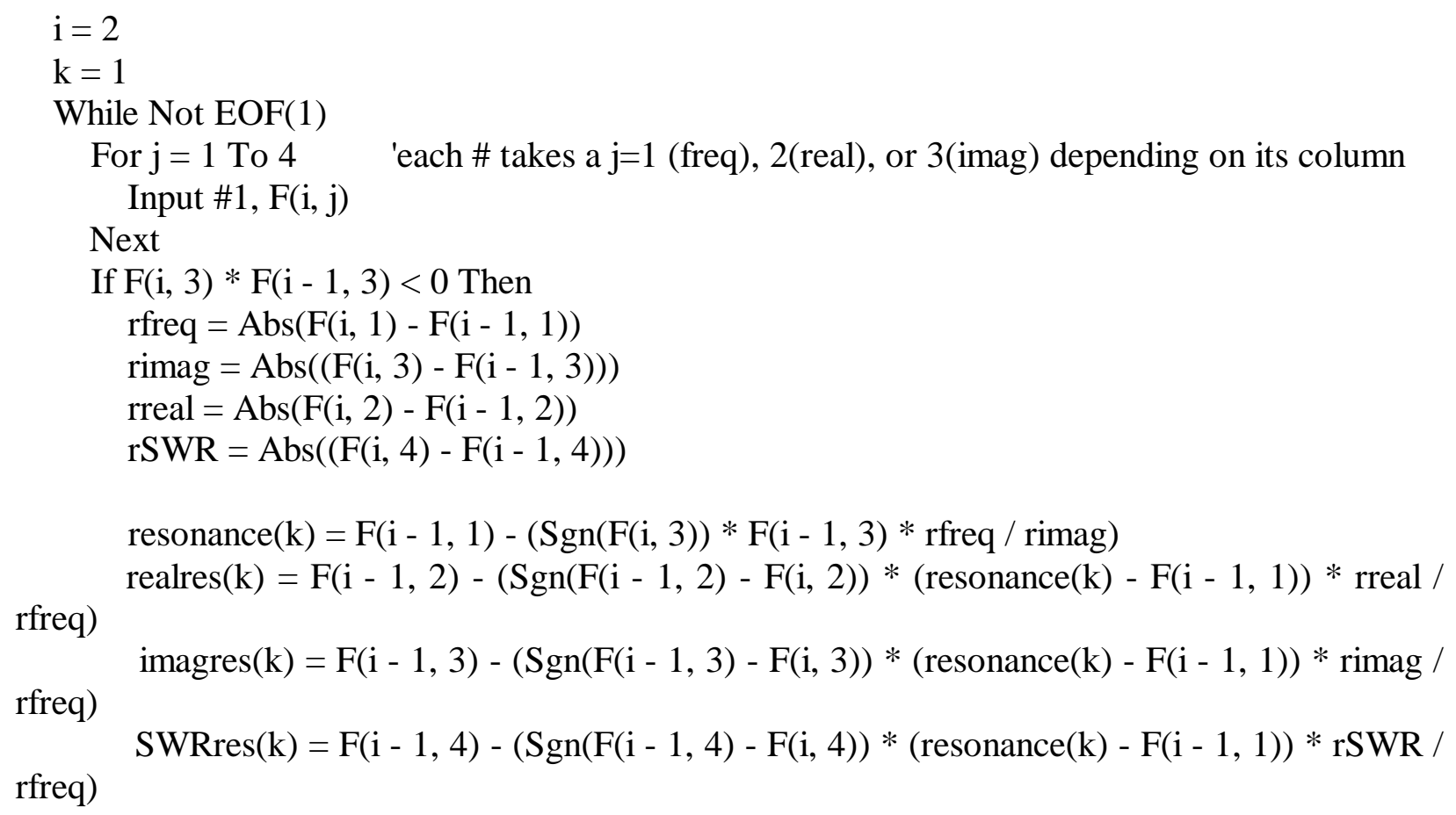

End Sub

\section{Form filename}

Private Sub Command1_Click()

$$
\text { End }
$$

End Sub

Private Sub Dir1_Change()

UpdateFile

End Sub

Private Sub Dir1_Click()

UpdateFile

End Sub

Private Sub file_txt_Click() 
File1.Pattern $=" * \cdot d 1 "$

file_txt.Text $=$ File1.filename

File1.Refresh

End Sub

Private Sub file_txt_Change()

File1.Pattern $={ }^{*} \cdot \mathrm{d} 1 "$

End Sub

Private Sub File1_Click()

UpdateFile

End Sub

Private Sub UpdateFile()

File1.Path = Dir1.Path

If Dir1.Path = "C:।" Or Dir1.Path = "A:।" Then

filename_txt.Text $=$ Dir1.Path

Else

filename_txt.Text $=$ Dir1.Path $+" \mid "$

End If

End Sub

Private Sub Form_Load()

File1.Pattern $=$ "*.d1"

File1.Path = Dir1.Path

filename_txt.Text = Dir1.Path + "I"

UpdateFile

End Sub

Private Sub ok_cmd_Click()

main

End Sub 


\section{Appendix E Photos for the Experimental Analysis Equipment}

This Appendix contains a collection of photos taken at the anechoic chamber site. These photos show the anechoic chamber and the equipment used for the experimental analysis that have been described in chapter 7 .

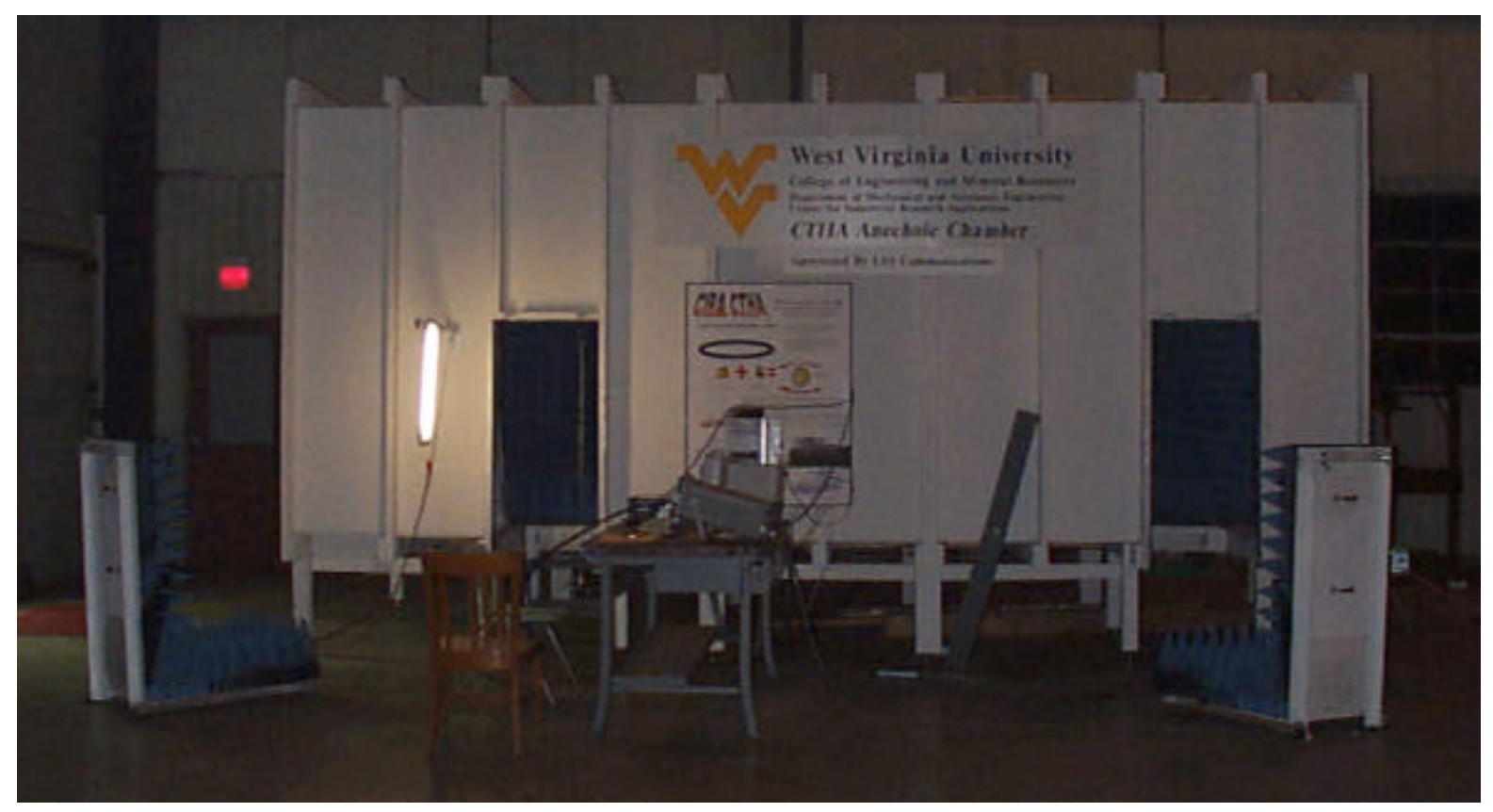

E.1 A full view of the anechoic chamber, with the two doors open and the computer and spectrum analyzer visible on the workbench in front of the chamber. The right side is where the transmitting antenna is located, while the left side is where the receiving antenna is located. The pole that carries the receiving antenna is visible through the left door. Also viewable is the fluorescent light used to illuminate the interior of the chamber. 


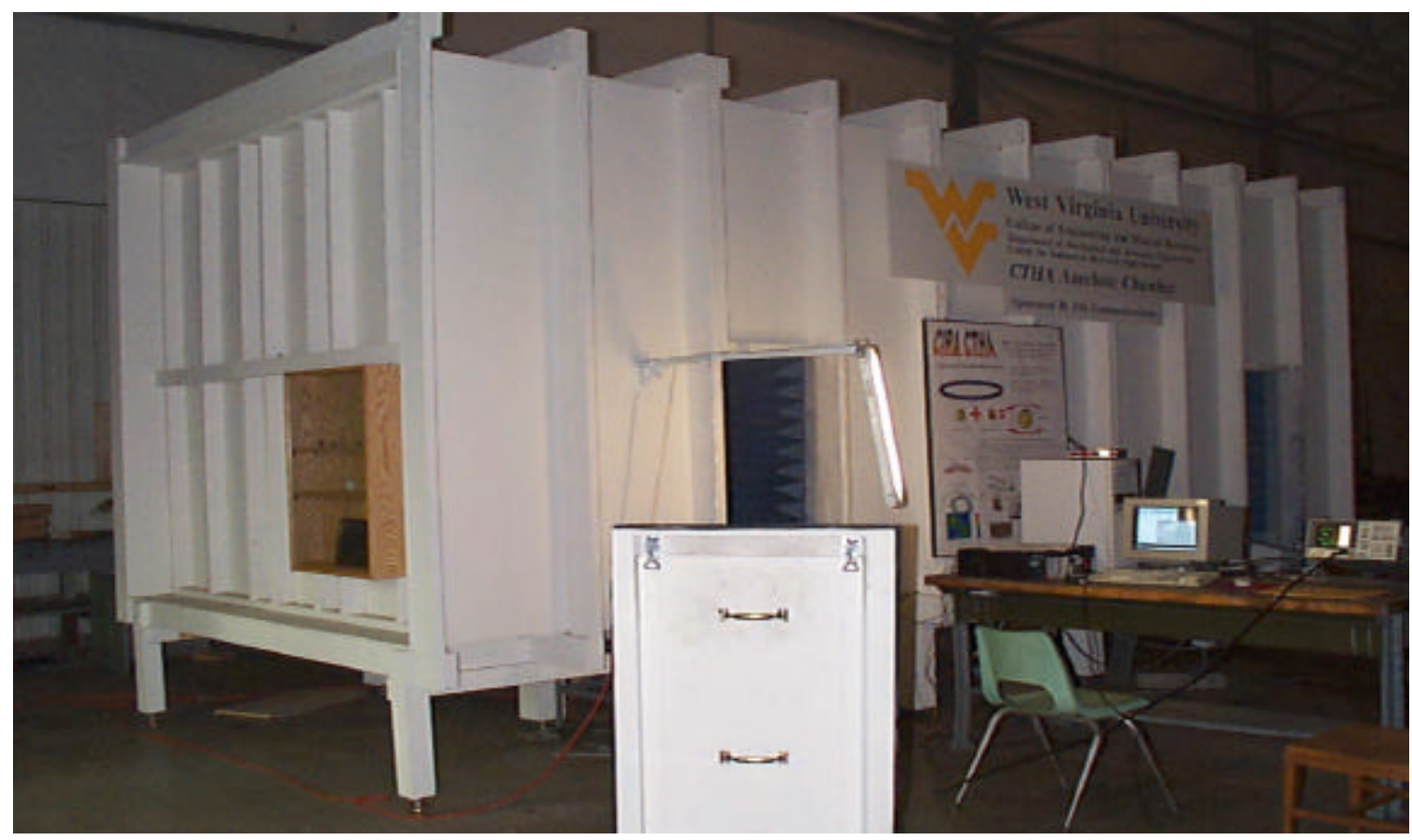

E.2 A side view of the chamber

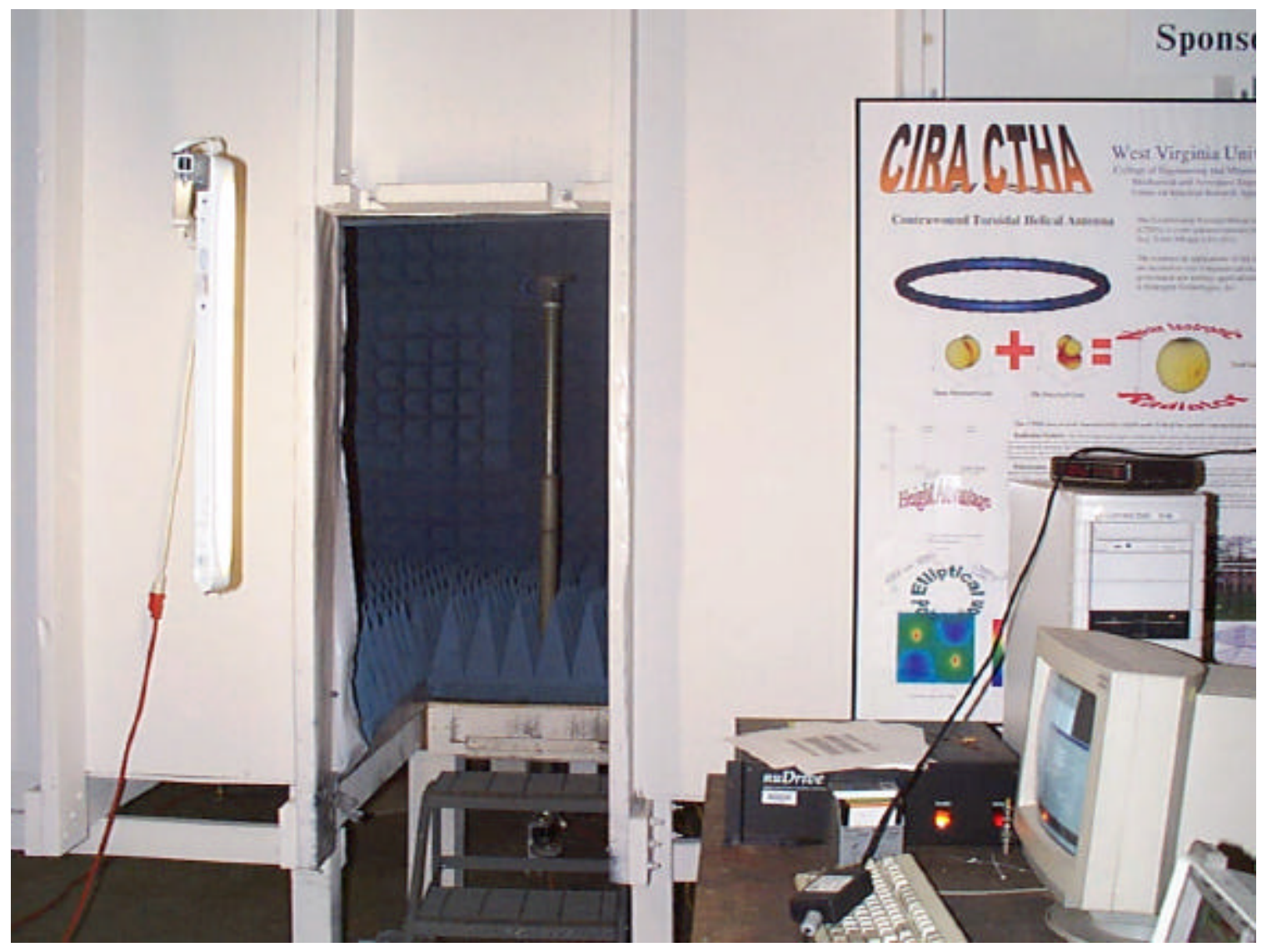

E.3 A closer look at the left door of the chamber 


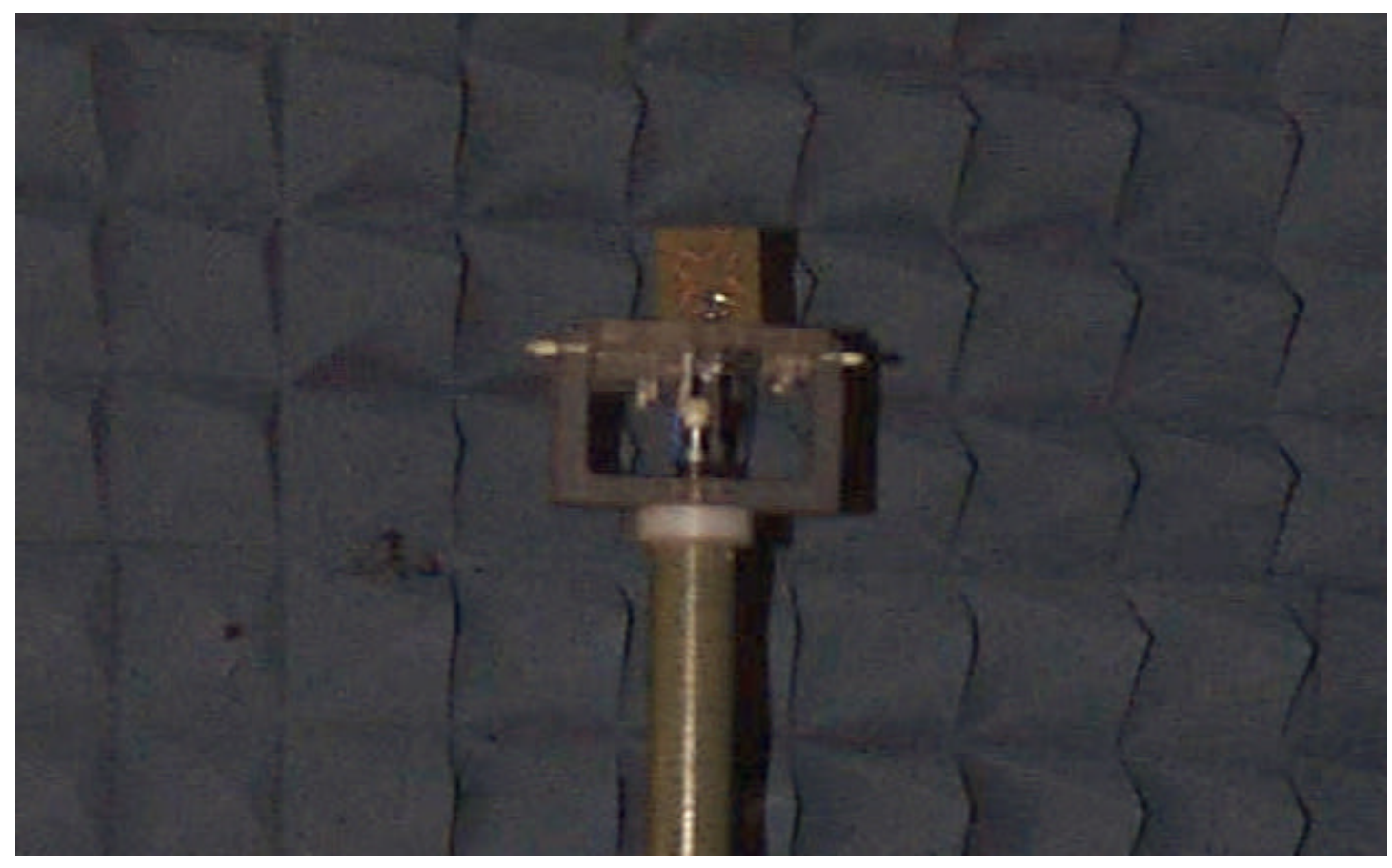

E.4 The receiving CTHA mounted vertically upon the pole. The rotation of the antenna is around the pole axis, thus this orientation would give the phi $=0$ plane of far fields for this antenna.

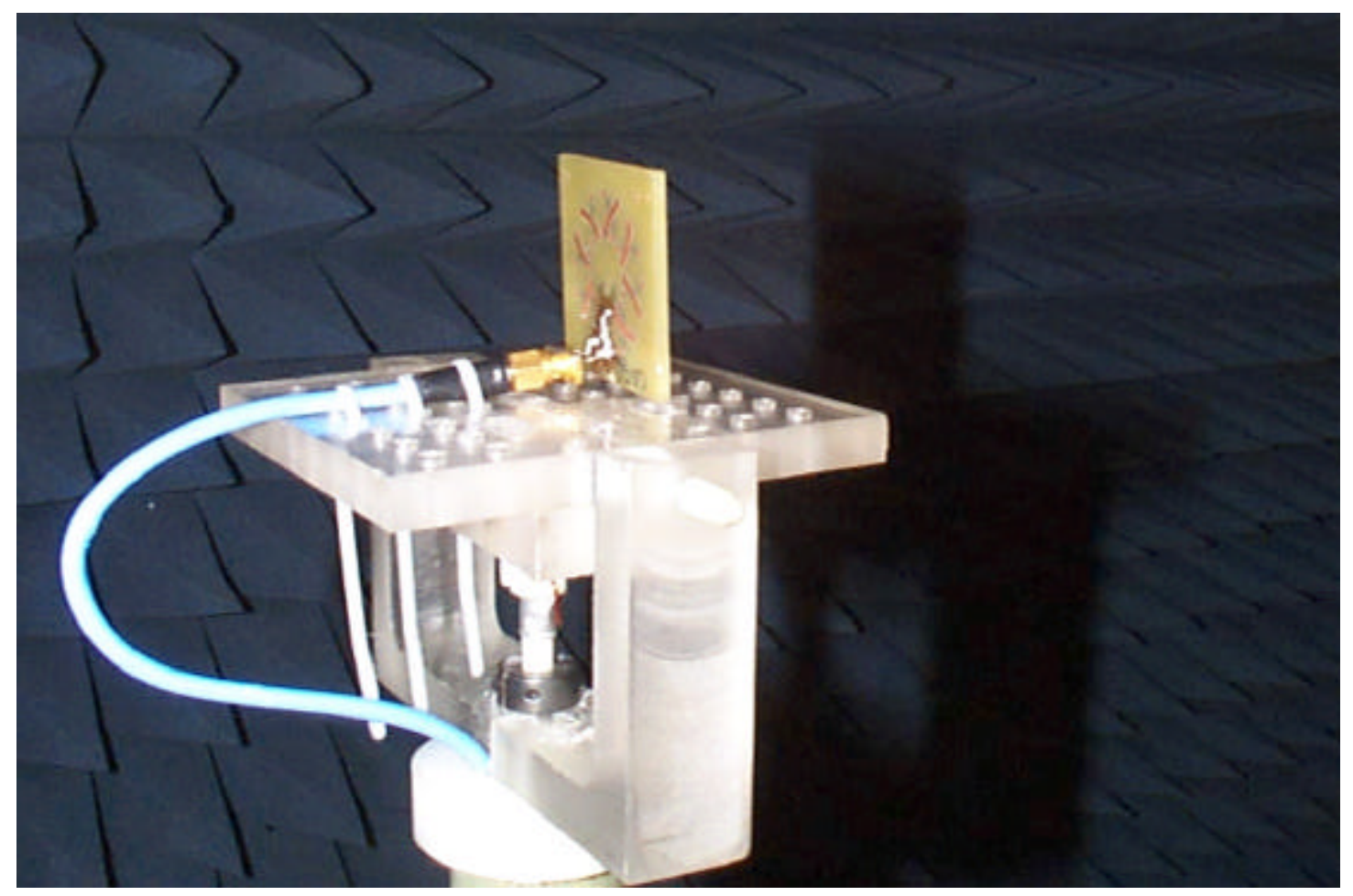

E.5 A closer view of the receiving CTHA mounted vertically. 


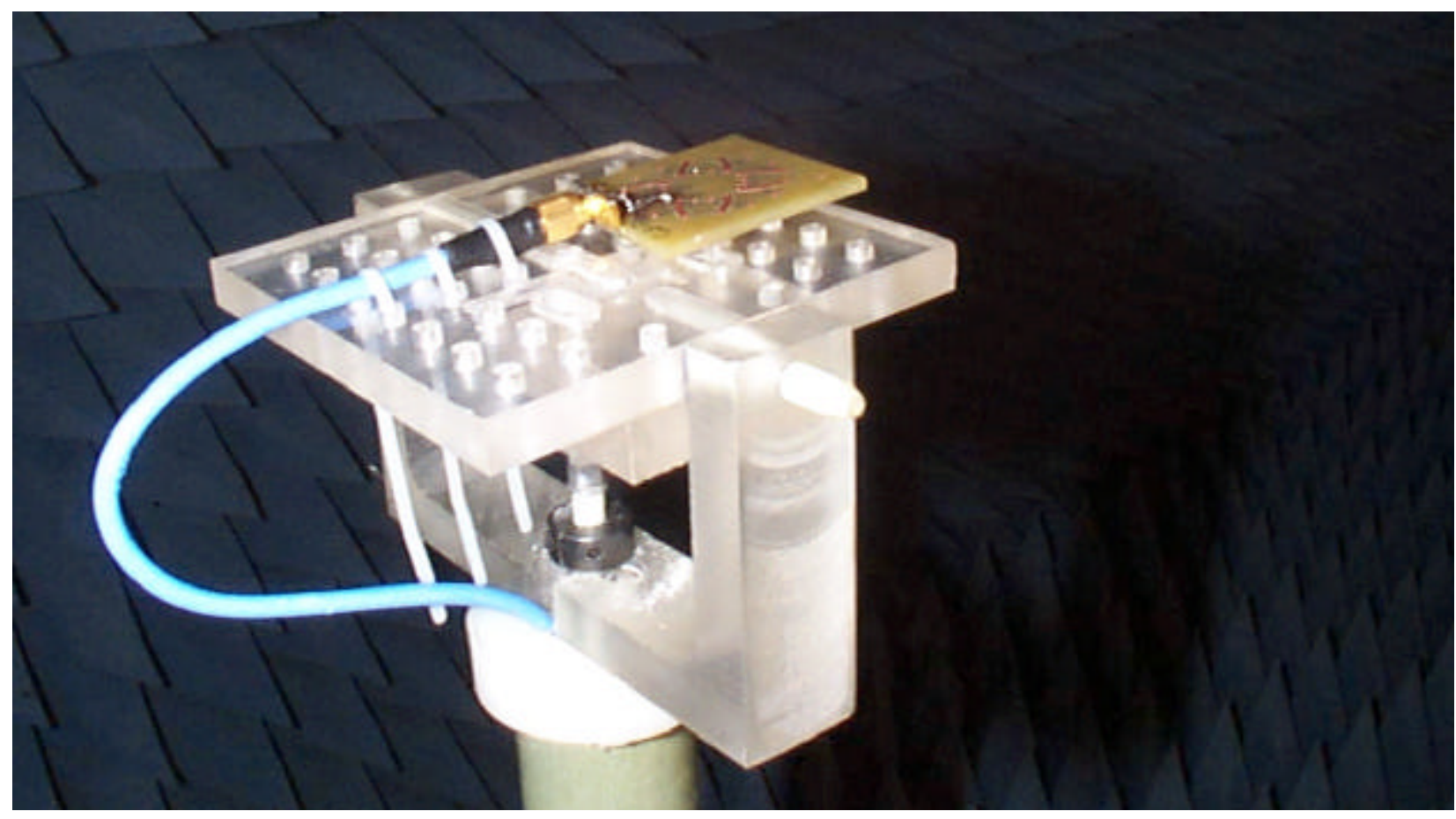

E.6 The receiving CTHA mounted horizontally. This orientation will give the theta $=90$ plane of far fields.

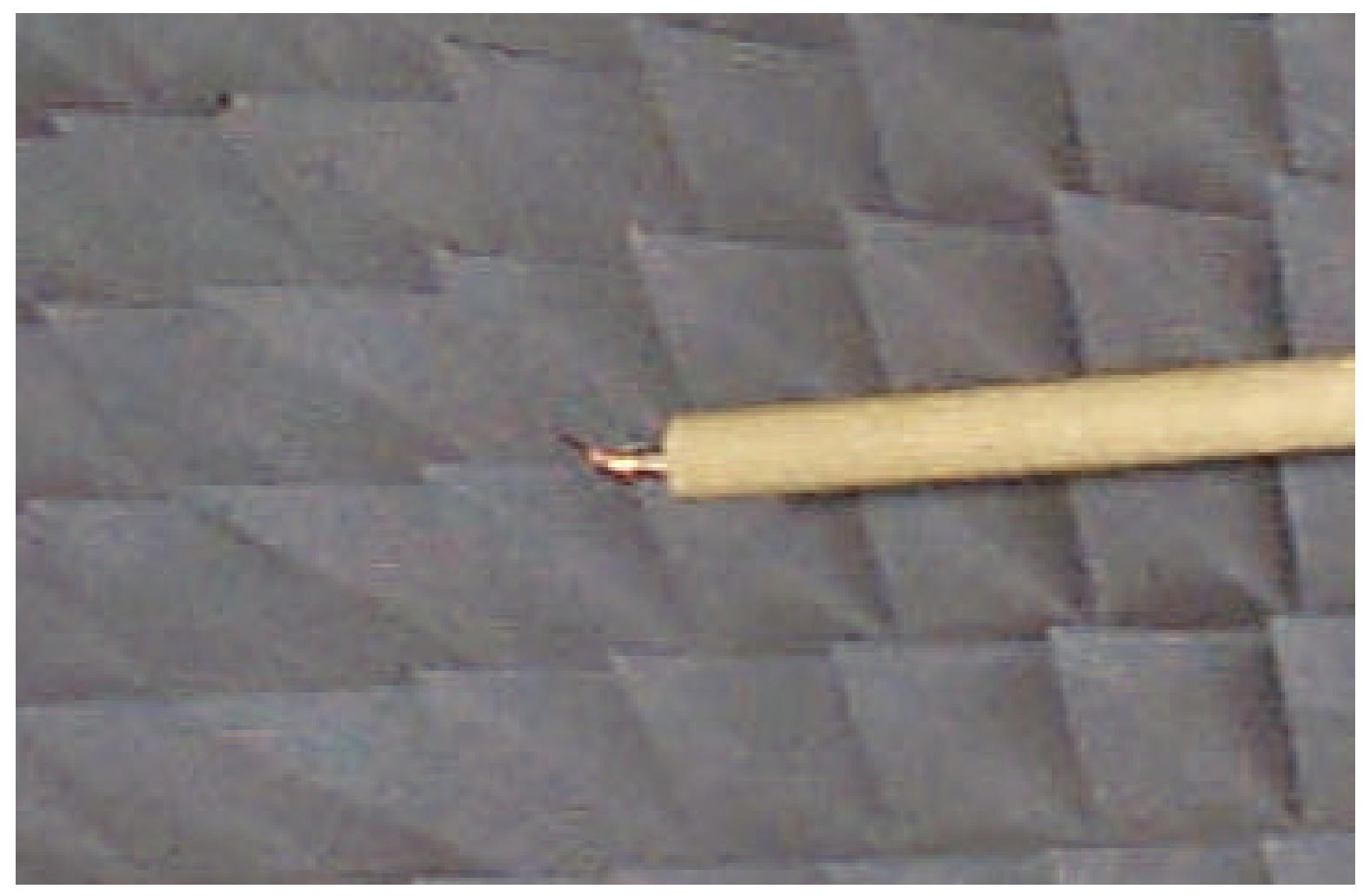

E.7 The transmitting dipole mounted horizontally 


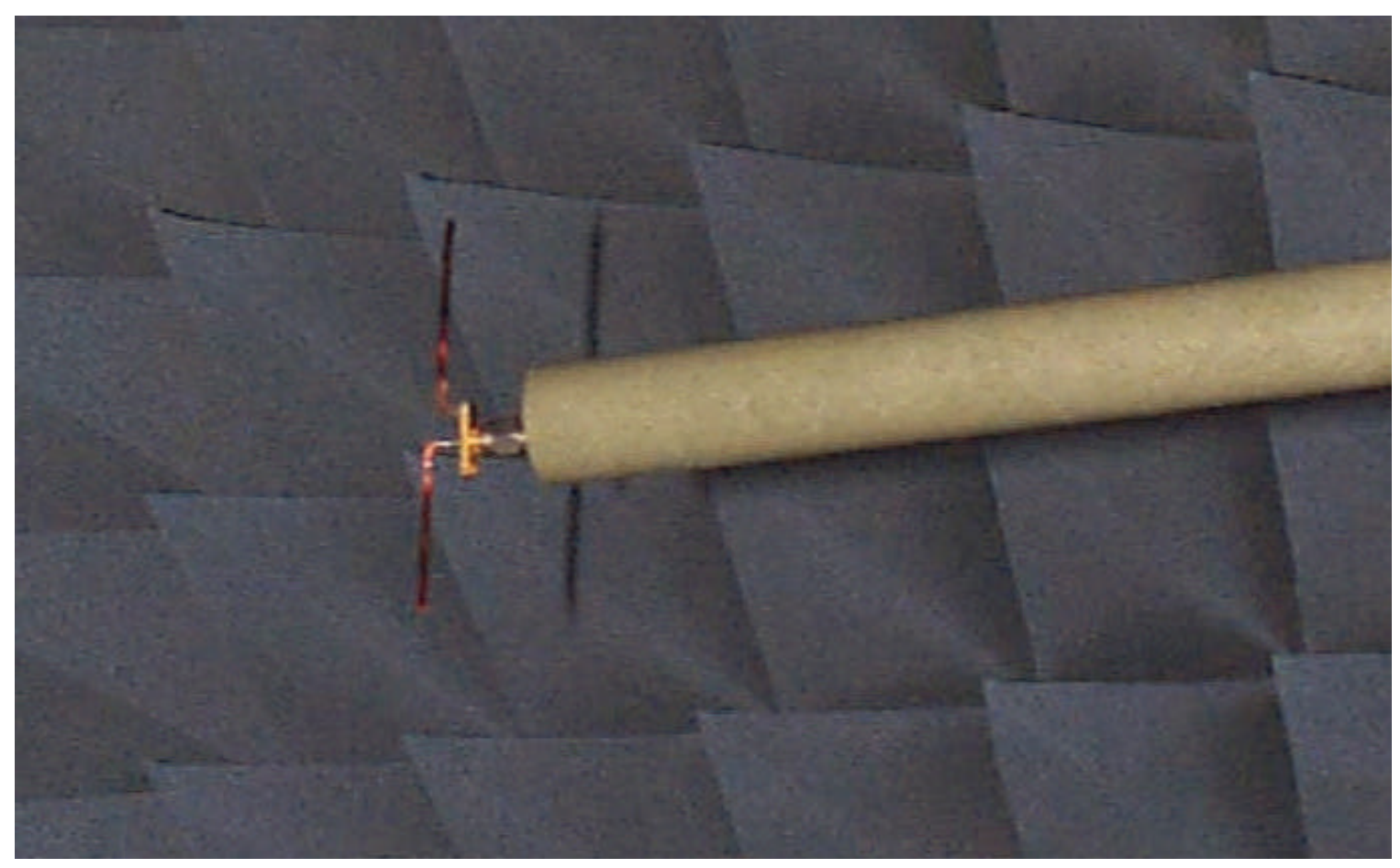

E.8 The transmitting dipole mounted vertically

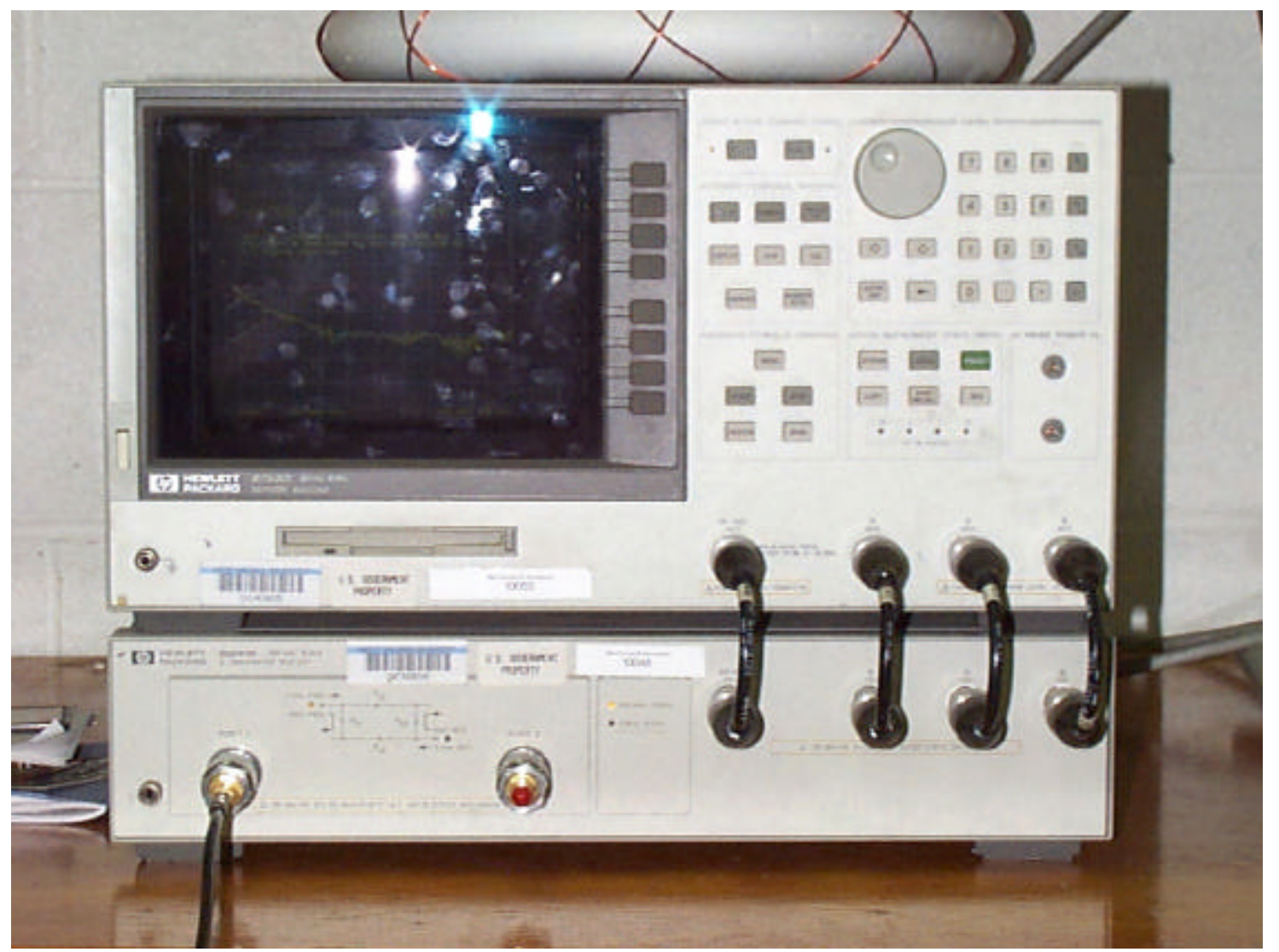

E.9 The network analyzer 


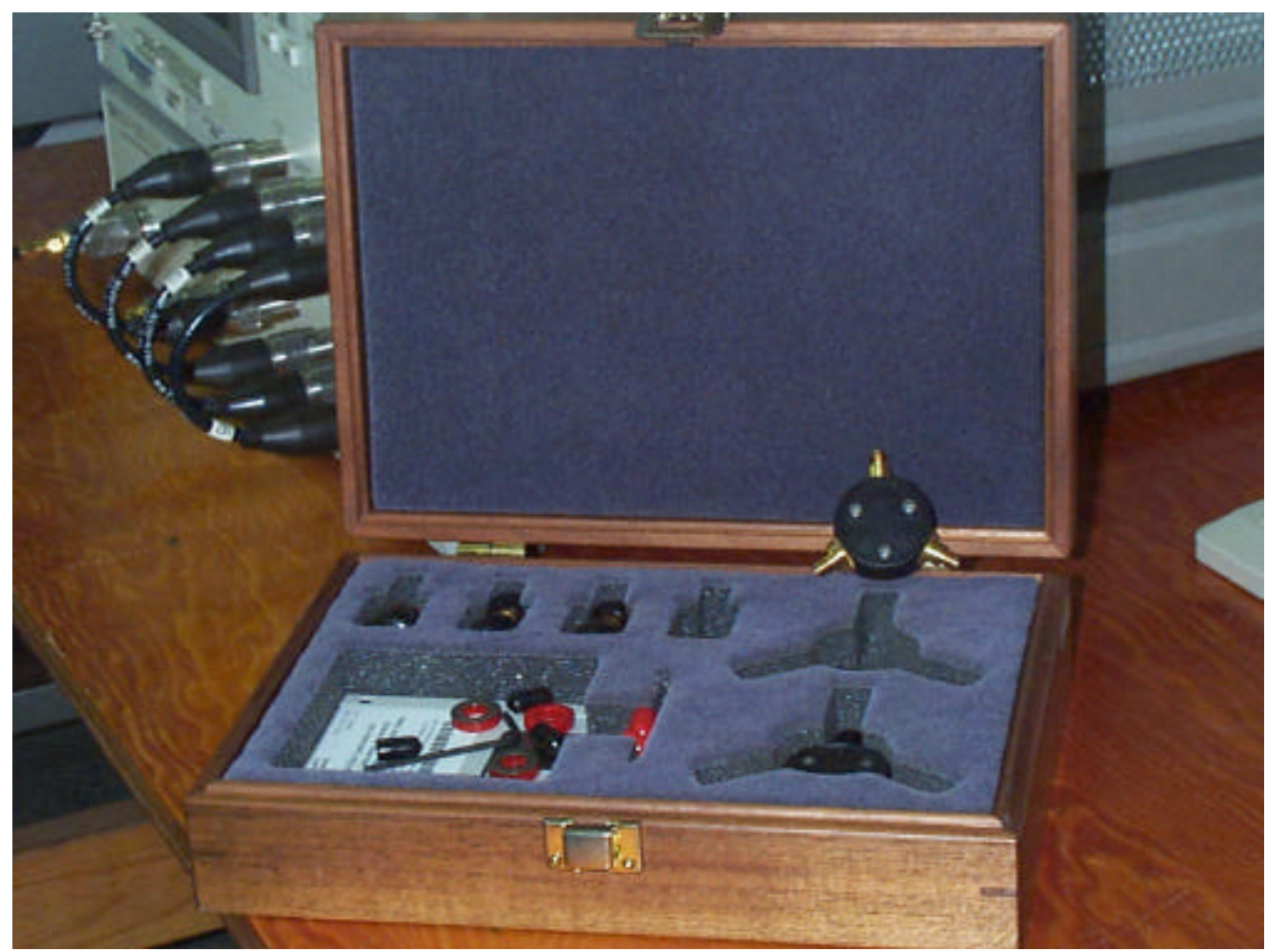

E.10 The calibration kit used to calibrate the network analyzer.

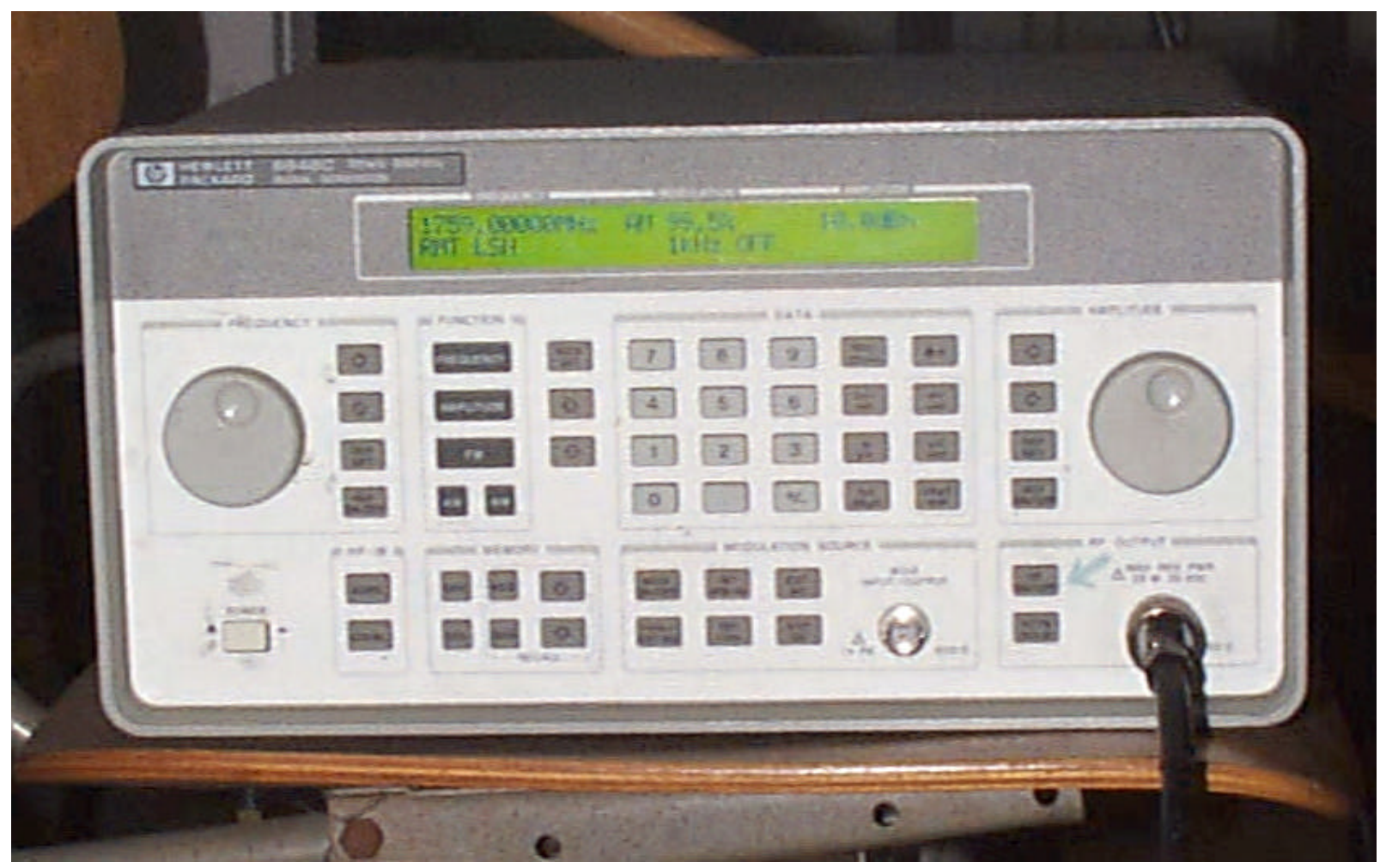

E.11 The signal generator 


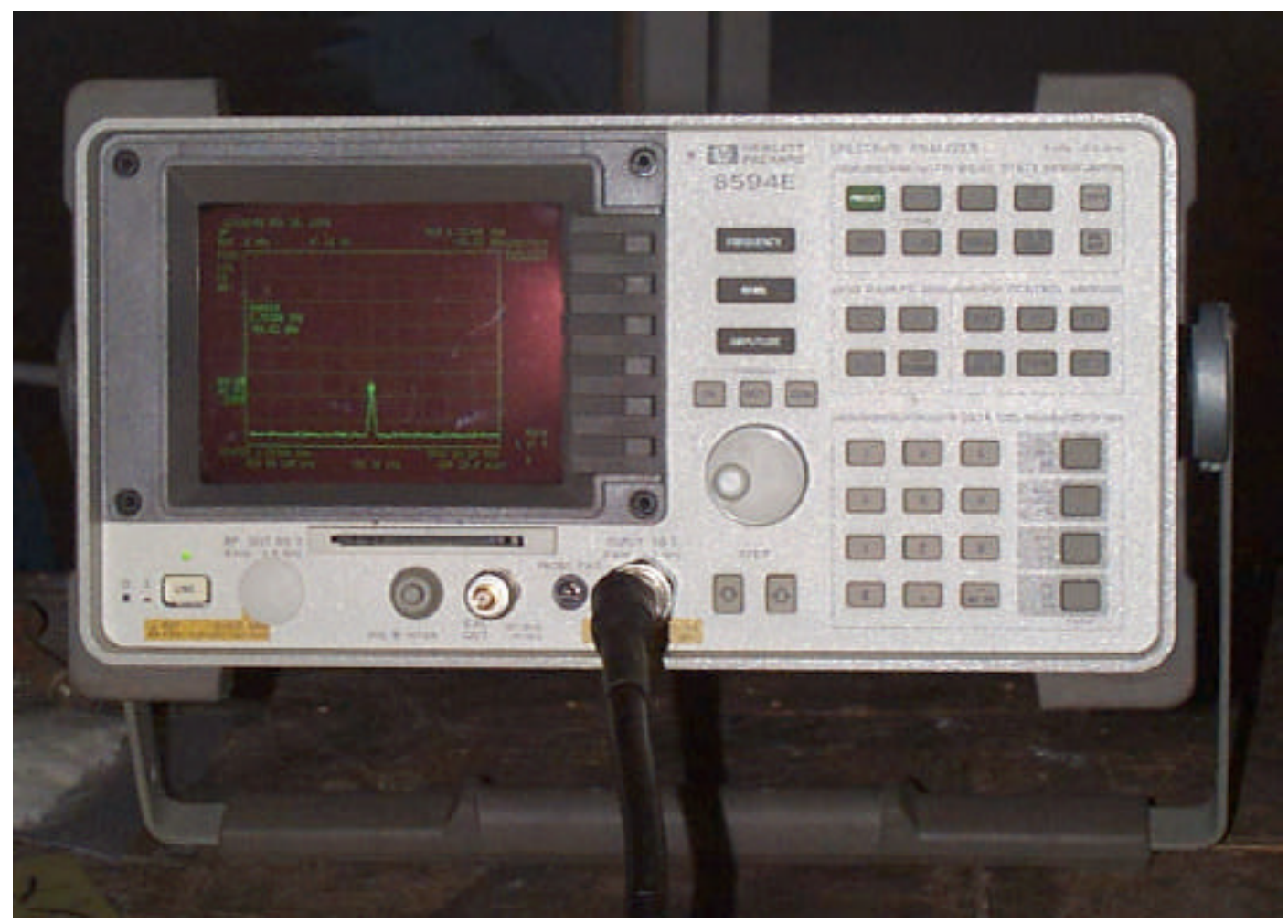

E.12 The Spectrum Analyzer

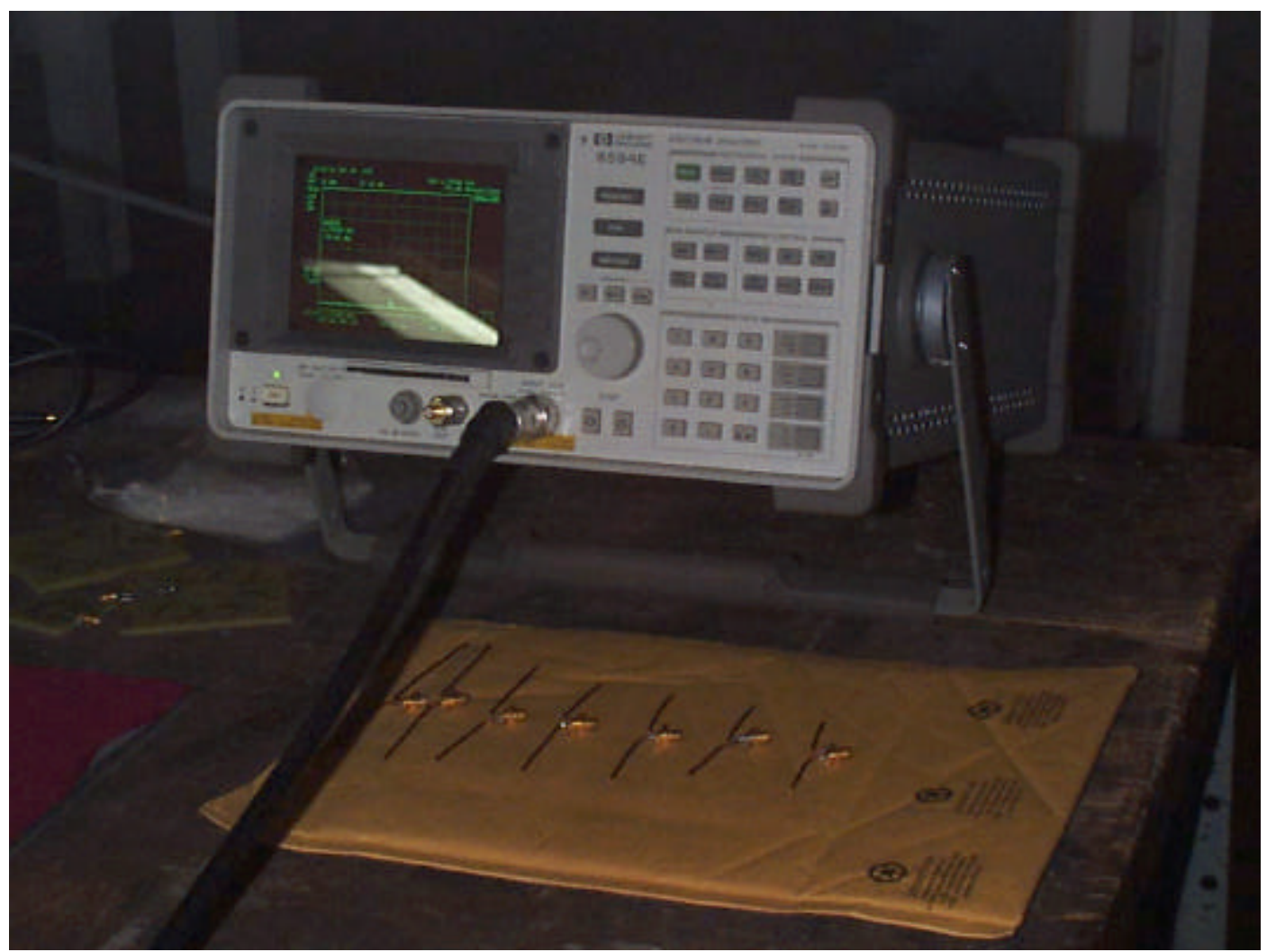

E.13 The Spectrum Analyzer, with the dipoles used for the experimental analysis shown. 


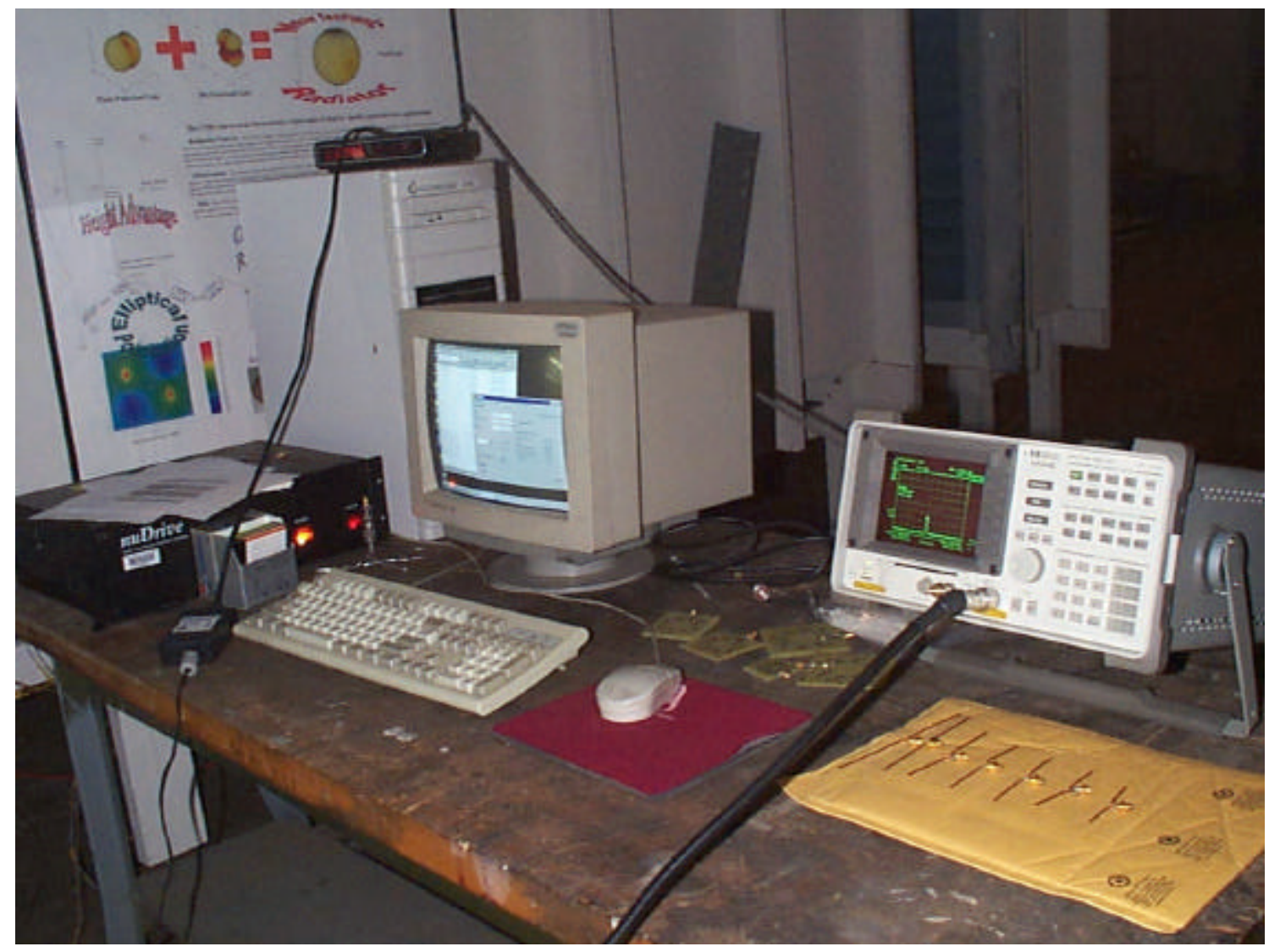

E14 The experimental workbench, with the computer, rotator motor, the spectrum analyzer, the CTHAs, and the dipoles visible. The anechoic chamber is also partially visible behind the workbench. 


\section{Appendix F: Analysis of All 13 Feeds}

As stated in Chapter 8, this Appendix will try to summarize the results for all 13 feeds in a simple shape. Each feed will be handled separately, with the powers and weaknesses of the prediction mentioned first, then general comments on the transient and steady state graphs mentioned after that. The feeds will be listed in order from feed 1 to feed 13 .

\section{Grading System for the Far Field Gain Charts}

The charts for the experimental case are provided in $\mathrm{dBm}$, while those for the numerical case are provided in $\mathrm{dBi}$. The comparison between both sets will be based on this understanding. Since the values on both sets of curves can not be compared, the comparison will be done in a qualitative fashion, where the following four ratings will be defined:

very good: This rating will be given to the numerical curve if it follows the experimental curve within an error of 10 degrees. So if all the high and low peaks on a numerical curve occur at the same angle as those of the experimental curve, or within 10 degrees from it, then the curve will be rated very good

good: This rating will be given to the numerical curve if the pattern of the numerical curve represents that of the experimental curve, but a shift of more than 10 degrees and less than 30 degrees exists. So if both curves have the same pattern, but an error between 10 and 30 degrees exist, this rating will be given.

fair: This rating will be given to a numerical curve if the pattern somewhat represents the experimental curve but some of the high and low peaks do not match or have an large error, over 30 degrees. 
poor: This rating will be given to a numerical curve if it does not represent at all the experimental curve. This would happen for example if a high peak on one curve coincides with a low peak on the other, etc.

From this point on within this text, all mention of the words "poor", "fair", "good", and "very good" will be understood to refer to the definitions stated above.

\section{Feed 1}

\section{Reactance Graph}

The numerical prediction of the imaginary component of the input impedance for feed 1 showed a good resemblance with the experimental graph between 0.8 and $1.8 \mathrm{GHz}$ as shown in Figure F.1. The numerical curve followed the experimental curve well within this range except for a low peak that showed up in the numerical curve at approximately $1.4 \mathrm{GHz}$ which has no match in the experimental curve. The resonant frequencies were also predicted fairly well, with an error of 2 to $8 \%$ within this range. The same accuracy in predicting the resonance frequencies applied for the whole range from 0.15 to $2 \mathrm{GHz}$, except for one anti resonance that showed a large error of $21 \%$ at $600 \mathrm{GHz}$.

The resonance frequencies for feed 1 did not show themselves to repeat accurately at constant intervals. Same type frequencies tended to repeat themselves at roughly every 660 $\mathrm{MHz}$, while the difference between the real and the following anti resonances was approximately $225 \mathrm{MHz}$ on the numerical curve, and between 150 and $200 \mathrm{MHz}$ on the experimental curve. These Figures are disregarding the resonances under $0.5 \mathrm{GHz}$ in Table F.2.

\section{Gain Graphs}

The numerical steady state curves for feed 1 showed a very poor resemblance to the experimental curves, especially the curves for the real resonance, where no resemblance at all 
was found. This can be mainly attributed to the fact that the Gain for all cases of the real resonance was almost equal to the noise level, since the noise level in the chamber was always around -75 to $-76 \mathrm{~dB}$. The Gain at the anti resonance was above the noise level, and ranged between -60 and $-75 \mathrm{dBm}$, but also showed no resemblance to the numerical predictions. The exception might be for the range from 180 to 360 degrees on the Theta $=90$ curve for the anti resonance, see Figure F.4, where the numerical and experimental curves showed a good match.

Overall it can be said that the numerical method failed to predict the steady state gain curves for feed 1 , and that the gain from this feed is very low, especially at the real resonance where the gain is as low as the noise.

\section{Feed 2}

\section{Reactance Graph}

Feed 2 showed one of the worst matches between the numerical and experimental transient curves. There was an approximate error of $6 \%$ within the range from 0.9 to $2 \mathrm{GHz}$. But at $1.17 \mathrm{GHz}$ the experimental curve showed a real then an anti resonance frequency, that were both not predicted by the numerical model. the numerical model did however get close to intersecting the $\mathrm{x}$-axis at this location, but it didn't actually get to cross it, thus no resonance was traced.

Both the numerical and the experimental curves also showed no definitive pattern for the input impedance. The resonance frequencies neither repeated themselves at regular intervals, nor did they have any uniqueness in their locations.

\section{Gain Graphs}

The numerical steady state curves for feed 2 were far better than those for feed 1 , and some of the curves showed excellent matching with the experimental curves. The Gain from 
these experimental curves was also relatively higher than the gain produced from feed 1, too. There was a poor match though for the steady state curves of both the real resonance at theta $=$ 90 , and for the anti resonance curves at $\mathrm{phi}=0$.

\section{Feed 3}

\section{Reactance Graph}

The transient curve for feed 3 followed well after the experimental curve, although it showed lots of fluctuations around the relatively straight experimental curve. The resonant frequencies were predicted fairly well, with an error between 2 and $4 \%$. The exception was the resonances predicted by the numerical curve at approximately $1.1 \mathrm{GHz}$ which resulted from the numerical curve fluctuations, this resonance was not found though on the experimental curve.

The feed 2 transient curve also showed no repetitive nature as that seen previously for feed 5, and the curve showed a very low impedance that seemed to crawl close to the $\mathrm{x}$ - axis and cross it from time to time.

\section{Gain Graphs}

Except for the phi $=0$ curves for the real resonance, the rest of the numerical curves showed a good resemblance of the experimental curves. The noticeable feature though, in these curves and in almost all the other feeds, is that even when the numerical curve follows the experimental curve closely, but the low peaks on the numerical curve go relatively much deeper than those for the experimental curves. This can be attributed to the fact that when the low peaks of the experimental curve drop to $-75 \mathrm{~dB}$ they reach the same level as the noise, thus the peaks go no lower, while the low peaks for the numerical curve are not restricted. This can be seen clearly for example on the theta $=90$ curves for the real resonance, where both the Ephi and the 
Etheta curves showed good resemblance, but the low peaks of the numerical curve were clearly deeper than those for the experimental curve.

The Ephi curve of the theta $=90$ steady state curves for the real resonance showed high gains that ranged between -54 and $-62 \mathrm{dBm}$, which are the highest among the three feeds

checked until now. The Etheta curve of the phi $=0$ plane for the real resonance also had most of the gain between -55 and $-60 \mathrm{dBm}$, except for the low peaks that reached as low as $-70 \mathrm{dBm}$. The rest of the curves were all between the -60 and $-75 \mathrm{dBm}$ as with the previous feeds.

\section{Feed 4}

\section{Reactance Graph}

This feed was one of the best predicted feeds, both transient and steady state. Both transient curves almost exactly matched, and the points where the curves intersected the x-axis, the resonant frequencies, were almost identical. The error for the resonant frequencies was between 0.5 to $3 \%$ for the whole range from 0.18 to $2 \mathrm{GHz}$. The two curves also followed each other very closely along all this range, the only exception was that the max and min impedance peaks on the numerical curve were always much less than those on the experimental curve. This was also what was noted on the curve for feed 5 described earlier, except that for feed 5 this happened on only two of the peaks, while here it happened on the first three peaks. On the last two peaks however the two values seemed to match closely.

The input impedance curve for feed 4 also shows a clear repetitive nature. The distance between each two resonances on both the numerical and the experimental curves was almost constant at $180 \mathrm{MHz}$, and the distance between each two resonances of the same type was also constant at about $360 \mathrm{MHz}$. This means that the resonances repeat themselves very uniformly, and that they are relatively much closer to each other than what was found on the previous feeds. 


\section{Gain Graphs}

Except for the Etheta curve for the phi $=0$ plane at the anti resonance, all the rest of the steady state far field gain patterns showed a very good match between the numerical and the experimental curves. The Ephi patterns for all four cases almost always got a v. good score, and the same also applied for the Etheta curves, except for the one mentioned above.

The four curves at the real resonance showed fairly high gains, compared to the gains obtained from the previous feeds, and even compared to the gains obtained at the anti resonance for this feed. The gains at the real resonance ranged between -50 and $-60 \mathrm{dBm}$, sometimes reaching $-70 \mathrm{dBm}$ at the low peaks tips. The gains at the anti resonance, on the other hand, ranged between -60 and $-75 \mathrm{dBm}$, which is the same range that was found for almost all the previous feeds.

In general, the results from feed 4 show that it is from among the best performing feeds, and also one of the best predicted feeds as well.

\section{Feed 5}

\section{Reactance Graph}

Although feed 5 was previously analyzed in detail, but it will be repeated again here briefly for the sake of completeness. The numerical input impedance curve matched the experimental curve very well, slightly less perfect compared to the match for the transient curves of feed 4. The error between both curves was between $1 \%$ and $3.5 \%$ for all the range from 0.3 $\mathrm{MHz}$ to $2 \mathrm{GHz}$, and showed an error of $6 \%$ at the very low frequency of $174 \mathrm{MHz}$.

Feed 5 also showed a repetitive behavior for its input impedance curve, a behavior that is almost identical to the behavior of feed 4 . The distance between consecutive impedances is 180 $\mathrm{MHz}$, with a distance of approximately $360 \mathrm{MHz}$ between impedances of the same type. This is 
exactly the same as was found for feed 4 . The interesting point, too, is that the location of the resonances was also found to be the same as the location of the resonances for feed 4 , only the type of resonances were reversed. So each real resonance on feed 4 would be at the same location as an anti resonance on feed 5, and vice versa. This can be seen clearly in Figure 8.3. In this Figure the first anti resonance at approximately $180 \mathrm{MHz}$ is for feed 5.

Looking at Figure 4.6 where the shapes of all thirteen feeds are shown, it can be noticed that both feeds have the feed point located between points $A$ on $B$, each on a different loop. The physical difference though between feeds 4 and 5 is that the other ends of the loops, points $\mathrm{C}$ and D, are shorted on feed 5 while they are left open on feed 4. From this it can be concluded that the shorting of the two loops kept the resonant points the same but flipped their type. This same behavior was seen between feeds 12 and 13, and is described later.

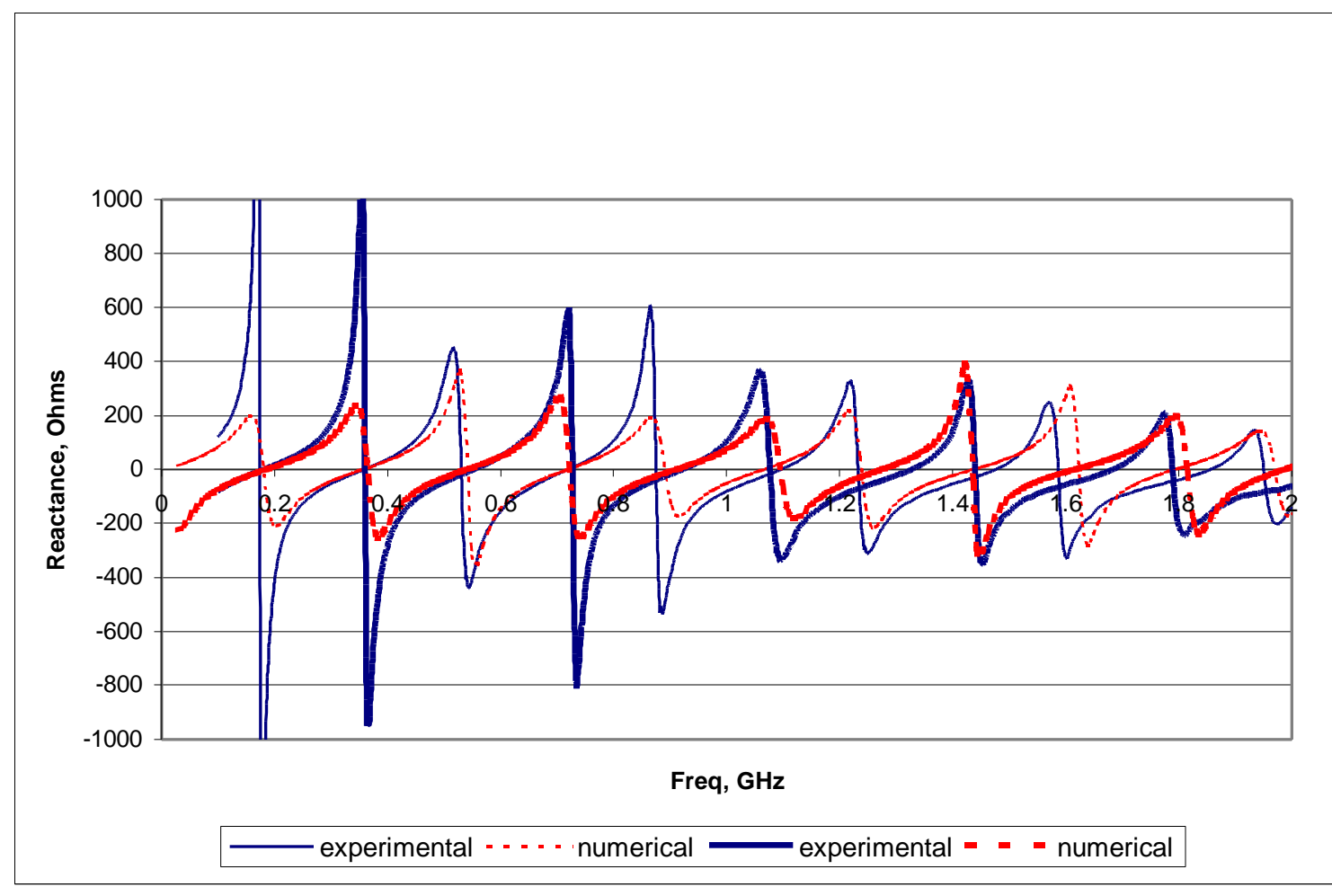

F.1 Comparing the Reactance for feeds 4 \& 5 


\section{Gain Graphs}

The steady state graphs for feed 5 did not show the same excellent match between the numerical and experimental curves as was found for feed 4, although the match was very good for most of the graphs, definitely much better than what as found for the first three feeds. The gains from this feed too were less than the gains obtained from feed 4, and was in average between -60 and $-75 \mathrm{dBm}$.

\section{Feed 6}

\section{Reactance Graph}

Feed 6 is the feed that was almost exclusively used for all CTHA experiments until this study. But surprisingly it is not from among the best performers, nor from the best predicted feeds. The match between the transient numerical and experimental feeds was among the average matches, where the two curves followed closely but not perfectly. The range of resonance frequency errors was from $1 \%$ all the way up to $9 \%$. Also a very clear extremely high impedance value, over $1000 \mathrm{Ohms}$, on the numerical curve at the anti resonance found at 1.6 $\mathrm{GHz}$ coincided with a regular $\pm 400 \mathrm{Ohm}$ anti resonance on the experimental curve. This kind of behavior was rarely seen among the other feeds.

The transient curve also showed a repetitive nature, although not as regular as the pattern found for feeds 4 and 5. The average distance between resonances was approximately $250 \mathrm{MHz}$, but this distance actually fluctuated between $210 \mathrm{MHz}$ up to over $400 \mathrm{MHz}$ sometimes, showing the inconsistency in the pattern. But overall the behavior should definitely be classified among the repetitive behaviors. 


\section{Gain Graphs}

The numerical prediction of the steady state curves for this feed were from among the poorest between all feeds. The pattern matches scored poor on most of the curves. The performance of the feed was also relatively poor. The real resonance curves all measured between -60 and $-75 \mathrm{dBm}$, while the anti resonance curves provided a slightly higher gain, between -55 and $-70 \mathrm{dBm}$.

\section{Feed 7}

\section{Reactance Graph}

The transient curves for this feed showed a very unique pattern, unlike all the patterns seen before for all the other feeds. And even from among the curves that are yet to come only feed 10 comes close to showing the same behavior. For all the range from $100 \mathrm{MHz}$ up to 1.5 $\mathrm{GHz}$, only one resonance frequency is found. This is an anti resonance at about $500 \mathrm{MHz}$. After this resonance, the rest of the curve crawls closely beside the $\mathrm{x}$-axis. At about $1.5 \mathrm{GHz}$ the experimental curve crosses the $\mathrm{x}$-axis providing a real resonance. At that same location, the numerical curve reaches very close to the $\mathrm{x}$-axis but does not cut it, thus no resonance frequency is predicted. The curves keep crawling beside the $\mathrm{x}$-axis until about $2 \mathrm{GHz}$ when the experimental curves returns and cuts back the $\mathrm{x}$ - axis to join the numerical curve once again. So practically only one clear resonance frequency is found, which is at $0.5 \mathrm{GHz}$. But tests can not be conducted at that frequency since it is way below the chamber limits, so tests were conducted at the two points where the experimental curve cuts the x-axis around 1.5 and $2 \mathrm{GHz}$. Since no numerical resonances are found at these two points, the same experimental frequencies were used for the numerical runs. This can be justified by the case that the numerical curve at these points was already very close to achieving a resonance. 
In spite of these unpredicted resonances, but the match between the numerical and experimental curves can still be classified among the best matches. The impedance values for both curves almost matched along the entire range, and the very unique behavior was perfectly predicted.

\section{Gain Graphs}

Most of the steady state curves for this feed were well predicted. The exceptions were The Ephi curves for both resonances in the phi $=0$ plane, and the Etheta curve for the anti resonance in the theta $=90$ plane. The Ephi gain for the real resonance in the theta $=90$ plane showed values between -55 and $-68 \mathrm{dBm}$. As for the rest of the curves, they all had values as regular between -60 and $-75 \mathrm{dBm}$. So despite the unique transient behavior for this feed, the steady state behavior showed high resemblance to all the other curves.

\section{Feed 8}

\section{Reactance Graph}

As with most of the previous feeds, the transient numerical curve well predicted the experimental curve, and matched it very well along the range from $500 \mathrm{MHz}$ up to over $2 \mathrm{GHz}$. The curve only showed some irregularity at the first anti resonance, at $890 \mathrm{MHz}$, where the numerical curve dived way below the experimental curve showing a very low min peak. This is despite the fact that the high peak for this resonance was very close in value to that of the experimental peak. This is contrary to what was seen in all of the other feeds where the high peak is usually close in value to the low peak. After the deep dive, the curve went up again, but another irregularity appeared where the curve showed another slight decline, then it went up again to follow the experimental curve. This fluctuation took about $300 \mathrm{MHz}$, from 0.9 to 1.2 $\mathrm{GHz}$. 
The resonant frequencies showed a regular repetitive pattern, where the difference between every two resonances of the same type had an average of $660 \mathrm{MHz}$. The distance between every real and the following anti resonance was approximately $180 \mathrm{MHz}$, while that between an anti and the following real resonance was $480 \mathrm{MHz}$.

\section{Gain Graphs}

The steady state behavior for this feed was from among the poorest in performance, and the poorest in prediction. All curves for the anti resonance fell below the $-70 \mathrm{dBm}$, which is very close to the noise level of -75 . The real resonance curves, on the other hand, showed a gain of 60 to -73 .

\section{Feed 9}

\section{Reactance Graph}

This feed clearly showed the worst match between the numerical and the experimental curves. Except for one resonant frequency that exactly matched, all the other resonance frequencies showed an error over 4\%. The numerical curve also showed lots of extra fluctuations that had no existence on the overall smooth and regular experimental curve.

The feed also showed a repetitive nature, but the repetitiveness seemed not as consistent as was seen in other feeds like feeds 4 and 5 .

\section{Gain Graphs}

The Ephi curves for this feed were predicted fairly well overall. The rating for most of the Ephi curves was either good or very good. The Etheta curves, on the other hand, were very poorly predicted, rating either poor or fair on all the curves. The gain for the real resonance curves all fell below $-62 \mathrm{dBm}$, while the gain for the anti resonance curves showed a higher gain which ranged between -55 and $-75 \mathrm{dBm}$. 
As an overall steady state performance for this curve, it can be said that both the prediction of the gains and the performance of the feed are slightly below the average found between all the feeds.

\section{Feed 10}

\section{Reactance Graph}

The transient behavior for this feed showed the same behavior seen with feed 7. Only one clear resonance at $483 \mathrm{MHz}$, then the impedance curve crawls close to the $\mathrm{x}$-axis all the way up to $1.5 \mathrm{GHz}$ where a real then an anti resonance exist, then once again the crawling remains until another resonance is found at approximately $1.9 \mathrm{GHz}$. The numerical curve predicted the experimental curve in an excellent fashion, showing the exact same curve, and giving a very low error in the prediction of the resonant frequencies between 1.2 and $2.7 \%$.

\section{Gain Graphs}

The average rating for the steady state numerical graphs predictability of the experimental graphs for this feed was fair, which is a rating that means that the curves are not totally off from the experimental curves, but can not be relied upon to predict the experimental behavior accurately.

The gain produced from this feed showed similar behavior to that viewed for the other feeds. The gain for the anti resonance curves ranged between -55 and $-75 \mathrm{dBm}$, while the real resonance curves ranged between -60 and $-75 \mathrm{dBm}$.

\section{Feed 11}

\section{Reactance Graph}

The match between the numerical and experimental curves for this feed were among the high performers. The numerical curve followed the path of the experimental curve along the 
whole range from $200 \mathrm{MHz}$ up to $2 \mathrm{GHz}$, and the error in predicting the resonant frequencies was between 0 and $3 \%$ for all resonances between $400 \mathrm{MHz}$ and $1.7 \mathrm{GHz}$. The numerical curve, though, did not show the same high and low peak impedances as the experimental curve, and it fluctuated between having a higher value and a lower value.

The behavior of the transient curve though was repetitive and consistent. The spacing between all the resonances, regardless of their type, had an average value of $172 \mathrm{MHz}$, with a mean of $342 \mathrm{MHz}$ between resonances of the same type.

\section{Gain Graphs}

Except for the Etheta curve for the anti resonance at the theta $=90$ plane, which received a very good rating for the prediction of the numerical pattern to the experimental pattern, the rest of the curves all received a fair to poor rating. But the real resonance curves for this feed showed some of the highest gains, reaching $-50 \mathrm{dBm}$ sometimes, which was not previously achieved by any other feed, and keeping above $-60 \mathrm{dBm}$ for most of the curve. This applied to all the real resonance curves except the Etheta curve for the theta $=90$ plane, which had a regular gain between -60 and $-75 \mathrm{dBm}$. The anti resonant curves, on the other hand, all showed values between -60 and -75 , similar to most of the other feeds.

\section{Feed 12}

\section{Reactance Graph}

Again, the numerical curve for the transient behavior was able to well predict the resonant frequencies found from the experimental curve. The error between the resonant frequencies found from both curves was between 0.2 and $3 \%$ for all the range from $500 \mathrm{MHz}$ to $2 \mathrm{GHz}$. The two curves also matched well along the rest of the curves, with the high and low 
peaks at the anti resonances not exactly matching, but not too far away as seen in other feeds. Over all, the transient matching between both curves can be classified as excellent.

The curves also showed a consistent repetitive behavior, with an average spacing of 350 $\mathrm{MHz}$ between resonances of the same type. These $350 \mathrm{MHz}$ are approximately split to $150 \mathrm{MHz}$ between a real resonance and the following anti resonance, and to $200 \mathrm{MHz}$ between that anti resonance and the following real resonance. This behavior was consistent almost along the whole range from $400 \mathrm{MHz}$ to $2 \mathrm{GHz}$, and was seen more clearly in the experimental curve than in the numerical curve.

A very interesting point is when the curves for feed 4 and feed 12 are compared. Shown below in Figure 8.4 is a comparison between the experimental curves for these two feeds. The numerical curves were taken out of this graph for clarity, but since both feeds showed an excellent match between the numerical and experimental curves, thenit is known that the behavior of the numerical curves is the same. Figure 8.4 shows that the impedance curves for both these feeds is almost identical. Although the high and low peaks did not give exact matches, but the resonant frequencies and the paths were identical. This shows that the transient behavior for both feeds is identical, and, as was shown from Figure 8.3, they are exact opposites of the behavior of feed 5 . 


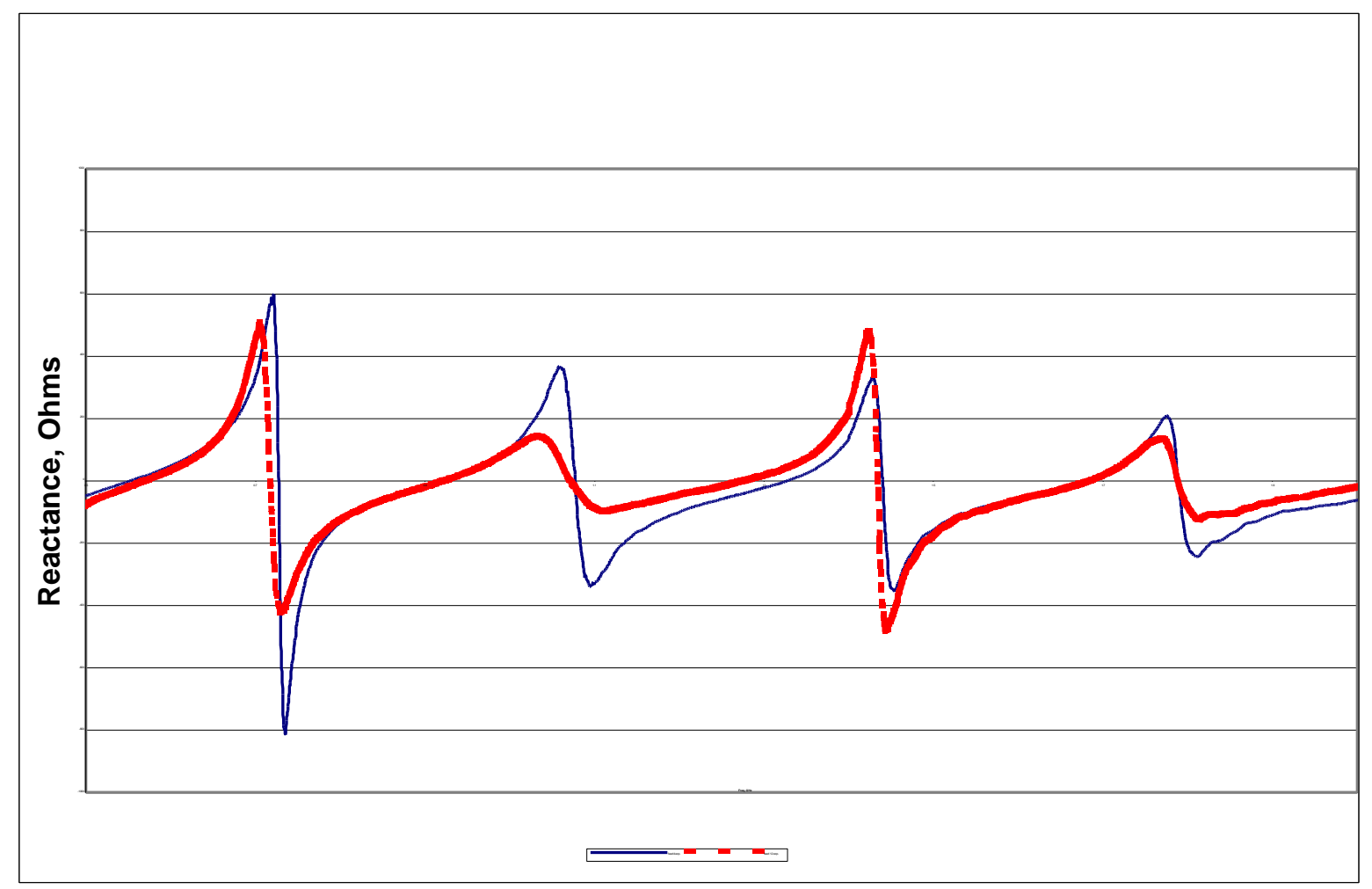

Figure F.2 Comparison Between the Reactance Curves for Feed 4 and Feed 12

\section{Gain Graphs}

The real resonance gains for this feed showed some of the highest gains found until now.

All four curves were between -50 and $-60 \mathrm{dBm}$ for most of the range, with some of the curves reaching values as high as $-48 \mathrm{dBm}$, which is a value seen for the very first time. The Ephi curve for the real resonance of the phi $=$ o plane showed exceptional behavior, where the gain was almost $-50 \mathrm{dBm}$ for the entire far field curve, with values only dropping under $-60 \mathrm{dBm}$ for a window of 30 degrees between 270 and 300 degrees. This behavior was also well predicted by the numerical curve, except for the small low peak window mentioned above that was not found on the numerical curve. The anti resonances, on the other hand, showed all gain values between -60 and $-75 \mathrm{dBm}$. 


\section{Feed 13}

\section{Reactance Graph}

This feed also showed a perfectly repetitive behavior, very similar to that found in feeds 4, 5, and 12. By comparing the graphs for this feed and for feed 5 it is clear that their transient behavior is identical. This behavior is clear from Figure 8.5 below. The Figure compares the experimental transient curves for both feed 5 and feed 13, and as shown, the result is a perfect match.

The resonant frequencies once again had a spacing of approximately $172 \mathrm{MHz}$, with the distance between resonances of the same type being approximately $345 \mathrm{MHz}$. The numerical curve for this feed also showed a perfect match with the experimental curve, with the error between both curves always below $2 \%$ along the whole range from $300 \mathrm{MHz}$ to $2 \mathrm{GHz}$, except once where the error reached $4.4 \%$. From this it can be understood that comparing the numerical curves for this feed with feed 5 would have yielded the same result as that found with the experimental curves, a perfect match.

\section{Gain Graphs}

Contrary to feed 12 , the anti resonances for this curve are the ones that showed high gains. The gain for all anti resonance curves reached as high as $-50 \mathrm{dBm}$ and as low as $-75 \mathrm{dBm}$, with the gain being between -50 and $-60 \mathrm{dBm}$ most of the time. The real resonance curves, on the other hand, showed a poor performance, with the gains between -60 and $-75 \mathrm{dBm}$. The matching between the numerical and experimental curves were average, with some curves, like the anti resonance Etheta curve for the theta $=90$ plane and the real resonance Ephi curve for the phi $=0$ plane, showing very good matches, while other curves showed poor matches. The overall steady state behavior of this feed is exactly opposite to that of feed 12 , just like was 
found for the transient behavior, with the performance of feed 12 slightly higher than that for feed 13 .

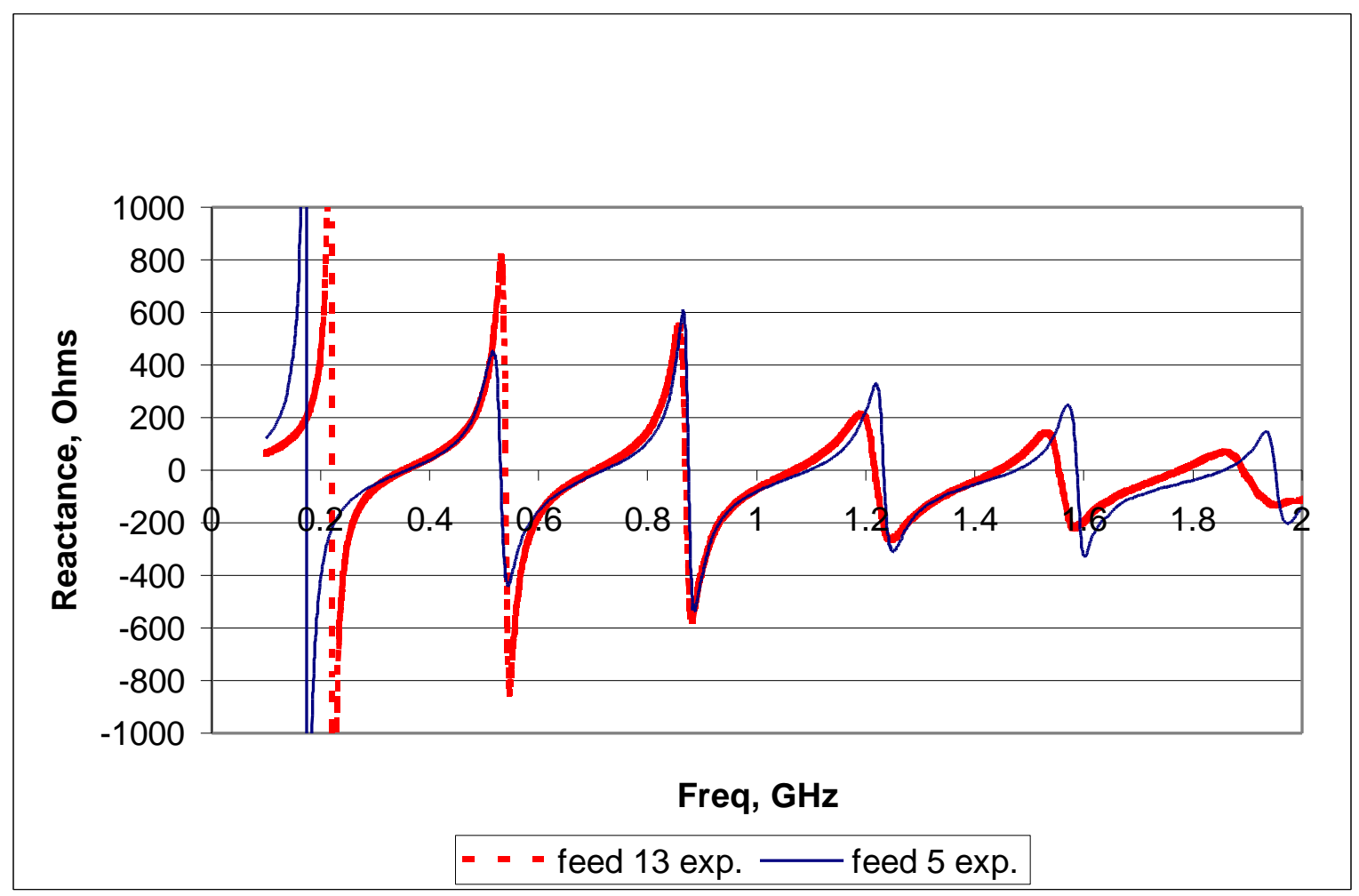

Figure F.3 Comparison Between the Reactance Curves for Feed 5 and Feed 13

This concludes the detailed analysis of the transient and steady state behavior and predictions for all 13 feeds.

\section{Far Field Gain Frequencies}

Following is a list of all the frequencies at which the far field gain runs were conducted. This table is provided as an easy access if needed. 
Table F.1 All frequencies at which the far field gain runs were conducted

\begin{tabular}{|c|c|c|c|}
\hline & res type & $\exp$ & num \\
\hline feed1 & $\begin{array}{r}\text { real } \\
\text { imaginary }\end{array}$ & $\begin{array}{l}1.103 \\
1.259\end{array}$ & $\begin{array}{l}1.009 \\
1.231\end{array}$ \\
\hline feed2 & $\begin{array}{r}\text { real } \\
\text { imaginary }\end{array}$ & $\begin{array}{l}1.467 \\
1.824\end{array}$ & $\begin{array}{l}1.551 \\
1.701\end{array}$ \\
\hline feed3 & $\begin{array}{r}\text { real } \\
\text { imaginary }\end{array}$ & $\begin{array}{l}1.401 \\
1.524\end{array}$ & $\begin{array}{l}1.371 \\
1.548\end{array}$ \\
\hline feed4 & $\begin{array}{r}\text { real } \\
\text { imaginary }\end{array}$ & $\begin{array}{l}1.327 \\
1.440 \\
\end{array}$ & $\begin{array}{l}1.291 \\
1.448 \\
\end{array}$ \\
\hline feed5 & $\begin{array}{r}\text { real } \\
\text { imaginary }\end{array}$ & $\begin{array}{l}1.096 \\
1.233\end{array}$ & $\begin{array}{l}1.086 \\
1.263\end{array}$ \\
\hline feed6 & $\begin{array}{r}\text { real } \\
\text { imaginary }\end{array}$ & $\begin{array}{l}1.326 \\
1.569\end{array}$ & $\begin{array}{l}1.206 \\
1.615\end{array}$ \\
\hline feed7 & $\begin{array}{r}\text { real } \\
\text { imaginary }\end{array}$ & $\begin{array}{l}1.489 \\
1.944 \\
\end{array}$ & * \\
\hline feed8 & $\begin{array}{r}\text { real } \\
\text { imaginary }\end{array}$ & $\begin{array}{l}1.409 \\
1.591 \\
\end{array}$ & $\begin{array}{l}1.387 \\
1.590 \\
\end{array}$ \\
\hline feed9 & $\begin{array}{r}\text { real } \\
\text { imaginary }\end{array}$ & $\begin{array}{l}0.929 \\
1.263 \\
\end{array}$ & $\begin{array}{l}0.978 \\
1.196 \\
\end{array}$ \\
\hline feed10 & $\begin{array}{r}\text { real } \\
\text { imaginary }\end{array}$ & $\begin{array}{l}1.525 \\
1.759\end{array}$ & $\begin{array}{l}1.564 \\
1.738\end{array}$ \\
\hline feed11 & $\begin{array}{r}\text { real } \\
\text { imaginary }\end{array}$ & $\begin{array}{l}0.900 \\
1.080\end{array}$ & $\begin{array}{l}0.900 \\
1.105\end{array}$ \\
\hline feed12 & $\begin{array}{r}\text { real } \\
\text { imaginary }\end{array}$ & $\begin{array}{l}1.275 \\
1.434\end{array}$ & $\begin{array}{l}1.290 \\
1.459\end{array}$ \\
\hline feed13 & $\begin{array}{r}\text { real } \\
\text { imaginary }\end{array}$ & $\begin{array}{l}1.070 \\
1.217\end{array}$ & $\begin{array}{l}1.041 \\
1.211\end{array}$ \\
\hline
\end{tabular}




\section{Feed 1}

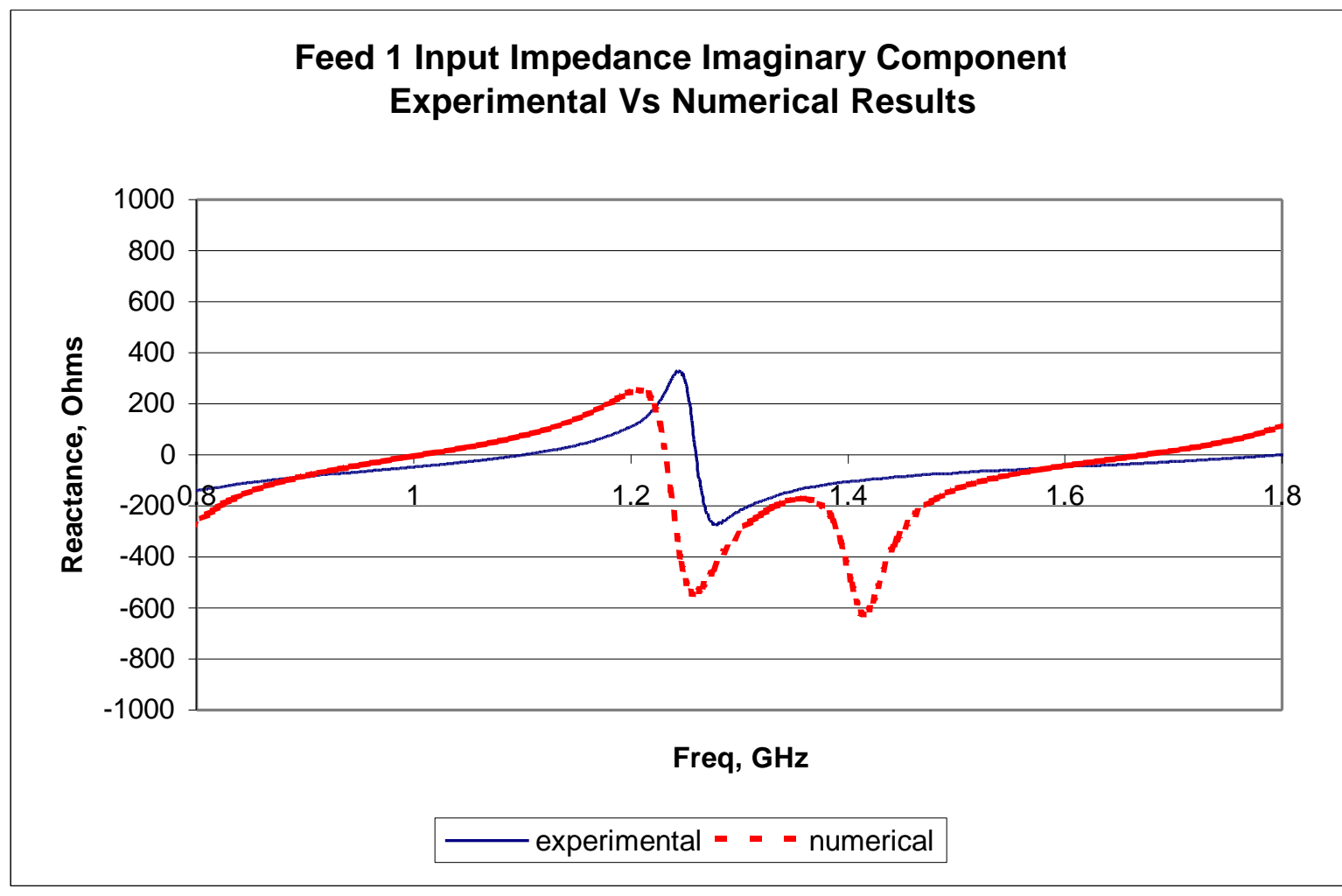

ure F.4 The imaginary component of the input impedance for feed 1

Table F.2 Analysis of resonance frequencies for feed 1

\begin{tabular}{|c|c|c|}
\hline $\begin{array}{c}\mathrm{f1} \text { exp. } \\
(\mathrm{GHz})\end{array}$ & $\begin{array}{c}\text { diff. between } \\
\text { res freq's }\end{array}$ & $\begin{array}{c}\text { diff. between } \\
\text { same type freq's }\end{array}$ \\
\hline 0.166 & & \\
\hline 0.367 & 0.201 & \\
\hline 0.602 & 0.235 & 0.436 \\
\hline 1.103 & 0.500 & 0.735 \\
\hline 1.259 & 0.157 & 0.657 \\
\hline 1.800 & 0.541 & 0.697 \\
\hline 1.996 & 0.195 & 0.736 \\
\hline mean & 0.305 & 0.652 \\
\hline
\end{tabular}

\begin{tabular}{|c|c|c|}
\hline $\begin{array}{c}\text { f1 num. } \\
(\mathrm{GHz})\end{array}$ & $\begin{array}{r}\text { diff between } \\
\text { res freq's }\end{array}$ & $\begin{array}{c}\text { diff between } \\
\text { same type freq's }\end{array}$ \\
\hline 0.179 & & \\
\hline 0.340 & 0.161 & \\
\hline 0.734 & 0.394 & 0.555 \\
\hline 1.009 & 0.275 & 0.669 \\
\hline 1.231 & 0.223 & 0.498 \\
\hline 1.674 & 0.443 & 0.666 \\
\hline 1.900 & 0.226 & 0.669 \\
\hline mean & 0.287 & 0.611 \\
\hline
\end{tabular}

\begin{tabular}{|c|}
\hline $\begin{array}{c}\text { error } \\
\%\end{array}$ \\
\hline 7.34 \\
\hline 7.47 \\
\hline 21.80 \\
\hline 8.53 \\
\hline 2.23 \\
\hline 6.97 \\
\hline 4.78 \\
\hline
\end{tabular}




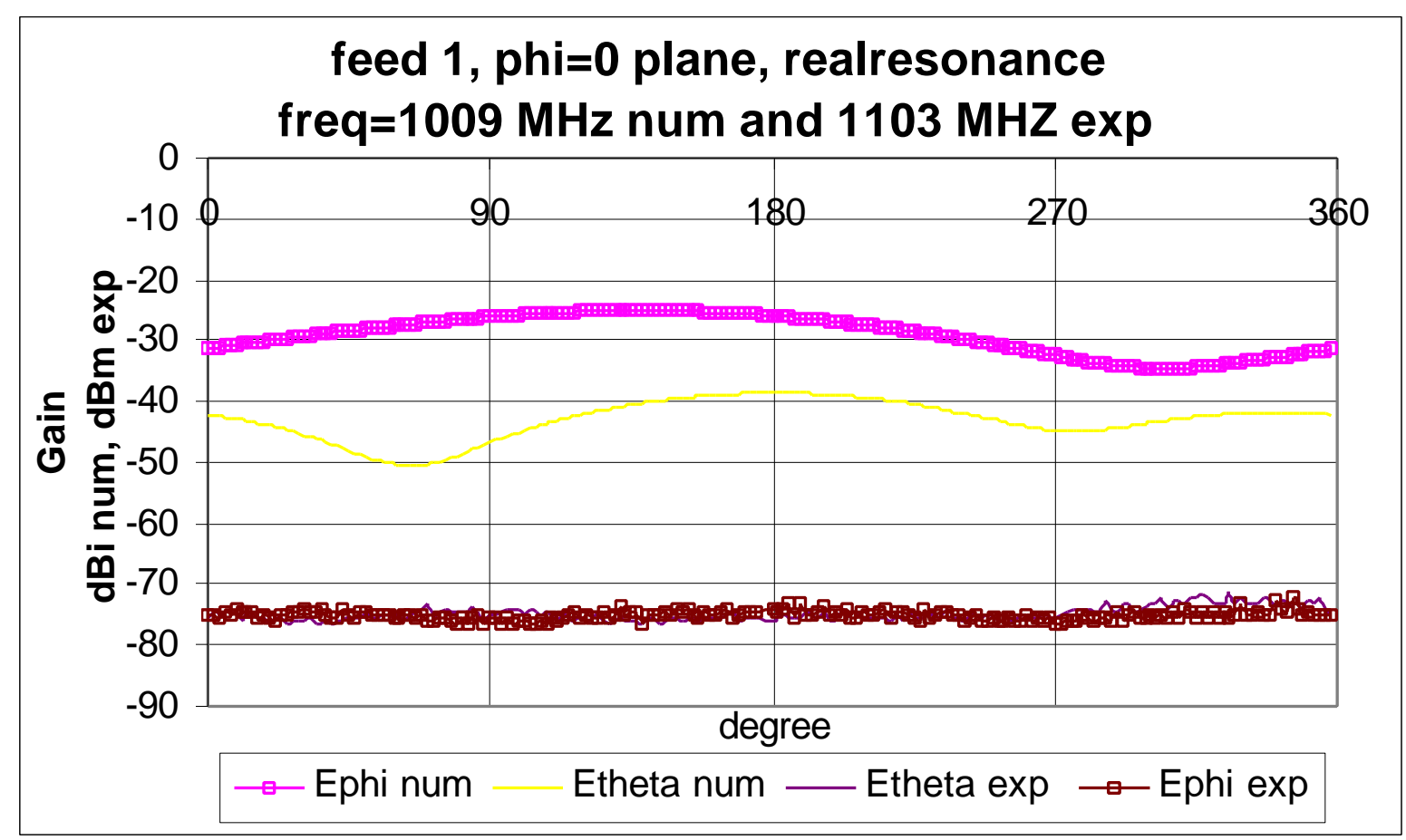

Figure F.5 The Phi = O plane for feed 1 at $1009 \mathrm{MHz}$ num and $1103 \mathrm{MHz}$ exp

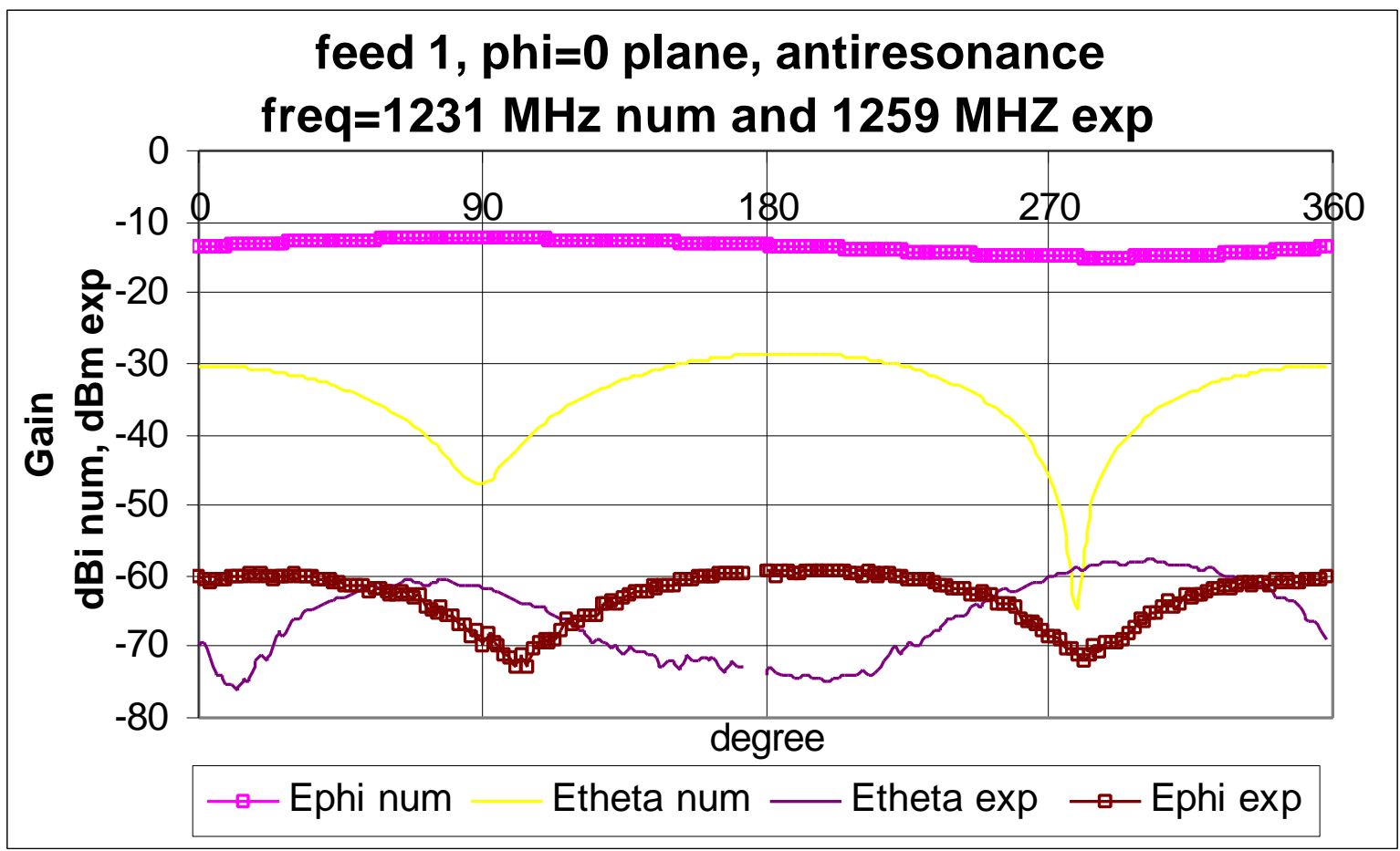

Figure F.6 The Phi = 0 plane for feed 1 at $1231 \mathrm{MHz}$ num and $1259 \mathrm{MHz}$ exp 


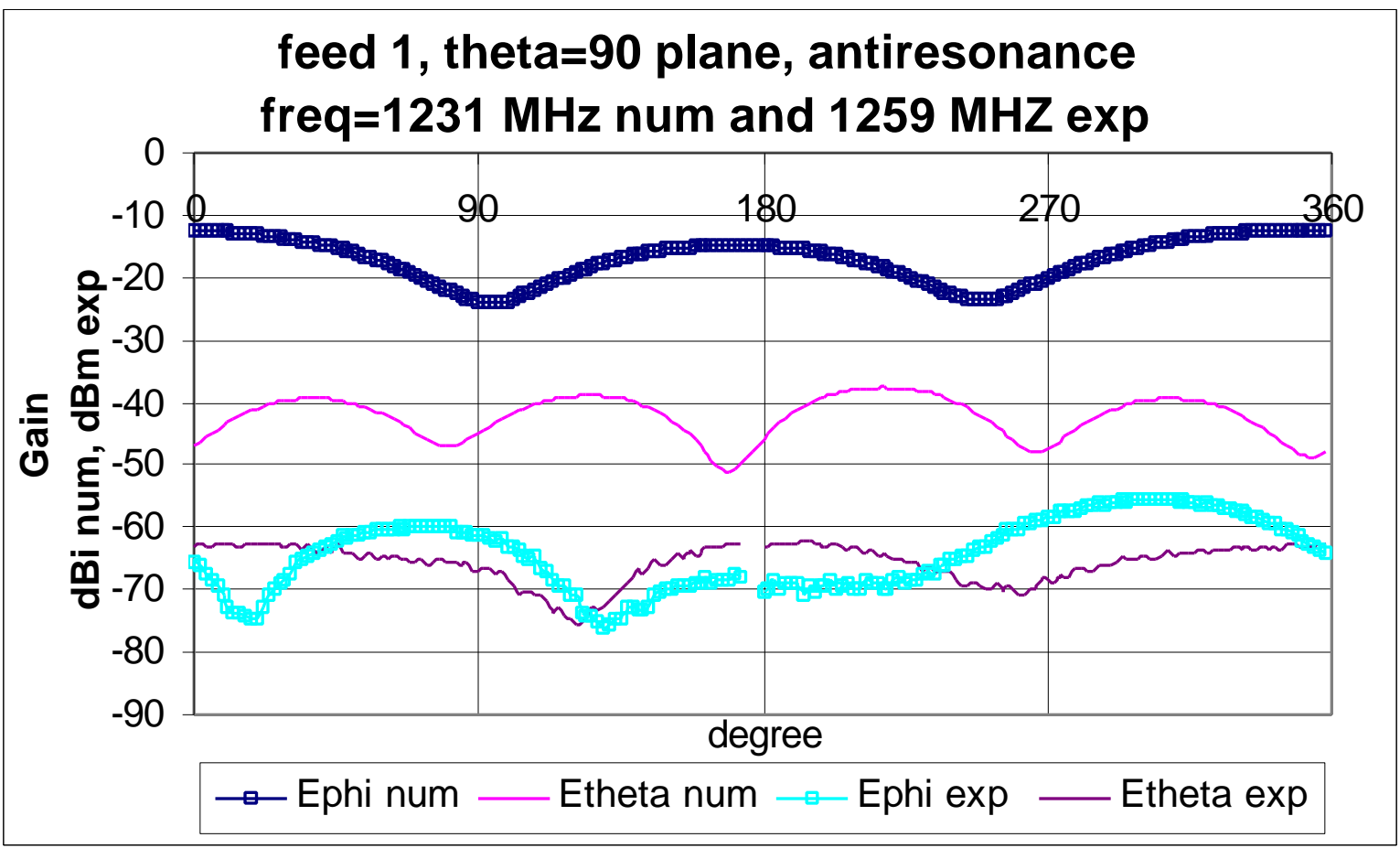

Figure F.7 The Theta $=90$ plane for feed 1 at $1231 \mathrm{MHz}$ num and $1259 \mathrm{MHz}$ exp

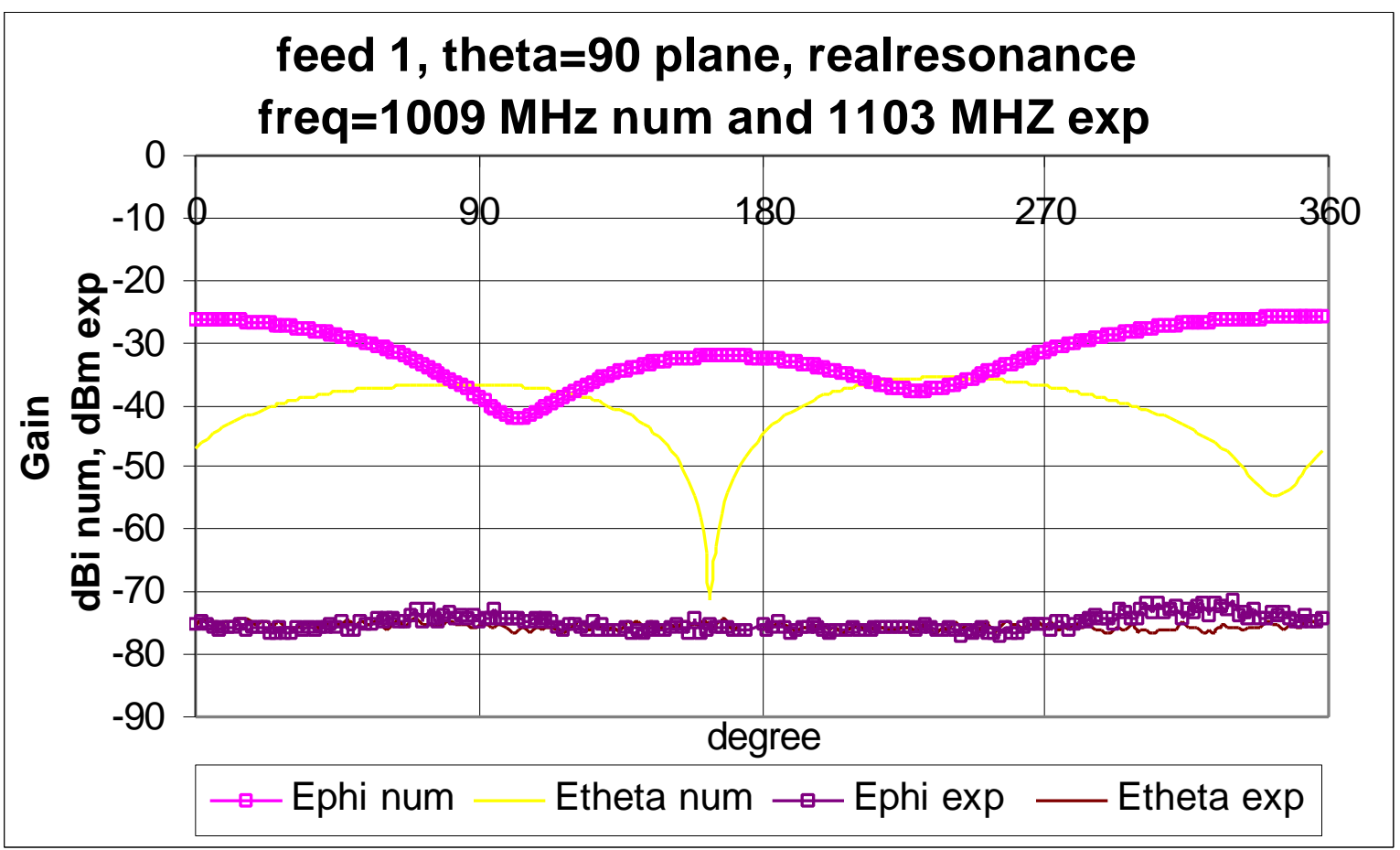

Figure F.8 The Theta $=90$ plane for feed 1 at $1009 \mathrm{MHz}$ num and $1103 \mathrm{MHz}$ exp 
Table F.3 Analysis of the steady state graphs for feed 1

\begin{tabular}{|c|c|c|c|c|c|c|}
\hline & & & & & & \\
\hline Plane & res. type & Graph & angles & score & $\min$ & $\max$ \\
\hline & & Enhi & $0-180$ & $\mathrm{~N} / \mathrm{A}$ & 43 & 51 \\
\hline & & EpnI & $180-360$ & $\mathrm{~N} / \mathrm{A}$ & 31 & 49 \\
\hline & real & Fthots & $0-180$ & $\mathrm{~N} / \mathrm{A}$ & 23 & 37 \\
\hline Phi - 0 & & Etneta & $180-360$ & $\mathrm{~N} / \mathrm{A}$ & 28 & 42 \\
\hline$r n I=0$ & & Fnhi & $0-180$ & Poor & 13 & 60 \\
\hline & anti & Ephi & $180-360$ & Poor & 14 & 57 \\
\hline & anti & & $0-180$ & Poor & 14 & 46 \\
\hline & & Etheta & $180-360$ & Poor & 2 & 46 \\
\hline & & & $0-180$ & $\mathrm{~N} / \mathrm{A}$ & 32 & 50 \\
\hline & ronl & Ephi & $180-360$ & $\mathrm{~N} / \mathrm{A}$ & 26 & 49 \\
\hline & real & Cthoto & $0-180$ & $\mathrm{~N} / \mathrm{A}$ & 4 & 48 \\
\hline Theta - 90 & & Etneta & $180-360$ & $\mathrm{~N} / \mathrm{A}$ & 21 & 48 \\
\hline Ineta $=90$ & & Enhi & $0-180$ & fair & 15 & 62 \\
\hline & & Ергі & $180-360$ & good & 12 & 55 \\
\hline & antl & & $0-180$ & poor & 11 & 49 \\
\hline & & E & $180-360$ & good & 14 & 48 \\
\hline
\end{tabular}




\section{Feed 2}

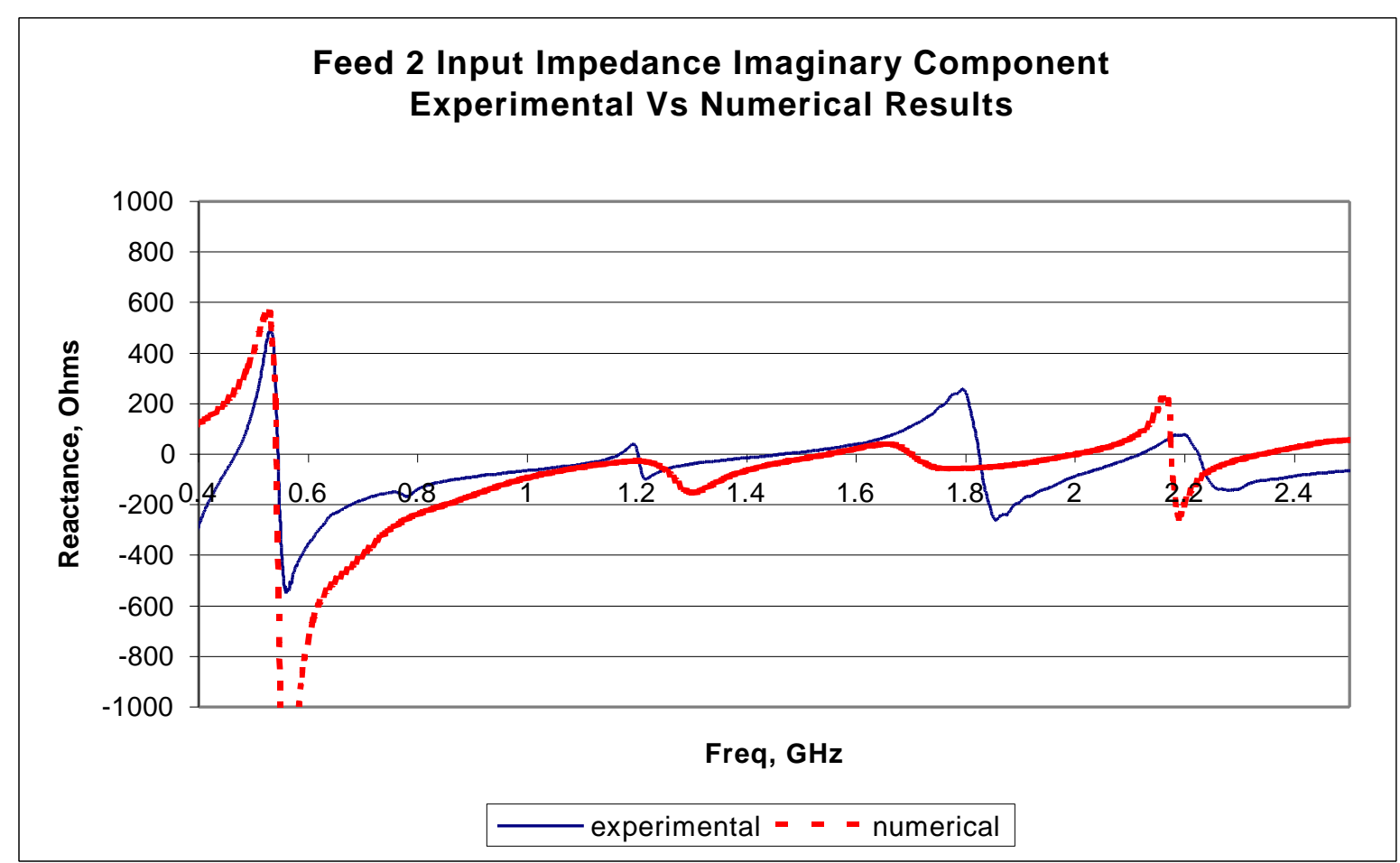

Figure F.9 The imaginary component of the input impedance for feed 2

Table F.4 Analysis of resonance frequencies for feed 2

\begin{tabular}{|c|c|c|}
\hline $\begin{array}{c}\text { f2 exp. } \\
(\mathrm{GHz})\end{array}$ & $\begin{array}{c}\text { f2 num. } \\
(\mathrm{GHz})\end{array}$ & $\begin{array}{c}\text { error } \\
\%\end{array}$ \\
\hline 0.545 & 0.541 & 0.65 \\
\hline 1.169 & & \\
\hline 1.201 & & \\
\hline 1.467 & 1.551 & 5.72 \\
\hline 1.824 & 1.701 & 6.73 \\
\hline
\end{tabular}




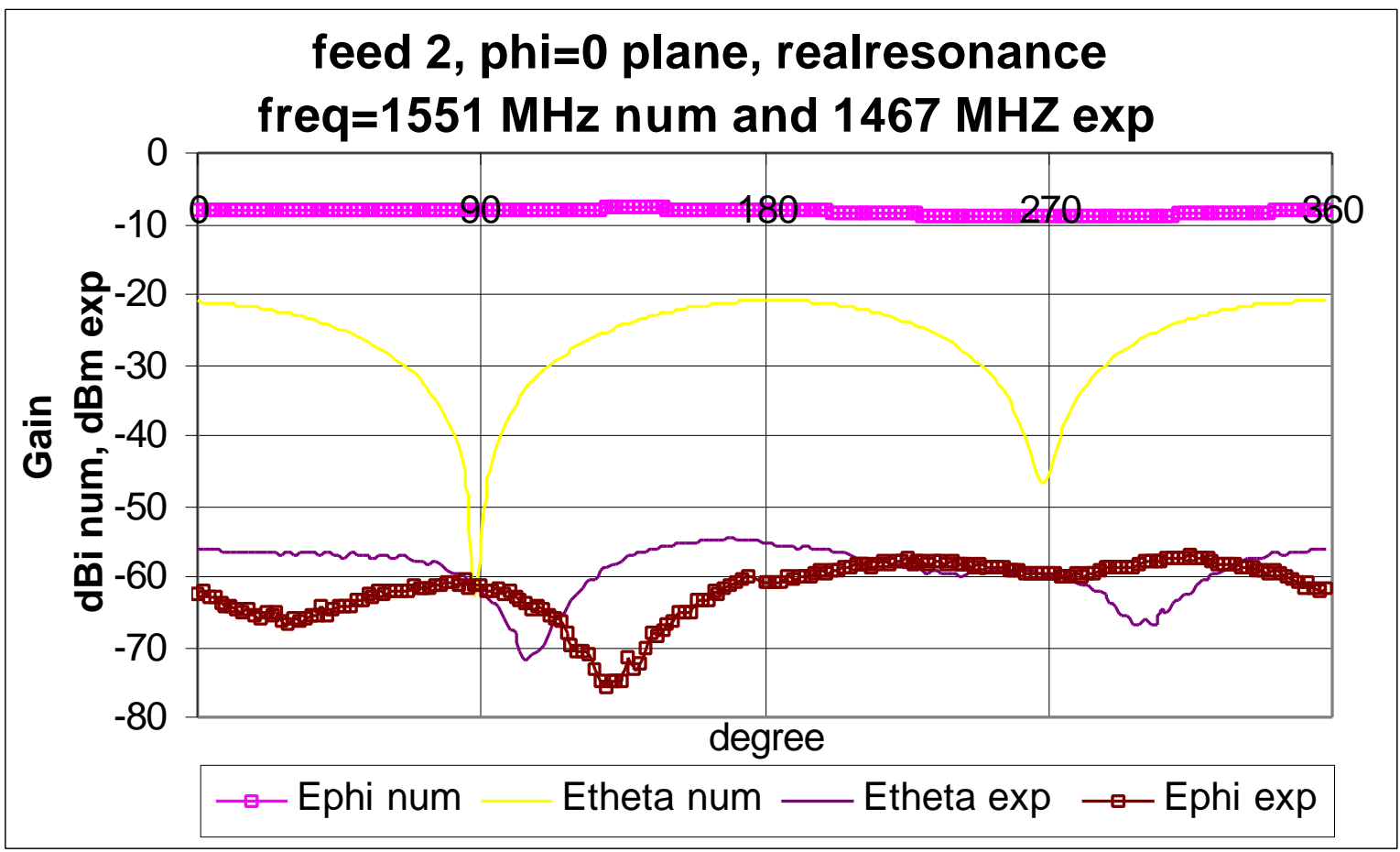

Figure F.10 The Phi = O plane for feed 2 at $1551 \mathrm{MHz}$ num and $1467 \mathrm{MHz}$ exp

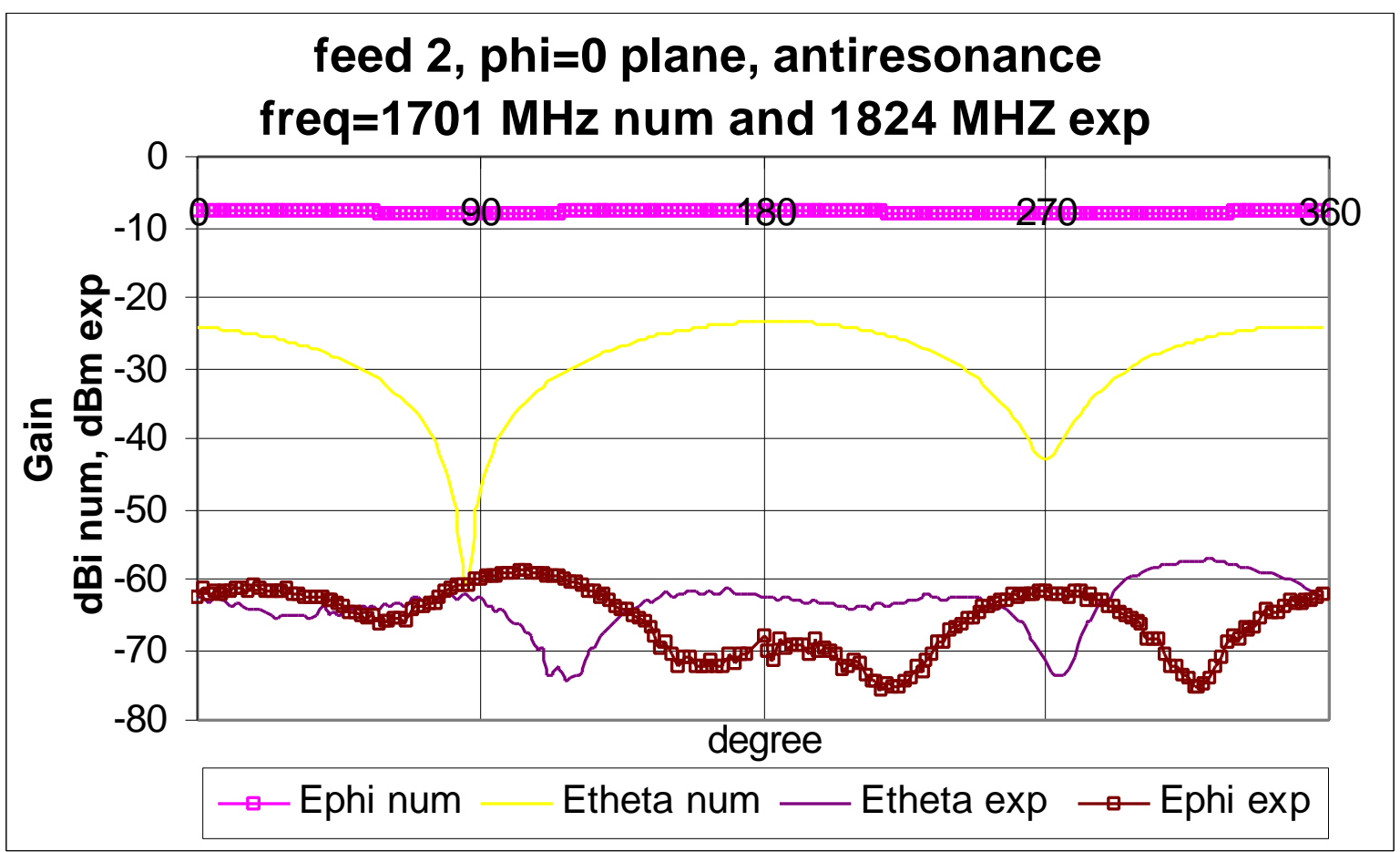

Figure F.11 The Phi = 0 plane for feed 2 at $1701 \mathrm{MHz}$ num and $1824 \mathrm{MHz}$ exp 


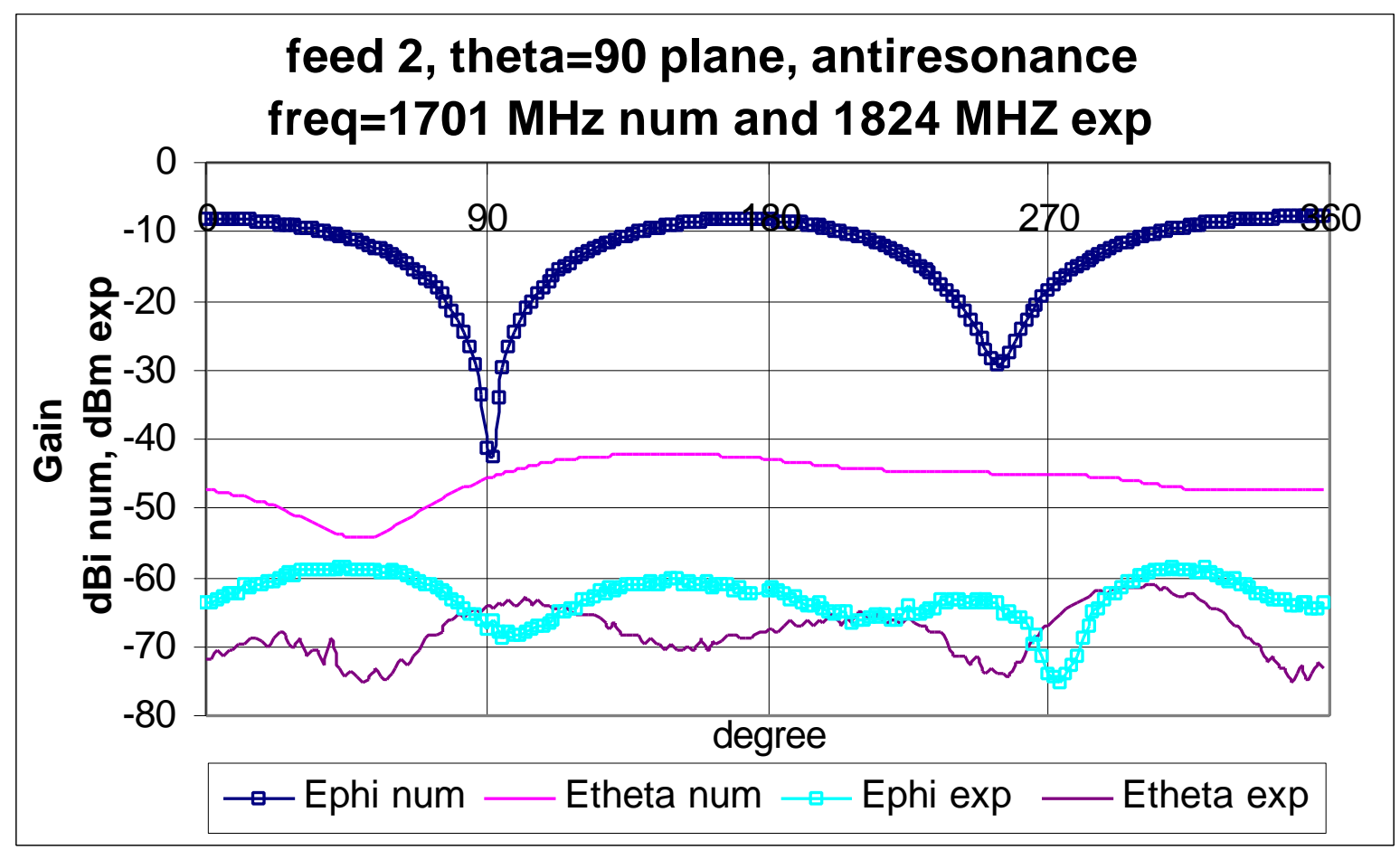

Figure F.12 The Theta $=90$ plane for feed 2 at $1701 \mathrm{MHz}$ num and $1824 \mathrm{MHz}$ exp

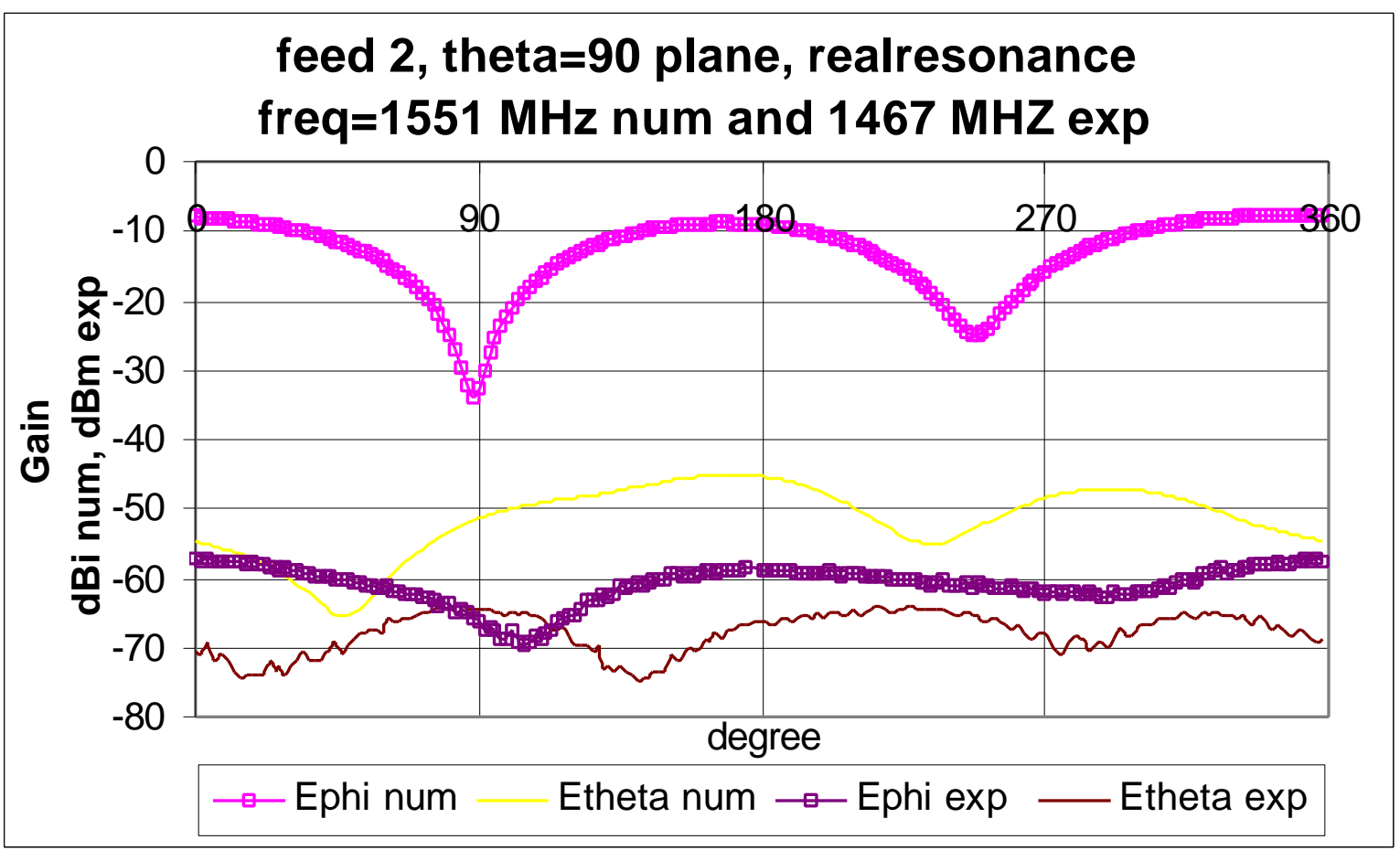

Figure F.13 The Theta $=90$ plane for feed 2 at $1551 \mathrm{MHz}$ num and $1467 \mathrm{MHz}$ exp 
Table F.5 Analysis of the steady state graphs for feed 2

\begin{tabular}{|c|c|c|c|c|c|c|}
\hline \multirow{2}{*}{ Plane } & \multirow[b]{2}{*}{ res. type } & \multirow[b]{2}{*}{ Graph } & \multirow[b]{2}{*}{ angles } & \multirow[b]{2}{*}{ score } & \multicolumn{2}{|c|}{$\mathrm{dBd}$} \\
\hline & & & & & $\min$ & $\max$ \\
\hline \multirow{8}{*}{$P h i=0$} & \multirow{4}{*}{ real } & \multirow{2}{*}{ Ephi } & $0-180$ & fair & 52 & 68 \\
\hline & & & $180-360$ & v. good & 48 & 55 \\
\hline & & \multirow{2}{*}{ Etheta } & $0-180$ & v. good & 2 & 39 \\
\hline & & & $180-360$ & v. good & 13 & $\overline{41}$ \\
\hline & \multirow{4}{*}{ anti } & \multirow{2}{*}{ Ephi } & $0-180$ & poor & 51 & 65 \\
\hline & & & $180-360$ & poor & 8 & $\overline{68}$ \\
\hline & & \multirow{2}{*}{ Etheta } & $0-180$ & fair & 2 & 44 \\
\hline & & & $180-360$ & V. good & 24 & 40 \\
\hline \multirow{8}{*}{ Theta $=90$} & \multirow{4}{*}{ real } & \multirow{2}{*}{ Ephi } & $0-180$ & v. good & 32 & 52 \\
\hline & & & $180-360$ & poor & 8 & 52 \\
\hline & & \multirow{2}{*}{ Etheta } & $0-180$ & poor & 4 & 28 \\
\hline & & & $180-360$ & poor & 9 & 55 \\
\hline & \multirow{4}{*}{ anti } & \multirow{2}{*}{ Ephi } & $0-180$ & v. good & 23 & 55 \\
\hline & & & $180-360$ & v. good & 8 & 57 \\
\hline & & \multirow{2}{*}{ Etheta } & $0-180$ & v. good & 15 & 28 \\
\hline & & & $180-360$ & poor & 15 & 47 \\
\hline
\end{tabular}




\section{Feed 3}

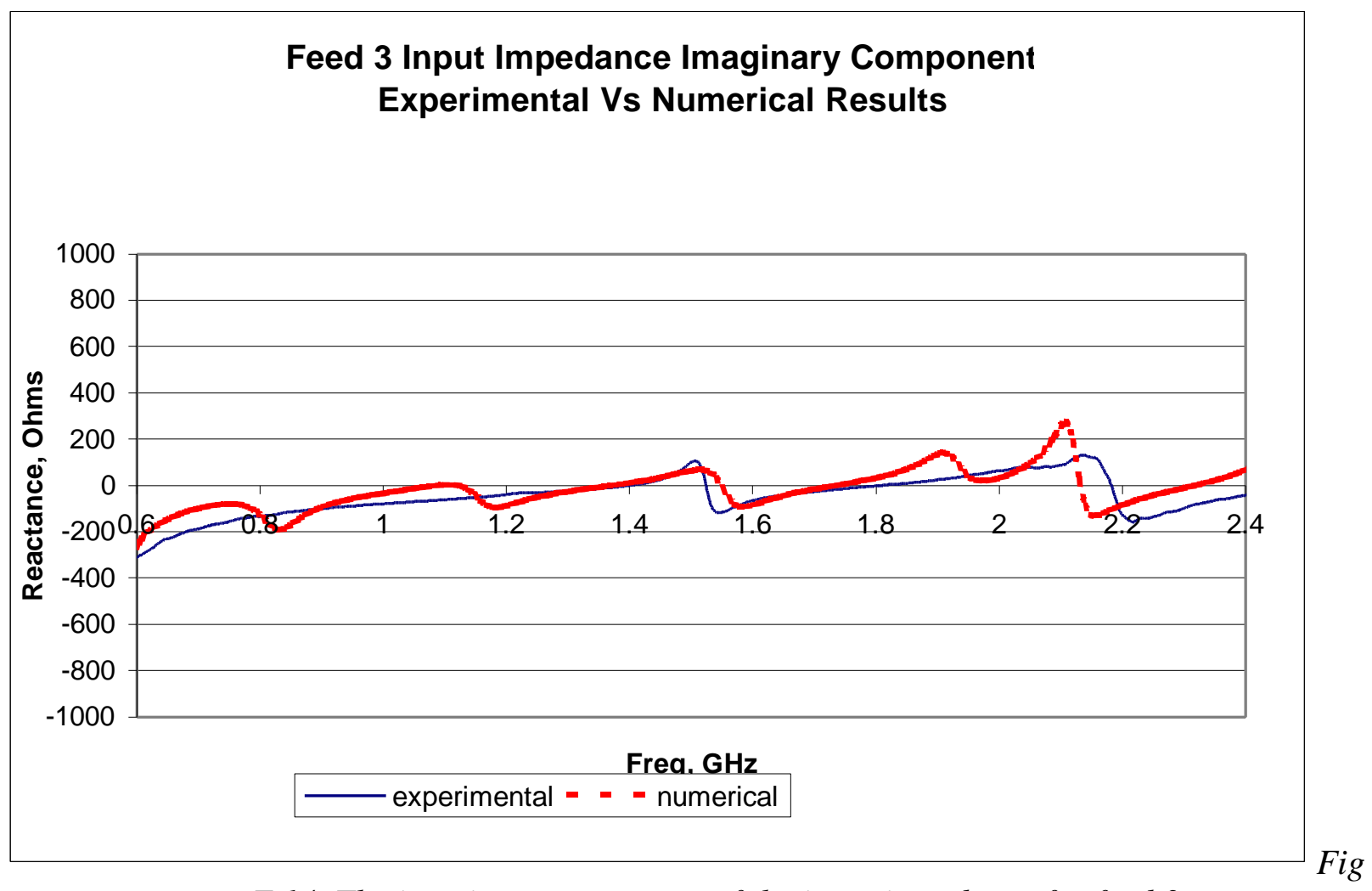

ure F.14 The imaginary component of the input impedance for feed 3

Table F.6 Analysis of resonance frequencies for feed 3

\begin{tabular}{|c|c|c|}
\hline \multicolumn{1}{|c|}{$\begin{array}{c}\text { f3 exp. } \\
(\mathrm{GHz})\end{array}$} & $\begin{array}{c}\text { f3 num. } \\
(\mathrm{GHz})\end{array}$ & $\begin{array}{c}\text { error } \\
\%\end{array}$ \\
\hline 0.479 & 0.548 & 14.40 \\
\hline & 1.088 & \\
\hline & 1.122 & \\
\hline 1.401 & 1.371 & 2.19 \\
\hline 1.524 & 1.548 & 1.59 \\
\hline 1.811 & 1.735 & 4.21 \\
\hline
\end{tabular}




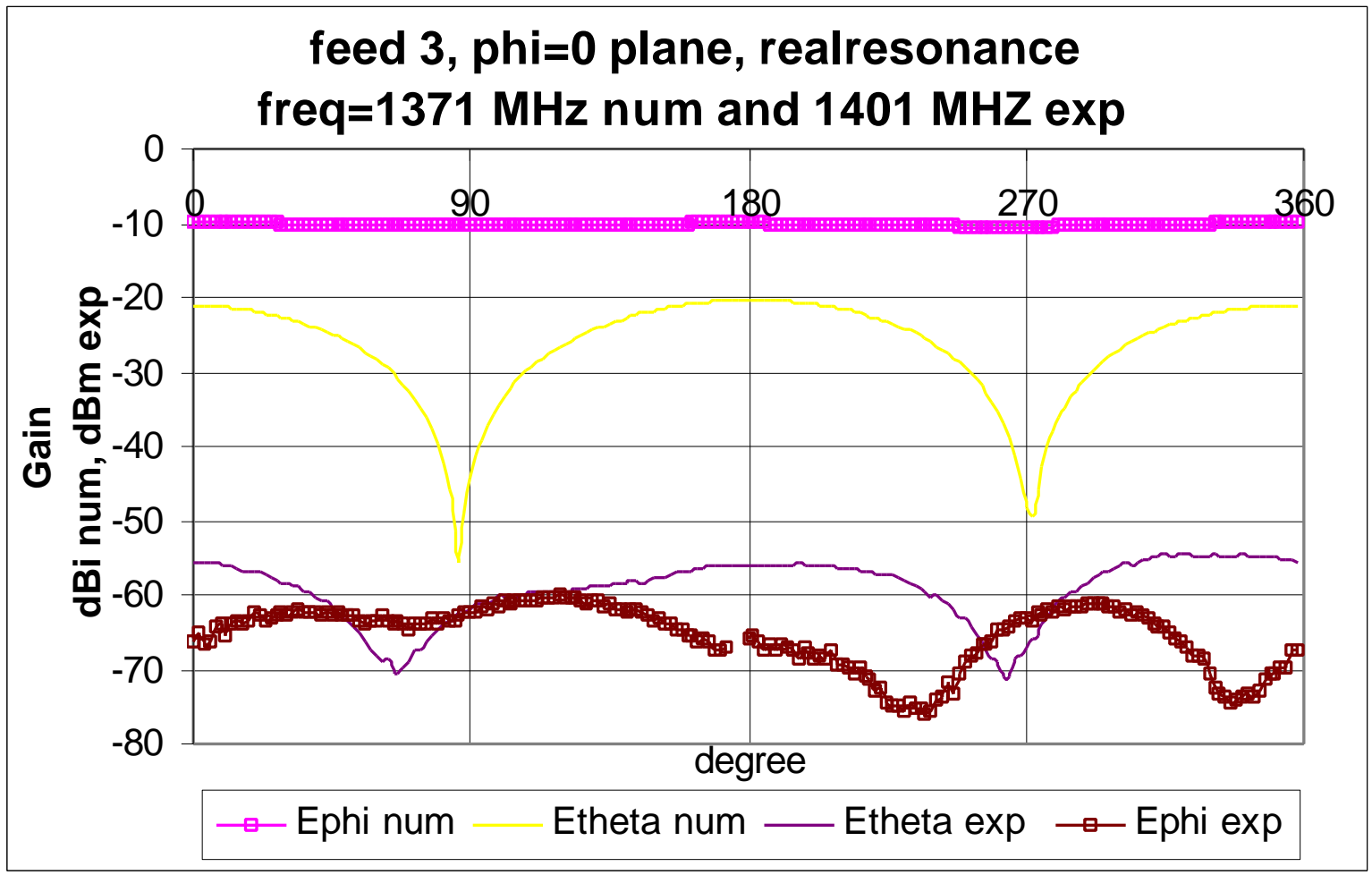

Figure F.15 The Phi = 0 plane for feed 3 at $1371 \mathrm{MHz}$ num and $1401 \mathrm{MHz}$ exp

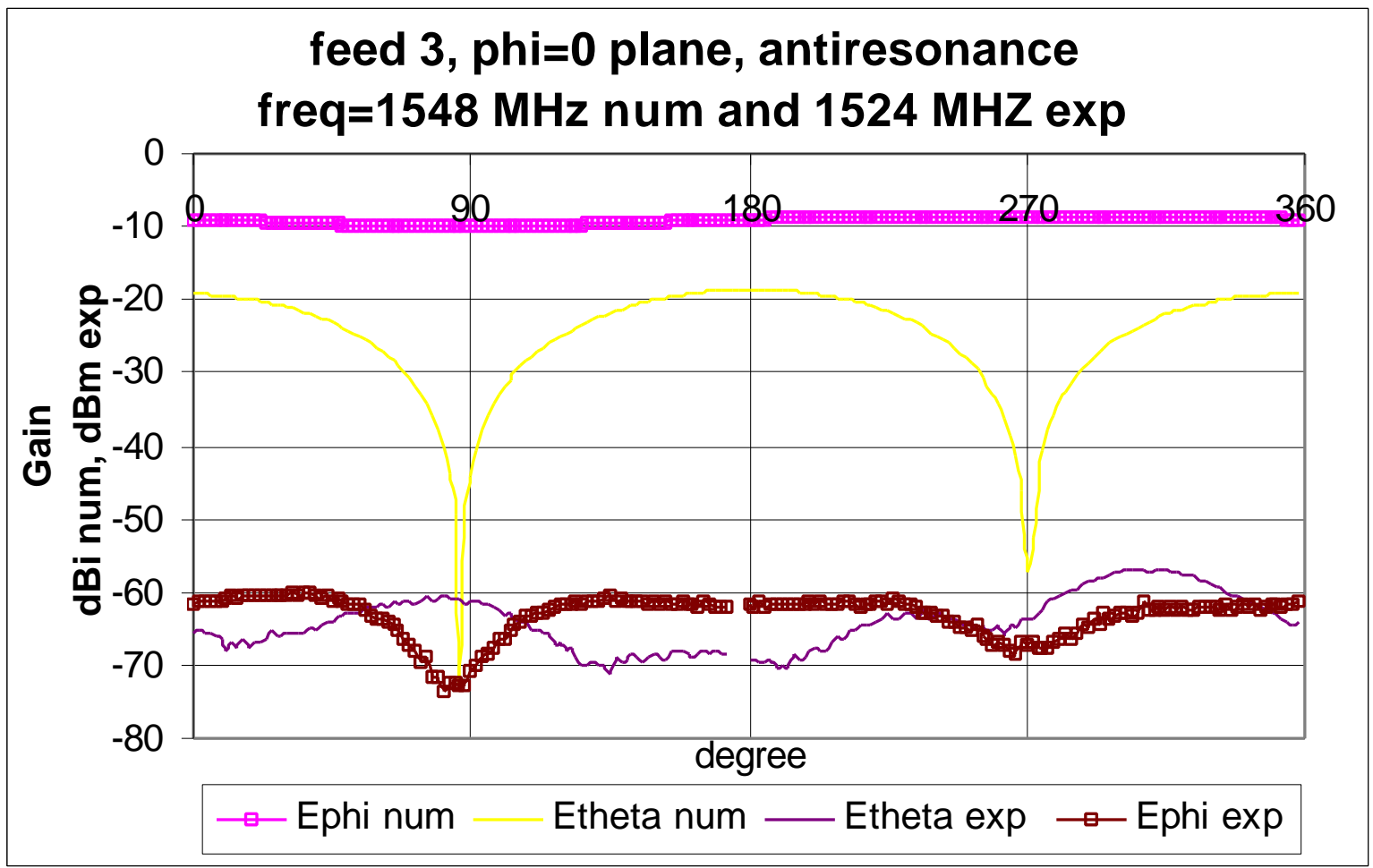

Figure F.16 The Phi = O plane for feed 3 at $1548 \mathrm{MHz}$ num and $1524 \mathrm{MHz}$ exp 


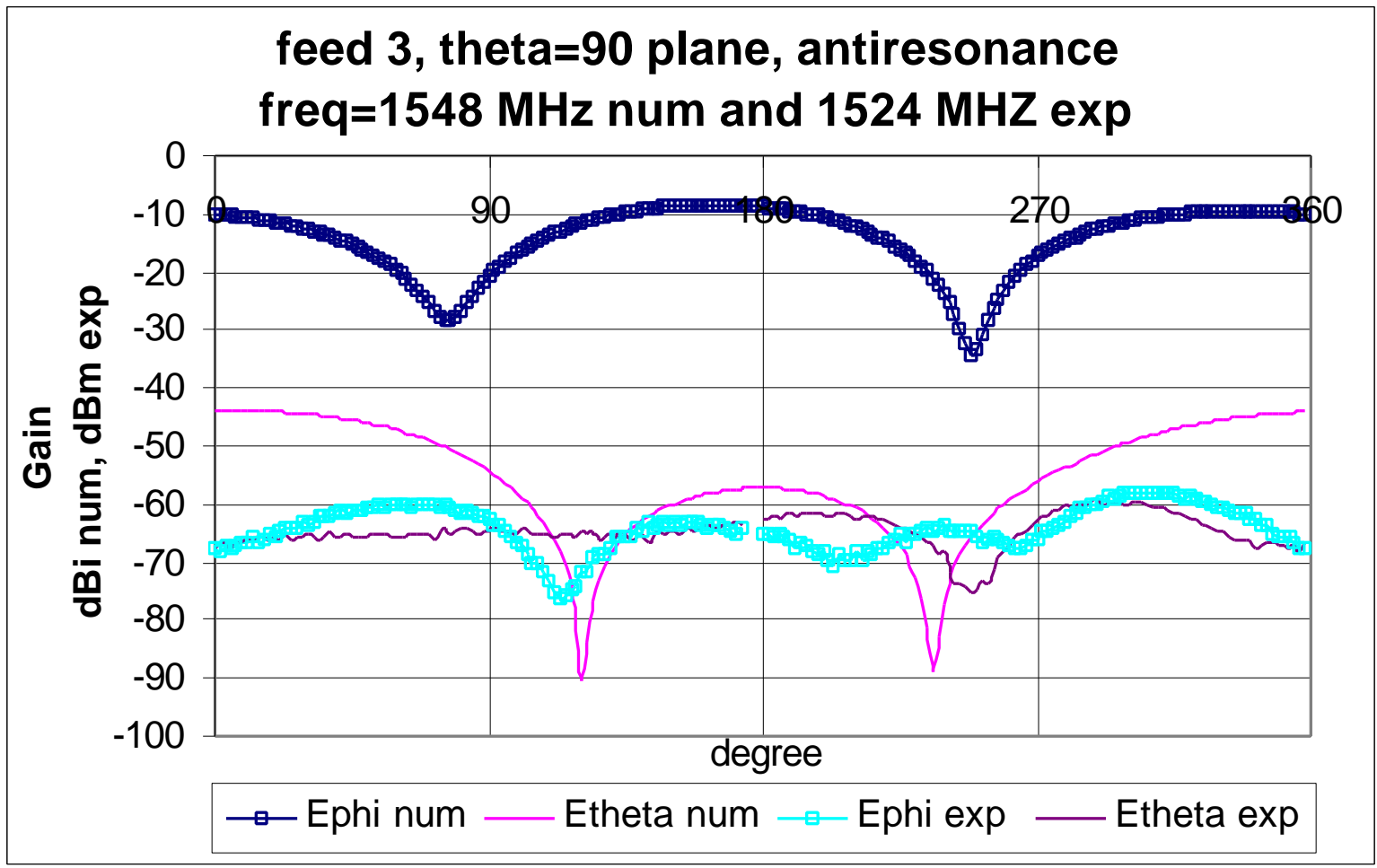

Figure F.17 The Theta $=90$ plane for feed 3 at $1548 \mathrm{MHz}$ num and $1524 \mathrm{MHz}$ exp

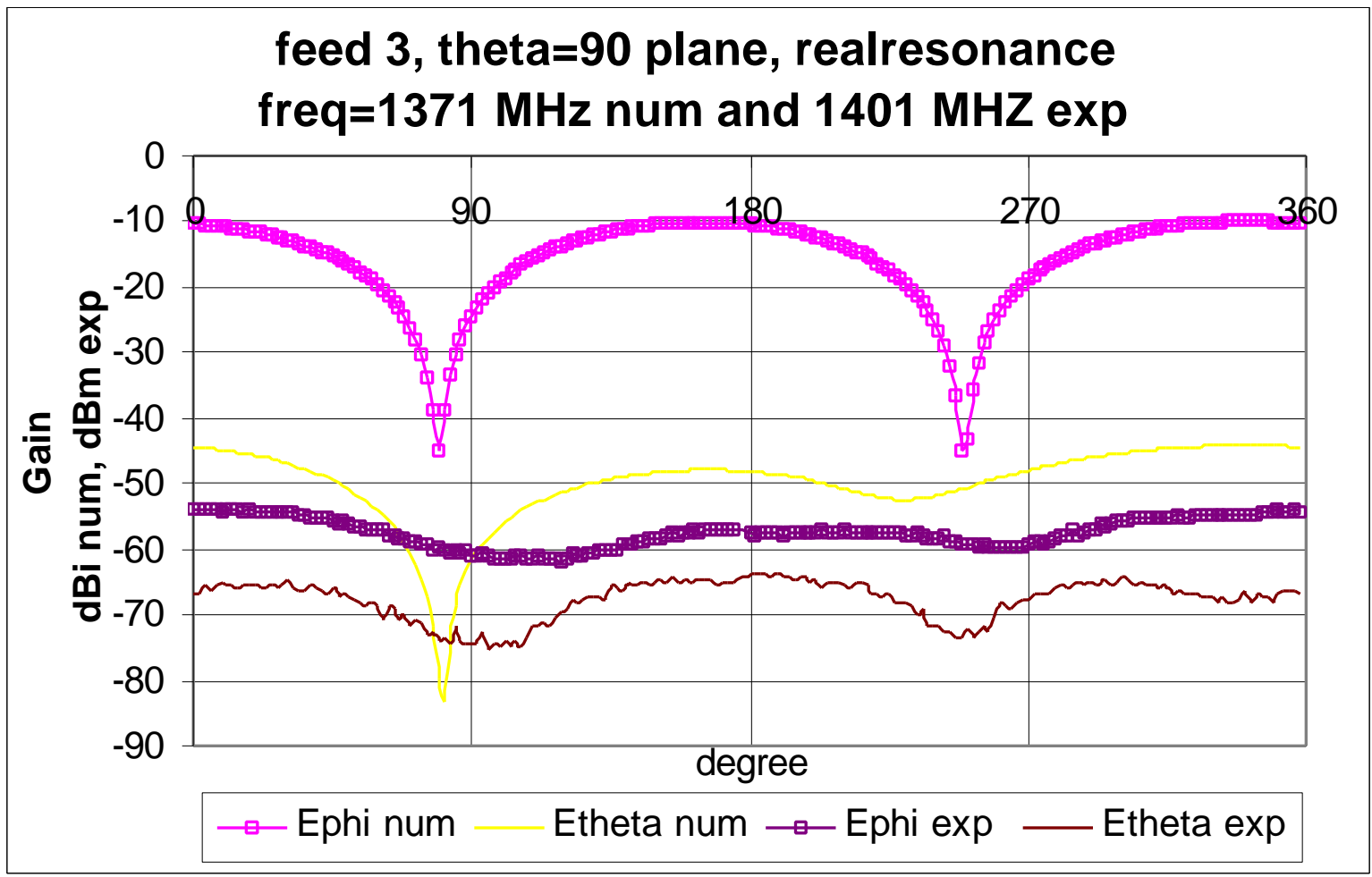

Figure F.18 The Theta $=90$ plane for feed 3 at $1371 \mathrm{MHz}$ num and $1401 \mathrm{MHz}$ exp 
Table F.7 Analysis of the steady state graphs for feed 3

\begin{tabular}{|c|c|c|c|c|c|c|}
\hline \multirow{2}{*}{ Plane } & \multirow[b]{2}{*}{ res. type } & \multirow[b]{2}{*}{ Graph } & \multirow[b]{2}{*}{ angles } & \multirow[b]{2}{*}{ score } & \multicolumn{2}{|c|}{$\mathrm{dBd}$} \\
\hline & & & & & $\min$ & $\max$ \\
\hline \multirow{8}{*}{$P h i=0$} & \multirow{4}{*}{ real } & \multirow{2}{*}{ Ephi } & $0-180$ & good & 56 & $\overline{71}$ \\
\hline & & & $180-360$ & poor & 55 & 71 \\
\hline & & \multirow{2}{*}{ Etheta } & $0-180$ & v. good & 54 & 65 \\
\hline & & & $180-360$ & v. good & 54 & $\overline{63}$ \\
\hline & \multirow{4}{*}{ anti } & \multirow{2}{*}{ Ephi } & $0-180$ & poor & 0 & 71 \\
\hline & & & $180-360$ & poor & 57 & 71 \\
\hline & & \multirow{2}{*}{ Etheta } & $0-180$ & poor & 9 & 58 \\
\hline & & & $180-360$ & poor & 51 & 67 \\
\hline \multirow{8}{*}{ Theta $=90$} & \multirow{4}{*}{ real } & \multirow{2}{*}{ Ephi } & $0-180$ & fair & 47 & 50 \\
\hline & & & $180-360$ & fair & 48 & 49 \\
\hline & & \multirow{2}{*}{ Etheta } & $0-180$ & good & 15 & 49 \\
\hline & & & $180-360$ & v. good & 14 & 47 \\
\hline & \multirow{4}{*}{ anti } & \multirow{2}{*}{ Ephi } & $0-180$ & v. good & 60 & 237 \\
\hline & & & $180-360$ & good & 239 & 411 \\
\hline & & \multirow{2}{*}{ Etheta } & $0-180$ & poor & 9 & 64 \\
\hline & & & $180-360$ & good & 32 & 60 \\
\hline
\end{tabular}




\section{Feed 4}

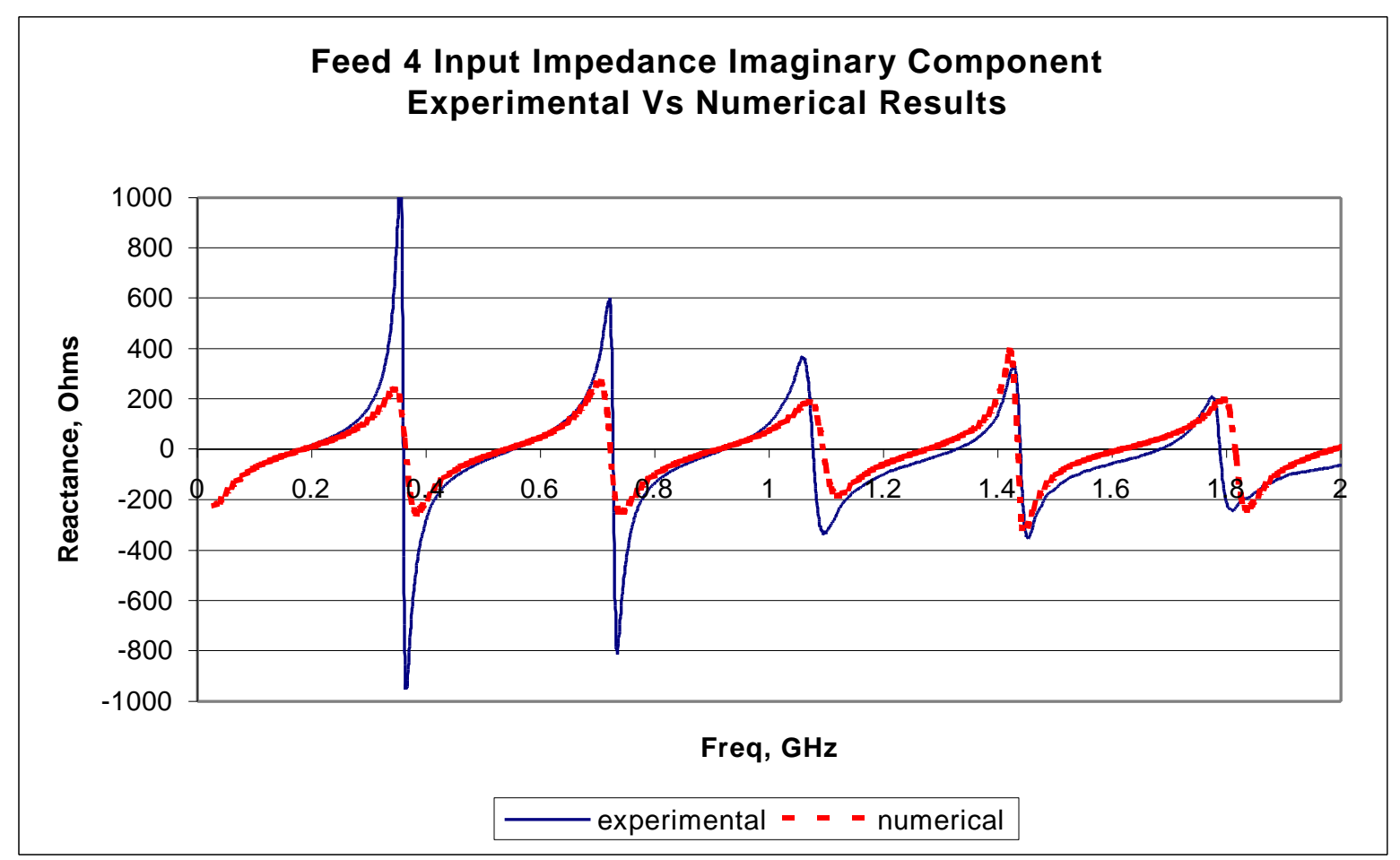

Figure F.19 The imaginary component of the input impedance for feed 4

Table F.8 Analysis of resonance frequencies for feed 4

\begin{tabular}{|c|c|c|}
\hline $\begin{array}{c}f 4 \text { exp. } \\
(\mathrm{GHz})\end{array}$ & $\begin{array}{c}\text { diff. between } \\
\text { res freq's }\end{array}$ & $\begin{array}{c}\text { diff. between } \\
\text { same type freq's }\end{array}$ \\
\hline 0.181 & & \\
\hline 0.360 & 0.180 & \\
\hline 0.552 & 0.192 & 0.371 \\
\hline 0.727 & 0.175 & 0.367 \\
\hline 0.922 & 0.194 & 0.369 \\
\hline 1.077 & 0.155 & 0.350 \\
\hline 1.327 & 0.250 & 0.406 \\
\hline 1.440 & 0.113 & 0.363 \\
\hline 1.683 & 0.243 & 0.356 \\
\hline 1.789 & 0.106 & 0.349 \\
\hline mean & 0.179 & 0.366 \\
\hline
\end{tabular}

\begin{tabular}{|c|c|c|}
\hline $\begin{array}{c}\text { f4 num. } \\
\text { (GHz) }\end{array}$ & $\begin{array}{l}\text { diff between } \\
\text { res freq's }\end{array}$ & $\begin{array}{c}\text { diff between } \\
\text { same type freq's }\end{array}$ \\
\hline \multicolumn{3}{|l|}{0.187} \\
\hline 0.366 & 0.179 & \\
\hline 0.537 & 0.171 & 0.350 \\
\hline 0.715 & 0.178 & 0.349 \\
\hline 0.915 & 0.200 & 0.378 \\
\hline 1.111 & 0.196 & 0.396 \\
\hline 1.291 & 0.180 & 0.376 \\
\hline 1.448 & 0.157 & 0.337 \\
\hline 1.627 & 0.178 & 0.335 \\
\hline 1.834 & 0.207 & 0.385 \\
\hline mean & 0.183 & 0.363 \\
\hline
\end{tabular}

\begin{tabular}{|c|}
\hline $\begin{array}{c}\text { error } \\
\%\end{array}$ \\
\hline 3.34 \\
\hline 1.59 \\
\hline 2.73 \\
\hline 1.71 \\
\hline 0.67 \\
\hline 3.16 \\
\hline 2.71 \\
\hline 0.59 \\
\hline 3.37 \\
\hline 2.50 \\
\hline
\end{tabular}




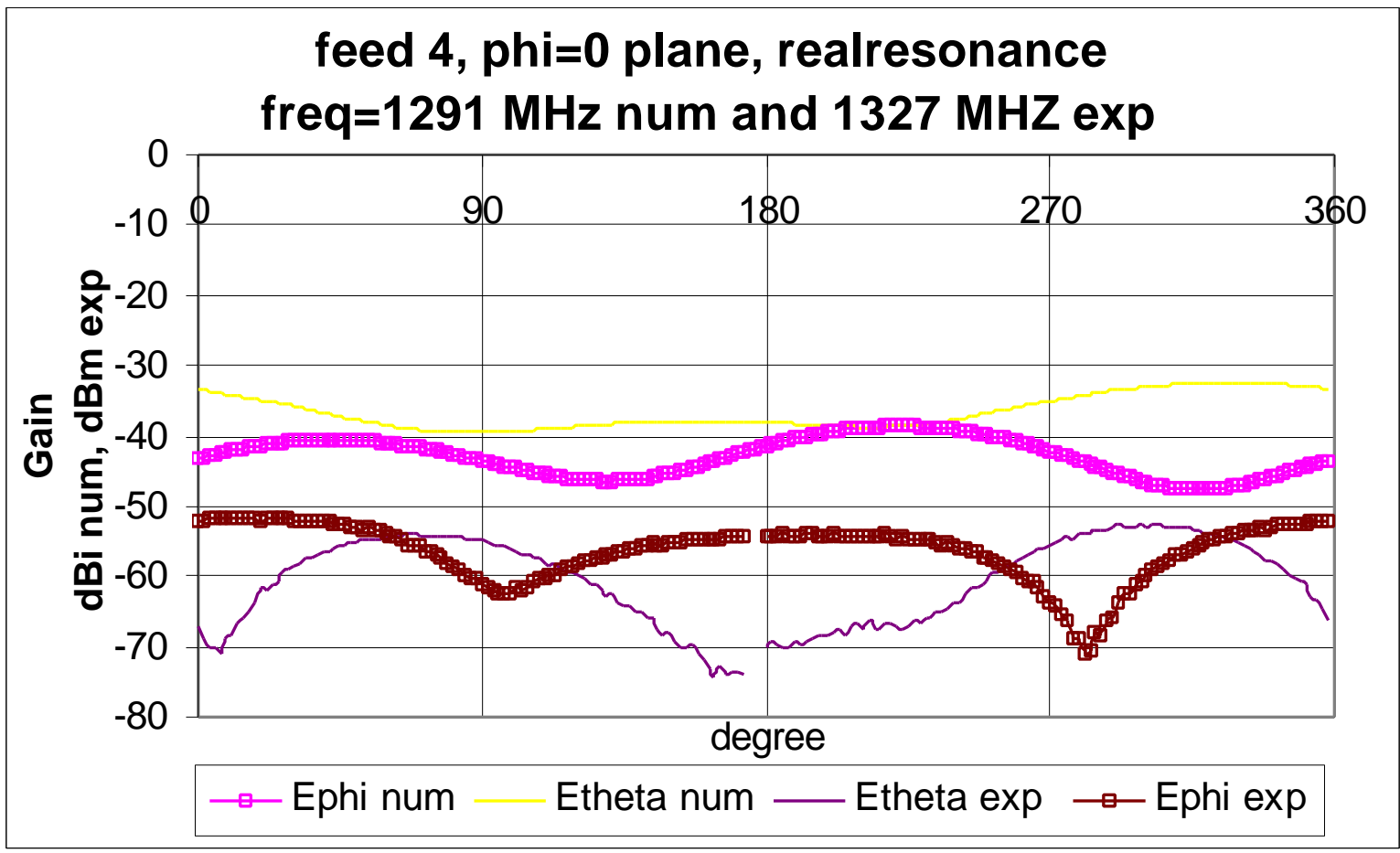

Figure F.20 The Phi = O plane for feed 4 at $1291 \mathrm{MHz}$ num and $1327 \mathrm{MHz}$ exp

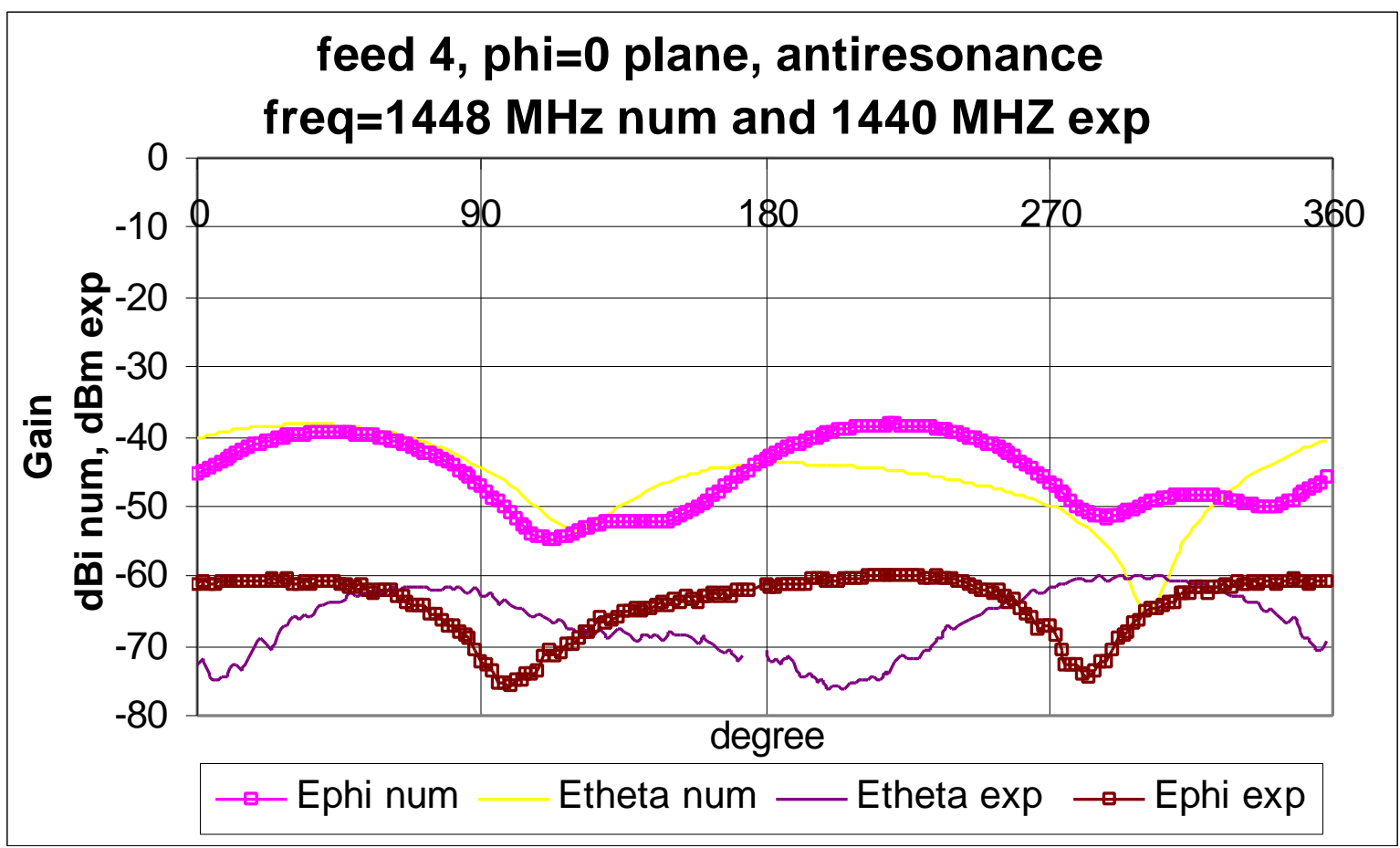

Figure F.21 The Phi $=0$ plane for feed 4 at $1448 \mathrm{MHz}$ num and $1440 \mathrm{MHz}$ exp 


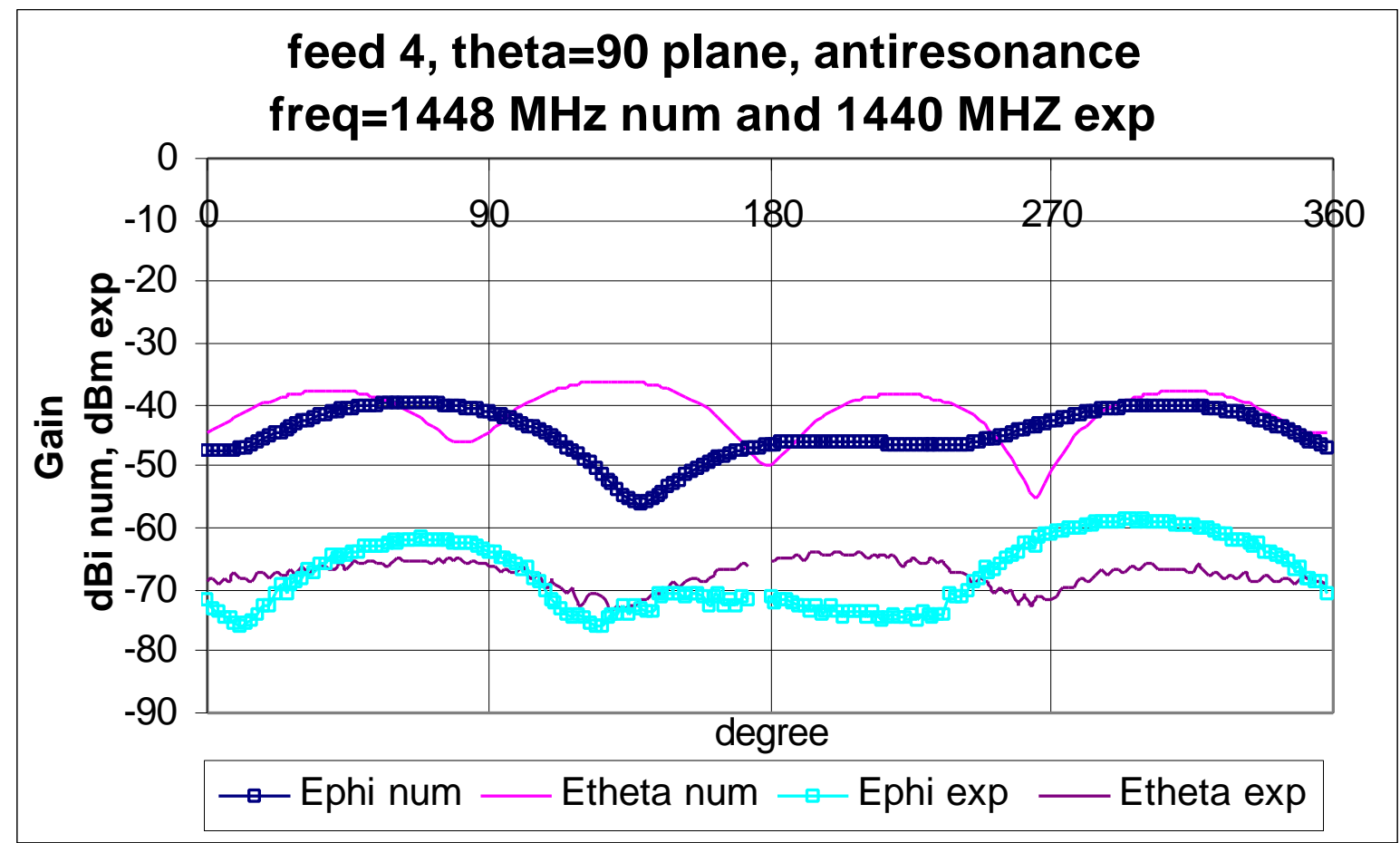

Figure F.22 The Theta $=90$ plane for feed 4 at $1448 \mathrm{MHz}$ num and $1440 \mathrm{MHz}$ exp

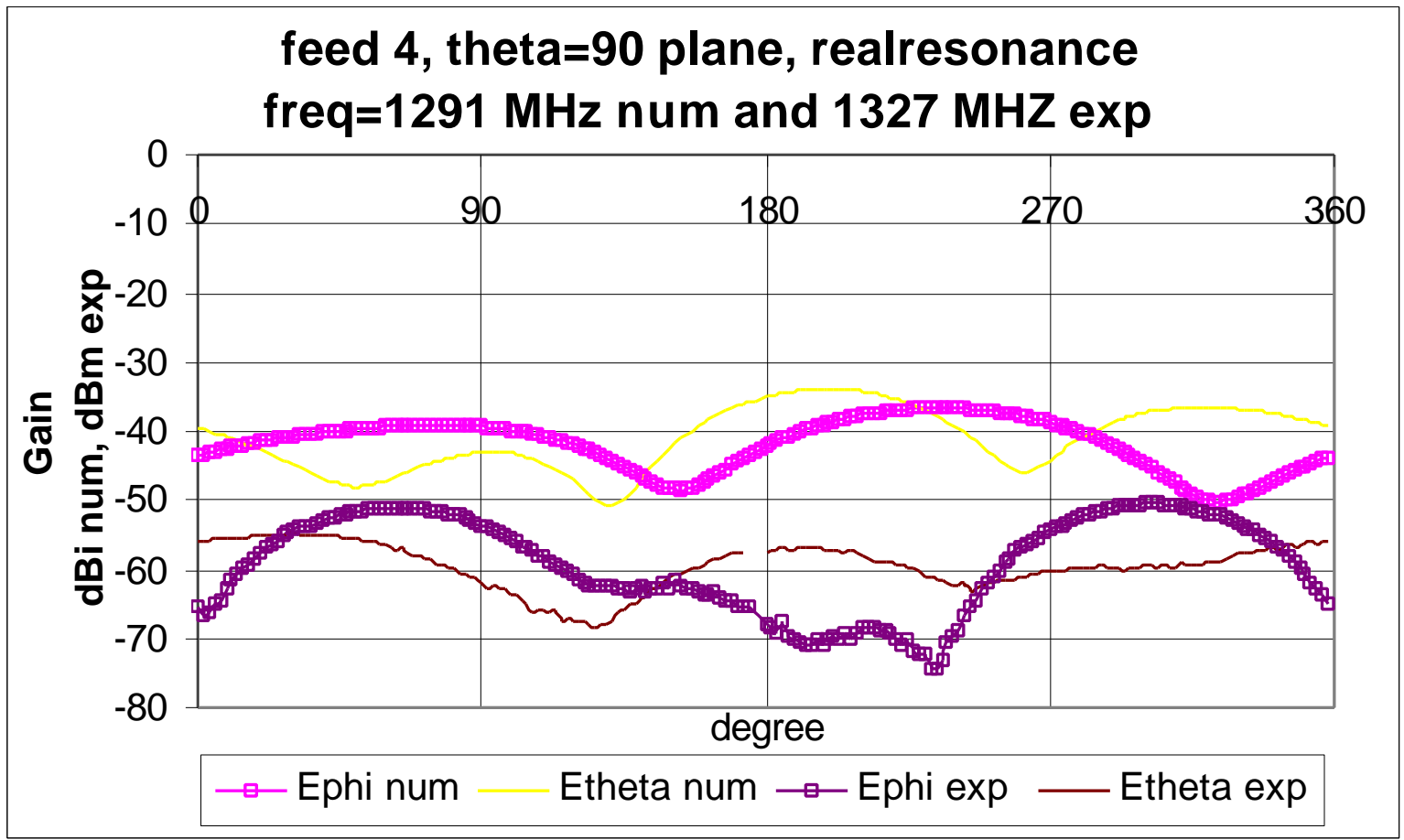

Figure F.23 The Theta $=90$ plane for feed 4 at $1291 \mathrm{MHz}$ num and $1327 \mathrm{MHz}$ exp 
Table F.9 Analysis of the steady state graphs for feed 4

\begin{tabular}{|c|c|c|c|c|c|c|}
\hline & & & & & & \\
\hline Plane & res. type & Graph & angles & score & $\min$ & $\max$ \\
\hline & & Enhi & $0-180$ & v. good & 9 & 42 \\
\hline & real & ᄃрmII & $180-360$ & good & 6 & 43 \\
\hline & real & Ftheta & $0-180$ & poor & 15 & 38 \\
\hline & & Cunta & $180-360$ & v. good & 19 & 34 \\
\hline $\mathrm{rnl}=0$ & & Enhi & $0-180$ & v. good & 12 & 26 \\
\hline & anti & ᄃрі11 & $180-360$ & v. good & 11 & 46 \\
\hline & anll & Ftheta & $0-180$ & fair & 13 & 44 \\
\hline & & Lutela & $180-360$ & poor & 1 & 41 \\
\hline & & Fnhi & $0-180$ & v. good & 12 & 28 \\
\hline & roal & 다이 & $180-360$ & fair & 2 & 44 \\
\hline & real & Ftheta & $0-180$ & v. good & 8 & 36 \\
\hline Theta $=90$ & & & $180-360$ & v. good & 15 & 40 \\
\hline metd $=90$ & & & $0-180$ & v. good & 16 & 47 \\
\hline & anti & Epni & $180-360$ & v. good & 18 & 47 \\
\hline & allu & Ftheta & $0-180$ & poor & 15 & 48 \\
\hline & & cmela & $180-360$ & v. good & 16 & 45 \\
\hline
\end{tabular}




\section{Feed 5}

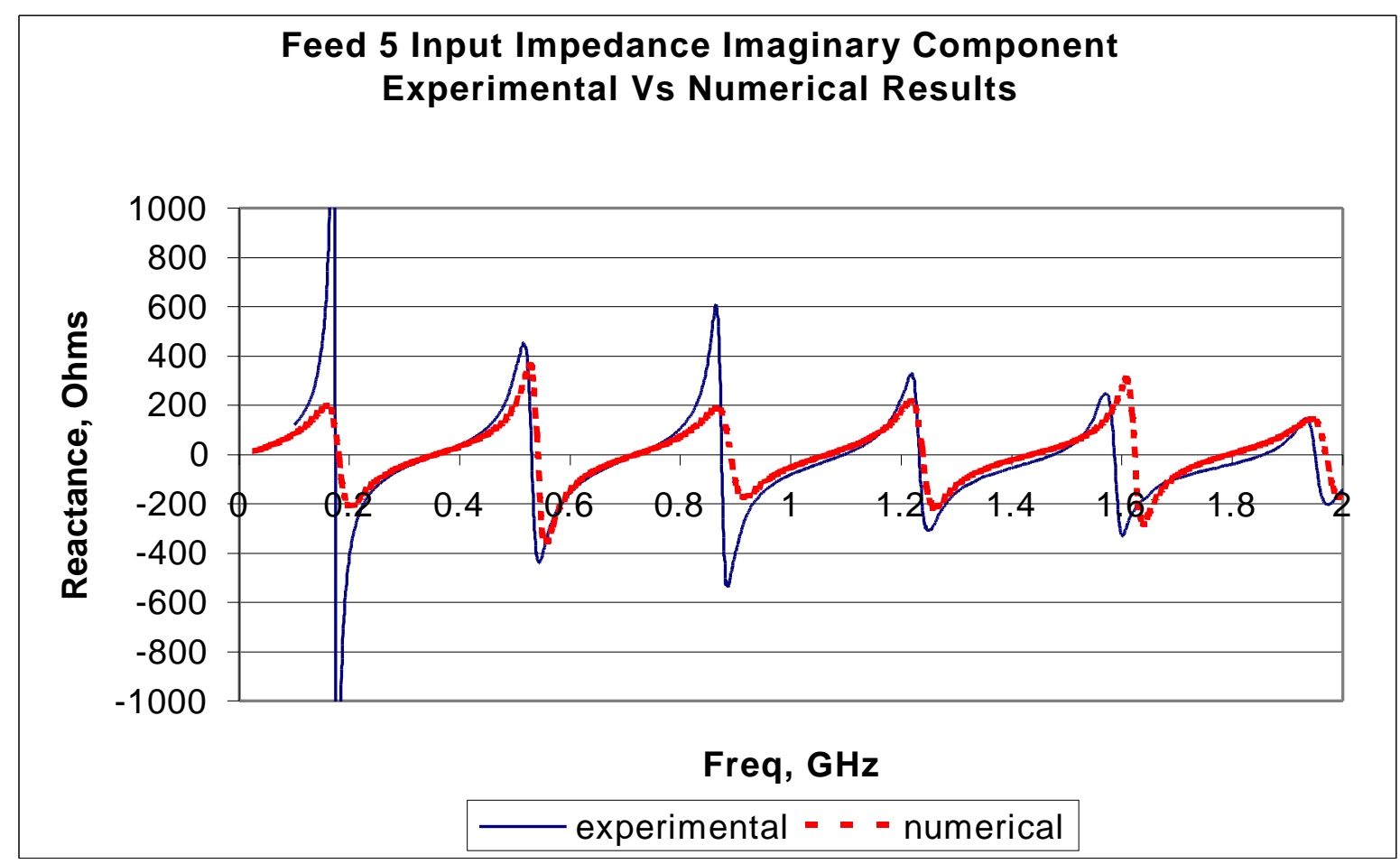

Figure F.24 The imaginary component of the input impedance for feed 5

Table F.10 Analysis of resonance frequencies for feed 5

\begin{tabular}{|c|c|c|}
\hline $\begin{array}{c}\text { f5 exp. } \\
(\mathrm{GHz})\end{array}$ & $\begin{array}{c}\text { diff. between } \\
\text { res freq's }\end{array}$ & $\begin{array}{c}\text { diff. between } \\
\text { same type freq's }\end{array}$ \\
\hline 0.174 & & \\
\hline 0.358 & 0.184 & \\
\hline 0.53 & 0.172 & 0.356 \\
\hline 0.719 & 0.189 & 0.361 \\
\hline 0.875 & 0.156 & 0.345 \\
\hline 1.096 & 0.221 & 0.377 \\
\hline 1.233 & 0.137 & 0.358 \\
\hline 1.476 & 0.243 & 0.38 \\
\hline 1.587 & 0.111 & 0.354 \\
\hline 1.856 & 0.269 & 0.38 \\
\hline mean & 0.187 & 0.364 \\
\hline
\end{tabular}

\begin{tabular}{|c|c|c|}
\hline $\begin{array}{c}\text { f5 num. } \\
(\mathrm{GHz})\end{array}$ & $\begin{array}{l}\text { Numerical } \\
\text { diff between } \\
\text { res freq's }\end{array}$ & $\begin{array}{c}\text { diff between } \\
\text { same type freq's }\end{array}$ \\
\hline 0.186 & & \\
\hline 0.366 & 0.18 & \\
\hline 0.549 & 0.183 & 0.363 \\
\hline 0.728 & 0.179 & 0.362 \\
\hline 0.904 & 0.176 & 0.355 \\
\hline 1.086 & 0.182 & 0.358 \\
\hline 1.263 & 0.177 & 0.359 \\
\hline 1.46 & 0.197 & 0.374 \\
\hline 1.646 & 0.186 & 0.383 \\
\hline 1.822 & 0.176 & 0.362 \\
\hline mean & 0.182 & 0.365 \\
\hline
\end{tabular}

\begin{tabular}{|c|}
\hline $\begin{array}{c}\text { error } \\
\%\end{array}$ \\
\hline 6.90 \\
\hline 2.23 \\
\hline 3.58 \\
\hline 1.25 \\
\hline 3.31 \\
\hline 0.91 \\
\hline 2.43 \\
\hline 1.08 \\
\hline 3.72 \\
\hline 1.83 \\
\hline
\end{tabular}




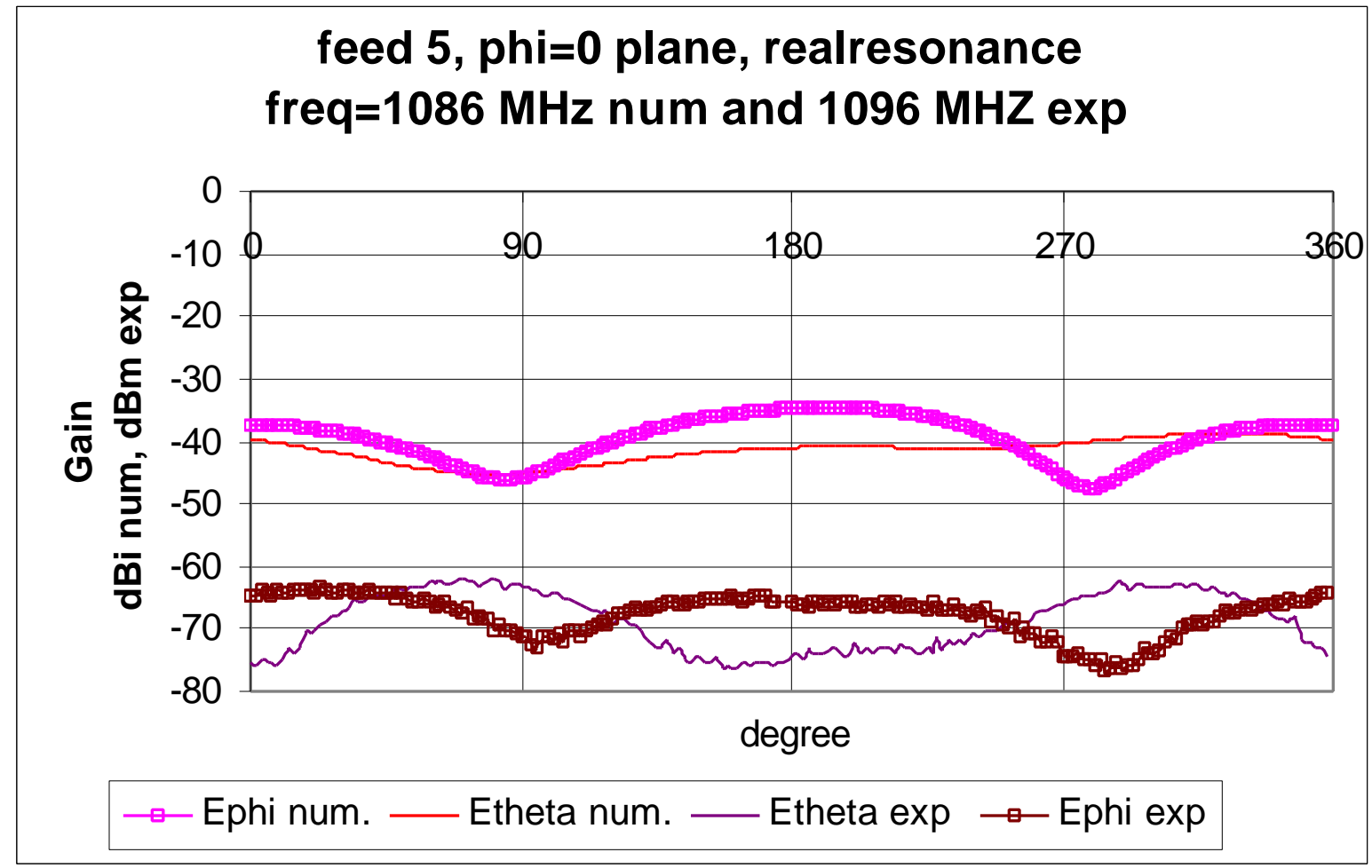

Figure F.25 The Phi = 0 plane for feed 5 at $1086 \mathrm{MHz}$ num and $1096 \mathrm{MHz}$ exp

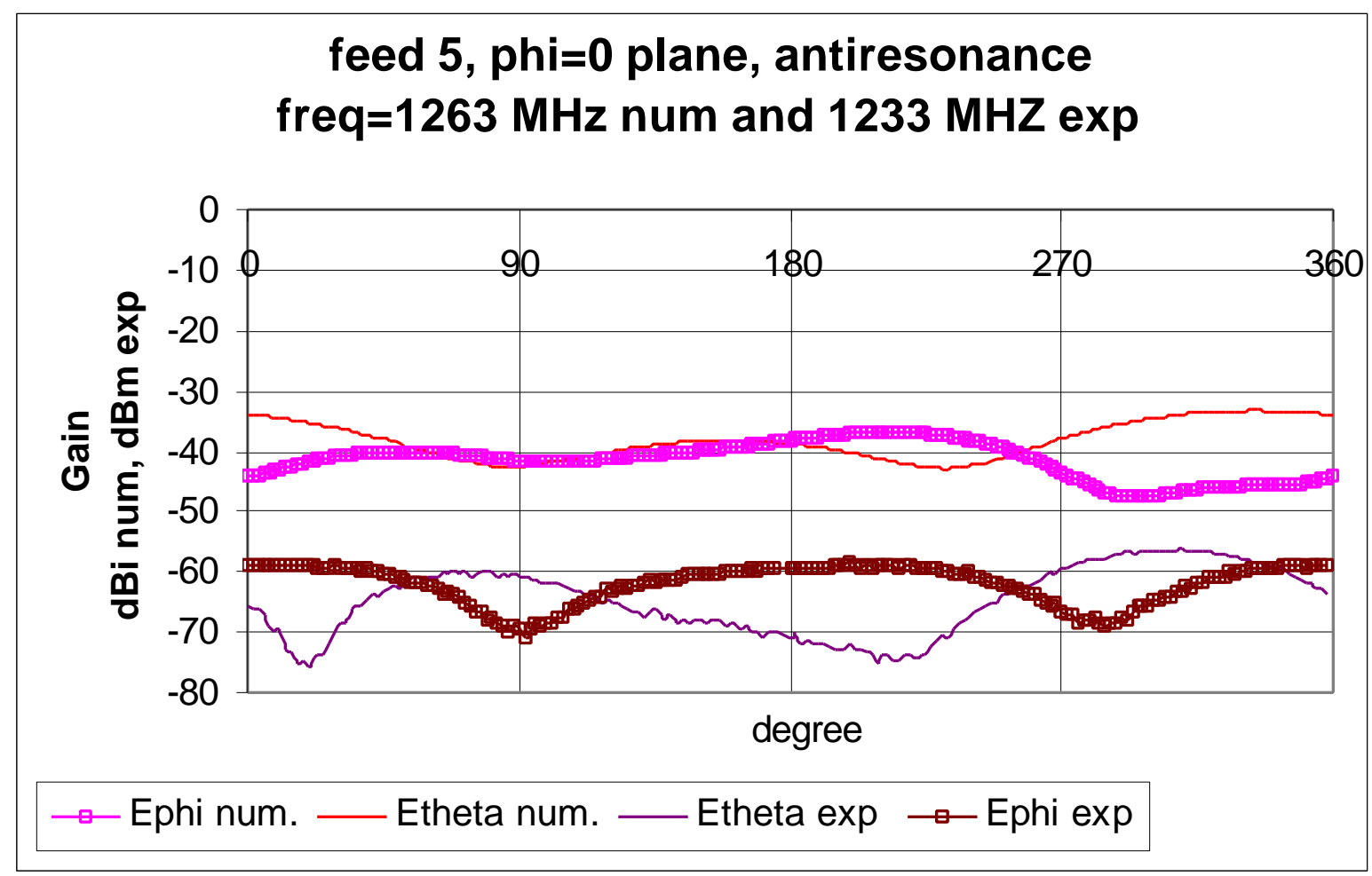

Figure F.26 The Phi = O plane for feed 5 at $1263 \mathrm{MHz}$ num and $1233 \mathrm{MHz}$ exp 


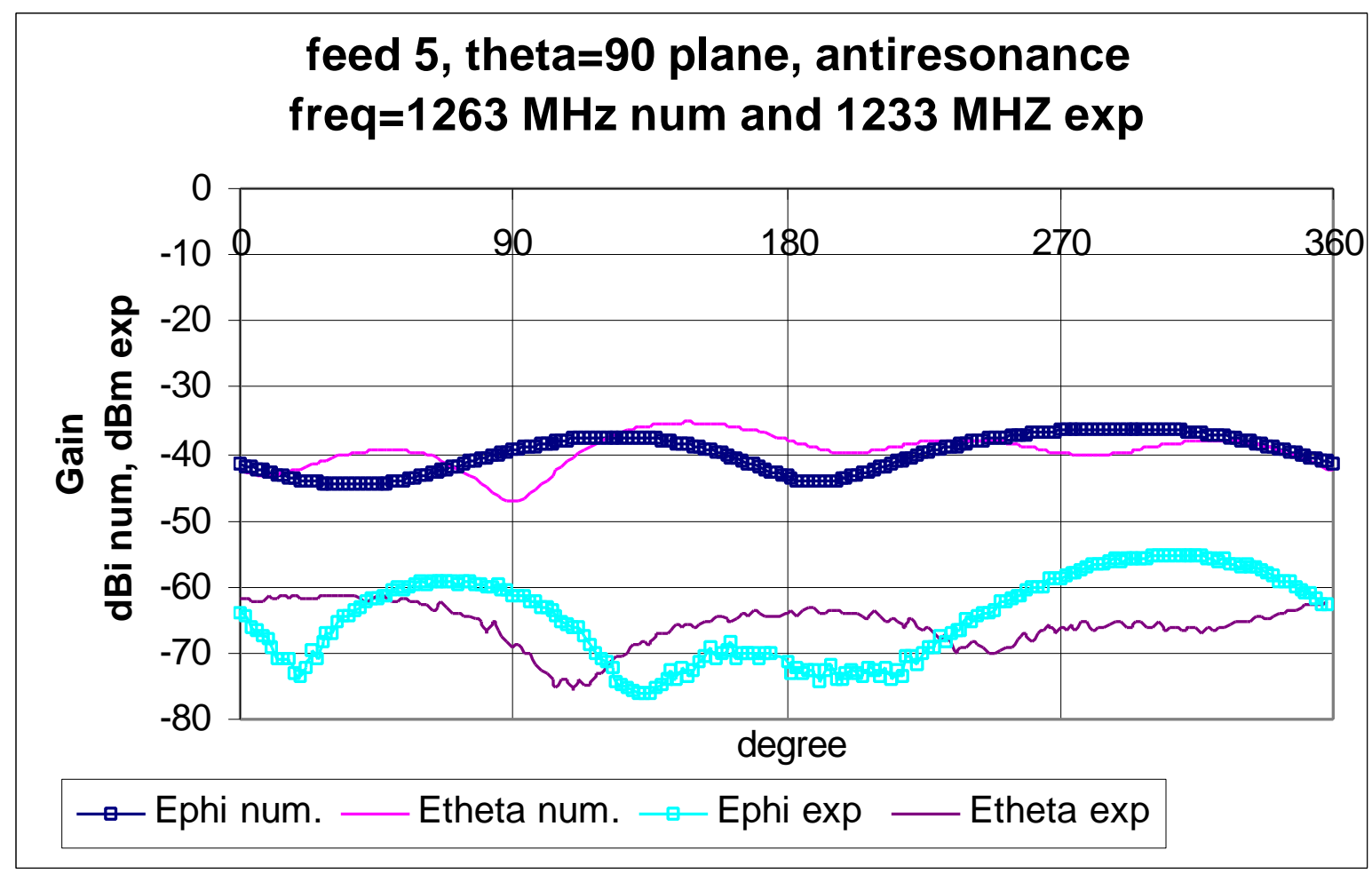

Figure F.27 The Theta $=90$ plane for feed 5 at $1263 \mathrm{MHz}$ num and $1233 \mathrm{MHz}$ exp

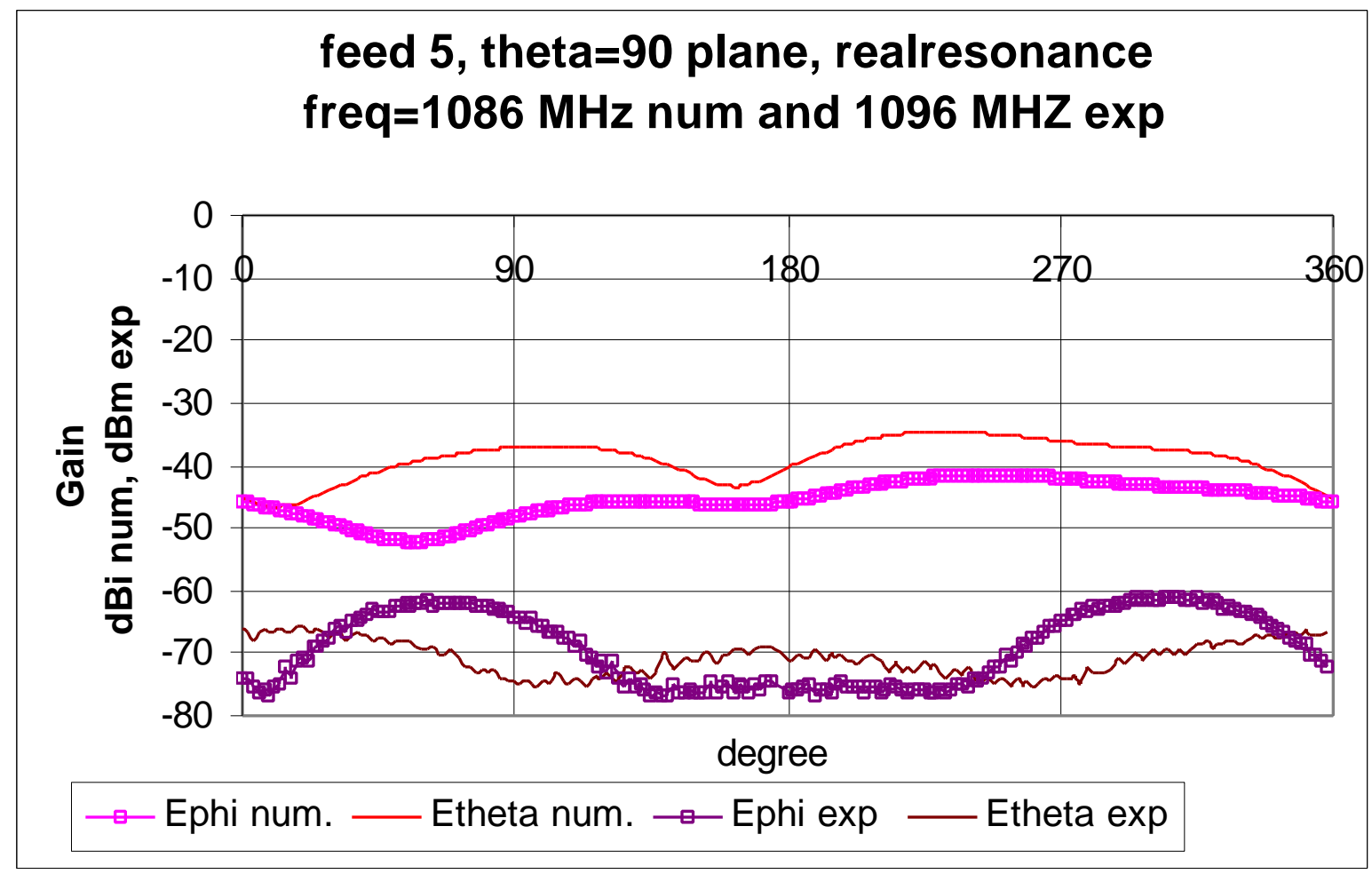

Figure F.28 The Theta $=90$ plane for feed 5 at $1263 \mathrm{MHz}$ num and $1233 \mathrm{MHz}$ exp 
Table F.11 Analysis of the steady state graphs for feed 5

\begin{tabular}{|c|c|c|c|c|c|c|}
\hline \multirow{2}{*}{ Plane } & \multirow[b]{2}{*}{ res. type } & \multirow[b]{2}{*}{ Graph } & \multirow[b]{2}{*}{ angles } & \multirow[b]{2}{*}{ score } & \multicolumn{2}{|c|}{$\mathrm{dBd}$} \\
\hline & & & & & $\min$ & $\max$ \\
\hline \multirow{8}{*}{$P h i=0$} & \multirow{4}{*}{ real } & \multirow{2}{*}{ Ephi } & $0-180$ & v. good & 21 & 32 \\
\hline & & & $180-360$ & v. good & 27 & 37 \\
\hline & & \multirow{2}{*}{ Etheta } & $0-180$ & poor & 17 & 35 \\
\hline & & & $180-360$ & fair & 23 & 40 \\
\hline & \multirow{4}{*}{ anti } & \multirow{2}{*}{ Ephi } & $0-180$ & good & 15 & 29 \\
\hline & & & $180-360$ & v. good & 13 & 45 \\
\hline & & \multirow{2}{*}{ Etheta } & $0-180$ & poor & 18 & 40 \\
\hline & & & $180-360$ & v. good & 21 & 34 \\
\hline \multirow{8}{*}{ Theta $=90$} & \multirow{4}{*}{ real } & \multirow{2}{*}{ Ephi } & $0-180$ & poor & 10 & 32 \\
\hline & & & $180-360$ & fair & 17 & 46 \\
\hline & & \multirow{2}{*}{ Etheta } & $0-180$ & poor & 19 & 39 \\
\hline & & & $180-360$ & poor & 22 & 45 \\
\hline & \multirow{4}{*}{ anti } & \multirow{2}{*}{ Ephi } & $0-180$ & fair & 16 & 38 \\
\hline & & & $180-360$ & v. good & 19 & 42 \\
\hline & & \multirow{2}{*}{ Etheta } & $0-180$ & v. good & 18 & 36 \\
\hline & & & $180-360$ & v. good & 20 & 42 \\
\hline
\end{tabular}




\section{Feed 6}

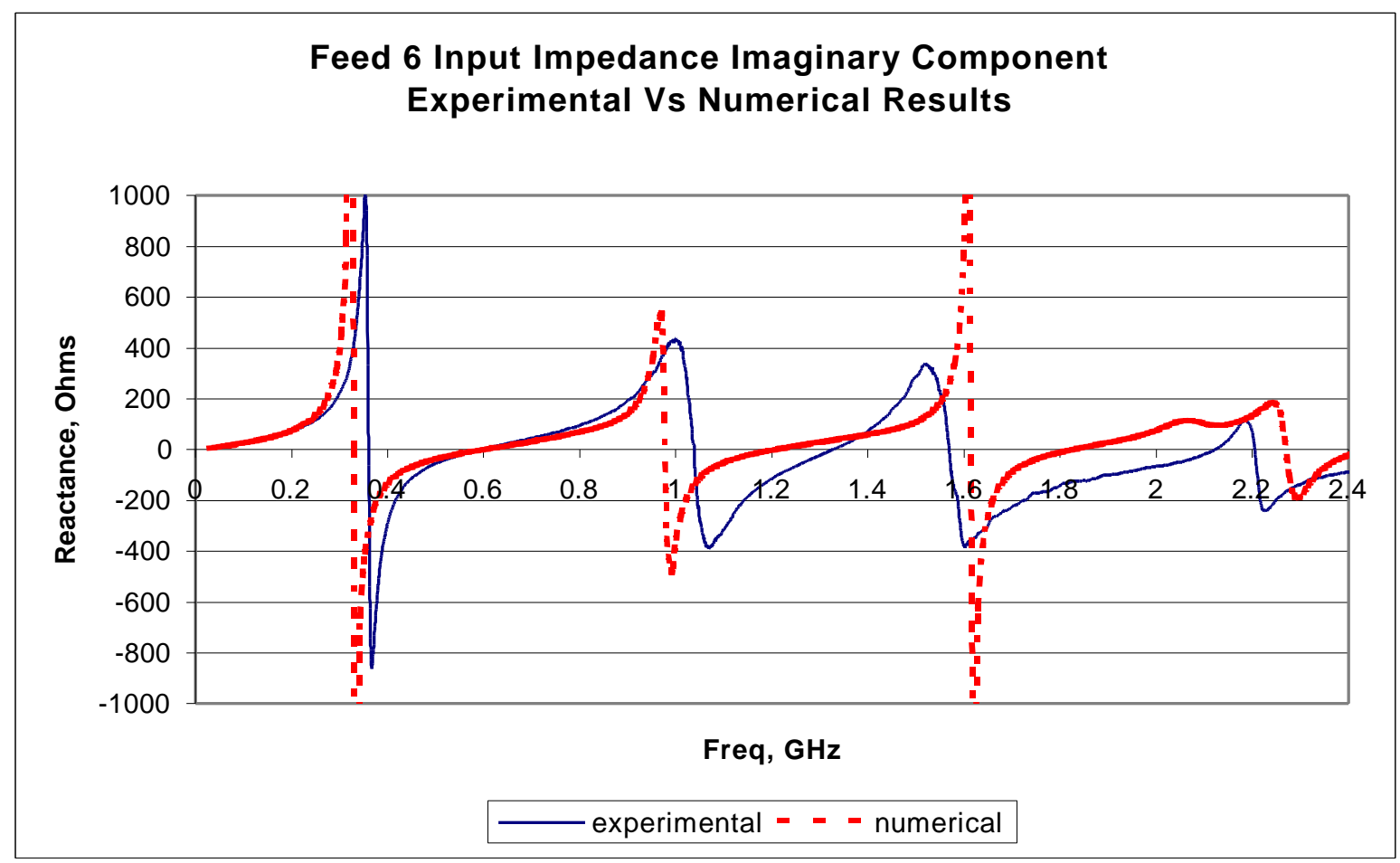

Figure F.29 The imaginary component of the input impedance for feed 6

Table F.12 Analysis of resonance frequencies for feed 6

\begin{tabular}{|c|c|c|}
\hline $\begin{array}{c}\text { f6 exp. } \\
(\mathrm{GHz})\end{array}$ & $\begin{array}{c}\text { diff. between } \\
\text { res freq's }\end{array}$ & $\begin{array}{c}\text { diff. between } \\
\text { same type freq's }\end{array}$ \\
\hline 0.361 & & \\
\hline 0.593 & 0.232 & \\
\hline 1.038 & 0.445 & 0.677 \\
\hline 1.326 & 0.289 & 0.734 \\
\hline 1.569 & 0.242 & 0.531 \\
\hline & & \\
\hline mean & 0.302 & 0.647 \\
\hline
\end{tabular}

\begin{tabular}{|c|c|c|}
\hline $\begin{array}{c}\text { f6 num. } \\
(\mathrm{GHz})\end{array}$ & $\begin{array}{c}\text { diff between } \\
\text { res freq's }\end{array}$ & $\begin{array}{c}\text { diff between } \\
\text { same type freq's }\end{array}$ \\
\hline 0.330 & & \\
\hline 0.598 & 0.268 & \\
\hline 0.978 & 0.379 & 0.647 \\
\hline 1.206 & 0.228 & 0.607 \\
\hline 1.615 & 0.409 & 0.637 \\
\hline 1.825 & 0.210 & 0.620 \\
\hline mean & 0.299 & 0.628 \\
\hline
\end{tabular}

\begin{tabular}{|c|}
\hline $\begin{array}{c}\text { error } \\
\%\end{array}$ \\
\hline 8.39 \\
\hline 0.92 \\
\hline 5.80 \\
\hline 9.12 \\
\hline 2.93 \\
\hline \\
\hline
\end{tabular}




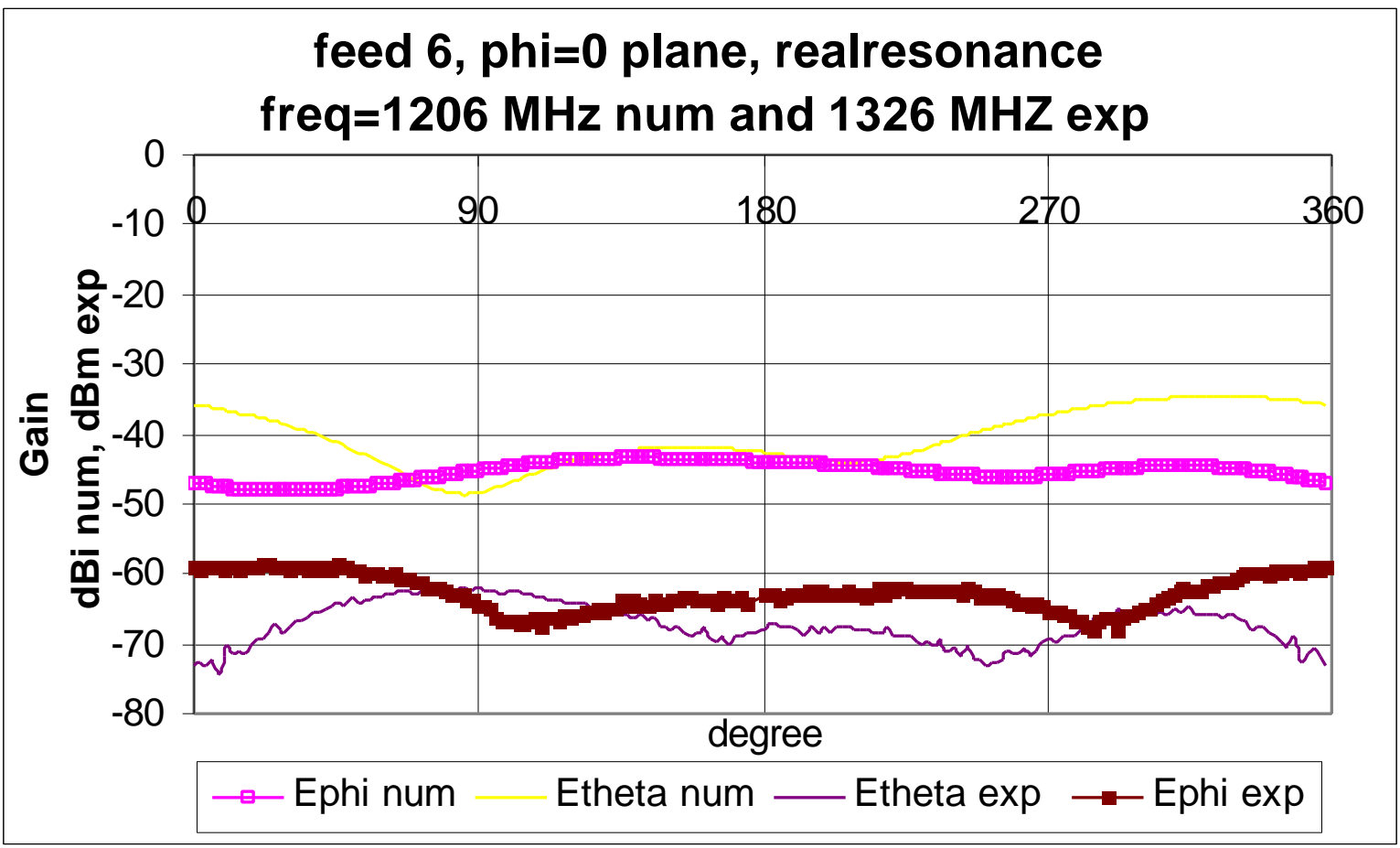

Figure F.30 The Phi = O plane for feed 6 at $1206 \mathrm{MHz}$ num and $1326 \mathrm{MHz}$ exp

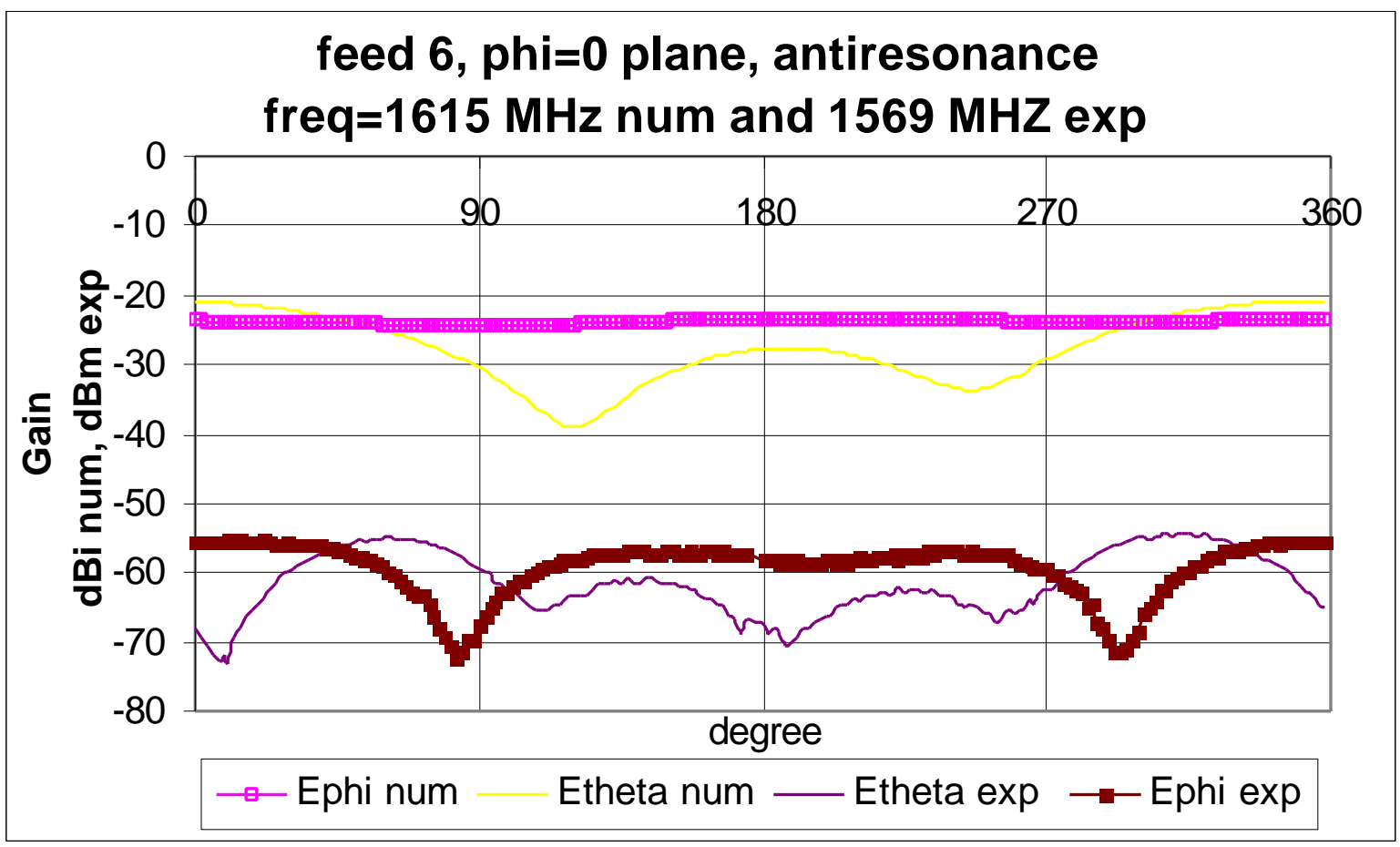

Figure F.31 The Phi =O plane for feed 6 at $1615 \mathrm{MHz}$ num and $1569 \mathrm{MHz}$ exp 


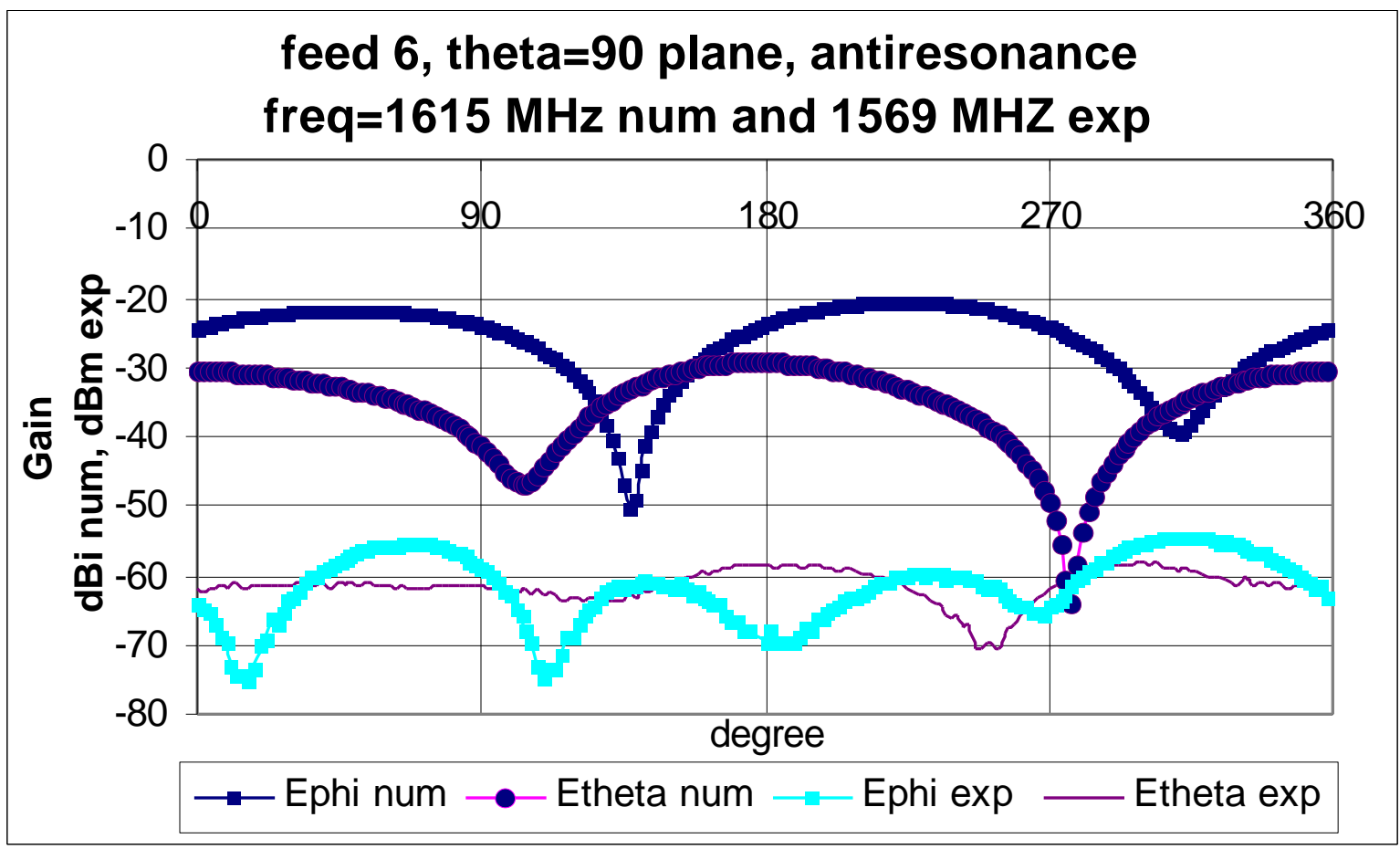

Figure F.32 The Theta $=90$ plane for feed 6 at $1615 \mathrm{MHz}$ num and $1569 \mathrm{MHz}$ exp

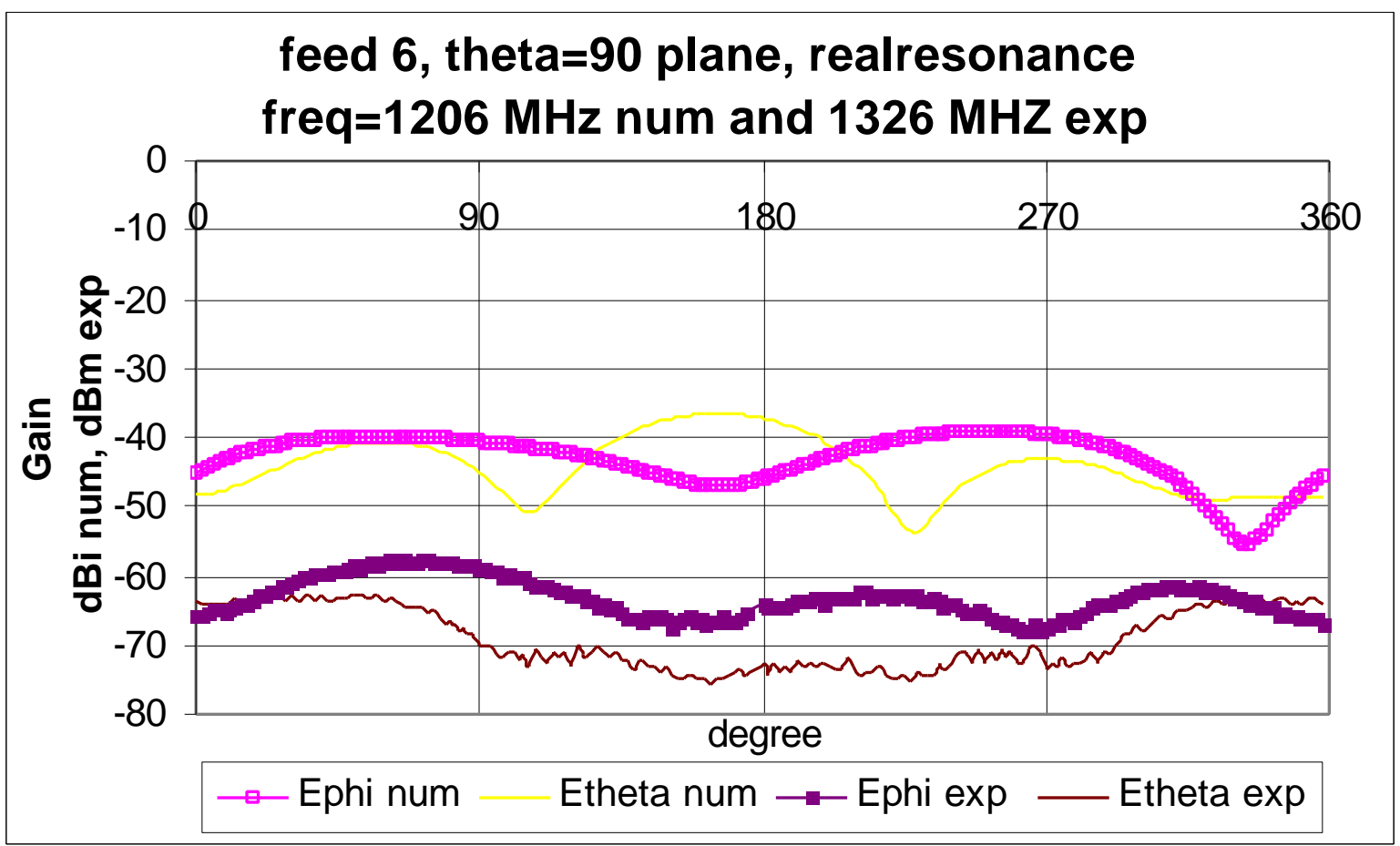

Figure F.33 The Theta $=90$ plane for feed 6 at $1206 \mathrm{MHz}$ num and $1326 \mathrm{MHz}$ exp 
Table F.13 Analysis of the steady state graphs for feed 6

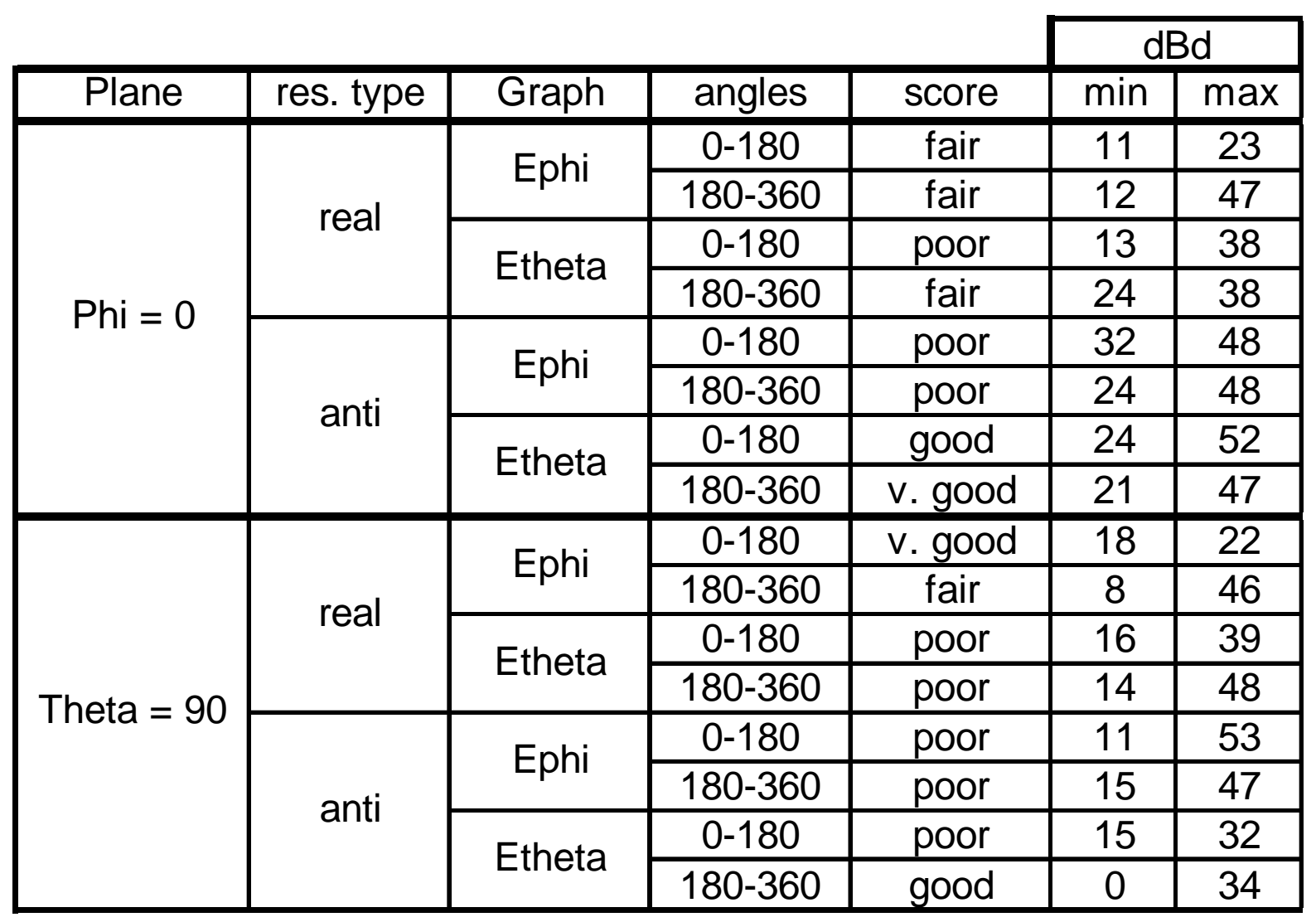




\section{Feed 7}

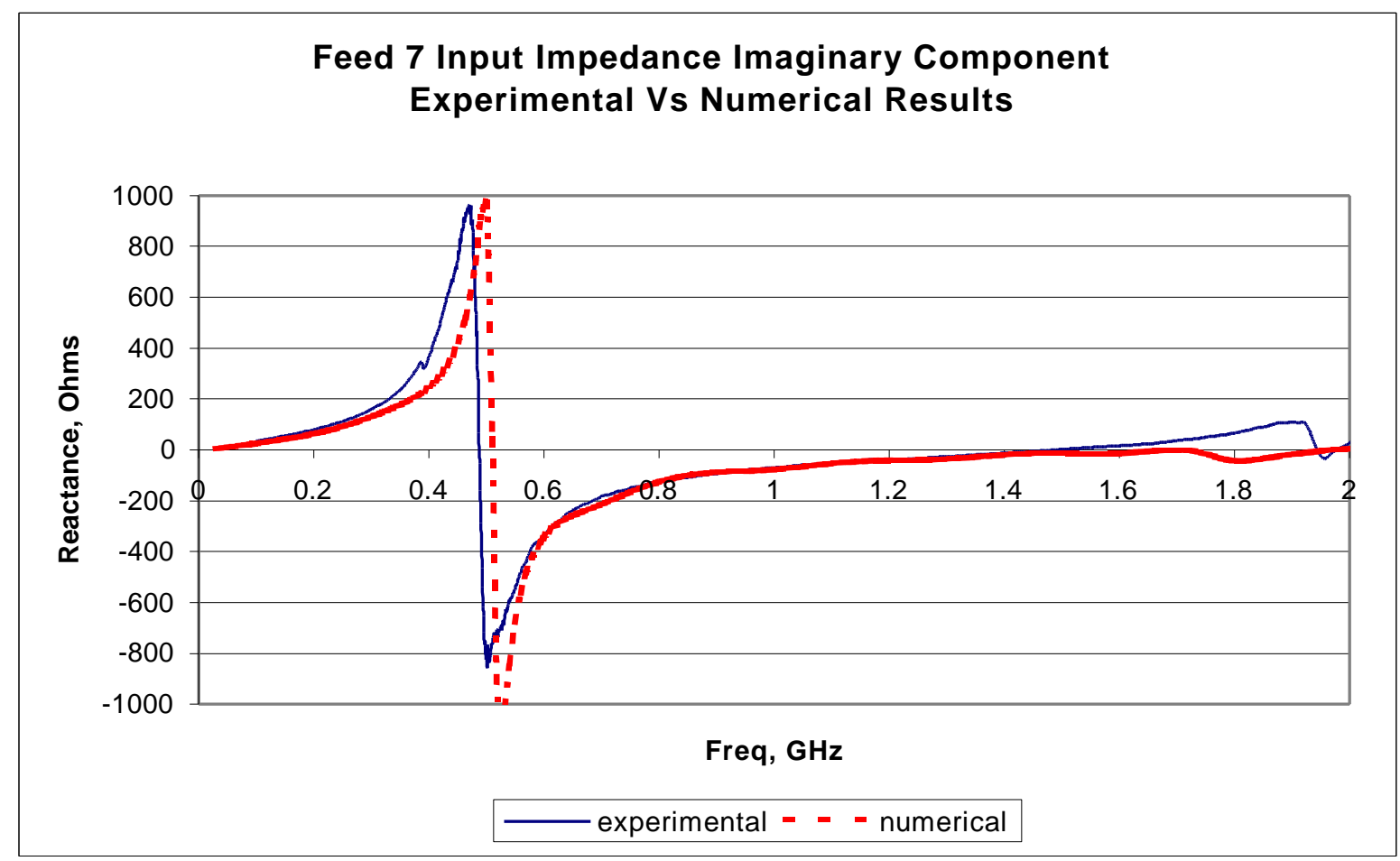

Figure F.34 The imaginary component of the input impedance for feed 7

Table F.14 Analysis of resonance frequencies for feed 7

\begin{tabular}{|c|c|c|}
\hline $\begin{array}{r}\text { f7 exp. } \\
(\mathrm{GHz})\end{array}$ & $\begin{array}{c}\text { f7 num. } \\
(\mathrm{GHz})\end{array}$ & $\begin{array}{c}\text { error } \\
\%\end{array}$ \\
\hline 0.488 & 0.511 & 4.75 \\
\hline 1.489 & & \\
\hline 1.944 & & \\
\hline 1.978 & 1.970 & 0.38 \\
\hline
\end{tabular}




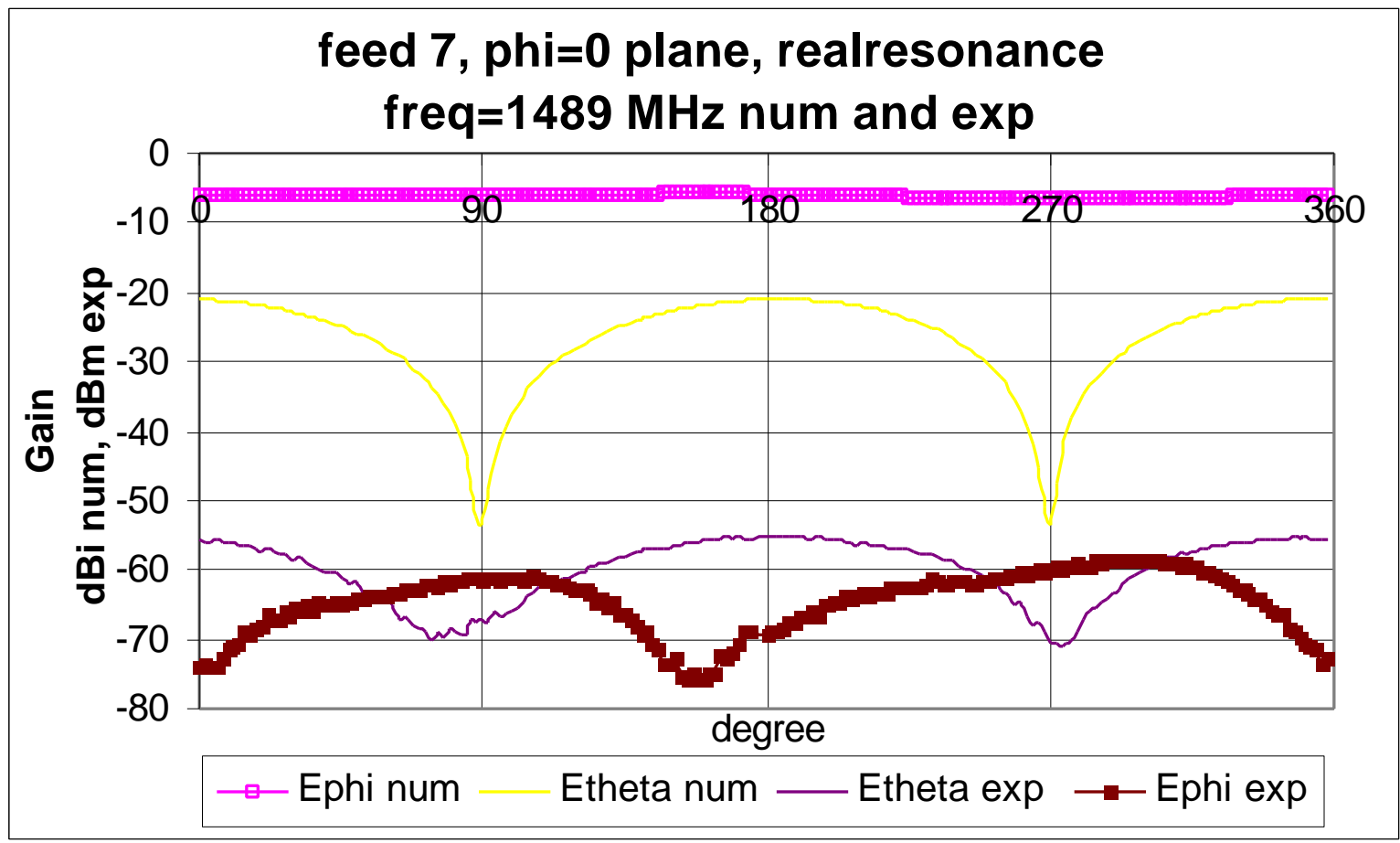

Figure F.35 The Phi $=0$ plane for feed 7 at $1489 \mathrm{MHz}$ num and exp

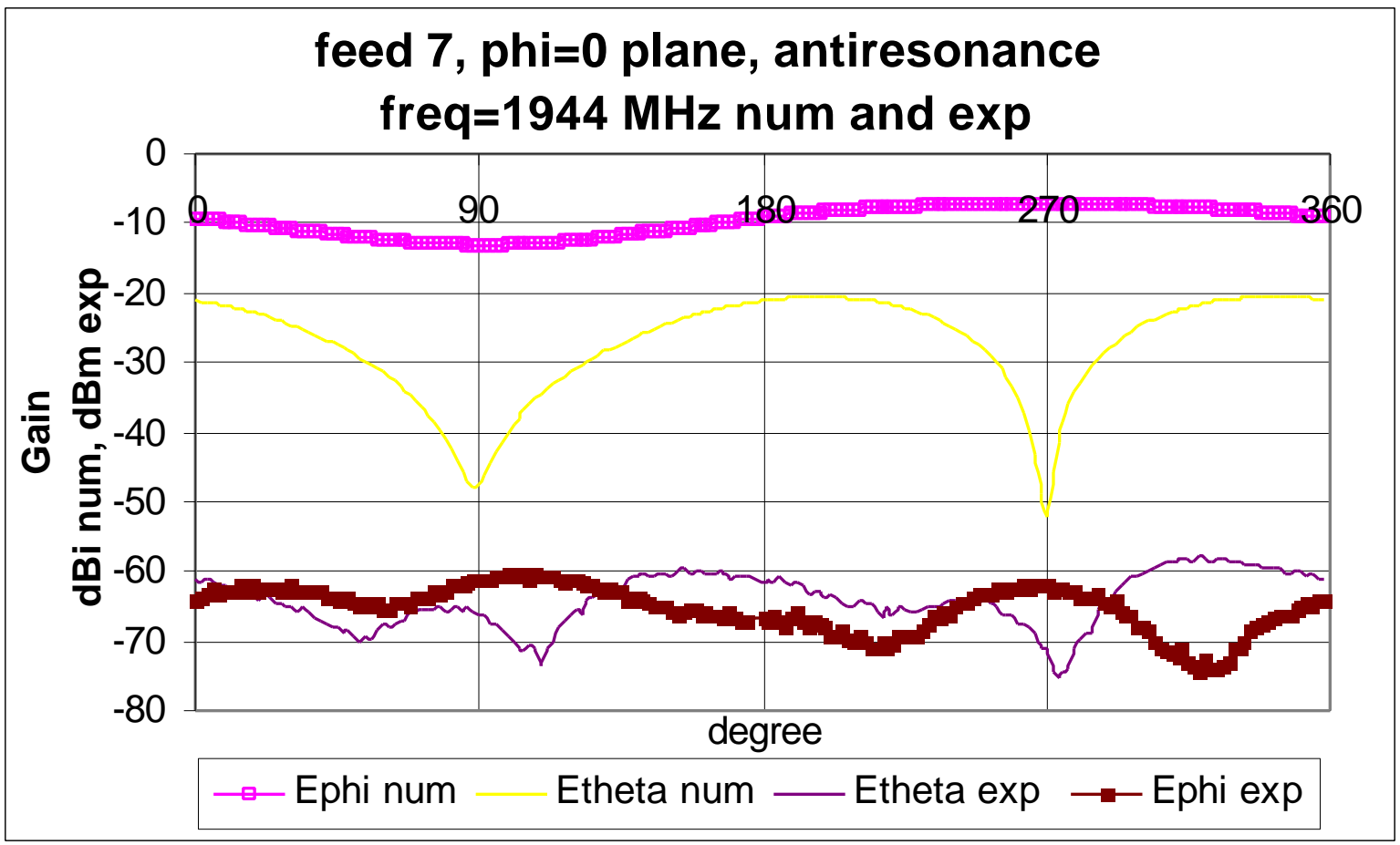

Figure F.36 The Phi = O plane for feed 7 at $1944 M H z$ num and exp 


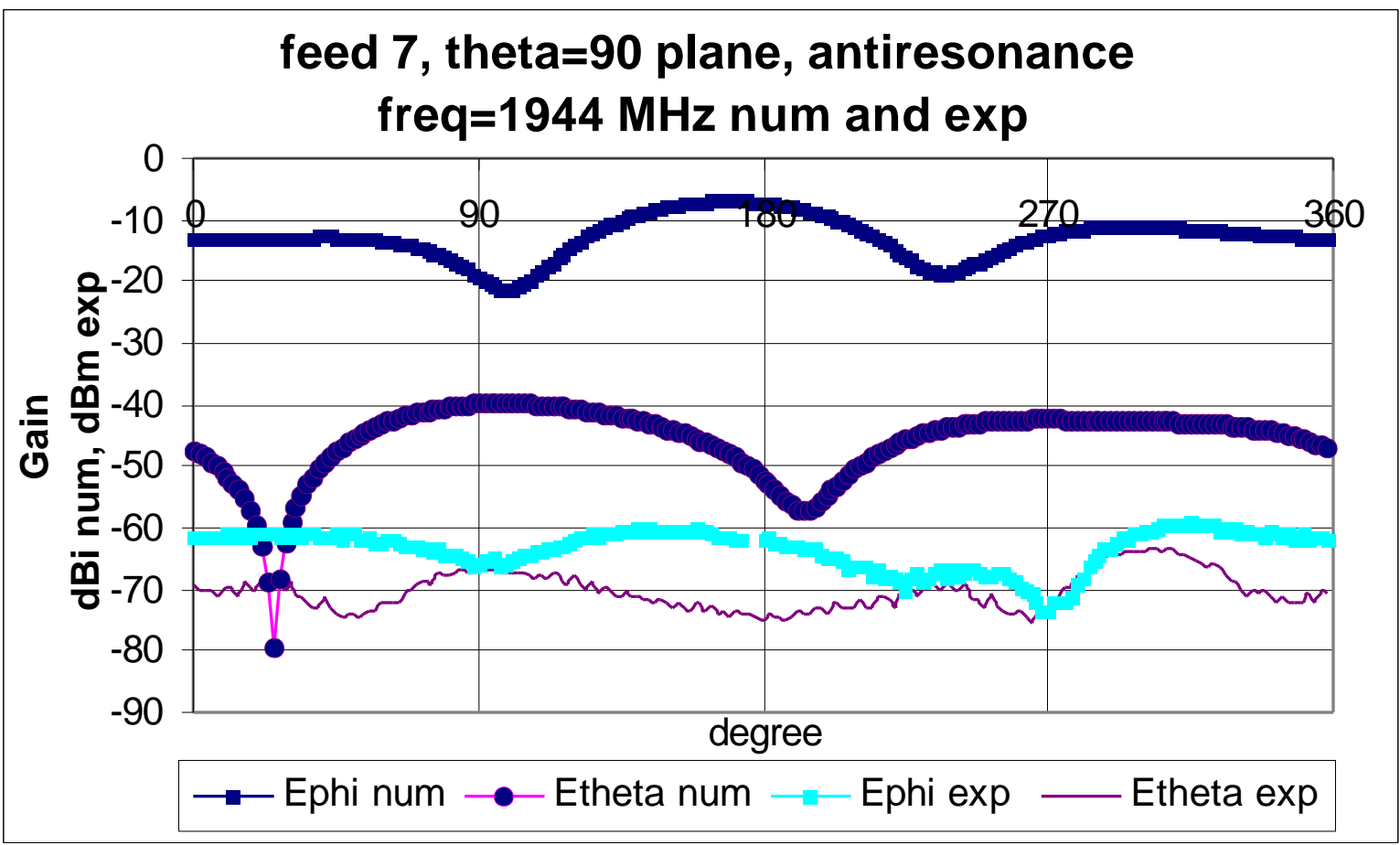

Figure F.37 The Theta $=90$ plane for feed 7 at $1944 \mathrm{MHz}$ num and exp

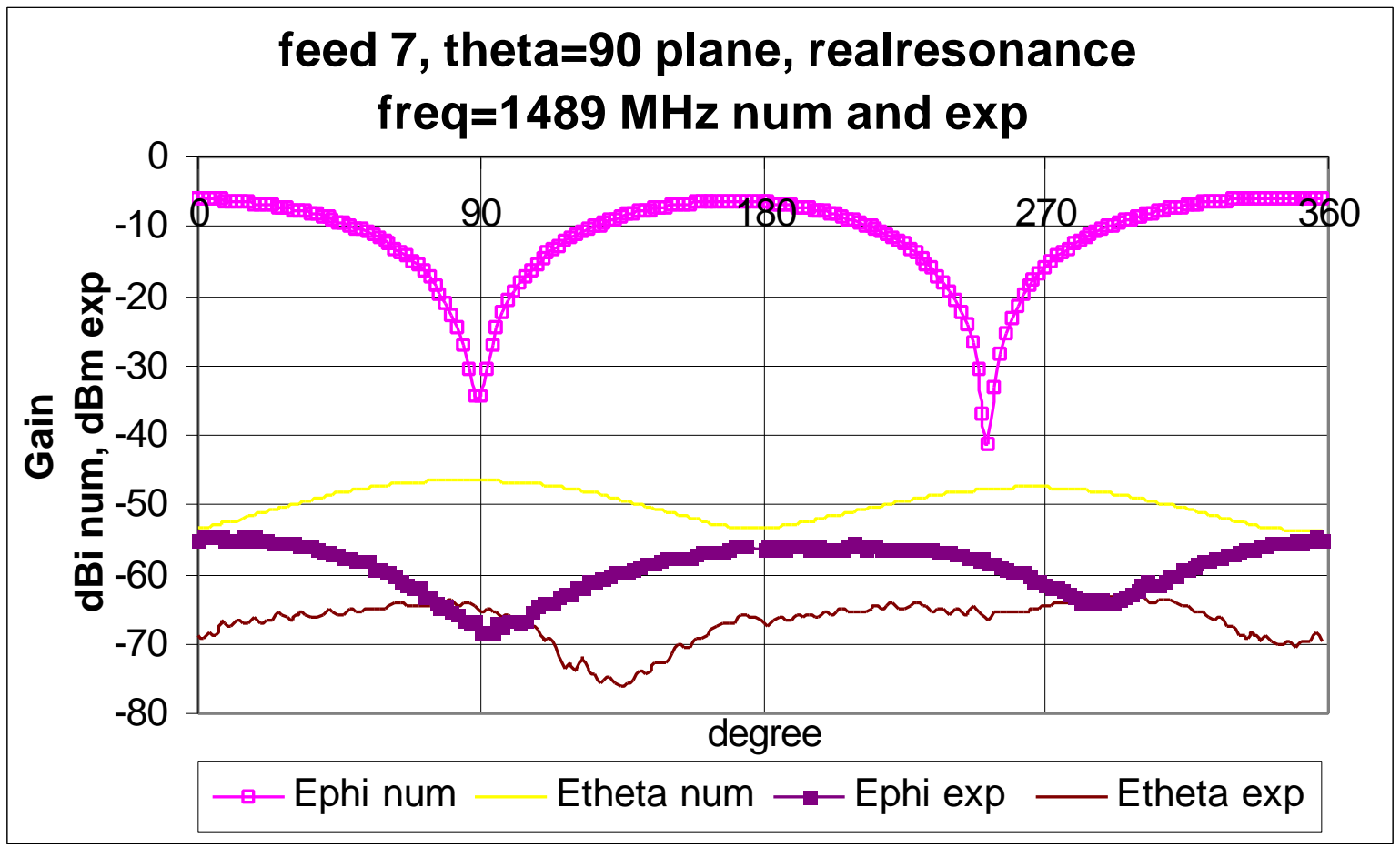

Figure F.38 The Theta $=90$ plane for feed 7 at $1489 \mathrm{MHz}$ num and exp 
Table F.15 Analysis of the steady state graphs for feed 7

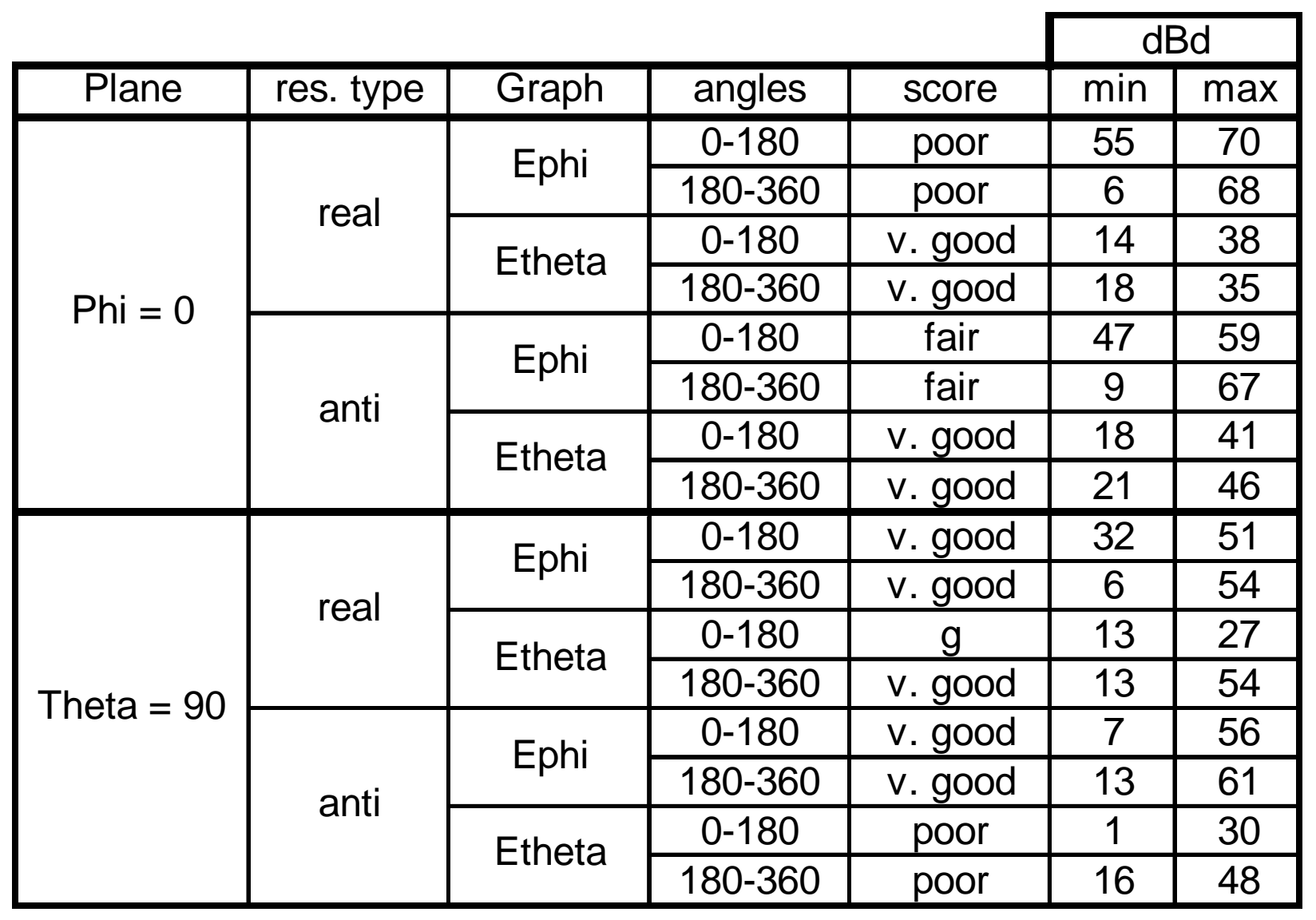




\section{Feed 8}

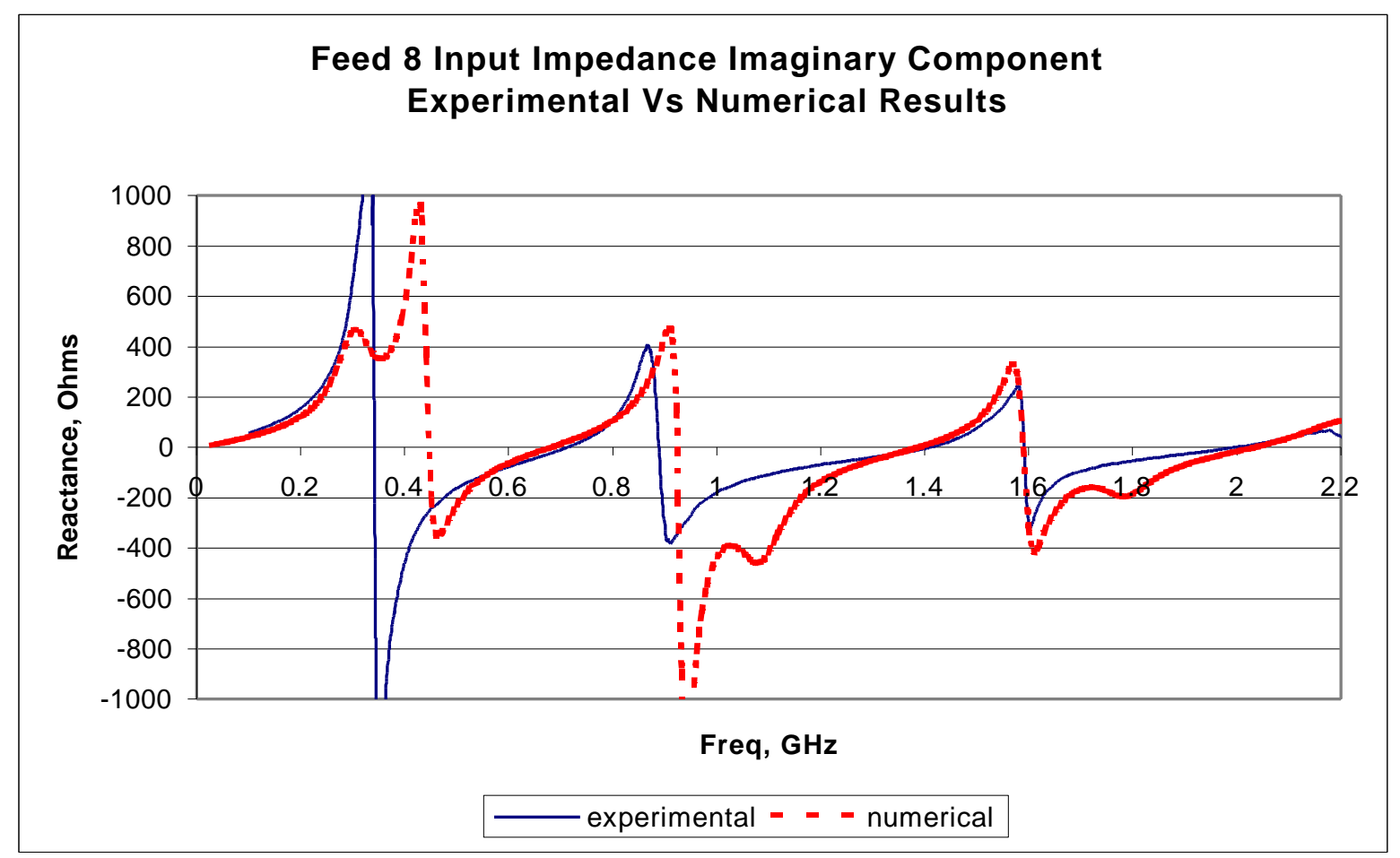

Figure F.39 The imaginary component of the input impedance for feed 8

Table F.16 Analysis of resonance frequencies for feed 8

\begin{tabular}{|c|c|c|}
\hline $\begin{array}{c}\text { f8 exp. } \\
(\mathrm{GHz})\end{array}$ & $\begin{array}{c}\text { Experimental } \\
\text { res freq's }\end{array}$ & $\begin{array}{c}\text { diff. between } \\
\text { same type freq's }\end{array}$ \\
\hline 0.712 & & \\
\hline 0.889 & 0.177 & \\
\hline 1.409 & 0.520 & 0.697 \\
\hline 1.591 & 0.182 & 0.702 \\
\hline 1.994 & 0.403 & 0.585 \\
\hline mean & 0.320 & 0.661 \\
\hline
\end{tabular}

\begin{tabular}{|c|c|c|}
\hline \multicolumn{3}{|c|}{ Numerical } \\
\hline $\begin{array}{c}\text { f8 num. } \\
(\mathrm{GHz})\end{array}$ & $\begin{array}{c}\text { diff between } \\
\text { res freq's }\end{array}$ & $\begin{array}{c}\text { diff between } \\
\text { same type freq's }\end{array}$ \\
\hline 0.681 & & \\
\hline 0.924 & 0.243 & \\
\hline 1.387 & 0.463 & 0.706 \\
\hline 1.590 & 0.203 & 0.666 \\
\hline 2.032 & 0.442 & 0.645 \\
\hline mean & 0.338 & 0.672 \\
\hline
\end{tabular}

\begin{tabular}{|c|}
\hline $\begin{array}{c}\text { error } \\
\%\end{array}$ \\
\hline 4.38 \\
\hline 3.95 \\
\hline 1.59 \\
\hline 0.10 \\
\hline 1.91 \\
\hline
\end{tabular}




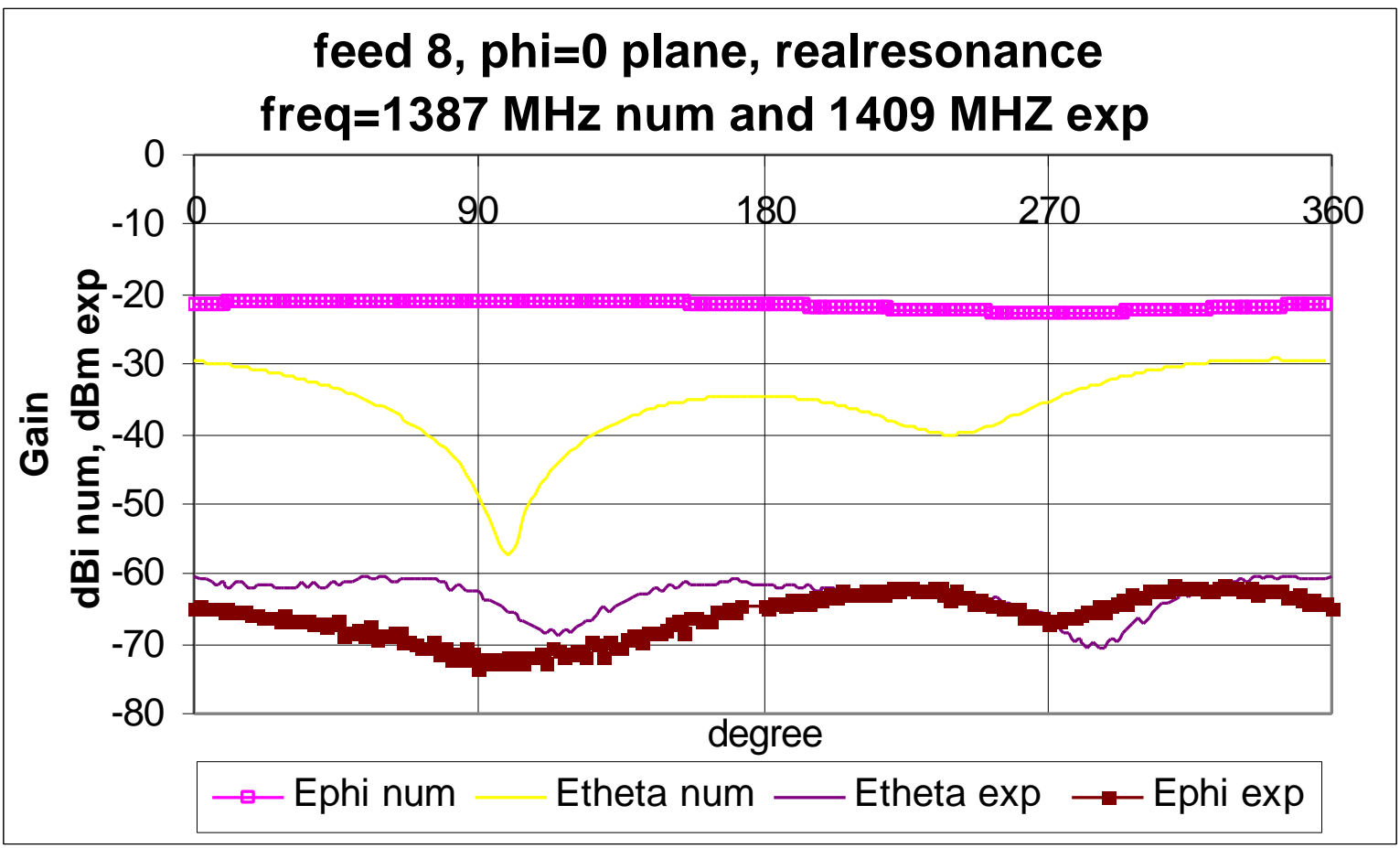

Figure F.40 The Phi = O plane for feed 8 at $1387 \mathrm{MHz}$ num and $1409 \mathrm{MHz}$ exp

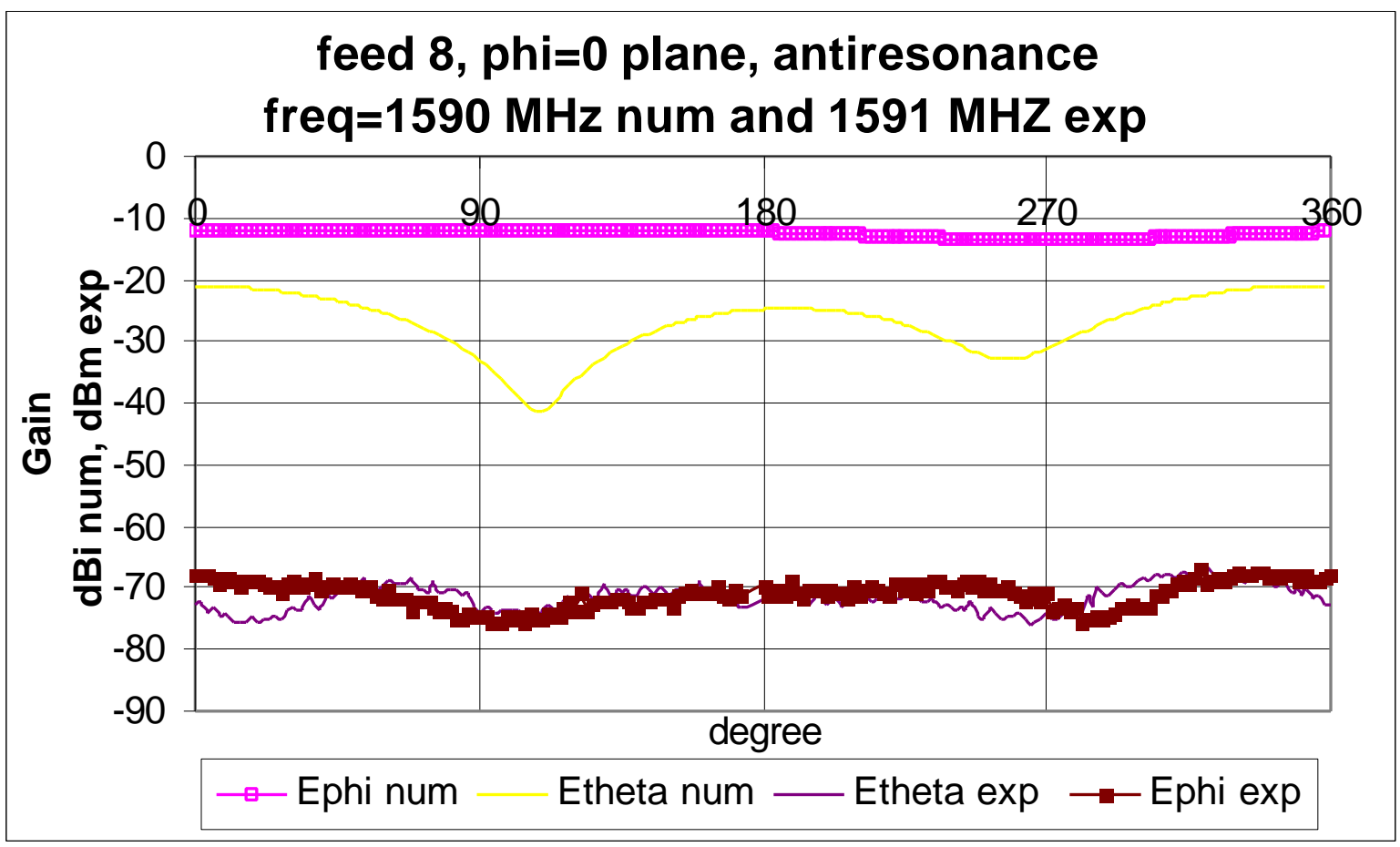

Figure F.41 The Phi =0 plane for feed 8 at 1590MHz num and $1591 \mathrm{MHz}$ exp 


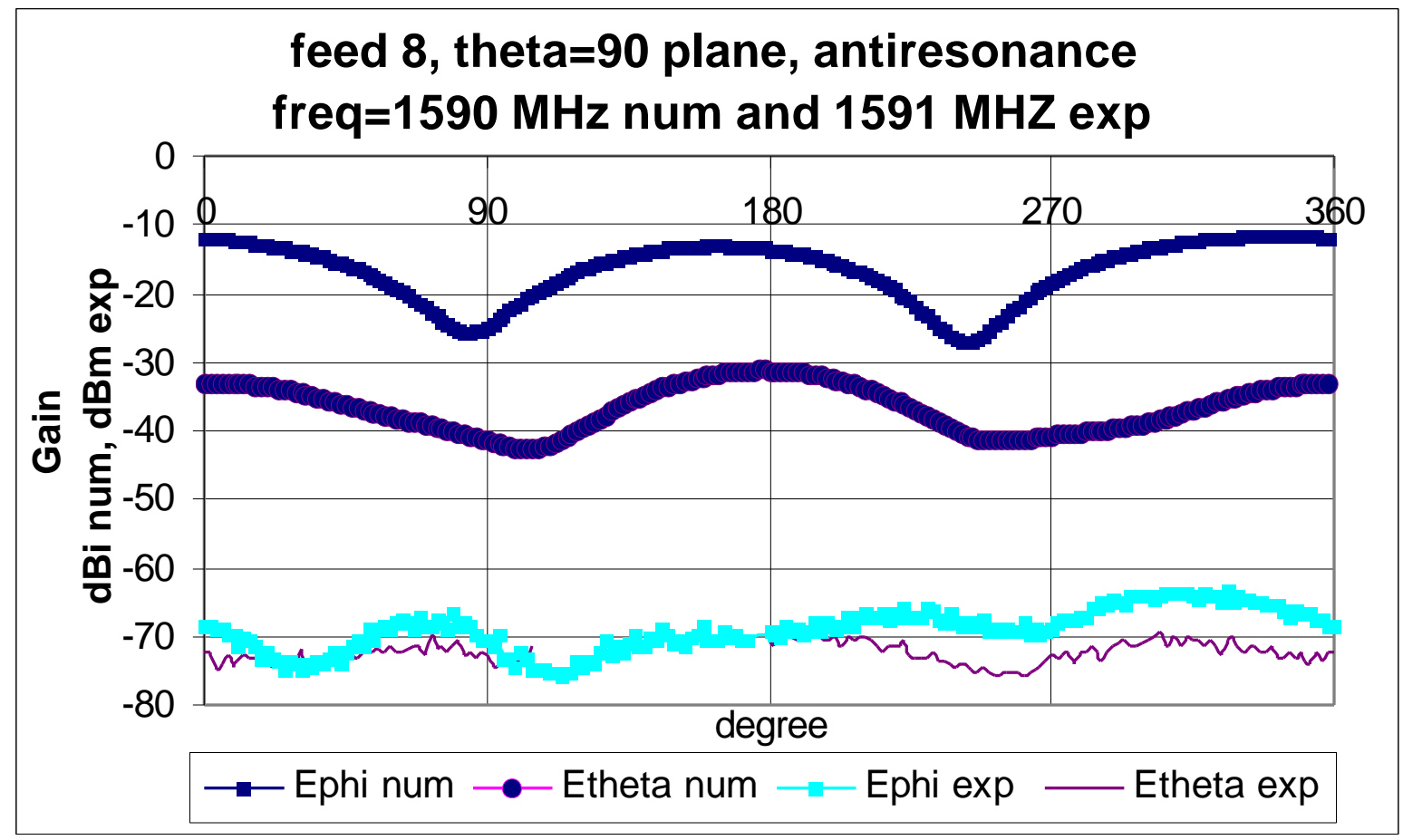

Figure F.42 The Theta $=90$ plane for feed 8 at $1590 \mathrm{MHz}$ num and $1591 \mathrm{MHz}$ exp

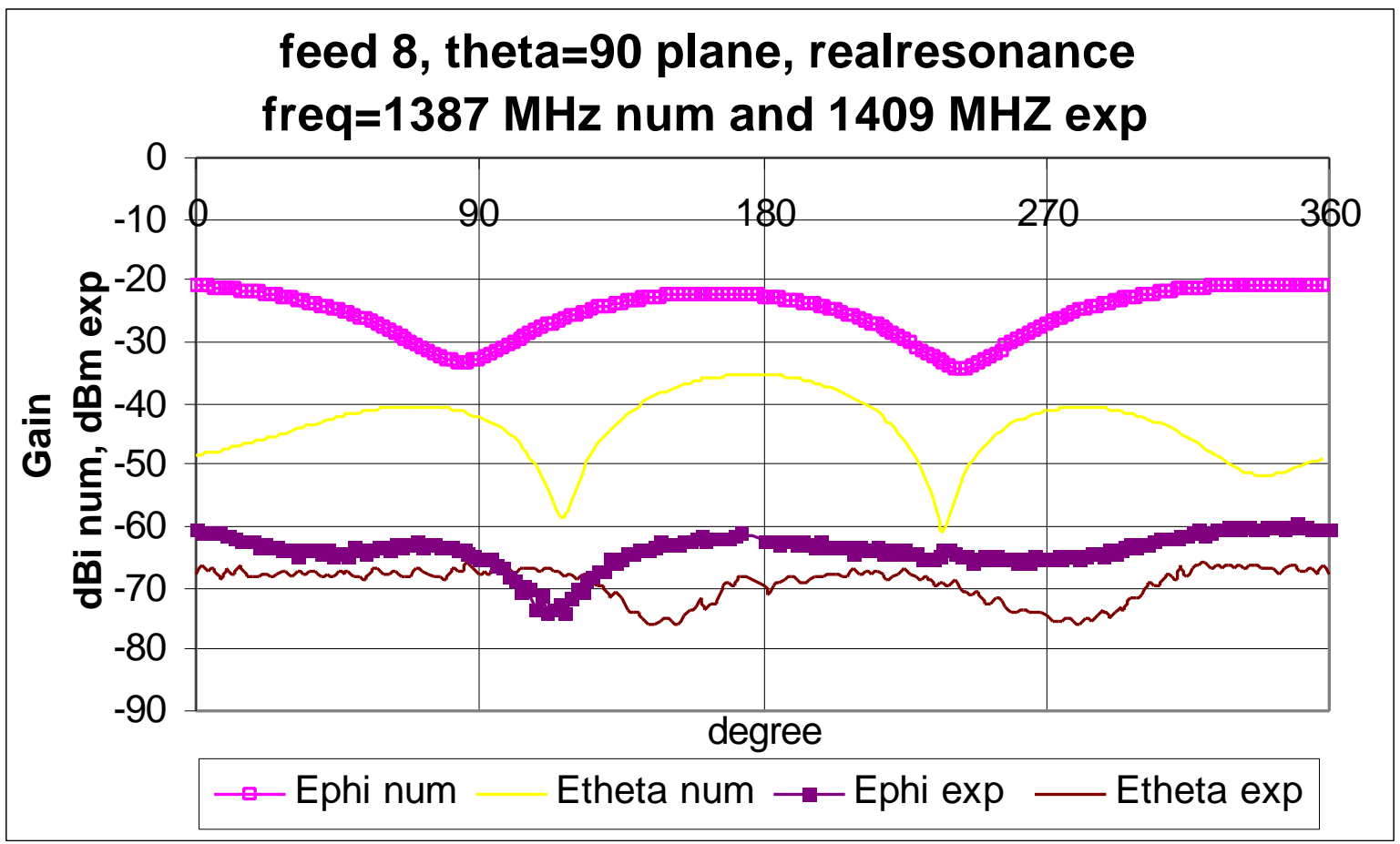

Figure F.43 The Theta $=90$ plane for feed 8 at $1387 \mathrm{MHz}$ num and $1409 \mathrm{MHz}$ exp 
Table F.17 Analysis of the steady state graphs for feed 8

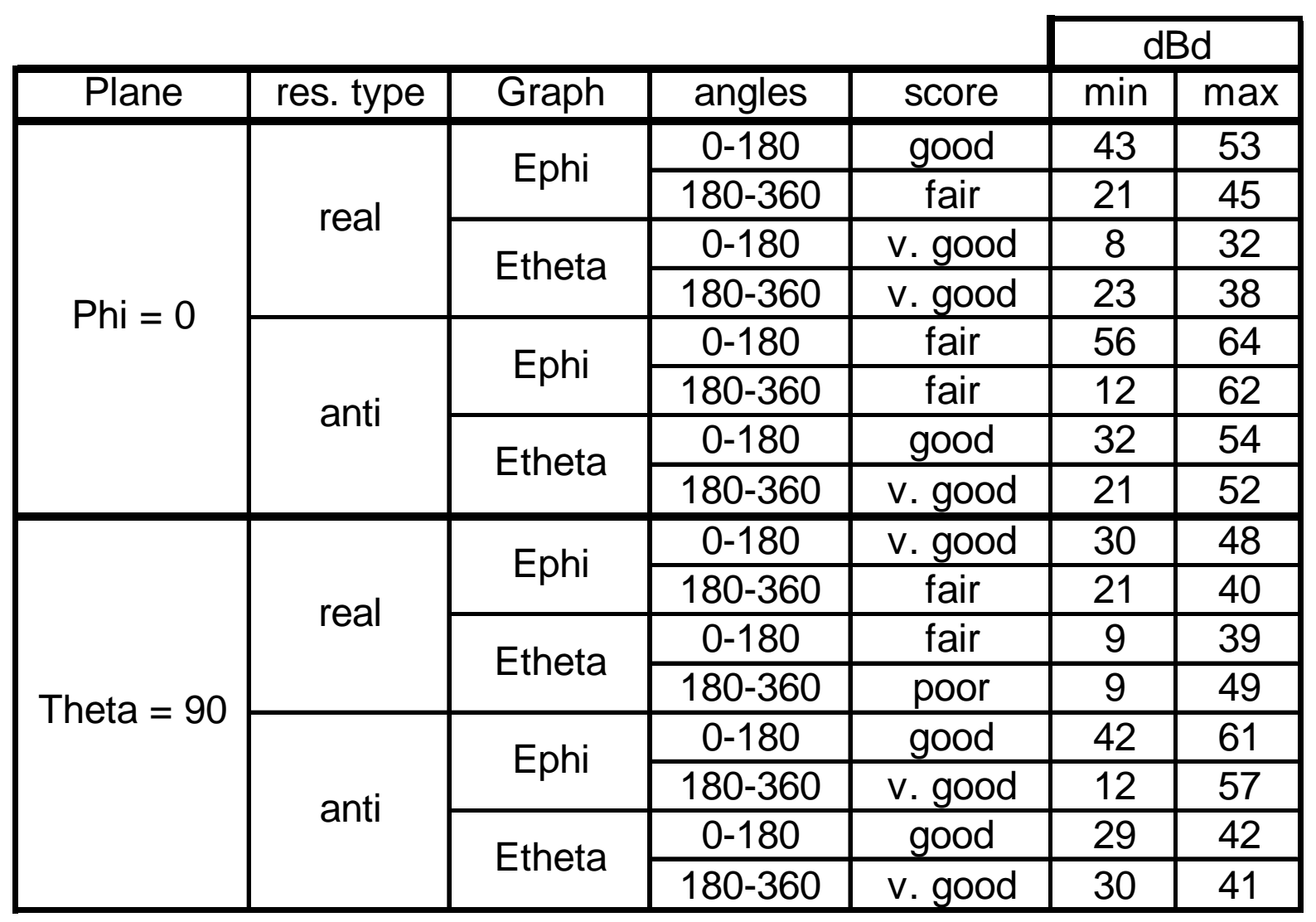




\section{Feed 9}

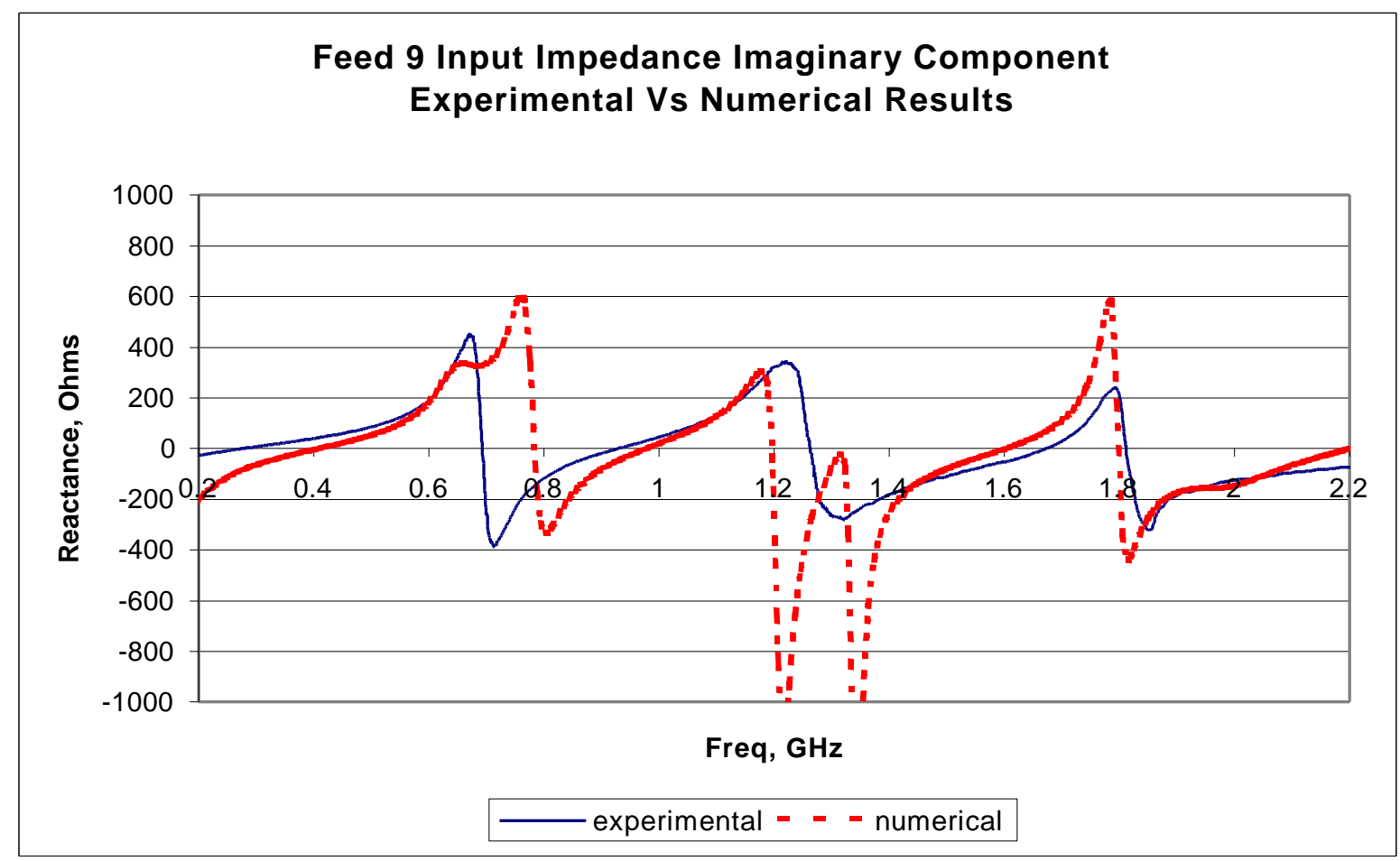

Figure F.44 The imaginary component of the input impedance for feed 9

Table F.18 Analysis of resonance frequencies for feed 9

\begin{tabular}{|c|c|c|}
\hline $\begin{array}{c}\text { f9 exp. } \\
(\mathrm{GHz})\end{array}$ & $\begin{array}{c}\text { Experimental } \\
\text { res freq's }\end{array}$ & $\begin{array}{c}\text { diff. between } \\
\text { same type freq's }\end{array}$ \\
\hline 0.693 & & \\
\hline 0.929 & 0.236 & \\
\hline 1.263 & 0.334 & 0.570 \\
\hline 1.673 & 0.410 & 0.744 \\
\hline 1.812 & 0.140 & 0.550 \\
\hline mean & 0.280 & 0.621 \\
\hline
\end{tabular}

\begin{tabular}{|c|c|c|}
\hline \multicolumn{3}{|c|}{ Numerical } \\
\hline $\begin{array}{c}\text { f9 num. } \\
\text { (GHz) }\end{array}$ & $\begin{array}{c}\text { diff between } \\
\text { res freq's }\end{array}$ & $\begin{array}{c}\text { diff between } \\
\text { same type freq's }\end{array}$ \\
\hline 0.784 & & \\
\hline 0.978 & 0.194 & \\
\hline 1.196 & 0.219 & 0.413 \\
\hline 1.603 & 0.406 & 0.625 \\
\hline 1.799 & 0.197 & 0.603 \\
\hline mean & 0.254 & 0.547 \\
\hline
\end{tabular}

\begin{tabular}{|c|}
\hline $\begin{array}{c}\text { error } \\
\%\end{array}$ \\
\hline 13.15 \\
\hline 5.28 \\
\hline 5.23 \\
\hline 4.18 \\
\hline 0.70 \\
\hline
\end{tabular}




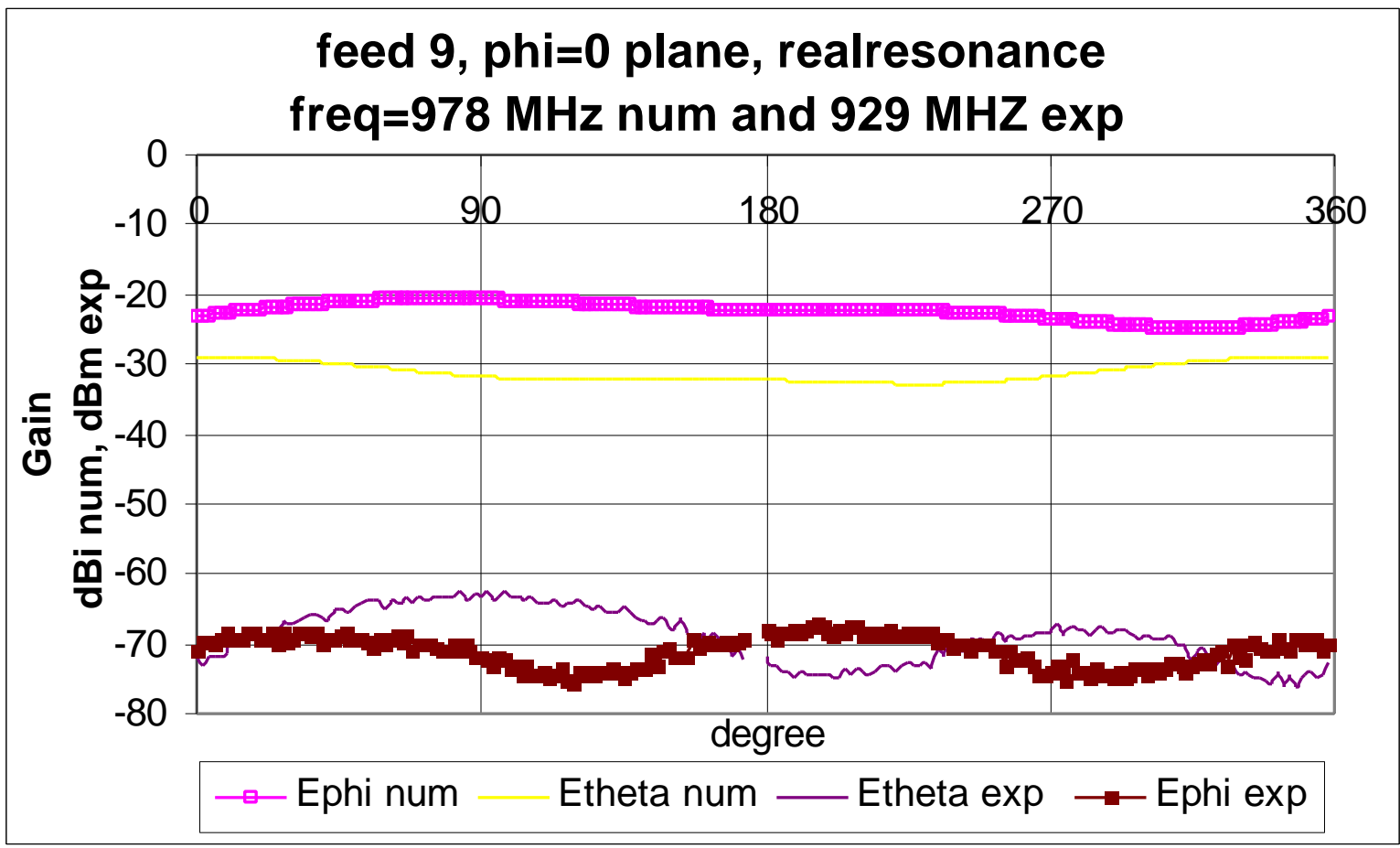

Figure F.45 The Phi = 0 plane for feed 9 at $978 \mathrm{MHz}$ num and $929 \mathrm{MHz}$ exp

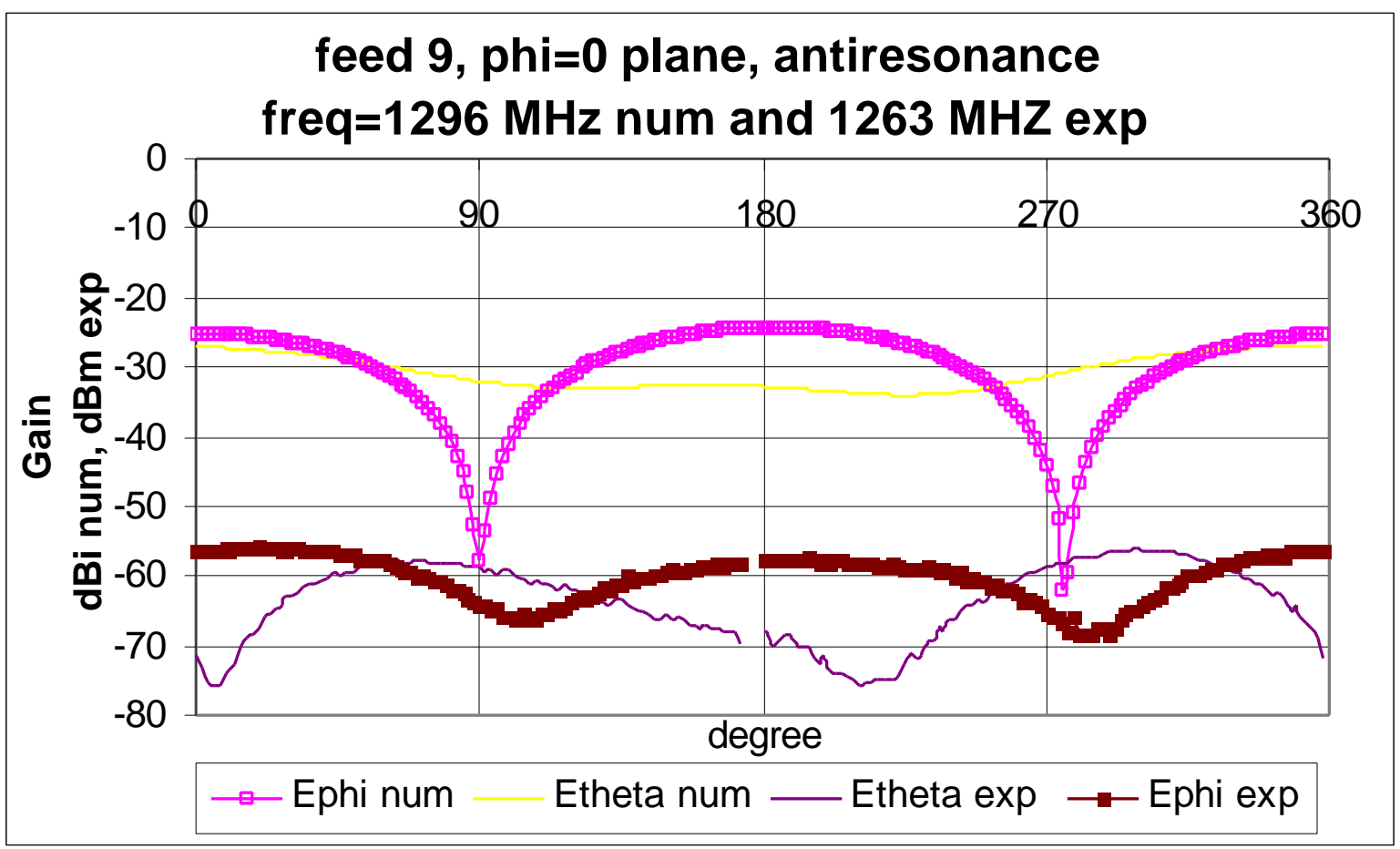

Figure F.46 The Phi = 0 plane for feed 9 at 1296MHz num and $1263 \mathrm{MHz}$ exp 


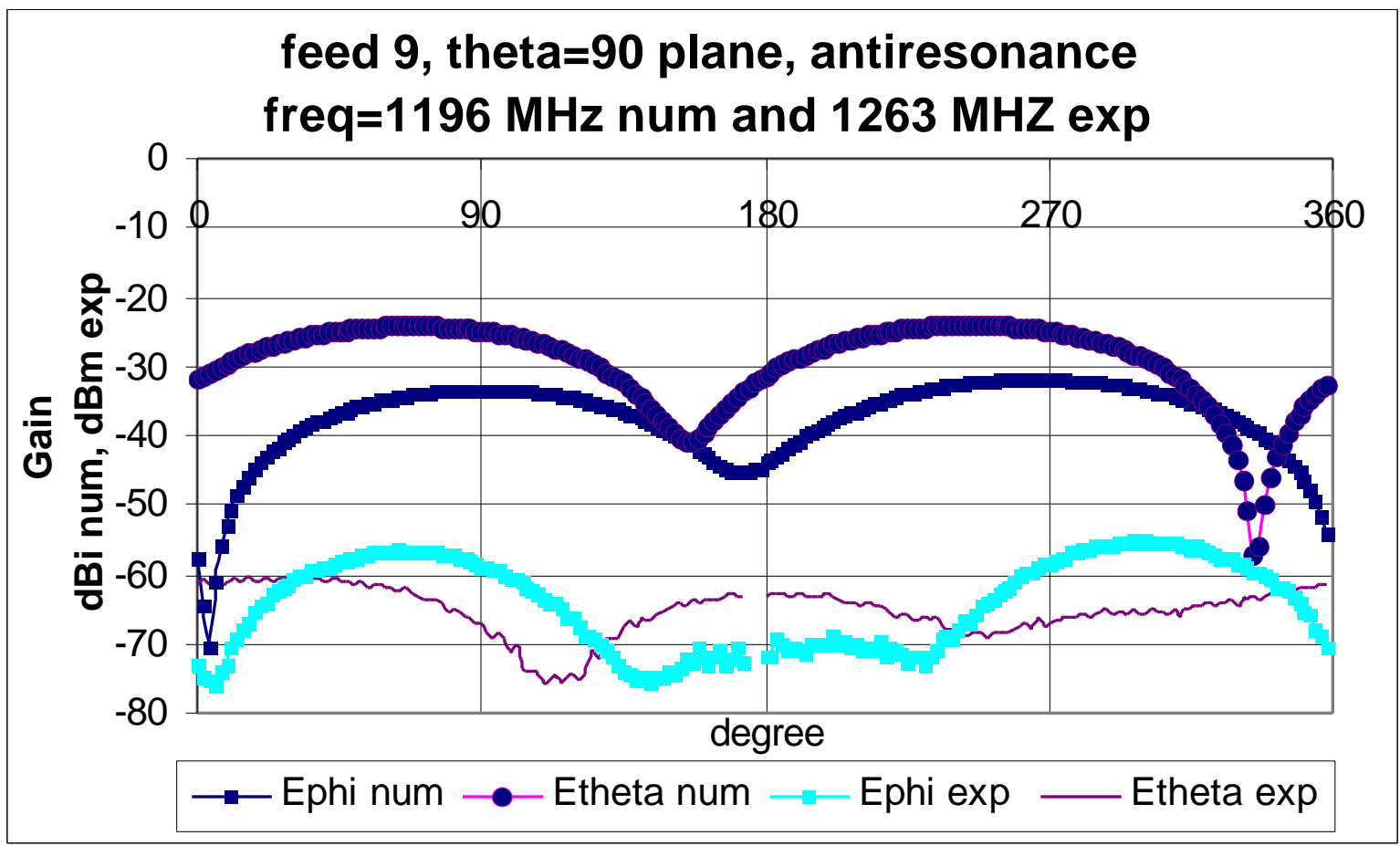

Figure F.47 The Theta $=90$ plane for feed 9 at $1296 \mathrm{MHz}$ num and $1263 \mathrm{MHz}$ exp

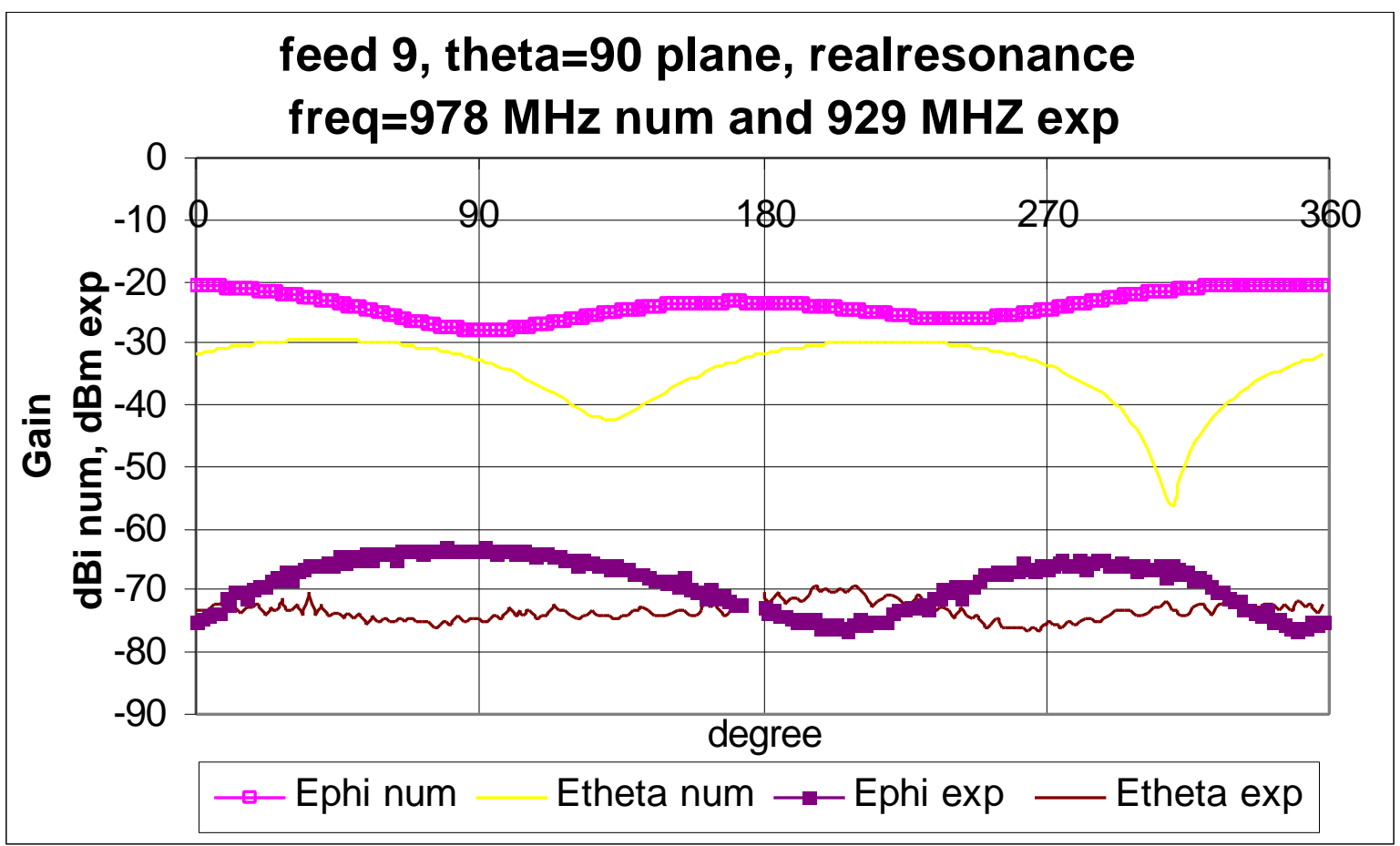

Figure F.48 The Theta $=90$ plane for feed 9 at 978MHz num and 929MHz exp 
Table F.19 Analysis of the steady state graphs for feed 9

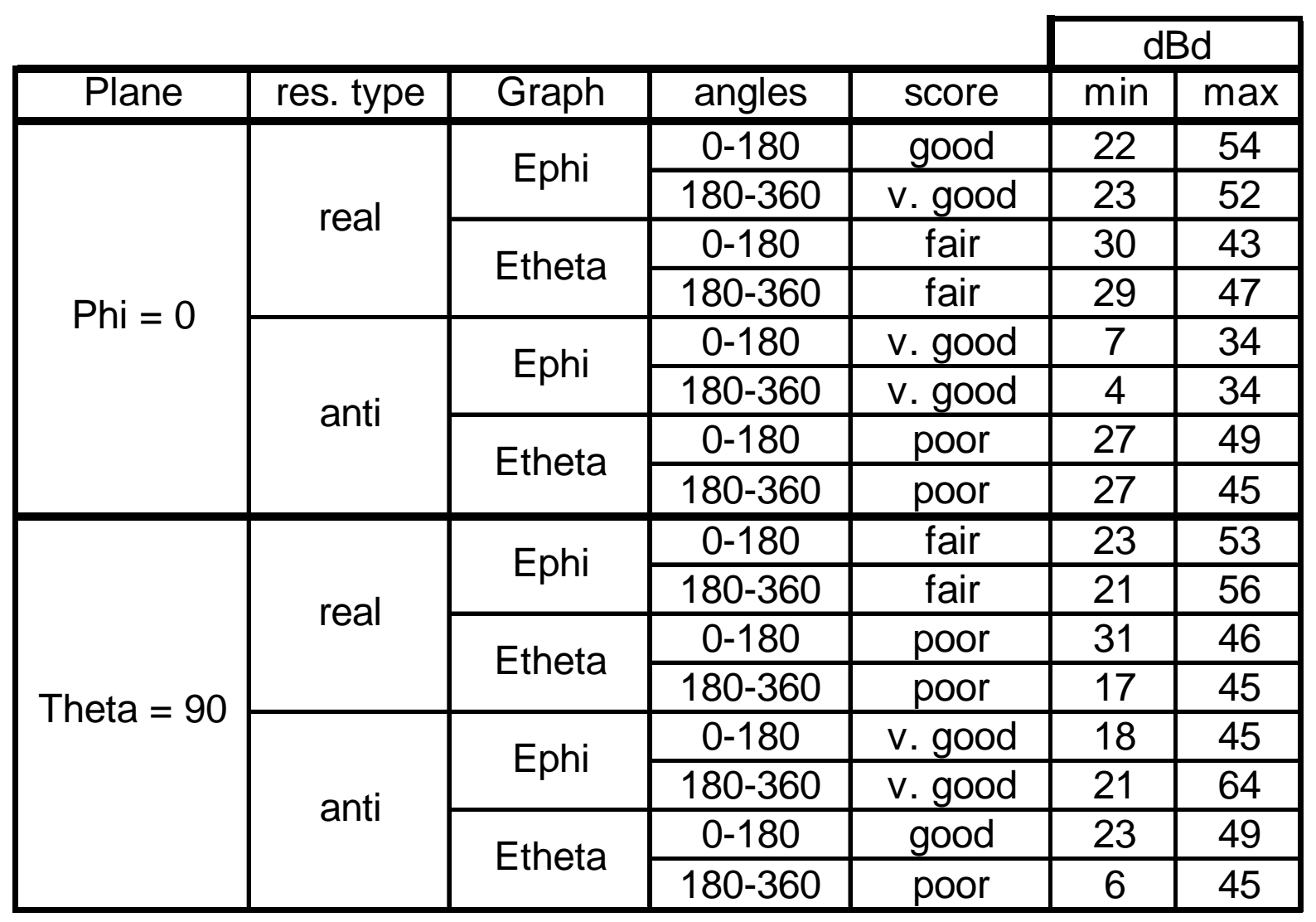




\section{Feed 10}

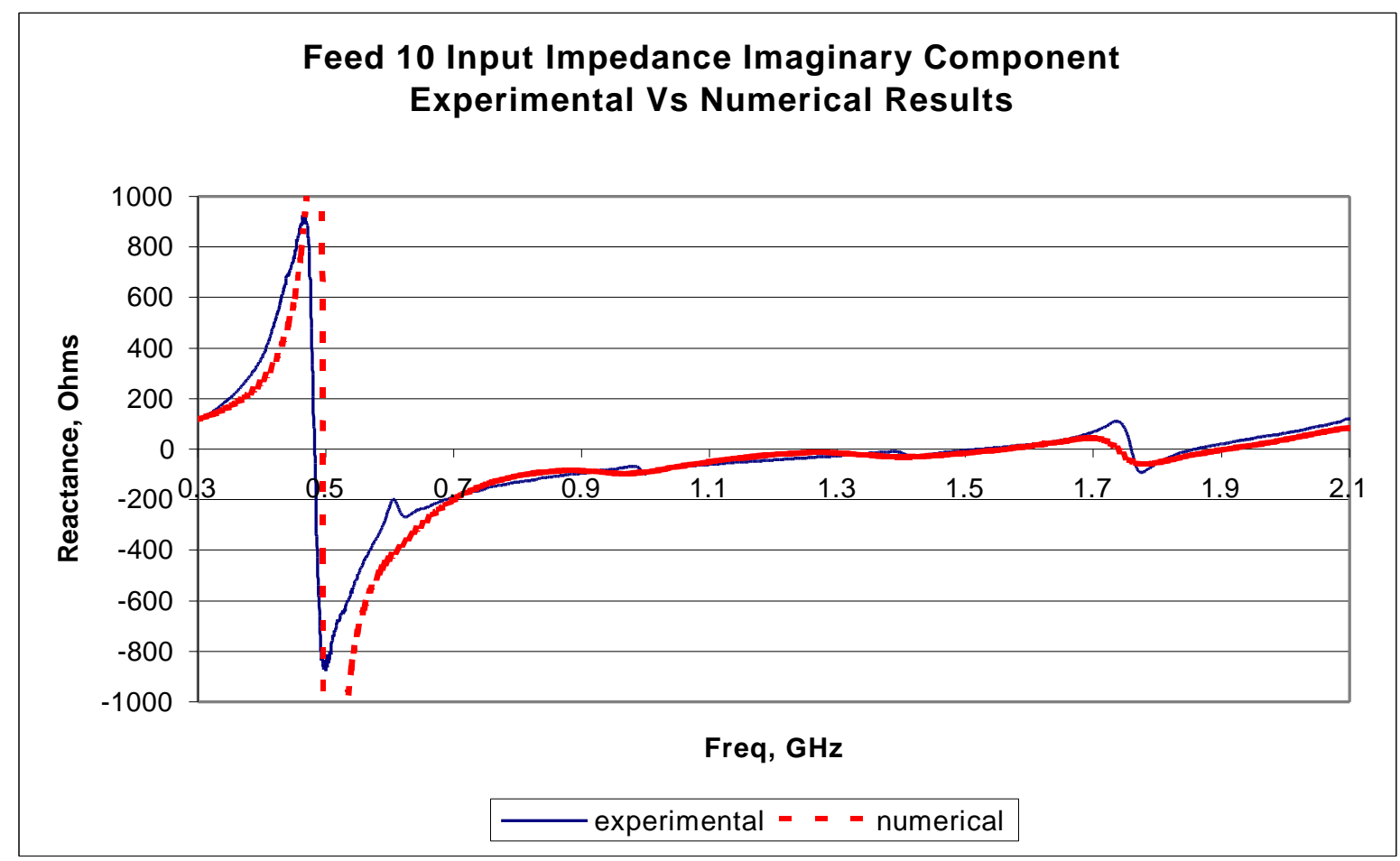

Figure F.49 The imaginary component of the input impedance for feed 10

Table F.20 Analysis of resonance frequencies for feed 10

\begin{tabular}{|c|c|c|}
\hline $\begin{array}{c}\mathrm{f} 10 \text { exp. } \\
(\mathrm{GHz})\end{array}$ & $\begin{array}{c}\mathrm{f} 10 \text { num. } \\
(\mathrm{GHz})\end{array}$ & $\begin{array}{c}\text { error } \\
\%\end{array}$ \\
\hline 0.483 & 0.493 & 2.07 \\
\hline 1.525 & 1.564 & 2.51 \\
\hline 1.759 & 1.738 & 1.23 \\
\hline 1.860 & 1.910 & 2.68 \\
\hline
\end{tabular}




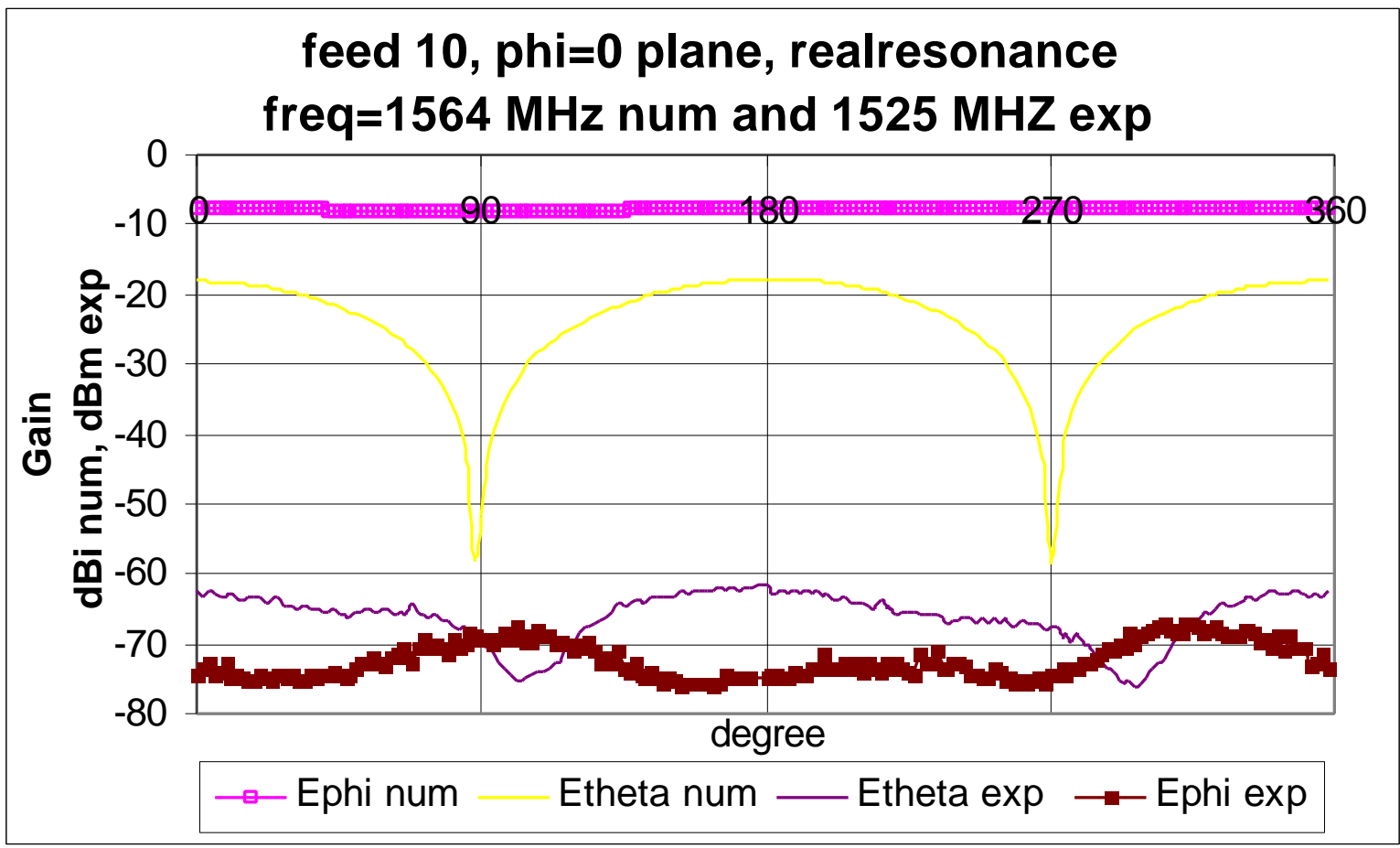

Figure F.50 The Phi =0 plane for feed 10 at $1564 \mathrm{MHz}$ num and $1525 \mathrm{MHz}$ exp

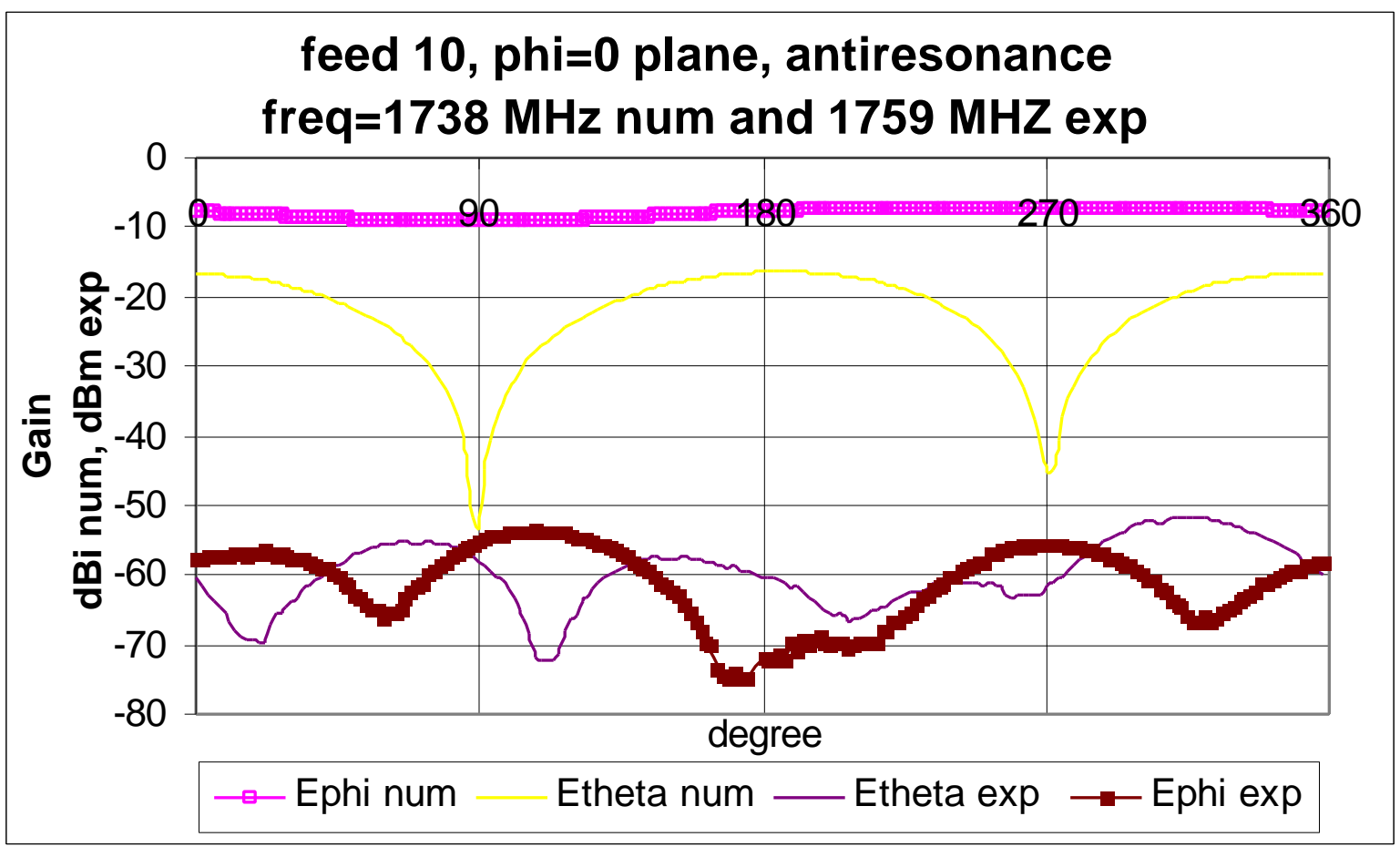

Figure F.51 The Phi =0 plane for feed 10 at $1738 \mathrm{MHz}$ num and $1759 \mathrm{MHz}$ exp 


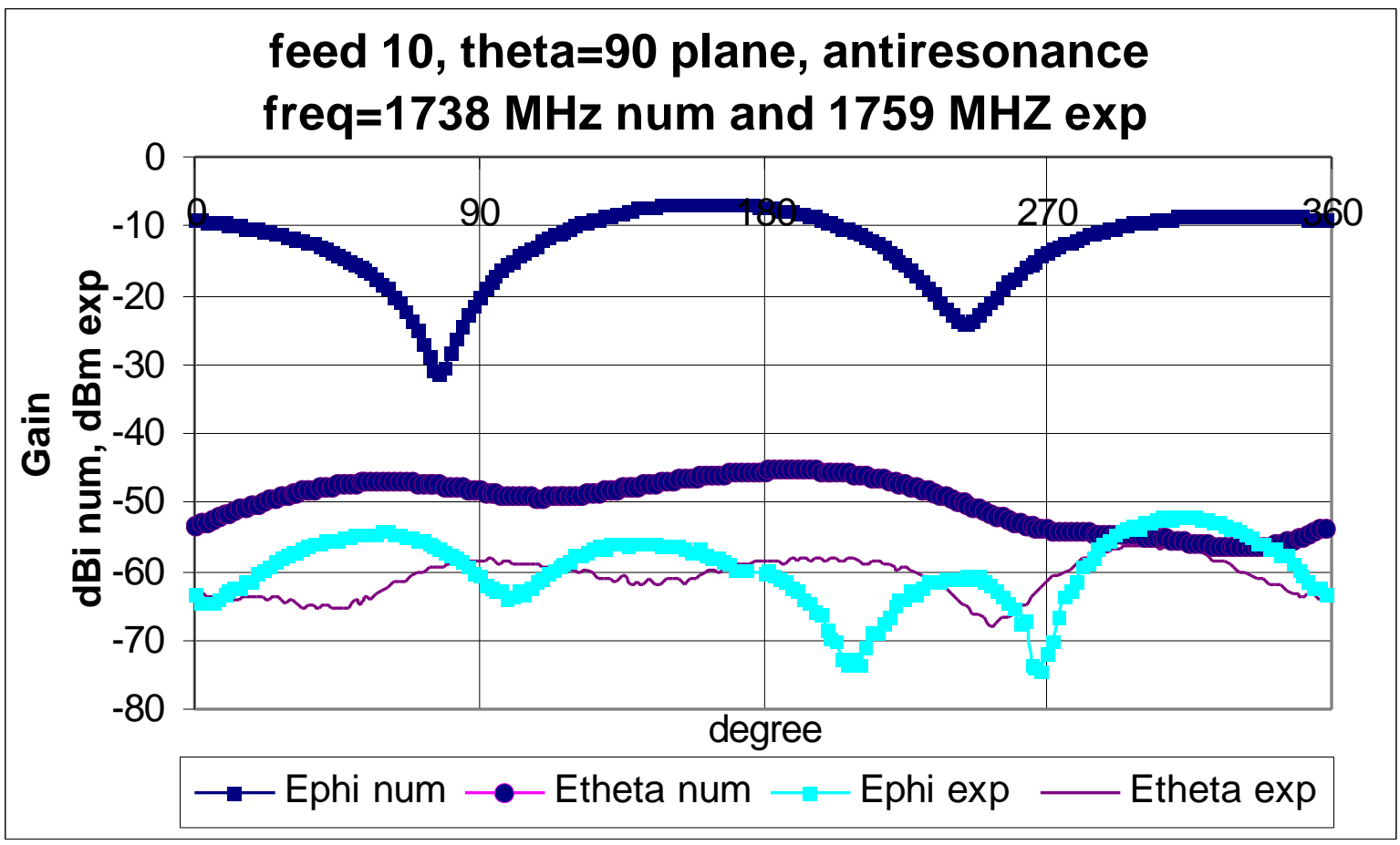

Figure F.52 The Theta $=90$ plane for feed 10 at $1738 \mathrm{MHz}$ num and $1759 \mathrm{MHz}$ exp

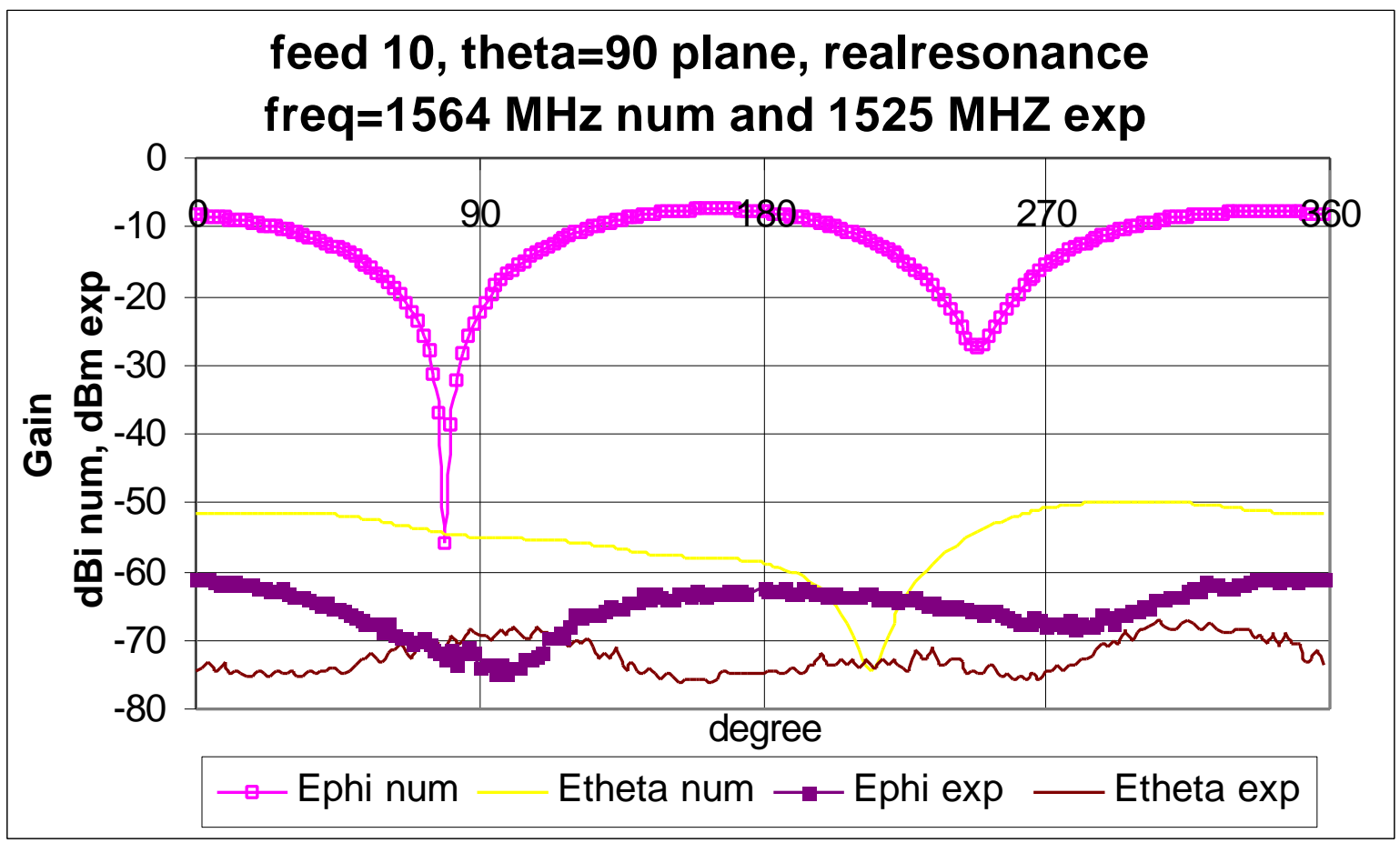

Figure F.53 The Theta $=90$ plane for feed 10 at $1564 \mathrm{MHz}$ num and $1525 \mathrm{MHz}$ exp 
Table F.21 Analysis of the steady state graphs for feed 10

\begin{tabular}{|c|c|c|c|c|c|c|}
\hline & & & & & & \\
\hline Plane & res. type & Graph & angles & score & $\min$ & $\max$ \\
\hline & & Enhi & $0-180$ & fair & 60 & 68 \\
\hline & real & ᄃр/II & $180-360$ & fair & 8 & 68 \\
\hline & real & Ftheta & $0-180$ & fair & 11 & 47 \\
\hline & & Cuneta & $180-360$ & fair & 10 & 50 \\
\hline $\mathrm{rnl}=0$ & & Enhi & $0-180$ & poor & 45 & 67 \\
\hline & anti & ᄃprII & $180-360$ & poor & 8 & 63 \\
\hline & anll & Ftheta & $0-180$ & fair & 5 & 52 \\
\hline & & Emeta & $180-360$ & fair & 15 & 50 \\
\hline & & Fnhi & $0-180$ & good & 17 & 59 \\
\hline & ral & 다이 & $180-360$ & good & 8 & 56 \\
\hline & real & Ftheta & $0-180$ & fair & 13 & 24 \\
\hline Theta $=c$ & & Cuneta & $180-360$ & poor & 0 & 52 \\
\hline meta $=90$ & & & $0-180$ & v. good & 25 & 55 \\
\hline & anti & Ephi & $180-360$ & fair & 9 & 64 \\
\hline & dillu & Ftheta & $0-180$ & fair & 10 & 18 \\
\hline & & Elneta & $180-360$ & poor & $\overline{1}$ & 54 \\
\hline
\end{tabular}




\section{Feed 11}

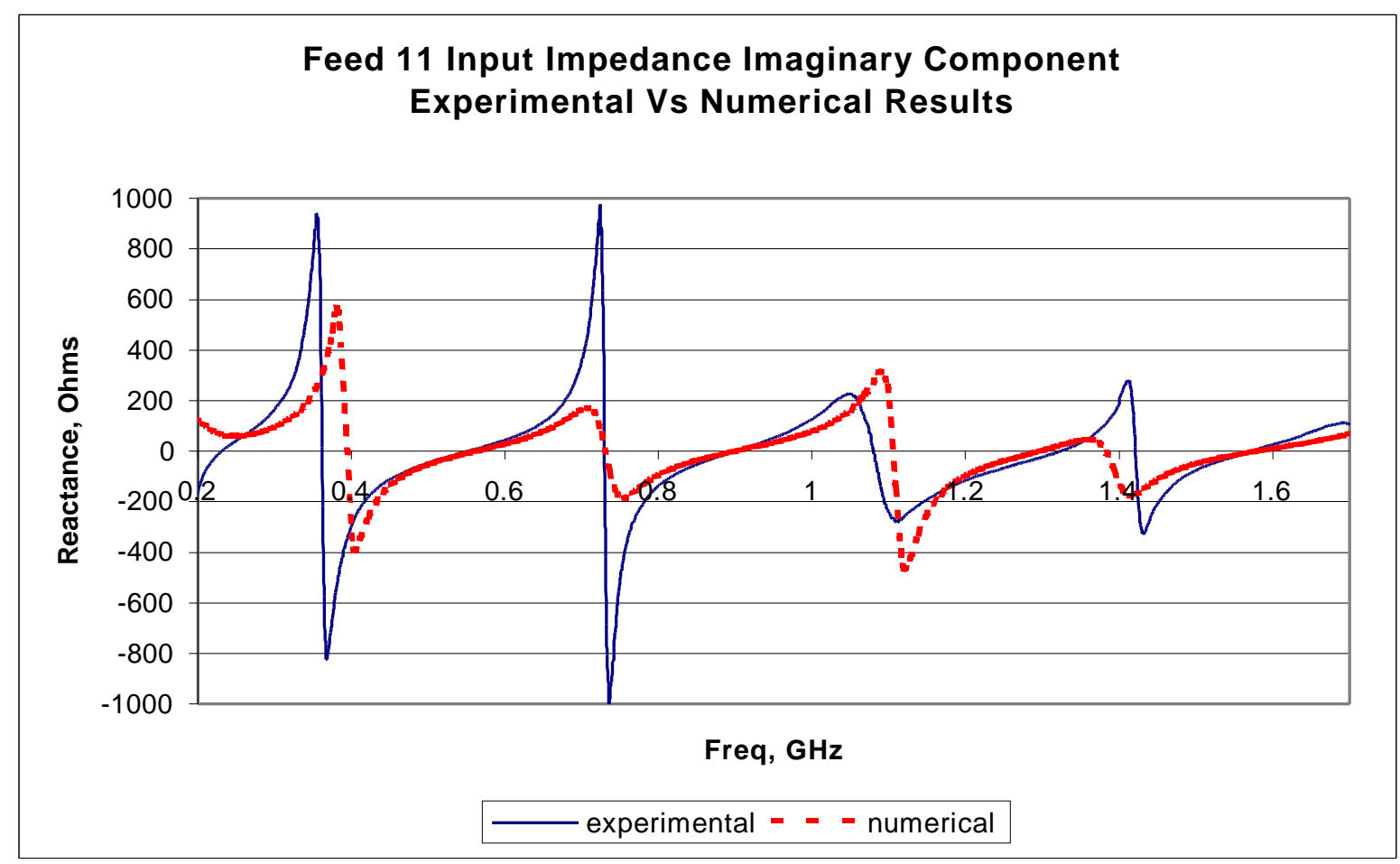

Figure F.54 The imaginary component of the input impedance for feed 11

Table F.22 Analysis of resonance frequencies for feed 11

\begin{tabular}{|c|c|c|}
\multicolumn{3}{c}{ Experimental } \\
\hline $\begin{array}{c}\text { f11 exp. } \\
(\mathrm{GHz})\end{array}$ & $\begin{array}{c}\text { diff. between } \\
\text { res freq's }\end{array}$ & $\begin{array}{c}\text { diff. between } \\
\text { same type freq's }\end{array}$ \\
\hline 0.362 & & \\
\hline 0.552 & 0.191 & \\
\hline 0.730 & 0.178 & 0.368 \\
\hline 0.900 & 0.171 & 0.348 \\
\hline 1.080 & 0.180 & 0.350 \\
\hline 1.324 & 0.244 & 0.424 \\
\hline 1.421 & 0.097 & 0.342 \\
\hline 1.573 & 0.152 & 0.249 \\
\hline 1.735 & 0.162 & 0.313 \\
\hline mean & 0.172 & 0.342 \\
\hline
\end{tabular}

\begin{tabular}{|c|c|c|}
\hline \multicolumn{3}{|c|}{ Numerical } \\
\hline $\begin{array}{c}\text { f11 num. } \\
(\mathrm{GHz})\end{array}$ & $\begin{array}{l}\text { diff between } \\
\text { res freq's }\end{array}$ & $\begin{array}{c}\text { diff between } \\
\text { same type freq's }\end{array}$ \\
\hline 0.394 & & \\
\hline 0.563 & 0.168 & \\
\hline 0.731 & 0.168 & 0.336 \\
\hline 0.900 & 0.169 & 0.337 \\
\hline 1.105 & 0.205 & 0.375 \\
\hline 1.296 & 0.191 & 0.396 \\
\hline 1.382 & 0.086 & 0.277 \\
\hline 1.580 & 0.198 & 0.284 \\
\hline 1.833 & 0.253 & 0.451 \\
\hline mean & 0.180 & 0.351 \\
\hline
\end{tabular}

\begin{tabular}{|c|}
\hline $\begin{array}{c}\text { error } \\
\%\end{array}$ \\
\hline 9.06 \\
\hline 1.88 \\
\hline 0.16 \\
\hline 0.04 \\
\hline 2.37 \\
\hline 2.12 \\
\hline 2.76 \\
\hline 0.45 \\
\hline 5.65 \\
\hline
\end{tabular}




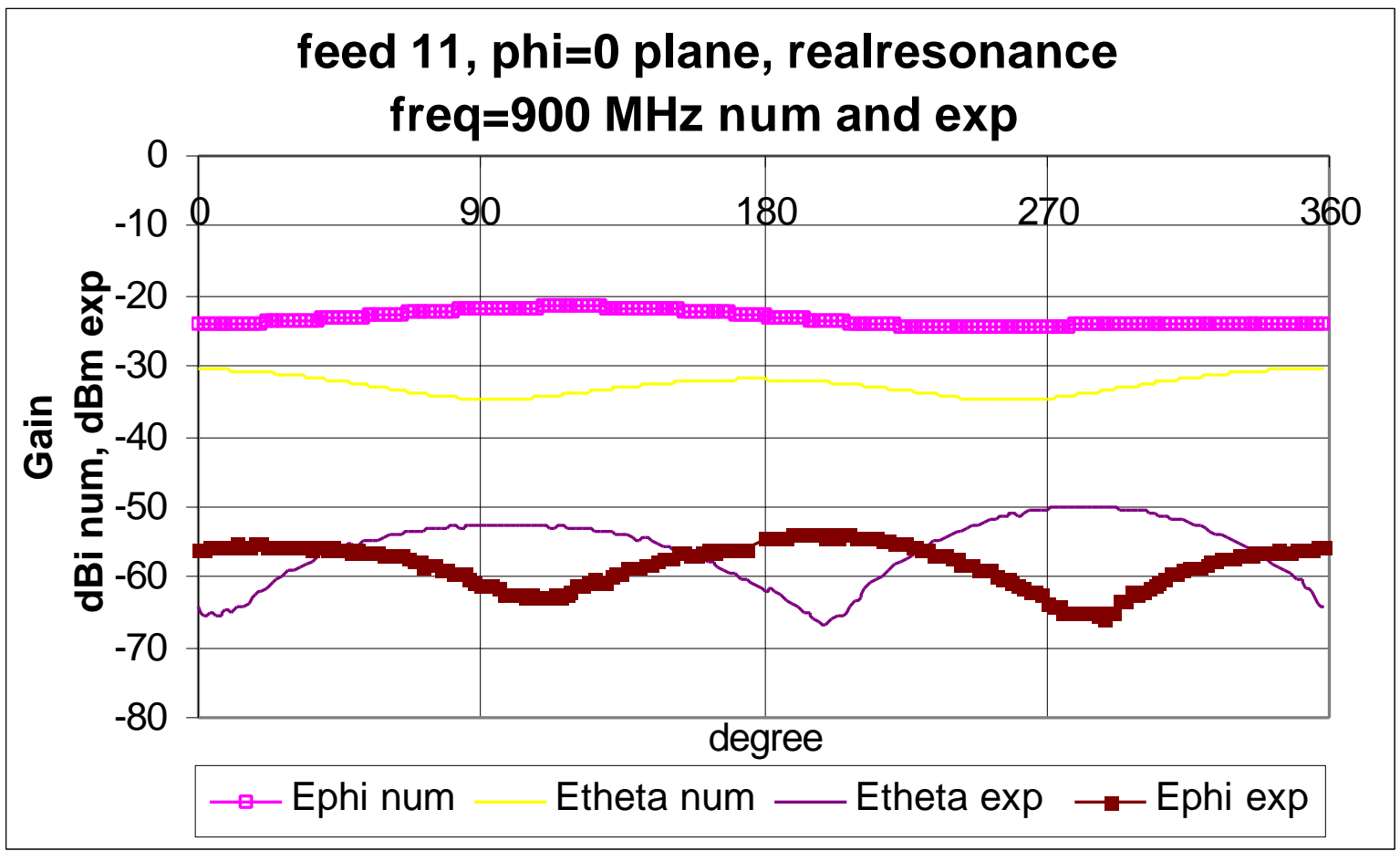

Figure F.55 The Phi =0 plane for feed 11 at 900MHz num and exp

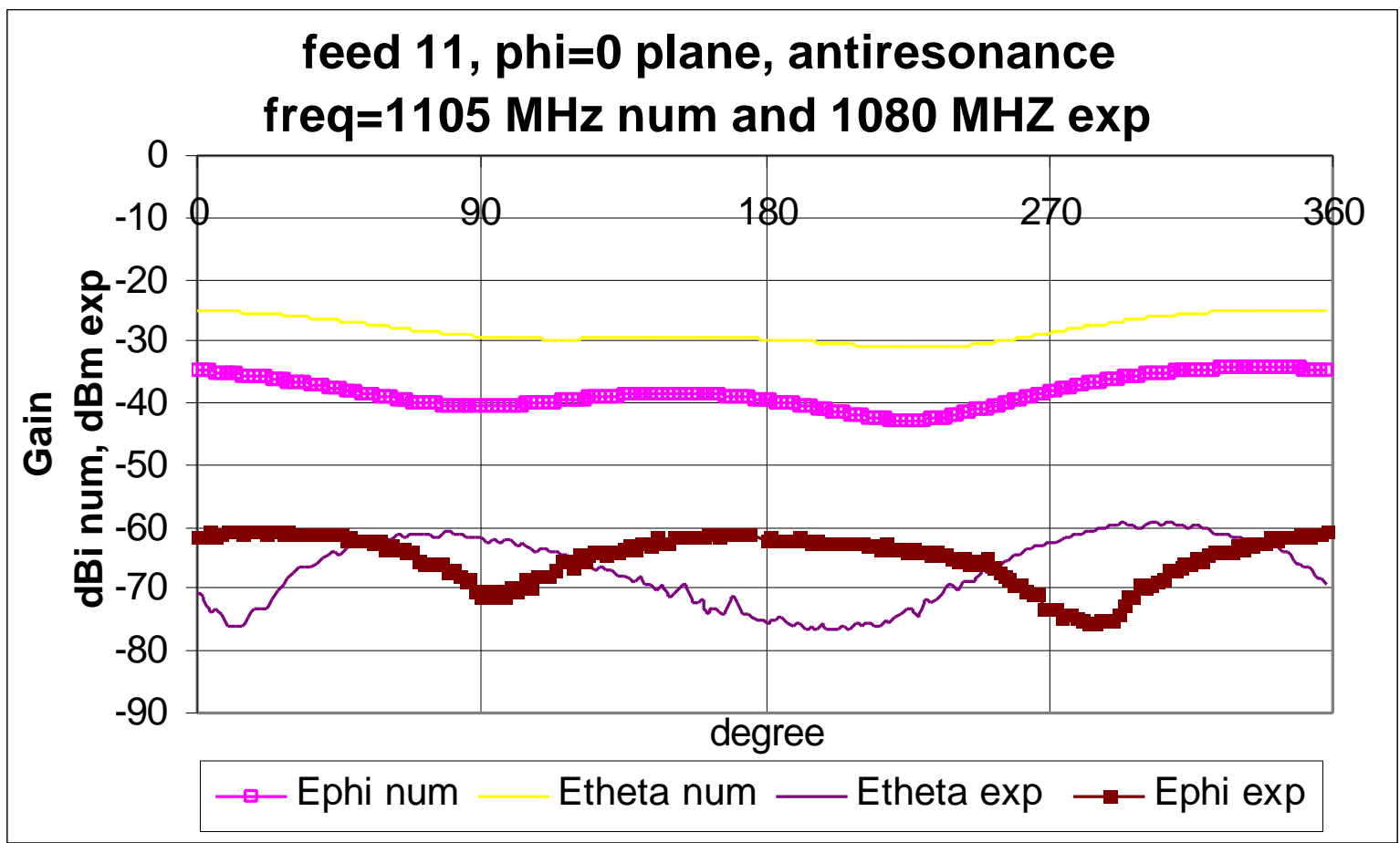

Figure F.56 The Phi = 0 plane for feed 11 at $1105 \mathrm{MHz}$ num and $1080 \mathrm{MHz}$ exp 


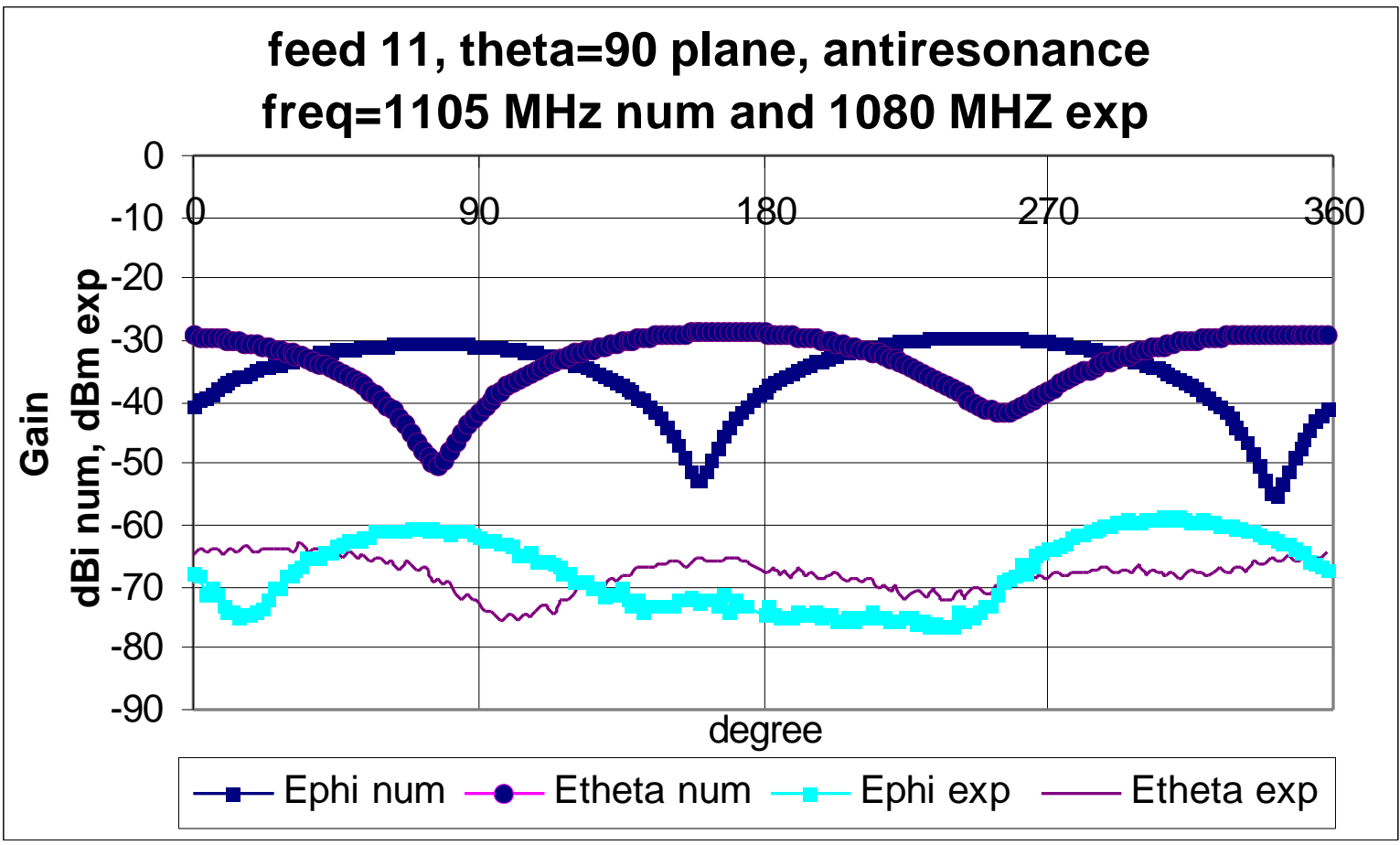

Figure F.57 The Theta $=90$ plane for feed 11 at $1105 \mathrm{MHz}$ num and $1080 \mathrm{MHz}$ exp

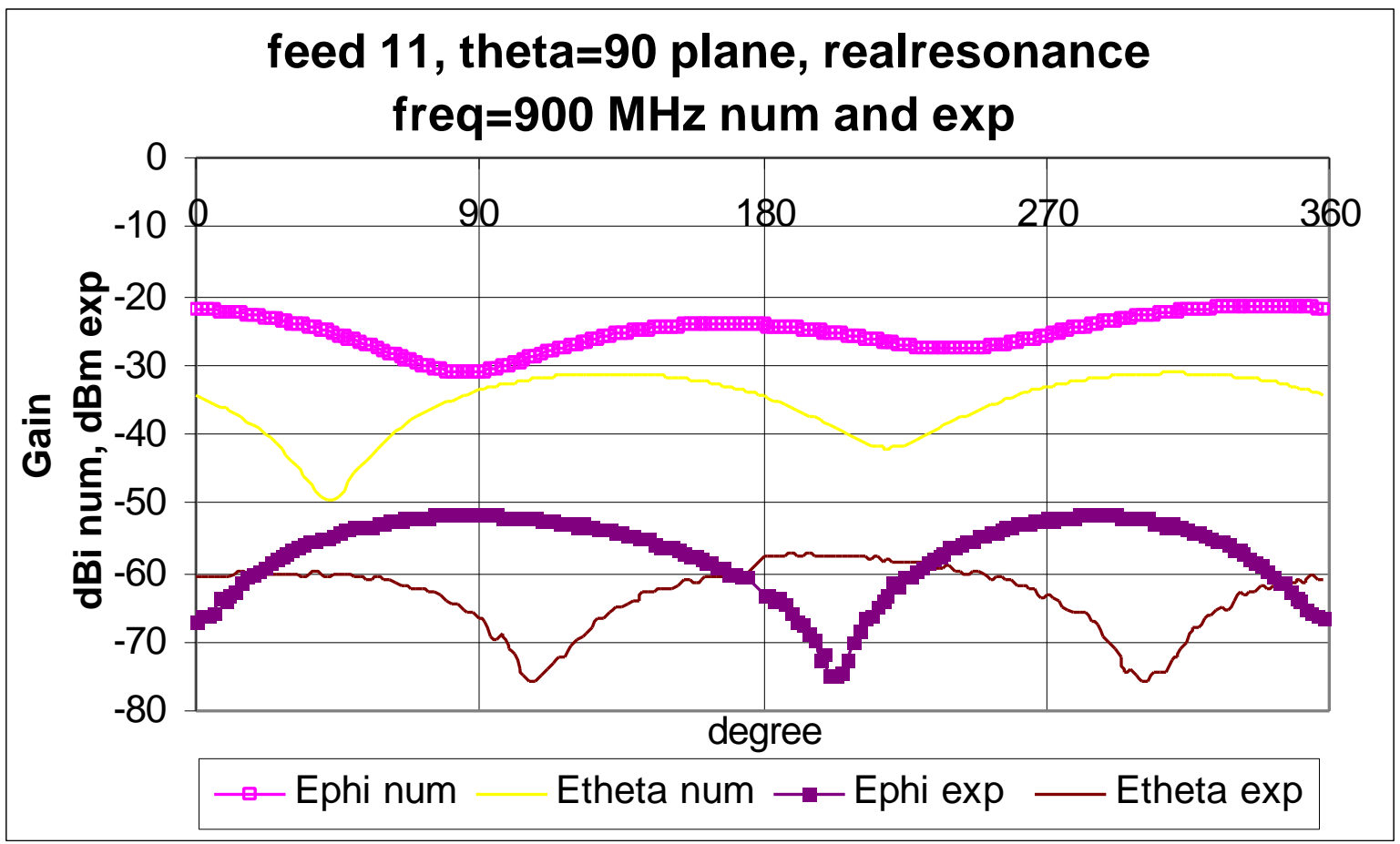

Figure F.58 The Theta $=90$ plane for feed 11 at $900 \mathrm{MHz}$ num and exp 
Table F.23 Analysis of the steady state graphs for feed 11

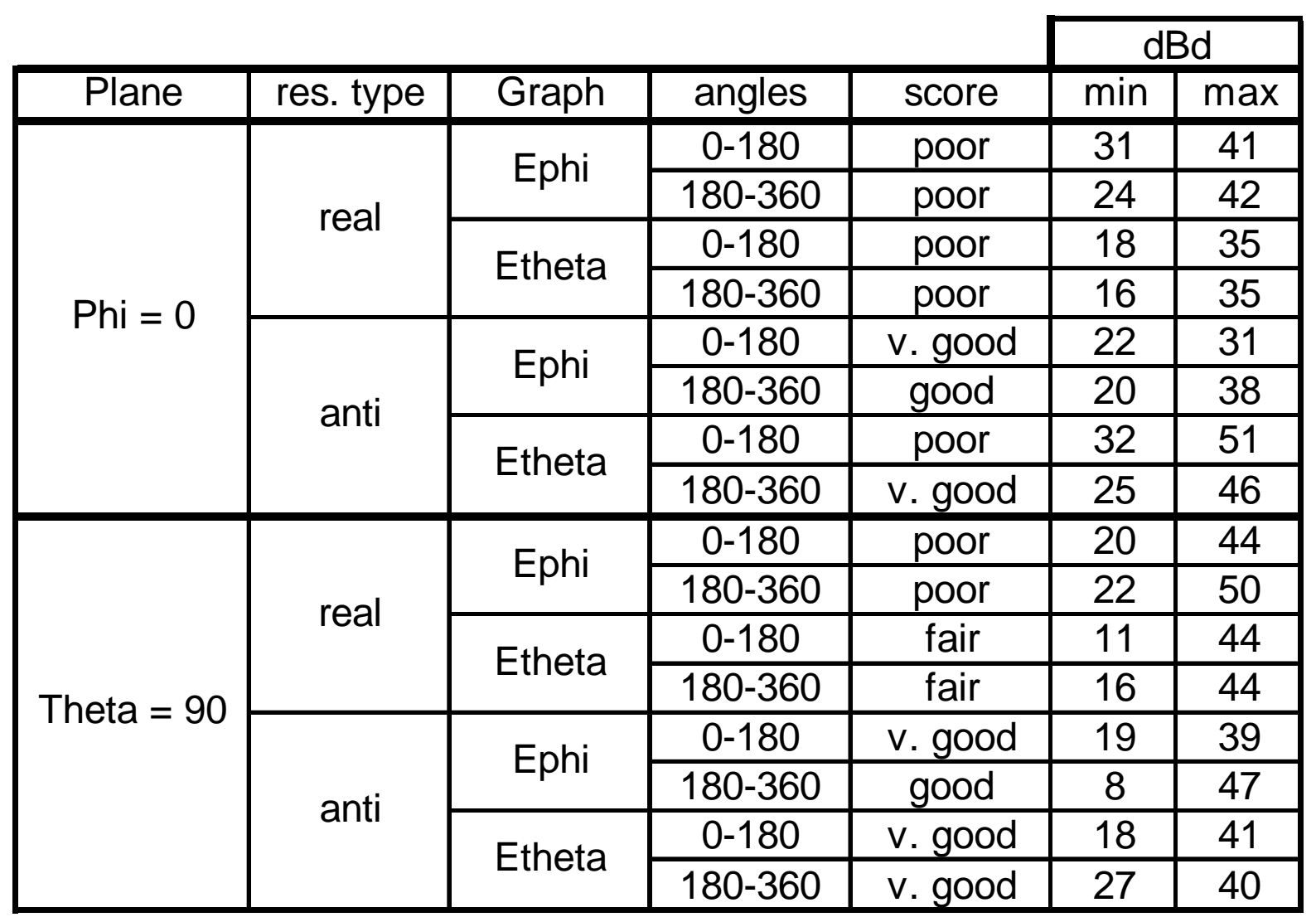




\section{Feed 12}

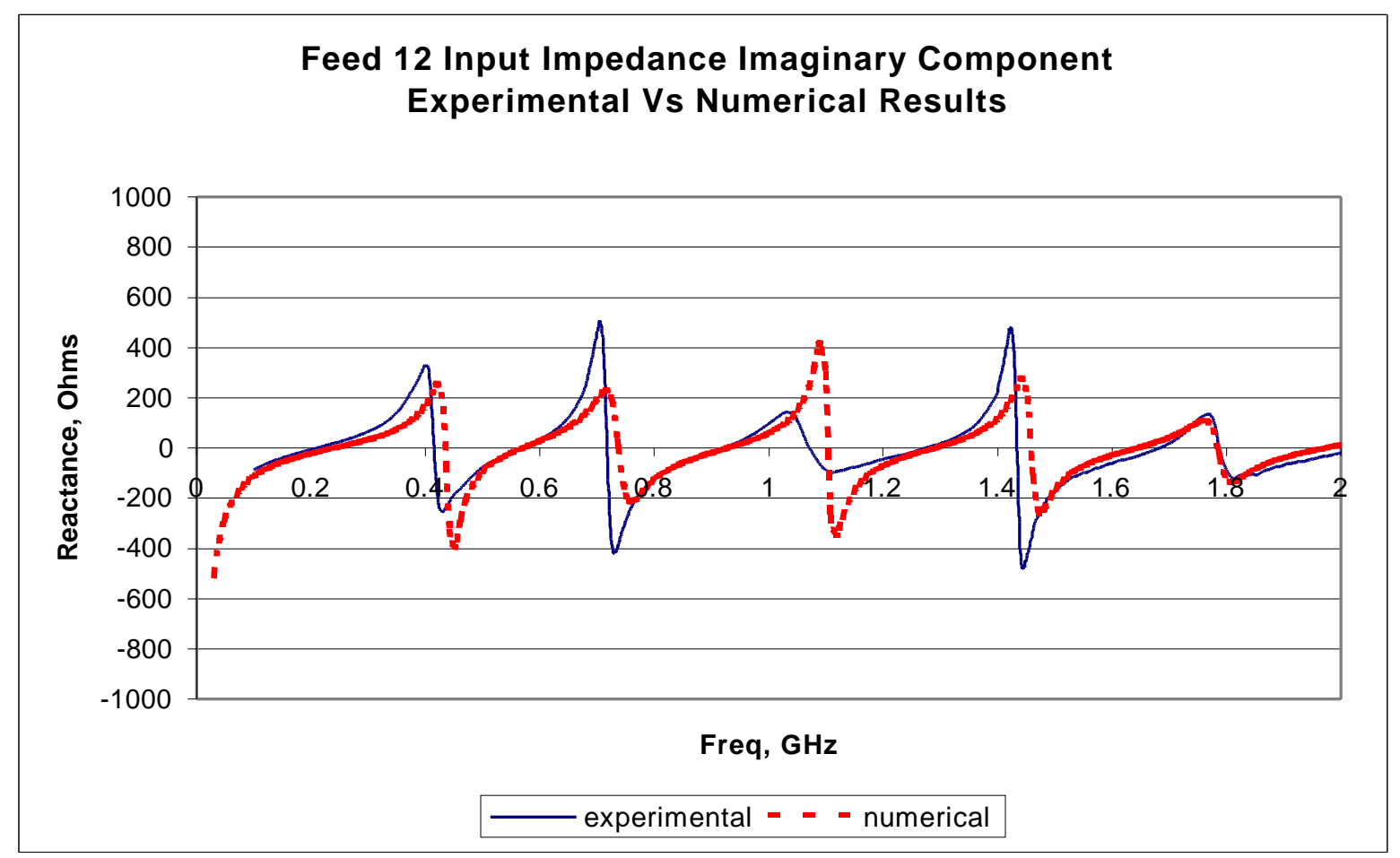

Figure F.59 The imaginary component of the input impedance for feed 12

Table F.24 Analysis of resonance frequencies for feed 12

\begin{tabular}{|c|c|c|}
\multicolumn{3}{c}{ Experimental } \\
\hline $\begin{array}{c}f 12 \text { exp. } \\
(\mathrm{GHz})\end{array}$ & $\begin{array}{c}\text { diff. between } \\
\text { res freq's }\end{array}$ & $\begin{array}{c}\text { diff. between } \\
\text { same type freq's }\end{array}$ \\
\hline 0.415 & & \\
\hline 0.569 & 0.154 & \\
\hline 0.717 & 0.149 & 0.302 \\
\hline 0.922 & 0.204 & 0.353 \\
\hline 1.071 & 0.150 & 0.354 \\
\hline 1.275 & 0.204 & 0.353 \\
\hline 1.434 & 0.159 & 0.363 \\
\hline 1.684 & 0.249 & 0.409 \\
\hline 1.788 & 0.104 & 0.354 \\
\hline mean & 0.172 & 0.355 \\
\hline
\end{tabular}

\begin{tabular}{|c|c|c|}
\hline \multicolumn{3}{|c|}{ Numerical } \\
\hline $\begin{array}{c}\text { f12 num. } \\
(\mathrm{GHz})\end{array}$ & $\begin{array}{l}\text { diff between } \\
\text { res freq's }\end{array}$ & $\begin{array}{c}\text { diff between } \\
\text { same type freq's }\end{array}$ \\
\hline 0.435 & & \\
\hline 0.570 & 0.135 & \\
\hline 0.738 & 0.168 & 0.303 \\
\hline 0.928 & 0.191 & 0.358 \\
\hline 1.104 & 0.176 & 0.367 \\
\hline 1.290 & 0.186 & 0.362 \\
\hline 1.459 & 0.168 & 0.355 \\
\hline 1.644 & 0.185 & 0.354 \\
\hline 1.785 & 0.141 & 0.326 \\
\hline mean & 0.169 & 0.346 \\
\hline
\end{tabular}

\begin{tabular}{|c|}
\hline $\begin{array}{c}\text { error } \\
\%\end{array}$ \\
\hline 4.76 \\
\hline 0.20 \\
\hline 2.84 \\
\hline 0.71 \\
\hline 3.08 \\
\hline 1.19 \\
\hline 1.72 \\
\hline 2.34 \\
\hline 0.18 \\
\hline
\end{tabular}




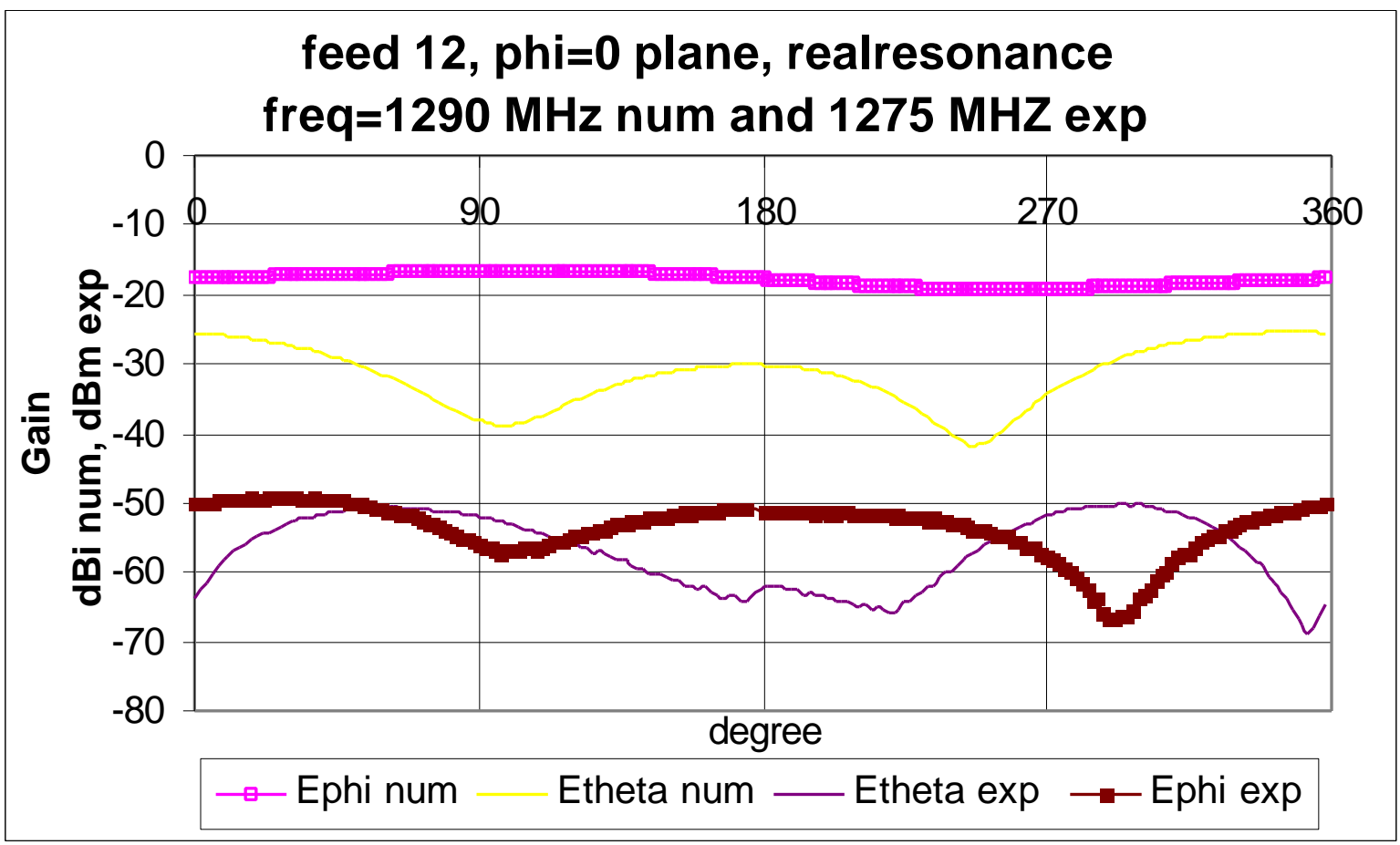

Figure F.60 The Phi = O plane for feed 12 at $1290 \mathrm{MHz}$ num and $1275 \mathrm{MHz}$ exp

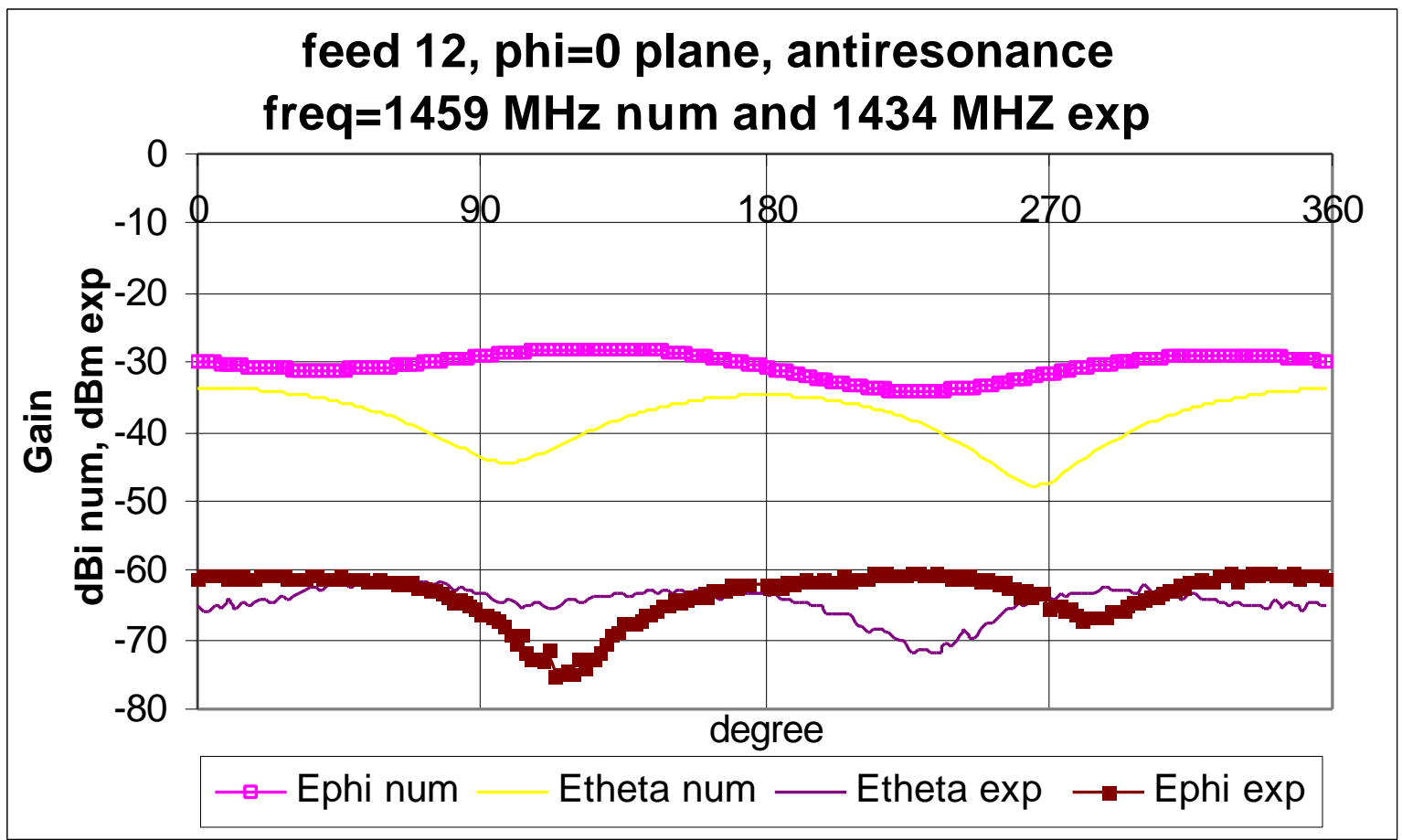

Figure F.61 The Phi = O plane for feed 12 at $1459 \mathrm{MHz}$ num and $1434 \mathrm{MHz}$ exp 


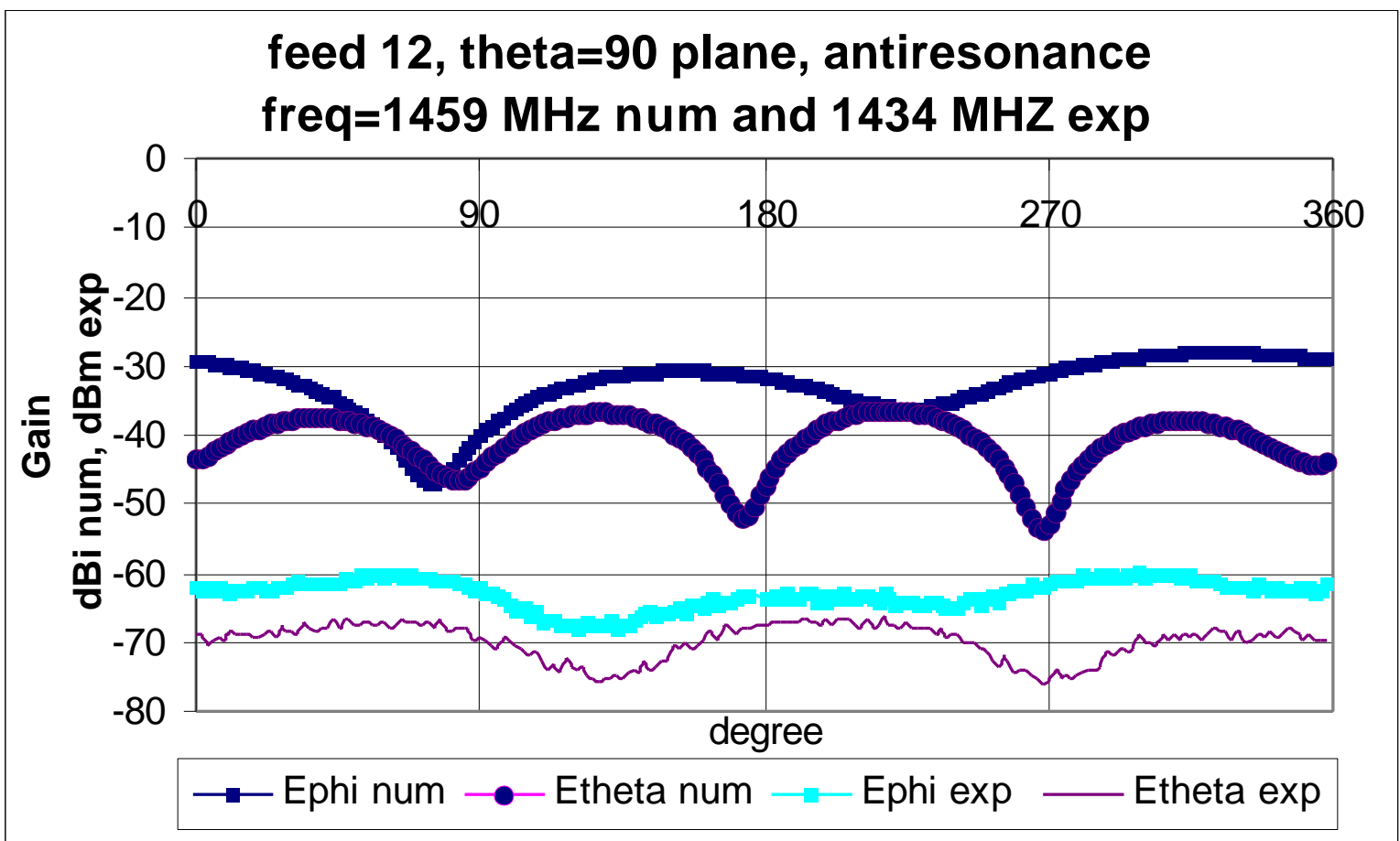

Figure F.62 The Theta $=90$ plane for feed 12 at $1459 \mathrm{MHz}$ num and $1434 \mathrm{MHz}$ exp

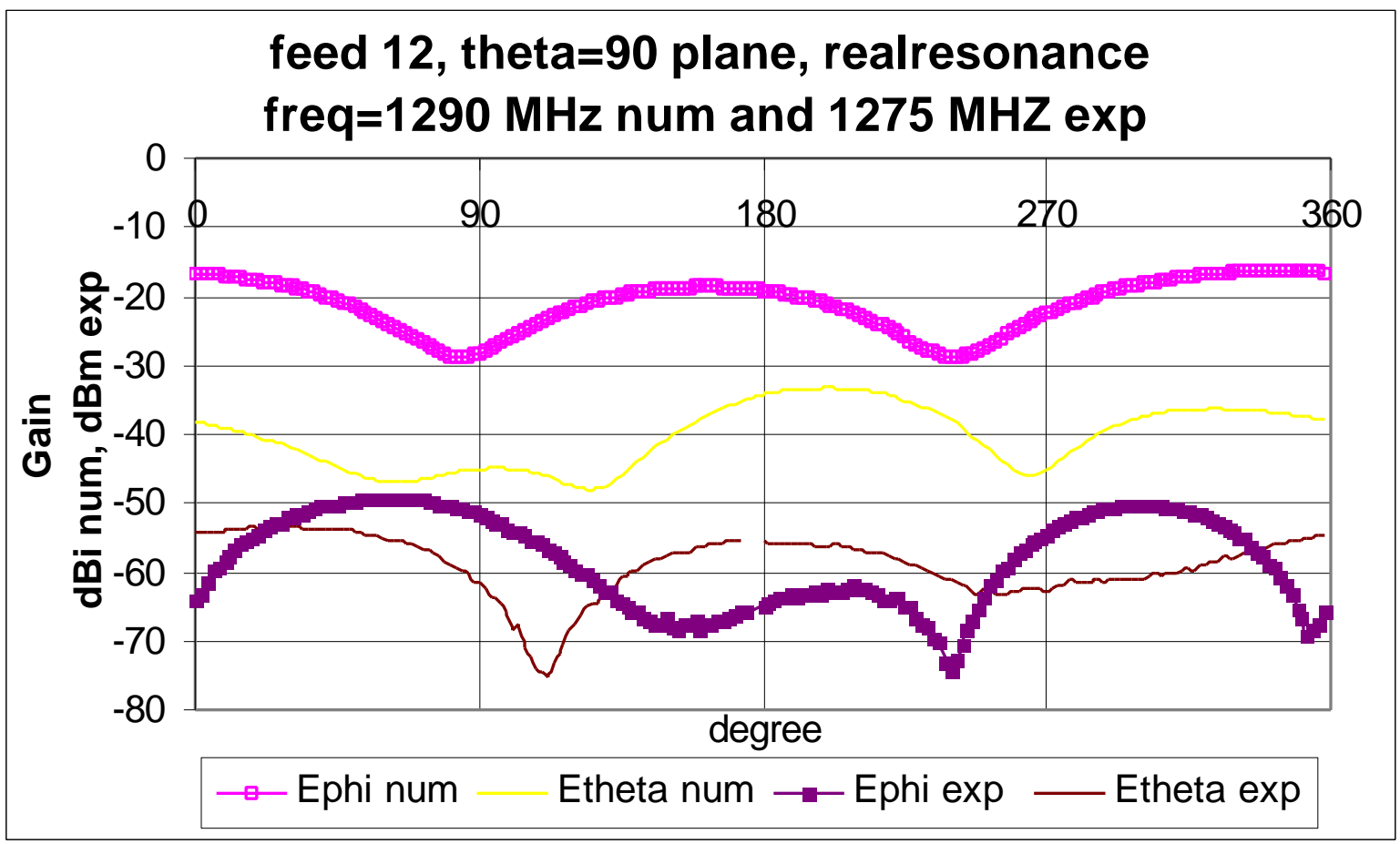

Figure F.63 The Theta $=90$ plane for feed 12 at $1290 \mathrm{MHz}$ num and $1275 \mathrm{MHz}$ exp 
Table F.25 Analysis of the steady state graphs for feed 12

\begin{tabular}{|c|c|c|c|c|c|c|}
\hline & & & & & & \\
\hline Plane & res. type & Graph & angles & score & $\min$ & $\max$ \\
\hline \multirow{8}{*}{$P h i=0$} & \multirow{4}{*}{ real } & \multirow{2}{*}{ Ephi } & $0-180$ & v. good & 32 & 41 \\
\hline & & & $180-360$ & v. good & 18 & 48 \\
\hline & & \multirow{2}{*}{ Etheta } & $0-180$ & poor & 14 & 38 \\
\hline & & & $180-360$ & fair & 14 & 43 \\
\hline & \multirow{4}{*}{ anti } & \multirow{2}{*}{ Ephi } & $0-180$ & good & 30 & 47 \\
\hline & & & $180-360$ & v. good & 26 & 36 \\
\hline & & \multirow{2}{*}{ Etheta } & $0-180$ & poor & 20 & 32 \\
\hline & & & $180-360$ & v. good & 16 & 34 \\
\hline \multirow{8}{*}{ Theta $=90$} & \multirow{4}{*}{ real } & \multirow{2}{*}{ Ephi } & $0-180$ & poor & 22 & 50 \\
\hline & & & $180-360$ & fair & 17 & 53 \\
\hline & & \multirow{2}{*}{ Etheta } & $0-180$ & poor & 8 & 35 \\
\hline & & & $180-360$ & poor & 16 & 38 \\
\hline & \multirow{4}{*}{ anti } & \multirow{2}{*}{ Ephi } & $0-180$ & fair & 14 & 37 \\
\hline & & & $180-360$ & v. good & 27 & 34 \\
\hline & & \multirow{2}{*}{ Etheta } & $0-180$ & v. good & 16 & 39 \\
\hline & & & $180-360$ & v. good & 20 & 44 \\
\hline
\end{tabular}




\section{Feed 13}

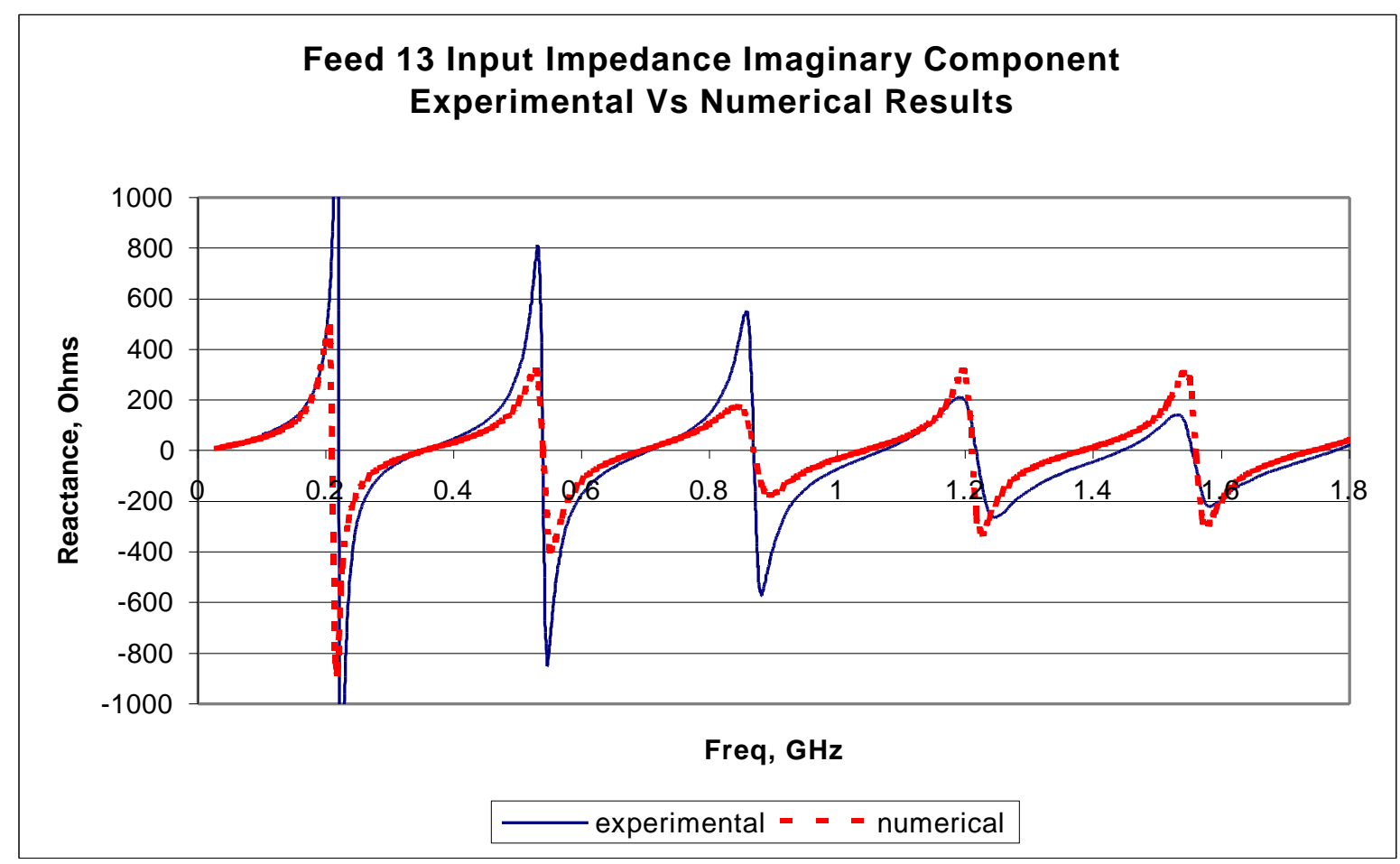

Figure F.64 The imaginary component of the input impedance for feed 13

Table F.26 Analysis of resonance frequencies for feed $\mathbf{1 3}$

\begin{tabular}{|c|c|c|}
\hline & \\
\hline $\begin{array}{c}\text { f13 exp. } \\
(\mathrm{GHz})\end{array}$ & $\begin{array}{l}\text { diff. between } \\
\text { res freq's }\end{array}$ & $\begin{array}{c}\text { diff. between } \\
\text { same type freq's }\end{array}$ \\
\hline 0.221 & & \\
\hline 0.354 & 0.133 & \\
\hline 0.539 & 0.185 & 0.319 \\
\hline 0.705 & 0.166 & 0.352 \\
\hline 0.869 & 0.164 & 0.330 \\
\hline 1.070 & 0.200 & 0.364 \\
\hline 1.217 & 0.147 & 0.347 \\
\hline 1.444 & 0.228 & 0.375 \\
\hline 1.554 & 0.110 & 0.337 \\
\hline 1.777 & 0.223 & 0.333 \\
\hline 1.891 & 0.114 & 0.337 \\
\hline mean & 0.173 & 0.345 \\
\hline
\end{tabular}

\begin{tabular}{|c|c|c|}
\hline \multicolumn{3}{|c|}{ Numerical } \\
\hline $\begin{array}{c}\text { f13 num. } \\
(\mathrm{GHz})\end{array}$ & $\begin{array}{l}\text { diff between } \\
\text { res freq's }\end{array}$ & $\begin{array}{c}\text { diff between } \\
\text { same type freq's }\end{array}$ \\
\hline 0.210 & & \\
\hline 0.354 & 0.144 & \\
\hline 0.540 & 0.186 & 0.330 \\
\hline 0.694 & 0.154 & 0.340 \\
\hline 0.869 & 0.175 & 0.329 \\
\hline 1.041 & 0.173 & 0.348 \\
\hline 1.211 & 0.169 & 0.342 \\
\hline 1.381 & 0.171 & 0.340 \\
\hline 1.560 & 0.179 & 0.349 \\
\hline 1.744 & 0.184 & 0.362 \\
\hline 1.927 & 0.183 & 0.367 \\
\hline mean & 0.170 & 0.343 \\
\hline
\end{tabular}

\begin{tabular}{|c|}
\hline $\begin{array}{c}\text { error } \\
\%\end{array}$ \\
\hline 4.95 \\
\hline 0.03 \\
\hline 0.15 \\
\hline 1.63 \\
\hline 0.04 \\
\hline 2.62 \\
\hline 0.48 \\
\hline 4.36 \\
\hline 0.39 \\
\hline 1.87 \\
\hline 1.91 \\
\hline
\end{tabular}




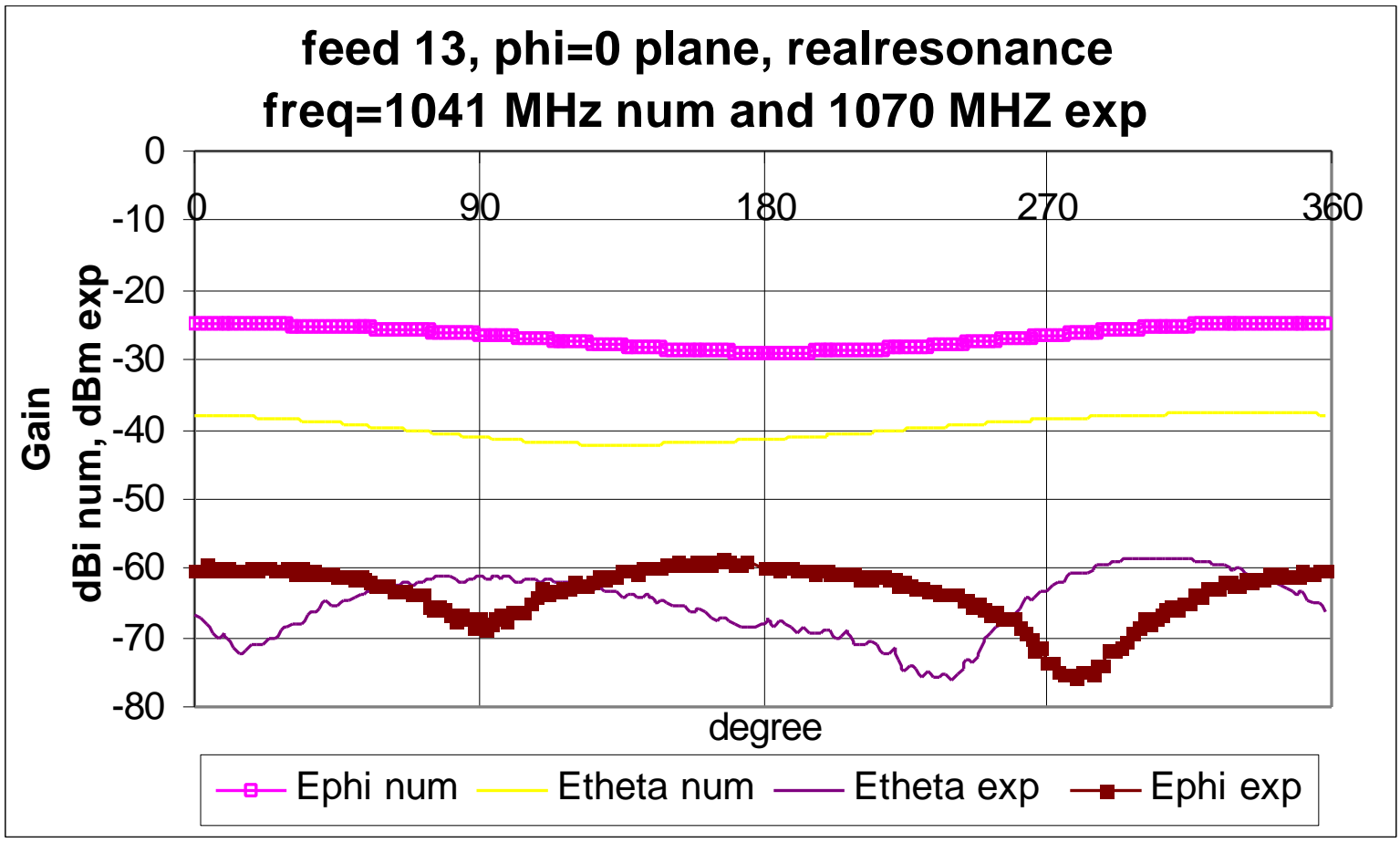

Figure F.65 The Phi = 0 plane for feed 13 at $1041 \mathrm{MHz}$ num and $1070 \mathrm{MHz}$ exp

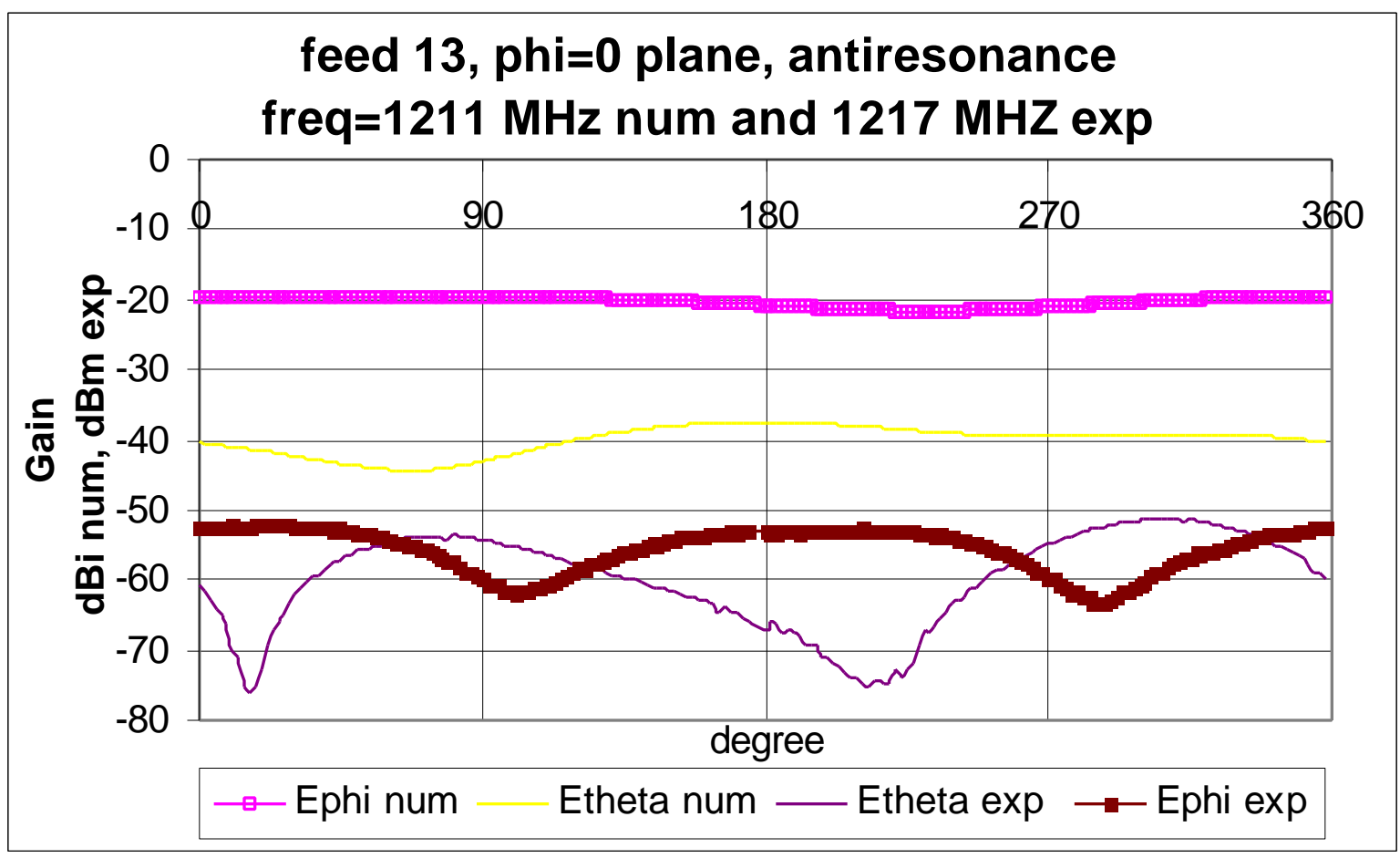

Figure F.66 The Phi =0 plane for feed 13 at $1211 \mathrm{MHz}$ num and $1217 \mathrm{MHz}$ exp 


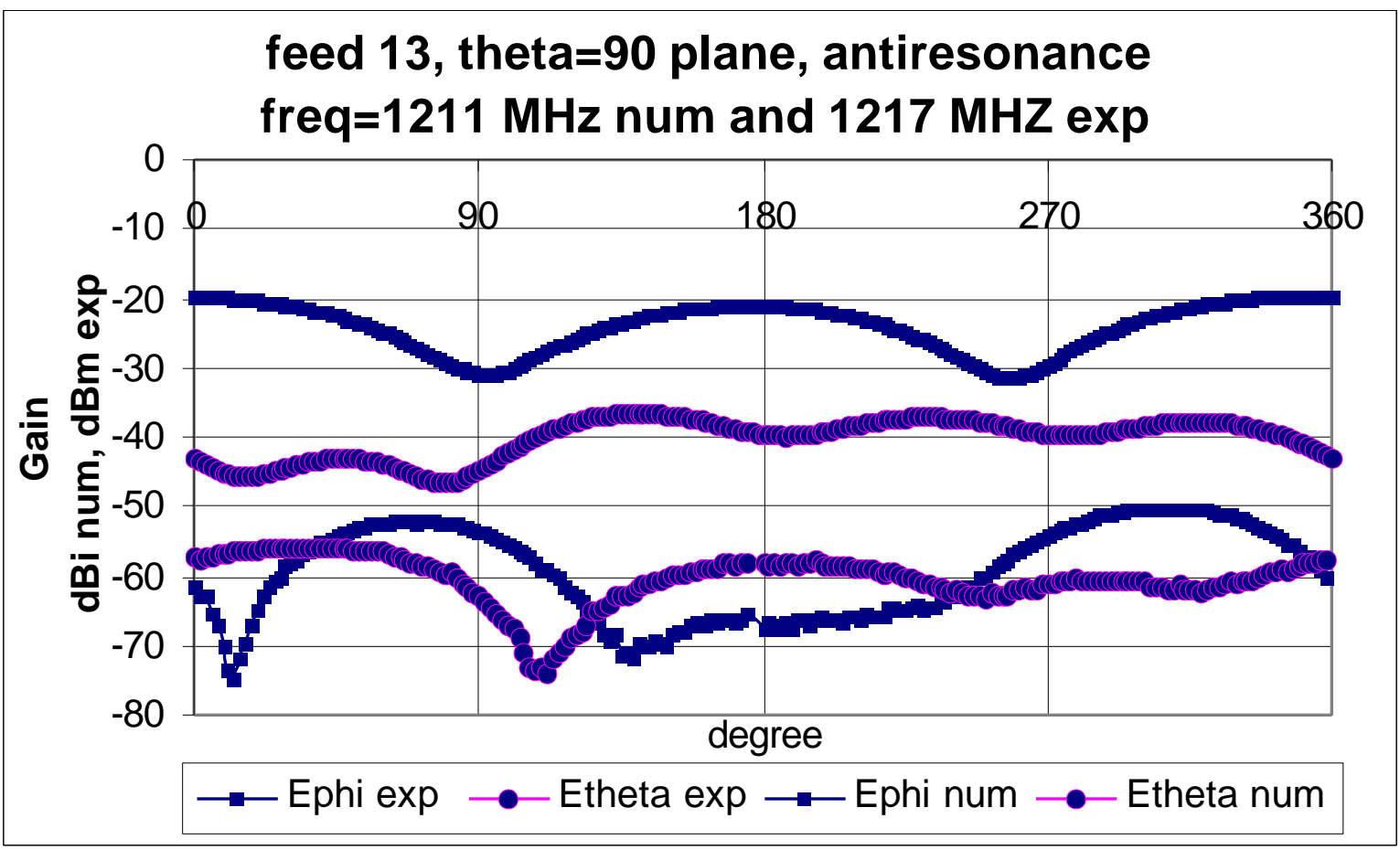

Figure F.67 The Theta $=90$ plane for feed 13 at $1211 \mathrm{MHz}$ num and $1217 \mathrm{MHz}$ exp

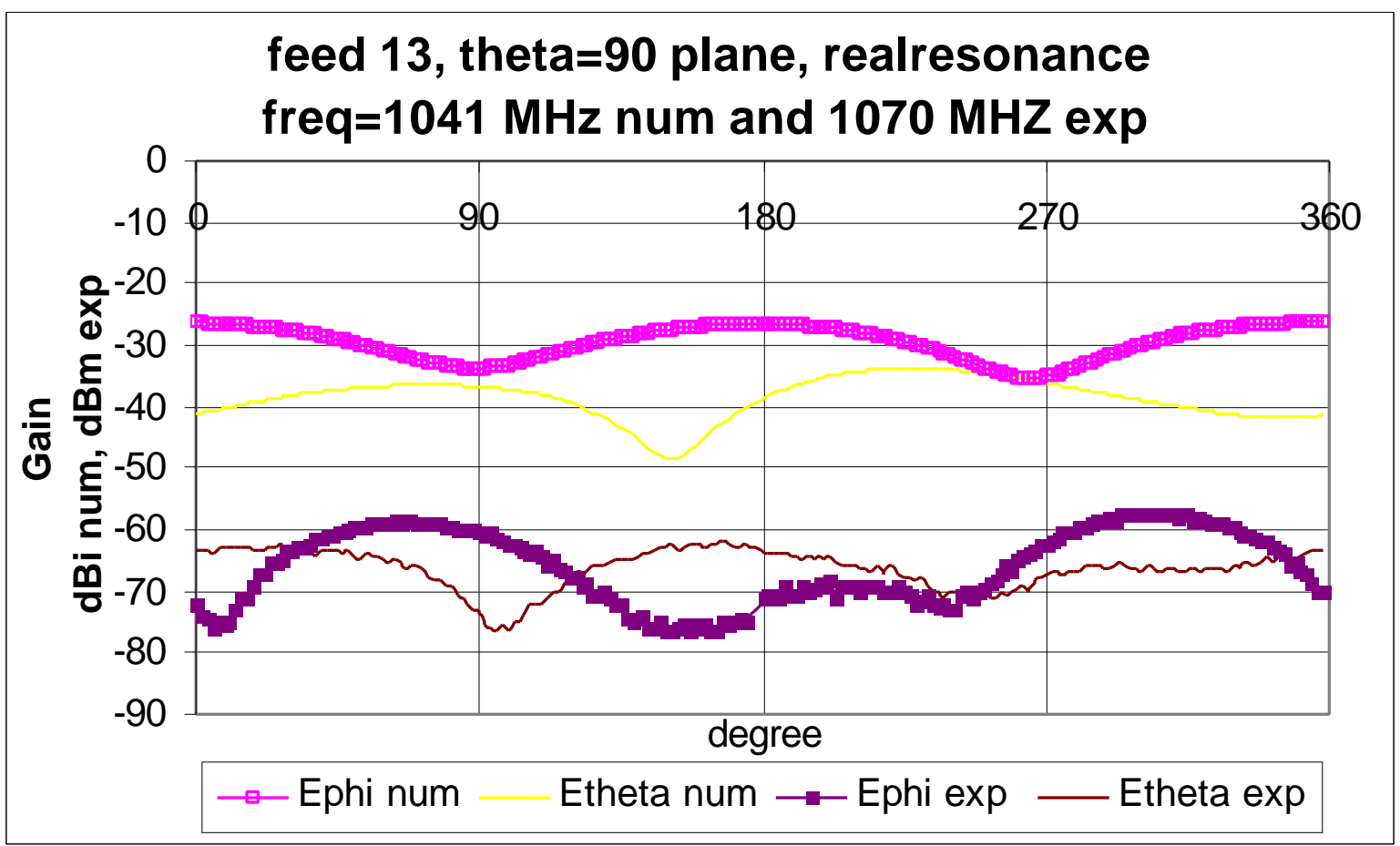

Figure F.68 The Theta $=90$ plane for feed 13 at $1041 \mathrm{MHz}$ num and $1070 \mathrm{MHz}$ exp 
Table F.27 Analysis of the steady state graphs for feed $\mathbf{1 3}$

\begin{tabular}{|c|c|c|c|c|c|c|}
\hline & & & & & & \\
\hline Plane & res. type & Graph & angles & score & $\min$ & $\max$ \\
\hline & & Enhi & $0-180$ & v. good & 30 & 42 \\
\hline & real & ᄃр/II & $180-360$ & v. good & 25 & 49 \\
\hline & real & Ftheta & $0-180$ & poor & 20 & 34 \\
\hline & & Cuneta & $180-360$ & fair & 20 & 38 \\
\hline $\mathrm{rnl}=0$ & & & $0-180$ & good & 32 & 42 \\
\hline & anti & Epni & $180-360$ & v. good & 20 & 43 \\
\hline & anll & Ftheta & $0-180$ & poor & 9 & 35 \\
\hline & & Emeta & $180-360$ & v. good & 12 & 40 \\
\hline & & Enhi & $0-180$ & poor & 26 & 50 \\
\hline & ral & ᄃp/II & $180-360$ & fair & 25 & 46 \\
\hline & real & Ftheta & $0-180$ & poor & 14 & 39 \\
\hline Theta $=c$ & & Lireta & $180-360$ & poor & 22 & 41 \\
\hline meta $=y$ & & & $0-180$ & fair & 22 & 55 \\
\hline & anti & Ephi & $180-360$ & v. good & 20 & 46 \\
\hline & dillu & Ftheta & $0-180$ & v. good & 11 & 35 \\
\hline & & Elneta & $180-360$ & v. good & 15 & 44 \\
\hline
\end{tabular}




\section{Appendix G: CIRA Internal Reports}

In the following pages, a copy of the internal reports mentioned in this study is provided.

The reports provided are:

- ElSherbini, K.M., "Design of a $433 \mathrm{MHz}$ PCB-CTHA," CTHA Internal Report Series 980806, Center for Industrial Research Applications, West Virginia University, 1998.

- ElSherbini, K.M., "Effect of the Material Properties and the Holes Geometry on the Input Impedance of a PCB-CTHA," CTHA Internal Report Series 980729, Center for Industrial Research Applications, West Virginia University, 1998.

- ElSherbini, K.M., "Experiments Conducted on Larry Hawks Antennas," CTHA Internal Report Series 980715, Center for Industrial Research Applications, West Virginia University, 1998.

- ElSherbini, K.M., "More Experiments on Larry Hawks Antennas," CTHA Internal Report Series 980722, Center for Industrial Research Applications, West Virginia University, 1998.

- Zheng, L., "Flexible Mesh Generator for CTHA," CTHA Internal Report Series 980805, Center for Industrial Research Applications, West Virginia University, 1998.

- Zheng, L., "Parametric Study on Resonance Frequency of CTHA, " CTHA Internal Report Series 980812, Center for Industrial Research Applications, West Virginia University, 1998.

- Craven, R., "CTHA feed study 1," CTHA Internal Report Series 980325, Center for Industrial Research Applications, West Virginia University, 1998. 
Center for Industrial Research Applications

West Virginia University

CTHA Internal Report Series 980805

Aug 5, 1998

Khaled ElSherbini

\section{Design of a $433 \mathrm{MHz}$ PCB-CTHA}

\section{Introduction}

In this report we go through the design process of a $433 \mathrm{MHz}$ printed circuit board CTHA. The aim is to design a PCB-CTHA with a first resonance frequency of, or as close as possible to, $433 \mathrm{MHz}$, and with the highest gain possible. The CTHA has a dimensional limitation of:

Maximum major diameter of $1.5 "$

Minimum minor diameter of 0.5 "

Thickness of 0.19 "

\section{Design methodology}

The following steps are to be followed in designing the CTHA:

1. The first antenna is to be designed with no binding material and with 8 turns

2. Then the same antenna is to be designed with a binding material with a dielectric constant of 4.0

3. For each of the above antennas we are to check for the first resonance frequency and for the gain at that frequency

4. If the desired frequency is not obtained, we start changing the number of turns between 5 and 10 depending on the desired effect.

5. If the desired frequency is still not obtained, we take the design giving the closest first resonance frequency to $433 \mathrm{MHz}$ and start fine tuning the frequency by controlling the ratio of major to minor diameters, the thickness of the four layers, the thickness of the traces, the diameter of the holes, etc... 


\section{Design Procedure}

The following are the all the runs required until the desired design was obtained.

Run1: The following are the design parameters of the first antenna

Length \& width: $38.1 \mathrm{~mm}$

Height: $\quad 4.826 \mathrm{~mm}$

Major diameter: $36 \mathrm{~mm}$

Minor diameter: $15 \mathrm{~mm}$

Lowest and highest planes of traces on bottom and top of the CTHA

Second plane of traces: $1.61 \mathrm{~mm}$

Third plane of traces: $3.22 \mathrm{~mm}$

Hollow holes

Hole diameter: $1.6764 \mathrm{~mm}$

Width of traces: $1.6764 \mathrm{~mm}$

Number of turns: 8

Binding material: air

Feed 6

Run2: this was exactly the same as run1 but with a number of turns of 5 .

Runs 3 to 8: These were all similar to runs 1 and 2 except that the number of turns and the dielectric constant of the binder material were changed, as shown in table 1:

\begin{tabular}{|c|c|c|c|c|c|c|c|c|}
\hline Run \# & 1 & 2 & 3 & 4 & 5 & 6 & 7 & 8 \\
\hline \# of turns & 8 & 5 & 8 & 5 & 6 & 6 & 7 & 7 \\
\hline Dielectric & 1 & 1 & 4 & 4 & 1 & 4 & 1 & 4 \\
\hline
\end{tabular}

Table 1. The differences in the design parameters of runs 1 through 8 
Where a dielectric of 4 was used since this is very close to the dielectric constant of the current PCB-CTHA's available. Table 2 shows the resonant frequencies of all 8 runs up to $2 \mathrm{GHz}$ :

\begin{tabular}{|c|c|c|c|c|c|c|c|c|}
\hline & run1 & run2 & run3 & run4 & run5 & run6 & run7 & run8 \\
\hline 1 & 518.7 & 864.1 & 300.8 & 517.1 & 739.4 & $\mathbf{4 3 4 . 1}$ & 605.4 & 349.1 \\
\hline 2 & 967.4 & 1533.0 & 555.5 & 895.4 & 1308.7 & 752.6 & 1114.6 & 634.6 \\
\hline 3 & 1600.7 & & 930.0 & 1569.3 & & 1293.2 & 1871.5 & 1082.5 \\
\hline 4 & & & 1167.8 & 1846.2 & & 1552.2 & & 1326.0 \\
\hline 5 & & & 1593.5 & & & & & 1839.8 \\
\hline 6 & & & 1796.9 & & & & & \\
\hline
\end{tabular}

Table 2. The resonant frequencies of the 8 runs in $\mathrm{MHz}$

As seen in table 2, run6, which has a dielectric constant of 4.0 and 6 turns, gave a first resonance frequency of $434.1 \mathrm{MHz}$ which is very close to the desired frequency. Thus all our concentration will be on run6 from here on.

Run6corrected: In this run, the previously mentioned run6 was used as is, but the model was cleared from any extra cells, and the minor diameter of the wires was made to be equal to the inner diameter of the CTHA. Figure 1 shows the top plane of the CTHA run6corr, with the main dimensions imposed upon it.

For this antenna we achieved a first resonance frequency of $433.5 \mathrm{MHz}$. The resonant frequencies up to $2 \mathrm{GHz}$ were as shown in table 3:

$\begin{array}{cc}1 & 433.5 \\ 2 & 765.3 \\ 3 & 1323.4 \\ 4 & 1590.2\end{array}$

Table 3. first four resonant frequencies of run6corr.

At this point we were satisfied with the frequency of $433.5 \mathrm{MHz}$, and the next steps of the design are to study the radiation patterns and gain of this specific design, run6corr, and compare it to the other designs in this study. 


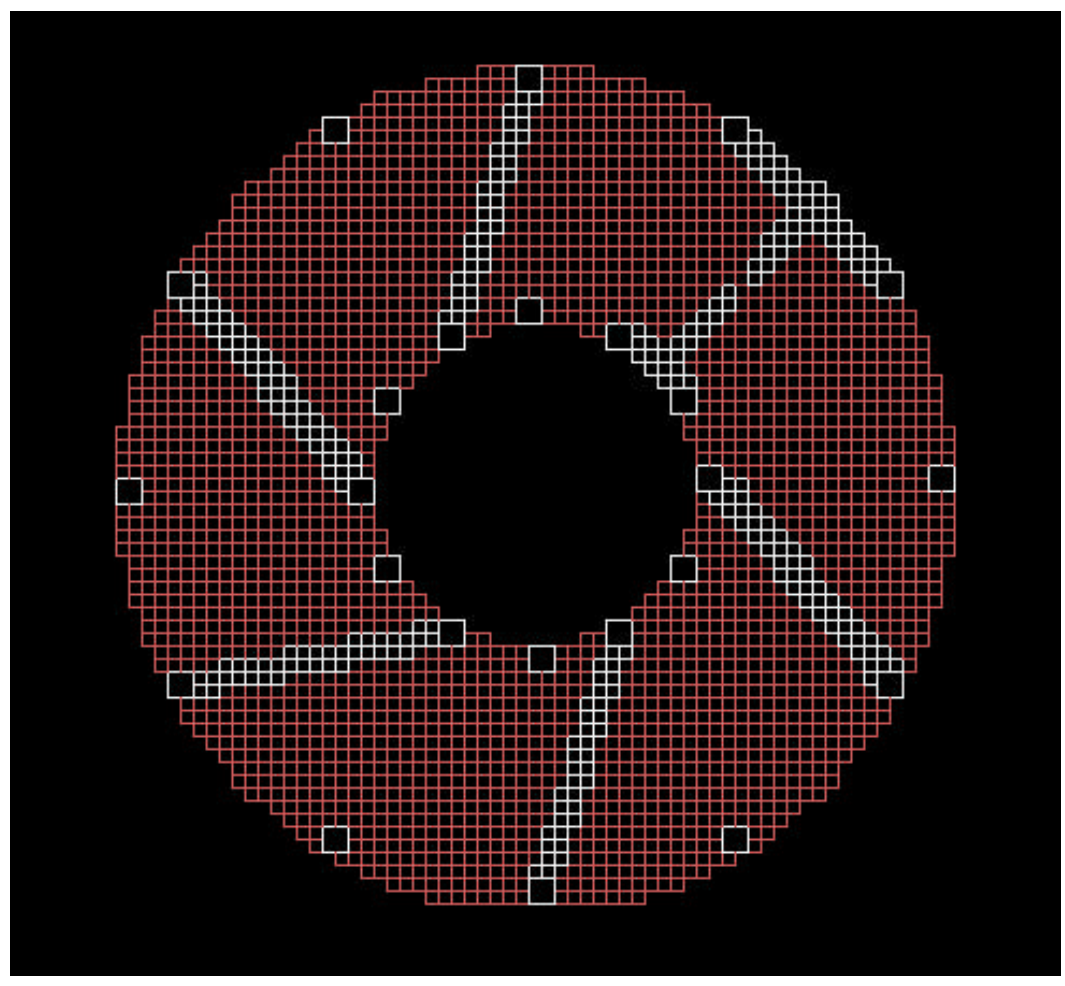

Figure 1. The top layer of the CTHA run6corr

\section{Performance of PCB run6corr}

All 8 circuit boards run1 to run8 were checked for performance at a frequency of $433 \mathrm{MHz}$, in addition to PCB run6corr. Although we are not yet confident of the gain outputs of xfdtd on absolute terms, but the comparison is to be made on relative terms between all 9 antennas. The comparisons will be made on the gain output of the Theta=90 plane, for phi between 0 and 360 degrees in steps of 5 degrees.

Figures 2 and 3 show the far field patterns for all runs. We can clearly see that all antennas almost show the same far field performance, with very slight differences. And that CTHA run6corr shows no superiority over any of the other CTHA's. Actually they show that all the variations of the parameters have a very minor effect on the far field gains. 


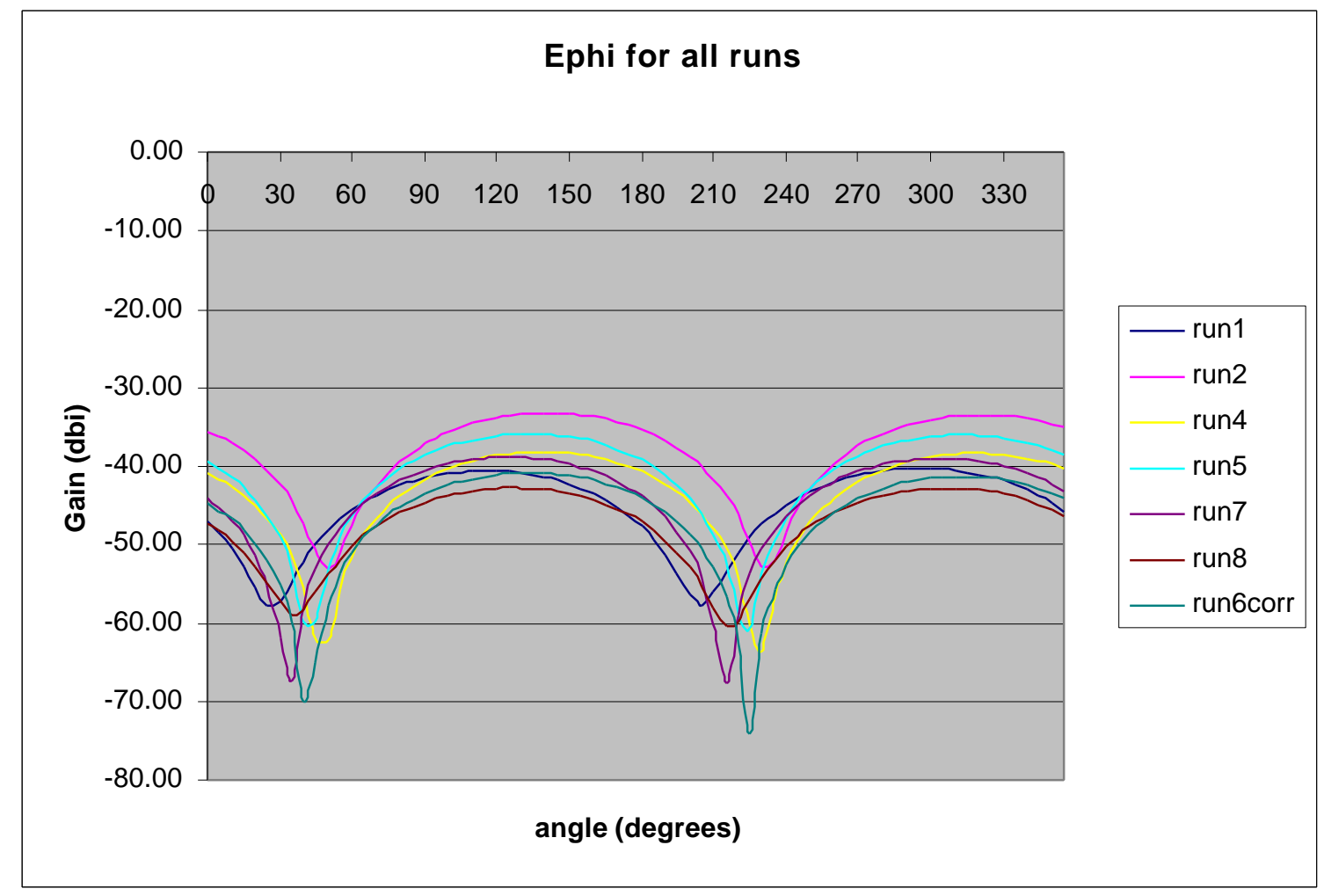

Figure 2. Ephi for all runs at plane theta $=90$

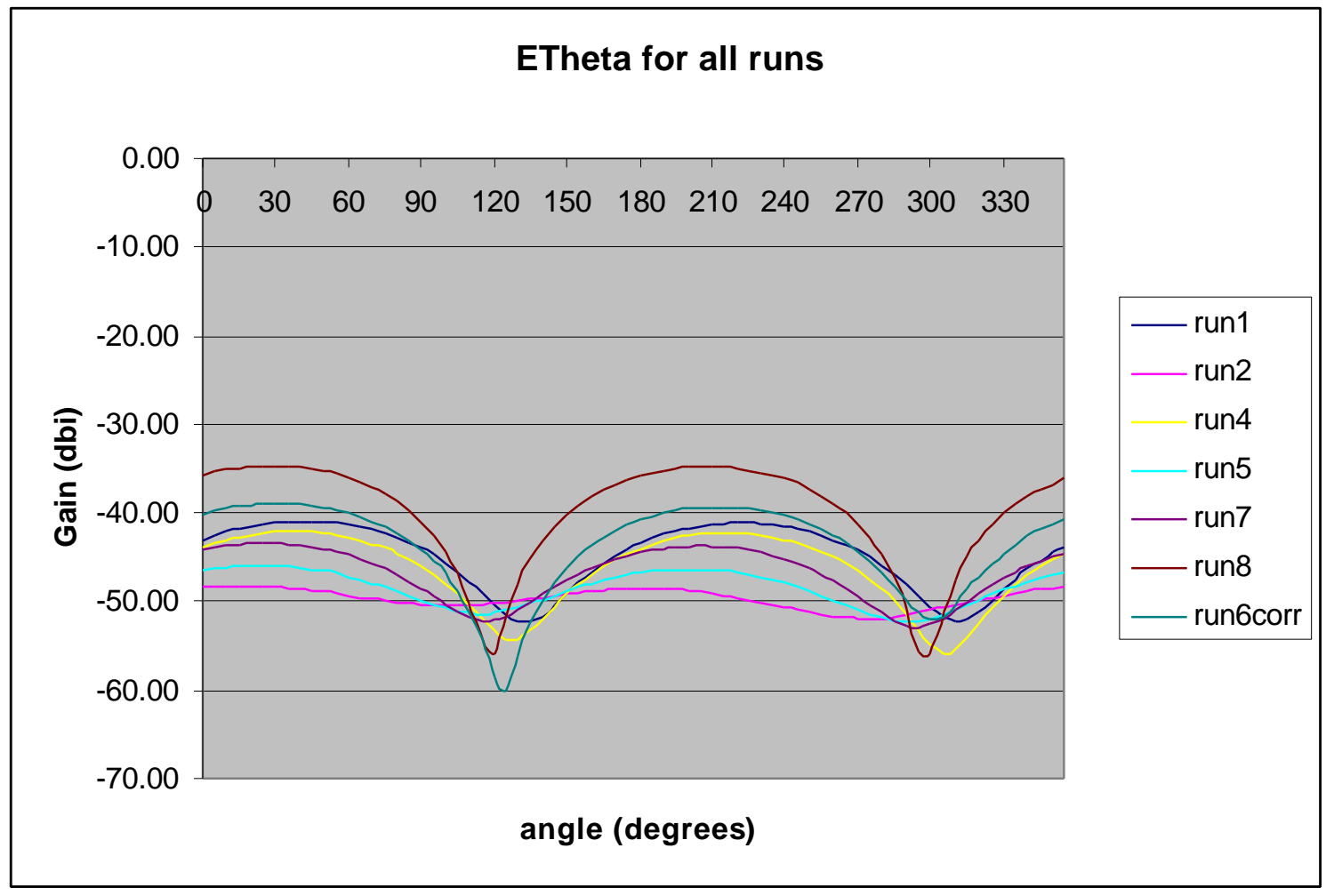

Figure 3. Etheta for all runs at plane theta $=90$ 
Center for Industrial Research Applications

West Virginia University

CTHA Internal Report Series 980729

July 29, 1998

Khaled ElSherbini

\section{Effect of the material properties and the holes geometry on the Input Impedance of a PCB-CTHA}

\section{Introduction}

In this report, the effect of the dielectric constant of the binder material in PCB-CTHA's on the input impedance and resonant frequencies of these antennas will be shown. Also, the effect of two less important parameters, namely the effect of filled/empty holes and the effect of through/nonthrough holes is provided. Then, in the analysis of the effect of the dielectric constant we will see how this effect is found to provide a smooth pattern that can be applied elsewhere. Also, a method of matching resonant frequencies for CTHA's of different dielectrics will be also shown. The gain effect study will be left for another report.

\section{Different Parameters}

The four antennas studied all had the same dimensions, thus the comparison between the effect of the different parameters can also be noted from the graphs. The following were the common geometrical parameters between all four antennas:

$\begin{array}{lllc}\text { Length \& width } & 30.3022 \mathrm{~mm} & \text { Height } & 3.2258 \mathrm{~mm} \\ \text { Holes diameter } & 1.1176 \mathrm{~mm} & \text { Width of traces } & 1.6764 \mathrm{~mm} \\ \text { Second layer at } & 0.8382 \mathrm{~mm} & \text { Major diameter } & 24.892 \mathrm{~mm} \\ \text { Third layer at } & 2.3876 \mathrm{~mm} & \text { Minor diameter } & 12.3698 \mathrm{~mm} \\ \text { \# holes per turn } & 8 \text { holes } & \text { empty space in all directions } 20 \text { cells } \\ \text { Feed type } & 6 & & \end{array}$

As for the differences, these were as follows:

Geometry 1: empty holes, no through holes

Geometry 2: $\quad$ empty holes, through holes 
Geometry 3: $\quad$ filled holes, no through holes

Geometry 4: $\quad$ filled holes, through holes.

Figure 1 shows the difference between through holes and no through holes, where a cross section of a PCB-CTHA is shown. For through holes, the hole goes from the top all way to the bottom in all cases. As for no through holes, the holes only go between the desired layers, i.e. either from layer 1 to 3 or 2 to 4 , except for the feed case.

As for filled and empty holes, this means if the holes are filled with the conducting material, or left empty of that material, i.e. have air in them, except for a very thin layer of material on the borders.
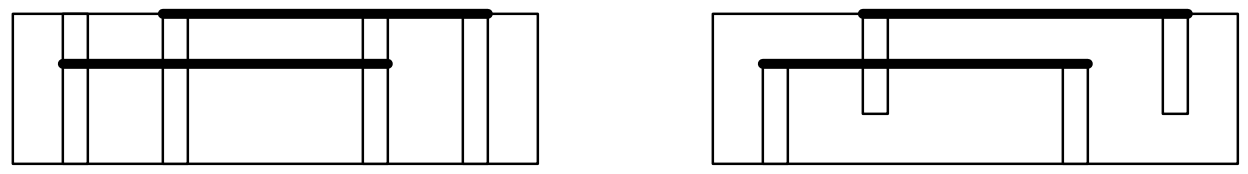

Figure 1. The difference between through holes and non through holes

\section{Results}

Figures 2 to 5 show the graphs and data for the resonant frequencies of each of the four geometries up to $4 \mathrm{~GB}$. From the four figures we can draw the following conclusions:

1. The effect of the dielectric constant: it is clear that the increase of the dielectric constant causes a continuous decrease in the resonance frequencies of all four antennas. To check that this effect is continuous and does not have an apparent peak where it will change its direction, we increased the dielectric in one of the geometries (geometry 3) until 15, and the same effect was also realized.

But, the higher the dielectrics go, the less the decrease in the resonant frequencies at constant dielectric intervals. For example, for geometry 1 at the first resonance frequency, an increase in the dielectric constant from 1 to 1.5 causes a decrease of $121 \mathrm{MHz}$ for the first resonance frequency from 760 to $639 \mathrm{MHz}$. While going from 1.5 to 2 only decreased it $77 \mathrm{MHz}$, then 55 $\mathrm{MHz}$, etc... A detailed analysis of this behavior is provided in the next section. 
2. The effect of through holes: comparing geometries 1 and 2, and also geometries 3 and 4 , we can see that the through holes cause a slight increase in the resonant frequencies over the non through holes. This increase is in the order of 2 to $3 \%$.

3. The effect of empty and filled holes: By comparing figures 1 and 3, and figures 2 and 4 , we can see that filling the holes or keeping them empty has no effect at all on the resonant frequencies of the PCB-CTHA's.

\section{Analysis of dielectric constant effect}

After analyzing the effects of the change of the resonance frequencies due to the change of the dielectric constant from the above section, it was found that the behavior provides a repetitive effect that can be applied to other geometry's as well.

Since the effect of the filled to empty holes was negligible, we will consider we have two geometry's to analyze, one with through holes and one without through holes. The resonance frequency at a dielectric constant of 1.0, properties of air, will be considered as our reference point, and all other resonance frequencies will be compared with that frequency.

For all cases, the dielectric constant relative effect was calculated using the following formula:

\section{Relative effect $=\left(\mathbf{R F}_{\text {air }}-\mathbf{R F}_{\text {freq }}\right) / \mathbf{R F}_{\text {air }}$}

RF: the resonance frequency, either at air or at the desired frequency.

The following tables, table $1 \mathrm{~A}$ and table $1 \mathrm{~B}$, show the relative effect of the dielectric constant on the resonant frequencies. The data in these tables is taken from figures 2 through 5 , and analyzed as discussed above.

Comparing the matching dielectric constants between tables $1 \mathrm{~A}$ and $1 \mathrm{~B}$, as dielectrics 1.5, 2, $2.5,3.5$, etc..., we see that the relative effect is constant irrespective of the geometry. Thus based on this data table 2 was developed. 


\begin{tabular}{|c|c|c|c|c|c|c|c|c|c|c|c|c|c|}
\hline Res \# & 1 & \multicolumn{2}{|c|}{1.5} & \multicolumn{2}{c|}{2} & \multicolumn{2}{c|}{2.5} & \multicolumn{2}{c|}{3} & \multicolumn{3}{c|}{3.5} & \multicolumn{2}{c|}{4} \\
\hline & $\mathrm{GHz}$ & $\mathrm{GHz}$ & effect & $\mathrm{GHz}$ & effect & GHz & effect & $\mathrm{GHz}$ & effect & GHz & effect & $\mathrm{GHz}$ & effect \\
\hline 1 & 0.760 & 0.639 & 0.16 & 0.563 & 0.26 & 0.508 & 0.33 & 0.469 & 0.38 & 0.436 & 0.43 & 0.408 & 0.46 \\
\hline 2 & 1.391 & 1.170 & 0.16 & 1.030 & 0.26 & 0.930 & 0.33 & 0.856 & 0.38 & 0.797 & 0.43 & 0.749 & 0.46 \\
\hline 3 & 2.327 & 1.959 & 0.16 & 1.731 & 0.26 & 1.570 & 0.33 & 1.442 & 0.38 & 1.340 & 0.42 & 1.261 & 0.46 \\
\hline 4 & 2.903 & 2.446 & 0.16 & 2.155 & 0.26 & 1.951 & 0.33 & 1.794 & 0.38 & 1.670 & 0.42 & 1.568 & 0.46 \\
\hline 5 & 3.979 & 3.364 & 0.15 & 2.979 & 0.25 & 2.697 & 0.32 & 2.479 & 0.38 & 2.311 & 0.42 & 2.173 & 0.45 \\
\hline ave & & & 0.16 & & 0.26 & & 0.33 & & 0.38 & & 0.42 & & 0.46 \\
\hline
\end{tabular}

\begin{tabular}{|c|c|c|c|c|c|}
\hline Res \# & 1 & \multicolumn{2}{|c|}{4.5} & \multicolumn{2}{c|}{5} \\
\hline & $\mathrm{GHz}$ & $\mathrm{GHz}$ & effect & $\mathrm{GHz}$ & effect \\
\hline 1 & 0.760 & 0.385 & 0.49 & 0.366 & 0.52 \\
\hline 2 & 1.391 & 0.711 & 0.49 & 0.673 & 0.52 \\
\hline 3 & 2.327 & 1.193 & 0.49 & 1.128 & 0.52 \\
\hline 4 & 2.903 & 1.488 & 0.49 & 1.418 & 0.51 \\
\hline 5 & 3.979 & 2.052 & 0.48 & 1.944 & 0.51 \\
\cline { 1 - 2 } ave & \multicolumn{3}{|l}{0.49} & & 0.51 \\
\hline
\end{tabular}

Table 1A. The relative effect of the dielectric constant on the resonant frequencies. No through holes

Dielectric constant

\begin{tabular}{|c|c|c|c|c|c|c|c|c|c|c|c|c|c|c|}
\hline Res \# & 1 & \multicolumn{2}{|c|}{1.5} & \multicolumn{2}{|c|}{2} & \multicolumn{2}{c|}{2.5} & \multicolumn{2}{c|}{3.1} & \multicolumn{2}{c|}{3.3} & \multicolumn{2}{c|}{3.5} \\
\hline & $\mathrm{GHz}$ & $\mathrm{GHz}$ & effect & $\mathrm{GHz}$ & effect & GHz & effect & GHz & effect & GHz & effect & $\mathrm{GHz}$ & effect \\
\hline 1 & 0.783 & 0.656 & 0.16 & 0.573 & 0.27 & 0.520 & 0.34 & 0.470 & 0.40 & 0.456 & 0.42 & 0.445 & 0.43 \\
\hline 2 & 1.436 & 1.201 & 0.16 & 1.055 & 0.27 & 0.952 & 0.34 & 0.860 & 0.40 & 0.835 & 0.42 & 0.811 & 0.44 \\
\hline 3 & 2.396 & 2.006 & 0.16 & 1.774 & 0.26 & 1.602 & 0.33 & 1.447 & 0.40 & 1.406 & 0.41 & 1.365 & 0.43 \\
\hline 4 & 2.998 & 2.513 & 0.16 & 2.208 & 0.26 & 1.994 & 0.33 & 1.804 & 0.40 & 1.751 & 0.42 & 1.703 & 0.43 \\
\hline 5 & 4.090 & 3.443 & 0.16 & 3.030 & 0.26 & 2.745 & 0.33 & 2.487 & 0.39 & 2.411 & 0.41 & 2.341 & 0.43 \\
\hline ave & & & 0.16 & & 0.26 & & 0.33 & & 0.40 & & 0.42 & & 0.43 \\
\hline
\end{tabular}

\begin{tabular}{|c|c|c|c|c|c|c|c|c|c|c|c|c|c|}
\hline Res \# & 1 & \multicolumn{2}{|c|}{3.7} & \multicolumn{2}{|c|}{3.9} & \multicolumn{2}{|c|}{4.10} & \multicolumn{2}{|c|}{4.30} & \multicolumn{2}{|c|}{4.50} & \multicolumn{2}{|c|}{15.00} \\
\hline & $\mathrm{GHz}$ & $\mathrm{GHz}$ & effect & $\mathrm{GHz}$ & effect & $\mathrm{GHz}$ & effect & $\mathrm{GHz}$ & effect & $\mathrm{GHz}$ & effect & $\mathrm{GHz}$ & effect \\
\hline 1 & 0.783 & 0.434 & 0.45 & 0.422 & 0.46 & 0.41 & 0.473 & 0.40 & 0.489 & 0.39 & 0.5 & 0.21 & 0.726 \\
\hline 2 & 1.436 & 0.792 & 0.45 & 0.771 & 0.46 & 0.75 & 0.475 & 0.74 & 0.485 & 0.72 & 0.496 & 0.42 & 0.707 \\
\hline 3 & 2.396 & 1.330 & 0.44 & 1.299 & 0.46 & 1.27 & 0.469 & 1.24 & 0.48 & 1.21 & 0.494 & 0.67 & 0.722 \\
\hline 4 & 2.998 & 1.661 & 0.45 & 1.618 & 0.46 & 1.58 & 0.473 & 1.54 & 0.485 & 1.51 & 0.495 & 0.87 & 0.71 \\
\hline 5 & 4.090 & 2.283 & 0.44 & 2.230 & 0.45 & 2.18 & 0.468 & 2.13 & 0.479 & 2.08 & 0.491 & 1.15 & 0.718 \\
\hline ave & & & 0.45 & & 0.46 & & 0.472 & & 0.484 & & 0.495 & & 0.717 \\
\hline
\end{tabular}

Table 1B. The relative effect of the dielectric constant on the resonant frequencies.

Through holes 


\begin{tabular}{|c|c|}
\hline DI & relative effect \\
\hline 1 & 0 \\
\hline 1.5 & 0.16 \\
\hline 2 & 0.26 \\
\hline 2.5 & 0.33 \\
\hline 3 & 0.38 \\
\hline 3.1 & 0.4 \\
\hline 3.3 & 0.42 \\
\hline 3.5 & 0.43 \\
\hline
\end{tabular}

\begin{tabular}{|c|c|}
\hline DI & relative effect \\
\hline 3.7 & 0.45 \\
\hline 3.9 & 0.46 \\
\hline 4 & 0.46 \\
\hline 4.1 & 0.47 \\
\hline 4.3 & 0.48 \\
\hline 4.5 & 0.49 \\
\hline 5 & 0.51 \\
\hline 15 & 0.72 \\
\hline
\end{tabular}

Table 2. The relative effect of the dielectric constant on the resonance frequencies

Table 2, shows the conclusion of the study. In it the predicted effect of each "studied" dielectric constant is tabulated. This table can be used as a powerful tool for design of PCBCTHA's, where an initial run can be made on the PCB-CTHA with no binder, then by referring to this table, the required binder material can be predicted.

\section{A method for matching resonant frequencies of antennas with different dielectric constants:}

The aim of this part of the report was to try to find a way to match the resonant frequencies of antennas with different dielectric constants. We will demonstrate our method on the first geometry by trying to match the resonant frequencies of the antennas with dielectrics of 1 and 4 . In order to keep all parameters of the antennas relatively fixed to each other, all we did was change the cell size for the geometry in all three directions by the same factor, thus indirectly changing the dimensions of the antenna in all directions with the exact same ratio.

\begin{tabular}{|c|c|c|c|c|c|}
\hline Res freq & DI4, $\mathrm{x} 0.5$ & Di4, $\mathrm{x} 1$ & $\mathrm{Di} 4, \mathrm{x} 2$ & $\mathrm{Di} 4, \mathrm{x} 0.5686$ & $\mathrm{Di} 1, \mathrm{x} 1$ \\
\hline 1 & 0.816 & 0.408 & 0.204 & 0.717 & 0.760 \\
\hline 2 & 1.487 & 0.749 & 0.372 & 1.308 & 1.391 \\
\hline 3 & 2.521 & 1.261 & 0.630 & 2.217 & 2.327 \\
\hline 4 & 3.125 & 1.568 & 0.781 & 2.748 & 2.903 \\
\hline 5 & 4.342 & 2.173 & 1.086 & 3.818 & 3.979 \\
\hline 6 & 4.863 & 2.437 & 1.216 & & \\
\hline 7 & 6.216 & 3.113 & 1.554 & & \\
\hline 8 & 6.675 & 3.343 & 1.669 & & \\
\hline 9 & 7.989 & 3.996 & 1.997 & & \\
\hline
\end{tabular}

Table 3: method for matching resonance frequencies. 
First we multiplied the cell sizes by 2 , then by 0.5 , i.e. double and half. Then from the results of the input impedance of these three geometries we found the behavior to be almost linear. Thus by interpolation we were able to determine the factor needed to be able to obtain the exact resonance frequencies required. Table 3 shows the results of this procedure. As we can see, the method produced a result with an error of less than $5 \%$. 
Center for Industrial Research Applications

West Virginia University

CTHA Internal Report Series 980715

July 15,1998

Khaled ElSherbini

\section{Experiments conducted on Larry Hawks Antennas}

\section{Introduction}

In his latest visit, Larry Hawks provided several CTHA antennas for testing. He coded the antennas $\mathrm{X} 1$ up to $\mathrm{X} 7$, in addition to a cone shaped CTHA that was coded "purple cone. In the following pages we provide the results of testing these antennas for input impedance and SWR. All tests were conducted at the CIRA-WVU anechoic chamber.

\section{Test Results}

The results are posted in both tabulated and graph shapes. Table 1 provides a detailed listing of all the experiments conducted, and the resonance frequencies obtained in each test. The tests were coded 00 to 16. Each two consecutive tests were identical, except for either using a 10ft RG-8 cable, provided by Hawks, or not. Thus each two consecutive experiments were grouped together to show the effect of the cable. The only exceptions are experiments 14 and 15 which are identical, thus they were grouped together with experiment 16 which is the same but with no cable.

Table 2, on the other hand, shows the real and imaginary components of the input impedance and the SWR for each test at a frequency of $27.21 \mathrm{MHz}$. This was the desired frequency when rapping these antennas, thus it is useful to show the data at this specific frequency. A listing of all 8 antennas is provided in table 3 , which gives a detailed description of each of these antennas. This table was provided by Larry Hawks.

In the pages following that, graphs are provided for the total shape of the test results. The first group of graphs, figures 1 to 17 , show the real and imaginary components of the 
input impedance for each of the 17 tests. Again, in the figures each two consecutive tests were put together on the same page to show the effect of adding the cable.

Finally, the last group of figures shows the SWR for all the antennas. Two graphs are provided for each antenna, the only difference between the two graphs is that one is plotted using a linear scale, while the other is plotted using a logarithmic scale. On each of these graphs, both the test with the cable and the test without the cable are plotted together, thus providing a clear comparison of the effect of the cable on the SWR of each antenna.

\section{Analysis}

Cable effect: the effect of the cable on all the experiments is clearly evident. The input impedance behavior, and the resonant frequencies are totally changed by the introduction of the cable. The effect on the SWR, on the other hand, is not so prominent, especially at the points where the antennas are well matched.

In all the SWR graphs we can see that the general effect of the cable was a better matching of the antenna, especially at the points where the antenna is badly matched. But the better the matching of the antenna, in other words the lower the SWR, the less the effect of introducing the cable. This behavior can be clearly detected from the SWR for antennas X4, X5, X7 and the purple cone.

Behavior of the antennas: From table 2 it is clear that at a frequency of 27.21 the best antennas were X4, the purple cone, and X7. Although X7 shows an SWR of about 5.0, but it is clear from the SWR graph that the bandwidth at the lowest SWR is very narrow, thus the least shift from the best matching point would lead to a much higher SWR. So by properly matching X7, which is a very small shift from its current shape, we can get SWRs as low as those for $\mathrm{X} 4$ and the purple cone..

The SWR figures for both X4 and the purple cone also show how the bandwidth at this specific frequency is very narrow, although not as narrow as $\mathrm{X} 7$. They also show that these two antennas are well matched for a frequency of 27.21.

Antennas X2, X3, and X6, although badly matched at a frequency of 27.21, but they all provide a large bandwidth around this frequency. The SWR graph is very flat around this frequency, especially for the case of including the cable with all three antennas. 


\begin{tabular}{|c|c|c|c|c|c|c|c|}
\hline Test \# & Antenna & Cable & res\# & freq(MHz) & real & imag & SWR \\
\hline 00 & $\mathrm{X} 3$ & $\mathrm{Y}$ & 1 & 2.541 & 6368.273 & $2.5 \mathrm{E}-11$ & 201.303 \\
\hline & & & 2 & 15.395 & 2.586 & $1.5 \mathrm{E}-16$ & 19.338 \\
\hline & & & 3 & 28.439 & 547.255 & $-1.9 \mathrm{E}-12$ & 10.949 \\
\hline 01 & $\mathrm{X} 3$ & $\mathrm{~N}$ & 1 & 7.734 & 8151.454 & $3.4 \mathrm{E}-12$ & 164.616 \\
\hline & & & 2 & 13.150 & 472.051 & $3.5 \mathrm{E}-14$ & 9.441 \\
\hline & & & 3 & 13.332 & 664.900 & $-5.9 \mathrm{E}-14$ & 13.298 \\
\hline
\end{tabular}

\begin{tabular}{|c|c|c|c|c|c|c|c|}
\hline 02 & $\mathrm{X} 2$ & $\mathrm{Y}$ & 1 & 11.337 & 3.235 & $1.6 \mathrm{E}-15$ & 15.456 \\
\hline & & & 2 & 14.325 & 340.216 & $5.1 \mathrm{E}-13$ & 6.866 \\
\hline & & & 3 & 19.679 & 2.111 & $9.2 \mathrm{E}-15$ & 23.684 \\
\hline 03 & $\mathrm{X} 2$ & $\mathrm{~N}$ & 1 & 13.945 & 6.223 & $-2.7 \mathrm{E}-14$ & 8.035 \\
\hline & & & 2 & 26.466 & 2973.107 & $4.3 \mathrm{E}-12$ & 59.503 \\
\hline
\end{tabular}

\begin{tabular}{|c|c|c|c|c|c|c|c|}
\hline 04 & $\mathrm{X} 5$ & $\mathrm{Y}$ & 1 & 17.145 & 1.403 & $7.3 \mathrm{E}-15$ & 35.642 \\
\hline & & & 2 & 25.387 & 222.775 & $-6.1 \mathrm{E}-13$ & 4.484 \\
\hline & & & 3 & 26.517 & 9.037 & $-6.6 \mathrm{E}-14$ & 5.537 \\
\hline & & & 4 & 34.535 & 430.609 & $5.4 \mathrm{E}-13$ & 8.613 \\
\hline 05 & $\mathrm{X} 5$ & $\mathrm{~N}$ & 1 & 15.676 & 1.916 & $-1.3 \mathrm{E}-14$ & 26.102 \\
\hline & & & 2 & 18.165 & 56.050 & $1.0 \mathrm{E}-13$ & 1.134 \\
\hline & & & 3 & 19.391 & 6.666 & $9.0 \mathrm{E}-15$ & 7.501 \\
\hline & & & 4 & 27.858 & 100.544 & $7.8 \mathrm{E}-14$ & 2.011 \\
\hline & & & 5 & 28.138 & 57.528 & $5.3 \mathrm{E}-14$ & 1.151 \\
\hline & & & 6 & 33.930 & 534.952 & $2.9 \mathrm{E}-12$ & 10.705 \\
\hline & & & 8 & 37.376 & 69.631 & $-7.8 \mathrm{E}-14$ & 1.393 \\
\hline
\end{tabular}

\begin{tabular}{|c|c|c|c|c|c|c|c|}
\hline 06 & $\mathrm{X} 7$ & $\mathrm{Y}$ & 1 & 15.676 & 1.916 & $-1.3 \mathrm{E}-14$ & 26.102 \\
\hline & & & 2 & 18.165 & 56.050 & $1.0 \mathrm{E}-13$ & 1.134 \\
\hline & & & 3 & 19.391 & 6.666 & $9.0 \mathrm{E}-15$ & 7.501 \\
\hline & & & 4 & 27.858 & 100.544 & $7.8 \mathrm{E}-14$ & 2.011 \\
\hline & & & 5 & 28.138 & 57.528 & $5.3 \mathrm{E}-14$ & 1.151 \\
\hline & & & 6 & 33.930 & 534.952 & $2.9 \mathrm{E}-12$ & 10.705 \\
\hline & & & 7 & 37.376 & 69.631 & $-7.8 \mathrm{E}-14$ & 1.393 \\
\hline 07 & $\mathrm{X} 7$ & $\mathrm{~N}$ & 1 & 37.974 & 170.722 & $-5.3 \mathrm{E}-13$ & 3.415 \\
\hline & & & 2 & 18.639 & 3473.025 & $8.8 \mathrm{E}-12$ & 69.947 \\
\hline & & & 3 & 21.210 & 21.218 & $-1.7 \mathrm{E}-13$ & 1.220 \\
\hline & & & 4 & 28.183 & 53.676 & $-9.9 \mathrm{E}-13$ & 43.678 \\
\hline & & & 5 & 30.942 & 844.245 & $2.7 \mathrm{E}-13$ & 1.078 \\
\hline & & & 6 & 37.286 & 58.337 & $1.7 \mathrm{E}-13$ & 1.173 \\
\hline
\end{tabular}

Table 1: The input impedance and SWR at the resonant frequencies for all tests 


\begin{tabular}{|c|c|c|c|c|c|c|c|}
\hline $\begin{array}{c}\text { Test } \\
\#\end{array}$ & Antenna & Cable & res\# & freq(MHz) & real & imag & SWR \\
\hline 08 & $\mathrm{X} 4$ & $\mathrm{Y}$ & 1 & 17.148 & 1.338 & $7.9 \mathrm{E}-15$ & 37.374 \\
\hline & & & 2 & 27.005 & 122.177 & $-2.6 \mathrm{E}-13$ & 2.446 \\
\hline & & & 3 & 27.726 & 29.040 & $-7.7 \mathrm{E}-14$ & 1.724 \\
\hline & & & 4 & 34.532 & 493.776 & $1.5 \mathrm{E}-12$ & 9.877 \\
\hline 09 & $\mathrm{X} 4$ & $\mathrm{~N}$ & 1 & 16.989 & 5052.482 & $-1.5 \mathrm{E}-11$ & 101.453 \\
\hline & & & 2 & 27.534 & 37.058 & $-4.6 \mathrm{E}-14$ & 1.352 \\
\hline & & & 3 & 34.397 & 3321.451 & $-1.7 \mathrm{E}-11$ & 66.488 \\
\hline
\end{tabular}

\begin{tabular}{|c|c|c|c|c|c|c|c|}
\hline 10 & Purple & $\mathrm{Y}$ & 1 & 18.758 & 1.055 & $-9.1 \mathrm{E}-15$ & 47.392 \\
\hline & cone & & 2 & 27.257 & 122.145 & $-2.2 \mathrm{E}-13$ & 2.445 \\
\hline & & & 3 & 28.057 & 25.439 & $3.2 \mathrm{E}-14$ & 1.967 \\
\hline & & & 4 & 35.629 & 1382.160 & $1.5 \mathrm{E}-12$ & 27.757 \\
\hline 11 & Purple & $\mathrm{N}$ & 1 & 23.037 & 3598.256 & $6.3 \mathrm{E}-13$ & 72.480 \\
\hline & cone & & 2 & 27.709 & 37.609 & $-2.1 \mathrm{E}-14$ & 1.331 \\
\hline
\end{tabular}

\begin{tabular}{|c|c|c|c|c|c|c|c|}
\hline 12 & $\mathrm{X} 6$ & $\mathrm{Y}$ & 1 & 17.847 & 2.235 & $-9.4 \mathrm{E}-15$ & 22.372 \\
\hline & & & 2 & 33.054 & 839.875 & $2.8 \mathrm{E}-14$ & 16.814 \\
\hline 13 & $\mathrm{X} 6$ & $\mathrm{~N}$ & 1 & 21.230 & 2056.781 & $5.5 \mathrm{E}-13$ & 41.170 \\
\hline
\end{tabular}

\begin{tabular}{|c|c|c|c|c|c|c|c|}
\hline 14 & $\mathrm{X} 1$ & $\mathrm{Y}$ & 1 & 103.598 & 855.227 & $-4.5 \mathrm{E}-12$ & 17.248 \\
\hline & & & 2 & 120.213 & 2.896 & $1.9 \mathrm{E}-14$ & 17.268 \\
\hline & & & 3 & 135.904 & 563.877 & $-8.0 \mathrm{E}-12$ & 11.320 \\
\hline & & & 4 & 153.463 & 5.032 & $-4.0 \mathrm{E}-14$ & 9.937 \\
\hline & & & 5 & 169.629 & 361.373 & $-2.6 \mathrm{E}-13$ & 7.229 \\
\hline 15 & $\mathrm{X} 1$ & $\mathrm{Y}$ & 1 & 103.478 & 817.254 & $9.6 \mathrm{E}-12$ & 16.457 \\
\hline & & & 2 & 120.272 & 3.982 & $-1.4 \mathrm{E}-14$ & 12.556 \\
\hline & & & 3 & 136.237 & 537.395 & $1.5 \mathrm{E}-12$ & 10.767 \\
\hline & & & 4 & 153.748 & 4.138 & $5.0 \mathrm{E}-14$ & 12.082 \\
\hline & & & 5 & 169.688 & 475.273 & $4.8 \mathrm{E}-12$ & 9.542 \\
\hline & & & 6 & 187.542 & 5.557 & $4.2 \mathrm{E}-14$ & 8.999 \\
\hline 16 & $\mathrm{X} 1$ & $\mathrm{~N}$ & none & & & & \\
\hline
\end{tabular}

Table 1 (continued) 


\begin{tabular}{|c|c|c|c|c|c|}
\hline Test \# & Antenna & Cable & Real & Imag & SWR \\
\hline 00 & X3 & Y & 52.86 & 172.84 & 13.23 \\
\hline 01 & X3 & N & 9.96 & -106.98 & 28.16 \\
\hline 02 & X2 & Y & 5.12 & 60.23 & 24.01 \\
\hline 03 & X2 & N & 1143.65 & -1554.79 & 65.18 \\
\hline 04 & X5 & Y & 5.62 & 21.81 & 10.61 \\
\hline 05 & X5 & N & 23.71 & 95.98 & 10.26 \\
\hline 06 & X7 & Y & 82.83 & 110.30 & 5.00 \\
\hline 07 & X7 & N & 56.51 & -99.93 & 5.36 \\
\hline 08 & X4 & Y & 80.10 & -20.68 & 1.77 \\
\hline 09 & X4 & N & 36.22 & -26.96 & 2.01 \\
\hline 10 & Purple Cone & Y & 129.86 & 11.31 & 2.62 \\
\hline 11 & Purple Cone & N & 38.99 & -43.15 & 2.64 \\
\hline 12 & X6 & Y & 8.61 & 67.78 & 16.59 \\
\hline 13 & X6 & N & 219.94 & -509.82 & 28.23 \\
\hline 14 & X1 & Y & n/a & & \\
\hline 15 & X1 & Y & n/a & & \\
\hline 16 & X1 & N & n/a & & \\
\hline
\end{tabular}

Table 2: Input impedance and SWR for all tests at 27.21 MHz

\begin{tabular}{|c|c|c|c|c|c|c|}
\hline Ant & $\mathrm{N}$ & $\mathrm{F}(\mathrm{MHz})$ & $\mathrm{L}$ & $\mathrm{Ra}$ & $\mathrm{Rb}$ & $\mathrm{Ga}$ \\
\hline $\mathrm{X} 1$ & 10 & 146 & 36.4 & 2.98 & 0.496 & 22 \\
\hline $\mathrm{X} 2$ & 10 & $3.8-29.5$ & 194 & 19 & 2.13 & 10 \\
\hline $\mathrm{X} 3$ & 16 & $3.8-29.5$ & 395 & 21.62 & 0.625 & 12 \\
\hline $\mathrm{X} 4$ & 30 & 27 & 196 & 6.14 & 1.02 & 22 \\
\hline $\mathrm{X} 5$ & 30 & 27 & 196 & 6.14 & 1.02 & 12 \\
\hline $\mathrm{X} 6$ & 16 & 27 & 107 & 5.42 & 0.75 & 12 \\
\hline $\mathrm{X} 7$ & 30 & 27 & 196 & 6.14 & 1.02 & 22 \\
\hline
\end{tabular}

Table 3: The antennae dimensions and specifications

$\mathrm{N}$ : number of turns

$\mathrm{F}$ : Designed frequency $(\mathrm{MHz})$

$L$ : length of the wire (inches)

$\mathrm{Ra}, \mathrm{Rb}$ : major and minor radii (inches)

Ga: wire gauge

$\mathrm{X} 2$ \& $\mathrm{X} 3$ are HF type utilizing a transmatch tuning to a $50 \mathrm{ohm}$ load $X 1$, and $X 4$ to $X 7$ were constructed to a specific frequency 


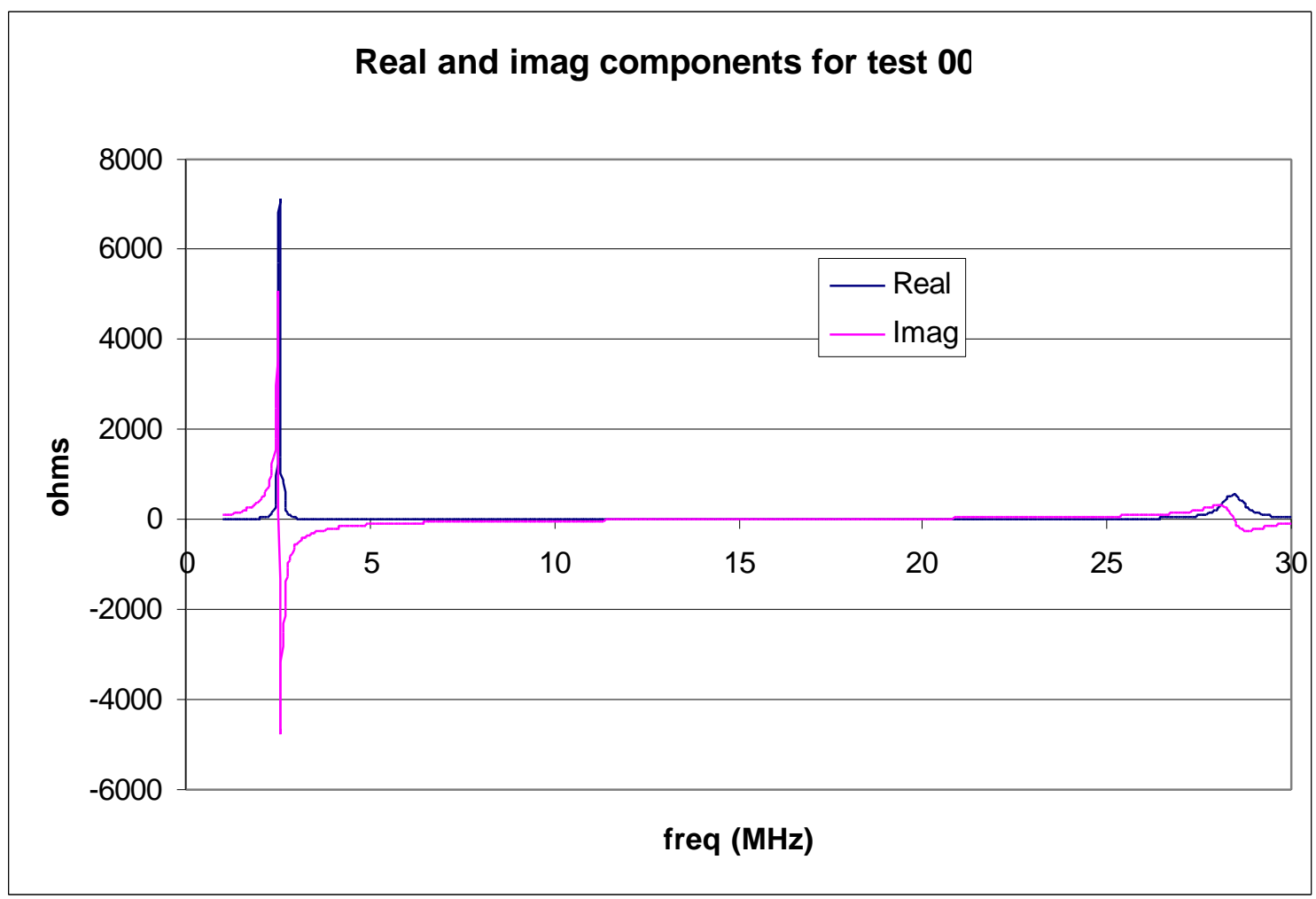

figure 1: antenna X3 with cable

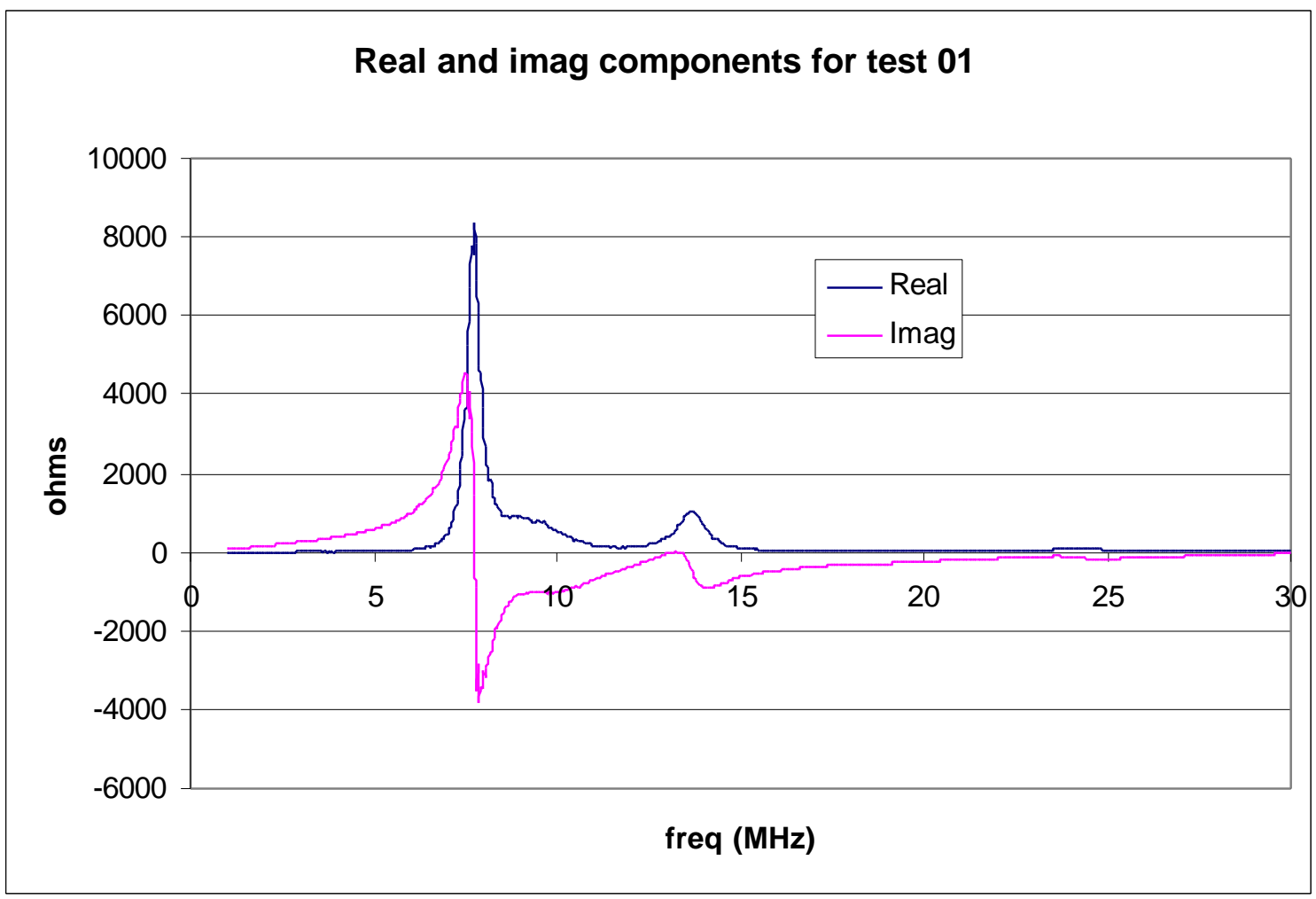

figure 2: antenna X3, no cable 


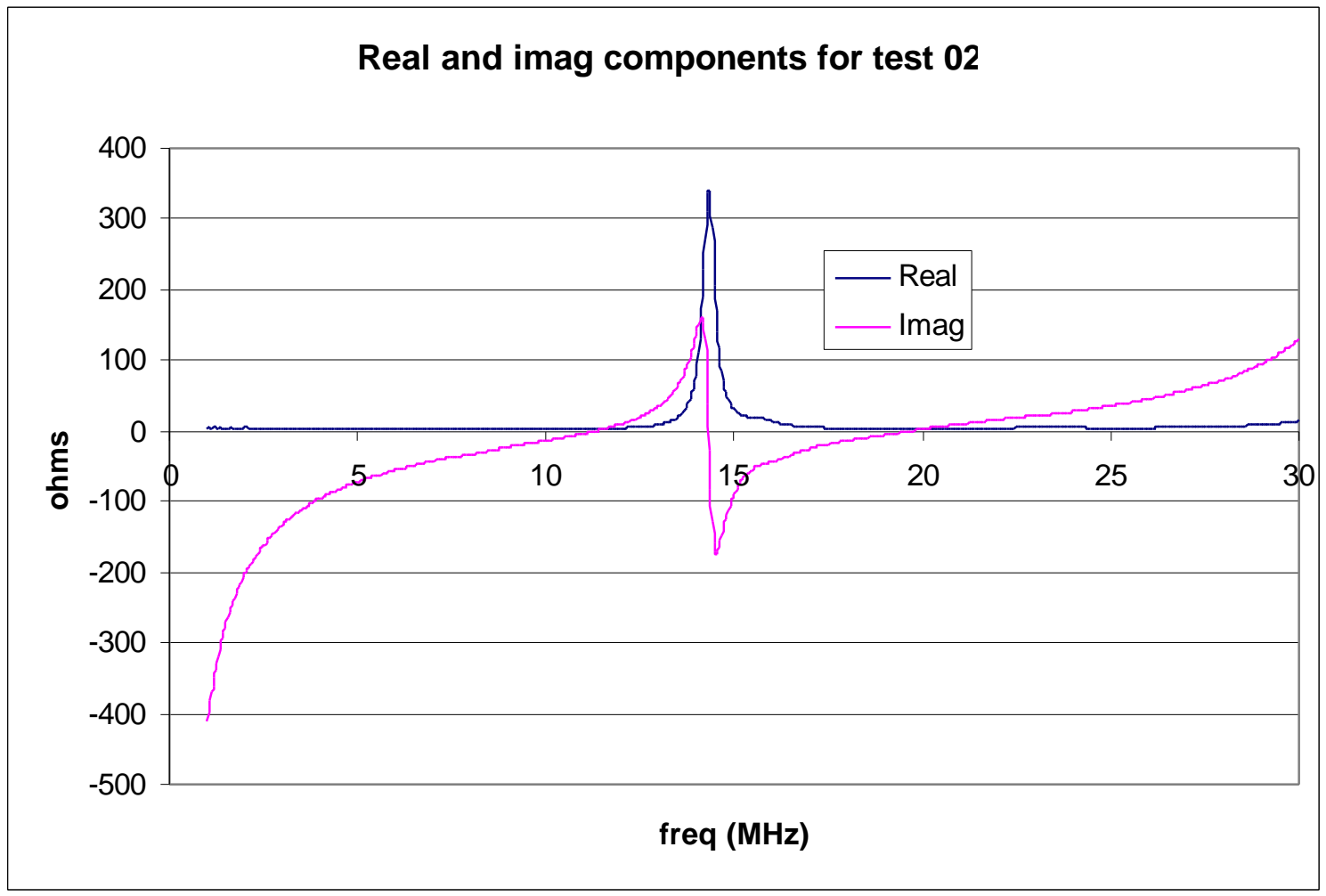

figure 3: antenna X2 with cable

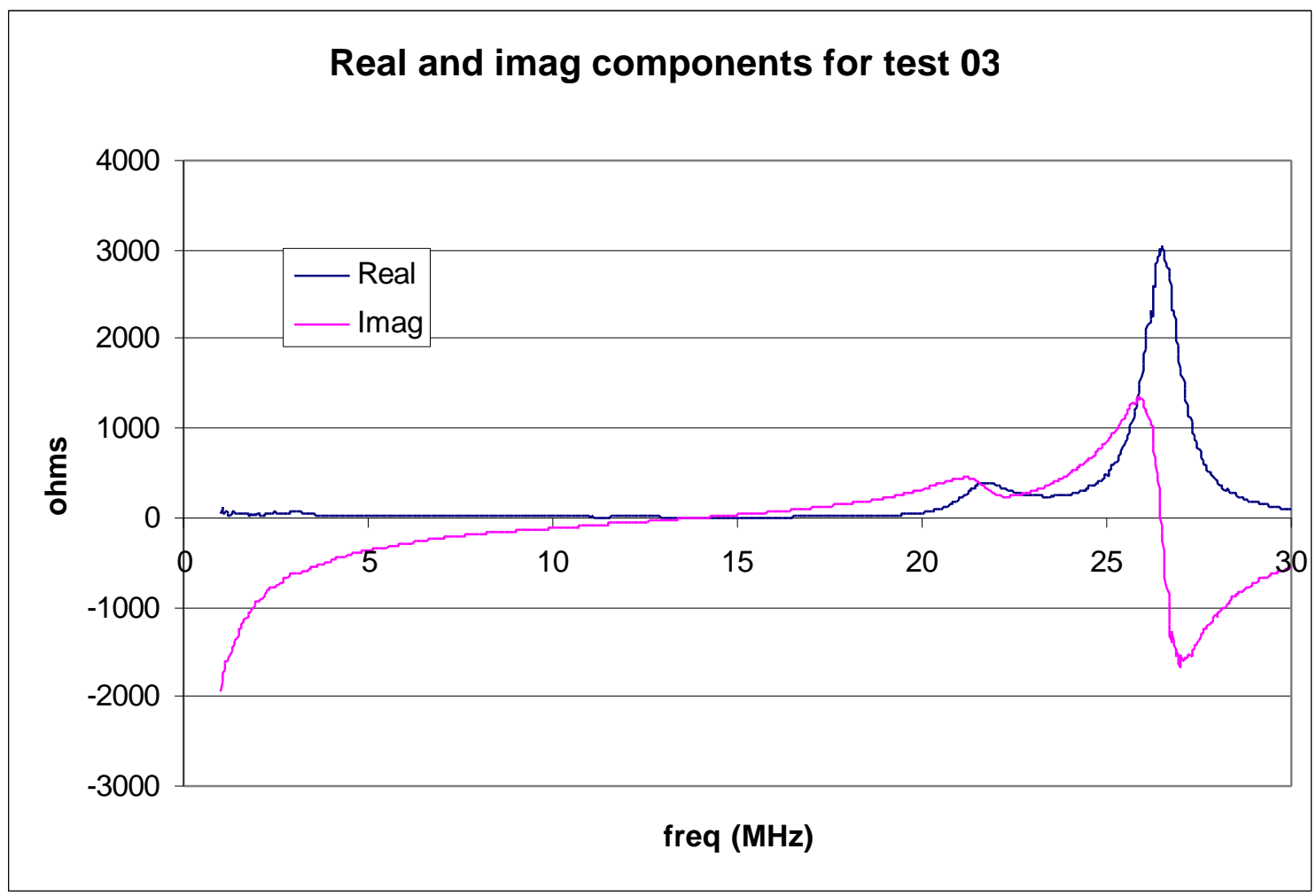

figure 4: antenna X2 no cable 


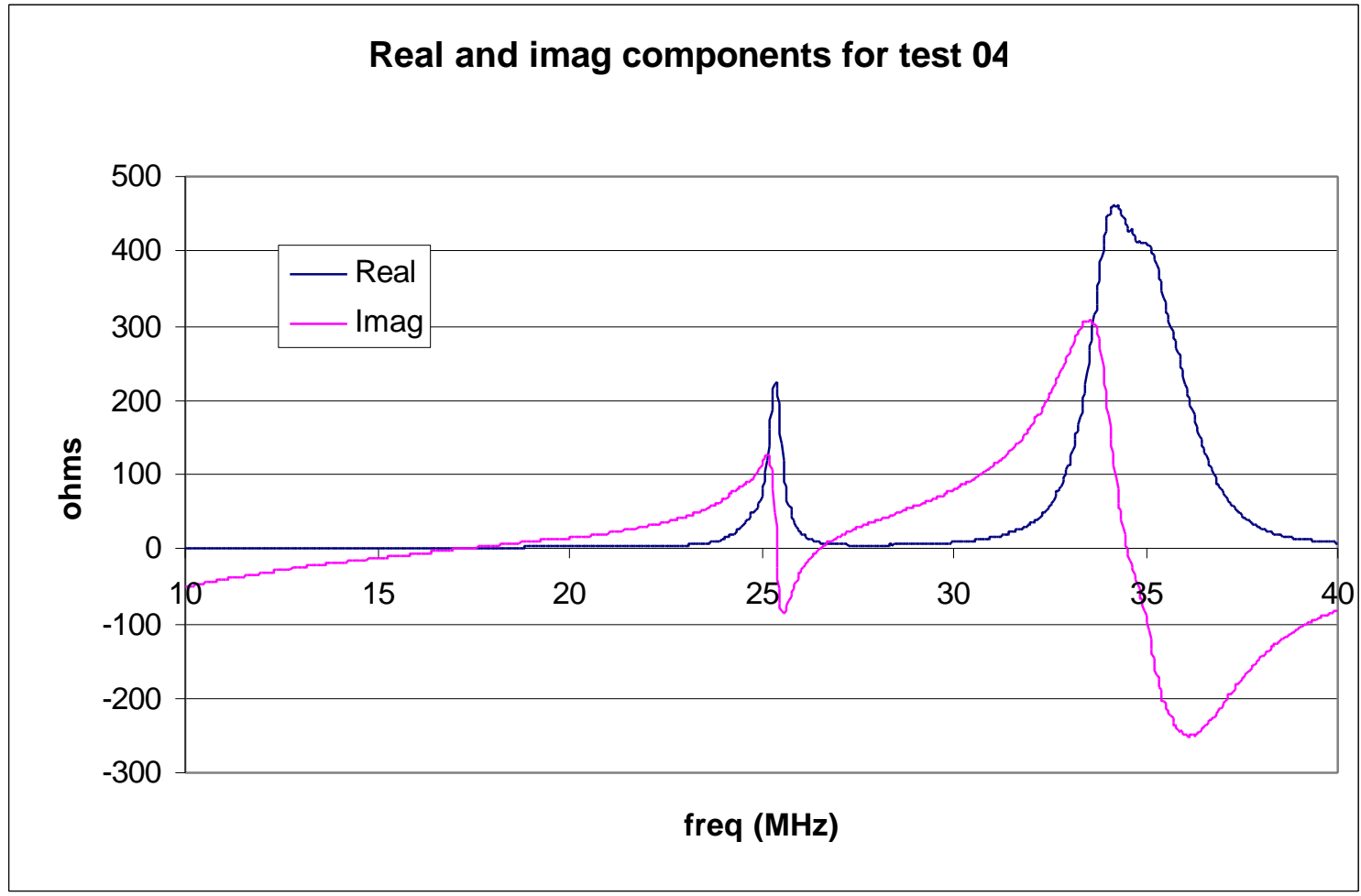

figure 5: antenna X5 with cable

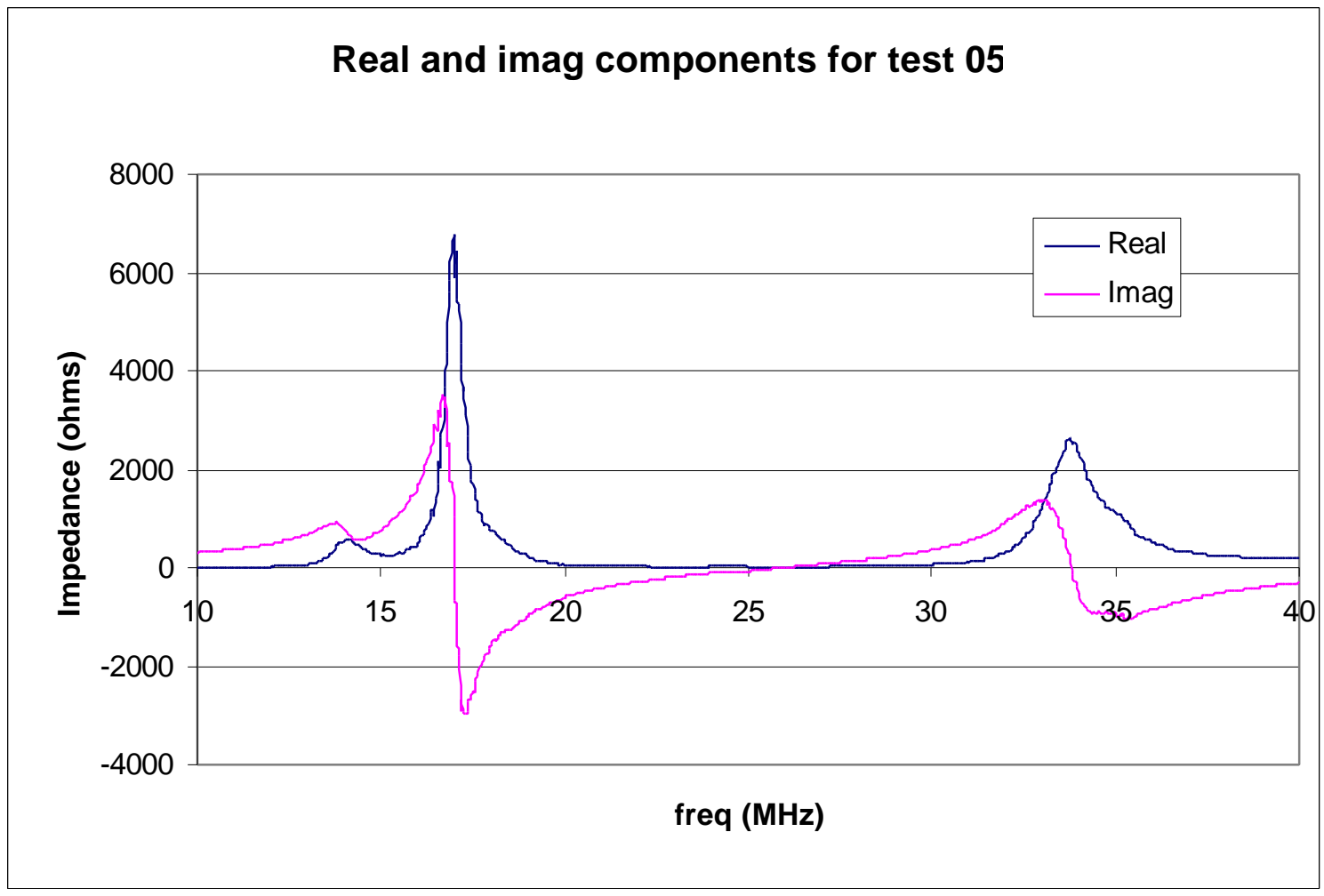

figure 6: antenna X5 no cable 


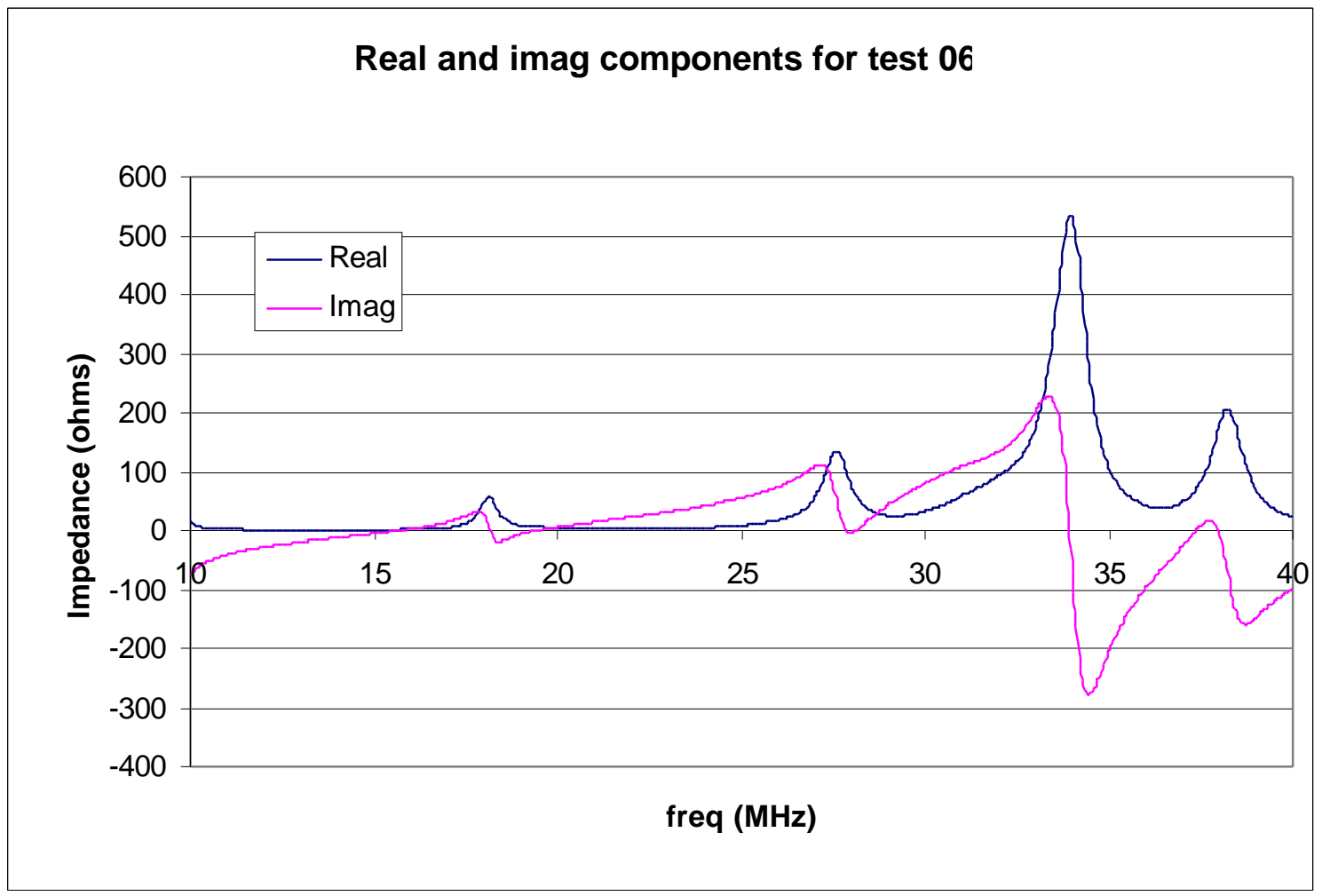

figure 7: antenna X7 with cable

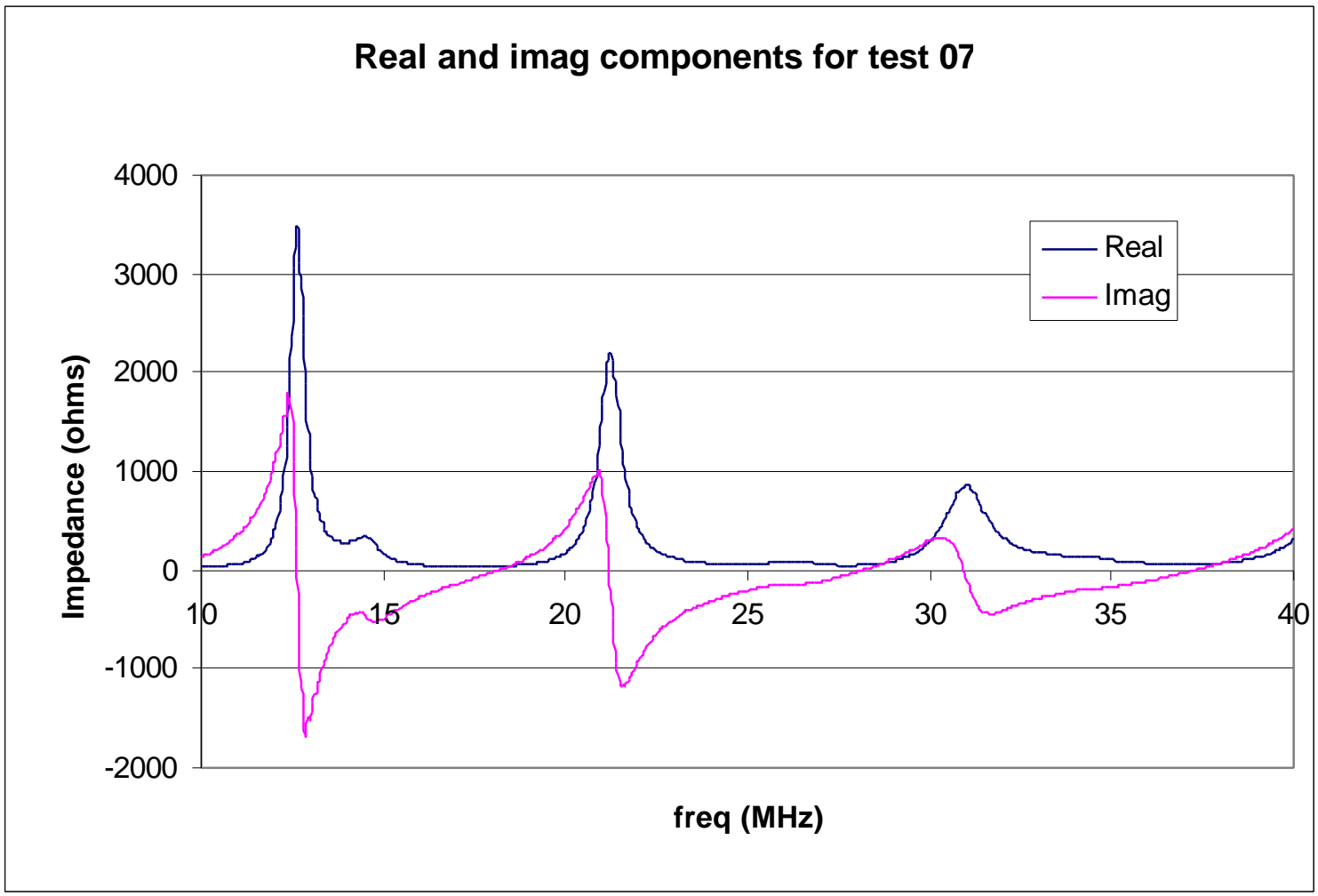

figure 8: antenna X7 no cable 


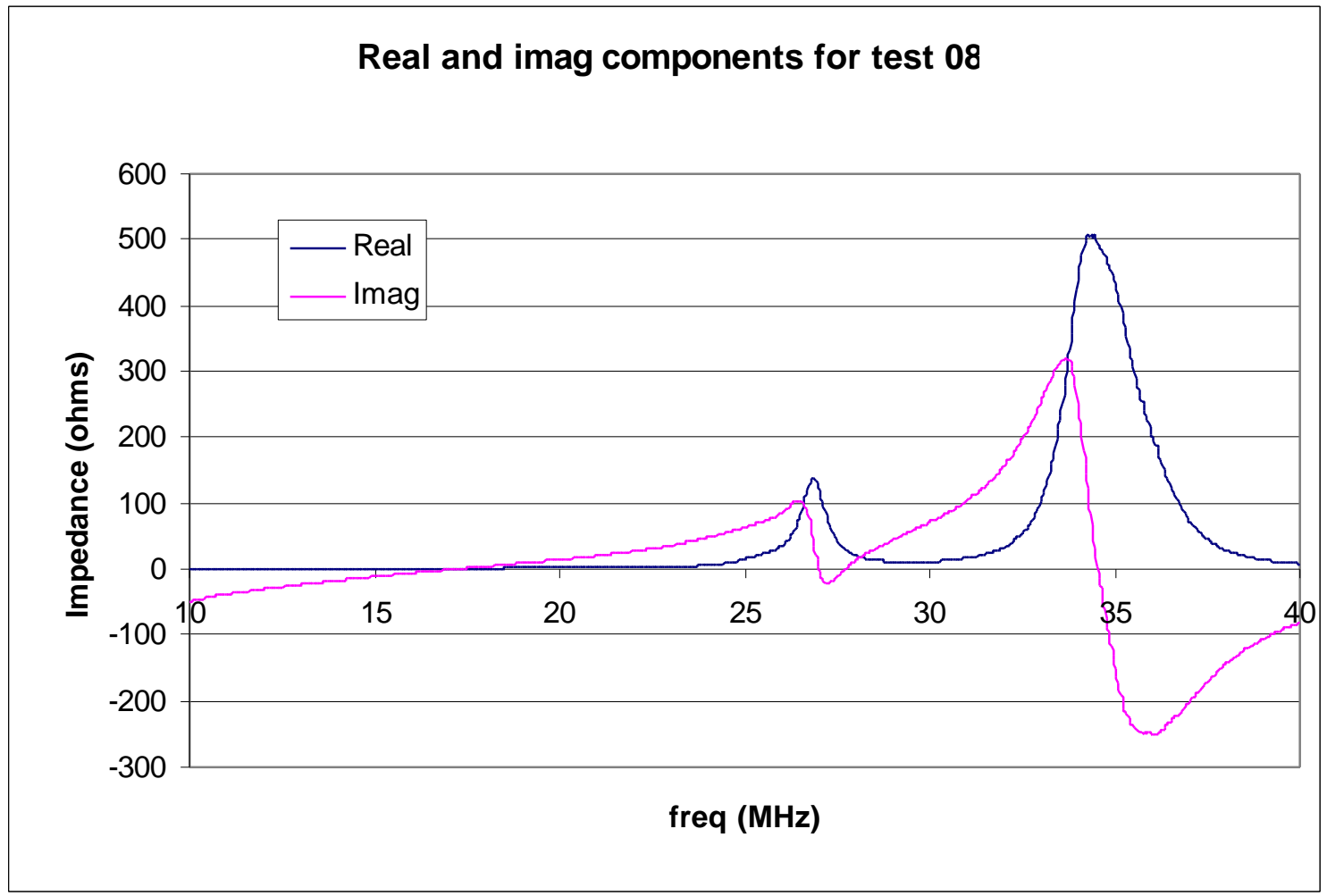

figure 9: antenna $X 4$ with cable

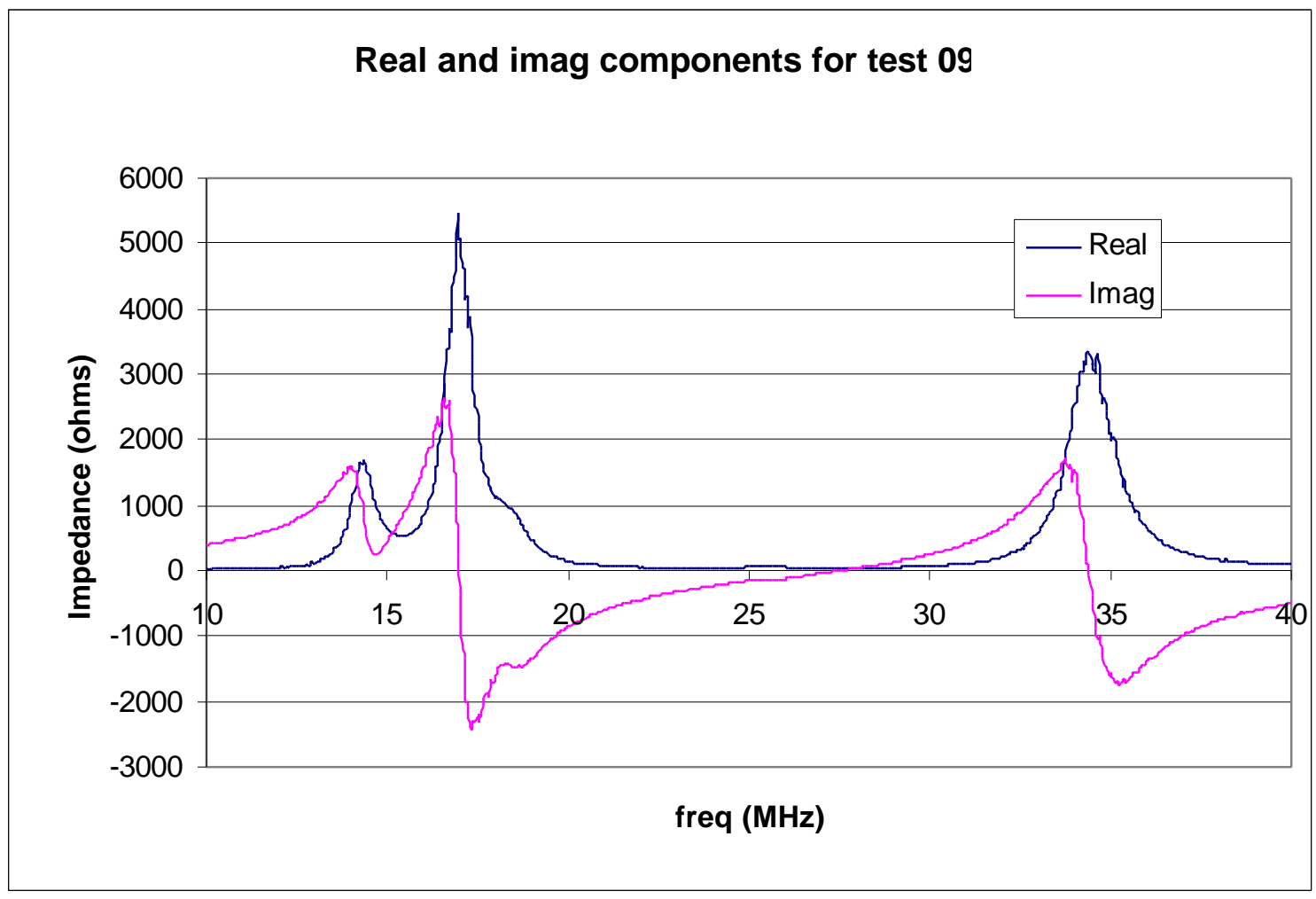

figure 10: antenna X4 no cable 


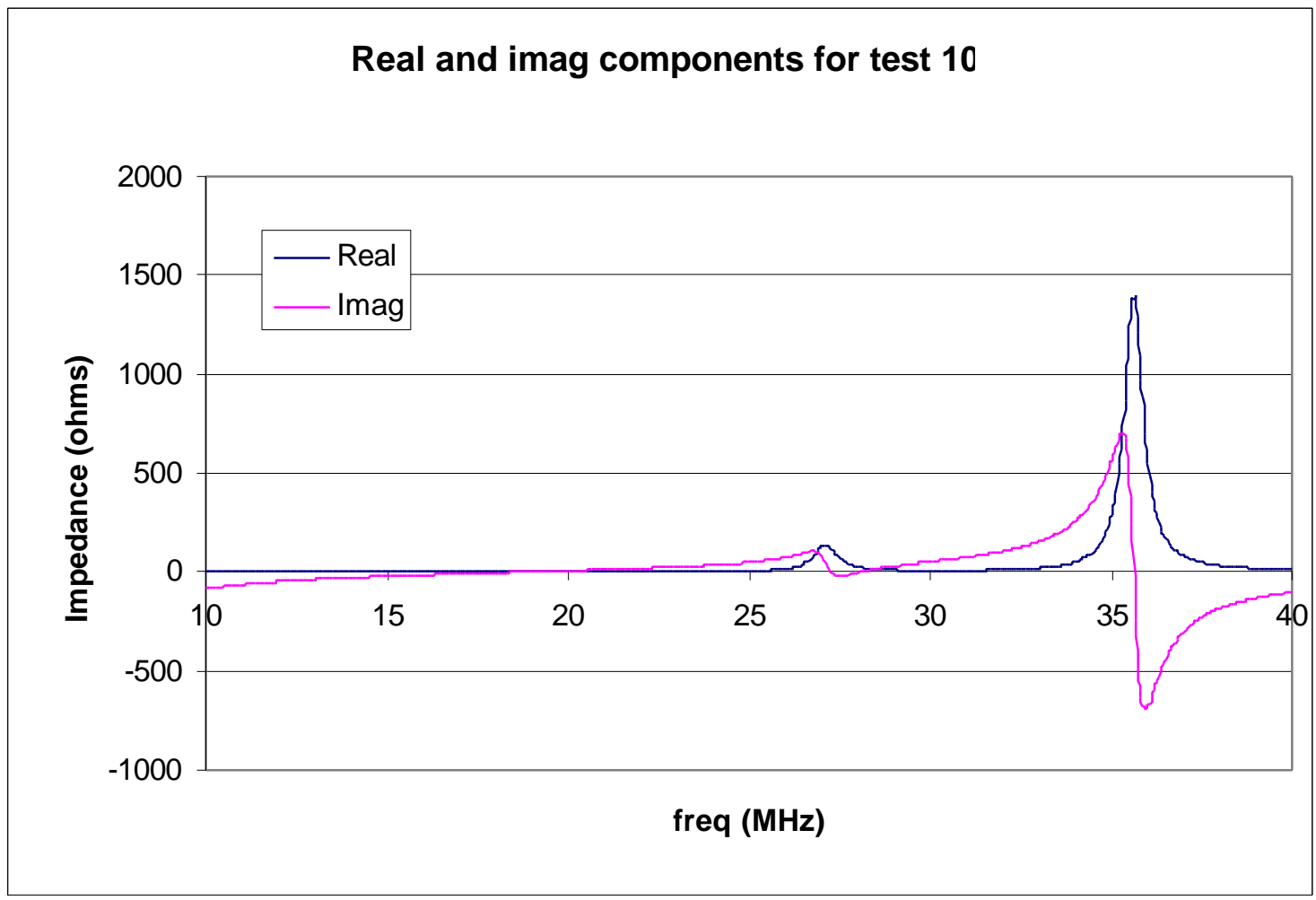

figure 11: antenna "purple cone" with cable

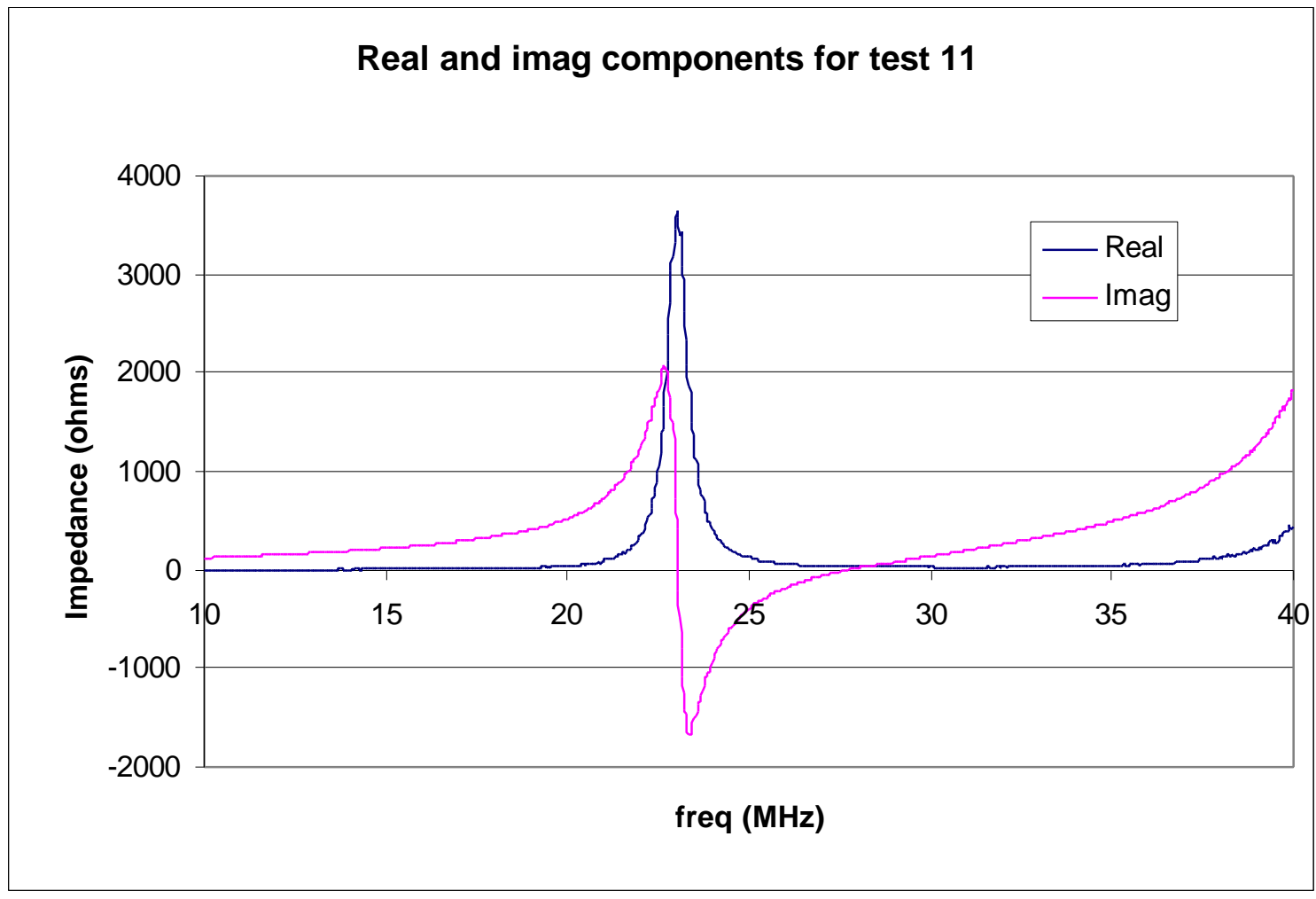

figure 12: antenna "purple cone" no cable 


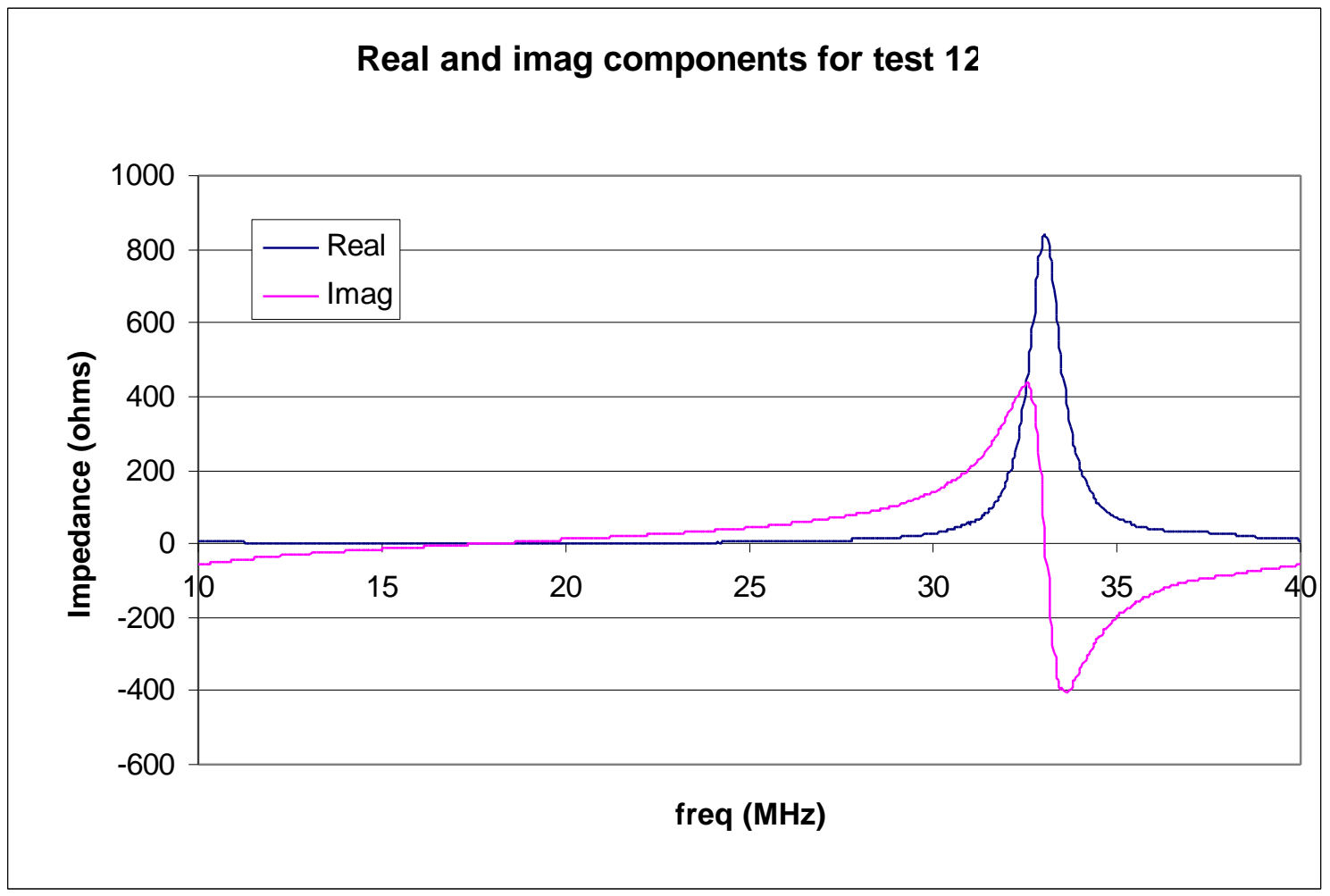

figure 13: antenna X6 with cable

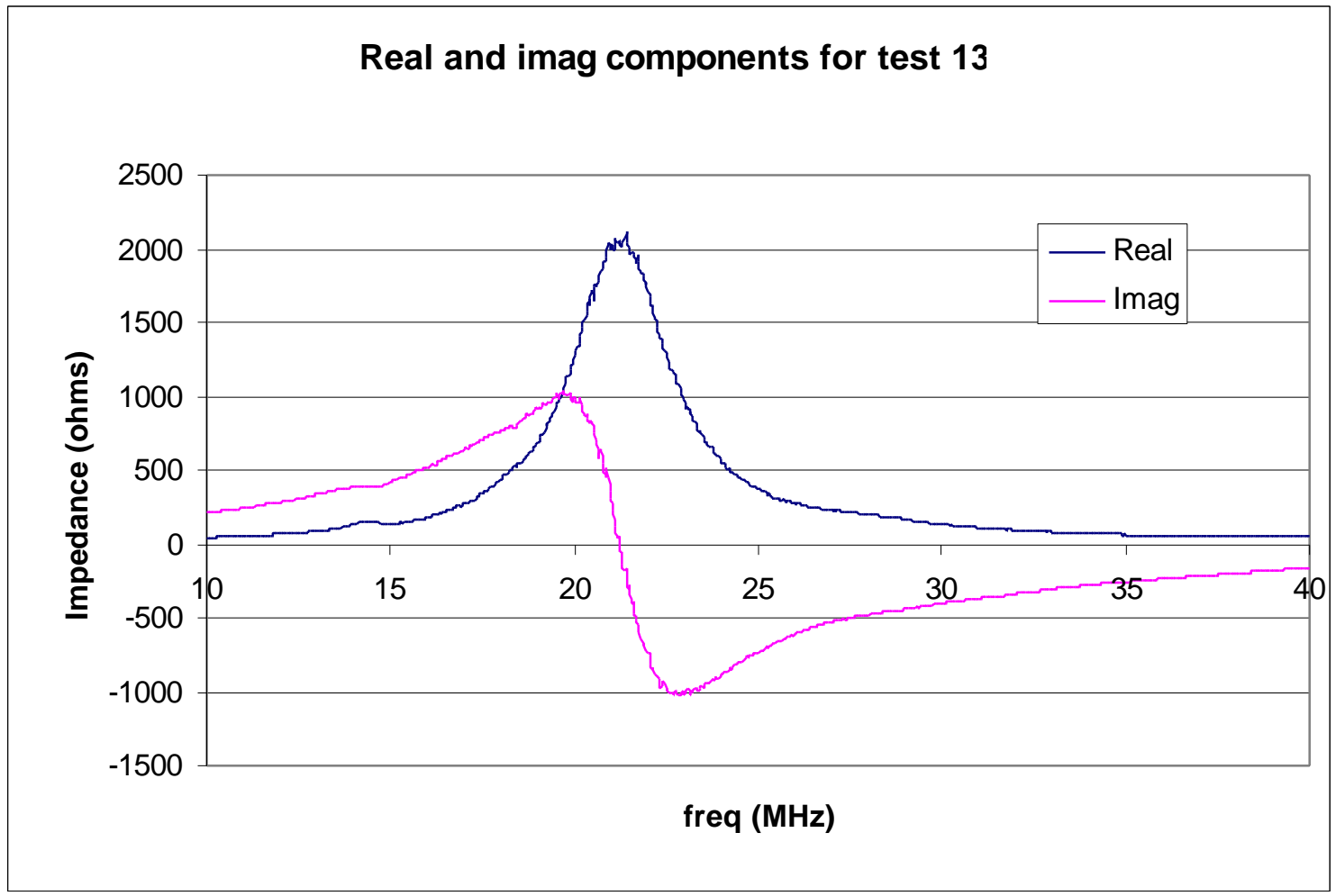

figure 14: antenna X6 no cable 


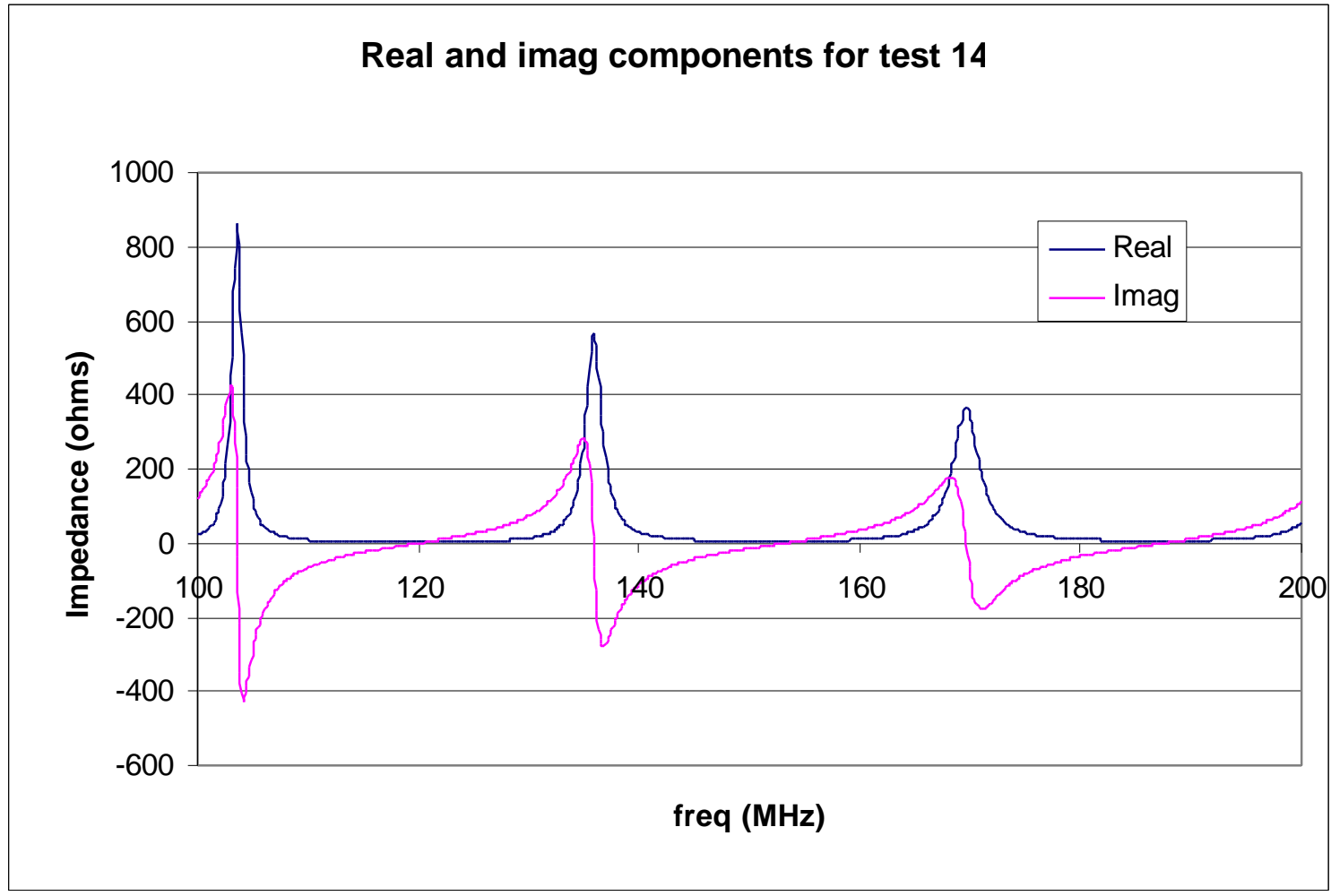

figure 15: antenna X1 with cable

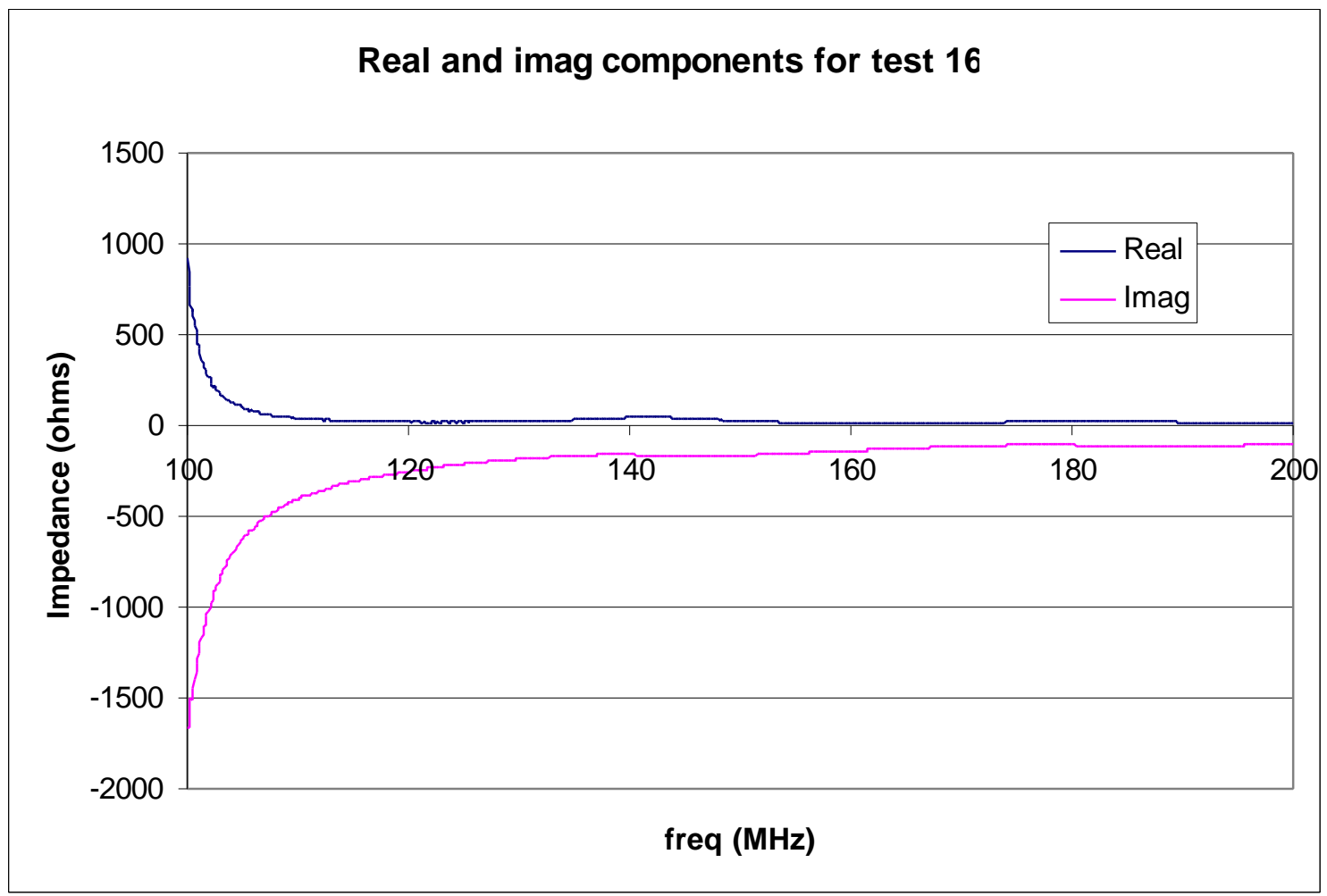

figure 16: antenna X1 no cable 


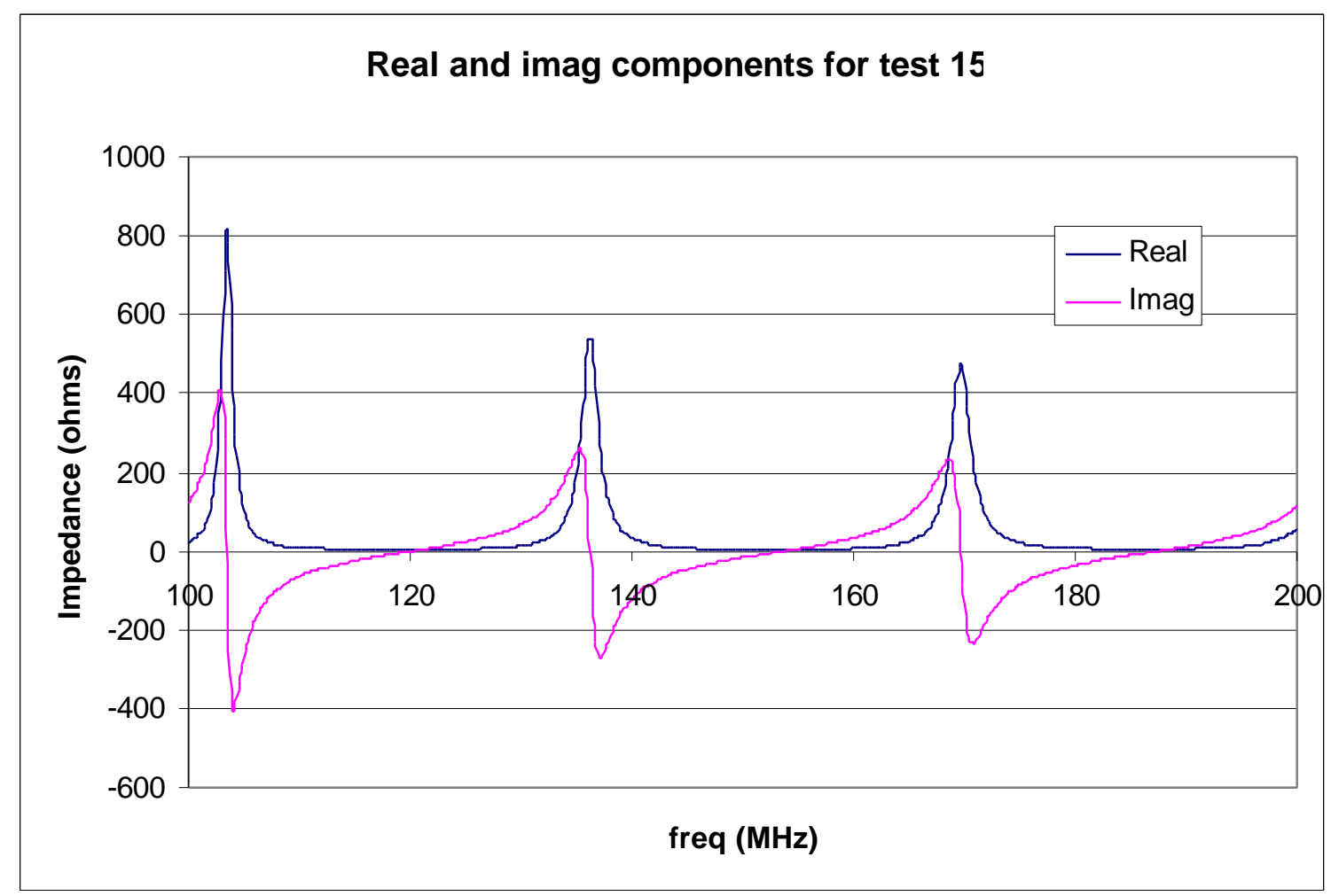

figure 17: antenna X1 with cable 
Center for Industrial Research Applications

West Virginia University

CTHA Internal Report Series 980722

July 22, 1998

Khaled ElSherbini

\section{More Tests on Larry Hawks' antennas}

\section{Introduction}

New tests were performed on two of Larry Hawks antennas that were previously discussed in report "CTHA 980715 Hawks Antennas", these are antennas X5 and X7. An additional antenna was also added, that is antenna AP1 provided by F. Pertl. The goal of the experiments was to compare AP1 to Hawks antennas.

Although the data for Hawks antennas already exists in the previously mentioned report, but tests were reconducted on them to eliminate any difference in experimental conditions, including environmental and equipment changes.

\section{Experiments}

The three above mentioned antennas were tested using two sets of connectors, SMA connectors and UHF connectors. A 20ft RG-58 cable extension was used with the SMA connectors, while a 10ft RG-8 cable extension, similar to the one used in the previous report, was used with the UHF connectors.

Thus, four experiments were conducted on each antenna as follows:

- $\quad$ Antenna with SMA connectors and 20ft RG-58 cable

- $\quad$ Antenna with SMA connectors and no cable

- $\quad$ Antenna with UHF connectors and 10ft RG-8 cable

- $\quad$ Antenna with UHF connectors and no cable

Table 1 provides a detailed listing of all 12 experiments conducted, and the resonance frequencies obtained in each test. The tests were coded 17 to 28 as a continuation of the 
previous tests that were coded 00 to 16 . All four experiments conducted on the same antenna were grouped together for a clearer comparison of results.

\section{Results}

Figures 1 through 12 show the real and imaginary components of the input impedance for each of the 12 tests, while figures 13 through 18 show the SWR for each of the three antennas on both a linear and a logarithmic scale.

\section{Analysis}

By comparing figures 1 to 4, 5 to 8 , and 9 to 12 , and by looking at figures 13 to 18 we can see that both the connectors and the cables have a very prominent effect on the input impedance and the SWR of all three antennas.

Also, by comparing the results for antennas X5 and X7 to those results provided in the previous report we can deduct several interesting points:

We can see that figure 8 from this report and figure 5 from the previous report, which are both for the X5 antenna with a $10 \mathrm{ft}$ RG-8 cable, both highly agree. The same also applies for figure 7 in the previous report and figure 11 of this report, which are both for the X7 antenna with a 10ft RG-8 cable. This shows that the experiments were well repeated under the same conditions.

Also figures 6 and 8 from the previous report, and figures 7 and 12 from this report, for antennas X5 and X7 without cable, show the same resemblance previously mentioned.

The results for the SMA connectors, on the other hand, show large differences from those conducted with the UHF connectors in both this set of experiments and in the previous set of experiments.

Comparing the SWR figures from this report and from the previous report also showed the high similarity between the cases of the UHF connector with cable and without cable in both sets of experiments, while the results with the SMA connectors were also far from those with the UHF connector. 
By looking at the results for AP1, we can see that the best condition for it to be matched well for a frequency for $27 \mathrm{MHz}$ is in the cases of the third resonance frequency of test 18, and the first resonance of test 23. The first is with SMA connectors and a $20 \mathrm{ft}$ RG-58 cable, and the second is UHF connectors and no cable. From table 1 we can see that in the first case we get a real impedance of 45 and an SWR of 1.13 at a frequency of 26.88, while in the second case we get a real impedance of 56 and an SWR of 1.19 at a frequency of 26.97.

\begin{tabular}{|c|c|c|c|c|c|c|c|c|}
\hline Test \# & Antenna & $\begin{array}{c}\text { connector } \\
\mathrm{s} \\
\end{array}$ & Cable & res\# & $\begin{array}{c}\text { freq }(\mathrm{MH} \\
\mathrm{z})\end{array}$ & real & imag & SWR \\
\hline \multirow[t]{2}{*}{17} & $\mathrm{AP1}$ & SMA & - & 1 & 31.07 & 51.44 & $\begin{array}{c}\text { 5.37E- } \\
14\end{array}$ & 1.09 \\
\hline & & & & 2 & 33.86 & 1121.06 & $\begin{array}{c}\text { 1.99E- } \\
12\end{array}$ & 22.43 \\
\hline \multirow[t]{6}{*}{18} & AP1 & SMA & $\begin{array}{c}\text { 20ft RG- } \\
58\end{array}$ & 1 & 19.46 & 1166.25 & $\begin{array}{c}-2.33 \mathrm{E}- \\
12\end{array}$ & 23.44 \\
\hline & & & & 2 & 26.68 & 21.50 & $\begin{array}{c}-2.42 \mathrm{E}- \\
14\end{array}$ & 2.34 \\
\hline & & & & 3 & 26.88 & 44.78 & $\begin{array}{c}-1.24 \mathrm{E}- \\
13\end{array}$ & 1.13 \\
\hline & & & & 4 & 29.83 & 3.54 & $\begin{array}{c}1.13 \mathrm{E}- \\
14\end{array}$ & 14.13 \\
\hline & & & & 5 & 39.60 & 738.61 & $\begin{array}{c}-4.48 \mathrm{E}- \\
12\end{array}$ & 14.78 \\
\hline & & & & 6 & 49.37 & 4.57 & $\begin{array}{c}2.61 \mathrm{E}- \\
15\end{array}$ & 10.93 \\
\hline \multirow[t]{2}{*}{23} & AP1 & UHF & - & 1 & 26.97 & 55.86 & $\begin{array}{c}-2.42 \mathrm{E}- \\
13\end{array}$ & 1.19 \\
\hline & & & & 2 & 28.81 & 2450.45 & $\begin{array}{c}-7.45 \mathrm{E}- \\
12\end{array}$ & 49.11 \\
\hline \multirow[t]{2}{*}{24} & AP1 & $\overline{\mathrm{UHF}}$ & 10ft RG-8 & $\overline{1}$ & 16.49 & 1.54 & $\begin{array}{c}1.89 \mathrm{E}- \\
15\end{array}$ & 32.54 \\
\hline & & & & 2 & 33.65 & 1245.05 & $\begin{array}{c}2.76 \mathrm{E}- \\
12\end{array}$ & 24.91 \\
\hline \multirow[t]{4}{*}{19} & $\times 5$ & SMA & $\begin{array}{c}\text { 20ft RG- } \\
58\end{array}$ & 1 & 10.81 & 2.03 & $\begin{array}{c}-5.68 \mathrm{E}- \\
15\end{array}$ & 24.58 \\
\hline & & & & 2 & 19.32 & 742.80 & $\begin{array}{c}-3.25 \mathrm{E}- \\
12\end{array}$ & 14.94 \\
\hline & & & & 3 & 25.12 & 9.67 & $\begin{array}{c}-6.47 \mathrm{E}- \\
14\end{array}$ & 5.17 \\
\hline & & & & 4 & 26.37 & 222.12 & $\begin{array}{c}-1.57 \mathrm{E}- \\
12\end{array}$ & 4.50 \\
\hline
\end{tabular}




\begin{tabular}{|c|c|c|c|c|c|c|c|c|}
\hline Test \# & Antenna & $\begin{array}{c}\text { connector } \\
\mathrm{s}\end{array}$ & Cable & res\# & $\begin{array}{c}\text { freq(MH } \\
\mathrm{z})\end{array}$ & real & imag & SWR \\
\hline & & & & 5 & 30.50 & 4.59 & $\begin{array}{c}-1.05 \mathrm{E}- \\
14\end{array}$ & 10.90 \\
\hline & & & & 6 & 39.50 & 627.63 & $\begin{array}{c}-4.43 \mathrm{E}- \\
12\end{array}$ & 12.56 \\
\hline & & & & 7 & 48.42 & 5.40 & $\begin{array}{c}6.38 \mathrm{E}- \\
15\end{array}$ & 9.27 \\
\hline \multirow[t]{3}{*}{20} & $\overline{X 5}$ & SMA & - & 1 & 16.28 & 8798.65 & $\begin{array}{c}5.00 \mathrm{E}- \\
12\end{array}$ & 178.30 \\
\hline & & & & 2 & 26.10 & 13.82 & $\begin{array}{c}-3.03 \mathrm{E}- \\
14\end{array}$ & 3.62 \\
\hline & & & & 3 & 32.82 & 1802.48 & $\begin{array}{c}4.82 \mathrm{E}- \\
12\end{array}$ & 36.07 \\
\hline \multirow[t]{3}{*}{25} & $\overline{X 5}$ & UHF & - & 1 & 16.01 & 6762.31 & $\begin{array}{c}-2.18 \mathrm{E}- \\
11\end{array}$ & 135.79 \\
\hline & & & & 2 & 25.85 & 16.06 & $\begin{array}{c}5.37 \mathrm{E}- \\
14\end{array}$ & 3.11 \\
\hline & & & & 3 & 34.44 & 1270.84 & $\begin{array}{c}6.96 \mathrm{E}- \\
13\end{array}$ & 25.42 \\
\hline \multirow[t]{4}{*}{26} & $\overline{X 5}$ & UHF & $10 \mathrm{ft}$ RG-8 & 1 & 17.53 & 2.24 & $\begin{array}{c}1.16 \mathrm{E}- \\
14\end{array}$ & 22.33 \\
\hline & & & & 2 & 25.32 & 241.47 & $\begin{array}{c}-5.76 \mathrm{E}- \\
13\end{array}$ & 4.88 \\
\hline & & & & 3 & 26.61 & 9.63 & $\begin{array}{c}5.60 \mathrm{E}- \\
14\end{array}$ & 5.19 \\
\hline & & & & 4 & 35.17 & 904.87 & $\begin{array}{c}1.58 \mathrm{E}- \\
12\end{array}$ & 18.14 \\
\hline \multirow[t]{8}{*}{21} & $\mathrm{X7}$ & SMA & - & 1 & 12.67 & 2017.33 & $\begin{array}{c}-7.11 \mathrm{E}- \\
15\end{array}$ & 40.36 \\
\hline & & & & 2 & 18.29 & 41.08 & $\begin{array}{c}6.93 \mathrm{E}- \\
14\end{array}$ & 1.23 \\
\hline & & & & 3 & 20.88 & 1637.81 & $\begin{array}{c}5.85 \mathrm{E}- \\
12\end{array}$ & 32.85 \\
\hline & & & & 4 & 28.32 & 56.12 & $\begin{array}{c}5.20 \mathrm{E}- \\
14\end{array}$ & 1.14 \\
\hline & & & & 5 & 30.11 & 353.30 & $\begin{array}{c}\text { 3.33E- } \\
13\end{array}$ & 7.07 \\
\hline & & & & 6 & 37.38 & 64.79 & $\begin{array}{c}-3.38 \mathrm{E}- \\
13\end{array}$ & 1.30 \\
\hline & & & & 7 & 40.16 & 1079.59 & $\begin{array}{c}-6.22 \mathrm{E}- \\
13\end{array}$ & 21.61 \\
\hline & & & & 8 & 48.37 & 81.33 & $\begin{array}{c}3.91 \mathrm{E}- \\
14\end{array}$ & 1.63 \\
\hline 22 & $\mathrm{X} 7$ & SMA & $\begin{array}{c}20 \mathrm{ft} \mathrm{RG}- \\
58\end{array}$ & 1 & 10.90 & 3.56 & $\begin{array}{c}8.66 \mathrm{E}- \\
15\end{array}$ & 14.03 \\
\hline
\end{tabular}




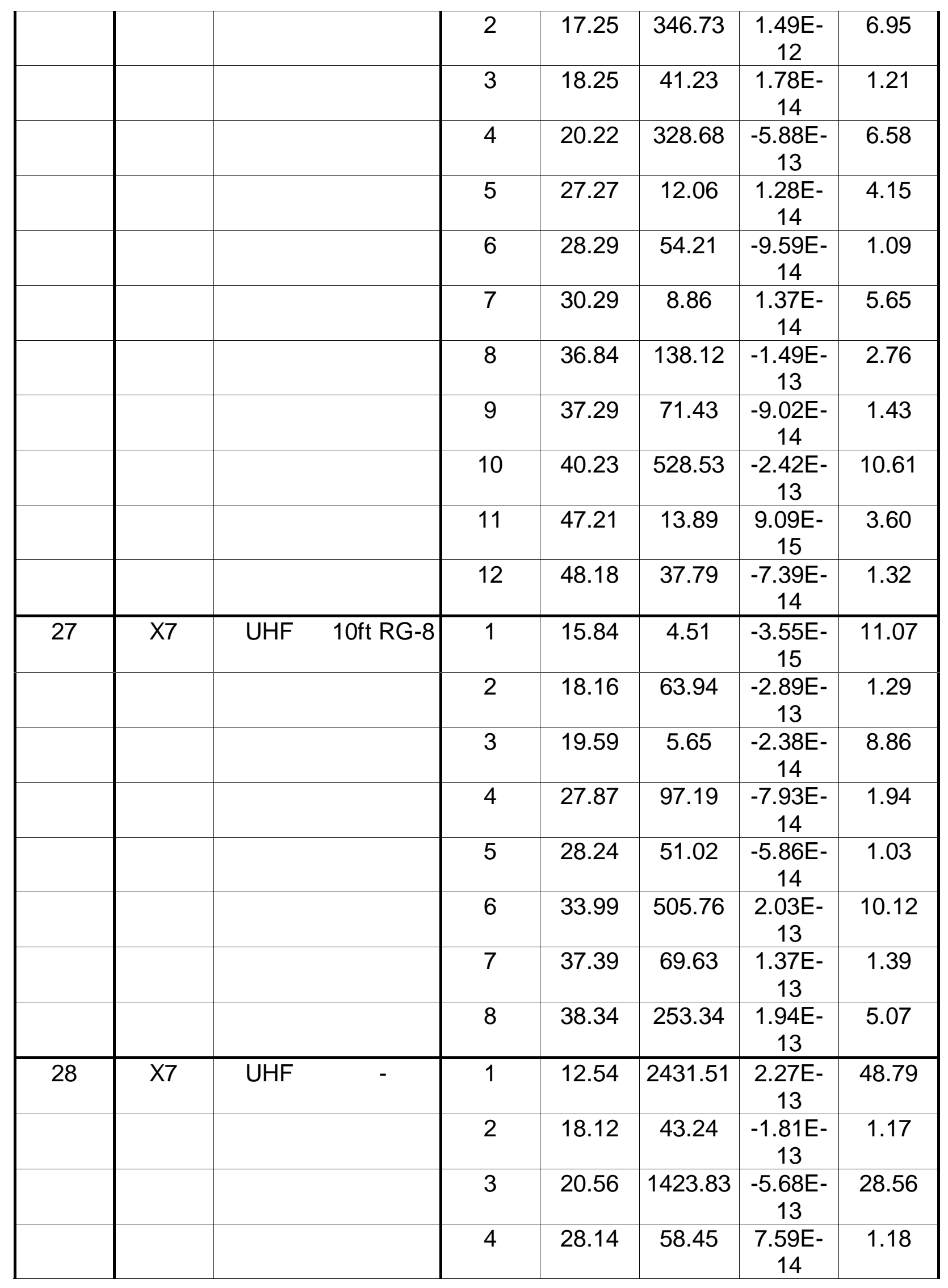




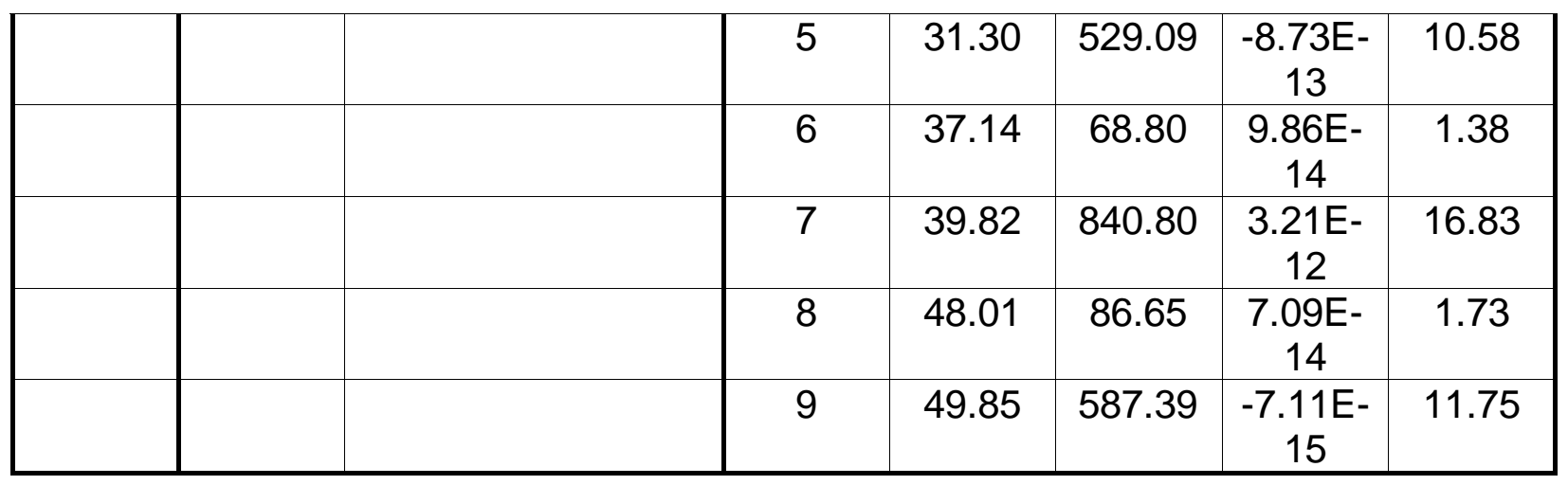

Table 1 


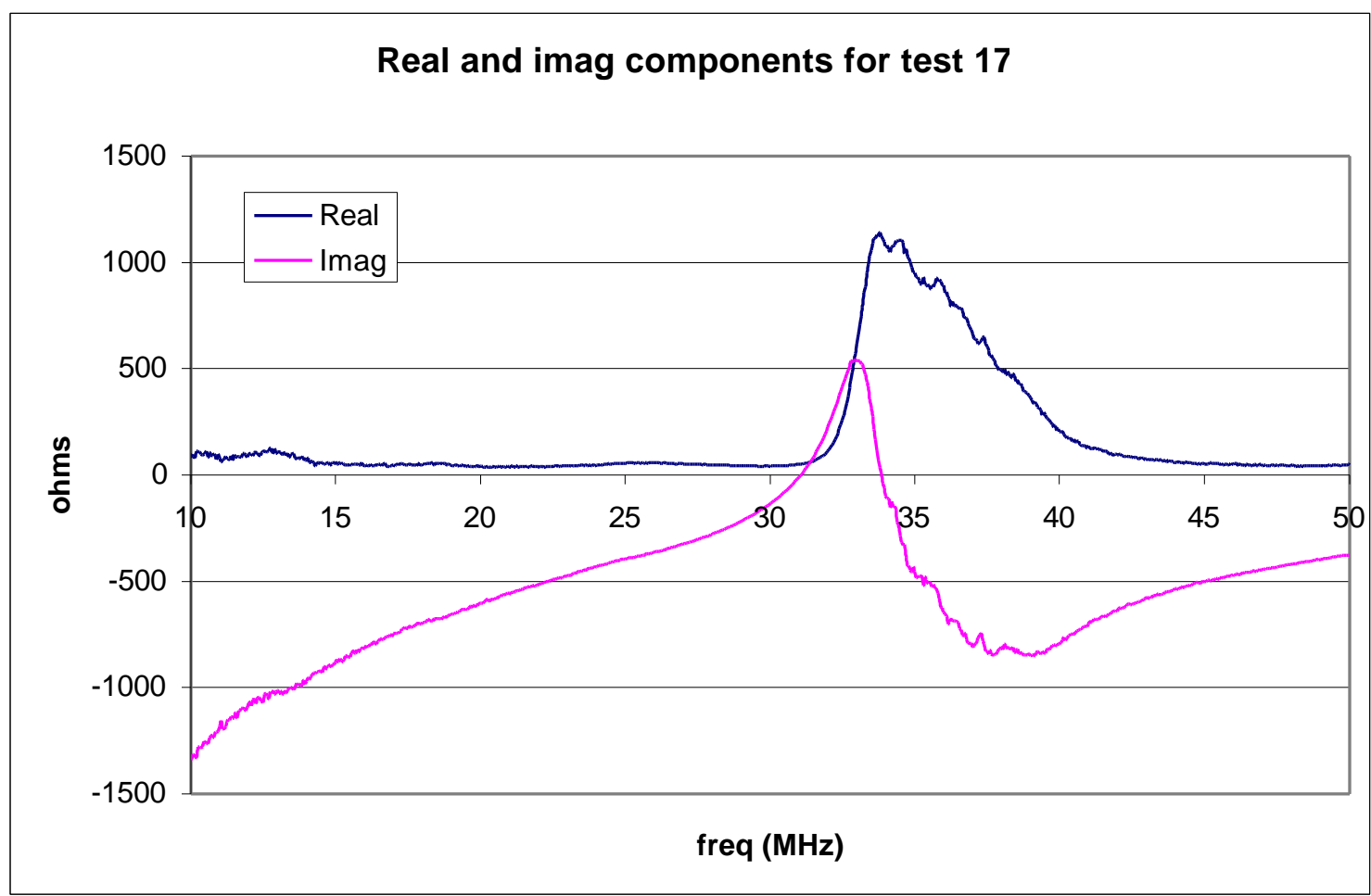

figure 1: antenna AP1 with SMA connectors, no cable

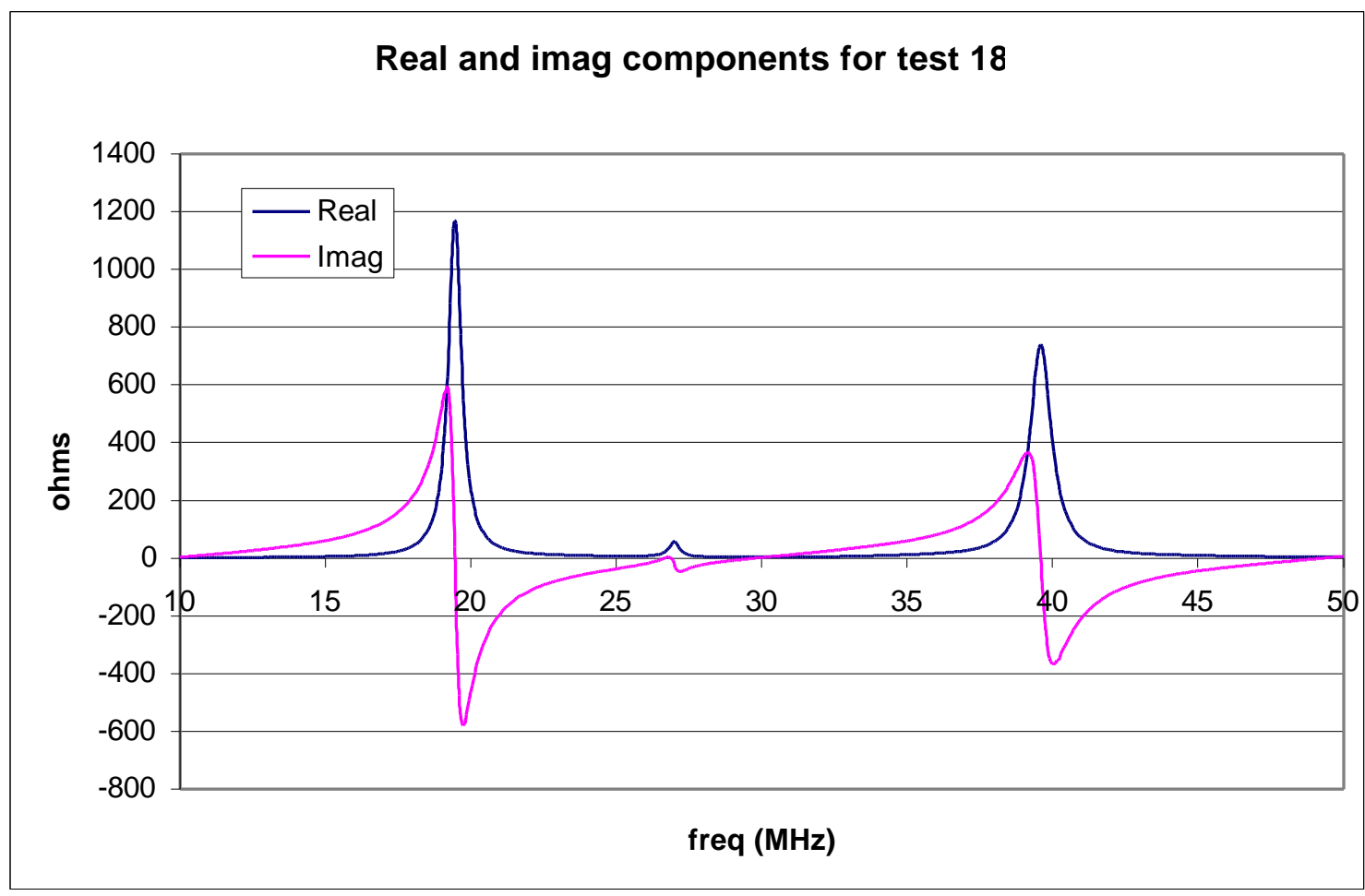

figure 2: antenna AP1 with SMA connectors and 20ft RG-58 cable. 


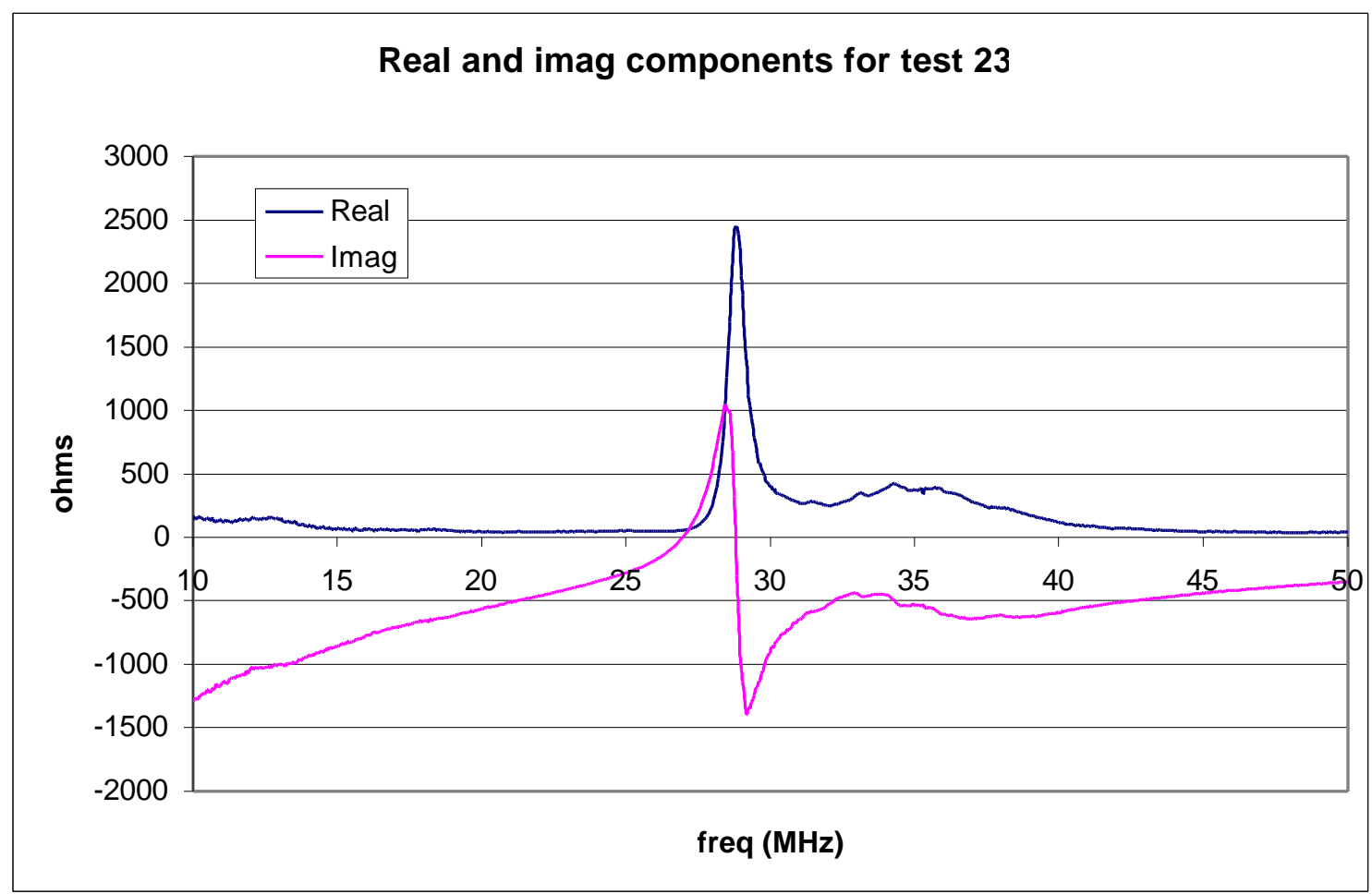

figure 3: antenna AP1 with UHF connectors, no cable.

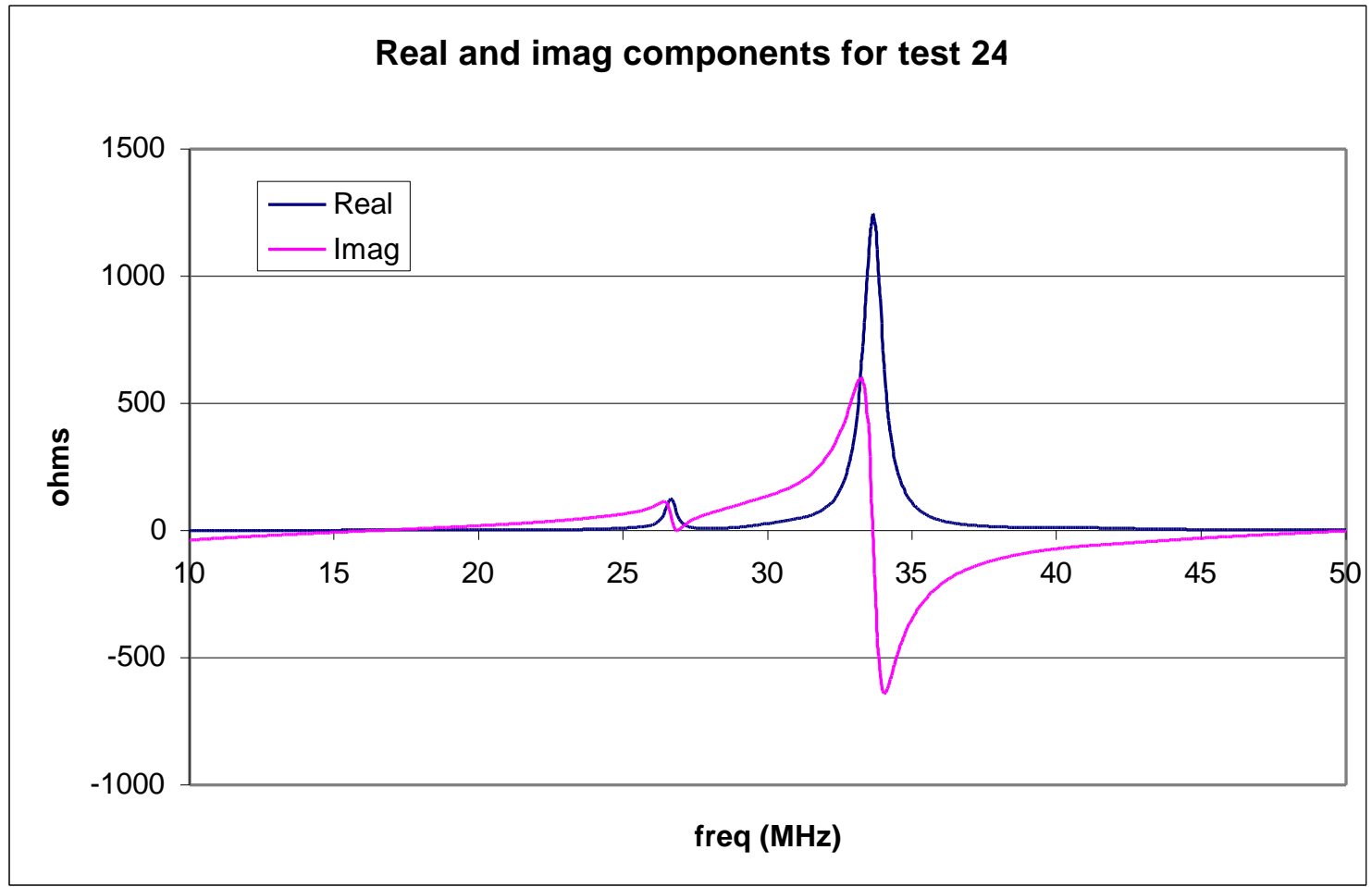

figure 4: antenna AP1 with UHF connectors and 10ft RG-8 cable. 


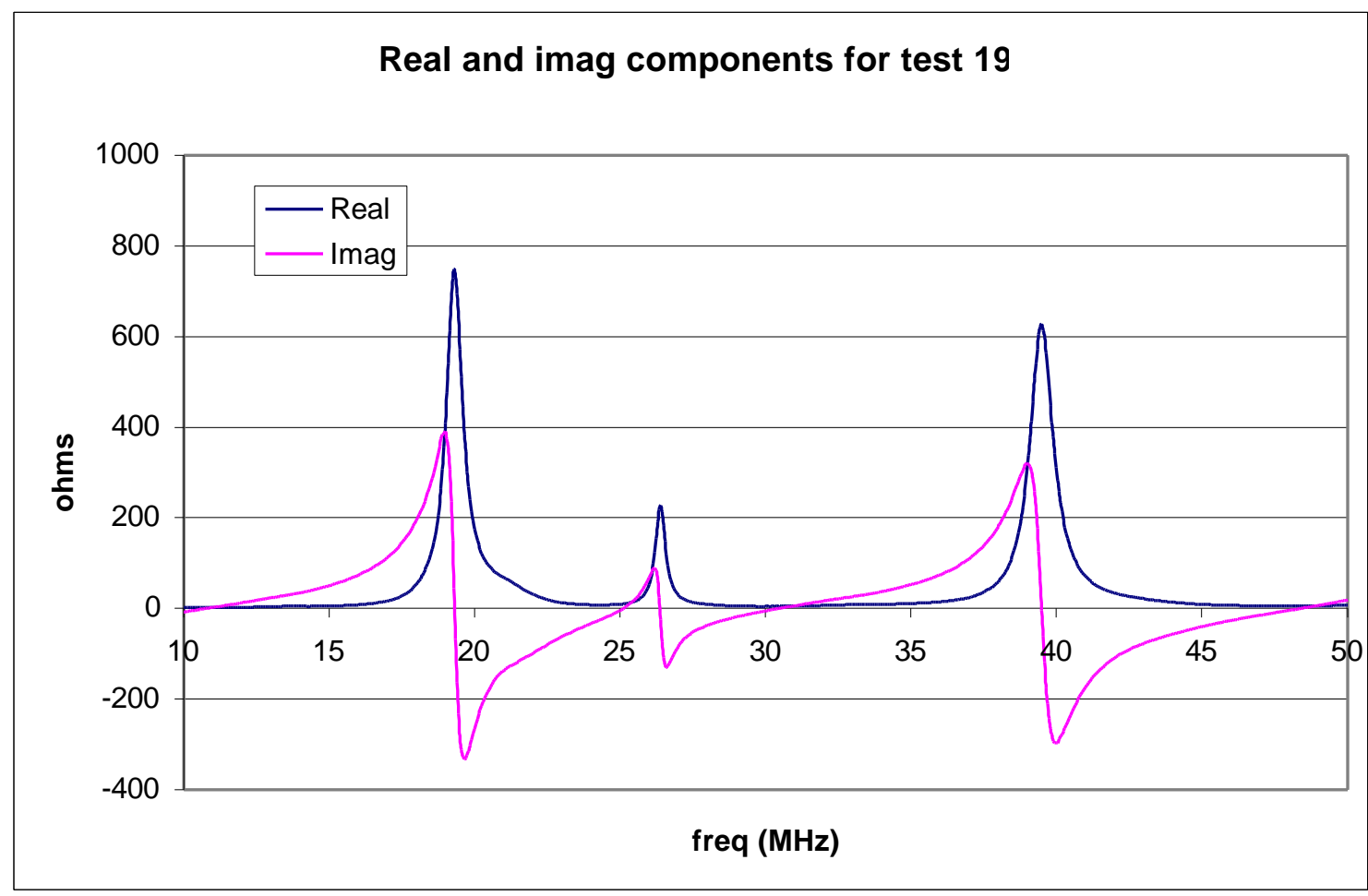

figure 5: antenna X5 with SMA connectors and 20ft RG-58 cable.

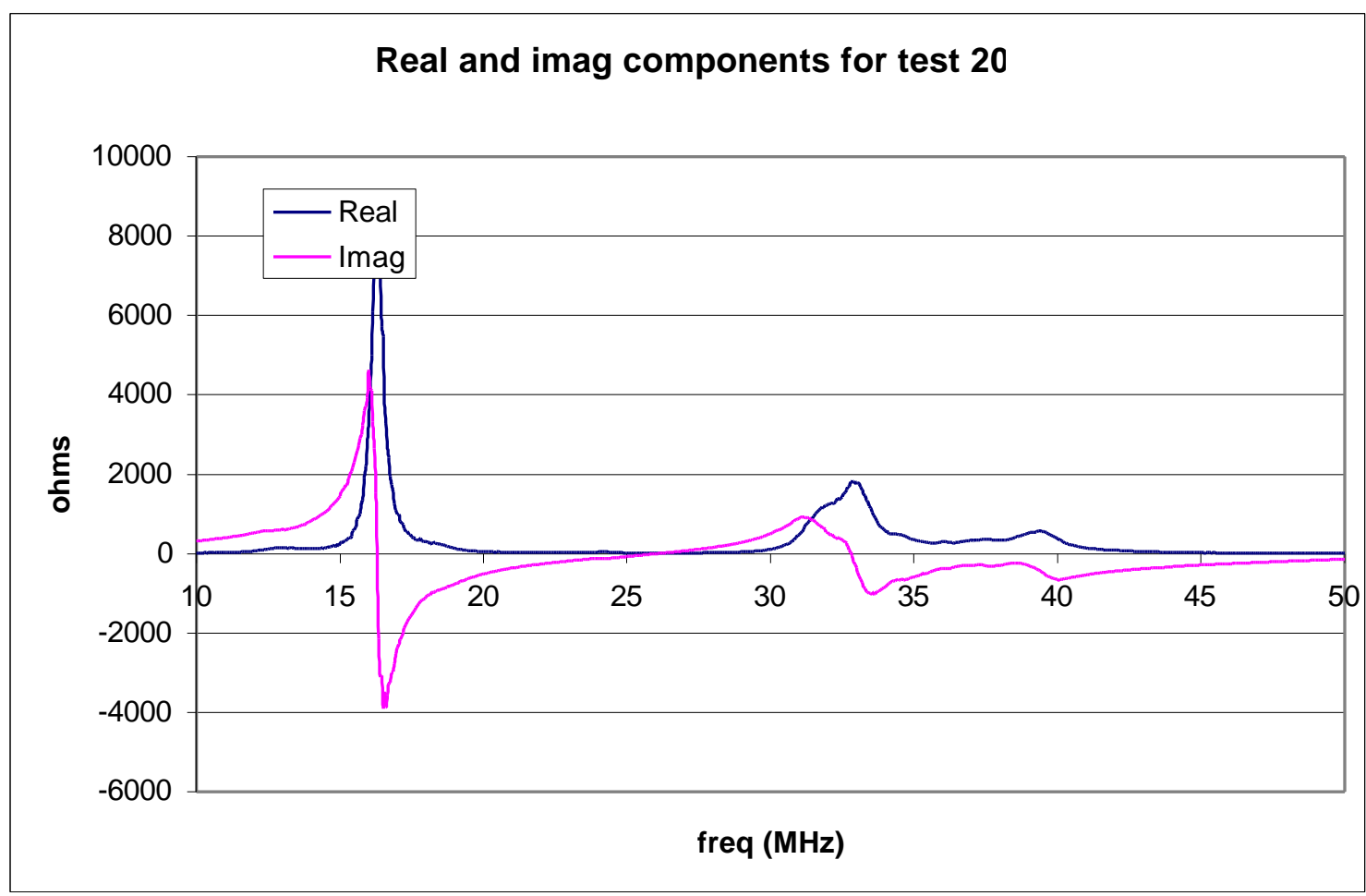

figure 6: antenna X5 with SMA connectors, no cable. 


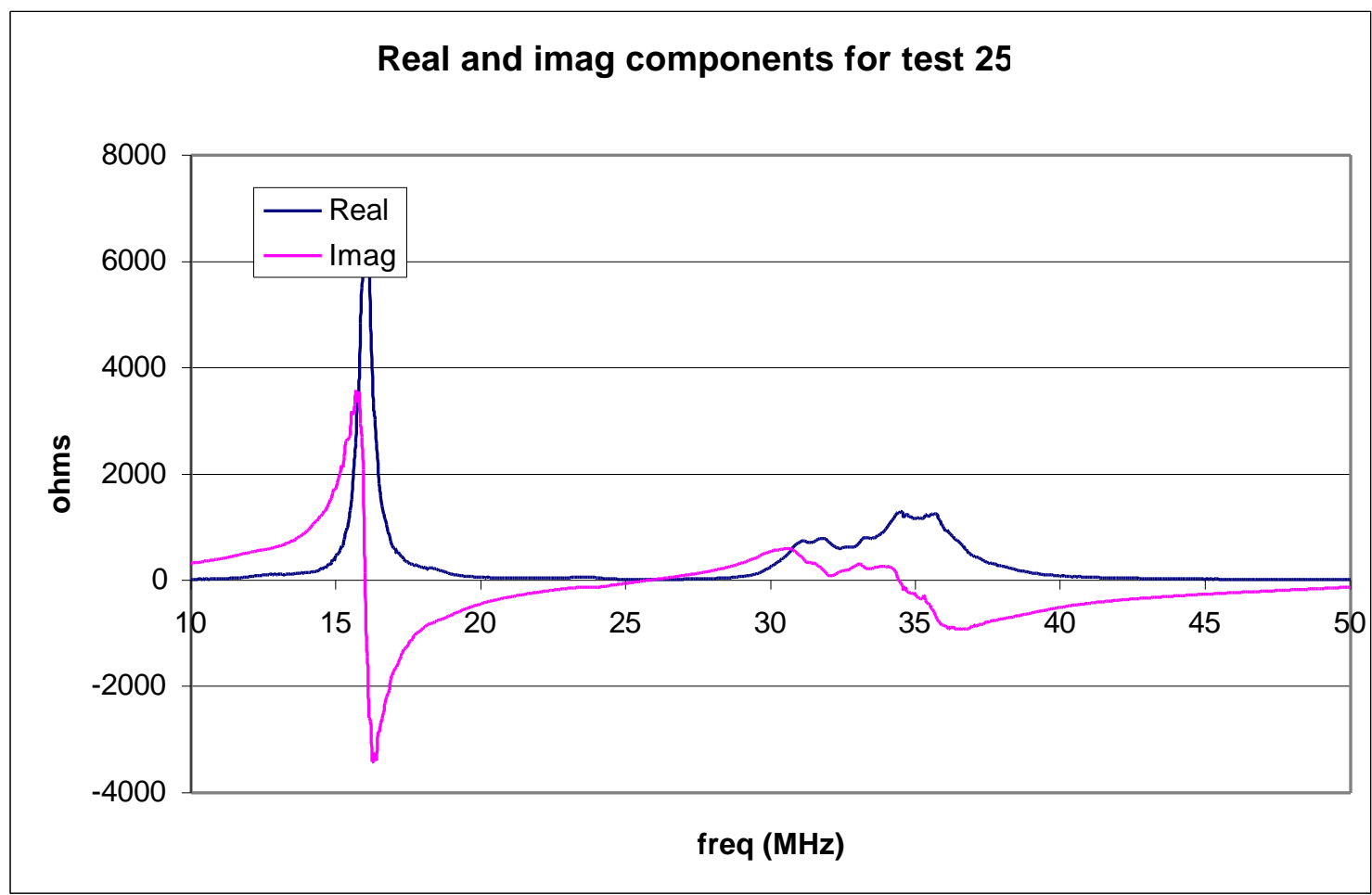

figure 7: antenna X5 with UHF connectors, no cable.

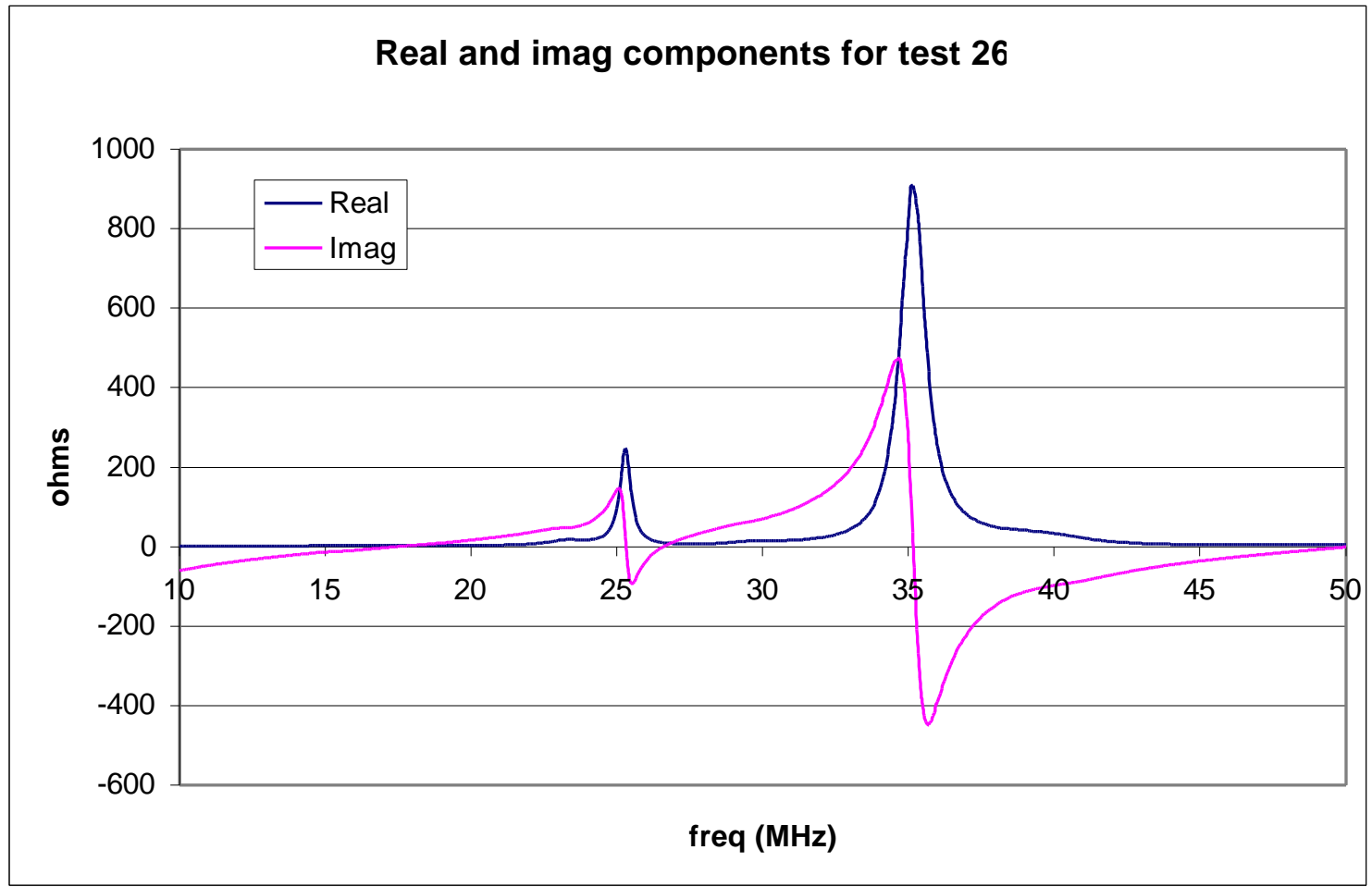

figure 8: antenna X5 with UHF connectors and 10ft RG-8 cable. 


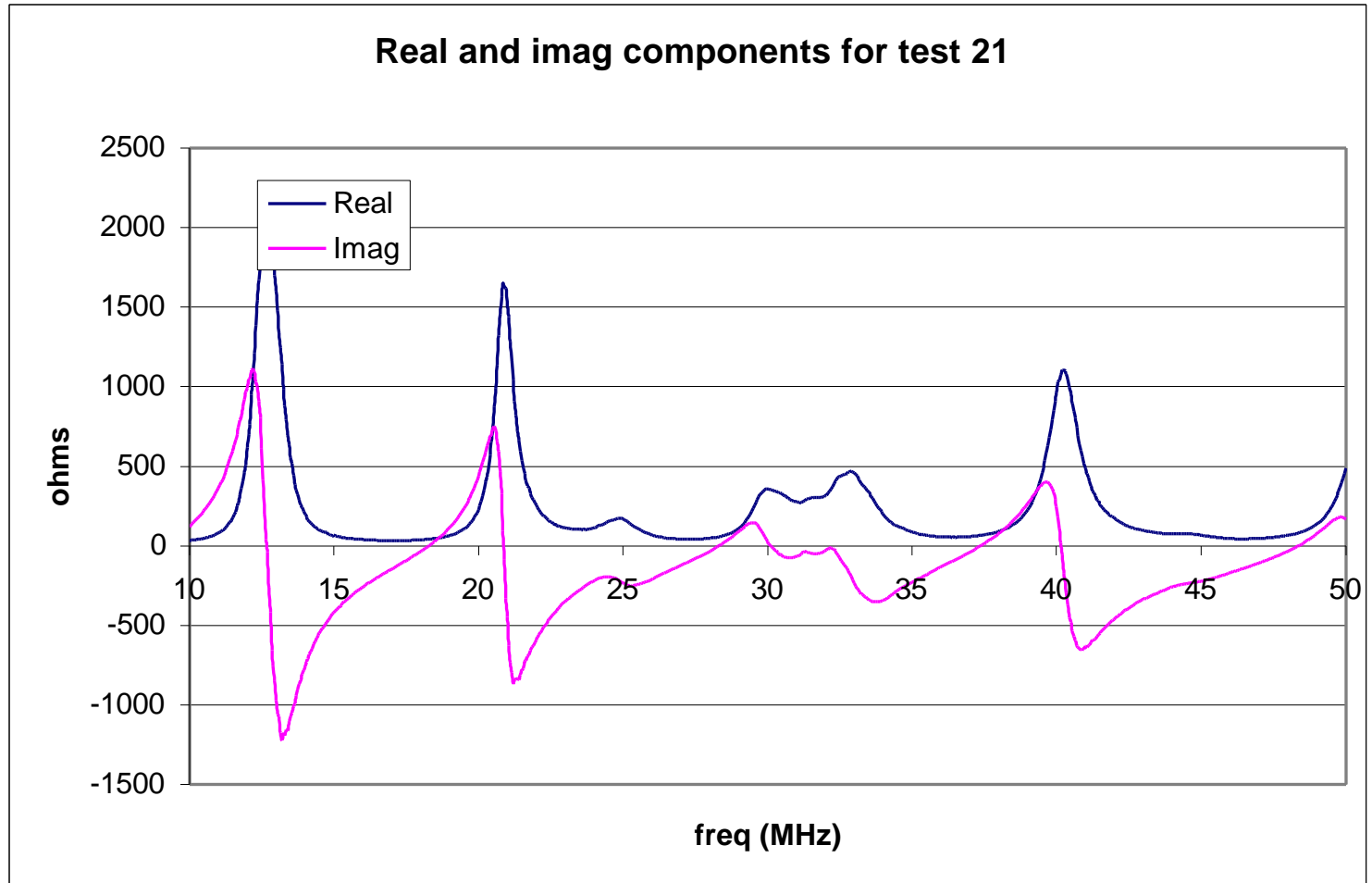

figure 9: antenna X7 with SMA connectors, no cable.

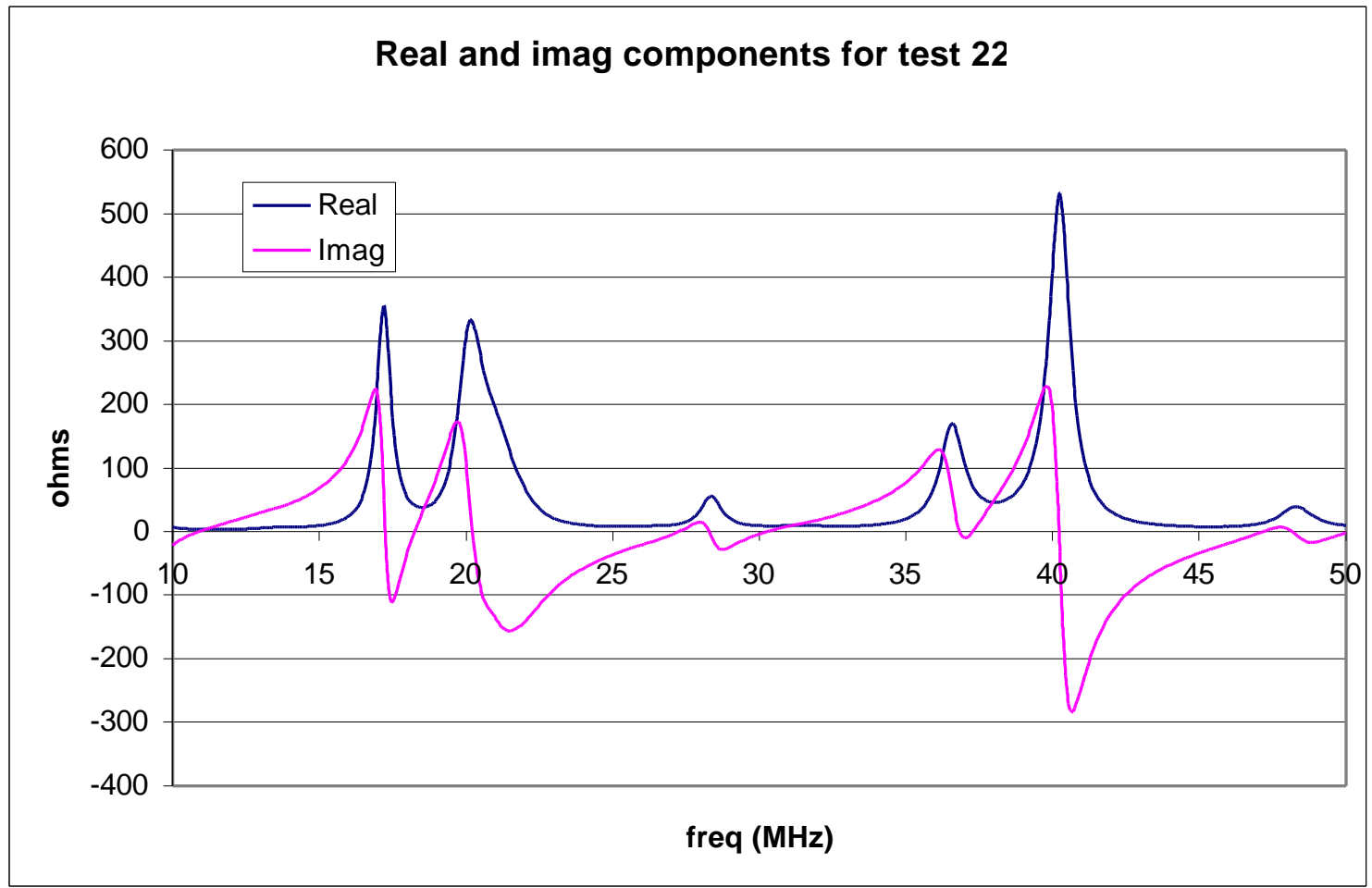

figure 10: antenna X7 with SMA connectors and 20ft RG-58 cable. 


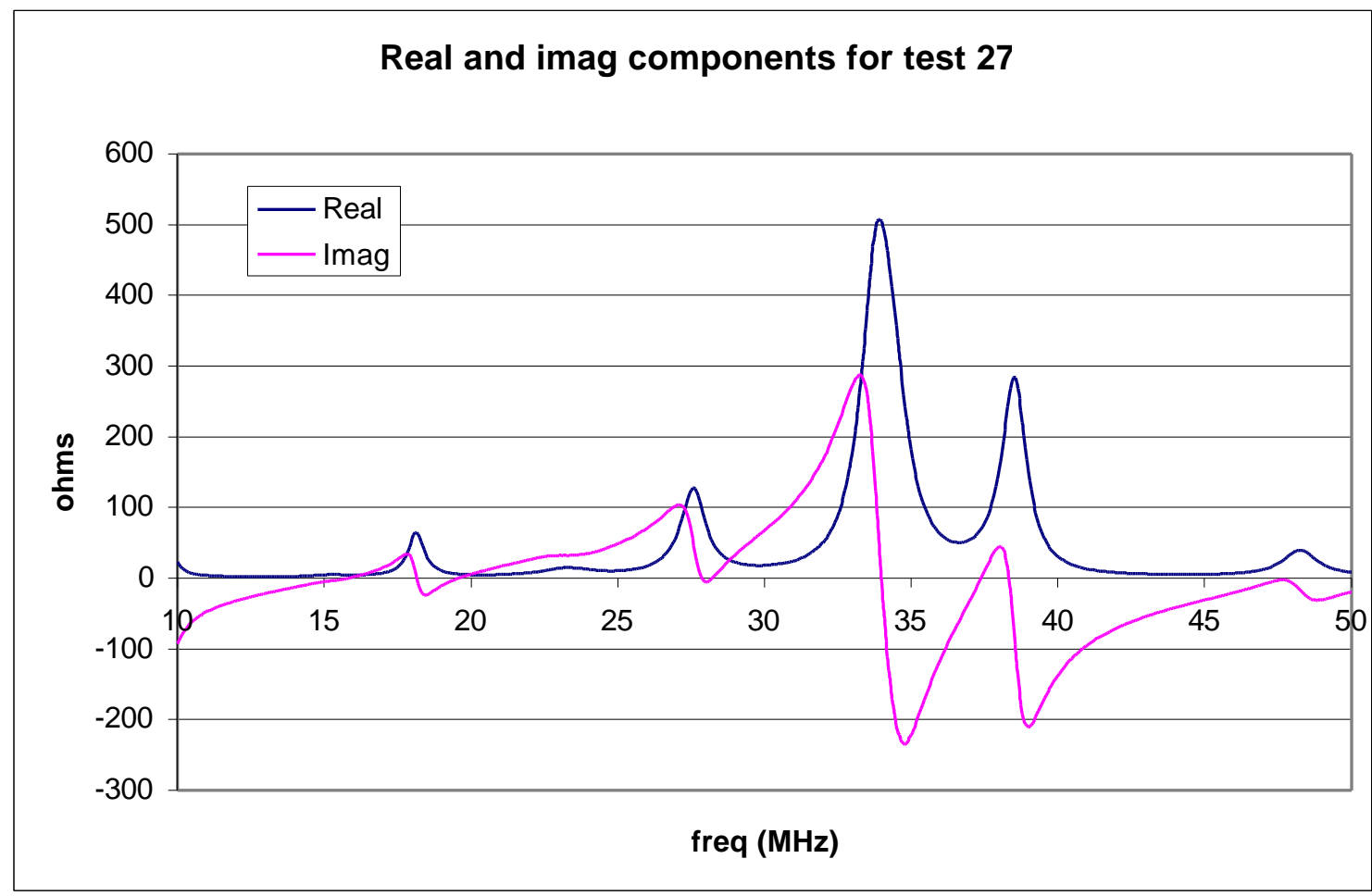

figure 11: antenna X7 with UHF connectors and 10ft RG-8 cable.

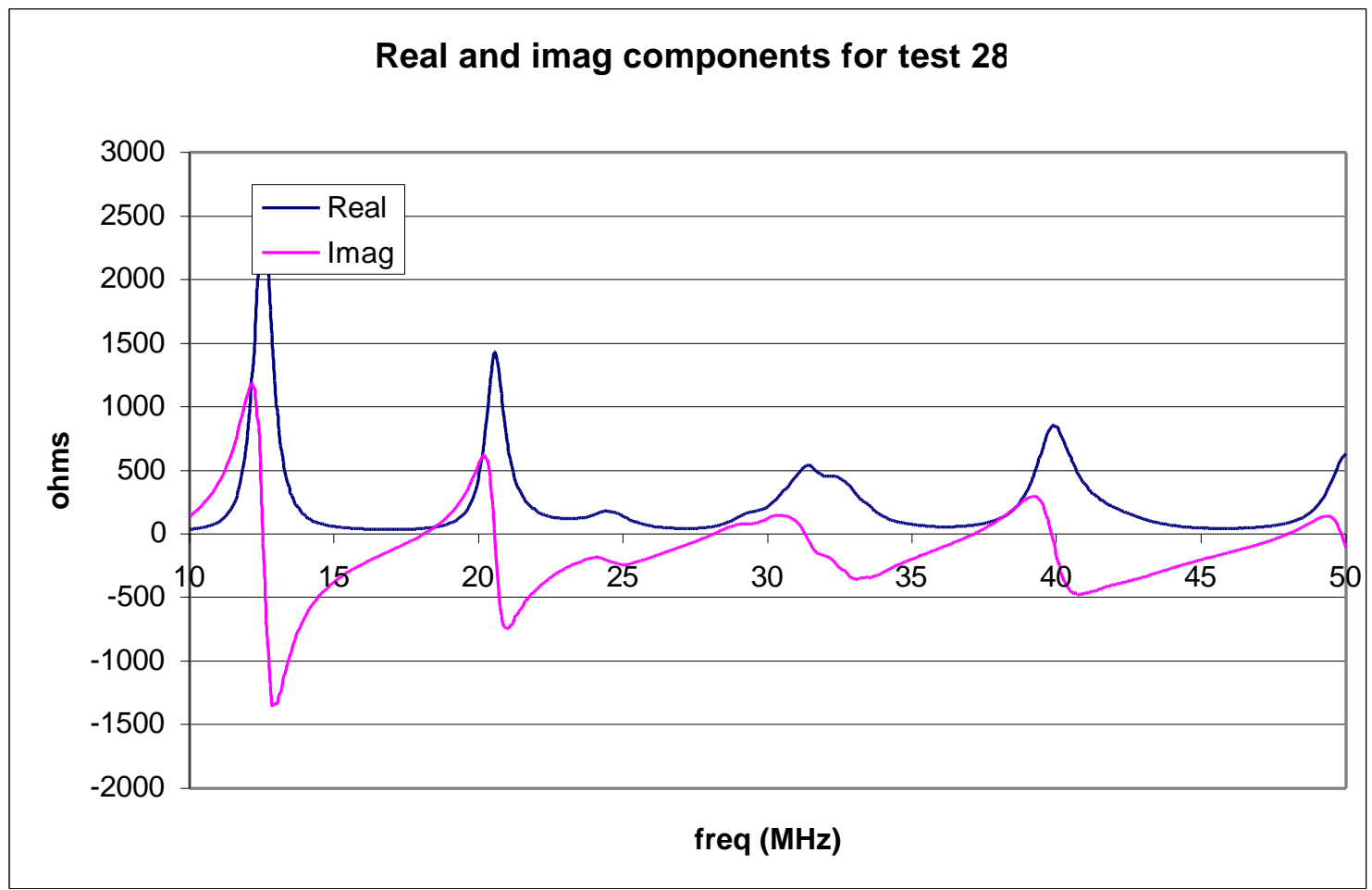

figure 12: antenna X7 with UHF connectors, no cable. 


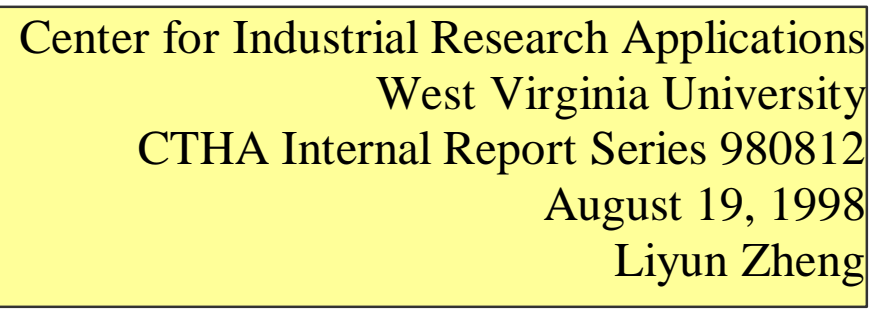

\section{Parametric Study on Resonance Frequency of CTHA (1)}

\section{Introduction}

The purpose of this work is to inspect the effect of certain parameters on the resonance frequency of the CTHA. Studied parameters include the permittivity of the base material, extra space between helixes, and the geometry of the base. The resonance frequencies computed from XFDTD are also compared with those from NEC and those from tests in the anechoic chamber to illustrate the difference between these modeling and simulation techniques.

\section{Modeling and Simulation}

CTHA342 is used to implement the parametric study. Geometric and material parameters of CTHA342 are listed in Table 5. Controlled parameters and their values, which define all the cases of modeling and simulation. Most cases are modeled and simulated in XFDTD. Only one duplicate case is modeled and simulated in NEC, and another real case is tested in the anechoic chamber. Simulation parameters for XFDTD that are kept identical for all cases are also listed in Table 5 for reference.

Table 5 General Parameters and Values for CTHA and Simulation

\begin{tabular}{|l|l|l|l|}
\hline \multicolumn{2}{|c|}{ Common Parameters for CTHA342 } & \multicolumn{2}{l|}{ Simulation Parameters for XFDTD } \\
\hline Major Radius & $26.67 \mathrm{~mm}$ & Source & Voltage \\
\hline Minor Radius & $4.70 \mathrm{~mm}$ & Source Form & Gaussian \\
\hline Wire Radius & $0.35 \mathrm{~mm}$ & Pulse Width & 64 steps \\
\hline Wraps & 10 & Amplitude & 1000 volts \\
\hline Feed Type & 6 & Time & 20000 steps \\
\hline Feed Position & Polar & Feed Resistance & 50 Ohms \\
\hline Wire Material & PFC & & \\
\hline Cell Size & $0.55 \mathrm{~mm}$ & & \\
\hline
\end{tabular}

Setting the base (or core material) to empty is equivalent to specifying a permittivity of 1.0 for the base material. When inspecting the effect of extra space between helixes, the base for the CTHA in all cases is set to empty. Discretization in XFDTD is limited to a Cartesian grid, so one cell is the minimum extra space that must be used to avoid 
unwanted connections between the two helixes. When studying the effect of the base geometry, the permittivity of the base material is set to 2.0.

\section{Results \\ Permittivity}

The resonance frequencies of CTHA342 with different base materials are listed in Table 3.

For each parametric level, $\mathrm{F} / \mathrm{F}_{\text {air }}$ for each resonance is calculated in the form of a percentage, as listed in the same table. Fair is the resonance frequency of an antenna with dielectric permittivity of 1.0 (Air), and $\mathrm{F}$ is the resonance frequency of the same antenna but with different dielectric permittivity. The mean and variance of the $F / F$ air are also calculated for each parametric level. ANOVA shows that the difference between these parametric levels is significant (confidence=0.999). Compared to the resonance frequency of the CTHA with the empty base, the resonance frequency decreases about $27.6 \%$ when permittivity increases to $2.0,40.4 \%$ when permittivity increases to 3.0 , and $48.2 \%$ when permittivity increases to 4.0 .

Table 6 Resonance Frequencies versus Permittivity of Base Material

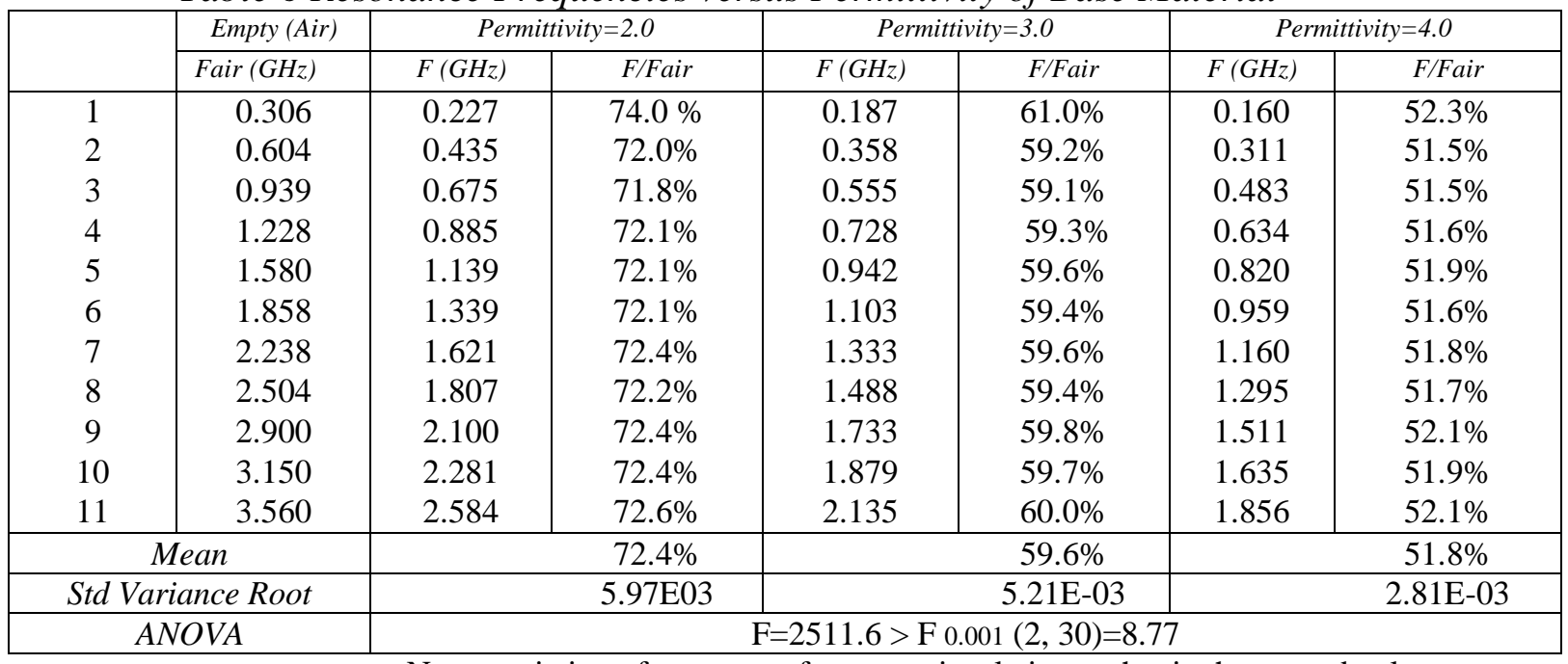

Note: variation of resonance frequency is relative to that in the second column

Theoretically, the effect of permittivity on the resonance frequency can be expressed as

$$
\mathrm{F} / \mathrm{F}_{\text {air }}=1 / \mathrm{Sqrt}(\mathrm{P})
$$

where $\mathrm{P}$ is the dielectric permittivity of an antenna.

As for $\mathrm{F} / \mathrm{F}$ air versus the permitivity listed in the Table 3, the following relationship can be obtained by regression analysis 


$$
\mathrm{F} / \mathrm{F}_{\text {air }}=0.0276+0.9829 / \mathrm{Sqrt}(\mathrm{P}) \quad(\text { Equation } 4)
$$

The Standard Error is about 0.001. Regression statistics show that the intercept term, while significant, is of far less contribution to the variation when compared to the second term ( $t=9.2$ for the intercept term, $t=215.7$ for the second term). A model of a secondorder polynomial with a $1 / \operatorname{Sqrt}(\mathrm{P})$ term is also used to fit the data; however statistics indicate both the first order and the second order term are of no significant contribution to $\mathrm{F} / \mathrm{F}_{\text {air }}(\mathrm{t}=0.015$ and $\mathrm{t}=-0.104$ respectively). These results conform to the theoretical conclusion, although systematic error exists, as shown. The fitted model using a polynomial of the second order is plotted in green; the fitted model only using $1 / \operatorname{Sqrt}(\mathrm{P})$ and constant term is plotted in green, and the theoretical model is plotted in blue. Visually, there is no significant difference between two fitted models, since they are seriously overlapped.

\section{Extra Space Between Helixes}

Resonance frequencies of CTHAs with different spacing between the two helixes are listed in Table 7. For each extra space level, $\mathrm{F} / \mathrm{F}_{1}$ for each resonance frequency is calculated in the form a percentage, where $F_{1}$ is the resonance frequency of CTHA342 with extra space of one cell, and F is the resonance frequency of the same CTHA but with different extra space. The mean and variance of $\mathrm{F} / \mathrm{F}_{1}$ for each parametric level are also computed. ANOVA shows that the difference between these parametric levels is significant (confidence=0.999). When compared to the reference, the resonance frequency increases by $7.1 \%$ when increasing extra space to two cells, $11.3 \%$ when increasing extra space to three cells, $14.6 \%$ when increasing extra space to 4 cells, and $17.4 \%$ when increasing extra space to 5 cells. 
Table 7 Resonance Frequencies versus Extra Space between Two Helixes

\begin{tabular}{|c|c|c|c|c|c|c|c|c|c|}
\hline \multirow[t]{2}{*}{ Res \# } & 1 cell & \multicolumn{2}{|c|}{2 cells } & \multicolumn{2}{|c|}{3 cells } & \multicolumn{2}{|c|}{4 cells } & \multicolumn{2}{|c|}{$5 \mathrm{cells}$} \\
\hline & $F 1$ & $F$ & $F / F 1$ & $F$ & $F / F 1$ & $F$ & $F / F 1$ & $F$ & $F / F 1$ \\
\hline 1 & 0.306 & 0.335 & $109.4 \%$ & 0.344 & $112.4 \%$ & 0.354 & $115.7 \%$ & 0.361 & $117.8 \%$ \\
\hline 2 & 0.604 & 0.646 & $106.9 \%$ & 0.676 & $111.8 \%$ & 0.696 & $115.1 \%$ & 0.717 & $118.6 \%$ \\
\hline 3 & 0.939 & 1.004 & $106.8 \%$ & 1.039 & $110.6 \%$ & 1.076 & $114.5 \%$ & 1.097 & $116.7 \%$ \\
\hline 4 & 1.228 & 1.311 & $106.7 \%$ & 1.370 & $111.6 \%$ & 1.409 & $114.7 \%$ & 1.451 & $118.1 \%$ \\
\hline 5 & 1.580 & 1.687 & $106.7 \%$ & 1.748 & $110.6 \%$ & 1.806 & $114.3 \%$ & 1.842 & $116.6 \%$ \\
\hline 6 & 1.858 & 1.983 & $106.8 \%$ & 2.072 & $111.6 \%$ & 2.130 & $114.6 \%$ & 2.190 & $117.9 \%$ \\
\hline 7 & 2.238 & 2.389 & $106.8 \%$ & 2.473 & $110.5 \%$ & 2.552 & $114.0 \%$ & 2.602 & $116.3 \%$ \\
\hline 8 & 2.504 & 2.672 & $106.7 \%$ & 2.790 & $111.4 \%$ & 2.860 & $114.2 \%$ & 2.940 & $117.4 \%$ \\
\hline & \multicolumn{2}{|r|}{$107.1 \%$} & \multicolumn{2}{|r|}{$111.3 \%$} & \multicolumn{2}{|r|}{$114.6 \%$} & \multicolumn{2}{|r|}{$117.4 \%$} \\
\hline & & & $9.26 \mathrm{E}-03$ & & 6.87E-03 & & $5.29 \mathrm{E}-03$ & & $8.29 \mathrm{E}-03$ \\
\hline \multicolumn{2}{|c|}{$A N O V A$} & \multicolumn{8}{|c|}{$\mathrm{F}=273.9>\mathrm{F} 0.001(3,28)=7.19$} \\
\hline
\end{tabular}

Note: variation of resonance frequency is relative to that in second column

$\mathrm{F} / \mathrm{F}_{1}$ versus extra space $(\mathrm{S})$ can be fitted using polynomial of second order as follow

$$
\mathrm{V}=0.9678+0.0587 * \mathrm{~S}-0.0035 * \mathrm{~S} \wedge 2
$$

\section{Base or Core Geometry}

As shown in Figure 2, three types of base (or core) geometry are specified to investigate the effect of base geometry on the resonance frequency. The results from simulation are shown in Figure 3. A t-test shows significant differences between the CTHA with a non-empty base and the CTHA with an empty base $(\mathrm{t}=153.2$, confidence $=0.999$ ). However, the difference between the CTHA with a partially filled base and the CTHA with the filled base is trivial $(\mathrm{t}=2.98)$. When compared to the CTHA with the empty base, the resonance frequency decreases by $27.0 \%$ for CTHA with the partially filled base and $27.6 \%$ for the CTHA with the filled base. A reasonable explanation is that only the base material near the helixes has a significant effect on the resonance frequency of the CTHA.
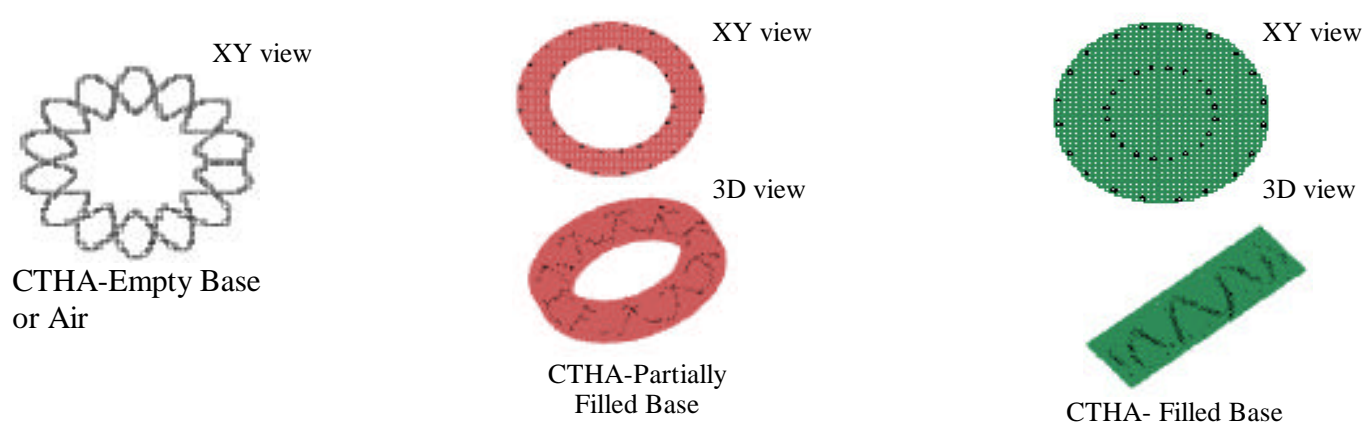

Figure 2 Three types of Base Geometry 


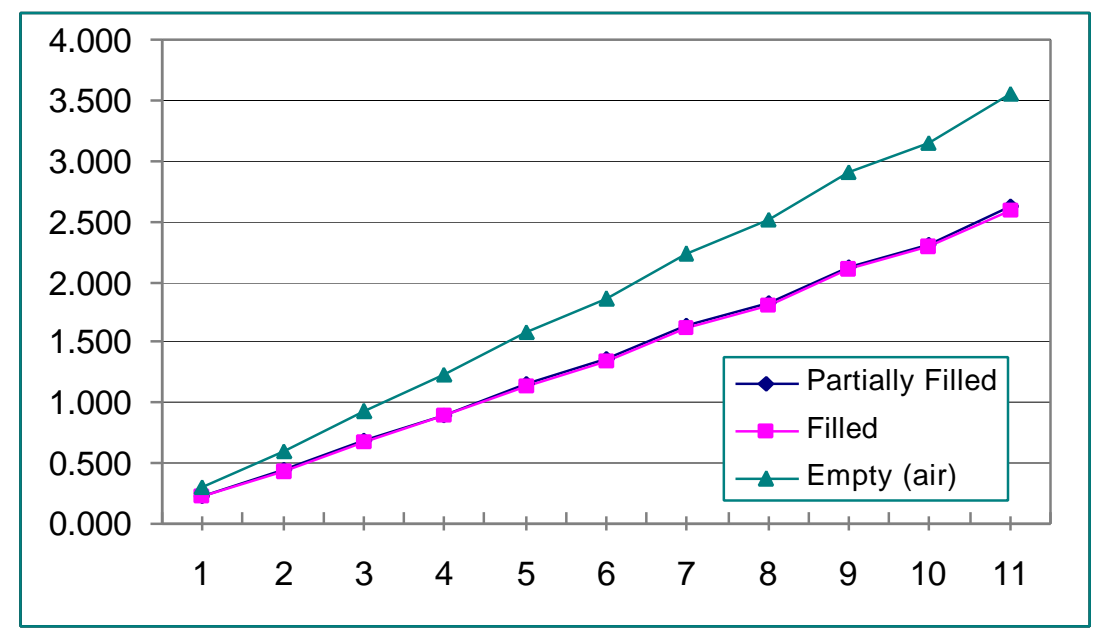

Figure 3 Resonance Frequencies versus Base Shape

\section{XFDTD Compared to NEC and Actual Measurements}

The resonance frequencies of CTHAs with the same geometry are also computed from NEC. Additionally, a real CTHA342 has been tested in the anechoic chamber and its resonance frequencies measured. The results are shown in Figure 4 together with those from XFDTD. The results from NEC are close to those from XFDTD when the base is specified as empty or air. The t-test couldn't find significant differences between these two techniques when simulating the CTHA with an empty base ( $t=1.82 \sim 2.32$ ). The curve of resonance versus frequency from the real test lies between that from the case with permittivity 2.0 and the case with permittivity 3.0, indicating the possible permittivity of the base material of the real one is between 2.0 and 3.0.

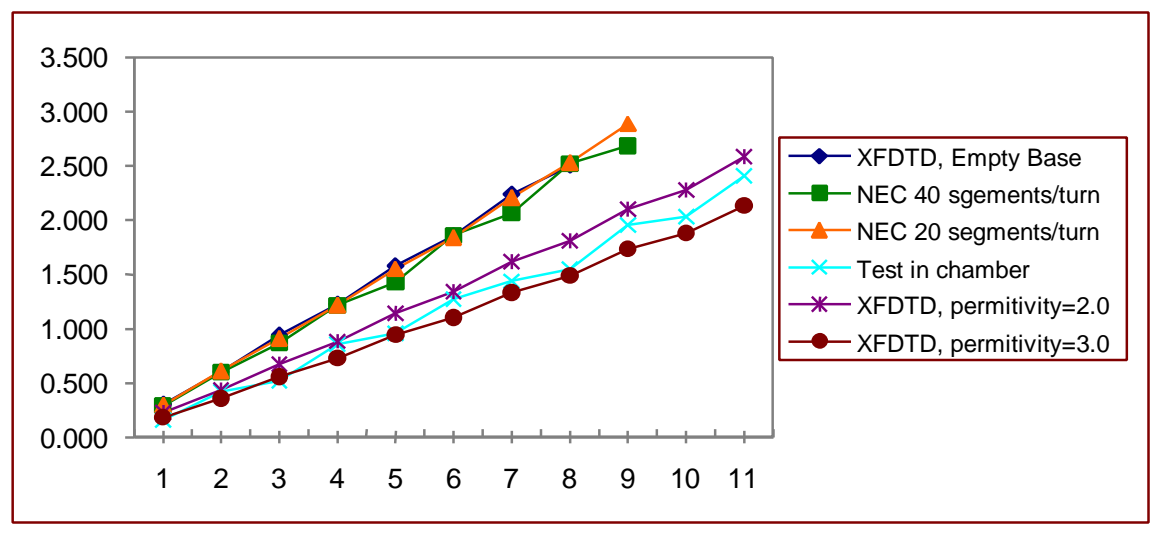

Figure 4 Resonance Frequencies from XFDTD, NEC, and Actual Test 


\section{Conclusion}

The permittivity of the base material has a significant effect on the resonance frequency of the CTHA. When permittivity increases, each resonance frequency decreases by approximately the same percentage, which is inversely proportional to the square root of the permittivity.

Extra space between the two helixes also has an effect on the resonance frequency of the CTHA, though not as significant as permittivity. When extra space increases, each resonance frequency increases by approximately the same percentage.

There is a significant difference between a CTHA with a non-empty base and a CTHA with an empty base when the resonance frequency is concerned. However, the difference between a CTHA with a partially filled base and a CTHA with a filled base is trivial. The reasonable explanation is only the base material near the helixes will greatly affect the resonance frequency of the CTHA.

NEC is as reliable as XFDTD for predicting the resonance frequency of a CTHA without the dielectric. However, when a dielectric is present, NEC doesn't predict the resonance frequency as accurately as XFDTD does. 


\section{Flexible Mesh Generator for CTHA}

\section{Introduction}

The Finite Difference Time Domain (FDTD) method and the software based on this method have received wide use for antenna electromagnetic calculation. To apply this method and use this kind of software, the geometry of an antenna must be modeled first. The geometry is usually modeled as discrete material cells, or rather as discrete nodes linked with edges, which may be defined with different dielectric properties. XFDTD, for example, uses a cubic cell as the discretization unit, meaning that the geometry can only be approximated as edges of a cube, surfaces of a cube, or a cube itself.

Software such as XFDTD usually provides some basic tools for geometry modeling, such as creating the mesh manually with a mouse or building the mesh from the basic shapes from the associated geometry element library.

For the CTHA, however, these tools are far from adequate due to its complex and diverse geometry. This complexity requires the development of a more efficient and flexible mesh generator for the CTHA.

\section{Basic Requirements of Mesh Generator for CTHA}

There are two points that need serious consideration in developing such a mesh generator. The first is the efficiency of the generator's algorithm, and the second and more important is the flexibility of the generator.

\section{Algorithm Efficiency}

Several aspects related to algorithm efficiency are the accuracy of the algorithm and the time and resources necessary to complete modelling using the algorithm.

In general, accuracy conflicts with the time and resources expended in modeling. Smaller resolution of the mesh is preferred for greater accuracy but requires more time and resources for modeling. Given that the desired resolution must be smaller than 
approximately 0.1 wavelength for accurate features, the algorithm must ensure the smoothness and connectivity of the modeled geometry element, which certainly affects the accuracy of the model.

Since the memory and the time required to model are significant, the algorithm should also save memory and reduce computation time, if possible.

\section{Generator Flexibility}

Flexibility is necessary for a CTHA mesh generator. First, changing the parameters of the CTHA should be convenient, ideally through a user-friendly, graphical interface. Second, the generator should be capable of modeling various kinds of CTHAs. Third, user-defined CTHAs or antennas should be easily derived and modeled within the existing frame of the mesh generator.

\section{Implementation of Mesh Generator}

\section{General Algorithm}

- Determine the boundary of the introduced CTHA and allocate the required memory for the nodes (represented as discrete integer data) that will be generated.

- Discretize each geometric element in the CTHA with a specified material. Three points with identical intervals per unit length are sampled for the relatively plain elements, but more points are sampled for elements with detailed geometric features. Since every geometric element has its own moving coordinate system as discussed later, the sampled points will be transformed to the base coordinate system before finally being approximated to the nodes.

- Refine the nodes to improve the smoothness, ensure the overall connectivity within the element or between different elements, and avoid unwanted connection between different elements.

- Save the nodes to the file in the format that is required for XFDTD.

\section{Class Hierarchy}

Though each CTHA model has some geometric features that distinguish it from others, each is derived from a set of basic models, either through a combination of some 
basic models, a series of transformations of a certain basic model, or the addition of new features. An object-oriented approach, therefore, is applied to embed modeling flexibility in the mesh generator.

The basic geometric element types, defined as classes, for the CTHA are the circle, cylinder, donut, and helix, where the cylinder, donut, and helix can all be derived from the circle. The feed of the CTHA is also defined as a class and is derived from a cylinder. The class Feed encompasses all kinds of feed structures. Other classes such as Point, Line, Rectangle, Box, Sphere, etc., are not necessary for the CTHA but may be used to model user-defined antennas (see Appendix). In each class, a function to discretize the element is defined. Therefore, any object instantiated from the above class has the ability to discretize itself.

Two other important classes in the mesh generator are Axis and NodeArray. These classes are developed to support the flexibility of the mesh generator. The class Axis represents the moving coordinate system that is used in the modeling. It stores the position of and the reference information about the geometric element and encapsulates the coordinate-related functionality of the element, such as translation, rotation, and so on. Since every class of geometric element has an Axis-type object, which stores its reference (an Axis-type object of other elements), each element in a model geometrically correlates with the others. More important, each element now possesses the ability of translation and rotation relative to other geometric elements. It is because of this mechanism that a complex geometric model can be created, typically through the combination of basic models or the serial coordinate transformation of the basic model.

NodeArray represents the geometric model in discrete form. It stores the integer data of every node generated from the geometric elements and encapsulates the functionality of refining nodes to improve the smoothness and connectivity, linking nodes with edges of appropriate material and saving nodes to the file in a certain format. After all the elements in a model are discretized, NodeArray takes charge of the remaining work. In refining the procedure, some nodes may be deleted while others may be added and the material type of some nodes may be changed. 


\section{Results and Discussion}

The mesh generator for the CTHA is implemented in Visual Basic 5.0. The capabilities of this mesh generator are discussed below.

As mentioned earlier, each geometric element class defines the function to discretize this type of element. Consequently, the mesh of 3D geometric models like Point, Line, Rectangle, Box, Circle, Cylinder, Helix, BiHelix, CTHA with cylinder base, or CTHA with donut base can now be directly generated after the object is instantiated from the corresponding class. For each type of element, variation from the basic shape of that type, for example the inner hollow for the cylinder, is allowed. Figure 5 provides examples of meshes created by the generator.

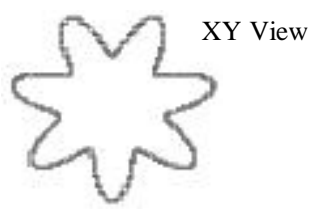

Helix

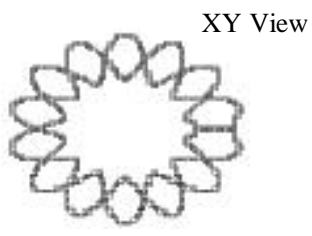

BiHelix
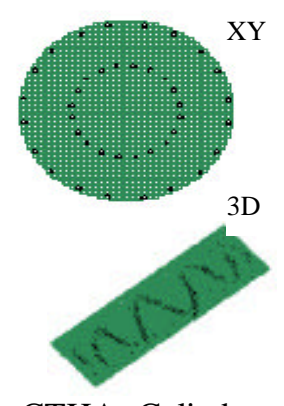

CTHA- Cylinder Base, Filled
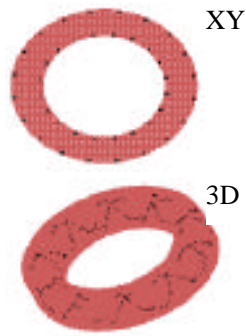

CTHA-Cylinder Base, Partially Filled

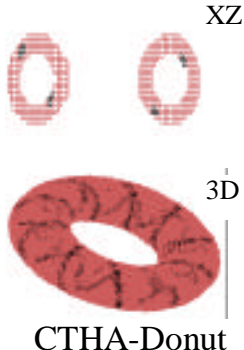

Base, Inner

Hollow

\section{Figure 5 Examples of the Mesh Created by the Generator}

Due to the generator's flexibility, new types of CTHAs or antennas can be easily defined and modeled based on existing geometric element classes. For example, a new CTHA-Array class can be derived from the above CTHA class through a combination of a certain number of CTHA objects in a certain geometric form.

Because of the refining work completed by the object instantiated from the class NodeArray, the quality of the mesh is significantly improved. The improvement is evidenced in the overall connectivity of the model, the improved smoothness under certain resolutions, and the lack of unwanted connections between two helixes.

It is good idea to use a linked list to store information about the nodes generated from geometric elements rather than an array, to save computer memory. 
Each geometric element is associated with its own object in the coordinate system to obtain modeling flexibility at the cost of more time for discretization since a great deal of coordinate transformation is needed. For the sake of modeling independent from the XFDTD, computation time is not as crucial as accuracy and flexibility.

Properties and methods of the classes

\begin{tabular}{|c|c|c|}
\hline Class Name & Properties or Methods & Description \\
\hline 1 Axis & $\begin{array}{l}\text { ReferAxis as Axis } \\
\text { Matrix(0 to 2, } 0 \text { to } 2) \text { as single } \\
\text { SetValue() } \\
\text { TransX() } \\
\text { TransY() } \\
\text { TransZ() } \\
\text { Move() } \\
\text { RotateByX() } \\
\text { RotateByY() } \\
\text { RotateByZ() }\end{array}$ & $\begin{array}{l}\text { Reference of the Axis } \\
\text { Position matrix } \\
\text { Initialize the Axis } \\
\text { Coordinate transformation of } \mathrm{X} \\
\text { Coordinate transformation of } \mathrm{Y} \\
\text { Coordinate transformation of } \mathrm{Z} \\
\text { Translation } \\
\text { Rotation around } \mathrm{X} \text { axis } \\
\text { Rotation around } \mathrm{Y} \text { axis } \\
\text { Rotation around } \mathrm{Z} \text { axis }\end{array}$ \\
\hline 2 NodeArray & $\begin{array}{l}\text { Resolution as single } \\
\text { Xlower as integer } \\
\text { Ylower as integer } \\
\text { Zlower as integer } \\
\text { Xupper as integer } \\
\text { Yupper as integer } \\
\text { Initialize() } \\
\text { LinkNodes() } \\
\text { SortBridgeNodes() } \\
\text { RefineNodes() } \\
\text { SaveNodes() }\end{array}$ & $\begin{array}{l}\text { Resolution of the mesh } \\
\text { Lower boundary of } \mathrm{X} \text { dimension } \\
\text { Lower boundary of } \mathrm{Y} \text { dimension } \\
\text { Lower boundary of } \mathrm{Z} \text { dimension } \\
\text { Upper boundary of } \mathrm{X} \text { dimension } \\
\text { Upper boundary of } \mathrm{Y} \text { dimension } \\
\text { Upper boundary of } \mathrm{Z} \text { dimension } \\
\text { Link the nodes with edge of approiate material } \\
\text { Add nodes that diminish the unconnectivity } \\
\text { Delete nodes to improve the smoothness } \\
\text { Save the information of nodes (dimension, } \\
\text { material) }\end{array}$ \\
\hline 3. Geometric Element (Common) & $\begin{array}{l}\text { EmbededAxis as Axis } \\
\text { Material as integer } \\
\text { Setvalue() } \\
\text { Discretize() }\end{array}$ & $\begin{array}{l}\text { Position information of the geometric element } \\
\text { Material type of the geometric element } \\
\text { Initialize the geometric element } \\
\text { Descretize the geometric element into mesh }\end{array}$ \\
\hline 3.1 Point & $\mathrm{X}, \mathrm{Y}, \mathrm{Z}$ as single & Project of point in $\mathrm{X}, \mathrm{Y}, \mathrm{Z}$ axis \\
\hline 3.2 Line & $\begin{array}{l}\text { Rx, Ry, Rz as single } \\
\text { Length as single }\end{array}$ & $\begin{array}{l}\text { Orientation of line referenced to } \mathrm{X} \text { axis } \\
\text { Length of the line }\end{array}$ \\
\hline 3.3 Plate & $\begin{array}{l}\mathrm{X} 1, \mathrm{Y} 1, \mathrm{Z} 1 \text { as single } \\
\mathrm{X} 2, \mathrm{Y} 2, \mathrm{Z} 2 \text { as single } \\
\text { Width as single }\end{array}$ & $\begin{array}{l}\text { Position of the first point } \\
\text { Position of the second point } \\
\text { Width of the plate }\end{array}$ \\
\hline 3.4 Rectangle & Length as single & Length of the rectangle \\
\hline
\end{tabular}




\begin{tabular}{|c|c|c|}
\hline & Width as single & Width of the rectangle \\
\hline 3.5 Box & $\begin{array}{l}\text { Length as single } \\
\text { Width as single } \\
\text { Heigth as single }\end{array}$ & $\begin{array}{l}\text { Legth of the box } \\
\text { Width of the box } \\
\text { Height of the box }\end{array}$ \\
\hline 3.6 Circle & $\begin{array}{l}\text { Radius as single } \\
\text { Innerradius as single } \\
\mathrm{Rx}, \mathrm{Ry}, \mathrm{Rz} \text { as single }\end{array}$ & $\begin{array}{l}\text { Radius of the circle } \\
\text { Radius of the inner circle } \\
\text { Orientation of the circle referenced to axes }\end{array}$ \\
\hline 3.7 Cylinder & $\begin{array}{l}\text { Radius as single } \\
\text { Innerradius as single } \\
\text { Heigth as single } \\
\mathrm{Rx}, \mathrm{Ry}, \mathrm{Rz} \text { as single }\end{array}$ & $\begin{array}{l}\text { Radius of the cylinder } \\
\text { Radius of the inner hollow cylinder } \\
\text { Heigth of the cylinder } \\
\text { Orientation of the cylinder referenced to axes }\end{array}$ \\
\hline 3.8 Sphere & $\begin{array}{l}\text { Radius as single } \\
\text { Innerradius as single } \\
\text { Height as single }\end{array}$ & $\begin{array}{l}\text { Radius of the sphere } \\
\text { Radius of the inner hollow } \\
\text { Height of the sphere }\end{array}$ \\
\hline 3.9 Donut & $\begin{array}{l}\text { Majorradius as single } \\
\text { Minorradius as single } \\
\text { Subminorradius as single }\end{array}$ & $\begin{array}{l}\text { Major radius of the donut } \\
\text { Minor radius of the donut } \\
\text { Sub minor radius of the donut }\end{array}$ \\
\hline 3.9 Helix & $\begin{array}{l}\text { Majorradius, Minorradius as } \\
\text { single } \\
\text { Helixradius as single } \\
\text { Feedposition as bool } \\
\text { Rings as integer } \\
\text { Direction as bool } \\
\text { Pti, ptf as Point }\end{array}$ & $\begin{array}{l}\text { Major and minor radius of the helix } \\
\text { Radius of the helix } \\
\text { Feed position } \\
\text { Wraps of the helix } \\
\text { Direction of the helix } \\
\text { Connecting points for feed }\end{array}$ \\
\hline 3.10 Bihelix & $\begin{array}{l}\text { Majorradius, Minorradius as } \\
\text { single } \\
\text { Helixradius as single } \\
\text { Helixconfiguration as bool } \\
\text { Xtraspace as integer } \\
\text { Feedposition as bool } \\
\text { Feedtype as integer } \\
\text { Rings as integer } \\
\text { PtA1, PtA2, PtB1, PtB2 as Point }\end{array}$ & $\begin{array}{l}\text { Major and minor radius of the helix } \\
\text { Radius of the helix } \\
\text { Helix configuration (Offset or Coaxial) } \\
\text { Extra space between two helix } \\
\text { Feed position } \\
\text { Feed type } \\
\text { Wraps of the helix } \\
\text { Connecting points for feed }\end{array}$ \\
\hline 3.11 Cylinder_CTHA & $\begin{array}{l}\text { Base as Cylinder } \\
\text { Mbihelix as Bihelix }\end{array}$ & $\begin{array}{l}\text { Base of CTHA } \\
\text { Bihelix of the CTHA }\end{array}$ \\
\hline 3.12 Donut_CTHA & $\begin{array}{l}\text { Base as Donut } \\
\text { Mbihelix as Bihelix }\end{array}$ & $\begin{array}{l}\text { Base of the CTHA } \\
\text { Bihelix of the CTHA }\end{array}$ \\
\hline 3.13 Feed & $\begin{array}{l}\text { Radius as single } \\
\text { Feedtype as integer, } \\
\text { PtA1, PtA2, PtB1, PtB2 as point }\end{array}$ & $\begin{array}{l}\text { Radius of the Feed } \\
\text { Feed type of the feed } \\
\text { Connecting points for the feed }\end{array}$ \\
\hline
\end{tabular}




\section{Center for Industrial Research Applications}

West Virginia University CTHA Internal Report Series 980325 August 12, 1998 Robert Craven

\section{CTHA Feed Study 1}

\section{Introduction}

Only a few feeds for the CTHA have been protected explicitly in the patents to date. This document is to teach why other feeds might be used and to promote intellectual protection for additional explicitly defined feeds and general protection for basic feed strategies as applied to the CTHA. This expansion of the CTHA could be considered analogous to different modes of traditional antennas. For instance, a simple linear helix can be designed to end-fire, that is radiate off the end of the helix predominately, or broadside fire. Similarly different modes of operation of the CTHA can be induced by geometric parameters or, as shown here, by different feed configurations.

Many factors must be considered when designing an antenna: the efficiency, the input impedance, the far-field radiation pattern, the polarization of the radiated energy, and the size and shape of the antenna. Different applications may stress different factors in the design process. The feed of the antenna gives the antenna designer an additional parameter to vary in trying to meet application-specific requirements.

The CTHA strengths lie in its short profile, which yields a nearly isotropic radiation pattern of predominately theta-polarized radiation. Not all communication tasks require this combination of characteristics, providing incentive to develop new characteristics by varying antenna parameters, including the feed. An antenna application might need phipolarized radiation, or it may be geometrically constrained into a vertical position but still need theta-polarized radiation. Note that even when the CTHA is in a vertical position (lollypop mode) it is still a compact, device that is smaller than traditional antennas such as a vertical loop antenna. 
In an application where cost is the dominating factor, like a disposable smart card, the need to obtain a $50 \mathrm{ohm}$ input impedance without the use of costly discrete components in a matching network may cause an antenna designer to sacrifice uniform radiation pattern or antenna efficiency in the quest for a naturally matched antenna. This case might occur for an in-room communication link connecting a portable device to a network via a wireless link. This document will show that a great deal of variety can be introduced both to the input impedance characteristics and to the polarization and radiation pattern of an antenna via the feed selection.

\section{Number of Possible Feeds}

To determine the number of possible feeds, a rigorous study has been conducted. Alternative methods of feeding a CTHA include the use of inductive loops, but the first examination concentrates on physical connections while noting that the other techniques can be applied alone or in conjunction with any of the physical connections to create a geometric expansion of the number of possible feeds.

The CTHA can have multiple sections, each with a potentially different feed, as in the four sections of the Quad-Contra configuration. For simplicity, this discussion is confined to a single (una) contrawound toroidal section while noting that the multiple section embodiments increase the possible feed configurations exponentially and of course should be protected as well.

A single section CTHA has four wire ends, each of which can be left alone, connected to another wire end, and/or connected to one of the two radio feed lines. Conversely, each of the two lines from the radio can be connected to a wire end, a group of wire ends, or to something completely different like a ground plane, reflector, or inductively coupled loop, or left unconnected.

Figure 6 shows the wire ends for two different CTHA antennas, the top one having "polar" crossings, i.e. top and bottom, while the bottom CTHA, which is shown in profile, has equatorial crossings. In either case a single wire, illustrated in blue or green, can be seen with both ends in the feed area. The blue wire has ends B and C while the green wire has ends marked A and D. The typical feed, as predominately practiced, connects $\mathrm{A}$ and $\mathrm{C}$ together with one of the two feed lines and $\mathrm{B}$ and $\mathrm{D}$ together with the other feed line. This configuration imparts contra-currents on the two helices; these add 
together to form a pseudo poloidal current, thereby reinforcing the loop magnetic flux. This arrangement is good for producing vertically polarized energy from a predominately horizontal structure.

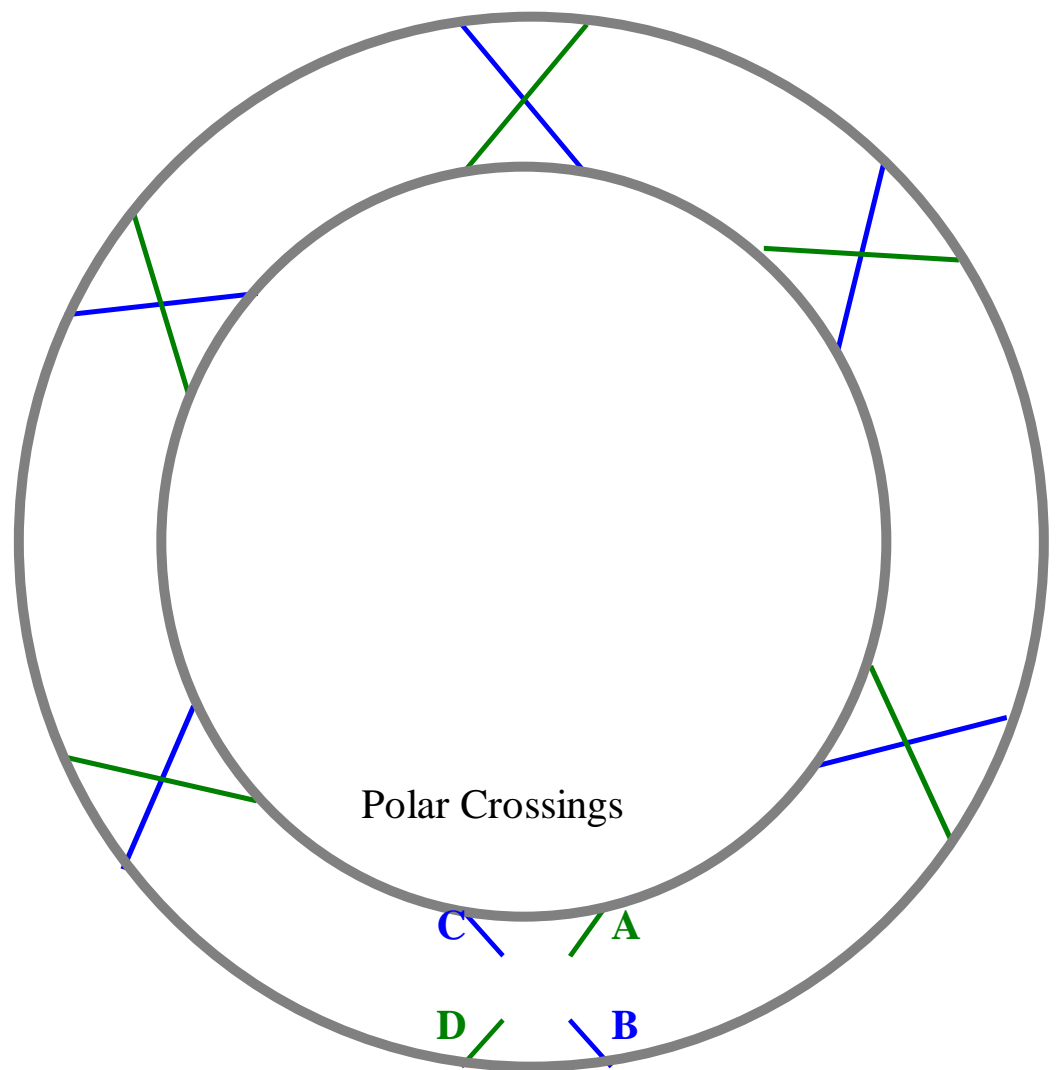

Equatorial Crossings

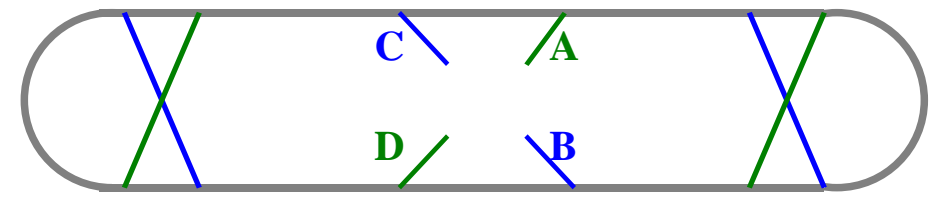

Figure 6 Wiring diagram for CTHA with either polar or equatorial crossings.

A computer program was written to explore the possible connections, and the concept of terminals was introduced to facilitate the algorithm. In actuality, terminals are not necessarily part of the CTHA but are used here as a means of logically describing connections. Four terminals are employed: terminals 1 and 2 represent the two feed lines and terminals 3 and 4 represent a means of connecting multiple wire ends that are not fed. Each of the four wire ends can therefore have five possible values: 0 for no connection or 1-4 indicating a terminal connection. Additional rules were added to the computer 
system to remove redundant and symmetrical configurations. While this procedure is not the only means for determining all possible configurations, it is sufficiently rigorous to ensure all configurations will be found. Note the following rules:

Operation Description

1.

If terminal 3 or 4 has a wire

connected to it, it must have more than one wire connected to it or it is redundant to a configuration with no connection.

2.

Terminals 3 and 4 are interchangeable (no logical difference).

3. interchangeable.

Terminals 1 and 2 are

4. for $\mathrm{C}$ and $\mathrm{D} .(\mathrm{A}<->\mathrm{C}, \mathrm{B}<->\mathrm{D})$ Symmetry

Wire ends A and B can be swapped

5. for $\mathrm{B}$ and $\mathrm{C}(\mathrm{A}<->\mathrm{B}, \mathrm{C}<->\mathrm{D})$ Symmetry

Wire ends A and D can be swapped

6.

Wire ends A and B can be swapped for $\mathrm{D}$ and $\mathrm{C}$. $(\mathrm{A}<->\mathrm{D}, \mathrm{B}<->\mathrm{C})$ (same as performing operation 4 and then operation 5) Symmetry

It is also necessary to perform combinations of these symmetry rules to remove all redundant configurations.

\section{Results}

Table 8 shows the effect of removing redundant feed configurations by applying successive symmetry rules. The program yields 35 physical ways to connect a pair of feed lines to four wire ends. Recall that these can be diversified by choosing multiple segment CTHAs or by using inductive loops, reflectors, ground planes, etc., in combination with the 35 direct feed configurations.

Table 8 Symmetry rules for unique feed finding algorithm

\begin{tabular}{|lc|}
\hline Operation & Combinations \\
\hline $\begin{array}{l}\text { Rule 1 (R1) } \\
\text { Rule 2, Rule 3, and Rule 2 then Rule 3 }\end{array}$ & 625 \\
(R2,R3,R2-R3) & 83 \\
R4, R4-R2, R4-R3, R4-R2-R3 & 51 \\
R5, R5-R2, R5-R3, R5-R2-R3 & 46 \\
R6, R6-R2, R6-R3, R6-R2-R3 & 35 \\
\hline
\end{tabular}


Table 9 breaks the 35 feed configurations into three main groups: two connection feeds, one connection feeds, and no physical connection feeds. The last category relies completely on alternative feed techniques, including inductive loops or antenna coupling of passive elements in an array, etc.

Table 9 Wire end terminal connections for various CTHA feeds

\begin{tabular}{|c|c|c|c|c|}
\hline \multicolumn{5}{|c|}{ Two Connections } \\
\hline \multicolumn{5}{|l|}{ Feed } \\
\hline & $\mathbf{A}$ & B & C & $\mathbf{D}$ \\
\hline 1 & 3 & 2 & 3 & 1 \\
\hline 2 & 1 & 0 & 0 & 1 \\
\hline 3 & 0 & 1 & 0 & 2 \\
\hline 4 & 2 & 1 & 0 & 0 \\
\hline 5 & 1 & 2 & 3 & 3 \\
\hline 6 & 2 & 1 & 2 & 1 \\
\hline 7 & 2 & 2 & 1 & 1 \\
\hline 8 & 2 & 3 & 3 & 1 \\
\hline 9 & 1 & 2 & 2 & 1 \\
\hline 10 & 2 & 2 & 0 & 1 \\
\hline 11 & 2 & 1 & 0 & 1 \\
\hline 12 & 1 & 2 & 9 & 2 \\
\hline 13 & 2 & 1 & 1 & 1 \\
\hline \multicolumn{5}{|c|}{ One Connection } \\
\hline \multicolumn{5}{|c|}{ Wire } \\
\hline & $\mathbf{A}$ & B & C & $\mathbf{D}$ \\
\hline 14 & 1 & 0 & 0 & 0 \\
\hline 15 & 1 & 1 & 0 & 0 \\
\hline 16 & 1 & 0 & 1 & 0 \\
\hline 17 & 0 & 1 & 1 & 0 \\
\hline 18 & 1 & 1 & 1 & 0 \\
\hline 19 & 3 & 3 & 1 & 0 \\
\hline 20 & 3 & 1 & 3 & 0 \\
\hline 21 & 1 & 3 & 3 & 0 \\
\hline 22 & 1 & 1 & 1 & 1 \\
\hline 23 & 3 & 3 & 1 & 1 \\
\hline 24 & 3 & 1 & 3 & 1 \\
\hline 25 & 1 & 3 & 3 & 1 \\
\hline 26 & 3 & 3 & 3 & 1 \\
\hline \multicolumn{5}{|c|}{ No Connections } \\
\hline Feed & & re & & \\
\hline & $\mathbf{A}$ & B & C & $\mathbf{D}$ \\
\hline 27 & 0 & 0 & 0 & 0 \\
\hline 28 & 3 & 3 & 0 & 0 \\
\hline 29 & 3 & 0 & 3 & 0 \\
\hline
\end{tabular}




\begin{tabular}{|lllll|}
30 & 0 & 3 & 3 & 0 \\
31 & 3 & 3 & 3 & 0 \\
32 & 3 & 3 & 3 & 3 \\
33 & 4 & 4 & 3 & 3 \\
34 & 4 & 3 & 4 & 3 \\
35 & 3 & 4 & 4 & 3 \\
\hline
\end{tabular}



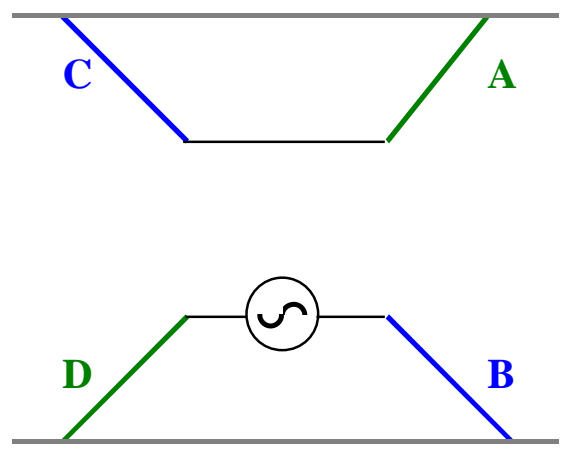

Feed 1
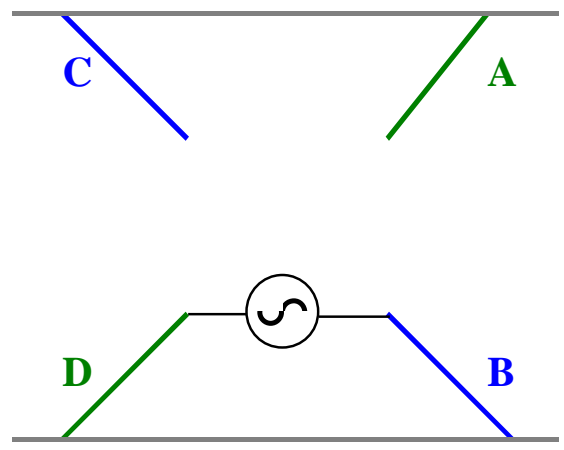

Feed 3

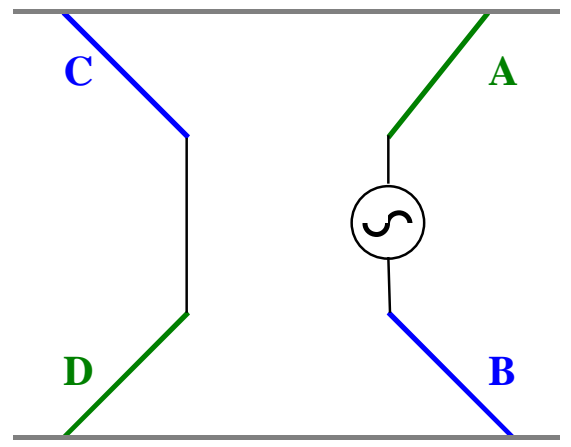

Feed 5

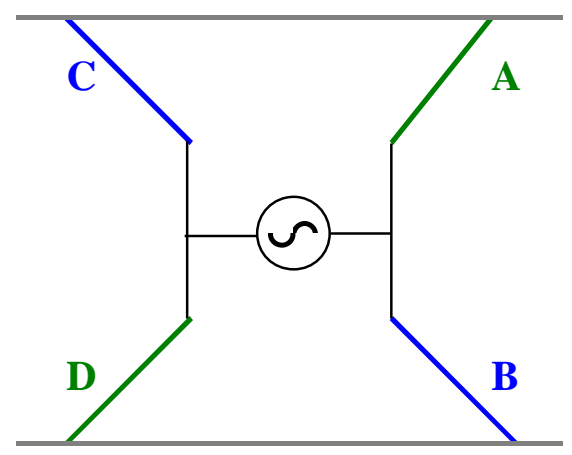

Feed 7

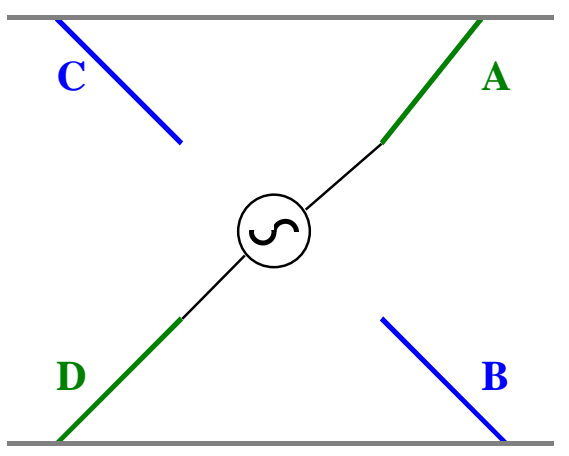

Feed 2

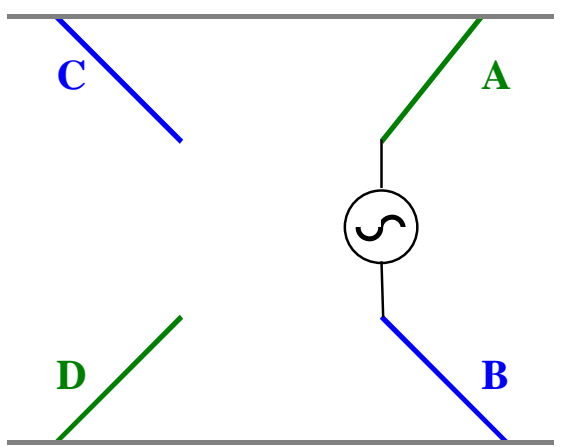

Feed 4

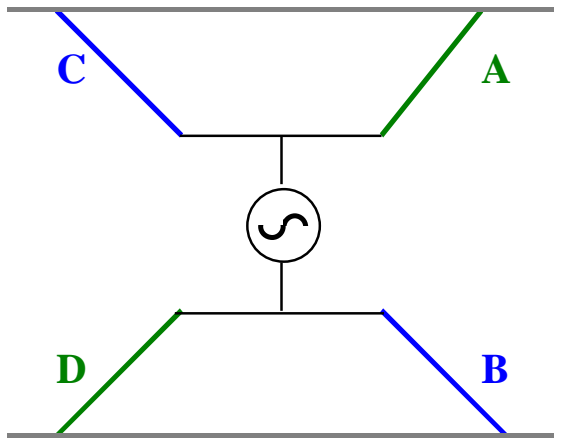

Feed 6

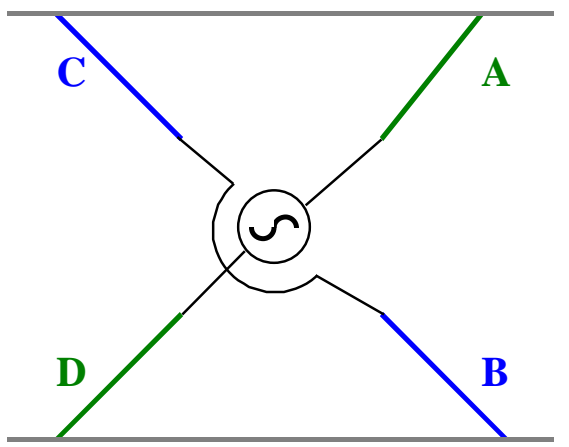

Feed 8 


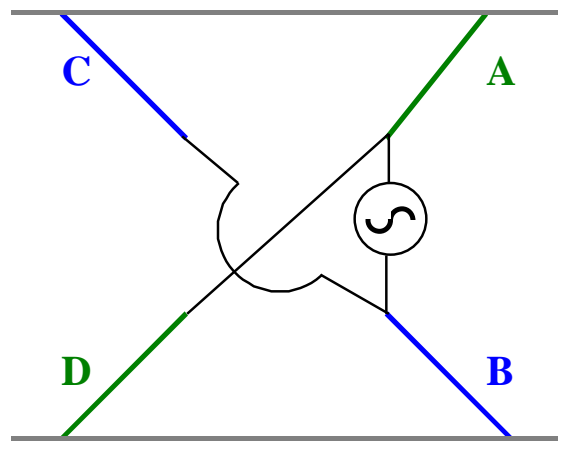

Feed 9

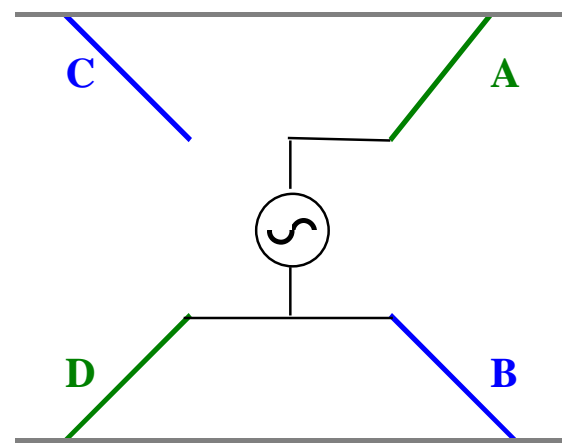

Feed 11

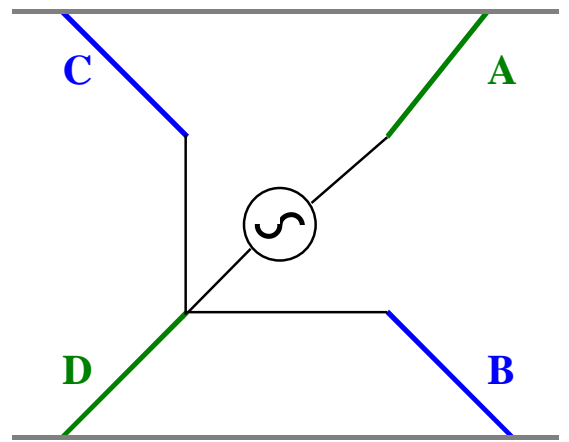

Feed 13

Figure 7 CTHA feeds employing two feed lines.

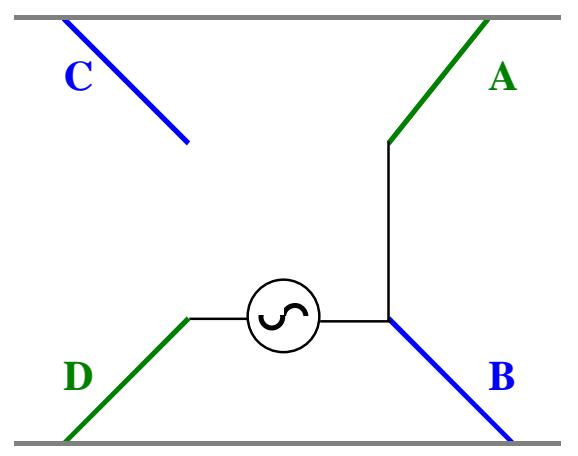

Feed 10

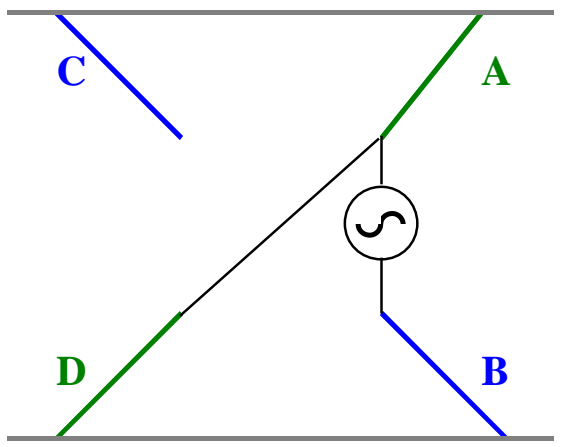

Feed 12 


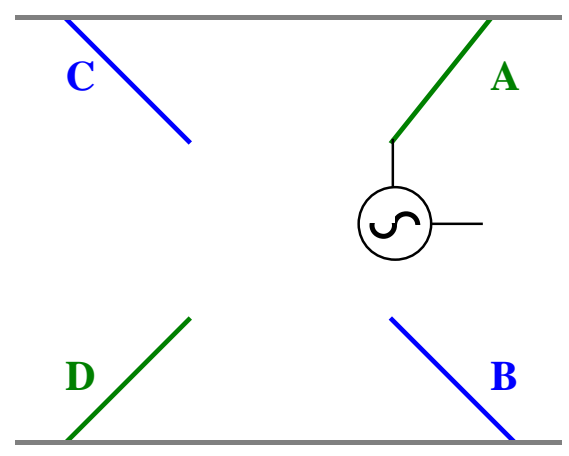

Feed 14

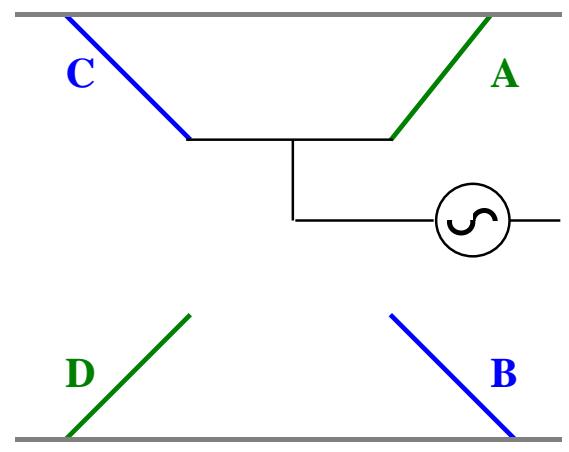

Feed 16

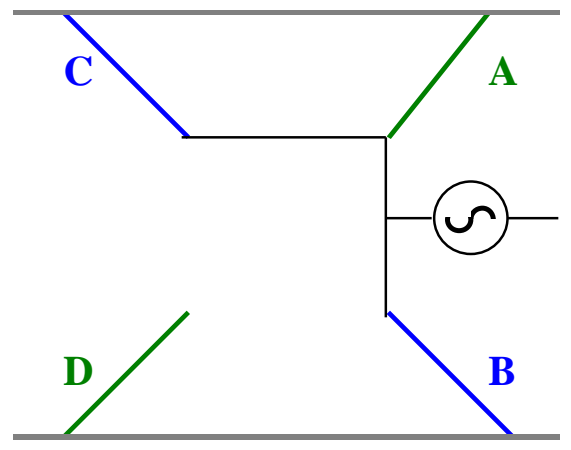

Feed 18
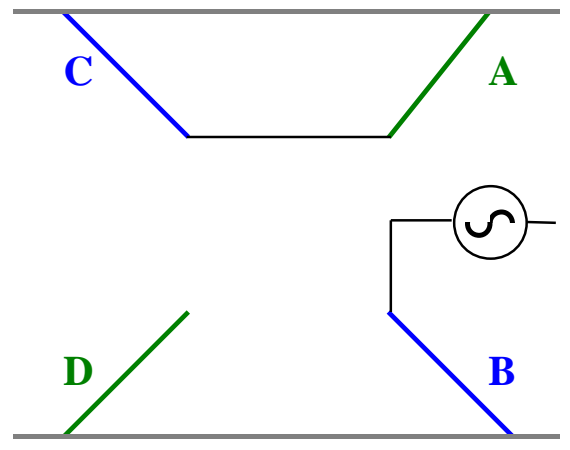

Feed 20

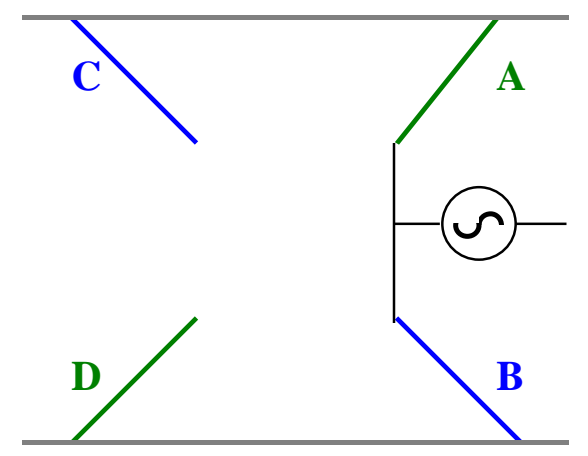

Feed 15

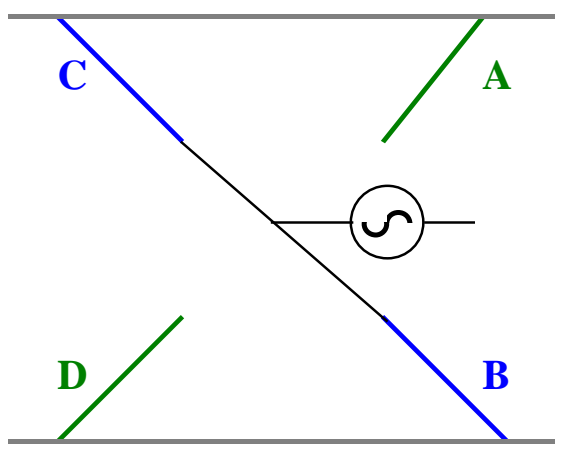

Feed 17

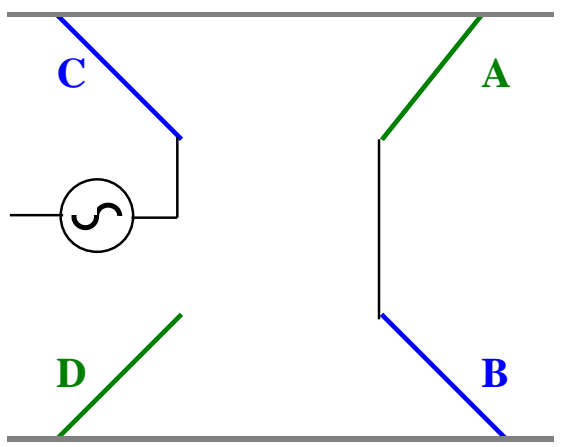

Feed 19

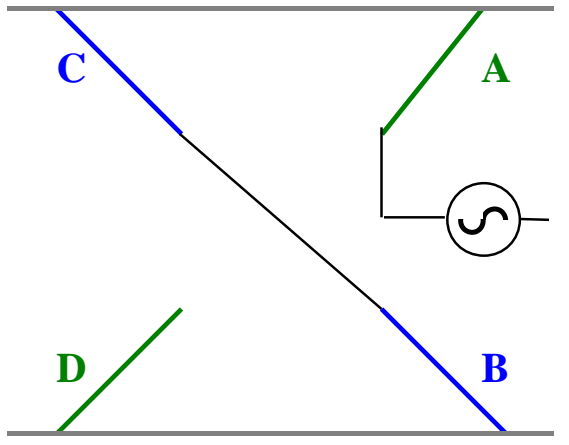

Feed 21 


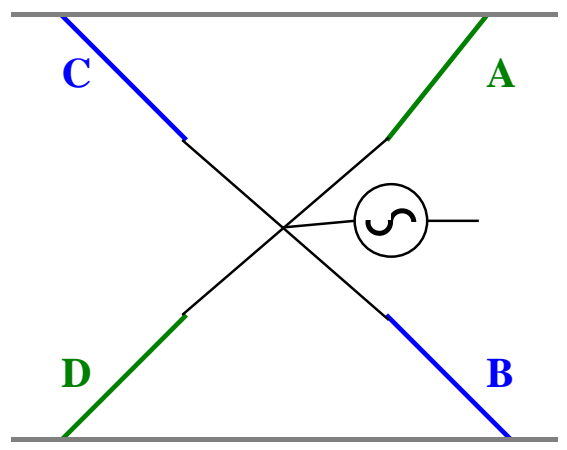

Feed 22

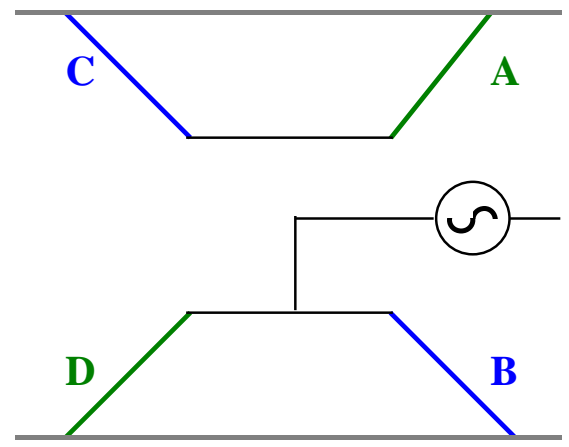

Feed 24

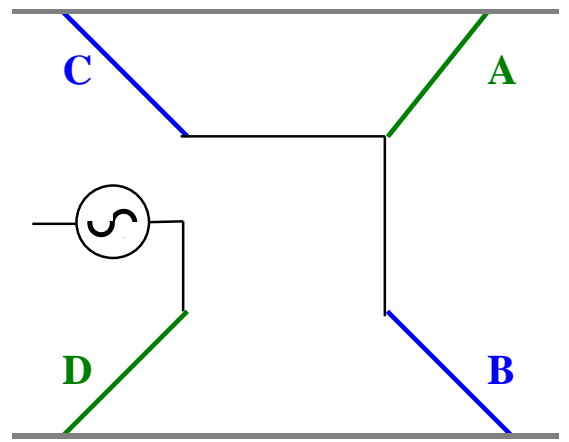

Feed 26

Figure 8 CTHA feeds employing only one direct feed connection.

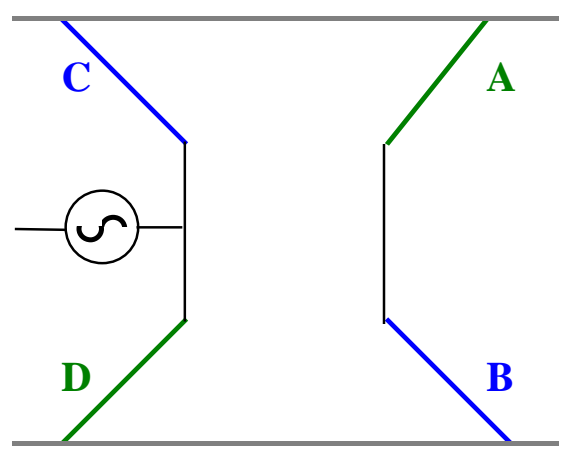

Feed 23

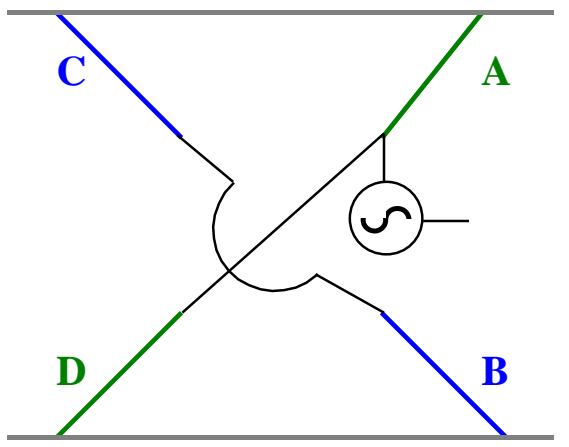

Feed 25 


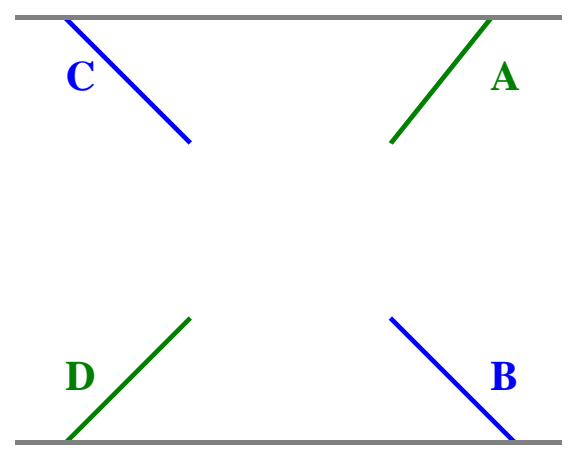

Feed 27

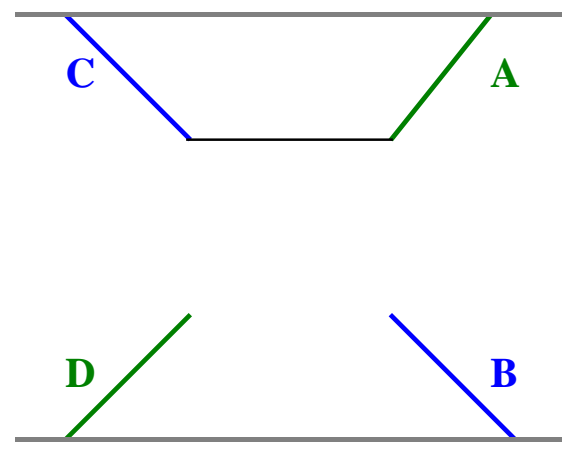

Feed 29

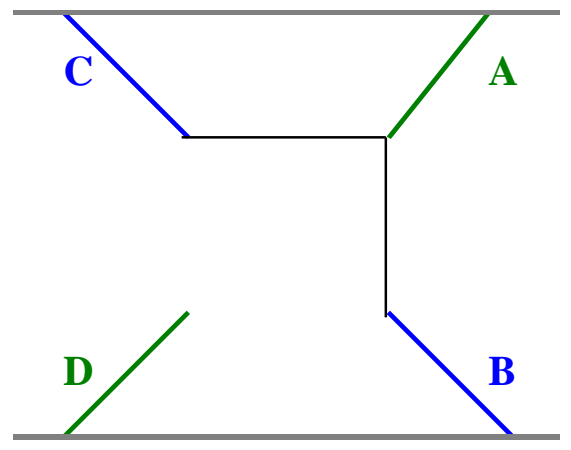

Feed 31

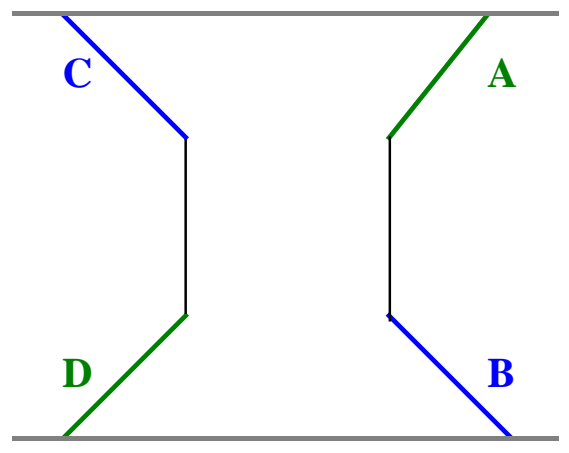

Feed 33

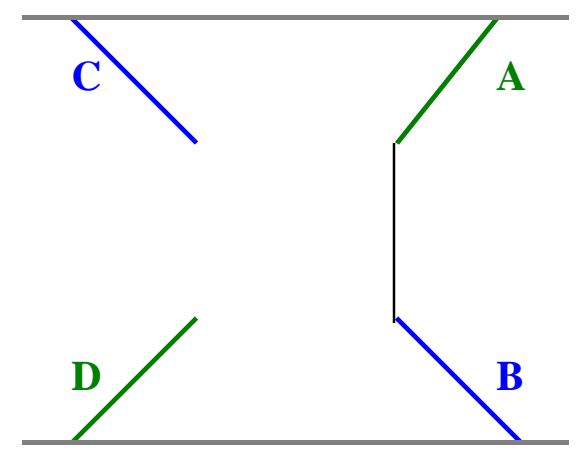

Feed 28

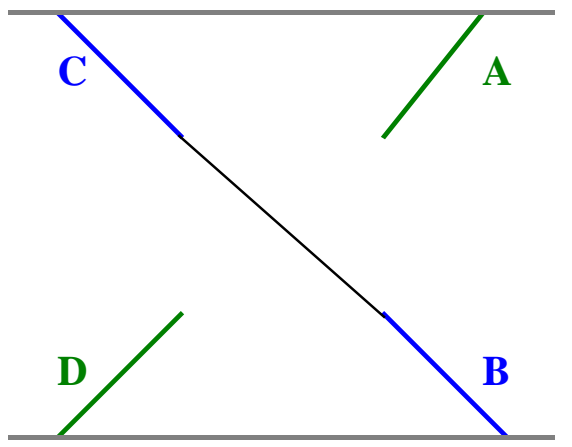

Feed 30

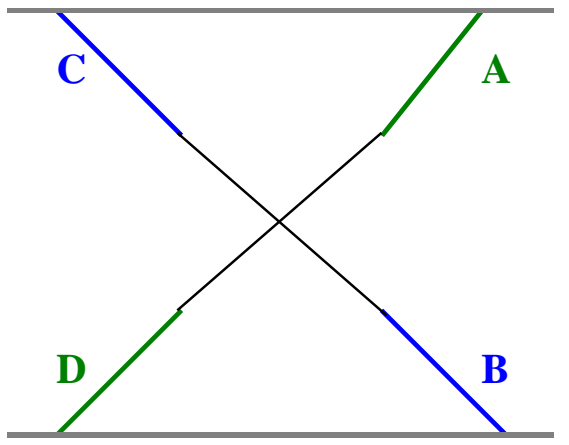

Feed 32
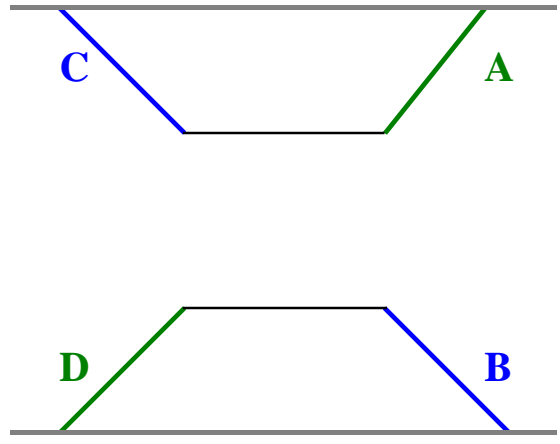

Feed 34 


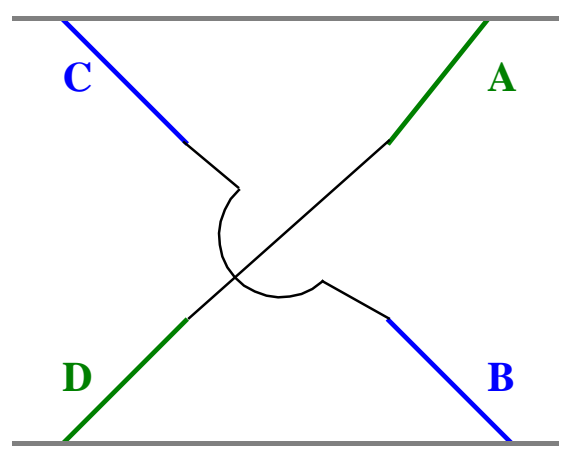

Feed 35

Figure 9 CTHA feeds employing no direct feed connections. 